\title{
Cadre d'évaluation et d'analyse du cycle PISA 2012
}

COMPÉTENCES EN MATHÉMATIQUES, EN COMPRÉHENSION DE L'ÉCRIT, EN SCIENCES, EN RÉSOLUTION DE PROBLĖMES ET EN MATIĖRES FINANCIÈRES

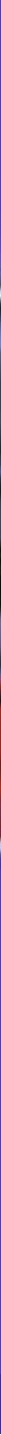

Q $1>O C D E$ 

PISA

\title{
Cadre d'évaluation et d'analyse du cycle PISA 2012
}

\author{
COMPÉTENCES EN MATHÉMATIQUES, \\ EN COMPRÉHENSION DE L'ÉCRIT, EN SCIENCES, \\ EN RÉSOLUTION DE PROBLÈMES \\ ET EN MATIÈRES FINANCIÈRES
}


Cet ouvrage est publié sous la responsabilité du Secrétaire général de l'OCDE. Les opinions et les interprétations exprimées ne reflètent pas nécessairement les vues de l'OCDE ou des gouvernements de ses pays membres.

Ce document et toute carte qu'il peut comprendre sont sans préjudice du statut de tout territoire, de la souveraineté s'exerçant sur ce dernier, du tracé des frontières et limites internationales, et du nom de tout territoire, ville ou région.

\section{Merci de citer cet ouvrage comme suit :}

OCDE (2013), Cadre d'évaluation et d'analyse du cycle PISA 2012 : Compétences en mathématiques, en compréhension de l'écrit, en sciences, en résolution de problèmes et en matières financières, Éditions OCDE. http://dx.doi.org/10.1787/9789264190559-fr

ISBN 978-92-64-19054-2 (imprimé)

ISBN 978-92-64-19055-9 (PDF)

Les données statistiques concernant Israël sont fournies par et sous la responsabilité des autorités israéliennes compétentes. L'utilisation de ces données par l'OCDE est sans préjudice du statut des hauteurs du Golan, de Jérusalem-Est et des colonies de peuplement israéliennes en Cisjordanie aux termes du droit international.

\section{Crédits photo:}

() khoa vu/Flickr/Getty Images

(c) Shutterstock/Kzenon

(c) Simon Jarratt/Corbis

C OCDE 2013

Vous êtes autorisés à copier, télécharger ou imprimer du contenu OCDE pour votre utilisation personnelle. Vous pouvez inclure des extraits des publications, des bases de données et produits multimédia de l'OCDE dans vos documents, présentations, blogs, sites Internet et matériel d'enseignement, sous réserve de faire mention de la source OCDE et du copyright. Les demandes pour usage public ou commercial ou de traduction devront être adressées à rights@oecd.org. Les demandes d'autorisation de photocopier une partie de ce contenu à des fins publiques ou commerciales peuvent être obtenues auprès du Copyright Clearance Center (CCC) info@copyright.com ou du Centre français d'exploitation du droit de copie (CFC) contact@cfcopies.com. 


\section{Avant-propos}

Créé en 1997, le programme international de l'OCDE pour le suivi des acquis des élèves (PISA) est l'expression concrète de l'engagement des gouvernements à suivre l'évolution des résultats produits par leur système d'éducation, en l'occurrence en évaluant la performance des élèves dans un cadre commun approuvé à l'échelle internationale. L'enquête PISA est avant tout le fruit d'un effort concerté qui met en synergie l'expertise scientifique des pays participants, et que les gouvernements de ces pays dirigent conjointement en fonction de préoccupations communes en matière d'action publique.

Les pays participants assument la responsabilité politique du projet. Des experts des pays participants siègent au sein de groupes de travail chargés de mettre au service des objectifs de l'enquête PISA les meilleures compétences techniques disponibles dans le domaine des évaluations comparatives internationales. En contribuant aux travaux de ces groupes d'experts, les pays garantissent la validité internationale des épreuves d'évaluation et la prise en considération des contextes culturels et éducatifs des pays membres de l'OCDE. Ils veillent aussi à ce que ces épreuves constituent d'excellents instruments de mesure, valides et conçus dans un souci d'authenticité pour les élèves.

Le cycle PISA 2012 poursuit la mise en œuvre de la stratégie adoptée en 1997 par les pays de l'OCDE en matière de collecte et d'exploitation des données. La culture mathématique est, comme en 2003, le domaine majeur d'évaluation, mais son cadre conceptuel a été totalement remanié et inclut désormais les trois processus mathématiques à la base de l'évolution du compte rendu des résultats PISA en mathématiques. Le cycle PISA 2012 a également inclus une épreuve informatisée de résolution de problèmes. Le cadre d'évaluation de la compréhension de l'écrit, révisé lors du cycle PISA 2009, est resté inchangé lors du cycle PISA 2012. Le cadre d'évaluation de la culture scientifique, élaboré en vue du cycle PISA 2006, est lui aussi resté inchangé lors du cycle PISA 2012. Le cadre d'analyse à la base de l'élaboration des différents questionnaires a été revu en prévision du cycle PISA 2012.

Le cycle PISA 2012 a vu l'inclusion d'une évaluation des compétences en résolution de problèmes et en matière financière. La résolution de problèmes a été retenue comme domaine d'évaluation PISA en 2003, mais pas en 2006 et en 2009. Toutefois, un nouveau cadre d'évaluation des compétences en résolution de problèmes a été élaboré en vue du cycle PISA 2012, et de nouvelles méthodes d'évaluation ont été appliquées, permettant entre autres l'enregistrement du cheminement des élèves en temps réel. Les épreuves de résolution de problèmes ont été administrées de manière informatisée lors du cycle PISA 2012, et les interactivités avec les élèves ont été placées au cœur de l'évaluation. La culture financière a été incluse pour la première fois dans les épreuves PISA. Son cadre d'évaluation propose un langage commun pour débattre de la culture financière, une définition opérationnelle du domaine et un plan structuré pour concevoir les items, et définit les contenus, processus et contextes à retenir pour évaluer les compétences des élèves de 15 ans dans ce domaine.

Cette publication expose les principes directeurs du cycle d'évaluation PISA 2012 et définit les connaissances que les élèves doivent acquérir, les processus qu'ils doivent appliquer et les contextes dans lesquels leurs savoirs et savoir-faire seront évalués. Elle illustre, par ailleurs, les domaines d'évaluation par des exemples de tâches.

Le cadre d'évaluation de la culture mathématique a été élaboré conjointement par l'Australian Council for Educational Research (ACER) et une organisation de recherche pédagogique basée aux États-Unis, Achieve Inc. Le cadre d'évaluation de tous les autres domaines cognitifs et le cadre conceptuel des questionnaires contextuels ont été élaborés par I'Australian Council for Educational Research. 
Les cadres d'évaluation sont le fruit des travaux menés par des panels d'experts sous la direction de Raymond Adams, Barry McCrae, Petra Lietz, Juliette Mendelovits, Dara Ramalingam et Ross Turner (ACER). Le groupe d'experts en charge de la culture mathématique est présidé par Kaye Stacey, de I'Université de Melbourne (Australie), celui en charge de la résolution de problèmes, par Joachim Funke, de I'Université d'Heidelberg (Allemagne), celui en charge de la compréhension de l'écrit, par Irwin Kirsch, de l'Educational Testing Service (États-Unis), celui en charge de la culture scientifique, par Rodger Bybee, auparavant du Biological Science Curriculum Study (États-Unis), celui en charge de la culture financière, par Annamaria Lusardi, de la School of Business de l'Université George Washington (États-Unis) et, enfin, celui en charge des questionnaires, par Eckhard Klieme, de l'Institut allemand de recherche pédagogique internationale (DIPF) (Allemagne). La liste des membres des groupes d'experts figure à l'annexe B. Les cadres d'évaluation ont également été passés en revue par des panels d'experts dans chacun des pays participants. Les chapitres ont été rédigés par leur groupe d'experts respectif sous la direction de leur président. Cette publication a été préparée par le Secrétariat de I'OCDE, en particulier par Michael Davidson, Sophie Vayssettes, Pablo Zoido, Giannina Rech, Elisabeth Villoutreix, Marilyn Achiron, Marika Boiron et Elizabeth Del Bourgo.

Le présent rapport est publié sous la responsabilité du Secrétaire général de l'OCDE. 


\section{Table des matières}

\section{INTRODUCTION}

Vue d'ensemble

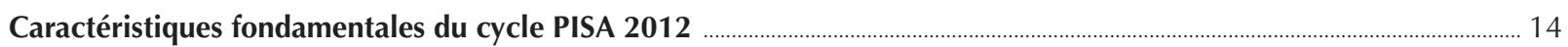

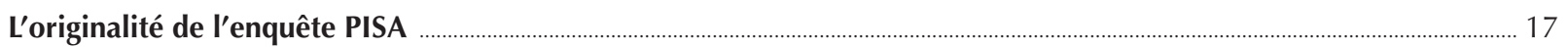

Aperçu des aspects évalués dans chaque domaines ………………………........................................................................................... 18

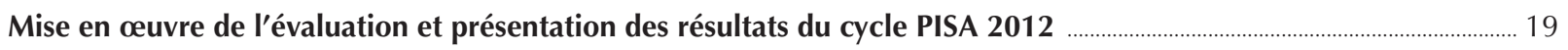

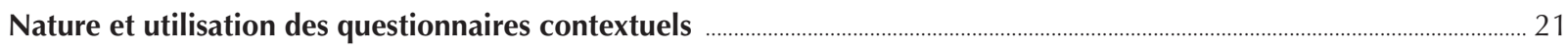

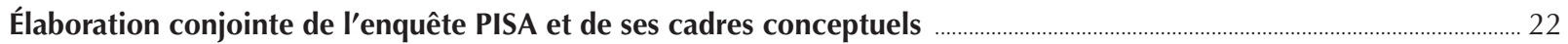

CHAPITRE 1 CADRE D'ÉVALUATION DE LA CULTURE MATHÉMATIQUE DU CYCLE PISA 2012 ................................ 25

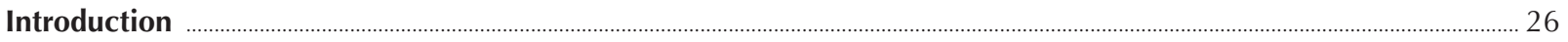

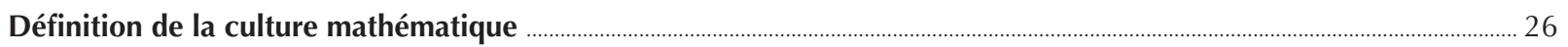

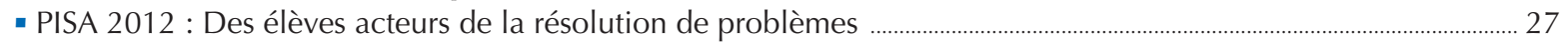

- Un lien explicite vers un éventail de contextes dans les problèmes du cycle PISA 2012 ................................................ 29

- Le rôle visible des outils mathématiques, y compris de la technologie, dans le cycle PISA 2012 ................................................ 29

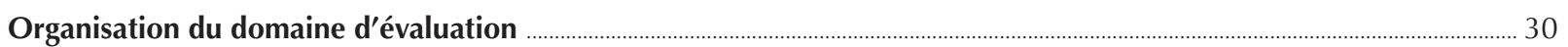

- Les processus mathématiques et les facultés mathématiques qui les sous-tendent ........................................................... 30

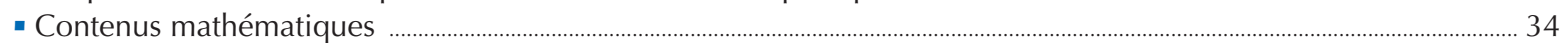

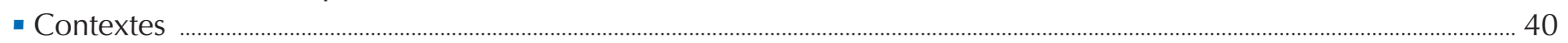

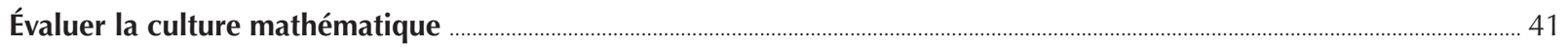

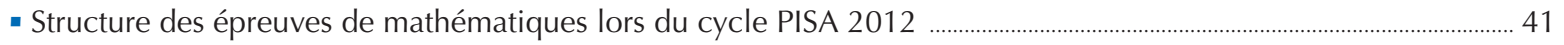

- Présentation du niveau de compétence en mathématiques ………………………....................................................................... 45

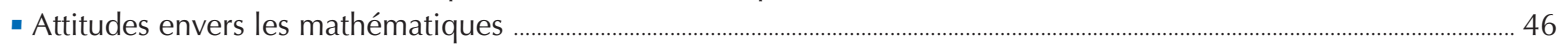

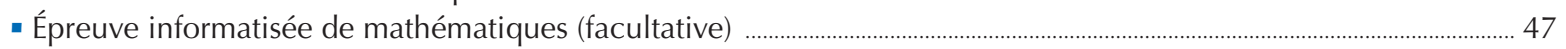

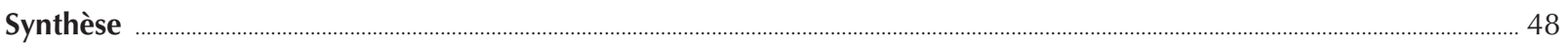

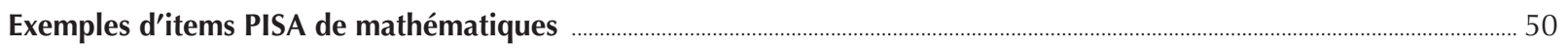

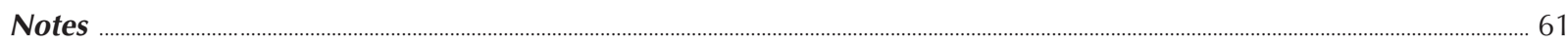

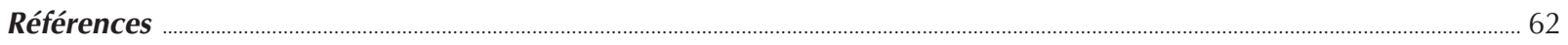

CHAPITRE 2 CADRE D'ÉVALUATION DE LA COMPRÉHENSION DE L'ÉCRIT DU CYCLE PISA 2012 ......................... 63

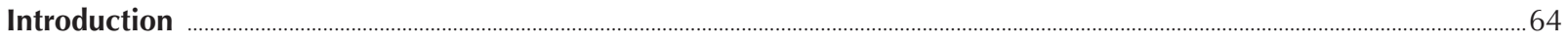

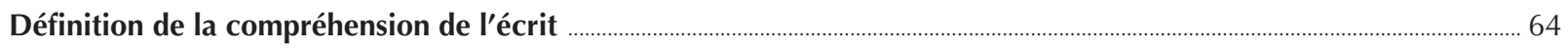

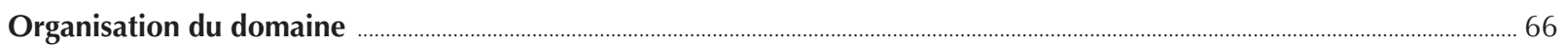

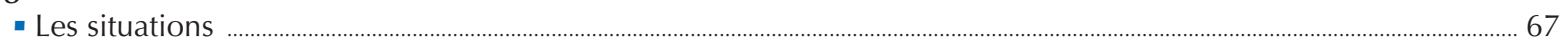

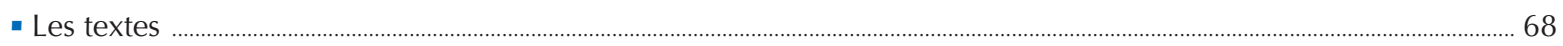

- Synthèse de la relation entre les tâches et les textes dans les épreuves papier-crayon

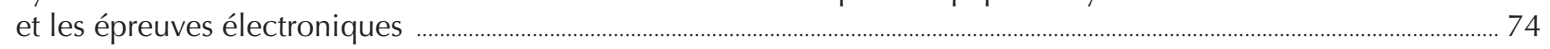

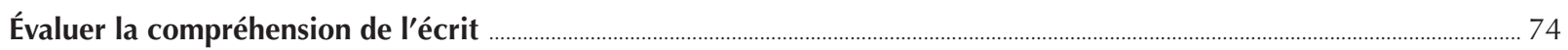

- Conception des tâches des épreuves papier-crayon ........................................................................................................................ 74

- Conception des tâches des épreuves électroniques .......................................................................................................................... 77

Exemples d'items PISA de compréhension de l'écrit électronique …………………......................................................................... 78

Présentation des résultats en compréhension de l'écrit sur papier et de l'écrit électronique ............................................ 84

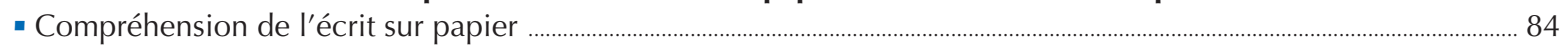

- Compréhension de l'écrit électronique ........................................................................................................................................................... 86 
Synthèse

Exemples d'items PISA en compréhension de l'écrit sur papier

Notes

Références

CHAPITRE 3 CADRE D'ÉVALUATION DE LA CULTURE SCIENTIFIQUE DU CYCLE PISA 2012

Introduction

Définition de la culture scientifique

- Explication de la définition

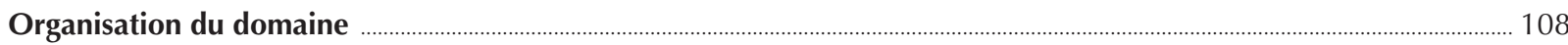

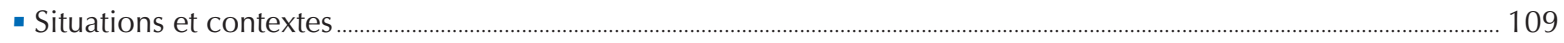

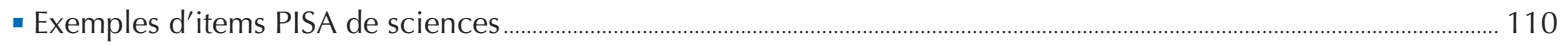

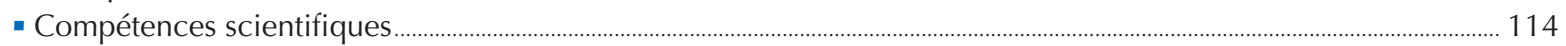

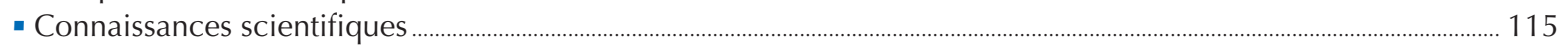

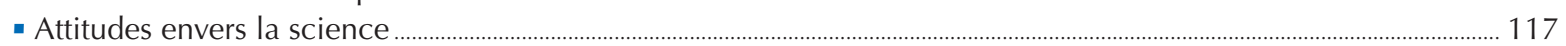

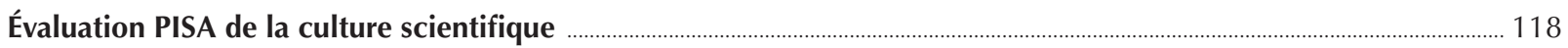

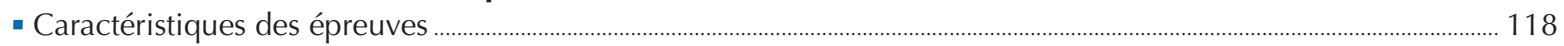

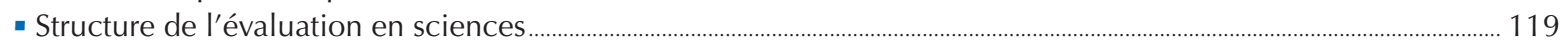

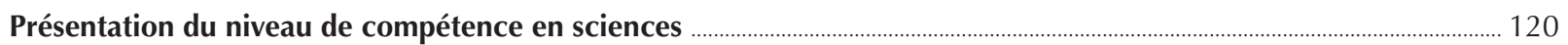

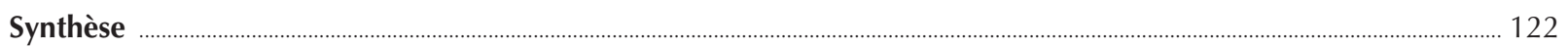

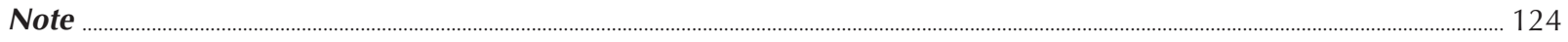

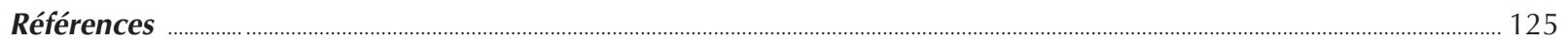

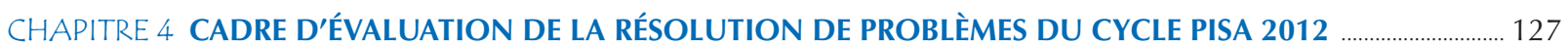

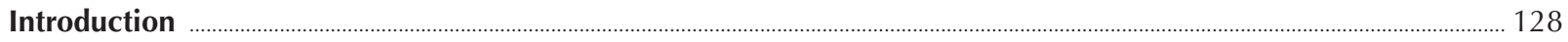

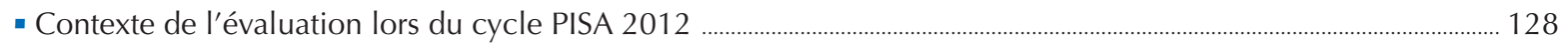

- La résolution de problèmes dans le programme de l'OCDE d'évaluation des compétences des adultes .................... 129

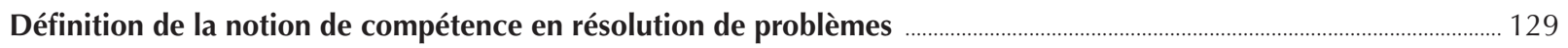

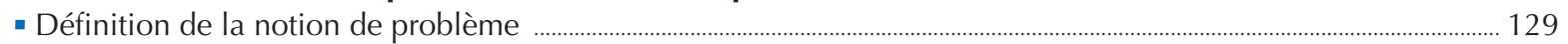

- Définition de la notion de résolution de problèmes ........................................................................................................................... 130

- Définition de la notion de compétence en résolution de problèmes ……….......................................................................... 130

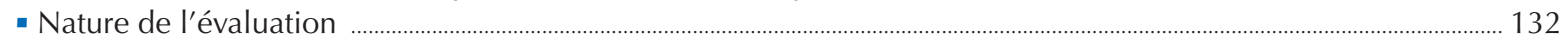

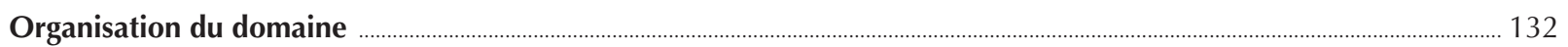

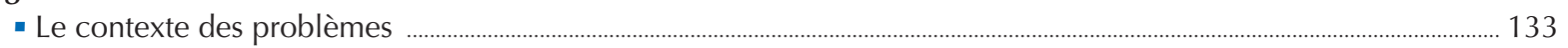

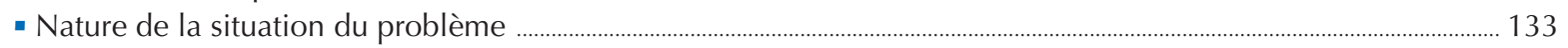

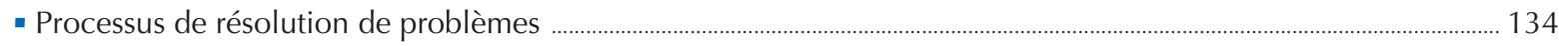

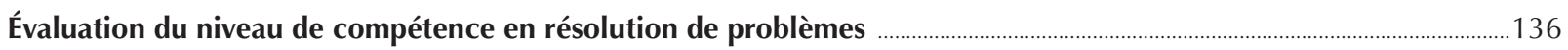

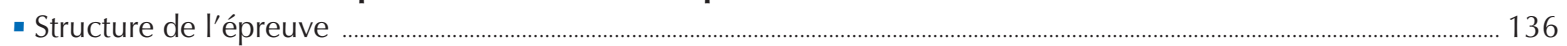

- Fonctionnalité de l'informatisation de l'épreuve ............................................................................................................................. 136

- Caractéristiques de l'épreuve et difficulté des items .................................................................................................................... 136

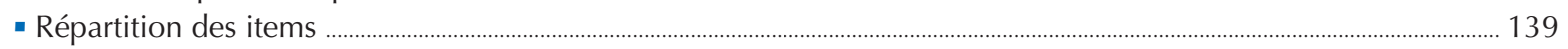

Présentation du niveau de compétence en résolution de problèmes ……………….............................................................. 140

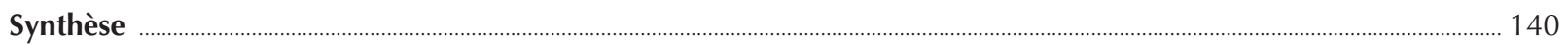

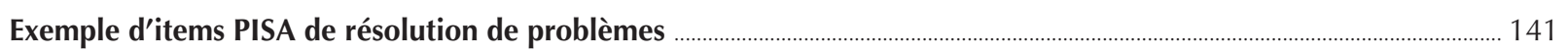

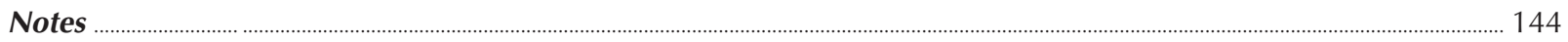

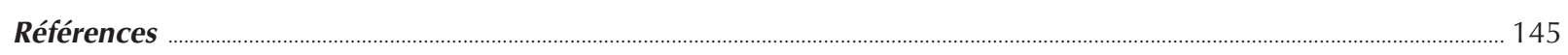

CHAPITRE 5 CADRE D'ÉVALUATION DE LA CULTURE FINANCIÈRE DU CYCLE PISA $2012 \ldots$

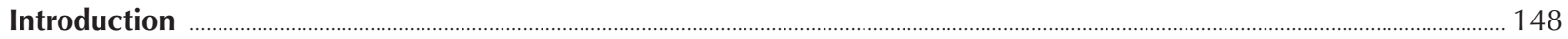

- L'importance de la culture financière ………......................................................................................................................... 148

- Avantages à attendre de l'éducation financière et de l'amélioration du niveau de culture financière ..................... 149

- Activités de l'OCDE en matière d'éducation financière ....................................................................................................... 149 
- Éducation financière des jeunes en milieu scolaire

- La nécessité de recueillir des données

- L'évaluation de la culture financière dans l'enquête PISA

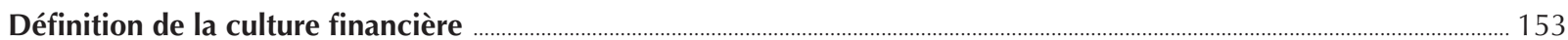

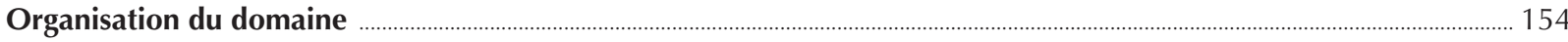

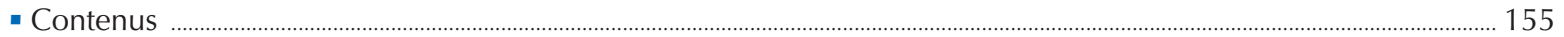

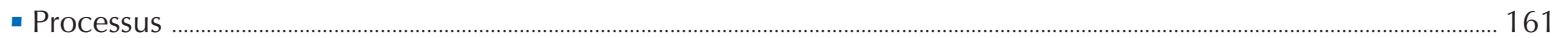

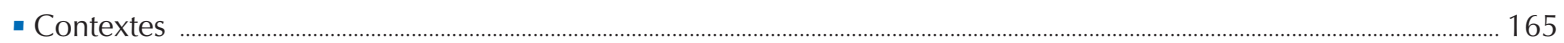

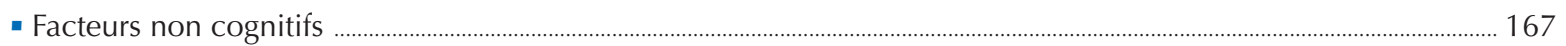

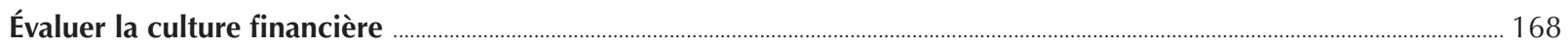

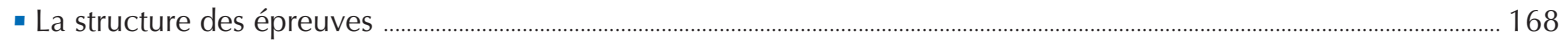

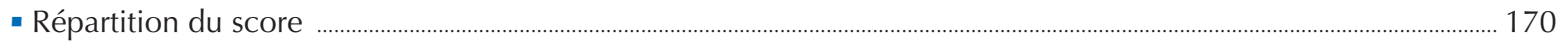

- L'impact des connaissances et compétences dans d'autres domaines sur la culture financière .................................... 171

- Recueillir des données à propos des expériences et des comportements dans le domaine financier ...................... 172

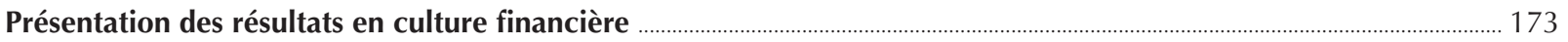

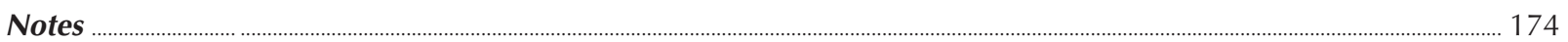

Références

CHAPITRE 6 CADRE CONCEPTUEL DES QUESTIONNAIRES CONTEXTUELS DU CYCLE PISA $2012 \ldots 1 . \ldots \ldots \ldots \ldots \ldots \ldots \ldots \ldots . . . . . . . . . .177$

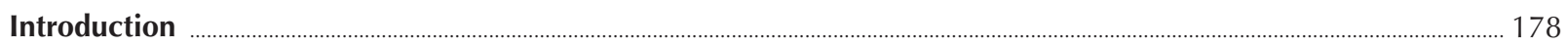

L'objectif général de l'enquête PISA et sa pertinence pour l'action publique ……........................................................... 179

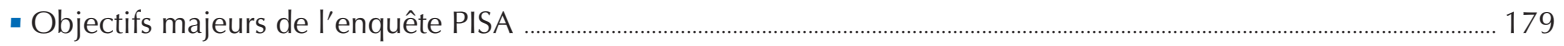

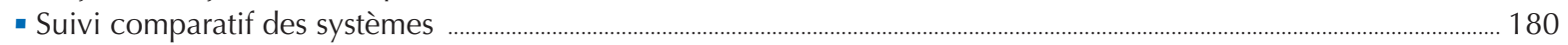

- Comprendre les modèles d'efficience dans les systèmes d'éducation ............................................................................... 181

- Créer une base de données durable au service des recherches pertinentes pour l'action publique .......................... 183

- Priorité à l'équité ........................................................................................................................................................................................ 184

La base de connaissances générales : La recherche sur l'efficience de l'éducation .............................................................. 184

- Un certain nombre de résultats de l'éducation et de variables prédictives .......................................................................... 184

- Intrants et processus à l'échelle des systèmes .......................................................................................................................... 187

- Intrants et processus à l'échelle des établissements d'enseignement .................................................................................... 189

- Intrants et processus à l'échelle des classes .............................................................................................................................. 191

- Intrants et processus à l'échelle des élèves ....................................................................................................................................... 193

Les conditions d'apprentissage au service de la culture mathématique …………………............................................................. 194

- La culture mathématique : Un défi en matière d'instruction et d'évaluation ...................................................................... 195

- Indicateurs de résultats en rapport avec la culture mathématique : Stratégies, convictions et motivation ............... 196

- Possibilités d'apprentissage et qualité de l'instruction : Évaluation de l'environnement d'apprentissage ............... 199

- Soutien à l'enseignement et à l'apprentissage des mathématiques à l'échelle du système et des établissements d'enseignement ..................................................................................................................................................... 202

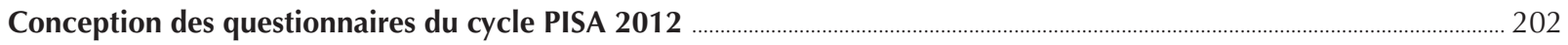

- Répartition des contenus entre les cycles PISA : Une structure globale pour les variables générales, les variables spécifiques au domaine d'évaluation et les variables d'extensions thématiques ..................................... 202

- Exploration des indicateurs du cycle PISA 2003 .................................................................................................................... 204

- Rotation des items dans les extensions du questionnaire « Élève » et les instruments informatisés ........................... 205

Garantir la validité des instruments PISA, leur pouvoir explicatif et leur pertinence pour l'action publique .............. 209

- Comment modéliser les effets des variables contextuelles sur l'apprentissage et la performance des élèves .................. 209

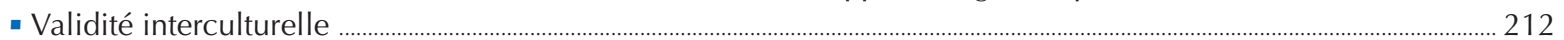

- Extension de l'échantillon pour étudier la variation au niveau Établissement (option internationale) …................. 213

- Extensions longitudinales de niveau Élève (recommandations pour les prochains cycles) ........................................... 213

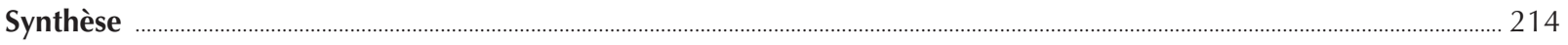

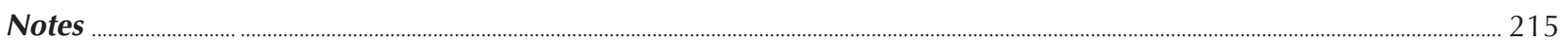

Références 
ANNEXE A QUESTIONNAIRES CONTEXTUELS

Questionnaire « Établissement »

Questionnaire "Élève »

Questionnaire sur le parcours scolaire

Questionnaire sur les TIC

Questionnaire « Parents» 


\section{ENCADRÉS}

Encadré 0.1 Qu'est-ce que l'enquête PISA ?

Encadré 0.2 Définition des domaines d'évaluation

Encadré 1.1 Les facultés mathématiques fondamentales et leur relation avec la difficulté des items

Encadré 3.1 Connaissances scientifiques : La définition de l'enquête PISA ……............................................................................. 106

Encadré 3.2 Culture scientifique : La définition de l'enquête PISA ….......................................................................... 106

\section{FIGURES}

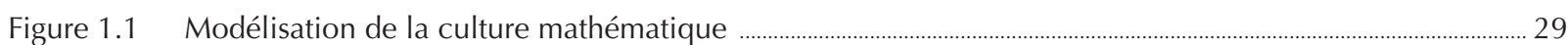

Figure 1.2 Relation entre les processus mathématiques et les facultés mathématiques fondamentales .......................... 35

Figure 1.3 Description de l'échelle de compétence en mathématiques (2003-2009) .............................................................. 45

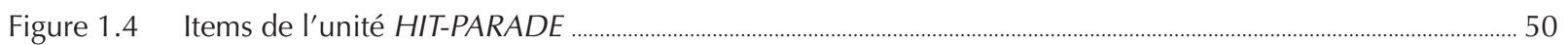

Figure 1.5 Items de l'unité ASCENSION DU MONT FUJI ............................................................................................ 52

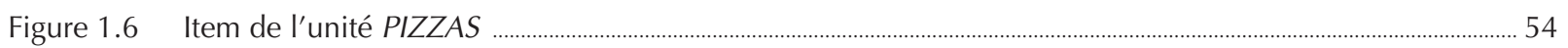

Figure 1.7 Exemple de réponse à l'item de l'unité PIZZAS ................................................................................................................ 55

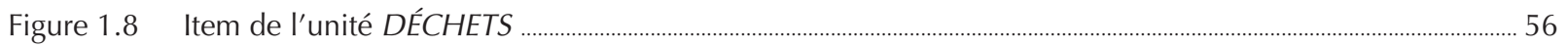

Figure 1.9 Item de l'unité CONCERT ROCK …….................................................................................................................................. 57

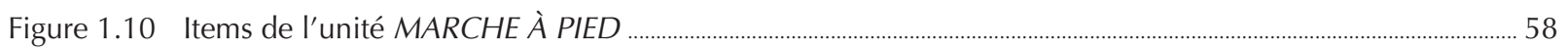

Figure 1.11 Item de l'unité MENUISIER .......................................................................................................................................................... 60

Figure 2.1 Relations entre les aspects du cadre d'évaluation et les sous-échelles d'aspect .................................................. 72

Figure 2.2 Description succincte des sept niveaux de compétence en compréhension de l'écrit sur papier lors du cycle PISA 2012

Figure 2.3 Description succincte des quatre niveaux de compétence en compréhension de l'écrit électronique lors du cycle PISA 2012 ........................................................................................................... 86

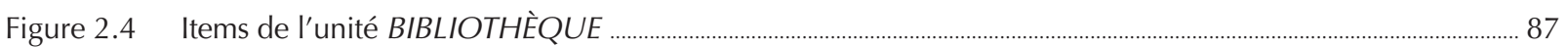

Figure 2.5 Items de l'unité AVIS AFFICHÉ AU SUPERMARCHÉ ................................................................................................... 91

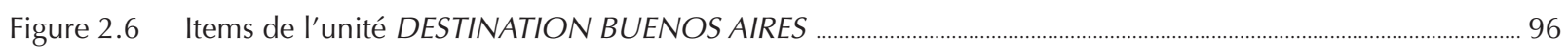

Figure 3.1 Cadre PISA d'évaluation de la culture scientifique ....................................................................................... 108

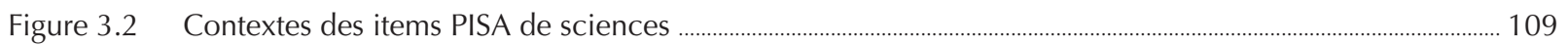

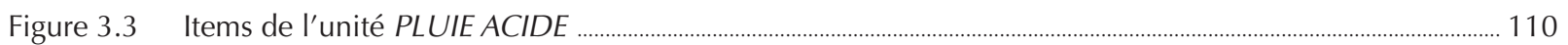

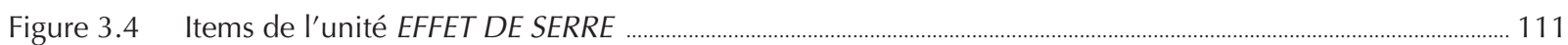

Figure 3.5 Items de l'unité EXERCICE PHYSIQUE …........................................................................................................................... 113

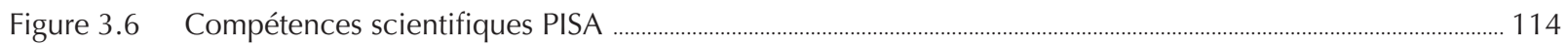

Figure 3.7 Catégories PISA de connaissances en sciences ................................................................................................................. 116

Figure 3.8 Catégories PISA de connaissances à propos de la science ........................................................................... 117

Figure 3.9 Outil d'élaboration et d'analyse des unités et des items d'évaluation ................................................................ 118

Figure 3.10 Description succincte des six niveaux de compétence de l'échelle PISA de culture scientifique ............. 121

Figure 3.11 Composantes principales de la définition PISA de la culture scientifique ............................................................. 122 


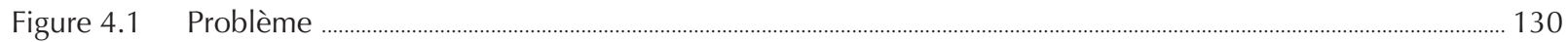

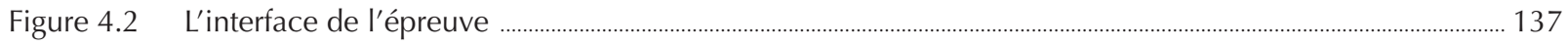

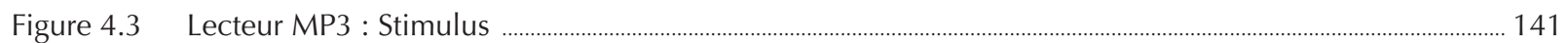

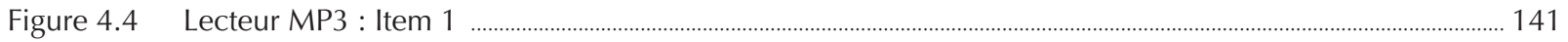

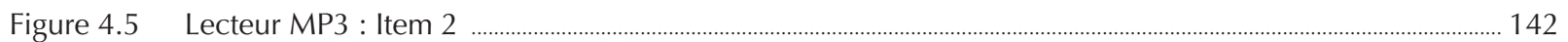

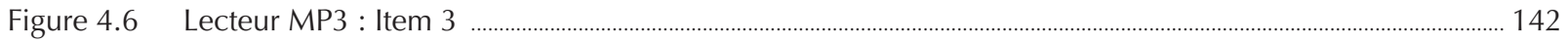

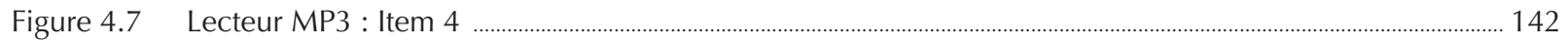

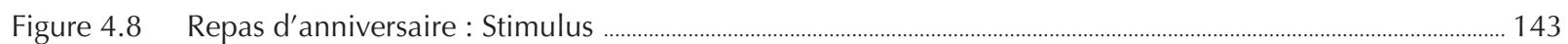

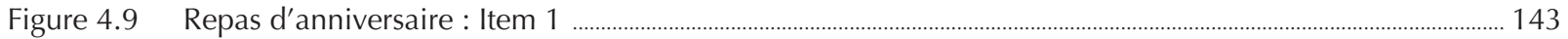

Figure 5.1 Modèle conceptuel d'organisation du domaine d'évaluation ................................................................................ 155

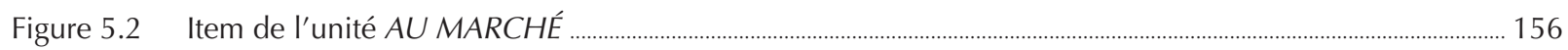

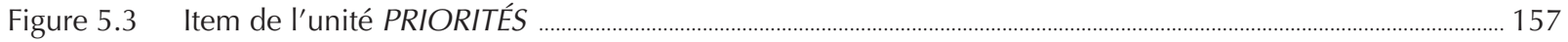

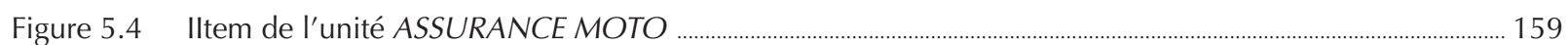

Figure 5.5 Item de l'unité ERREUR BANCAIRE ………………………......................................................................................... 160

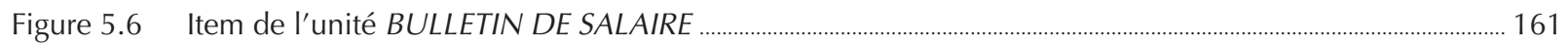

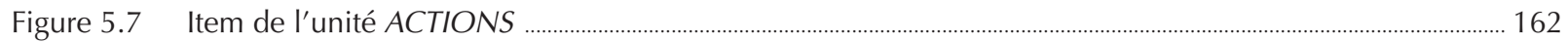

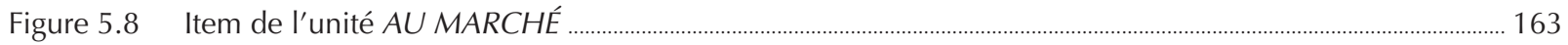

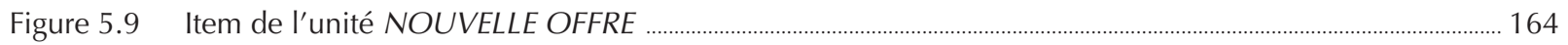

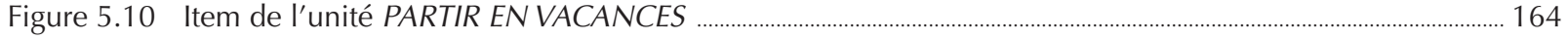

Figure 5.11 Item de l'unité NOUVELLE CARTE BANCAIRE _................................................................................................. 166

Figure 5.12 Relation entre les contenus de culture mathématique et de culture financière dans l'enquête PISA ....... 172

Figure 6.1 La théorie du comportement planifié ............................................................................................................................. 198

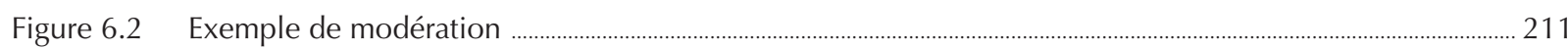

\section{TABLEAUX}

Tableau 1.1 Répartition approximative du score en mathématiques entre les catégories de processus …............................... 42

Tableau 1.2 Répartition approximative du score en mathématiques entre les catégories de contenus ............................... 42

Tableau 1.3 Répartition approximative du score en mathématiques entre les catégories de contextes ................................ 42

Tableau 2.1 Répartition approximative du score en compréhension de l'écrit entre les types de situations ..................... 68

Tableau 2.2 Répartition approximative du score en compréhension de l'écrit électronique

Tableau 2.3 Répartition approximative du score en compréhension de l'écrit entre les formats de texte ......................... 70

Tableau 2.4 Répartition approximative du score en compréhension de l'écrit entre les aspects ..............................................74

Tableau 2.5 Similitudes et différences entre les épreuves papier-crayon et les épreuves électroniques, selon les caractéristiques retenues dans le cadre d'évaluation

Tableau 2.6 Répartition approximative du score en compréhension de l'écrit par modalités de codage dans chaque aspect de la compréhension de l'écrit

Tableau 2.7 Analyse d'un item de I'unité OSONS PARLER de l'évaluation de la compréhension de l'écrit électronique 
Tableau 3.1 Répartition approximative des points de score en sciences entre les catégories de connaissances scientifiques

Tableau 3.2 Répartition approximative des points de score en sciences entre les compétences scientifiques

Tableau 3.3 Répartition approximative des points de score en sciences entre les types d'item

Tableau 4.1 Caractéristiques des tâches 138

Tableau 4.2 Répartition approximative du score en résolution de problèmes entre les processus cognitifs

Tableau 4.3 Répartition approximative du score en résolution de problèmes par type de situation et de contexte du problème

Tableau 5.1 Répartition approximative du score en culture financière entre les catégories de contenus 170

Tableau 5.2 Répartition approximative du score en culture financière entre les catégories de processus 170

Tableau 5.3 Répartition approximative du score en culture financière entre les catégories de contextes

Tableau 6.1 Taxinomie bidimensionnelle des résultats de l'éducation et de leurs variables prédictives 186

Tableau 6.2 Classification des questionnaires du cycle PISA 2003

Tableau 6.3 Partie commune du questionnaire «Élève »

Tableau 6.4 Rotation A, B et C du questionnaire «Élève » 



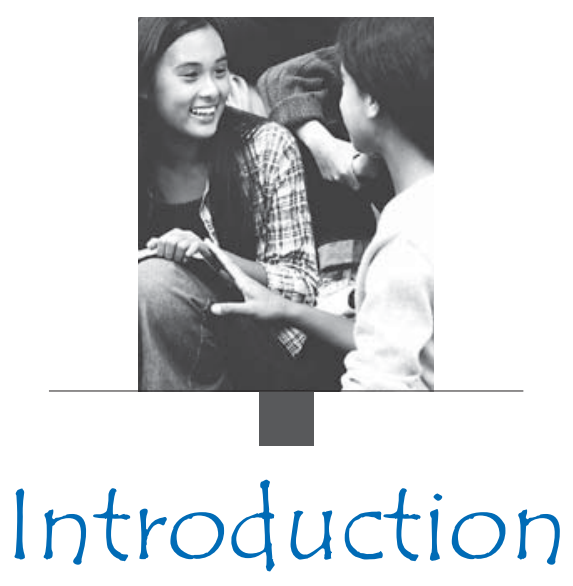

\section{VUE D'ENSEMBLE}

Parents, élèves, gouvernements, grand public, tous veulent savoir si le système d'éducation de leur pays prépare bien les jeunes à affronter les situations de la vie courante. De nombreux pays suivent de près l'apprentissage de leurs élèves pour répondre à cette question. Les évaluations comparatives internationales peuvent étoffer l'état des lieux dressé à l'échelle nationale en offrant un cadre plus étendu d'interprétation de la performance. Elles peuvent montrer ce qu'il est possible d'accomplir pour améliorer la qualité des résultats de l'apprentissage et l'égalité des chances en matière d'éducation. Elles peuvent aider à définir les objectifs de l'action publique en fournissant des objectifs quantifiables atteints par d'autres pays, et à choisir des pistes de réforme. Enfin, elles peuvent aussi aider les pays à mieux cerner leurs points forts et leurs points faibles, et à suivre leurs progrès.

C'est en 1997 que l'Organisation de coopération et de développement économiques (OCDE) a mis en œuvre le Programme international pour le suivi des acquis des élèves (PISA) dans le but de combler ce besoin de données comparables entre les pays sur la performance des élèves. L'enquête PISA est l'expression concrète de l'engagement des gouvernements à suivre l'évolution des résultats produits par leur système d'éducation, en l'occurrence en évaluant la performance des élèves, à intervalles réguliers et dans un cadre commun approuvé par les pays participants. Grâce à son approche novatrice, qui se focalise sur les compétences jugées pertinentes pour la vie adulte, l'enquête PISA entend poser de nouvelles bases pour alimenter le débat sur l'action publique et pour encourager la collaboration lors de la définition et de la mise en œuvre des objectifs de l'éducation.

L'enquête PISA est le fruit d'un effort concerté de la part des pays participants - les États membres de l'OCDE ainsi que plus d'une trentaine de pays et économies partenaires - pour déterminer dans quelle mesure les élèves âgés de 15 ans sont préparés à relever les défis que l'avenir leur réserve. L'âge de 15 ans a été retenu, car c'est celui auquel les élèves approchent du terme de leur scolarité obligatoire dans la plupart des pays de I'OCDE. L'enquête PISA, dont les orientations sont définies en concertation par les pays et économies participants, leur apporte l'expertise scientifique nationale et internationale sur les thématiques d'action publique qui les intéressent. Elle évalue les connaissances, les compétences et les attitudes des élèves de 15 ans depuis plus d'une dizaine d'années, permettant ainsi de montrer l'évolution de la situation des pays au fil du temps.

L'enquête PISA se fonde sur une conception de l'évaluation des connaissances, des compétences et des attitudes qui reflète l'évolution des programmes d'enseignement : elle va au-delà des acquis purement scolaires et se concentre sur la mise en œuvre des savoirs et savoir-faire dans des tâches et des défis quotidiens, que ce soit en famille ou dans le monde du travail. Elle repose sur un modèle dynamique d'apprentissage qui veut que les individus acquièrent, de façon continue au cours de leur vie, de nouvelles connaissances et compétences dont ils ont besoin pour s'adapter dans un monde en constante évolution. L'enquête PISA cible des activités que les élèves âgés de 15 ans auront à accomplir dans l'avenir et cherche à identifier ce qu'ils sont capables de faire avec ce qu'ils ont appris - c'est-à-dire leur faculté à continuer à apprendre tout au long de leur vie en appliquant ce qu'ils ont appris à l'école et ailleurs, de porter un regard critique sur leurs choix et de prendre des décisions. Les épreuves sont conçues à la lumière du dénominateur commun des programmes scolaires des pays participants, sans toutefois s'y cantonner. Elles servent à évaluer les connaissances des 
élèves, certes, mais aussi leur faculté de réflexion et leur capacité à appliquer leurs connaissances et leurs expériences dans des situations qui s'inspirent du monde réel. Pour comprendre et juger un avis scientifique sur la sécurité sanitaire des produits alimentaires, par exemple, un adulte doit avoir des connaissances élémentaires concernant la composition des nutriments et doit aussi être capable de les utiliser. Les termes « compréhension » et « culture » décrivent mieux cette conception plus large des connaissances et compétences : I'enquête PISA cherche à déterminer dans quelle mesure les élèves âgés de 15 ans sont capables d'activer divers processus cognitifs pour utiliser comme il se doit les connaissances et compétences en compréhension de l'écrit, en mathématiques et en sciences qu'ils ont acquises durant leur scolarité et leurs autres expériences d'apprentissage.

L'enquête PISA procède, tous les trois ans, à la collecte de données sur les connaissances et compétences des élèves au travers d'épreuves en compréhension de l'écrit, en mathématiques et en sciences, sur les établissements d'enseignement et sur les pays. Elle combine les résultats des épreuves en compréhension de l'écrit, en mathématiques et en sciences à des informations sur le milieu familial des élèves, leurs approches à l'égard de l'apprentissage, leurs cadres d'apprentissage et leur degré de maîtrise de l'informatique, afin d'établir des relations entre leur niveau de performance et ces variables contextuelles. Elle permet donc de mieux cerner les facteurs qui influent sur l'acquisition des connaissances et des compétences et sur le développement des attitudes à l'école et dans le milieu familial, et d'étudier leurs interactions et leurs implications pour l'action publique.

L'enquête PISA se distingue par plusieurs atouts : i) des mécanismes stricts de contrôle de la qualité lors de l'échantillonnage, de la traduction et de l'administration des épreuves; ii) des dispositions qui garantissent la diversité culturelle et linguistique des épreuves, grâce, en particulier, à la participation des pays aux processus d'élaboration et de révision lors de la production des items; et iii) I'application d'une méthodologie et de technologies de pointe lors du traitement des données. Ces atouts se conjuguent pour aboutir à des instruments de mesure de grande qualité et à des résultats dont la validité et la fiabilité sont élevées, qui permettent de mieux comprendre les systèmes d'éducation et de mieux cerner les connaissances, les compétences et les attitudes des élèves.

Cette publication présente le cadre conceptuel du cycle PISA 2012, dont une version remaniée et enrichie du cadre d'évaluation de la culture mathématique, qui intègre les processus dans lesquels les élèves s'engagent lorsqu'ils résolvent des problèmes, ce qui constitue en soi une nouvelle source d'informations. Elle décrit également la nouvelle option facultative d'épreuve informatisée en mathématiques (Computer-Based Assessment of Mathematics, CBAM), qui reflète I'importance des technologies de l'information et de la communication (TIC) pour l'utilisation des mathématiques dans les sociétés modernes. Elle présente, par ailleurs, le cadre d'évaluation de la compréhension de l'écrit et de la culture scientifique. Le cadre conceptuel de chaque domaine d'évaluation définit les connaissances que les élèves doivent acquérir, les processus qu'ils doivent mettre en œuvre et les contextes dans lesquels ils doivent appliquer leurs savoirs et savoir-faire. Des exemples de tâches illustrent les domaines d'évaluation et leurs différentes composantes. Enfin, cette publication présente le cadre conceptuel des questionnaires contextuels destinés à recueillir des informations auprès des chefs d'établissement, des élèves et de leurs parents sur leur milieu familial, leurs attitudes, leur parcours scolaire et leur environnement d'apprentissage à l'école.

\section{CARACTÉRISTIQUES FONDAMENTALES DU CYCLE PISA 2012}

Le cycle PISA 2012 est le cinquième du programme stratégique de collecte de données défini par les pays participants en 1997. Les publications Mesurer les connaissances et compétences des élèves - Un nouveau cadre d'évaluation (OCDE, 1999), Cadre d'évaluation de PISA 2003 - Connaissances et compétences en mathématiques, lecture, science et résolution de problèmes (OCDE, 2003), Compétences en sciences, lecture et mathématiques - Le cadre d'évaluation de PISA 2006 (OCDE, 2006) et Le cadre d'évaluation de PISA 2009 - Les compétences clés en compréhension de l'écrit, en mathématiques et en sciences (OCDE, 2009) exposent le cadre conceptuel d'évaluation des quatre premiers cycles de l'enquête PISA. Les résultats de ces trois cycles ont été présentés dans les rapports Connaissances et compétences : Des atouts pour la vie Premiers résultats de PISA 2000 (OCDE, 2001), Apprendre aujourd'hui, réussir demain - Premiers résultats de PISA 2003 (OCDE, 2004), PISA 2006 - Les compétences scientifiques, un atout pour l'avenir (OCDE, 2007) et Résultats du PISA 2009 : Savoirs et savoir-faire des élèves (volumes I à VI) (OCDE, 2010). Toutes ces publications sont également accessibles en ligne, sur le site web de l'enquête PISA (www.pisa.oecd.org). Grâce à ces résultats, les décideurs politiques peuvent comparer la performance de leur système d'éducation à celle d'autres pays. Comme les cycles précédents, le cycle PISA 2012 porte sur les mathématiques, son domaine majeur d'évaluation, la compréhension de l'écrit et les sciences. Un questionnaire contextuel a été soumis aux élèves, et des informations supplémentaires ont été demandées aux chefs d'établissement. Des informations ont également été recueillies auprès des parents d'élèves dans 11 pays et économies. Soixante-six pays et économies, dont les 34 pays membres de I'OCDE, ont participé au cycle PISA 2012. 


\section{Encadré 0.1 Qu'est-ce que l'enquête PISA ?}

\section{Description}

- L'enquête PISA est une évaluation normalisée à l'échelle internationale élaborée conjointement par les pays participants et administrée aux jeunes de 15 ans qui sont scolarisés.

- Le premier cycle a été mis en œuvre dans 43 pays et économies (32 en 2000 et 11 en 2002), le deuxième cycle, dans 41 pays et économies (2003), le troisième cycle, dans 57 pays et économies (2006) et le quatrième cycle, dans 75 pays et économies (65 en 2009 et 10 en 2010). Le cycle PISA 2012 a vu la participation de 66 pays et économies.

- Dans chaque pays/économie, ce sont entre 4500 et 10000 élèves qui sont soumis aux épreuves PISA.

\section{Domaines d'évaluation}

- Le cycle PISA 2012 porte sur les mathématiques, la compréhension de l'écrit et les sciences. L'évaluation vise à déterminer non seulement si les élèves sont capables de restituer des acquis scolaires, mais aussi s'ils sont capables de faire des extrapolations à partir de ce qu'ils ont appris et d'appliquer leurs connaissances dans des situations inédites. Deux autres domaines d'évaluation sont inclus dans le cycle PISA 2012 : la résolution de problèmes, - des épreuves que les pays n'ont pas tous administrées pour des raisons techniques -, et la culture financière, administrée par certains pays à titre d'option.

- La priorité est accordée à la maîtrise des processus, à la compréhension des concepts et à la faculté d'affronter diverses situations dans chaque domaine d'évaluation.

\section{Méthodologie}

- Les épreuves sont de type " papier-crayon » et durent deux heures en tout par élève. Dans un certain nombre de pays et d'économies, des épreuves électroniques d'une durée de 40 minutes sont ajoutées en mathématiques et en compréhension de l'écrit.

- Les épreuves contiennent des items à choix multiple ainsi que des items qui demandent aux élèves d'argumenter leur réponse. Elles comportent un certain nombre de groupes d'items se rapportant à la description d'une situation qui s'inspire du monde réel.

- La batterie complète d'items représente une durée totale de 390 minutes, et les épreuves soumises aux élèves n'en reprennent qu'une partie, selon diverses combinaisons.

- Les élèves doivent également remplir un questionnaire contextuel d'une demi-heure pour fournir des informations sur eux et leur milieu familial. Les chefs d'établissement doivent quant à eux remplir un questionnaire d'une vingtaine de minutes sur leur établissement. Enfin, dans certains pays et économies, de courts questionnaires facultatifs sont administrés : i) aux parents, qui sont invités à fournir des informations sur les habitudes concernant les mathématiques dans leur foyer ; et ii) aux élèves, qui sont invités à fournir des informations sur leur accès à I'informatique et l'usage qu'ils en font, ainsi que sur leur parcours scolaire et leurs aspirations.

\section{Cycle d'évaluation}

- L'évaluation a lieu tous les trois ans et son programme stratégique prévoit d'ores et déjà sa mise en œuvre jusqu'à I'horizon 2015.

- Chaque cycle prévoit l'évaluation en profondeur d'un des trois domaines, dont les épreuves représentent deux tiers de la durée totale. Les deux autres domaines d'évaluation font l'objet d'une description synthétique du profil de compétence des élèves. Le domaine majeur d'évaluation était la compréhension de l'écrit en 2000 et en 2009, les mathématiques en 2003 et les sciences en 2006. En 2012, les mathématiques constituent une nouvelle fois le domaine majeur d'évaluation.

\section{Résultats}

- Profil général des connaissances et compétences des élèves de 15 ans.

- Indicateurs contextuels reliant les résultats des épreuves cognitives à des caractéristiques propres aux élèves et aux établissements d'enseignement. Indicateurs tendanciels montrant l'évolution des résultats au fil du temps.

- Une précieuse base de connaissances au service de la recherche et de l'analyse de la politique de l'éducation. 
Comme l'objectif de l'enquête PISA est d'évaluer le rendement cumulé des systèmes d'éducation à un âge où la scolarisation est encore généralisée, elle cible les jeunes de 15 ans inscrits en filière générale ou en filière professionnelle. Dans chaque pays, entre 4500 et 10000 élèves sont échantillonnés dans au moins 150 établissements d'enseignement pour passer les épreuves. Ces larges échantillons fournissent des données statistiquement fiables qui permettent ensuite des analyses pointues en fonction d'un certain nombre de critères.

L'enquête PISA cherche avant tout à déterminer dans quelle mesure les jeunes ont acquis, en compréhension de l'écrit, en mathématiques et en sciences, les connaissances et compétences plus générales dont ils auront besoin une fois adultes. L'évaluation des compétences transversales fait partie intégrante du cycle PISA 2012. Plusieurs raisons fondamentales expliquent le choix de cette approche holistique :

- L'acquisition de connaissances spécifiques durant la scolarité est importante, certes, mais l'exploitation de ces connaissances à l'âge adulte passe par l'acquisition de concepts et de compétences plus vastes. En compréhension de l'écrit, interpréter des textes, réfléchir à leur contenu et juger de leurs qualités sont autant de compétences essentielles. En mathématiques, être capable de raisonner en termes quantitatifs et de représenter des relations ou des dépendances est plus pertinent lorsqu'il s'agit d'exploiter des compétences mathématiques dans la vie courante que de savoir répondre aux questions habituellement posées dans les manuels scolaires. En sciences, posséder des connaissances spécifiques, savoir le nom de plantes ou d'animaux par exemple, présente moins d'intérêt que de comprendre de grands concepts scientifiques, tels que la consommation d'énergie, la biodiversité et la santé humaine, pour réfléchir aux questions qui font débat chez les adultes.

- Dans le cadre d'une évaluation internationale, privilégier le contenu des programmes scolaires aurait pour effet de polariser les épreuves sur des éléments de ces programmes qui sont communs à tous les pays ou à la plupart d'entre eux. Cette approche nécessiterait de nombreux compromis et aboutirait à une évaluation trop limitée pour être utile aux gouvernements désireux d'en apprendre davantage sur les points forts et les aspects novateurs des systèmes d'éducation d'autres pays.

- Il est essentiel de développer un certain nombre de compétences générales chez les élèves : ils doivent pouvoir communiquer, s'adapter, faire preuve de souplesse, résoudre des problèmes et maîtriser l'informatique. Comme ces compétences sont inculquées dans l'ensemble des programmes scolaires, leur évaluation requiert l'adoption d'une approche transversale.

L'enquête PISA n'est pas une évaluation internationale ponctuelle des compétences des élèves âgés de 15 ans en compréhension de l'écrit, en mathématiques et en sciences. Il s'agit d'un programme continu d'évaluation, conçu pour constituer à long terme une base de données qui permet de suivre l'évolution des connaissances et compétences des élèves dans différents pays, ainsi que celles de différents sous-groupes de la population dans chaque pays. Lors de chaque cycle, un domaine fait l'objet d'une évaluation plus approfondie, et les épreuves le concernant représentent deux tiers environ de la durée totale des tests. Cette stratégie de collecte de données permet de réaliser une analyse approfondie de chacun des domaines d'évaluation tous les neuf ans et de rendre compte de leur évolution globale tous les trois ans. La compréhension de l'écrit a été déclarée domaine majeur d'évaluation en 2000 et en 2009, les mathématiques, en 2003 et les sciences, en 2006. Les mathématiques sont de nouveau le domaine majeur d'évaluation en 2012, sur la base d'un cadre d'évaluation remanié : il inclut les processus mathématiques dans lesquels les élèves s'engagent lorsqu'ils mobilisent leur culture mathématique et les facultés mathématiques fondamentales qui sous-tendent ces processus (voir le chapitre 1). En compréhension de l'écrit et en sciences, les cadres d'évaluation du cycle PISA 2012 sont identiques à ceux des cycles précédents (voir les chapitres 2 et 3, respectivement).

Comme lors des cycles précédents, les épreuves du cycle PISA 2012 durent 2 heures par élève et sont constituées de 4 blocs d'items de 30 minutes chacun dans un ou plusieurs domaines cognitifs. La batterie d'items représente toutefois au total 390 minutes de test et se divise en 13 carnets de test. La culture financière, une option des épreuves papiercrayon, représente deux blocs d'items, soit 60 minutes de test, lors de la campagne définitive du cycle PISA 2012. Chaque carnet de test est administré à un nombre suffisant d'élèves dans chaque pays pour produire des estimations fiables de la performance tous items confondus, d'une part, pour l'ensemble des élèves et, d'autre part, pour des sousgroupes d'élèves (les garçons et les filles, et les élèves issus de milieux socio-économiques différents, par exemple). Les élèves passent également 30 minutes à répondre à un questionnaire contextuel. La rotation des items du questionnaire permet d'utiliser davantage de matériel. Certaines questions ont été posées à tous les élèves, comme lors des cycles précédents, alors que d'autres n'ont été posées qu'à des sous-groupes d'élèves.

L'évaluation comprend de surcroît une épreuve informatisée de résolution de problèmes dans 44 pays et économies, dont 32 ont administré des épreuves informatisées en compréhension de l'écrit et en mathématiques. Ces épreuves 
informatisées représentent 40 minutes de test. Au total, 4 blocs de 20 minutes de test ont été constitués à partir des 80 minutes de test en résolution de problèmes. Les élèves des pays ne participant pas aux épreuves informatisées de mathématiques et de compréhension de l'écrit ont répondu à deux des blocs d'items en résolution de problèmes, selon un modèle de rotation. Les élèves de pays participants aux épreuves informatisées de mathématiques et de compréhension de l'écrit ont répondu à deux, à un ou à aucun des quatre blocs d'items, selon un autre modèle de rotation. Les épreuves informatisées représentent au total 80 minutes de test en mathématiques et 80 minutes de test en compréhension de l'écrit. Les items ont été répartis en 4 blocs de 20 minutes de test dans chacun des deux domaines d'évaluation. Tous les items des épreuves informatisées ont été répartis en carnets selon un modèle de rotation, à raison de deux blocs par carnet. Chaque élève a répondu à un carnet, soit 40 minutes de test au total.

Les résultats de l'enquête PISA sont de trois types différents :

- des indicateurs de base qui dressent le profil général des connaissances et compétences des élèves ;

- des indicateurs contextuels qui montrent en quoi le profil de compétence des élèves est lié à des variables importantes d'ordre démographique, social, économique et scolaire ; et

- des indicateurs tendanciels que la nature cyclique de la collecte des données permet de produire et qui montrent les changements dans le niveau général de performance, dans la répartition des élèves par niveau de compétence, et dans les relations entre ces résultats et des variables spécifiques aux élèves et aux établissements d'enseignement.

Ces indicateurs permettent d'attirer l'attention sur des thématiques importantes, mais pas de répondre aux questions que les décideurs politiques se posent. C'est pourquoi l'enquête PISA a également mis au point un programme d'analyse pertinent dans le domaine de la politique de l'éducation, qui va au-delà de la simple présentation d'indicateurs.

\section{L'ORIGINALITÉ DE L'ENQUÊTE PISA}

L'enquête PISA cherche à évaluer la capacité des jeunes à utiliser leurs connaissances et compétences pour relever les défis du monde réel. Cette approche reflète l'évolution des objectifs des programmes de cours : la priorité va désormais à ce que les élèves savent faire avec ce qu'ils ont appris à l'école plutôt qu'à la mesure dans laquelle ils ont assimilé des matières spécifiques.

L'enquête PISA est unique en son genre, comme le montrent ses grands principes :

- Son bien-fondé pour l'action publique : les données sur les acquis des élèves sont rapportées à des données sur leurs caractéristiques personnelles et sur des facteurs clés qui façonnent leur apprentissage à l'école et ailleurs pour repérer des différences dans les profils de compétence et identifier les caractéristiques des élèves, des établissements d'enseignement et des systèmes d'éducation qui se distinguent par des niveaux élevés de performance.

- Son approche novatrice basée sur la notion de «littératie », qui renvoie à la capacité des élèves d'exploiter des savoirs et savoir-faire dans des matières clés, et d'analyser, de raisonner et de communiquer lorsqu'ils énoncent, résolvent et interprètent des problèmes qui s'inscrivent dans divers contextes.

- Sa pertinence par rapport à l'apprentissage tout au long de la vie : I'enquête PISA ne se limite pas à évaluer les compétences des élèves dans des matières scolaires, mais demande également à ceux-ci de décrire leur envie d'apprendre, leur perception d'eux-mêmes et leurs stratégies d'apprentissage.

- Sa périodicité, qui permet aux pays de suivre leurs progrès sur la voie de l'accomplissement d'objectifs clés de l'apprentissage.

- Sa grande couverture géographique et son principe de collaboration : les 34 pays membres de I'OCDE ainsi que plus de 30 pays et économies partenaires ont participé au cycle PISA 2012.

La pertinence des savoirs et savoir-faire évalués par l'enquête PISA a été confirmée par de récentes études sur le parcours des élèves durant les années suivant I'administration des tests PISA. Ces études, menées en Australie, au Canada et au Danemark, montrent l'existence d'une forte corrélation entre la performance en compréhension de l'écrit à l'âge de 15 ans lors du cycle PISA 2000 et la probabilité qu'un élève termine avec succès ses études secondaires et poursuive son parcours dans I'enseignement post-secondaire à l'âge de 19 ans. Ainsi, les élèves canadiens qui s'étaient classés au niveau 5 de l'échelle de compréhension de l'écrit à l'âge de 15 ans étaient 16 fois plus susceptibles de poursuivre des études post-secondaires à l'âge de 19 ans que ceux qui se situaient sous le niveau 1 de cette échelle.

L'enquête PISA est l'évaluation internationale la plus complète et la plus rigoureuse pour la mesure de la performance des élèves et la collecte des données sur ces derniers, leurs familles et les facteurs institutionnels pouvant aider à 
expliquer les différences de performance. Les décisions concernant la nature et l'étendue des évaluations, ainsi que les données contextuelles à collecter, sont prises par d'éminents experts des pays participants, sous la direction conjointe des gouvernements de ces pays en fonction de préoccupations communes en matière d'action publique. Des efforts et des ressources importants sont déployés pour garantir la diversité culturelle et linguistique, ainsi que l'équilibre des épreuves. Des mécanismes stricts de contrôle de la qualité sont appliqués lors de l'échantillonnage, de la traduction et de la collecte des données. En conséquence, les résultats de l'enquête PISA présentent un degré élevé de validité et de fiabilité, et peuvent contribuer à améliorer de façon significative la compréhension des systèmes d'éducation des pays les plus développés, ainsi que d'un nombre croissant de pays en développement.

Les décideurs du monde entier utilisent les résultats de l'enquête PISA aux fins suivantes : comparer le niveau de compétence de leurs élèves à celui des élèves des autres pays participants ; se fixer des objectifs d'amélioration, par exemple atteindre les scores moyens d'autres pays ou leur degré plus élevé d'équité en termes de perspectives et de résultats dans l'éducation ; et enfin, comprendre les points forts et les points faibles de leur système d'éducation. Les innombrables rapports rédigés dans les pays participants attestent de l'intérêt que suscite l'enquête PISA. Par ailleurs, les résultats de l'enquête PISA sont abondamment cités dans les débats publics et foisonnent dans les médias du monde entier.

\section{APERÇU DES ASPECTS ÉVALUÉS DANS CHAQUE DOMAINE}

L'encadré 0.2 définit les trois domaines d'évaluation du cycle PISA 2012. Ces définitions mettent toutes en évidence les savoirs et savoir-faire fonctionnels qui permettent à l'individu de participer pleinement à la vie de la société. Pour ce faire, il ne suffit pas d'être capable d'exécuter des tâches imposées de l'extérieur, par un employeur par exemple. II faut aussi avoir le bagage nécessaire pour prendre part aux processus de prise de décision. Les tâches les plus complexes des épreuves PISA demandent aux élèves de réfléchir à ce qu'ils lisent et de l'évaluer, et pas uniquement de répondre à des questions auxquelles il n'y a qu'une seule réponse correcte. Ces définitions traitent de la capacité des élèves à faire des extrapolations à partir de ce qu'ils ont appris et d'appliquer leurs connaissances dans des situations inédites. Y figure également la capacité des élèves à analyser, à raisonner et à communiquer lorsqu'ils énoncent, résolvent et interprètent des problèmes dans diverses situations.

\section{Encadré 0.2 Définition des domaines d'évaluation}

Culture mathématique : la culture mathématique est l'aptitude d'un individu à formuler, employer et interpréter des mathématiques dans un éventail de contextes, c'est à dire à raisonner en termes mathématiques et à utiliser des concepts, procédures, faits et outils mathématiques pour décrire, expliquer et prévoir des phénomènes. Elle aide les individus à comprendre le rôle que les mathématiques jouent dans le monde et à se comporter en citoyens constructifs, engagés et réfléchis, c'est-à-dire à poser des jugements et à prendre des décisions en toute connaissance de cause.

Compréhension de l'écrit : comprendre l'écrit, c'est non seulement comprendre et utiliser des textes écrits, mais aussi réfléchir à leur propos et s'y engager. Cette capacité devrait permettre à chacun de réaliser ses objectifs, de développer ses connaissances et son potentiel, et de prendre une part active dans la société.

Culture scientifique : les connaissances scientifiques de l'individu et sa capacité d'utiliser ces connaissances pour identifier les questions auxquelles la science peut apporter une réponse, pour acquérir de nouvelles connaissances, pour expliquer des phénomènes scientifiques et pour tirer des conclusions fondées sur des faits à propos de questions à caractère scientifique; la compréhension des éléments caractéristiques de la science en tant que forme de recherche et de connaissance humaine ; la conscience du rôle de la science et de la technologie dans la constitution de notre environnement matériel, intellectuel et culturel ; et enfin, la volonté de s'engager en qualité de citoyen réfléchi sur des problèmes à caractère scientifique et touchant à des notions relatives à la science.

La culture mathématique (décrite en détail au chapitre 1) renvoie à la capacité des élèves à analyser, à raisonner et à communiquer efficacement leurs idées lorsqu'ils énoncent, formulent et résolvent des problèmes mathématiques relevant de situations variées, et qu'ils en interprètent les solutions. L'évaluation PISA de la culture mathématique s'articule autour des aspects suivants :

- Les processus : trois catégories de processus (formuler des situations de façon mathématique ; employer des concepts, faits, procédures et raisonnements mathématiques; et interpréter, appliquer et évaluer des résultats mathématiques soit, sous forme abrégée, formuler, appliquer et interpréter) décrivent le cheminement des individus lorsqu'ils tentent d'établir un lien entre le contexte d'un problème et le monde des mathématiques et donc, de résoudre le problème. Ces trois processus se basent sur les sept facultés mathématiques fondamentales (communication ; mathématisation ; 
représentation ; raisonnement et argumentation ; conception de stratégies de résolution de problèmes ; utilisation d'opérations et d'un langage symbolique, formel et technique; et utilisation d'outils mathématiques) que les individus doivent à leurs connaissances mathématiques dans des sujets donnés.

- Les contenus : les contenus se rapportent à quatre idées majeures (les variations et les relations ; l'espace et les formes ; la quantité ; et l'incertitude et les données) qui sont liées aux disciplines mathématiques (telles que I'arithmétique, I'algèbre et la géométrie), et qui se chevauchent de façon complexe.

- Les contextes : les contextes se définissent en fonction des aspects du monde dans lesquels les problèmes se situent. Le cadre d'évaluation identifie quatre catégories, à savoir les contextes personnels, professionnels, sociétaux et scientifiques.

La compréhension de l'écrit (décrite en détail au chapitre 2) renvoie à la capacité des élèves à comprendre l'écrit, à I'utiliser et à y réfléchir pour réaliser leurs objectifs. L'évaluation PISA de la compréhension de l'écrit s'articule autour des points suivants :

- Le format des textes : I'enquête PISA utilise des textes continus, en prose, constitués de phrases et de paragraphes, ainsi que des textes non continus qui présentent l'information sous d'autres formes, par exemple dans des listes, des formulaires, des graphiques ou des schémas. Elle établit également une distinction entre les différents types de textes en prose, tels que les textes de narration, d'information et d'argumentation.

- Les processus (aspects) : les épreuves ne sont pas conçues pour évaluer les compétences les plus élémentaires en lecture, dans la mesure où la plupart des élèves âgés de 15 ans les ont acquises, mais demandent aux élèves de montrer qu'ils sont capables de localiser et extraire l'information, de comprendre le sens global d'un texte, de développer une interprétation et de réfléchir au contenu et à la forme d'un texte.

- Les situations : elles sont définies en fonction de l'usage pour lequel les textes ont été rédigés. Ainsi, les romans, les lettres personnelles ou les biographies sont écrits en vue d'une lecture à usage privé ; les documents ou les avis officiels sont destinés à un usage public ; les manuels d'entretien ou les rapports sont destinés à une lecture à des fins professionnelles; et les manuels scolaires ou les fiches d'exercices sont destinés à une utilisation scolaire. Comme certains groupes d'élèves peuvent obtenir de meilleurs résultats dans une situation de lecture plutôt que dans une autre, il est souhaitable que les épreuves contiennent un éventail de situations de lecture.

La culture scientifique (décrite en détail au chapitre 3) renvoie à la capacité des élèves d'utiliser des connaissances et des processus scientifiques non seulement pour comprendre le monde naturel, mais aussi pour participer à des décisions le concernant. L'évaluation PISA de la culture scientifique s'articule autour des aspects suivants :

- Les connaissances ou concepts scientifiques : ils aident à comprendre les liens entre divers phénomènes scientifiques. Les concepts retenus dans l'enquête PISA sont des concepts bien connus, relevant de la physique, de la chimie, de la biologie, des sciences de la terre et des sciences de l'univers; pour autant, les items ne portent pas sur leur simple restitution, mais requièrent de les appliquer à un contenu donné.

- Les processus scientifiques : ils sont axés sur la capacité de recueillir des faits, de les interpréter et d'agir en conséquence. Les trois processus retenus dans l'enquête PISA consistent à décrire, expliquer et prévoir des phénomènes scientifiques ; comprendre la démarche utilisée dans les recherches scientifiques; et interpréter des résultats scientifiques et les conclusions qui en sont tirées.

- Les contextes scientifiques : ils définissent le cadre dans lequel les connaissances et les processus scientifiques sont appliqués. Le cadre d'évaluation PISA identifie trois champs d'application principaux : les sciences liées à la vie et à la santé ; les sciences liées à la terre et à l'environnement; et les sciences liées à la technologie.

\section{MISE EN GEUVRE DE L'ÉVALUATION ET PRÉSENTATION DES RÉSULTATS DU CYCLE PISA 2012}

Le cycle PISA 2012 comporte essentiellement des épreuves papier-crayon comme lors des cycles précédents, mais des épreuves informatisées de compréhension de l'écrit électronique ont été administrées dans un certain nombre de pays et économies. Les épreuves papier-crayon et les épreuves informatisées sont constituées de différents types de questions. Dans certaines questions, les élèves doivent choisir ou produire des réponses simples qui peuvent être comparées directement à l'unique réponse correcte, c'est le cas dans les items à choix multiple et les items à réponse construite fermée. La réponse à ces questions, qui servent généralement à évaluer des compétences d'un niveau peu élevé, est soit correcte, soit incorrecte. D'autres questions sont plus ouvertes : les élèves doivent construire leur propre réponse. Elles servent à mesurer des dimensions plus générales que celles cernées par les enquêtes classiques. Dans ces questions, l'éventail de réponses acceptables est plus large, ce qui nécessite des grilles de correction plus complexes qui prévoient dans certains cas l'attribution d'un crédit partiel pour les réponses en partie correctes. 
Les élèves ne doivent pas répondre à la totalité des items. Les épreuves papier-crayon administrées lors du cycle PISA 2012 en mathématiques, en compréhension de l'écrit et en sciences sont constituées de blocs d'items qui représentent 30 minutes de test. Les carnets de test des épreuves papier-crayon sont composés de quatre blocs d'items (à l'exception d'un carnet propre aux élèves ayant des besoins spécifiques d'éducation qui est composé de deux blocs).

En mathématiques, les pays pouvaient choisir d'administrer l'une des deux séries de carnets de test alternatifs prévues à l'occasion du cycle PISA 2012. Les 13 carnets de la première série sont constitués d'items dont le degré de difficulté varie comme lors des cycles précédents. Ces carnets comprennent quatre blocs d'items choisis par rotation entre les sept blocs de mathématiques, les trois blocs de compréhension de l'écrit et les trois blocs de sciences. Chaque carnet comportait au moins un bloc de mathématiques. Les carnets de la deuxième série contiennent eux aussi des items dont le degré de difficulté varie, mais inclut de surcroît des items plus faciles, ce qui permettra d'obtenir des informations descriptives plus probantes sur ce que les élèves dont le niveau de compétence est moins élevé sont capables de comprendre et de faire en mathématiques. Tous les pays et économies participants ont administré 11 blocs communs d'items : 5 blocs de mathématiques, 3 blocs de compréhension de l'écrit et 3 blocs de sciences. Ils ont en outre administré l'une des deux séries alternatives d’items en mathématiques. La performance des élèves sera présentée sur une échelle commune de culture mathématique, quels que soient les blocs d'items choisis par les pays. De plus, deux blocs d'items de culture financière et un bloc d'items à l'intention des élèves ayant des besoins spécifiques d'éducation ont été prévus pour les pays intéressés.

Quant aux épreuves informatisées que certains pays et économies ont administrées, elles sont constituées de 2 blocs de 20 minutes de test chacun. Dans les pays et économies qui ont choisi d'administrer uniquement l'épreuve informatisée de résolution de problèmes, les carnets de test étaient constitués de deux blocs d'items par rotation. Chaque élève a répondu à un des huit carnets de test comportant deux blocs chacun. Dans les pays et économies qui ont choisi d'administrer des épreuves informatisées en mathématiques, en compréhension de l'écrit et en résolution de problèmes, les 24 carnets de test étaient constitués de 2 blocs d'items, choisis par rotation entre les 4 blocs de résolution de problèmes, les 4 blocs de mathématiques et les 2 blocs de compréhension de l'écrit.

Que ce soit dans les épreuves papier-crayon ou dans les épreuves informatisées, les connaissances et les compétences sont évaluées au moyen d'unités constituées d'un stimulus (par exemple, un texte, un tableau, un graphique, un schéma, etc.) et d'une série d'items se rapportant à ce stimulus. C'est une caractéristique importante des épreuves dans la mesure où des questions plus approfondies peuvent être posées, ce qui ne pourrait être envisagé si chaque question s'inscrivait dans un contexte totalement nouveau. Cette approche donne aux élèves le temps d'assimiler le stimulus, qui peut être utilisé pour évaluer de nombreux aspects de la performance.

Les résultats des épreuves PISA sont rapportés sur des échelles de compétence dont le score moyen est fixé à 500 points et l'écart type, à 100 points, ce qui signifie que le score de deux tiers des élèves des pays de l'OCDE se situe entre 400 et 600 points. Ces scores correspondent à des niveaux de compétence dans chaque domaine. Les échelles de compréhension de l'écrit, domaine majeur d'évaluation du cycle PISA 2000, ont été divisées en cinq niveaux de connaissances et compétences. Cette approche a pour principal mérite de décrire ce qu'une grande partie des élèves sont capables de faire dans la mesure où les tâches associées à chaque niveau de difficulté sont indiquées. De plus, les résultats sont rapportés sur trois sous-échelles de compétence : localiser et extraire l'information ; intégrer et interpréter le texte; et réfléchir et évaluer. Les résultats des épreuves de mathématiques et de sciences ont également été rapportés sur une échelle de compétence, mais sans distinction de niveaux, les données étant plus limitées puisqu'il s'agissait de domaines mineurs d'évaluation. Lors du cycle PISA 2003, l'échelle de culture mathématique a été divisée en six niveaux, selon la même approche qu'en compréhension de l'écrit, et quatre sous-échelles de compétence ont été élaborées en fonction des contenus : espace et formes; variations et relations ; quantité ; et incertitude. De même, lors du cycle PISA 2006, I'échelle de culture scientifique a été divisée en six niveaux de compétence et trois sous-échelles de compétence ont été élaborées : identifier des questions d’ordre scientifique ; expliquer des phénomènes de manière scientifique; et utiliser des faits scientifiques. De plus, la performance des pays a été comparée dans deux dimensions : les connaissances en sciences et les connaissances à propos des sciences. Les trois grandes catégories de connaissances en sciences sont les systèmes physiques, les systèmes vivants et les systèmes de la Terre et de l'univers.

La compréhension de l'écrit était le domaine majeur d'évaluation du cycle PISA 2009 : c'est la première fois qu'un domaine majeur a été revisité. Le cycle PISA 2009 a permis de présenter des résultats tendanciels dans les trois domaines d'évaluation, c'est-à-dire en compréhension de l'écrit, en mathématiques et en sciences. Lors du cycle PISA 2009, l'échelle de compréhension de l'écrit élaborée en fonction des résultats aux évaluations successive a été étoffée. Au- 
dessus du niveau 5, le niveau le plus élevé de compétence jusque-là, est venu s'ajouter le niveau 6 pour décrire des performances très élevées. Le niveau le moins élevé de compétence, le niveau 1, a été renommé " niveau 1a ", et un nouveau niveau, le niveau $1 \mathrm{~b}$, a été ajouté pour décrire les performances des élèves précédemment classés « sous le niveau 1 » qui parviennent à mener à bien une série de tâches plus faciles que celles incluses dans les épreuves PISA administrées lors des cycles précédents. Ces changements ont permis aux pays d'en apprendre davantage sur ce que les élèves très performants ou très peu performants sont capables de faire. Lors du cycle PISA 2009, la description des autres niveaux, soit les niveaux 2, 3, 4 et 5, est restée inchangée par rapport aux cycles précédents.

Les mathématiques sont évaluées pour la deuxième fois à titre de domaine majeur lors du cycle PISA 2012. À côté des sous-échelles de contenu (la sous-échelle Incertitude devenant Incertitude et données pour plus de clarté), trois souséchelles ont été élaborées pour décrire les processus dans lesquels les élèves s'engagent lorsqu'ils résolvent activement des problèmes. Ces trois sous-échelles de processus sont : formuler des situations de façon mathématique ; employer des concepts, faits, procédures et raisonnements mathématiques; et interpréter, appliquer et évaluer des résultats mathématiques - soit, sous forme abrégée, formuler, appliquer et interpréter.

\section{NATURE ET UTILISATION DES QUESTIONNAIRES CONTEXTUELS}

Pour recueillir des informations contextuelles, I'enquête PISA demande aux élèves et aux chefs d'établissement de répondre à des questionnaires d'une durée de 30 minutes environ. Ces questionnaires jouent un rôle central dans l'analyse des résultats, car ils permettent de mettre en évidence une série de caractéristiques propres aux élèves et aux établissements qu'ils fréquentent. Le chapitre 6 décrit en détail le cadre conceptuel de ces questionnaires. Les questionnaires utilisés lors de tous les cycles d'évaluation (PISA 2000, 2003, 2006, 2009 et 2012) sont accessibles en ligne sur le site de l'enquête PISA (www.pisa.oecd.org). Ces questionnaires sont destinés à recueillir des informations concernant :

- Les élèves et leur milieu familial, notamment le capital économique, social et culturel des élèves et de leur famille.

- Divers aspects de la vie des élèves, notamment leurs attitudes à l'égard de l'apprentissage, leurs habitudes et leur mode de vie à l'école et chez eux.

- Les établissements d'enseignement, notamment la qualité des ressources humaines et matérielles, le mode de gestion et de financement (public ou privé), les processus de prise de décision, le recrutement du personnel enseignant, les contenus privilégiés dans les programmes d'enseignement et les activités extrascolaires proposées.

- Le cadre d'enseignement, notamment les structures institutionnelles, la taille des classes, le climat dans l'établissement et en classe, et les activités de mathématiques en classe.

- Divers aspects relatifs à l'enseignement et à l'apprentissage des mathématiques, notamment l'intérêt des élèves, leur motivation et leur engagement.

Les trois autres questionnaires suivants sont proposés à titre d'option internationale :

- Le questionnaire sur la maîtrise de l'informatique est destiné à recueillir des données sur l'accès des élèves aux technologies de l'information et de la communication (TIC) et l'usage qu'ils en font, notamment l'endroit où ils utilisent le plus souvent les TIC, sur leur capacité à effectuer des tâches sur ordinateur et enfin, sur leurs attitudes à l'égard de I'informatique.

- Le questionnaire sur le parcours scolaire vise à recueillir des informations supplémentaires sur les éventuelles interruptions de la scolarité des élèves, leur préparation à leur carrière professionnelle et le soutien à l'apprentissage linguistique.

- Le questionnaire « Parents » porte sur diverses thématiques, notamment leur opinion sur l'établissement d'enseignement fréquenté par leur enfant et leur implication dans la vie de cet établissement, leur soutien à l'apprentissage de leur enfant, le choix de l'établissement, leurs attentes concernant la profession de leur enfant, en particulier en rapport avec les mathématiques, et leur ascendance allochtone.

Les informations contextuelles recueillies via les questionnaires "Élève » et "Établissement », et via les questionnaires facultatifs sur la maîtrise de l'informatique, le parcours scolaire et le point de vue des parents, ne représentent qu'une partie des données dont dispose l'enquête PISA. Des indicateurs décrivant la structure générale des systèmes d'éducation et leur contexte démographique et économique (par exemple, les coûts, les effectifs d'élèves, les caractéristiques des établissements d'enseignement et des enseignants, et des informations sur un certain nombre de pratiques pédagogiques), ainsi que leur impact sur le marché du travail, sont régulièrement mis à jour par l'OCDE (voir, par exemple, le rapport annuel de l'OCDE Regards sur l'éducation). 


\section{ÉLABORATION CONJOINTE DE L'ENQUÊTE PISA ET DE SES CADRES CONCEPTUELS}

L'enquête PISA est le fruit d'un effort concerté de la part des gouvernements des pays membres de l'OCDE dans le but de mettre en place une nouvelle forme d'évaluation cyclique des acquis des élèves. Les évaluations sont élaborées et approuvées conjointement par les pays participants, puis mises en œuvre par des organisations nationales. La participation constructive des élèves, des enseignants et des chefs d'établissement impliqués dans l'opération a été déterminante pour la réussite de toutes les phases de l'élaboration et de la mise en œuvre de l'enquête PISA.

Le Comité directeur PISA, au sein duquel siègent des délégués représentant les autorités de l'éducation de chaque pays, définit les priorités d'action de l'enquête PISA dans le cadre des objectifs de l'OCDE et veille au respect de ces priorités au cours de la mise en œuvre de l'enquête. Il est chargé de déterminer les priorités concernant l'élaboration des indicateurs, la mise au point des instruments d'évaluation et la présentation des résultats. Des experts des pays participants siègent aussi dans les divers groupes de travail chargés de mettre au service des objectifs de l'enquête PISA les meilleures compétences techniques disponibles à l'échelon international dans les différents domaines d'évaluation. En contribuant aux travaux de ces groupes d'experts, les pays garantissent la validité internationale des instruments d'évaluation et la prise en considération des contextes culturels et éducatifs des pays membres de l'OCDE. Ils veillent aussi à ce que les épreuves constituent d'excellents instruments de mesure, valides et conçus dans un souci d'authenticité pour les élèves.

Les pays participants mettent en œuvre l'enquête PISA à l'échelon national par l'intermédiaire des directeurs nationaux de projet, dans le respect des procédures d'administration convenues. Les directeurs nationaux de projet jouent un rôle de premier plan pour garantir le bon déroulement du projet, et pour contrôler et évaluer les résultats, les analyses, les rapports et les publications.

La conception et la mise en œuvre des évaluations en compréhension de l'écrit, en mathématiques, en sciences, en résolution de problèmes et en culture financière, ainsi que la conception et la mise en œuvre des questionnaires et de la présente enquête, sont sous la responsabilité d'un consortium international dirigé par l'Australian Council for Educational Research (ACER) dans le respect du cadre fixé par le Comité directeur PISA. Les partenaires du consortium sont cApStAn Linguistic Quality Control, le département de pédagogie théorique et expérimentale de I'Université de Liège, en Belgique, le Deutsches Institut für Internationale Pädagogische Forschung (DIPF), en Allemagne, I'Institut national de recherche sur la politique de l'éducation (NIER), au Japon, WESTAT et Educational Testing Service (ETS), aux États-Unis, I'Institutt for Lærerutdanning og Skoleutvikling (ILS), en Norvège, I'Institut Leibnitz de pédagogie (IPN), en Allemagne, et l'initiative TAO (CRP Henri Tudor et Unité EMACS de l'Université de Luxembourg), au Luxembourg. Le Secrétariat de I'OCDE est responsable de la gestion globale de l'enquête. Il suit sa mise en ouvre au jour le jour, assure le secrétariat du Comité directeur PISA, facilite la recherche de consensus entre les pays participants et sert d'interlocuteur entre le Comité directeur PISA et le consortium international chargé de la mise en œuvre des activités. C'est également au Secrétariat de I'OCDE qu'il incombe de produire les indicateurs, de procéder aux analyses et de préparer les publications et les rapports internationaux en collaboration avec le consortium chargé de I'enquête PISA et en concertation avec les pays de l'OCDE, en ce qui concerne les orientations politiques (par l'entremise du Comité directeur PISA) et la réalisation (par l'intermédiaire des directeurs nationaux de projet).

Les cadres d'évaluation PISA sont le fruit d'efforts soutenus depuis la création de l'enquête en 1997. Leur élaboration s'inscrit dans un processus en plusieurs étapes :

- L'élaboration de la définition opérationnelle des domaines d'évaluation et la description des hypothèses qui soustendent cette définition.

- L'étude du mode d'organisation des tâches conçues pour rendre compte de la performance des élèves dans chaque domaine d'évaluation aux décideurs politiques et aux chercheurs, et l'identification des caractéristiques clés à prendre en considération lors de l'élaboration des tâches utilisées à l'échelle internationale.

- L'opérationnalisation des caractéristiques clés utilisées lors de la conception des épreuves, à la lumière des définitions présentes dans la littérature et de l'expérience acquise lors de la mise en œuvre d'autres enquêtes à grande échelle.

- La validation des variables sélectionnées et l'analyse de la contribution de chacune d'entre elles à la description de la difficulté des tâches dans les pays participants.

- La préparation d'un programme d'interprétation des résultats. 
Élaborer et valider un cadre conceptuel dans chacun des domaines d'évaluation a pour principal objectif d'améliorer la qualité des mesures, mais cette approche présente également d'autres avantages potentiels :

- Le cadre conceptuel propose une structure et un langage communs pour débattre de la finalité et de l'objet de l'évaluation. Ces débats facilitent l'établissement d'un consensus sur le cadre conceptuel et les objectifs de l'évaluation.

- L'analyse des types de savoirs et savoir-faire associés aux bonnes performances sert de base à la définition de normes ou de niveaux de compétence. Mieux comprendre ce qui est mesuré et interpréter plus finement la signification des scores sur une échelle de compétence permet de constituer une base empirique pour communiquer un ensemble plus riche d'informations aux divers publics visés.

- Identifier et comprendre les variables spécifiques qui sont associées à de bonnes performances permet d'affiner la méthodologie et d'apporter des améliorations aux évaluations ultérieures.

- Comprendre l'objet des mesures et leur rapport avec les conclusions tirées à propos des élèves est important pour établir des liens entre l'action publique, l'évaluation et la recherche, ce qui, en retour, améliore la pertinence des données recueillies. 



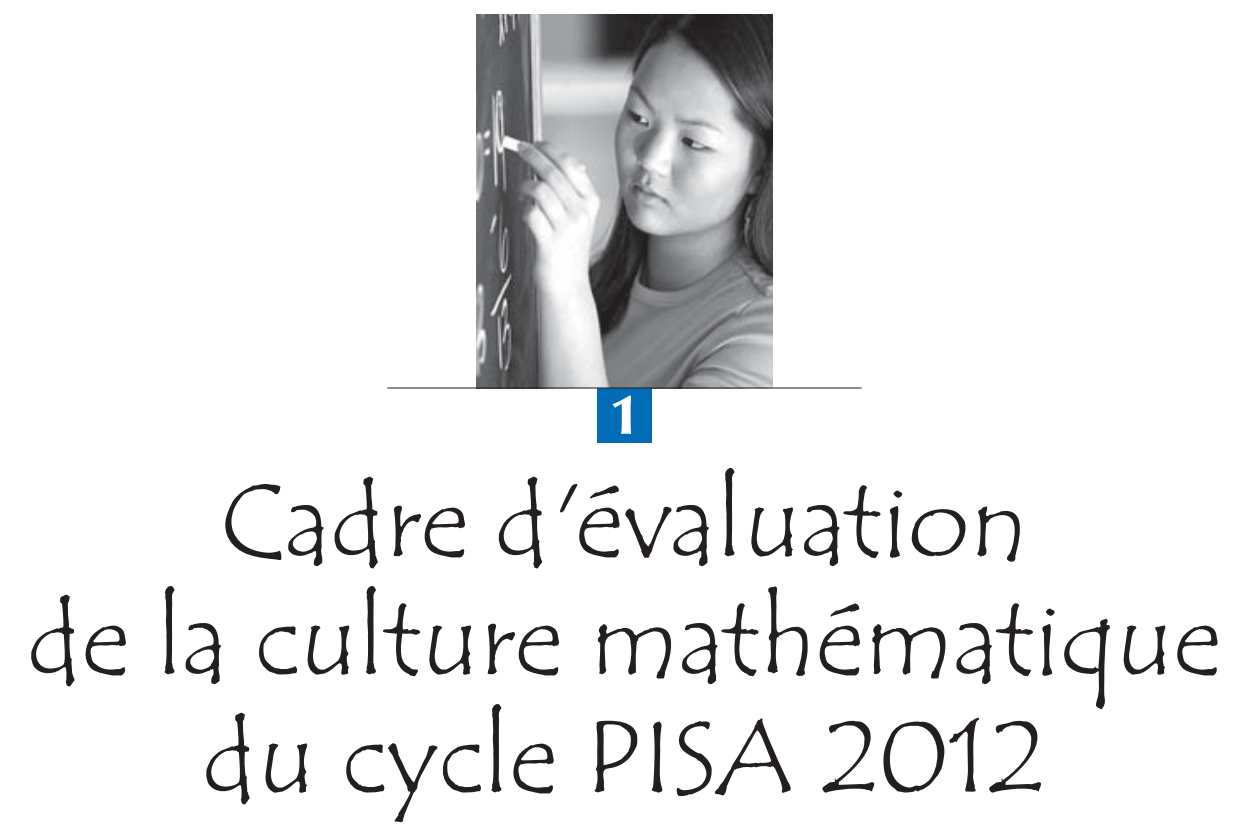

Le cadre conceptuel du cycle PISA 2012 explique les fondements théoriques des épreuves PISA de mathématiques. II actualise la définition de la culture mathématique et décrit les processus mathématiques que les élèves effectuent lorsqu'ils utilisent leur culture mathématique et les facultés mathématiques fondamentales qui sous-tendent ces processus. II présente la répartition des connaissances mathématiques entre quatre catégories de contenus mathématiques ainsi que les connaissances qu'il est pertinent d'évaluer en mathématiques chez les élèves âgés de 15 ans. II décrit les quatre catégories de contextes, c'est-à-dire les situations dans lesquelles les élèves auront à mener à bien des tâches mathématiques. II indique les pourcentages d'items par catégorie de contenus et de contextes, par format d'items et par processus, et décrit la rotation des unités par carnet. Chaque carnet est constitué d'items dont le degré de difficulté varie. Par ailleurs, le présent cadre décrit l'épreuve informatisée de mathématiques et explique la logique qui a présidé à sa conception ainsi que son potentiel de développement. Sept unités administrées lors d'essais de terrain ou de campagnes définitives sont proposées pour illustrer les catégories retenues. De nombreuses mesures de contrôle de la qualité sont décrites. L'évaluation PISA permettra de déterminer dans quelle mesure les pays préparent leurs élèves à utiliser les mathématiques dans tous les aspects de leur vie personnelle, civique et professionnelle, dans le cadre d'une citoyenneté constructive, engagée et réfléchie. 


\section{INTRODUCTION}

L'évaluation de la culture mathématique revêt une importance particulière dans le cadre du cycle PISA 2012, car c'est son domaine majeur d'évaluation. L'enquête PISA a déjà évalué la culture mathématique en 2000, 2003, 2006 et 2009, mais une fois seulement à titre de domaine majeur d'évaluation, en l'occurrence en 2003.

Comme les mathématiques sont une nouvelle fois le domaine majeur d'évaluation d'un cycle PISA, c'est l'occasion de suivre l'évolution de la performance des élèves au fil du temps et de réexaminer les aspects évalués à la lumière des changements intervenus dans la discipline ainsi que dans les politiques et pratiques pédagogiques en la matière. Tout l'enjeu est donc d'actualiser le cadre d'évaluation de la culture mathématique, tout en conservant les liens psychométriques avec les évaluations antérieures au travers d'une ligne tendancielle prospective et rétrospective. Le cadre d'évaluation du cycle PISA 2012 cherche à décrire de façon plus claire et explicite les aspects mathématiques pertinents pour des élèves âgés de 15 ans, tout en assurant que les items administrés s'inscrivent dans des contextes authentiques et porteurs de sens. Le cycle de modélisation mathématique utilisé dans les cadres précédents (voir, par exemple, OCDE, 2003) pour décrire les étapes que les individus enchaînent pour résoudre des problèmes contextualisés reste la clé de voûte du cadre d'évaluation du cycle PISA 2012. II est employé pour définir les processus mathématiques dans lesquels les élèves s'engagent quand ils résolvent des problèmes - ces processus seront utilisés pour la première fois comme dimension première de compte rendu. Une nouvelle option d'épreuve informatisée de mathématiques (Computer-Based Assessment of Mathematics, CBAM) est également proposée en 2012.

Le cadre d'évaluation de la culture mathématique du cycle PISA 2012 se divise en plusieurs grandes sections. La première section, "Définition de la culture mathématique », décrit les fondements théoriques de l'épreuve PISA de mathématiques et définit le construct ${ }^{1}$ de culture mathématique. La deuxième section, "Organisation du domaine », décrit trois aspects : i) les processus mathématiques et les facultés mathématiques fondamentales (appelées « compétences » dans les cadres d'évalutaion antérieurs) qui sous-tendent ces processus ; ii) les catégories de contenus mathématiques pertinentes pour les élèves âgés de 15 ans et la façon dont elles s'organisent dans le cadre d'évaluation du cycle PISA 2012 (les scores des élèves sont rapportés à la fois en fonction des trois catégories de processus mathématiques et en fonction des quatre catégories de contenus mathématiques); et iii) les contextes dans lesquels s'inscrivent les tâches mathématiques soumises aux élèves. La troisième section, "Évaluation de la culture mathématique ", décrit des enjeux structurels de l'épreuve et fournit des informations d'ordre technique. Les annexes décrivent les facultés mathématiques fondamentales de manière plus détaillée et proposent des items PISA à titre d'exemple. Les références bibliographiques figurent également en annexe.

Ce cadre a été rédigé sous la direction du groupe d'experts chargé des mathématiques (Mathematics Expert Group, MEG), constitué par les principaux contractants PISA après approbation du Comité directeur PISA (PISA Governing Board, PGB). Siègent dans ce groupe de dix membres des mathématiciens, des professeurs de mathématiques et des spécialistes en évaluation, en technologie et en pédagogie de divers pays. Par ailleurs, une version préliminaire de ce cadre d'évaluation de la culture mathématique a été soumise pour commentaires à plus de 170 experts en mathématiques de plus d'une quarantaine de pays, dans le but de susciter l'apport de contributions plus larges et de garantir sa révision approfondie. Achieve et l'Australian Council for Educational Research (ACER), les deux organisations choisies par l'Organisation de coopération et de développement économiques (OCDE) pour gérer l'élaboration du cadre d'évaluation, ont également mené diverses recherches pour éclairer la conception du cadre et l'étayer. L'enquête PISA et l'élaboration du cadre d'évaluation ont bénéficié des travaux actuels dans des pays participants (par exemple, les recherches décrites dans la publication de I'OCDE Pathways to Success: How Knowledge and Skills at Age 15 Shape Future Lives in Canada, 2010).

\section{DÉFINITION DE LA CULTURE MATHÉMATIQUE}

Comprendre les mathématiques est essentiel pour préparer les jeunes à vivre dans une société moderne. Il faut s'appuyer sur un certain degré de compréhension des mathématiques et sur des facultés de raisonnement mathématique et d'utilisation des mathématiques pour pouvoir appréhender un nombre croissant de situations et de problèmes qui surviennent dans la vie courante, y compris dans le cadre professionnel, et pouvoir y faire face. Les mathématiques sont un outil indispensable pour permettre aux jeunes d'apprhénender les problèmes qui surviennent dans différents contextes - personnel, professionnel, sociétal ou scientifique. Dans ce contexte, il est important de déterminer dans quelle mesure les jeunes approchant du terme de leur scolarité sont préparés à utiliser les mathématiques pour comprendre des enjeux importants et résoudre des problèmes. Évaluer les jeunes à l'âge de 15 ans permet de montrer par anticipation comment les individus sont susceptibles de réagir plus tard dans la vie dans un large éventail de situations en rapport avec les mathématiques. 
Il est raisonnable de se poser la question suivante pour déterminer ce qui doit être à la base d'une évaluation internationale à administrer aux élèves âgés de 15 ans : "Qu'est-ce qu'il est important que les citoyens sachent et soient capables de faire dans des situations en rapport avec les mathématiques? ». Ou plus précisément, que signifie la culture mathématique à l'âge de 15 ans, que ce soit dans la perspective de quitter l'école, de suivre une formation professionnelle ou d'entrer à l'université ? Il est important d'expliquer ici que le construct de culture mathématique, qui est utilisé dans ce rapport pour évoquer la capacité des individus à formuler, employer et interpréter les mathématiques dans divers contextes, ne peut s'assimiler à un niveau minimal, peu élevé, de connaissances et compétences. Au contraire, ce construct cherche à définir la capacité des individus à mener un raisonnement mathématique et à utiliser des concepts, procédures, faits et outils mathématiques pour décrire, expliquer et prévoir des phénomènes. Cette conception de la culture mathématique confirme combien il est important que les élèves parviennent à bien comprendre des concepts de mathématiques pures et à se rendre compte des avantages qu'il y a à s'engager dans l'exploration du monde abstrait des mathématiques. Le construct PISA de culture mathématique insiste fortement sur la nécessité de développer chez les élèves la faculté d'utiliser les mathématiques en contexte; il est important qu'ils vivent de riches expériences lors de leurs cours de mathématiques pour y parvenir. C'est vrai pour tous les élèves âgés de 15 ans, qu'ils approchent du terme de leur formation en mathématiques ou qu'ils la poursuivent. De plus, beaucoup estiment que la plupart des élèves sont plus motivés à l'idée d'apprendre les mathématiques lorsqu'ils sont conscients que ce qu'ils apprennent est utile non seulement dans d'autres matières, mais également en dehors du cadre scolaire.

La culture mathématique transcende bien entendu les groupes d'âge. Toutefois, pour l'évaluer chez les élèves âgés de 15 ans, il faut tenir compte de caractéristiques pertinentes propres à ces élèves ; d'où la nécessité d'identifier des contenus appropriés à leur âge, leur langage et leurs contextes. Ce cadre distingue plusieurs grandes catégories de contenus qui sont importantes dans la culture mathématique des individus en général et de contenus spécifiques qui sont appropriés pour les élèves âgés de 15 ans. La culture mathématique n'est pas un attribut que les individus possèdent ou non. C'est un attribut qui se situe sur un continuum, certains individus possédant une plus grande culture mathématique que d'autres - mais tous les individus peuvent la développer.

La culture mathématique se définit comme suit dans le cadre du cycle PISA 2012 :

La culture mathématique est l'aptitude d'un individu à formuler, employer et interpréter des mathématiques dans un éventail de contextes, soit de se livrer à un raisonnement mathématique et d'utiliser des concepts, procédures, faits et outils mathématiques pour décrire, expliquer et prévoir des phénomènes. Elle aide les individus à comprendre le rôle que les mathématiques jouent dans le monde et à se comporter en citoyens constructifs, engagés et réfléchis, c'est-àdire à poser des jugements et à prendre des décisions en toute connaissance de cause.

Quelques explications sont fournies ci-dessous pour clarifier et mettre en lumière certains aspects de la définition qui sont particulièrement importants.

\section{PISA 2012 : Des élèves acteurs de la résolution de problèmes}

La définition de la culture mathématique insiste sur la notion d'engagement actif dans les mathématiques et vise le raisonnement mathématique et l'utilisation de concepts, procédures, faits et outils mathématiques pour décrire, expliquer et prévoir des phénomènes. Les verbes «formuler », " employer » et " interpréter » désignent plus particulièrement les trois processus dans lesquels les élèves s'engagent en tant qu'acteurs de la résolution de problèmes. Le fait de formuler les choses de façon mathématique consiste à identifier des possibilités d'appliquer et d'utiliser les mathématiques - c'est-àdire de se rendre compte que les mathématiques peuvent servir à comprendre ou résoudre tel ou tel problème-, soit appréhender une situation telle qu'elle se présente, la traduire sous une forme qui se prête à un traitement mathématique, générer une structure ou des représentations mathématiques, identifier des variables et faire des hypothèses simplificatrices qui aideront à résoudre le problème ou à relever le défi. Le fait d'employer les mathématiques consiste à suivre un raisonnement mathématique et à utiliser des concepts, procédures, faits et outils mathématiques pour dégager une solution mathématique, c'est-à-dire à effectuer des calculs, à manipuler des opérations algébriques, des équations ou d'autres modèles mathématiques, à analyser des données de façon mathématique dans des graphiques ou des diagrammes mathématiques, à élaborer des descriptions ou des explications mathématiques et à utiliser des outils mathématiques pour résoudre des problèmes. Le fait d'interpréter les mathématiques consiste à réfléchir à des résultats ou des solutions mathématiques et à les interpréter dans le contexte d'un problème ou d'un défi, c'est-à-dire à évaluer des solutions mathématiques ou à raisonner de façon mathématique dans le contexte d'un problème, et à déterminer si les résultats sont plausibles et conviennent à la situation. 
Cette définition de la culture mathématique retenue en vue du cycle PISA 2012 vise également à intégrer la notion de modélisation mathématique, qui est depuis toujours une pierre angulaire du cadre d'évaluation de la culture mathématqiue de l'enquête PISA (OCDE, 2003). Les individus enchaînent les étapes à mesure qu'ils utilisent les mathématiques et les outils mathématiques pour résoudre des problèmes contextualisés. La figure 1.1 donne un aperçu des constructs majeurs de ce cadre et indique les relations entre chacun d'entre eux.

- Le cadre extérieur de la figure 1.1 montre que la culture mathématique s'exprime dans le contexte d'un défi ou d'un problème qui se pose dans le monde réel. Dans ce cadre, ces défis ou problèmes se caractérisent en fonction de deux dimensions. La première dimension est le contexte - dont les catégories sont décrites ci-après - qui identifie le domaine de la vie dans lequel le problème se produit. Le contexte peut être personnel, auquel cas les problèmes ou défis sont ceux qu'un individu peut rencontrer dans sa vie personnelle ou familiale, ou ses relations avec des proches. Les problèmes peuvent aussi s'inscrire dans un contexte sociétal (la communauté locale, nationale ou mondiale), professionnel (en rapport avec le monde du travail) ou scientifique (en rapport avec l'application des mathématiques au monde naturel ou technologique). La seconde dimension qui caractérise les problèmes ou les défis est la nature du phénomène mathématique qui les sous-tend. Les quatre catégories de contenus mathématiques identifient de grands groupes de phénomènes pour l'analyse desquels les mathématiques ont été créées. Ces catégories de contenus mathématiques (Quantité, Incertitude et données, Variations et relations et enfin, Espace et formes) sont également identifiées dans le cadre extérieur de la figure 1.1.

- Pour résoudre des problèmes contextualisés, les individus doivent se livrer à des raisonnements et des actions mathématiques, que le présent cadre caractérise en fonction de trois dimensions. En premier lieu, la figure 1.1 reconnaît que les individus doivent se baser sur divers concepts, savoirs et savoir-faire mathématiques. Ces connaissances en mathématiques interviennent lorsque les individus représentent la situation et la formulent de façon mathématique, élaborent des stratégies, formulent des raisons et des arguments, et ainsi de suite. Le cadre conceptuel caractérise ces actes mathématiques en fonction des sept facultés mathématiques fondamentales qui sont reprises dans la figure 1.1 et décrites en détail ci-après. Lorsqu'un individu travaille à la résolution d'un problème - ce qui requiert de sa part qu'il le formule, qu'il emploie des concepts ou des procédures mathématiques, ou qu'il interprète une solution mathématique -, il exploite successivement ou simultanément des facultés mathématiques fondamentales, et se base sur des contenus mathématiques appropriés pour créer une solution.

- La représentation graphique du cycle de modélisation mathématique qui figure dans le cadre intérieur de la figure 1.1 est une version idéalisée et simplifiée des étapes qu'enchaîne celui qui résout un problème à l'aide de sa culture mathématique. C'est une série idéalisée d'étapes qui commence par le "problème contextualisé ». L'individu qui s'attelle à la résolution du problème tente d'identifier les aspects mathématiques de la situation et de formuler celle-ci de façon mathématique en fonction des concepts et des relations qu'il a repérés, et des hypothèses simplificatrices qu'il a élaborées. Il traduit donc le " problème contextualisé » en un "problème mathématique » qui se prête à un traitement mathématique. Dans la figure 1.1, la flèche vers le bas décrit le travail entrepris lorsque l'individu emploie des concepts, procédures, faits et outils mathématiques pour obtenir des "résultats mathématiques ». Cette étape consiste généralement à raisonner, à manipuler, à transformer et à calculer. Lors de l'étape suivante, les « résultats mathématiques » doivent être contextualisés, c'est-à-dire interprétés en fonction du problème initial (ce qui aboutit aux « résultats contextualisés »). Pour ce faire, l'individu doit interpréter, appliquer et évaluer les résultats mathématiques, et juger de leur plausibilité dans le contexte du problème qui s'inscrit dans le monde réel. Ces processus qui consistent à formuler, à employer et à interpréter de façon mathématique sont des composantes majeures du cycle de modélisation mathématique et des composantes majeures de la définition de culture mathématique. Ces trois processus reposent sur des facultés mathématiques fondamentales, qui reposent à leur tour sur les connaissances mathématiques détaillées de l'individu à propos de thématiques spécifiques.

Le cycle de modélisation est au cœur de la conception PISA des élèves, en l'occurrence des acteurs de la résolution de problèmes. Toutefois, il est souvent inutile de se livrer à chaque étape du cycle de modélisation, en particulier lors d'une évaluation (Niss et al., 2007). Il est fréquent, en effet, qu'une partie substantielle du cycle de modélisation ait été effectuée par d'autres et que celui qui tente de résoudre le problème n'ait qu'à entreprendre quelques-unes des étapes. Ainsi, dans certains cas, des représentations mathématiques, telles que des graphiques ou des équations, sont fournies et peuvent être manipulées directement pour répondre à des questions ou tirer des conclusions. C'est la raison pour laquelle de nombreux items PISA n'impliquent qu'une partie du cycle de modélisation. Dans la réalité, les individus doivent aussi passer d'un processus à l'autre et revenir en arrière pour corriger des décisions ou des hypothèses. Chaque processus peut être relativement exigeant et plusieurs itérations peuvent se révéler nécessaires au cours du cycle de modélisation. 


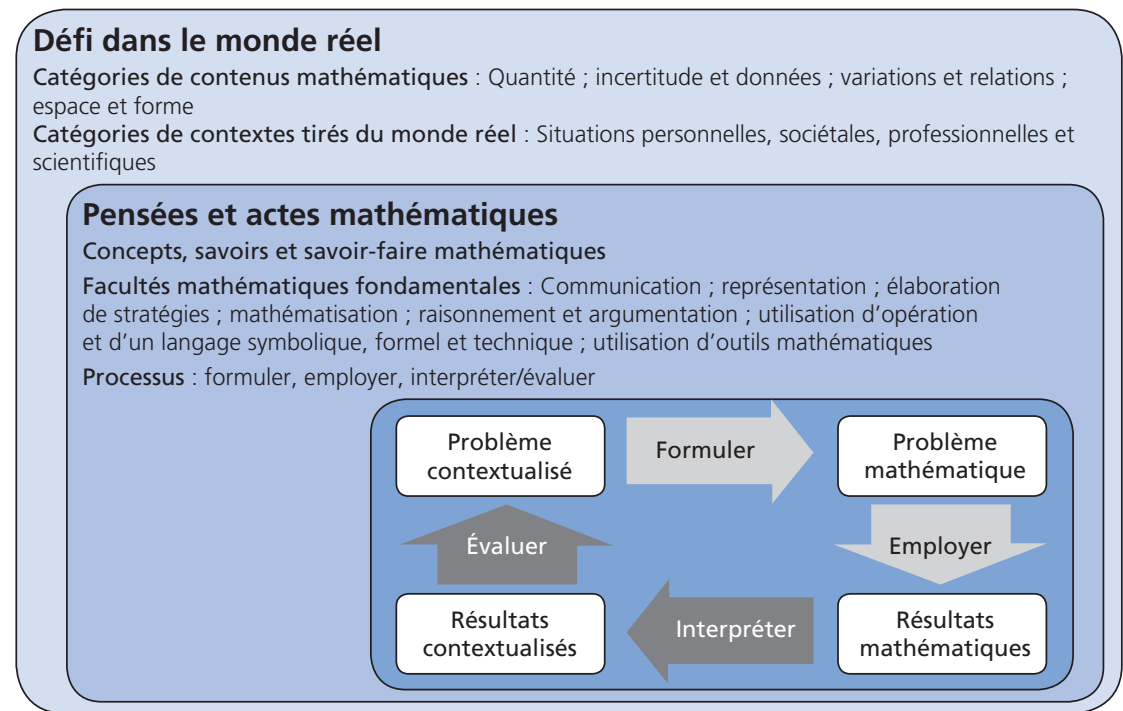

\section{Un lien explicite vers un éventail de contextes dans les problèmes du cycle PISA 2012}

C'est à dessein qu'il est fait référence à un « éventail de contextes » dans la définition de la culture mathématique, en l'occurrence pour établir un lien avec les contextes spécifiques qui sont décrits de façon plus détaillée ci-après, exemples à l'appui. En soi, les contextes spécifiques ne revêtent pas beaucoup d'importance ; en revanche, les catégories de contextes retenus (personnels, professionnels, sociétaux et scientifiques) reflètent une grande diversité de situations en rapport avec les mathématiques. La définition reconnaît également que la culture mathématique aide les individus à comprendre le rôle que les mathématiques jouent dans le monde, ainsi qu'à poser des jugements fondés et à prendre des décisions en toute connaissance de cause, ce que l'on attend de citoyens constructifs, engagés et réfléchis.

\section{Le rôle visible des outils mathématiques, y compris de la technologie, dans le cycle PISA 2012}

La définition de la culture mathématique inclut explicitement l'utilisation d'outils mathématiques, notamment des appareils, des équipements numériques, des logiciels et des systèmes de calcul². Parmi ces outils mathématiques, les outils informatiques sont $d^{\prime}$ 'usage courant dans le cadre professionnel au XXI'e siècle et le seront encore plus à l'avenir. La nature des problèmes et des raisonnements logiques propres au monde du travail s'est développée avec cette évolution - créant du même coup des attentes plus grandes en matière de culture mathématique.

L'épreuve informatisée de mathématiques est une première dans l'enquête PISA ; les pays participants qui le souhaitent peuvent l'administrer lors du cycle PISA 2012. S'agissant de cette épreuve, la référence aux outils mathématiques dans la définition de la culture mathématique est particulièrement pertinente. L'utilisation de calculatrices a été autorisée lors de toutes les épreuves PISA de mathématiques administrées à ce jour dans les pays où cela était conforme aux principes en vigueur. Par le passé, les items PISA de mathématiques étaient autant que possible conçus pour ne pas être sensibles à l'usage ou non d'une calculatrice. Dans certains items de mathématiques soumis aux élèves dans l'épreuve papier-crayon lors du cycle PISA 2012, I'usage d'une calculatrice peut apporter un plus; et dans l'épreuve informatisée de mathématiques, des outils mathématiques tels qu'une calculatrice en ligne sont fournis dans le cadre de certains items. Comme les items PISA s'inspirent de problèmes qui surviennent dans des contextes personnels, professionnels, sociétaux et scientifiques où il est d'usage d'utiliser une calculatrice, une calculatrice peut être utile pour répondre à certains items. L'épreuve informatisée de mathématiques offre l'occasion de fournir un éventail plus large d'outils mathématiques - par exemple, des logiciels d'analyse statistique, de construction géométrique, de visualisation, de mesure, etc. - dans les items. Cela reflète le média que de plus en plus d'individus utilisent lorsqu'ils sont interaction avec le monde qui les entoure et qu'ils résolvent des problèmes. C'est également l'occasion d'évaluer certains aspects de la culture mathématique qu'il est difficile d'appréhender lors d’une épreuve papier-crayon classique. 


\section{ORGANISATION DU DOMAINE D'ÉVALUATION}

Le cadre PISA de mathématiques définit le domaine des mathématiques tel que l'enquête PISA l'évalue et décrit I'approche adoptée pour mesurer la culture mathématique des adolescents de 15 ans. En fait, I'enquête PISA cherche à déterminer dans quelle mesure les élèves âgés de 15 ans sont capables d'utiliser les mathématiques dans les situations et problèmes (dont la plupart s'inscrivent dans des contextes s'inspirant du monde réel) qui se présentent à eux.

La définition de la culture mathématique retenue à l'occasion du cycle PISA 2012 englobe trois aspects interdépendants :

- les processus mathématiques, qui décrivent ce que font des individus pour établir un lien entre le contexte du problème et les mathématiques et, donc, pour résoudre le problème, ainsi que les facultés qui sous-tendent ces processus ;

- les contenus mathématiques, qui doivent être utilisés pour répondre aux items ; et

- les contextes dans lesquels les items s'inscrivent.

Les sections suivantes développent ces aspects. Le cadre d'évaluation de la culture mathématique du cycle PISA 2012 met ces aspects en évidence de manière à ce que les items mis au point reflètent un éventail varié de processus, de contenus et de contextes, et qu'ensemble, ils traduisent concrètement et fidèlement la culture mathématique telle qu'elle est définie ici. Plusieurs questions dérivées de la définition de la culture mathématique retenue à l'occasion du cycle PISA 2012 sont à la base de cette section :

- Quels sont les processus dans lesquels les individus s'engagent lorsqu'ils résolvent des problèmes de mathématiques en contexte? Quelles facultés attendons-nous qu'ils exploitent à mesure qu'ils enrichissent leur culture mathématique?

- Quels contenus mathématiques attendons-nous que les individus - en particulier les élèves âgés de 15 ans - maîtrisent?

- Dans quels contextes la culture mathématique peut-elle s'observer et s'évaluer?

\section{Les processus mathématiques et les facultés mathématiques qui les sous-tendent}

\section{Les processus mathématiques}

Par définition, la culture mathématique renvoie à la capacité des individus de formuler, d'employer et d'interpréter les mathématiques. Ces trois verbes, «formuler», « employer » et « interpréter », constituent à eux seuls une structure signifiante qui permet de définir les processus mathématiques qui décrivent ce que les individus font pour établir un lien entre le contexte d'un problème et les mathématiques et, donc, pour résoudre le problème. Les épreuves de mathématiques du cycle PISA 2012 permettront pour la première fois de rendre compte des résultats des élèves en fonction de ces processus mathématiques, une structure qui fournira des catégories utiles et pertinentes pour l'action publique. Ces catégories qui seront utilisées pour rendre compte des résultats sont les suivantes :

- formuler des situations de façon mathématique ;

- employer des concepts, faits, procédures et raisonnements mathématiques ; et

- interpréter, appliquer et évaluer des résultats mathématiques.

Il est important, tant pour les décideurs que pour les professionnels impliqués plus directement dans l'instruction des élèves, de savoir dans quelle mesure les adolescents sont capables de s'engager dans ces processus. Les résultats de I'enquête PISA concernant le processus de formulation montreront dans quelle mesure les élèves sont capables d'identifier et de reconnaître des possibilités d'utiliser les mathématiques dans le contexte d'un problème, puis de proposer la structure mathématique requise pour formuler le problème contextualisé sous la forme d'un problème mathématique. Les résultats de l'enquête PISA concernant le processus d'emploi des mathématiques montreront dans quelle mesure les élèves sont capables d'effectuer des calculs et des manipulations, et d'appliquer les concepts et les faits qu'ils connaissent pour proposer une solution mathématique à un problème formulé de façon mathématique. Les résultats de l'enquête PISA concernant le processus d'interprétation montreront dans quelle mesure les élèves sont capables de réfléchir à des conclusions ou des solutions mathématiques, de les interpréter dans le contexte d’un problème qui s'inspire du monde réel et de déterminer si les conclusions ou les résultats sont plausibles. La facilité avec laquelle les élèves appliquent les mathématiques dans des problèmes ou des situations dépend de compétences inhérentes à ces trois processus. Évaluer leur aisance par processus peut éclairer les décideurs, alimenter leurs débats et les aider à prendre des décisions plus en prise avec ce qui se passe en classe.

\section{Formuler des situations de façon mathématique}

Dans la définition de la culture mathématique, le verbe « formuler » renvoie à la capacité des individus d’identifier et de reconnaître des possibilités d'utiliser les mathématiques dans le contexte d'un problème, puis de structurer sous forme 
mathématique un problème présenté jusqu'à un certain point sous une forme contextualisée. Lors de ce processus de formulation mathématique, les individus déterminent les mathématiques essentielles à utiliser pour analyser, configurer et résoudre le problème. Ils transposent dans le domaine des mathématiques un problème qui s'inscrit dans un contexte tiré du monde réel, et lui donnent une structure, une représentation et une spécificité d'ordre mathématique. Ils réfléchissent aux contraintes et aux hypothèses, en découvrent le sens et raisonnent à leur sujet. Plus précisément, le processus qui consiste à formuler des situations de façon mathématique englobe des activités telles que celles énumérées ci-dessous :

- identifier les aspects mathématiques et les variables significatives d'un problème se situant dans un contexte tiré du monde réel ;

- reconnaître des structures mathématiques (des régularités, des relations, des récurrences, etc.) dans des problèmes ou des situations ;

- simplifier une situation ou un problème pour qu'il se prête à une analyse mathématique ;

- identifier les contraintes et les hypothèses qui sous-tendent toute modélisation mathématique et les simplifications extraites du contexte ;

- représenter la situation de façon mathématique à l'aide de variables, de symboles, de diagrammes et de modèles appropriés ;

- représenter le problème d'une autre façon, notamment l'organiser en fonction de concepts mathématiques et élaborer les hypothèses appropriées ;

- comprendre et expliquer les relations entre le langage spécifique au contexte employé pour décrire le problème et le langage symbolique et formel indispensable pour le représenter sous une forme mathématique ;

- traduire le problème en langage ou en représentation mathématique ;

- reconnaître les aspects du problème qui correspondent à des problèmes connus ou à des concepts, faits et procédures mathématiques; et

- utiliser la technologie (un tableur ou les fonctions d'une calculatrice graphique) pour décrire une relation mathématique inhérente, dans un problème contextualisé.

L'item PIZZAS (voir la section «Exemples d'items PISA » en fin de chapitre) fait largement appel aux compétences des élèves en matière de formulation mathématique. Pour répondre à cet item, les élèves doivent aussi effectuer des calculs et interpréter les résultats de leurs calculs pour déterminer quelle pizza est meilleur marché, mais tout l'enjeu cognitif de l'item réside dans leur capacité à formuler un modèle mathématique qui décrit la notion de rapport quantité-prix. Les élèves doivent donc comprendre qu'étant donné que les pizzas sont de la même épaisseur, mais d'un diamètre différent, ils doivent concentrer leur analyse sur la surface circulaire des pizzas. La relation entre la quantité de pizza et la somme d'argent peut alors être intégrée dans la notion du rapport quantité-prix, qui est modélisée en fonction d'un coût par unité de surface. L'item CONCERT ROCK (voir la section «Exemples d'items PISA » en fin de chapitre) illustre lui aussi les items dont la résolution dépend essentiellement de la capacité des élèves à formuler une situation de façon mathématique (en l'espèce, la superficie et la forme du terrain, le fait que le concert est complet et que les fans sont debout) et à traduire ces informations sous une forme mathématique sensée pour estimer le nombre de personnes qui assistent au concert.

\section{Employer des concepts, faits, procédures et raisonnements mathématiques}

Dans la définition de la culture mathématique, le verbe « employer » renvoie à la capacité des individus d'appliquer des concepts, faits, procédures et raisonnements mathématiques pour résoudre des problèmes énoncés de façon mathématique afin d'aboutir à des conclusions mathématiques. Au cours de ce processus qui consiste à employer des concepts, faits, procédures et raisonnements mathématiques, les individus appliquent les procédures mathématiques requises pour dériver des résultats et trouver une solution mathématique (effectuer des opérations arithmétiques, résoudre des équations, faire des déductions logiques à partir d'hypothèses mathématiques, faire des manipulations symboliques, extraire des informations de tableaux et graphiques, représenter et manipuler des formes dans l'espace, et analyser des données). Ils travaillent sur un modèle de la situation du problème, identifient des récurrences et des relations entre des entités mathématiques, et formulent des arguments mathématiques. Ce processus qui consiste à employer des concepts, faits, procédures et raisonnements mathématiques englobe des activités telles que celles énumérées ci-dessous :

- concevoir et appliquer des stratégies en vue de trouver des solutions mathématiques ;

- utiliser des outils mathématiques, dont des applications technologiques, pour faciliter la recherche d'une solution précise ou approximative ; 
- appliquer des faits, des lois, des algorithmes et des structures mathématiques à la recherche de la solution ;

- manipuler des nombres, des informations et des données graphiques et statistiques, des équations et des expressions algébriques, ainsi que des représentations géométriques ;

- élaborer des structures, des diagrammes et des graphiques mathématiques, et en extraire des informations mathématiques ;

- utiliser différentes représentations et passer de l'une à l'autre durant le processus de résolution du problème ;

- faire des généralisations à partir des résultats de l'application de procédures mathématiques pour trouver des solutions ; et

- réfléchir à des arguments mathématiques, et expliquer et justifier des résultats mathématiques.

Parmi les unités PISA rendues publiques, I'unité MARCHE À PIED (voir la section «Exemples d'items PISA »en fin de chapitre) comporte des items qui demandent essentiellement aux élèves d'employer des concepts, faits, procédures et raisonnements mathématiques. Les deux items de cette unité dépendent de l'utilisation d'un modèle donné, en l'espèce une formule, pour déterminer la longueur de pas (dans le premier item) et la vitesse de marche (dans le deuxième item). Les deux questions sont formulées en des termes qui se présentent déjà sous une structure mathématique, et les élèves doivent faire des manipulations et des opérations algébriques pour trouver la solution. Parmi les unités PISA publiées, I'unité MENUISIER (voir la section «Exemples d'items PISA » en fin de chapitre) demande essentiellement aussi aux élèves d'employer des concepts, faits, procédures et raisonnements mathématiques. La plus grande difficulté cognitive est de concevoir une stratégie pour trouver des informations au sujet de la longueur totale d'un tracé composé de segments dont la longueur est inconnue, puis de se livrer à un raisonnement pour comparer les longueurs. Les élèves doivent aussi établir un lien entre les diagrammes, le jardin et les périmètres, d'une part, et la longueur des planches disponibles, d'autre part, mais ce processus de formulation est nettement moins difficile que le raisonnement à propos de la longueur des périmètres.

\section{Interpréter, appliquer et évaluer des résultats mathématiques}

Dans la définition de la culture mathématique, le verbe « interpréter » renvoie à la capacité des individus de réfléchir à des solutions, des résultats ou des conclusions mathématiques, et de les interpréter dans le cadre de problèmes tirés du monde réel. Ce processus consiste à traduire des solutions mathématiques ou à replacer le raisonnement dans le contexte du problème, et à déterminer si les résultats sont plausibles et sont appropriés dans le contexte du problème. Ce processus mathématique est représenté par les flèches "Interpréter » et "Évaluer » dans le modèle de culture mathématique décrit à la figure 1.1. Les individus qui s'engagent dans ce processus peuvent être amenés à formuler et communiquer des explications et des arguments dans le contexte du problème, en réfléchissant au processus de modélisation et à ses résultats. Ce processus qui consiste à interpréter, appliquer et évaluer des résultats mathématiques englobe des activités telles que celles énumérées ci-dessous :

- interpréter un résultat mathématique en fonction de la situation initiale du problème ;

- évaluer la plausibilité d'une solution mathématique dans le contexte d'un problème qui s'inspire du monde réel ;

- comprendre en quoi le monde réel a un impact sur les résultats et les calculs d'un modèle ou d'une procédure mathématique pour poser des jugements en contexte sur la façon d'appliquer ou d'ajuster les résultats ;

- expliquer pourquoi une conclusion ou un résultat mathématique est ou n'est pas plausible dans le contexte d’un problème ;

- comprendre la portée et les limites de concepts et de résultats mathématiques; et

- critiquer le modèle utilisé pour résoudre le problème et en identifier les limites.

Parmi les items PISA rendus public, l'item DÉCHETS (voir la section « Exemples d'items PISA » en fin de chapitre) demande essentiellement aux élèves d'exploiter leur capacité d'interpréter, appliquer et évaluer des résultats mathématiques, en l'occurrence de déterminer si un résultat mathématique - en l'espèce un diagramme en bâtons qu'ils ont dessiné ou imaginé - est pertinent pour décrire les données présentées au sujet du temps de décomposition de plusieurs types de déchets. Les élèves doivent se livrer à un raisonnement à propos des données fournies et à une réflexion mathématique à propos de la relation entre les données et la façon dont elles sont présentées, puis évaluer les résultats. Ils doivent expliquer pourquoi un diagramme en bâtons n'est pas approprié pour présenter les données fournies.

\section{Facultés mathématiques fondamentales sous-tendant les processus mathématiques}

Les dix années passées à concevoir des items PISA et à analyser la façon dont les élèves y répondent ont révélé l'existence d'une série de facultés mathématiques fondamentales qui sous-tendent concrètement chacun des processus 
mathématiques retenus et la culture mathématique. Dans le cadre de leurs travaux, Mogens Niss et ses collègues danois (Niss, 2003 ; Niss et Jensen, 2002 ; Niss et Højgaard, 2011) ont identifié huit facultés - appelées « compétences » par Niss et également désignées de cette manière dans le cadre d'évaluation de la culture mathématique du cycle PISA 2003 (OCDE, 2003) - qui jouent un rôle primordial dans le comportement mathématique. Le cadre du cycle PISA 2012 reprend cette série de facultés, mais les condense pour les limiter à sept sur la base des recherches menées par le MEG sur leur utilisation lors de la résolution d'items déjà administrés (Turner et al., à paraître). Il est communément admis qu'il est indispensable d'identifier une série de facultés mathématiques générales en complément du rôle des contenus mathématiques spécifiques dans I'apprentissage des mathématiques. Citons, entre autres exemples connus, les huit pratiques mathématiques que prévoient les Common Core State Standards aux États-Unis (2010), les quatre processus mathématiques fondamentaux (représenter, analyser, interpréter et évaluer, et enfin, communiquer et réfléchir) du programme national de mathématiques en Angleterre (Qualifications and Curriculum Authority, 2007) et les processus de base retenus par le National Council of Teachers of Mathematics (NCTM) (Principles and Standards for School Mathematics, NCTM, 2000). Ces facultés cognitives peuvent être apprises par les individus pour comprendre le monde et s'y impliquer d'une façon mathématique ou résoudre des problèmes. À mesure que le degré de culture mathématique des individus augmente, ils peuvent faire appel de manière plus poussée à des facultés mathématiques fondamentales (Turner et Adams, 2012). L'activation croissante des facultés mathématiques fondamentales est donc associée à la difficulté croissante des items. Cette observation est à la base de la description des différents niveaux de compétence en culture mathématique retenus lors des cycles précédents de I'enquête PISA. Nous y reviendrons ultérieurement, dans l'encadré 1.1.

Les sept facultés mathématiques fondamentales retenues dans ce cadre d'évaluation sont les suivantes :

- Communication : la culture mathématique inclut la communication. Les individus perçoivent l'existence d'un défi, ce qui les stimule pour reconnaître et comprendre un problème contextualisé. Lire, décoder et interpréter des énoncés, des questions, des tâches ou des données permet aux individus de se construire un modèle mental de la situation, ce qui constitue une étape importante sur la voie de la compréhension, de la clarification et de la formulation d'un problème. Lors du processus de résolution, les individus peuvent avoir à résumer et présenter des résultats intermédiaires. Ensuite, lorsqu'ils ont trouvé une solution, ils peuvent avoir à présenter cette solution à d'autres, voire à l'expliquer ou à la justifier.

- Mathématisation : les individus sont souvent amenés à transposer un problème défini en fonction du monde réel sous une forme strictement mathématique (en faisant appel à des processus de structuration, de conceptualisation, d'élaboration d'hypothèses et/ou de formulation de modèle), et à interpréter ou évaluer un résultat ou un modèle mathématique en fonction du problème initial. Le terme de «mathématisation » est employé pour décrire les activités fondamentales de ce type.

- Représentation : les individus sont très souvent amenés à se représenter des situations ou objets mathématiques, ce qui peut consister à sélectionner, interpréter et utiliser diverses représentations pour se faire une idée du problème, à passer d'une représentation à l'autre, à entrer en interaction avec le problème ou à présenter leur travail. Par représentations, on entend des graphiques, des tableaux, des diagrammes, des images, des équations, des formules et des matériaux concrets.

- Raisonnement et argumentation : les facultés de raisonnement et d'argumentation interviennent au cours des différentes étapes et activités associées à la culture mathématique. Cette compétence implique des processus logiques approfondis et permet d'explorer et de relier des éléments du problème pour en dégager des inférences, vérifier une justification fournie ou justifier une affirmation ou une solution.

- Conception de stratégies de résolution de problèmes : les individus sont souvent amenés à concevoir des stratégies pour résoudre des problèmes de façon mathématique. Cela passe par une série de processus de contrôles critiques, qui guident les individus pour les aider à reconnaître, formuler et résoudre des problèmes. Cette compétence permet aux individus de sélectionner ou de concevoir une approche ou une stratégie permettant d’utiliser les mathématiques pour résoudre les problèmes qui se posent dans une tâche ou dans un contexte, mais aussi de guider sa mise en œuvre. Cette compétence mathématique peut intervenir à n'importe quel stade du processus de résolution de problèmes.

- Utilisation d'opérations et d'un langage symbolique, formel et technique : les individus qui exploitent leur culture mathématique doivent utiliser des opérations et un langage symbolique, formel et technique, ce qui consiste à comprendre, interpréter, manipuler et employer des expressions symboliques (y compris des opérations et des expressions arithmétiques) dans un contexte mathématique régi par des conventions et des règles mathématiques. Cela implique aussi de comprendre et d'utiliser des constructs formels basés sur des définitions, des règles et des systèmes formels, et d'employer des algorithmes avec ces entités. Les symboles, les règles et les systèmes utilisés varient en fonction des contenus mathématiques spécifiques requis dans une tâche pour formuler ou résoudre le problème, ou en interpréter les aspects mathématiques. 
- Utilisation d'outils mathématiques : la dernière compétence mathématique qui sous-tend concrètement la culture mathématique est la capacité d'utiliser des outils mathématiques. Par outils mathématiques, on entend les appareils tels que les instruments de mesure, ainsi que les calculatrices et les outils informatiques qui se généralisent. Les individus qui possèdent cette compétence connaissent divers outils susceptibles de les aider durant une activité mathématique, sont capables de les utiliser et sont conscients de leurs limites. Les outils mathématiques peuvent jouer un rôle important lors de la communication des résultats. Lors des cycles précédents, il n’a été possible de les inclure que de manière minimale, en raison de l'administration d'épreuves papier-crayon. L'épreuve informatisée de mathématiques, une composante facultative du cycle PISA 2012, offre davantage de possibilités pour faire en sorte que les élèves se servent des outils mathématiques, ce qui permettra d'observer la façon dont ils les utilisent durant l'épreuve.

Ces facultés interviennent à des degrés divers dans chacun des processus utilisés pour rendre compte des résultats. La façon dont ces facultés se manifestent dans les trois processus est décrite dans la figure 1.2. L'encadré 1.1 «Les facultés mathématiques fondamentales et leur relation avec la difficulté des items », en fin de chapitre, explique ces facultés de façon plus détaillée et revient en particulier sur leur lien avec la difficulté des items. De plus, les exemples proposés dans la section «Exemples d'items PISA » décrivent la façon dont les élèves peuvent utiliser ces facultés lorsqu'ils résolvent un problème spécifique.

\section{Contenus mathématiques}

Acquérir des connaissances en mathématiques - et savoir les appliquer pour résoudre des problèmes qui se posent dans le monde réel - est important pour les citoyens dans les sociétés modernes. Il faut en effet pouvoir s'appuyer sur des connaissances mathématiques et sur une certaine compréhension des mathématiques pour résoudre des problèmes et interpréter des situations en rapport avec la vie personnelle et professionnelle, la société et la science.

Les structures mathématiques ont été développées au fil du temps pour comprendre et interpréter des phénomènes naturels et sociaux. À l'école, les programmes de mathématiques sont organisés autour d'un découpage logique des matières (I'arithmétique, I'algèbre, la géométrie, etc.), qui reflète les diverses branches, historiquement bien établies, des mathématiques et contribue à la définition d’une progression structurée. Or, en dehors des cours de mathématiques, les problèmes qui se posent ne s'accompagnent pas d'une série de règles et de principes qui montrent comment les surmonter. Il faut généralement une certaine créativité pour déterminer comment utiliser les mathématiques pour les résoudre et les formuler de façon mathématique. Il est fréquent que la même situation puisse s'aborder avec des concepts, procédures, faits ou outils mathématiques différents.

Comme l'enquête PISA a pour objet d'évaluer la culture mathématique, une structure des connaissances mathématiques est proposée sur la base des phénomènes mathématiques qui sous-tendent un large éventail de problèmes et qui ont motivé l'élaboration de concepts et procédures mathématiques spécifiques. Ainsi, des phénomènes mathématiques tels que l'incertitude et la variation sous-tendent de nombreuses situations courantes, et des stratégies et des outils mathématiques ont été conçus pour analyser ce type de situations. Cette organisation des connaissances mathématiques n'est pas neuve, comme le montrent deux ouvrages célèbres : On the Shoulders of Giants: New Approaches to Numeracy (Steen, 1990) et Mathematics: The Science of Patterns (Devlin, 1994).

Comme les programmes nationaux de cours de mathématiques sont conçus pour inculquer aux élèves des connaissances et des compétences en rapport avec ces mêmes phénomènes mathématiques, les contenus mathématiques qui ressortent de l'organisation du domaine d'évaluation sont très proches de la structure des programmes de cours. Pour orienter les développeurs d'items, le présent cadre identifie aussi quelques thématiques appropriées pour évaluer la culture mathématique des élèves âgés de 15 ans, sur la base de l'analyse des normes en vigueur en la matière dans 11 pays ${ }^{3}$.

Pour organiser le domaine des mathématiques en vue d'évaluer la culture mathématique, il est important de choisir une structure conforme à l'évolution historique des mathématiques, qui soit suffisamment variée et approfondie pour révéler l'essence des mathématiques et qui, de surcroît, représente - ou inclue - les branches conventionnelles des mathématiques d'une manière acceptable. Historiquement, les mathématiques se sont muées en l'étude intégrée des nombres, des formes, des variations et des relations avec l'invention, au XVII siècle, du calcul et de la géométrie analytique; I'analyse de phénomènes tels que l'aléatoire et l'incertitude est devenue déterminante pour la résolution de problèmes au XIXe et au XXe siècle. C'est dans cet esprit que des catégories de contenus qui reflètent des phénomènes mathématiques ont été retenues dans le cadre d'évaluation du cycle PISA 2012. Ces catégories sont cohérentes avec celles utilisées lors des cycles PISA précédents. 
- Figure 1.2 -

Relation entre les processus mathématiques et les facultés mathématiques fondamentales

\begin{tabular}{|c|c|c|c|}
\hline & $\begin{array}{l}\text { Formuler des situations de façon } \\
\text { mathématique }\end{array}$ & $\begin{array}{c}\text { Employer des concepts, faits, } \\
\text { procédures et raisonnements } \\
\text { mathématiques }\end{array}$ & $\begin{array}{l}\text { Interpréter, appliquer et évaluer } \\
\text { des résultats mathématiques }\end{array}$ \\
\hline Communication & $\begin{array}{l}\text { Lire, décoder et comprendre des } \\
\text { questions, des tâches, des objets, } \\
\text { des images ou des animations (dans } \\
\text { l'épreuve informatisée) pour élaborer } \\
\text { un modèle mental de la situation }\end{array}$ & $\begin{array}{l}\text { Articuler une solution, expliquer le } \\
\text { cheminement vers la solution, et/ou } \\
\text { résumer et présenter des résultats } \\
\text { mathématiques intermédiaires }\end{array}$ & $\begin{array}{l}\text { Construire et communiquer des } \\
\text { explications et des arguments au } \\
\text { sujet du problème contextualisé }\end{array}$ \\
\hline Mathématisation & $\begin{array}{l}\text { Identifier les structures et les variables } \\
\text { mathématiques dans le problème } \\
\text { tel qu'il se pose dans le monde réel, } \\
\text { et formuler des hypothèses pour } \\
\text { pouvoir les utiliser }\end{array}$ & $\begin{array}{l}\text { Se baser sur la compréhension } \\
\text { du contexte pour orienter ou } \\
\text { effectuer le processus de résolution } \\
\text { mathématique, par exemple, } \\
\text { travailler avec un degré de précision } \\
\text { approprié au contexte }\end{array}$ & $\begin{array}{l}\text { Comprendre la portée et les limites } \\
\text { d'une solution mathématique qui } \\
\text { découlent du modèle mathématique } \\
\text { employé }\end{array}$ \\
\hline Représentation & $\begin{array}{l}\text { Créer une représentation } \\
\text { mathématique des données du } \\
\text { problème tel qu'il se pose dans le } \\
\text { monde réel }\end{array}$ & $\begin{array}{l}\text { Comprendre, relier et utiliser une } \\
\text { série de représentations lors de } \\
\text { l'interaction avec le problème }\end{array}$ & $\begin{array}{l}\text { Interpréter des résultats } \\
\text { mathématiques dans une série de } \\
\text { formats en rapport avec une situation } \\
\text { ou une utilisation ; comparer ou } \\
\text { évaluer plusieurs représentations en } \\
\text { fonction d'une situation }\end{array}$ \\
\hline $\begin{array}{l}\text { Raisonnement et } \\
\text { argumentation }\end{array}$ & $\begin{array}{l}\text { Expliquer, défendre ou justifier la } \\
\text { représentation identifiée ou conçue } \\
\text { de la situation du problème tel qu'il } \\
\text { se pose dans le monde réel }\end{array}$ & $\begin{array}{l}\text { Expliquer, défendre ou justifier les } \\
\text { procédures ou processus utilisés pour } \\
\text { chercher une solution ou un résultat } \\
\text { mathématique } \\
\text { Établir un lien entre des fragments } \\
\text { d'information pour parvenir à } \\
\text { une solution mathématique, faire } \\
\text { des généralisations ou créer une } \\
\text { argumentation en plusieurs étapes }\end{array}$ & $\begin{array}{l}\text { Réfléchir aux solutions } \\
\text { mathématiques et fournir des } \\
\text { explications et des arguments } \\
\text { pour étayer, réfuter ou confirmer } \\
\text { une solution mathématique à un } \\
\text { problème tel qu'il se pose dans le } \\
\text { monde réel }\end{array}$ \\
\hline $\begin{array}{l}\text { Conception } \\
\text { de stratégies } \\
\text { de résolution } \\
\text { de problèmes }\end{array}$ & $\begin{array}{l}\text { Choisir ou concevoir une approche } \\
\text { ou une stratégie pour situer des } \\
\text { problèmes contextualisés dans un } \\
\text { cadre mathématique }\end{array}$ & $\begin{array}{l}\text { Actionner des mécanismes efficaces } \\
\text { de contrôle pendant une procédure } \\
\text { en plusieurs étapes qui doit mener à } \\
\text { une généralisation, une conclusion } \\
\text { ou une solution mathématique }\end{array}$ & $\begin{array}{l}\text { Concevoir et appliquer une stratégie } \\
\text { pour interpréter, évaluer et valider } \\
\text { une solution mathématique à un } \\
\text { problème qui se pose dans le monde } \\
\text { réel }\end{array}$ \\
\hline $\begin{array}{l}\text { Utilisation } \\
\text { d'opérations } \\
\text { et d'un langage } \\
\text { symbolique, } \\
\text { formel et } \\
\text { technique }\end{array}$ & $\begin{array}{l}\text { Utiliser des modèles standard, des } \\
\text { diagrammes, des symboles et des } \\
\text { variables ad hoc pour énoncer dans } \\
\text { un langage symbolique ou formel un } \\
\text { problème qui se pose dans le monde } \\
\text { réel }\end{array}$ & $\begin{array}{l}\text { Comprendre et utiliser des constructs } \\
\text { formels sur la base de définitions, } \\
\text { de règles et de systèmes formels; } \\
\text { utiliser des algorithmes }\end{array}$ & $\begin{array}{l}\text { Utiliser des outils mathématiques } \\
\text { pour établir la plausibilité d'une } \\
\text { solution mathématique et identifier } \\
\text { d'éventuelles limites ou contraintes } \\
\text { à propos de la solution, compte tenu } \\
\text { du problème tel qu'il se présente dans } \\
\text { le monde réel }\end{array}$ \\
\hline $\begin{array}{l}\text { Utilisation d'outils } \\
\text { mathématiques }\end{array}$ & $\begin{array}{l}\text { Utiliser des outils mathématiques } \\
\text { pour identifier des structures } \\
\text { mathématiques ou décrire des } \\
\text { relations mathématiques }\end{array}$ & $\begin{array}{l}\text { Connaître et savoir utiliser comme } \\
\text { il se doit divers outils, pour faciliter } \\
\text { la mise en œuvre de processus et } \\
\text { de procédures à la recherche de } \\
\text { solutions }\end{array}$ & $\begin{array}{l}\text { Utiliser des outils mathématiques } \\
\text { pour établir la plausibilité d'une } \\
\text { solution mathématique et identifier } \\
\text { d'éventuelles limites ou contraintes } \\
\text { à propos de la solution, compte tenu } \\
\text { du problème tel qu'il se présente } \\
\text { dans le monde réel }\end{array}$ \\
\hline
\end{tabular}


Les catégories de contenus suivantes ont été retenues en vue du cycle PISA 2012 dans le respect de l'évolution historique des mathématiques pour bien refléter le domaine des mathématiques et les phénomènes sous-jacents qui ont motivé leur développement ainsi que les grandes branches des programmes de cours. Ces quatre catégories caractérisent les contenus mathématiques au cœur de la discipline et illustrent les contenus qui ont présidé au développement des items du cycle PISA 2012 :

- les variations et les relations;

- l'espace et les formes;

- la quantité ; et

- l'incertitude et les données ${ }^{4}$.

Ces quatre catégories permettent d'organiser le domaine d'évaluation de sorte que les items se répartissent bien dans I'ensemble du domaine et se concentrent sur des phénomènes mathématiques importants, tout en évitant une division trop fine qui irait à l'encontre de la volonté de proposer des problèmes mathématiques riches et passionnants qui s'inspirent du monde réel. Cette classification par contenu est importante pour concevoir et choisir les items ainsi que pour rendre compte des résultats, mais il faut souligner ici que certains contenus spécifiques peuvent se retrouver dans plus d'une catégorie. L'un des items de l'unité PISA PIZZAS implique, par exemple, de déterminer laquelle parmi deux pizzas rondes de même diamètre, mais d'épaisseur différente, est la meilleure marché (cet item et ses attributs sont décrits dans la section «Exemples d'items PISA » en fin de chapitre). Cet item intègre diverses branches des mathématiques, dont la mesure, la quantification (valeurs, proportions et opérations arithmétiques) et, enfin, les variations et les relations (relations entre les variables et variations des propriétés de la petite et de la grande pizza). Cet item a finalement été classé dans la catégorie de contenu Variations et relations car pour le résoudre, les élèves doivent essentiellement être capables d'établir une correspondance entre la variation de la taille de deux pizzas (la variation de diamètre) et la variation du prix. Un autre item en rapport avec un cercle aurait de toute évidence pu être classé dans la catégorie de contenu Espace et formes. Les liens entre des aspects qui s'étendent sur les quatre catégories contribuent à la cohérence des mathématiques en tant que discipline et sont visibles dans certains items retenus pour constituer les épreuves de mathématiques du cycle PISA 2012.

Les catégories de contenus et les concepts spécifiquement adaptés aux élèves âgés de 15 ans décrits ci-après permettent de cerner le niveau et la portée des contenus susceptibles d'être inclus dans l'enquête PISA. En premier lieu, les catégories de contenus sont décrites de façon globale et leur pertinence pour résoudre des problèmes est expliquée. En second lieu, les types de contenus qu'il est approprié d'inclure dans une évaluation de la culture mathématique des élèves âgés de 15 ans sont décrits de manière plus spécifique. Ces thématiques spécifiques reflètent des aspects communs qui se retrouvent dans les attentes d'un certain nombre de pays et de systèmes d'éducation. Les normes examinées pour identifier ces thématiques sont considérées non seulement comme des éléments qui montrent ce qui est enseigné en mathématiques dans ces pays, mais également comme des indicateurs des connaissances et compétences que ces pays jugent important d'inculquer aux élèves de cet âge pour les préparer à devenir des citoyens constructifs, engagés et réfléchis.

Les catégories de contenus mathématiques - variations et relations, ; espace et formes ; quantité ; et incertitude et données - sont décrites ci-dessous.

\section{Variations et relations}

Le monde naturel et le monde façonné par l'homme affichent une multitude de relations provisoires et permanentes entre les objets et les circonstances, dans lesquelles des changements interviennent dans des systèmes d'objets interdépendants ou dans des circonstances où les éléments s'influencent les uns les autres. Dans de nombreux cas, ces changements se produisent avec le temps. Il arrive aussi que des changements qui affectent un objet ou une quantité soient en rapport avec des changements qui ont eu lieu sur un autre objet ou quantité. II s'agit de changements tantôt ponctuels, tantôt continus. Certaines relations sont de nature permanente. Pour mieux comprendre les variations et les relations, il faut tout d'abord comprendre les types fondamentaux de changement et les reconnaître lorsqu'ils se produisent. C'est essentiel pour utiliser des modèles mathématiques adaptés qui permettent de décrire et prévoir les changements. En termes mathématiques, cela revient à modéliser les variations et les relations grâce à des fonctions et équations appropriées, ainsi qu'à créer, interpréter et traduire des représentations graphiques et symboliques des relations. 
Les variations et les relations s'observent dans des contextes très divers : la croissance des organismes, la musique, le cycle des saisons, les tendances météorologiques, le taux d'emploi et la conjoncture économique, par exemple. Certains aspects mathématiques traditionnels des fonctions et de l'algèbre, notamment les expressions algébriques, les équations et les inégalités ou les représentations sous forme de graphiques et de tableaux, sont essentiels pour décrire, modéliser et interpréter les phénomènes de variation. Parmi les unités PISA qui ont été publiées, I'unité MARCHE À PIED (voir la section "Exemples d'items PISA » en fin de chapitre) contient, par exemple, deux items typiques de la catégorie Variations et relations, car ils portent sur des relations algébriques entre deux variables et demandent aux élèves d'exploiter leurs connaissances et leurs compétences en algèbre. Les élèves doivent utiliser une formule donnée - sous forme algébrique - pour calculer la longueur de pas dans le premier item et la vitesse de marche dans le second item. Les représentations statistiques de données et de relations sont souvent utilisées pour décrire et interpréter des variations et des relations. Une bonne maîtrise des nombres et des unités est également essentielle pour définir et interpréter des variations et des relations. Quelques relations intéressantes se dégagent du mesurage géométrique, par exemple le fait que des changements de périmètre dans une famille de formes peuvent se traduire par des changements de superficie, ou encore les relations entre les longueurs des côtés de triangles. Parmi les unités PISA qui ont été publiées, I'unité PIZZAS (voir la section «Exemples d'items PISA » en fin de chapitre) illustre la catégorie Variations et relations.

L'épreuve informatisée de mathématiques, une option du cycle PISA 2012, permet de soumettre aux élèves des images dynamiques et plusieurs représentations en lien dynamique, et leur donne la possibilité de manipuler des fonctions. Ainsi, l'évolution dans le temps (la croissance ou le mouvement, par exemple) peut être décrite directement par des animations et des simulations, et représentée par des fonctions, des graphiques et des tableaux de données liés entre eux. Le processus qui consiste à trouver, puis utiliser des modèles mathématiques de changement est plus étendu, car les individus peuvent explorer et décrire le changement à l'aide de logiciels qui permettent de dessiner des fonctions, de manipuler des paramètres, de générer des tableaux de données, d'utiliser des relations géométriques, d’organiser des données, et d'en faire des graphiques et d'effectuer des calculs sur la base de formules. Les fonctionnalités des tableurs, qui permettent de travailler avec des formules et de transposer des données dans des graphiques, sont particulièrement pertinentes.

\section{Espace et formes}

La catégorie de contenus Espace et formes englobe un large éventail de phénomènes omniprésents dans notre environnement visuel et physique : les régularités, les propriétés des objets, les positions et les orientations, les représentations d'objets, l'encodage et le décodage d'informations visuelles, la navigation et les interactions dynamiques avec des formes réelles ainsi qu'avec leur représentation. La géométrie est un fondement essentiel de la catégorie Espace et formes, qui s'étend toutefois au-delà des limites de cette branche en termes de contenu, de signification et de méthode, et intègre d'autres branches des mathématiques, telles que la visualisation dans l'espace, le mesurage et l'algèbre. Ainsi, des formes peuvent se déformer et un point peut se déplacer dans l'espace, ce qui fait intervenir des concepts de fonction. Les formules de mesure sont centrales. La manipulation et l'interprétation de formes contextualisées qui passent par l'utilisation d'outils tels que des logiciels de géométrie dynamique ou de géolocalisation sont incluses dans cette catégorie de contenus.

L'enquête PISA part du principe que la maîtrise d'une série de compétences et de concepts fondamentaux est essentielle pour démontrer sa culture mathématique dans la catégorie Espace et formes, ce qui implique un large éventail d'activités, notamment comprendre la notion de perspective (dans des peintures, par exemple), créer et lire des cartes, transformer des formes avec ou sans aide technologique, interpréter des vues de scènes en trois dimensions sous diverses perspectives et construire des représentations de formes. L'item MENUISIER (voir la section « Exemples d'items PISA » en fin de chapitre) relève de cette catégorie Espace et formes, car il porte sur un autre de ses aspects majeurs, à savoir les propriétés des formes. Dans cet item à choix multiple complexe, les élèves doivent identifier parmi les quatre tracés de bordure de jardin qui leur sont présentés celui ou ceux qu'il est possible de fabriquer avec 32 mètres de planches. Pour y répondre, ils doivent exploiter leurs facultés de raisonnement et leurs connaissances en géométrie. Ils disposent de suffisamment d'informations pour calculer directement le périmètre précis de la bordure dans trois tracés, mais des informations incorrectes leur sont fournies dans un tracé, ce qui les oblige à utiliser leurs facultés de raisonnement en géométrie qualitative.

L'épreuve informatisée de mathématiques permet aux élèves de manipuler des représentations dynamiques de formes et d'explorer des relations entre des objets géométriques en trois dimensions, qu'il est possible de faire pivoter virtuellement pour obtenir une représentation mentale précise. Les élèves peuvent utiliser des cartes dont ils peuvent accroître ou réduire le degré de précision, ou qu'ils peuvent faire pivoter pour se construire une image mentale d'un endroit, puis les employer pour établir un itinéraire, par exemple. Ils peuvent choisir et utiliser des outils pour prendre 
des mesures (d'angles, de segments, etc.) sur des plans, des images et des modèles, puis utiliser ces données dans des calculs. La technologie permet aux élèves de mêler leurs connaissances en géométrie à des informations visuelles pour construire un modèle mental précis. Ainsi, pour déterminer le volume d'une tasse, ils peuvent manipuler une image pour déterminer s'il s'agit d'un cône tronqué, en connaître la hauteur perpendiculaire et savoir où la mesurer, et comprendre que ce qui ressemble à des ellipses en bas et en haut de l'image en deux dimensions est en fait un cercle dans l'espace en trois dimensions.

\section{Quantité}

La notion de quantité est peut-être l'aspect mathématique le plus répandu et le plus essentiel de l'engagement et du fonctionnement dans notre monde. Elle englobe la quantification d'attributs d'objets, de relations, de situations et d'entités dans le monde, la compréhension de diverses représentations de ces quantifications, et l'évaluation d'interprétations et d'arguments fondés sur la quantité. Pour appréhender la quantification, il faut comprendre le mesurage, le comptage, la magnitude, les unités, les indicateurs, la taille relative, les tendances numériques et les régularités. Certains aspects du raisonnement quantitatif - le sens des nombres, les représentations multiples des nombres, l'élégance des calculs, le calcul mental, les estimations et l'évaluation de la plausibilité des résultats - sont l'essence même de la culture mathématique dans la catégorie Quantité.

La quantification est la principale méthode qui existe pour décrire et mesurer un grand nombre des attributs d'objets dans le monde. Elle permet de modéliser des situations, d'examiner les variations et les relations, de décrire et de manipuler l'espace et les formes, d'organiser et d'interpréter les données, et de mesurer et d'évaluer l'incertitude. Dans la catégorie Quantité, la culture mathématique consiste à utiliser des connaissances relatives aux nombres et aux opérations avec des nombres dans un large éventail de contextes. L'item CONCERT ROCK (vois la section « Exemples d'items PISA » en fin de chapitre) est un exemple typique d'items relevant de la catégorie Quantité. Dans cet item, les élèves doivent estimer le nombre total de spectateurs qui assistent à un concert en fonction des dimensions du terrain en forme de rectangle où s'organise ce concert. Cet item comporte certains éléments qui le rapprochent de la catégorie Espace et formes, mais il demande avant tout aux élèves de formuler une hypothèse sur l'espace qu'il est raisonnable de prévoir par spectateur et d'utiliser la superficie totale disponible pour estimer le nombre de spectateurs qu'il est possible d'accueillir. Comme il s'agit d'un item à choix multiple, les élèves peuvent aussi travailler à rebours sur la base de la superficie et de chaque option de réponse pour calculer l'espace correspondant par personne et, ainsi, déterminer l'option de réponse la plus plausible. Comme les options de réponse sont exprimées en milliers de spectateurs (2 000 ou 5000 spectateurs, par exemple), cet item fait aussi appel aux facultés d'estimation numérique des élèves.

L'épreuve informatisée de mathématiques permet aux élèves de tirer parti de la grande puissance de calcul des technologies modernes. Il importe de souligner que si les individus peuvent, grâce à la technologie, éviter de faire des calculs et libérer certaines de leurs ressources cognitives pour leur permettre de se concentrer sur le sens et la stratégie lorsqu'ils résolvent des problèmes, ils doivent comprendre les mathématiques de manière approfondie pour démontrer leur culture mathématique. Les individus qui n'ont pas cette compréhension des mathématiques peuvent au mieux utiliser la technologie pour effectuer des tâches routinières, ce qui ne cadre pas avec la définition de la culture mathématique retenue dans le cadre du cycle PISA 2012. De plus, I'intégration de la technologie dans l'épreuve informatisée de mathématiques permet l'inclusion d'items qui font appel à des niveaux de compétence en calcul numérique et statistique qu'il serait impossible de gérer dans une épreuve papier-crayon.

\section{Incertitude et données}

L'incertitude est une donnée en sciences, dans la technologie et dans la vie de tous les jours. Le phénomène d'incertitude est donc au cœur de l'analyse mathématique de nombreux problèmes, et la théorie de la probabilité et la statistique ont été créées pour y répondre. Dans la catégorie de contenu Incertitude et données, il s'agit de reconnaître la place de la variation dans les processus, de comprendre l'ampleur de cette variation, d'admettre la notion d'incertitude et d'erreur dans le mesurage, et de connaître le concept de chance. Il faut également formuler, interpréter et évaluer des conclusions dans des situations où règne l'incertitude. La présentation et l'interprétation des données sont essentielles dans cette catégorie (Moore, 1997).

L'incertitude entoure les prévisions scientifiques, les résultats de scrutins électoraux, les prévisions météorologiques et les modèles économiques. Les notes à un examen, les résultats de sondages et les processus de fabrication varient, et la chance est fondamentale dans de nombreuses activités récréatives auxquelles les individus se livrent pendant leurs loisirs. Les branches traditionnelles de la probabilité et de la statistique sont des moyens formels de décrire, modéliser 
et interpréter une certaine catégorie de phénomènes, et de dégager des inférences. Par ailleurs, la connaissance des nombres et de certains aspects de l'algèbre comme les graphiques et les représentations symboliques facilite la tâche aux individus qui s'attaquent à des problèmes relevant de cette catégorie. L'item DÉCHETS (voir la section « Exemples d'items PISA » en fin de chapitre) est classé dans la catégorie Incertitude et données. Dans cet item, les élèves doivent examiner des données qui leur sont présentées dans un tableau et expliquer pourquoi un diagramme en bâtons n'est pas approprié pour les communiquer. La présentation et l'interprétation des données constituent un aspect important de la catégorie Incertitude et données.

L'épreuve informatisée de mathématiques permet de soumettre aux élèves des volumes plus importants de données et leur offre la puissance de calcul et des fonctions de traitement des données dont ils ont besoin pour les traiter. Ils ont la possibilité de choisir des outils pour manipuler, analyser et représenter des données, et de prélever des échantillons dans des séries de données. Des représentations interdépendantes leur permettent d'examiner et de décrire ces données de différentes façons. La faculté de générer des résultats aléatoires, dont des nombres, leur permet d'explorer des situations en rapport avec les probabilités par le biais de la simulation, par exemple la probabilité empirique d'événements et de propriétés d'échantillons.

\section{Thématiques refenues pour orienter l'évaluation de la culture mathématique des élèves âgés de 15 ans}

Pour bien comprendre, puis résoudre des problèmes contextualisés en rapport avec les catégories Variations et relations, Espace et formes, Quantité, et Incertitude et données, il faut pouvoir se baser sur une série de concepts, procédures, faits et outils mathématiques, et ce, avec un certain niveau de maîtrise et de sophistication. L'épreuve PISA de culture mathématique cherche à évaluer des niveaux et des contenus mathématiques appropriés aux élèves âgés de 15 ans pendant leur cheminement vers une citoyenneté constructive, engagée et réfléchie, qui leur permet de poser des jugements et de prendre des décisions en toute connaissance de cause. L'enquête PISA, qui n'est pas conçue pour évaluer des matières précises du programme de cours, tente de refléter les connaissances et compétences que les élèves ont eu la possibilité d'acquérir jusqu'à l'âge de 15 ans.

Nous avons analysé une série de normes prévues en mathématiques dans 11 pays pour déterminer ce qui est enseigné aux élèves dans le monde et ce que les pays jugent réaliste et important de leur inculquer pour les préparer alors qu'ils s'apprêtent à entrer dans le monde du travail ou à suivre des études supérieures, avec en ligne de mire la volonté de concevoir une épreuve qui soit non seulement prospective, mais également révélatrice des mathématiques qui sont enseignées jusqu'à l'âge de 15 ans. Les contenus mathématiques qu'il a été jugé pertinent d'inclure dans une évaluation de la culture mathématique des élèves de 15 ans sur la base des points communs relevés lors de ces analyses et des jugements d'experts en mathématiques sont décrits ci-dessous.

Les quatre catégories de contenu Variations et relations, Espace et formes, Quantité, et Incertitude et données ont servi de base à l'identification des différents contenus, même s'il n'y a pas de mise en correspondance précise entre des thématiques et ces catégories. Ainsi, le raisonnement proportionnel intervient dans des contextes très variés, notamment la conversion de mesures, I'analyse de relations linéaires, le calcul de probabilité ou la comparaison de la longueur des côtés de formes similaires. Les contenus décrits ci-dessous montrent que bon nombre de ces concepts sont au cœur des quatre catégories et confirment la cohérence des mathématiques en tant que discipline. Il s'agit plus d'exemples de contenus inclus dans l'épreuve de mathématiques du cycle PISA 2012 que d'une liste exhaustive :

- Fonctions : le concept de fonction, notamment (mais pas exclusivement) les fonctions linéaires, leurs propriétés et une série de descriptions et de représentations. Les représentations verbales, symboliques et graphiques, ainsi que les représentations sous forme de tableaux, sont souvent utilisées.

- Expressions algébriques : I'interprétation verbale et la manipulation d'expressions algébriques, comprenant des nombres, des symboles, des opérations arithmétiques, des puissances et des racines simples.

- Équations et inéquations : des équations et inéquations linéaires, des équations simples du second degré, et des méthodes analytiques et non analytiques de résolution.

- Systèmes de coordonnées : la représentation et la description de données, de positions et de relations.

- Relations dans et entre des objets géométriques en deux et en trois dimensions : des relations statiques telles que des liens algébriques entre des éléments de figures (par exemple, le théorème de Pythagore, qui définit la relation entre la longueur des côtés d'un triangle rectangle), les positions relatives, la similitude et la congruence, et les relations dynamiques impliquant la transformation et le mouvement d'objets, ainsi que les correspondances entre objets en deux et en trois dimensions. 
- Mesure : la quantification de formes et d'objets et de certains de leurs aspects, par exemple l'angle, la distance, la longueur, le périmètre, la circonférence, la superficie et le volume.

- Nombres et unités : les concepts, les représentations de nombres et les systèmes de numération, dont les propriétés de nombres entiers et rationnels, des aspects pertinents des nombres irrationnels, ainsi que des quantités et des unités en rapport avec des phénomènes tels que le temps, l'argent, le poids, la température, la distance, la superficie et le volume, ainsi que des quantités dérivées et leur description numérique.

- Opérations arithmétiques : la nature et les propriétés des opérations numériques, et les conventions d'écriture qui s’y rapportent.

- Pourcentages, ratios et proportions : la description numérique de grandeur relative et le raisonnement fondé sur les proportions pour résoudre des problèmes.

- Principes de comptage : les permutations et les combinaisons simples.

- Estimation : I'approximation dans un but particulier de quantités et expressions numériques, notamment les chiffres significatifs et les arrondis.

- Collecte, représentation et interprétation de données : la nature, l'origine et la collecte de divers types de données, et les différents modes de représentation et d'interprétation des données.

- Variabilité des données et descriptionde cette dernière : les concepts tels que la variabilité, la distribution et les tendances principales dans des groupes de données, les modes de description et d'interprétation de ces concepts en termes quantitatifs.

- Échantillonnage et échantillons : les concepts d'échantillonnage dans des groupes de données, notamment la formulation d'inférences simples sur la base des propriétés des échantillons.

- Risque et probabilité : les concepts tels que les événements aléatoires, la variation aléatoire et sa représentation, le risque et la fréquence des événements, et les aspects fondamentaux du concept de probabilité.

\section{Contextes}

L'un des aspects importants de la culture mathématique réside dans l'utilisation des mathématiques pour résoudre des problèmes en contexte. Par contexte, on entend la place des problèmes dans le monde des individus. Le choix de représentations et stratégies mathématiques appropriées dépend souvent du contexte dans lequel les problèmes se posent. De l'avis général, situer les problèmes dans un contexte permet d'accroître la difficulté des items (voir les conclusions en rapport avec la statistique de Watson et Callingham, 2003). La grande diversité des contextes utilisés est un aspect important de l'enquête PISA, car elle permet de potentiellement mettre en correspondance les centres d'intérêt des individus et les situations dans lesquelles les individus fonctionnent au XXIe siècle.

Le cadre d'évaluation de la culture mathématique du cycle PISA 2012 définit quatre catégories de contextes qui sont utilisées pour répartir les items constituant les épreuves PISA :

- Personnel : les problèmes classés dans cette catégorie portent sur les activités des individus, de leur famille et de leurs pairs. Parmi les contextes à considérer comme personnels, citons notamment la préparation des repas, les achats, les jeux, la santé individuelle, les moyens de transport, le sport, les voyages, l'emploi du temps et le budget personnel. L'item PIZZAS (voir la section "Exemples d'items PISA » en fin de chapitre) se situe dans un contexte personnel, car les élèves doivent déterminer quelle pizza est la plus avantageuse en termes de rapport quantité-prix. II en va de même pour les deux items de l'unité PISA MARCHE À PIED (voir la section « Exemples d'items PISA » en fin de chapitre), qui se situent dans un contexte personnel. Les élèves doivent utiliser une même formule mathématique pour calculer la longueur de pas d'un individu dans le premier item et la vitesse de marche d'un autre individu dans le deuxième item.

- Professionnel : les problèmes classés dans la catégorie des contextes professionnels se situent dans le monde du travail. Parmi les contextes à considérer comme professionnels, citons notamment ceux en rapport avec le mesurage, les devis et les commandes de matériaux de construction, par exemple, la comptabilité et la gestion des salaires, le contrôle de la qualité, les inventaires et les prévisions, le design et l'architecture, et la prise de décisions dans le cadre de la vie professionnelle. Les contextes professionnels peuvent concerner toutes les classes de main-d'œuvre, des travailleurs non qualifiés à ceux qui exercent les plus hautes fonctions, même si les items PISA doivent être accessibles à des élèves âgés de 15 ans. L'item MENUISIER (voir la section «Exemples d'items PISA » en fin de chapitre) se classe dans la catégorie des contextes professionnels, car il porte sur une bordure qu'un menuisier doit fabriquer autour d'un jardin. L'item de l'unité PISA PIZZAS évoqué ci-dessus se classerait également dans cette catégorie s'il portait sur le point de vue du vendeur de pizzas et non de l'acheteur de pizzas. 
- Sociétal : les problèmes classés dans la catégorie des contextes sociétaux se situent dans la communauté (locale, nationale ou mondiale). Parmi les contextes à considérer comme sociétaux, citons notamment ceux en rapport avec les systèmes électoraux, les transports publics, les gouvernements, les pouvoirs publics, la démographie, la publicité, les statistiques nationales et l'économie. Les individus sont impliqués dans tous ces contextes à titre personnel, mais les problèmes relevant de cette catégorie se présentent avant tout sous l'angle de la collectivité. L'item CONCERT ROCK (voir la section «Exemples d'items PISA » en fin de chapitre) est un exemple d'item classé dans la catégorie des contextes sociétaux, car il se situe dans le cadre de l'organisation d'un concert de rock, même s'il se base sur une expérience personnelle, en l'occurrence se trouver dans une foule.

- Scientifique: les problèmes classés dans la catégorie des contextes scientifiques traitent de l'application des mathématiques dans le monde naturel, ainsi que dans des thématiques en rapport avec la science et la technologie. Parmi les contextes à considérer comme scientifiques, citons notamment les contextes en rapport avec la météorologie ou le climat, l'écologie, la médecine, I'espace, la génétique, le mesurage et les mathématiques. L'item publié de l'unité PISA DÉCHETS (voir la section "Exemples d'items PISA » en fin de chapitre) est un exemple d'item classé dans la catégorie des contextes scientifiques, car il porte sur des aspects scientifiques en rapport avec l'environnement, plus précisément sur le temps de décomposition des déchets. Les items intramathématiques, dont tous les éléments ont trait au monde des mathématiques, se classent dans la catégorie des contextes scientifiques.

Les items PISA qui partagent le même stimulus sont regroupés par unité. Il est dès lors courant que tous les items d'une même unité se classent dans la même catégorie de contextes. Il y a toutefois quelques exceptions : il se peut, par exemple, que le stimulus d'une unité soit analysé sous l'angle individuel dans un item, mais sous l'angle sociétal dans un autre item. Si un item inclut uniquement des constructs mathématiques sans la moindre référence à des éléments contextuels de l'unité dont il relève, il se classe dans la catégorie de contextes à laquelle l'unité appartient. Dans les rares cas où une unité inclut uniquement des constructs mathématiques sans la moindre référence à un contexte sans rapport avec les mathématiques, cette unité se classe dans la catégorie des contextes scientifiques.

L'utilisation de ces catégories de contextes permet de constituer une gamme de contextes d'item et de garantir que les épreuves reflètent un large éventail d'applications des mathématiques, des applications dans la vie courante à celles requises pour résoudre des problèmes mondiaux. De plus, il est important que chaque catégorie de contextes comprenne des items dont le degré de difficulté varie. Comme ces catégories ont été définies pour soumettre aux élèves des problèmes dans des contextes très différents, chaque catégorie doit largement contribuer à l'évaluation de la culture mathématique. Il faut éviter que le degré de difficulté des items d'une catégorie soit systématiquement plus ou moins élevé que celui des items d'une autre catégorie.

Lors de l'identification des contextes qui peuvent se révéler pertinents, il est essentiel de garder présent à l'esprit que les épreuves ont pour but d'évaluer dans quelle mesure les élèves sont capables d'utiliser les contenus, processus et facultés mathématiques qu'ils ont acquis jusqu'à l'âge de 15 ans. C'est la raison pour laquelle les contextes des items sont choisis pour leur pertinence par rapport aux centres d'intérêt et à la vie des élèves, et en fonction des défis qu'ils auront à relever dès qu'ils commenceront à avoir un rôle social en tant que citoyens constructifs, engagés et réfléchis. Les directeurs nationaux de projet des pays qui participent à I'enquête PISA contribuent à l'évaluation de ce degré de pertinence.

\section{ÉVALUER LA CULTURE MATHÉMATIQUE}

Cette section décrit l'approche adoptée pour traduire concrètement les aspects du cadre d'évaluation du cycle PISA 2012 présentés ci-dessus, notamment la structure des épreuves PISA de mathématiques, la façon de rendre compte des niveaux de compétence en mathématiques, les savoirs et savoir-faire explorés dans le domaine de la culture mathématique, et les dispositions prises pour administrer l'épreuve informatisée de mathématiques.

\section{Structure des épreuves de mathématiques lors du cycle PISA 2012}

En vertu de la définition de la culture mathématique, les items constituant les épreuves PISA, qu'il s'agisse de l'épreuve papiercrayon ou de l'épreuve informatisée, doivent se situer dans un contexte. Pour répondre aux items, les élèves âgés de 15 ans doivent appliquer d'importants concepts, savoirs et savoir-faire en mathématiques (les contenus mathématiques) à un niveau approprié à leur âge, comme cela a été expliqué ci-dessus. Le cadre d'évaluation sert à orienter la structure et le contenu de l'évaluation, et il est important que les épreuves, qu'il s'agisse de l'épreuve papier-crayon ou de l'épreuve informatisée, soient constituées d'items qui reflètent les composantes du cadre d'évaluation de la culture mathématique. 


\section{Répartition souhaitée du score entre les processus mathématiques}

De plus, les items des épreuves de mathématiques du cycle PISA 2012 sont associés à I'un des trois processus mathématiques. L'objectif lors de la conception des épreuves, c'est d'obtenir un équilibre par lequel la pondération est assez égale entre les deux processus qui consistent à établir un lien entre le monde réel et le monde des mathématiques, et le processus qui demande aux élèves de résoudre un problème énoncé sous une forme mathématique.

Tableau 1.1

Répartition approximative du score en mathématiques entre les catégories de processus

\begin{tabular}{l|c}
\multicolumn{1}{c|}{ Catégorie de processus } & Pourcentage de points \\
\hline Formuler des situations de façon mathématique & $25 \%$ environ \\
\hline $\begin{array}{l}\text { Employer des concepts, faits, procédures et raisonnements } \\
\text { mathématiques }\end{array}$ & $50 \%$ environ \\
\hline Interpréter, appliquer et évaluer des résultats mathématiques & $25 \%$ environ \\
\hline TOTAL & $100 \%$ \\
\hline
\end{tabular}

Il importe de souligner ici que dans chaque catégorie, les items s'étendent sur tout le spectre de difficulté.

\section{Répartition souhaitée du score entre les catégories de contenus}

Les items PISA de mathématiques sont choisis pour refléter les contenus mathématiques décrits ci-avant. Les items choisis lors du cycle PISA 2012 se répartissent entre les quatre catégories de contenus mathématiques comme le montre le tableau 1.2. Lors de la conception des épreuves, l'objectif est d'obtenir une répartition des items qui soit la plus équilibrée possible entre les contenus mathématiques, car tous ces domaines sont importants pour des citoyens constructifs, engagés et réfléchis.

Tableau 1.2

Répartition approximative du score en mathématiques entre les catégories de contenus

\begin{tabular}{l|c}
\hline \multicolumn{1}{c|}{ Catégorie de contenus } & Pourcentage de points \\
\hline Variations et relations & $25 \%$ environ \\
\hline Espace et formes & $25 \%$ environ \\
\hline Quantité & $25 \%$ environ \\
\hline Incertitude et données & $25 \%$ environ \\
\hline TOTAL & $100 \%$ \\
\hline
\end{tabular}

Il importe de souligner ici que dans chaque catégorie, les items s'étendent sur tout le spectre de difficulté.

\section{Répartition souhaitée du score entre les catégories de contextes}

Dans l'enquête PISA 2012, tous les items se classent dans I'une des quatre catégories de contextes. Les items retenus pour constituer les épreuves de mathématiques du cycle PISA 2012 se répartissent entre ces catégories de contextes, comme le montre le tableau 1.3. Cette répartition équilibrée permet de faire en sorte qu'aucun type de contexte ne prédomine : les élèves se voient donc présenter des items dans un large éventail de contextes correspondant à un grand nombre de centres d'intérêt personnels et un grand nombre de situations que les individus sont susceptibles de rencontrer dans la vie.

Tableau 1.3.

Répartition approximative du score en mathématiques entre les catégories de contextes

\begin{tabular}{l|c}
\multicolumn{1}{c|}{ Catégorie de contextes } & Pourcentage de points \\
\hline Contextes personnels & $25 \%$ environ \\
\hline Contextes professionnels & $25 \%$ environ \\
\hline Contextes sociétaux & $25 \%$ environ \\
\hline Contextes scientifiques & $25 \%$ environ \\
\hline TOTAL & $100 \%$ \\
\hline
\end{tabular}

Il importe de souligner ici que dans chaque catégorie, les items s'étendent sur tout le spectre de difficulté. 


\section{Le spectre de difficulté des items}

Les épreuves de mathématiques du cycle PISA 2012 sont constituées d'items dont le degré de difficulté varie, comme les facultés des élèves âgés de 15 ans. Elles comportent des items difficiles même pour les élèves les plus brillants, ainsi que des items adaptés aux élèves les moins performants. D'un point de vue psychométrique, une évaluation destinée à évaluer une cohorte spécifique d'individus est la plus efficace et la plus efficiente lorsque la difficulté des items est semblable à la faculté des sujets évalués. De plus, les échelles de compétence qui sont utilisées pour rendre compte des résultats ne sont révélatrices des facultés de tous les élèves que si les items à partir desquels les compétences sont décrites s'étendent sur le spectre de compétence décrit. Les échelles de compétence se basent sur des niveaux croissants d'activation des facultés fondamentales en mathématiques, qui sont décrites de façon détaillée dans l'encadré 1.1 "Les facultés mathématiques fondamentales et leur relation avec la difficulté des items ». II ressort des cycles PISA précédents qu'ensemble, ces facultés sont des indicateurs de la charge cognitive et, donc, qu'elles contribuent à la difficulté des items (Turner, 2012 ; Turner et al., à paraître). Une échelle de compétence PISA a été élaborée après l'essai de terrain du cycle PISA 2012 sur la base de la description de l'activation de ces compétences. Cette échelle permet de mesurer, de façon empirique, la charge cognitive de chaque item.

\section{Structure des instruments d'évaluation}

En mathématiques, les épreuves papier-crayon du cycle PISA 2012 sont constituées à partir d'une batterie d'items de 270 minutes. Cette batterie est divisée en neuf blocs d'items, de 30 minutes de test chacun. Sur ces neuf blocs, trois (soit 90 minutes de test) contiennent des items d'ancrage, qui ont été administrés lors de cycles PISA antérieurs, quatre blocs standard (soit 120 minutes de test) contiennent de nouveaux items dont le degré de difficulté varie sensiblement, et deux (soit 60 minutes de test) contiennent des items « faciles », dont le degré de difficulté est inférieur.

Chaque pays participant administre sept des neuf blocs d'items : les trois blocs contenant des items d'ancrage, deux des blocs contenant de nouveaux items et soit les deux autres blocs "standard», soit les deux blocs "faciles ». Proposer au choix les blocs "faciles » et "standard " permet de mieux cibler l'évaluation dans chaque pays participant. Toutefois, les items sont mis à l'échelle de sorte que le score d'un pays ne pâtira pas que les blocs «faciles » ou «standard » soient administrés. Les blocs d'items sont répartis par rotation entre des carnets de test, de sorte que les épreuves contiennent quatre blocs d'items en mathématiques, en compréhension de l'écrit et en sciences. Chaque élève répond aux items d'un carnet, ce qui représente 120 minutes de test au total.

L'épreuve informatisée de mathématiques (Computer-Based Component of Mathematics, CBAM) contient 80 minutes de test au total. Les items sont répartis en quatre blocs de 20 minutes de test. Les carnets de test sont constitués par rotation des blocs et contiennent deux blocs d'items chacun, ainsi que du matériel destiné à l'administration informatisée de l'épreuve. Chaque élève répond aux items d'un carnet, ce qui représente 40 minutes de test.

\section{Structure des épreuves de mathématiques lors du cycle PISA 2012}

Trois formats d'items sont utilisés dans l'épreuve papier-crayon d'évaluation de la culture mathématique conçue pour le cycle PISA 2012 : des items à réponse construite ouverte, des items à réponse construite fermée et des items à choix multiple. Dans les items à réponse construite ouverte, les élèves doivent fournir une réponse écrite un tant soit peu élaborée, et ils doivent aussi parfois expliquer le cheminement vers leur réponse ou montrer les étapes qu'ils ont enchainées pour y aboutir. Ces items sont corrigés manuellement par des correcteurs spécialement formés à cet effet. Les items à réponse construite fermée offrent aux élèves un cadre plus structuré pour présenter leur réponse, qui peut dès lors être jugée plus facilement comme correcte ou incorrecte. Souvent, les réponses des élèves à ces items peuvent être saisies dans une base de données, puis codées automatiquement, mais il arrive que des correcteurs spécialement formés doivent intervenir. Les réponses construites fermées se résument la plupart du temps à un chiffre. Dans les items à choix multiple, les élèves doivent choisir une ou plusieurs options de réponse. Leurs réponses peuvent généralement être codées automatiquement. Les trois formats d'items sont représentés en proportions similaires dans les épreuves.

D'autres formats d'items sont utilisés dans l'épreuve informatisée de mathématiques. L'environnement informatique se prête à une plus grande variété de modes de réponse que dans les épreuves papier-crayon et facilite l'évaluation de certains aspects de la culture mathématique, notamment la manipulation et la rotation de formes en trois dimensions qu'il n'est pas aussi aisé d'évaluer sur papier. Il permet également d'améliorer la présentation des items. Ainsi, on peut utiliser un stimulus mobile, des représentations d'objets en trois dimensions qu'il est possible de faire pivoter, ou des moyens d'accès plus flexibles à des données ou des informations pertinentes. Cet environnement offre également la possibilité d'employer des formats d'items associés à un éventail plus large de types de réponse. Par exemple, les fonctions "glisser-déplacer » ou l'utilisation de points sur une image permettent aux élèves de répondre à davantage d'items de façon non verbale, ce qui donne une image plus précise d'une culture mathématique moins associée à 
I'expression. Une certaine interactivité est possible dans certains cas. De plus, le codage automatisé des réponses permet d'éviter une partie du travail manuel. Le plus important réside dans le fait que ce type d'épreuves permet de faciliter le codage de caractéristiques dans les schémas que réalisent les élèves, les pages qu'ils affichent et les procédures qu'ils utilisent, ce qui est impossible dans d'autres environnements (Stacey et Wiliam, à paraître).

Les épreuves PISA de mathématiques sont constituées d'unités qui comprennent le stimulus verbal et souvent d'autres d'informations, par exemple des tableaux, des diagrammes, des cartes ou des graphiques, ainsi que un ou plusieurs items en rapport avec le stimulus. Grâce à ce format, les élèves ont la possibilité de se plonger dans le contexte ou le problème en répondant à plusieurs items. Toutefois, le modèle de mesure utilisé pour analyser les données PISA suppose que les items sont indépendants les uns des autres : dès qu'une unité compte plus d'un item, les développeurs de test s'efforcent d'assurer que les items soient les plus indépendants possible les uns des autres. Cette structure par unité a été retenue dans l'enquête PISA, car elle facilite l'utilisation de contextes aussi réalistes que possible qui reflètent la complexité des situations dans le monde réel tout en exploitant au mieux le temps de passation des épreuves. Toutefois, il est important de garantir un éventail adéquat de contextes pour minimiser le risque de biais dû aux contextes choisis et optimiser l'indépendance des items. Les concepteurs des instruments PISA cherchent donc à concilier ces deux impératifs contradictoires.

Les items retenus pour constituer les épreuves PISA se répartissent sur un spectre étendu de difficulté, correspondant au spectre étendu de compétences des élèves. De plus, les grandes catégories de contenus, de processus et de contextes sont autant que possible représentées par des items dont le degré de difficulté varie fortement. Le degré de difficulté des items est déterminé entre autres propriétés lors d'un essai de terrain à grande échelle avant la sélection des items qui seront administrés lors de la campagne définitive. Les items sont sélectionnés pour constituer les épreuves PISA en fonction de leur adéquation avec les catégories définies dans le cadre et de leurs propriétés en matière d'évaluation.

De plus, le niveau de compétence en compréhension de l'écrit requis pour réussir à aborder un item fait l'objet d'une grande attention lors de la conception et de la sélection des items. Lors de la conception des items, on s'efforce de formuler les items de la façon la plus simple et la plus directe possible. On s'emploie également à éviter des contextes qui créeraient un biais culturel et tous les choix sont vérifiés avec les équipes nationales. La traduction des items dans de nombreuses langues différentes est un processus qui est mené avec grand soin et qui prévoit de nombreuses procédures de rétro traduction et autres. Le risque de biais a fait l'objet d'une plus grande attention encore lors du cycle PISA 2012, dans la mesure où l'épreuve informatisée pourrait poser des difficultés aux élèves qui n'ont pas accès à un ordinateur en cours de mathématiques.

\section{Outils mathématiques}

Selon I'approche retenue dans I'enquête PISA, les élèves peuvent utiliser une calculatrice pour répondre aux épreuves papier-crayon si c'est d'usage dans leur établissement. C'est ce qui permet d'évaluer de la manière la plus authentique ce que les élèves sont capables de faire et d'effectuer la comparaison la plus riche en informations entre les systèmes d'éducation. Le fait qu'un système d'éducation ait choisi d'autoriser ou non ses élèves à utiliser une calculatrice ne diffère en rien, en principe, de toutes les autres orientations de la politique de l'éducation, sur lesquelles l'enquête PISA n'exerce pas de contrôle. Lors du cycle PISA 2012, c'est la première fois que certains items des épreuves papier-crayon de mathématiques ont été conçus de sorte que l'usage d'une calculatrice facilite et accélère les calculs - en d'autres termes, il est probable que l'usage d'une calculatrice avantage de nombreux élèves dans certains items. Dans ces épreuves papiercrayon de mathématiques, une calculatrice de base avec fonctions arithmétiques suffit.

Dans l'épreuve informatisée de mathématiques proposée à titre d'option lors du cycle PISA 2012, les élèves ont accès à un logiciel de calcul ou une calculatrice en ligne pour répondre aux items où cela est pertinent. Ils peuvent également utiliser une calculatrice si leur système d'éducation l'autorise à l'âge de 15 ans. Ils peuvent être amenés à utiliser d'autres outils pendant les épreuves, par exemple des instruments de mesure, des fonctions élémentaires de tableurs et des outils de représentation graphique ou de visualisation.

\section{Codage des items}

La majorité des items sont corrigés de façon dichotomique (les réponses valent ou non un crédit). Certains des items à réponse construite ouverte peuvent toutefois valoir un crédit partiel, en fonction de la mesure dans laquelle les réponses sont correctes. Des consignes détaillées de correction (crédit complet, crédit partiel et pas de crédit) sont fournies aux correcteurs formés pour coder les réponses à ces items, ce qui permet de garantir que le codage des items est effectué d'une façon uniforme et fiable dans tous les pays participants. 


\section{Présentation du niveau de compétence en mathématiques}

Les résultats aux épreuves PISA de mathématiques sont présentés de différentes façons. Le niveau global de culture mathématique est estimé sur une échelle de compétence à partir des échantillons d'élèves dans chaque pays. Le degré de culture mathématique associé à chaque niveau de l'échelle de compétence est décrit. De plus, des aspects de la culture mathématique pertinents pour I'action publique dans les pays participants sont identifiés et le niveau de compétence des élèves est estimé sur des échelles correspondant à ces différents aspects. Les aspects susceptibles d'être utilisés pour rendre compte des résultats peuvent se définir de différentes façons. Lors du cycle PISA 2003, les quatre grandes catégories de contenu ont été retenues pour construire des échelles de compétence. La figure 1.3 décrit les six niveaux de compétence de l'échelle globale de compétence en mathématiques utilisée pour rendre compte des résultats aux épreuves PISA en 2003, 2006 et 2009. Ces niveaux sont à la base de l'échelle PISA de compétence en mathématiques du cycle PISA 2012.

- Figure 1.3 .

Description de l'échelle de compétence en mathématiques (2003-2009)

\begin{tabular}{|c|c|}
\hline Niveau & \\
\hline 6 & $\begin{array}{l}\text { Au niveau 6, les élèves sont capables de conceptualiser, de généraliser et d'utiliser des informations sur la base de leurs propres } \\
\text { recherches et de la modélisation de problèmes complexes. Ils peuvent établir des liens entre différentes représentations et } \\
\text { sources d'information, et passer de l'une à l'autre sans difficulté. Ils peuvent se livrer à des raisonnements et à des } \\
\text { réflexions mathématiques difficiles. Ils peuvent s'appuyer sur leur compréhension approfondie et leur maîtrise des relations } \\
\text { symboliques et des opérations mathématiques classiques pour élaborer de nouvelles approches et de nouvelles stratégies à } \\
\text { appliquer lorsqu'ils sont face à des situations qu'ils n'ont jamais rencontrées. Ils peuvent décrire clairement et communiquer } \\
\text { avec précision leurs actes et les fruits de leur réflexion - résultats, interprétations, arguments - qui sont en adéquation avec } \\
\text { les situations initiales. }\end{array}$ \\
\hline 5 & $\begin{array}{l}\text { Au niveau 5, les élèves peuvent élaborer et utiliser des modèles dans des situations complexes pour identifier des contraintes } \\
\text { et construire des hypothèses. Ils sont capables de choisir, de comparer et d'évaluer des stratégies de résolution de problèmes } \\
\text { leur permettant de s'attaquer à des problèmes complexes en rapport avec ces modèles. Ils peuvent aborder les situations } \\
\text { sous un angle stratégique en mettant en œuvre un grand éventail de compétences pointues de raisonnement et de } \\
\text { réflexion, en utilisant les caractérisations symboliques et formelles et les représentations y afférentes, et en s'appuyant sur } \\
\text { leur compréhension approfondie de ces situations. Ils peuvent réfléchir à leurs actes, et formuler et communiquer leurs } \\
\text { interprétations et leur raisonnement. }\end{array}$ \\
\hline 4 & $\begin{array}{l}\text { Au niveau 4, les élèves sont capables d'utiliser des modèles explicites pour faire face à des situations concrètes complexes } \\
\text { qui peuvent leur demander de tenir compte de contraintes ou de construire des hypothèses. Ils peuvent choisir et intégrer } \\
\text { différentes représentations, dont des représentations symboliques, et les relier directement à certains aspects de situations } \\
\text { tirées du monde réel. Ils peuvent mettre en œuvre un éventail de compétences pointues dans ces situations et raisonner avec } \\
\text { une certaine souplesse en s'appuyant sur leur compréhension de ces contextes. Ils peuvent formuler des explications et des } \\
\text { arguments sur la base de leurs interprétations et de leurs actions, et les communiquer. }\end{array}$ \\
\hline 3 & $\begin{array}{l}\text { Au niveau 3, les élèves peuvent appliquer des procédures bien définies, dont celles qui leur demandent des décisions } \\
\text { séquentielles. Ils peuvent choisir et mettre en œuvre des stratégies simples de résolution de problèmes. Ils peuvent interpréter } \\
\text { et utiliser des représentations basées sur différentes sources d'information, et construire leur raisonnement directement sur } \\
\text { cette base. Ils peuvent rendre compte succinctement de leurs interprétations, de leurs résultats et de leur raisonnement. }\end{array}$ \\
\hline 2 & $\begin{array}{l}\text { Au niveau 2, les élèves peuvent interpréter et reconnaître des situations dans des contextes qui leur demandent tout au plus } \\
\text { d'établir des inférences directes. Ils ne peuvent puiser des informations pertinentes que dans une seule source d'information et } \\
\text { n'utiliser qu'un seul mode de représentation. Ils sont capables d'utiliser des algorithmes, des formules, des procédures ou des } \\
\text { conventions élémentaires. Ils peuvent se livrer à un raisonnement direct et interpréter les résultats de manière littérale. }\end{array}$ \\
\hline 1 & $\begin{array}{l}\text { Au niveau 1, les élèves peuvent répondre à des questions s'inscrivant dans des contextes familiers, dont la résolution ne } \\
\text { demande pas d'autres informations que celles présentes et qui sont énoncées de manière explicite. Ils sont capables d'identifier } \\
\text { les informations et d'appliquer des procédures de routine sur la base de consignes directes dans des situations explicites. Ils } \\
\text { peuvent exécuter des actions qui vont de soi et qui découlent directement du stimulus donné. }\end{array}$ \\
\hline
\end{tabular}

Trois sous-échelles de compétence ont été élaborées de la même façon que l'échelle globale de compétence en mathématiques, après l'essai de terrain, sur la base des trois processus mathématiques décrits auparavant : formuler des situations de façon mathématique ; employer des concepts, faits, procédures et raisonnements mathématiques ; et enfin, interpréter, appliquer et évaluer des résultats mathématiques.

Les facultés mathématiques fondamentales jouent un rôle central dans la définition des différents niveaux de l'échelle globale de culture mathématique et des sous-échelles de processus - elles définissent l'accroissement de compétence dans tous ces aspects de la culture mathématique. Par exemple, dans la description du niveau 4 de l'échelle de compétence (voir la figure 1.3), la deuxième phrase souligne des aspects de la mathématisation et de la représentation 
qui sont évidents à ce niveau de compétence, et la dernière phrase met l'accent sur les facultés de communication, de raisonnement et d'argumentation caractéristiques de ce niveau, les comparant à celles, inférieures et supérieures respectivement, associées au niveau 3 et au niveau 5. À la fin de ce chapitre, l'encadré 1.1 « Les facultés mathématiques fondamentales et leur relation avec la difficulté des items » décrit les facultés mathématiques fondamentales et explique leur lien avec les niveaux de culture mathématique. Les processus mathématiques ont été décrits auparavant dans ce chapitre et dans la figure 1.2 en fonction des facultés mathématiques fondamentales qu'ils font intervenir.

Des échelles de compétence ont été également définies pour les quatre catégories de contenus - Quantité, Espace et formes, Variations et relations, et enfin, Incertitude et données - par souci de cohérence avec la présentation des résultats de mathématiques lors du cycle PISA 2003, soit la première fois où les mathématiques ont été domaine majeur d'évaluation, et en raison de leur pertinence pour éclairer l'action publique. Ces échelles restent intéressantes pour les pays, car elles sont révélatrices de certains aspects de la culture mathématique en rapport avec des priorités spécifiques des programmes de cours.

\section{Attitudes envers les mathématiques}

Les attitudes, les convictions et les émotions des individus peuvent jouer un rôle important dans l'intérêt qu'ils portent aux mathématiques, ainsi que dans la façon dont ils y réagissent en général et les utilisent dans leur vie. Les élèves qui se sentent plus à l'aise en mathématiques sont, par exemple, plus susceptibles que les autres d'utiliser les mathématiques dans les différentes situations qu'ils rencontrent. Les élèves chez qui les mathématiques suscitent des émotions positives sont plus susceptibles de mieux apprendre les mathématiques que ceux chez qui elles créent de l'anxiété. L'un des objectifs des cours de mathématiques doit donc être de faire naître chez les élèves des attitudes, des convictions et des émotions qui les rendent plus susceptibles d'utiliser les notions de mathématiques qu'ils connaissent et d'en apprendre davantage dans cette matière, par intérêt personnel et social.

L'attention accordée à ces variables dans les épreuves de mathématiques du cycle PISA 2012 découle de la thèse selon laquelle le développement de convictions, d'émotions et d'attitudes positives envers les mathématiques est en soi un résultat précieux des cours, et prédispose les élèves à utiliser les mathématiques dans la vie. Ces variables peuvent aussi contribuer à expliquer la variation de la culture mathématique entre les élèves. C'est pourquoi les épreuves PISA de mathématiques contiennent des items en rapport avec ces variables. De plus, I'enquête PISA enregistre une série de variables contextuelles qui permettent de rendre compte de la culture mathématique et de l'analyser dans des sousgroupes importants d'élèves (par exemple, en fonction du sexe, de la langue parlée ou de l'ascendance allochtone).

Pour recueillir des informations contextuelles, les élèves et les chefs d'établissement sont invités à répondre à un questionnaire, ce qui leur prend entre 20 et 30 minutes. Ces questionnaires sont au cœur de la présentation et de l'analyse des résultats en fonction des profils des élèves et des établissements.

Deux grandes catégories d'attitudes envers les mathématiques qui prédisposent les élèves à s'intéresser aux mathématiques de façon productive ont été identifiées pour leur intérêt potentiel et ont été ajoutées aux épreuves de mathématiques du cycle PISA 2012. Il s'agit de l'intérêt des élèves pour les mathématiques et de leur volonté de s'y engager.

L'intérêt pour les mathématiques recèle des aspects en rapport avec l'activité présente et à venir. Parmi les items qui $s^{\prime} y$ rapportent, citons ceux qui cherchent à déterminer dans quelle mesure les élèves s'intéressent aux mathématiques à l'école, jugent que les mathématiques sont utiles dans la vie et envisagent d'exercer une profession en lien avec les mathématiques. Ce dernier point est préoccupant dans le monde : de nombreux pays participants voient en effet diminuer le pourcentage d'individus qui choisissent de suivre des études en mathématiques, alors que la demande de diplômés dans cette matière augmente.

La volonté des élèves de faire des mathématiques dépend d'attitudes, d'émotions et de convictions qui disposent les élèves à tirer parti ou non de la culture mathématique qu'ils ont acquise. Les élèves qui prennent plaisir à faire des mathématiques et qui se sentent à l'aise dans cette matière sont plus susceptibles d'utiliser les mathématiques pour réfléchir aux situations qu'ils rencontrent dans les différentes facettes de leur vie, à l'école ou ailleurs. Les constructs retenus dans l'enquête PISA à ce sujet sont les suivants : le plaisir, la confiance, l'anxiété (ou l'absence d'anxiété), l'image de soi et l'efficacité perçue. Selon une analyse récente du parcours ultérieur de jeunes Australiens qui avaient obtenu de piètres résultats aux épreuves PISA à l'âge de 15 ans, les individus parmi eux qui reconnaissent la valeur des mathématiques pour leur réussite à l'avenir sont plus susceptibles de concrétiser cette réussite, notamment de retirer de la satisfaction de nombreux aspects de leur vie personnelle et professionnelle (Thomson et Hillman, 2010, p. 31). Cette étude recommande d'insister sur les applications pratiques des mathématiques dans la vie de tous les jours, car cela pourrait améliorer les perspectives qui s'offrent à ces élèves peu performants. 
Le questionnaire «Élève » inclut également des séries d'items sur les possibilités d'apprentissage. Ces items se rapportent à l'expérience des élèves au sujet de problèmes de mathématiques appliquées de différents types, à la mesure dans laquelle le nom de concepts mathématiques est familier aux élèves (des leurres ont été prévus pour obtenir des résultats probants) et à l'expérience vécue par les élèves en classe ou durant des épreuves de mathématiques telles que les épreuves PISA. Ces variables permettront d'approfondir l'analyse des résultats de l'enquête PISA.

Les résultats du cycle PISA 2012 fourniront des informations importantes aux responsables de la politique de l'éducation dans les pays participants au sujet du rendement des élèves ainsi que de leurs attitudes. Combiner les résultats aux épreuves PISA de mathématiques et les données sur les attitudes, les convictions et les émotions qui prédisposent les élèves à utiliser leur culture mathématique permet de brosser un tableau plus complet.

\section{Épreuve informatisée de mathématiques (facultative)}

Le cycle PISA 2012 inclut une épreuve informatisée de mathématiques. Cette épreuve est proposée à titre d'option aux pays participants (étant donné que leurs capacités technologiques sont variables). Deux raisons ont présidé à son inclusion dans le cycle PISA 2012. En premier lieu, I'ordinateur est désormais d'usage sur le lieu de travail et dans la vie quotidienne, de sorte que le degré de culture mathématique au XXIe siècle implique I'utilisation de I'informatique (Hoyles et al., 2002). L'informatique intervient dans la vie des individus au quotidien, dans leurs activités personnelles, professionnelles et scientifiques. Elle leur offre des outils pour calculer, se représenter un large éventail de phénomènes, processus ou objets mathématiques, les visualiser, les modifier, les explorer et les expérimenter. La définition de la culture mathématique retenue en vue du cycle PISA 2012 reconnaît l'importance des outils informatiques en soulignant que les individus sont censés les utiliser pour décrire, expliquer et prévoir des phénomènes. Dans cette définition, le terme " outils » désigne les calculatrices, les ordinateurs, ainsi que tout autre objet, comme une règle ou un rapporteur, utilisé comme instrument de mesure ou de construction. La deuxième raison réside dans le fait que l'informatique offre aux concepteurs d'épreuves la possibilité de créer des items plus interactifs, authentiques et intéressants (Stacey et Wiliam, à paraître). Ils peuvent notamment inventer de nouveaux formats d'items ("glisser-déplacer »), soumettre aux élèves des données qui s'inspirent du monde réel (de longues séries de données qu'il est possible de trier) ou utiliser des couleurs et des graphiques pour rendre les épreuves plus attrayantes.

Face à ces phénomènes, l'épreuve informatisée de mathématiques est une innovation majeure du cycle PISA 2012. Les unités PISA spécialement créées à cet effet sont présentées sur ordinateur, et les élèves y répondent par ordinateur. Les élèves peuvent également prendre des notes sur papier pour retracer leurs processus de réflexion. Des items informatisés plus sophistiqués pourraient être proposés lors des cycles PISA suivants, à mesure que les développeurs d'items s'immergeront dans l'environnement des évaluations informatisées. Le cycle PISA 2012 marque uniquement le début de l'exploration du potentiel des épreuves informatisées de mathématiques.

Le fait d'utiliser les fonctionnalités offertes par l'informatique permet de proposer aux élèves des items plus attrayants, plus colorés et plus faciles à comprendre. Il est par exemple possible de soumettre aux élèves un stimulus mobile et des représentations d'objets en trois dimensions qu'ils peuvent faire pivoter, ou de leur donner un accès plus flexible à des informations pertinentes. De nouveaux formats d'items, tels que ceux qui invitent les élèves à " glisser-déplacer " des informations ou à utiliser les «points» d'images, sont conçus pour amener les élèves à s'engager et offrent la possibilité de proposer un éventail plus large de types de réponses et de présenter un tableau plus complet de la culture mathématique.

Des recherches montrent que les exigences mathématiques dans le milieu professionnel augmentent dans les environnements électroniques de sorte que la culture mathématique et l'usage de l'informatique se combinent (Hoyles et al., 2002). Tous les travailleurs, quelle que soit leur place dans la hiérarchie, font face à l'interdépendance de la culture mathématique et de l'usage de l'informatique, une relation que l'épreuve informatisée de l'enquête PISA permet d'explorer. L'un des enjeux majeurs est d'établir une distinction entre les exigences mathématiques d'un item PISA soumis de façon informatisée et les exigences sans lien avec le niveau de compétence en mathématiques, notamment les exigences en technologies de l'information et de la communication (TIC) et les nouveaux formats de présentation. II est important de souligner à propos des épreuves informatisées du cycle PISA 2012 que tout est mis en œuvre pour que les exigences associées à l'utilisation d'un outil soient nettement inférieures aux exigences mathématiques. Des recherches ont été menées sur l'impact de l'informatisation d'épreuves sur les performances des élèves (Bennett, 2003 ; Bennett et al., 2008 ; Mason et al., 2001 ; Richardson et al., 2002 ; Sandene et al., 2008). Le cycle PISA 2012 est l'occasion d'approfondir ces recherches, en particulier d'enrichir les connaissances au sujet du développement des items en vue des prochaines épreuves informatisées, en 2015 et au-delà. Les items de l'épreuve informatisée ne se baseront pas tous sur de nouveaux formats, ce qui pourrait être utile pour déterminer l'impact (positif ou négatif) que les nouveaux formats ont sur la performance. 
Pour contrôler les caractéristiques de l'épreuve informatisée, trois aspects sont décrits dans chaque item :

- Les compétences mathématiques évaluées, qui comprennent des aspects de la culture mathématique applicables dans n'importe quel environnement - pas uniquement informatique - et qui sont mesurées dans chaque item informatisé.

- Les compétences en mathématiques et en informatique, qui consistent à faire des mathématiques avec l'aide d'outils informatiques ou autres. Ces compétences sont évaluées dans certains des items informatisés (mais pas tous). L'épreuve informatisée peut évaluer les compétences suivantes :

- créer un graphique (à secteurs, en bâtons, en courbes) à partir de données figurant dans un tableau par exemple, à l'aide $d^{\prime}$ « assistants » simples ;

- générer des graphiques de fonctions et les utiliser pour répondre à des questions sur les fonctions ;

- trier des données et prévoir des stratégies efficientes de tri ;

- utiliser des calculatrices de poche ou des calculatrices sur ordinateur ;

- utiliser des instruments virtuels, tels qu'une règle ou un rapporteur virtuel ; et

- transformer des images à l'aide d'une boîte de dialogue ou de la souris, par exemple faire pivoter des images, les réfléchir ou les traduire.

- Les compétences en technologies de l'information et de la communication (TIC), qui sont fondamentales pour répondre à une épreuve informatisée, au même titre que des compétences d'utilisation de l'écrit sont fondamentales pour répondre à une épreuve papier-crayon. Parmi ces compétences, citons la capacité à utiliser du matériel de base (la souris, le clavier, etc.) et la connaissance de conventions élémentaires (les flèches pour se déplacer et les boutons pour exécuter des commandes, par exemple). L'intention est de limiter autant que faire se peut la nécessité d'utiliser ces compétences dans toutes les épreuves informatisées.

\section{SYNTHĖSE}

L'objectif de I'enquête PISA concernant la culture mathématique est d'élaborer des indicateurs montrant l'efficacité avec laquelle les pays préparent leurs élèves à utiliser les mathématiques dans tous les aspects de leur vie personnelle, civique et professionnelle, en tant que citoyens constructifs, engagés et réfléchis. Pour ce faire, nous avons défini la culture mathématique dont les composantes importantes sont décrites dans le présent cadre d'évaluation. Les items de mathématiques qui ont été conçus, puis choisis pour constituer les épreuves du cycle PISA 2012 sur la base de cette définition et de ce cadre d'évaluation reflètent l'éventail de contextes, contenus et processus mathématiques pertinents. Ces items permettent de déterminer dans quelle mesure les élèves sont capables d'utiliser ce qu'ils ont appris. Ils amènent les élèves à utiliser leurs connaissances, à s'engager dans des processus et à exploiter leurs facultés pour résoudre des problèmes qui se posent dans des expériences du monde réel. Les problèmes sont présentés sous divers formats et sont accompagnés d'orientations et d'indices sur la structure qui sont plus ou moins explicites, mais l'accent est mis sur l'aspect pratique des problèmes, auxquels les élèves doivent réfléchir eux-mêmes.

\section{Encadré 1.1. Les facultés mathématiques fondamentales et leur relation avec la difficulté des items}

Analyser les aspects des facultés mathématiques fondamentales qui sont requis pour concevoir, puis appliquer une solution est un bon moyen de déterminer la difficulté empirique des items (Turner, 2012 ; Turner et Adams, 2012 ; Turner et al., à paraître). Les items les plus faciles requièrent l'exploitation de quelques facultés à peine, d'une façon relativement directe. Les items les plus difficiles requièrent l'exploitation complexe de plusieurs facultés. Pour estimer a priori la difficulté des items, il faut tenir compte à la fois du nombre de facultés à mettre en œuvre et de la complexité de leur activation. Les sections suivantes décrivent les caractéristiques qui rendent l'activation d'une faculté plus ou moins complexe (voir également Turner, 2012).

Communication : divers facteurs déterminent le niveau et l'ampleur des exigences d'une tâche en matière de communication, et la capacité des individus à répondre à ces exigences indique la mesure dans laquelle ils possèdent la faculté de communication. Côté réception, ces facteurs sont notamment la longueur et la complexité du texte ou de l'objet à lire et à interpréter, le caractère familier des idées ou des informations auxquelles il est fait référence dans le texte ou l'objet, la mesure dans laquelle il faut dégager les informations pertinentes d'un enchevêtrement d'informations, l'ordre des informations et la mesure dans laquelle cet ordre correspond à l'ordre des processus à mener pour interpréter et utiliser les informations, et la diversité des éléments (texte, graphique, diagramme, tableau, etc.) à interpréter les uns par rapport aux autres. Côté expression, les items les plus faciles sont ceux qui appellent simplement une réponse sous forme de chiffre. La difficulté de la communication augmente lorsque la solution aux items doit être construite, par exemple lorsque les élèves doivent expliquer ou justifier leur résultats oralement ou par écrit. 
Mathématisation : certaines tâches ne requièrent pas de mathématisation - que ce soit parce que le problème se présente déjà sous une forme suffisamment mathématique ou parce que la relation entre le modèle et la situation qu'il représente n'est pas nécessaire pour résoudre le problème. La mathématisation est la moins difficile lorsque les élèves doivent interpréter un modèle donné et faire des inférences directement ou traduire directement une situation en mathématiques (par exemple, structurer et conceptualiser la situation d'une façon pertinente, identifier et sélectionner des variables pertinentes, réunir des mesures pertinentes et/ou faire des diagrammes). La mathématisation devient plus difficile lorsque les élèves doivent modifier ou utiliser un modèle donné pour comprendre l'évolution de conditions ou interpréter des relations inférées, choisir un modèle familier dans le respect de contraintes limitées et clairement articulées, ou créer un modèle où les variables, les relations et les contraintes sont explicites et claires. À un degré de difficulté plus élevé encore, la mathématisation consiste à créer ou interpréter un modèle dans une situation où de nombreuses hypothèses, variables, relations et contraintes doivent être identifiées ou définies, et à vérifier que le modèle est conforme aux exigences de la tâche ou à évaluer ou comparer des modèles.

Représentation : cette faculté mathématique est la moins difficile à mettre en œuvre lorsque les tâches consistent à manipuler directement une représentation, par exemple passer directement du texte aux chiffres, ou lire directement une valeur dans un graphique ou un tableau. La charge cognitive des tâches augmente en matière de représentation lorsqu'elles requièrent la sélection et l'interprétation d'une représentation standard ou familière par rapport à une situation, et plus encore lorsqu'elles consistent à utiliser ou traduire deux ou plusieurs représentations par rapport à une situation, y compris à modifier une représentation, ou à comprendre et utiliser une représentation non standard au travers $d^{\prime}$ un processus complexe de décodage et d'interprétation, à concevoir une représentation qui englobe les aspects clés d'une situation complexe, ou à comparer ou à évaluer des représentations différentes.

Raisonnement et argumentation : dans les tâches où l'exploitation de cette faculté est minime, le raisonnement peut consister simplement à suivre les instructions fournies. Les items plus difficiles demandent une certaine réflexion pour établir des liens entre différents fragments d'information afin de faire des inférences (par exemple, établir un lien entre des composantes distinctes d'un problème ou se livrer à un raisonnement direct dans un seul aspect du problème). À un degré de difficulté plus élevé, les items requièrent l'analyse d'informations pour suivre ou créer une argumentation en plusieurs étapes, établir un lien entre plusieurs variables ou raisonner à partir de sources d'information différentes. À un niveau plus élevé encore, les élèves doivent résumer et évaluer des informations, utiliser ou créer un raisonnement en plusieurs étapes pour justifier des inférences, ou faire des généralisations en se basant sur plusieurs fragments d'information et en les combinant dans un but précis.

Conception de stratégies : les tâches où l'exploitation de cette faculté est minime demandent souvent simplement aux élèves de poser des actes, selon une stratégie qui est décrite ou qui va de soi. À un degré de difficulté légèrement plus élevé, les élèves peuvent avoir à choisir une stratégie adaptée compte tenu des informations pertinentes qui leur sont fournies pour aboutir à une conclusion. La charge cognitive est plus élevée lorsque les élèves doivent concevoir et construire une stratégie pour transformer des informations fournies afin d'aboutir à une conclusion. Dans les tâches encore plus difficiles, les élèves doivent construire une stratégie complexe pour trouver une solution exhaustive ou une conclusion généralisée, ou évaluer ou comparer plusieurs stratégies possibles.

Utilisation d'opérations et d'un langage symbolique, formel et technique : la difficulté de l'exploitation de cette faculté varie énormément selon les tâches. Dans les tâches les plus simples, les élèves ne doivent pas utiliser de règles mathématiques ou d'expressions symboliques qui vont au-delà des opérations arithmétiques fondamentales, avec des chiffres peu élevés ou faciles à manipuler. Dans les tâches plus difficiles, ils peuvent avoir à enchaîner des opérations arithmétiques, à utiliser directement une relation fonctionnelle simple soit implicite, soit explicite (une relation linéaire familière, par exemple), à utiliser des symboles mathématiques formels (par substitution directe ou via un enchaînement d'opérations arithmétiques impliquant des fractions et des décimales), ou à utiliser directement une définition formelle, une convention ou un concept symbolique de mathématiques. La charge cognitive augmente lorsque les élèves doivent utiliser et manipuler explicitement des symboles (recomposer une formule de façon algébrique, par exemple) ou utiliser des formules, des règles, des définitions, des conventions ou des procédures mathématiques sur la base de la combinaison de plusieurs relations ou concepts symboliques. À un degré de difficulté plus élevé encore, les élèves doivent appliquer des procédures mathématiques formelles en plusieurs étapes, utiliser avec souplesse des relations fonctionnelles ou algébriques, ou utiliser des connaissances et des techniques mathématiques pour produire des résultats. 
Utilisation d'outils mathématiques : dans les tâches qui ne demandent qu'une exploitation minime de cette faculté, les élèves peuvent avoir à utiliser directement des outils familiers (un instrument de mesure, par exemple) dans des situations où ces outils sont d'usage courant. La difficulté augmente lorsque l'utilisation de l'outil implique d'enchaîner des processus ou d'établir des liens entre des informations différentes, que les outils sont moins familiers ou que la situation dans laquelle l'outil doit être utilisé est moins familière. Elle augmente encore lorsque l'outil doit être utilisé pour traiter de nombreux fragments d'information et établir des liens entre eux, que l'outil doit l'être dans une situation qui est assez différente de celle où il est d'usage courant, que l'outil est complexe en soi et offre de nombreuses possibilités, ou que les élèves doivent réfléchir pour comprendre et évaluer les mérites et les limites de l'outil.

\section{EXEMPLES D'ITEMS PISA DE MATHÉMATIQUES}

Les items PISA qui suivent ont été rendus publics. Ils illustrent des nuances et des aspects pertinents du cadre d'évaluation du cycle PISA 2012. Les sept items proposés ont été sélectionnés pour montrer l'éventail de types d'items, de processus, de contenus et de contextes, et pour décrire l'activation des facultés mathématiques fondamentales, mais ils ne donnent pas toute la mesure de chaque aspect.

\section{HIT-PARADE}

La première unité proposée à titre d'exemple s'intitule HIT-PARADE. Elle est constituée d'un stimulus, en l'occurrence un texte et un graphique en bâtons qui montre les ventes de CD de quatre groupes pendant six mois, et de trois items à choix multiple (voir la figure 1.4).

- Figure 1.4 -

\section{Items de l'unité HIT-PARADE}

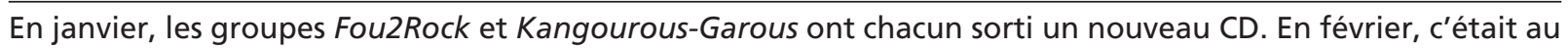
tour des groupes Seul au Monde et Cowboys Hurlants de sortir chacun leur CD. Le diagramme suivant montre les ventes de ces CD de janvier à juin.

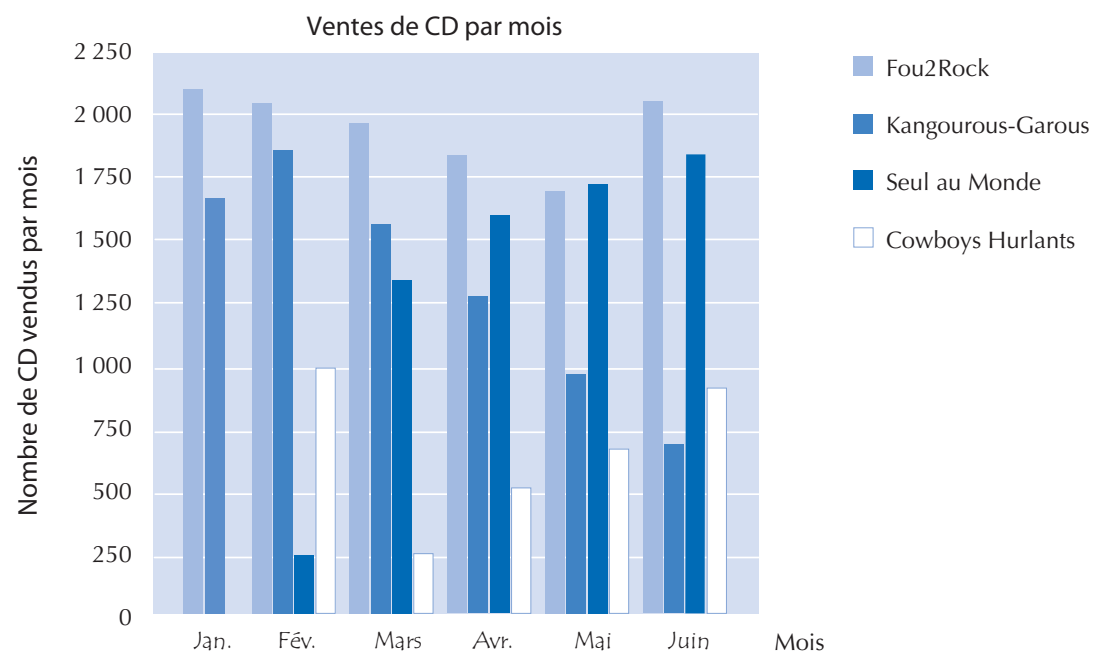




\section{QUESTION 1}

Combien de CD le groupe Cowboys

Hurlants a-t-il vendus en avril?

A. 250

B. 500

C. 1000

D. 1270
QUESTION 2

Au cours de quel mois le groupe Seul au Monde a-t-il vendu, pour la première fois, plus de CD que le groupe Kangourous-Garous?

A. Aucun mois

B. Mars

C. Avril

D. Mai

\section{QUESTION 3}

Le producteur des KangourousGarous s'inquiète car le nombre de CD qu'ils ont vendus a diminué de février à juin.

À combien peut-on estimer leurs ventes du mois de juillet si cette tendance à la baisse continue?

A. $70 C D$

B. $370 \mathrm{CD}$

C. $670 \mathrm{CD}$

D. $1340 C D$

Les pays PISA ont été invités à remplacer les noms des groupes par des noms de groupes fictifs, adaptés à leur contexte local, lors de la préparation de leur version nationale.

L'unité HIT-PARADE a été administrée lors de la campagne définitive du cycle PISA 2012. Les trois items de cette unité se classent dans la catégorie de contenus Incertitude et données puisque les élèves doivent lire, interpréter et utiliser des données présentées sous une forme graphique d'ordre mathématique, et dans la catégorie de Contextes sociétaux, car les données se rapportent à des informations publiques à propos de ventes de disques que l'on peut trouver dans un journal, un magazine de musique ou en ligne. Les deux premiers items se classent dans la catégorie de processus Interpréter, appliquer et évaluer des résultats mathématiques, car ils consistent à interpréter les informations mathématiques présentées dans le graphique par rapport à des caractéristiques du contexte, alors que le troisième item se classe dans la catégorie Employer des concepts, faits, procédures et raisonnements mathématiques, car il consiste à appliquer des connaissances en matière de procédures pour manipuler la représentation mathématique, puis faire des inférences. Ces trois items comptent parmi les plus faciles administrés lors de la campagne définitive du cycle PISA 2012.

Dans la question 1 (reprise dans la figure 1.4), les élèves doivent lire directement des données dans le graphique pour répondre à une question en rapport avec le contexte. Ils doivent s'orienter vers les informations fournies, identifier la série de données qui indique les ventes du groupe concerné et le segment qui représente le mois concerné dans cette série de données, puis lire directement la valeur « 500 CD » en ordonnée. Comme le texte est simple et clair, I'item appelle une activation minime de la faculté de communication. La stratégie à utiliser va de soi : il suffit de localiser les informations demandées dans le graphique. La mathématisation consiste à faire une inférence à propos des ventes directement à partir du modèle. La faculté de représentation intervient peu, car il suffit de lire une valeur directement dans un graphique. Le format du graphique est censé être familier pour la plupart des adolescents de 15 ans, auxquels le seul effort demandé est de lire l'intitulé des données. L'un des axes du graphique représente des catégories (mois) et la hauteur du bâton est précisée (500), de sorte que les élèves n'ont pas à comprendre d'échelle. Les connaissances techniques requises sont minimes, si ce n'est que le format du graphique doit être familier, et seule une inférence directe doit être faite, $d^{\prime}$ 'où le niveau très faible de raisonnement et d'argumentation. Cet item est extrêmement facile : 87 \% des élèves y répondu correctement (option B).

La question 2 n'est que légèrement plus difficile : 78 \% des élèves environ y ont répondu correctement (option C). Pour y répondre, les élèves doivent observer la relation entre deux séries de données présentées dans le graphique et comprendre son évolution au fil du temps pour réaliser que la situation concernée est apparue pour la première fois en avril.

La difficulté de cet item est similaire à celle du premier item en matière de communication. L'activation de la faculté de stratégie est légèrement plus difficile, car les élèves doivent associer plusieurs éléments provenant des deux séries de données. La mathématisation consiste ici aussi à faire une inférence à propos des ventes, de manière assez directe, à partir du graphique. Cet item est légèrement plus difficile en matière de représentation que le premier, car les élèves doivent établir un lien entre les deux séries de données et tenir compte de la variable du temps. Il est aussi facile que le premier en matière d'utilisation d'opérations et d'un langage symbolique, formel et technique, car seule une comparaison quantitative est requise. Enfin, il est légèrement plus difficile en matière de raisonnement et d'argumentation, car les élèves doivent enchaîner plusieurs étapes de raisonnement.

La question 3 est assez différente des deux premières, dans la mesure où elle demande essentiellement aux élèves de comprendre une relation mathématique décrite dans le graphique, puis de l'extrapoler pour prévoir la valeur d'un mois 
à venir. Le lien avec le contexte est toujours bien présent, mais les élèves doivent essentiellement travailler sur la base des informations mathématiques qui leur sont fournies. Pour répondre à la question, ils peuvent, par exemple, lire les valeurs de chaque mois dans la série concernée, estimer la réduction moyenne de la valeur de chaque mois, puis appliquer cette réduction à la valeur du dernier mois. La faculté de communication intervient peu. Tout l'enjeu est d'éviter de se faire distraire par les séries de données des autres groupes. Toutefois, la seule réponse incorrecte courante, l'option C, est vraisemblablement due à une mauvaise compréhension du passage " si cette tendance à la baisse continue »:15\% des élèves ont choisi cette option estimant que les ventes seraient les mêmes en juillet qu'en juin. Ils ont peut-être choisi la valeur constante parce qu'elle perpétuait les mauvais chiffres de vente de juin en juillet. La faculté de stratégie intervient de toute évidence davantage que dans les deux premières questions et son exploitation requiert un certain suivi. II y a des décisions à prendre, notamment celle de savoir s'il vaut mieux utiliser les chiffres de ce groupe entre février et juin ou se baser sur la variation moyenne entre février et juin, $\mathrm{s}^{\prime}$ il faut faire un calcul exact, dessiner ou visualiser une courbe de tendance, ou utiliser des estimations approximatives et remarquer que les ventes diminuent chaque mois $d^{\prime}$ un peu plus d'un niveau sur l'échelle en ordonnée. La mathématisation consiste à manipuler légèrement le modèle fourni par rapport au contexte, des calculs s'imposent (faire plusieurs soustractions de nombres à plusieurs chiffres, lire l'évolution sur une échelle), ce qui ajoute à la difficulté en matière d'utilisation d'opérations et d'un langage symbolique, formel et technique. L'item consiste à faire une inférence à propos de la relation tendancielle décrite dans le graphique en matière de représentation, et à enchaîner quelques étapes de raisonnement. Toutefois, cet item est relativement facile aussi : 76 \% des élèves y ont répondu correctement (option B) lors de la campagne définitive du cycle PISA 2012.

- Figure 1.5 -

Items de l'unité ASCENSION DU MONT FUJI

\section{ASCENSION DU MONT FUJ}

Le mount Fuji est un célèbre volcan éteint, situé au Japon.

QUESTION 1

Le mont Fuji n'est accessible au public que du 1 1er juillet au 27 août chaque année. Environ 200 OOO personnes font l'ascension du mont Fuji pendant cette période.

En moyenne, combien de personnes environ font l'ascension du mont Fuji chaque jour?

A. 340

B. 710

C. 3400

D. 7100

E. 7400

\section{QUESTION 2}

La voie Gotemba, qui conduit au sommet du mont Fuji, fait environ 9 kilomètres ( km) de long.

Les marcheurs doivent être de retour de la randonnée de $18 \mathrm{~km}$ pour 20 heures.

Toshi estime qu'il peut gravir la montagne à une vitesse moyenne de 1,5 kilomètre/heure, et en redescendre en doublant cette vitesse. Ces vitesses tiennent compte des pauses-repas et des temps de repos.

D’après les vitesses estimées par Toshi, à quelle heure au plus tard doit-il commencer sa randonnée afin de pouvoir être de retour pour 20 heures?

\section{QUESTION 3}

Lors de sa randonnée sur la voie Gotemba, Toshi portait un podomètre pour comptabiliser ses pas.

Son podomètre indique qu'il a fait 22500 pas lors de la montée.

Estimez la longueur moyenne des pas de Toshi lors de la montée de 9 kilomètres de la voie Gotemba. Donnez votre réponse en centimètres $(\mathrm{cm})$.

Réponse..................... cm 


\section{ASCENSION DU MONT FUJI}

La deuxième unité proposée à titre d'exemple s'intitule ASCENSION DU MONT FUJI (voir la figure 1.5). La première question est un item à choix multiple et les deux dernières, des items à réponse construite appelant une réponse sous forme de chiffre. Le troisième item permet l'octroi d'un crédit partiel, une forme de codage utilisée dans un pourcentage limité d'items PISA auxquels des réponses de qualité variable peuvent être données et sont associées à un niveau de compétence nettement différent.

L'unité ASCENSION DU MONT FUII a été administrée lors de la campagne définitive du cycle PISA 2012 avant d'être rendue publique. Les questions 1 et 3 se classent dans la catégorie de contenus Quantité, car elles demandent aux élèves de faire des calculs sur la base de dates et de mesures, et de faire des conversions. La question 2 est basée sur le thème de la vitesse, ce qui la classe dans la catégorie de contenus Variations et relations.

Ces questions se classent toutes dans la catégorie de Contextes sociétaux, car les données se rapportent à des informations sur l'accès du public au mont Fuji. Les deux premières questions illustrent le processus qui consiste à formuler des situations de façon mathématique, puisqu'elles demandent essentiellement aux élèves de créer un modèle mathématique pour répondre à la question.

La question 3 se classe dans la catégorie de processus Employer des concepts, faits, procédures et raisonnements mathématiques parce qu'elle demande essentiellement aux élèves de calculer une moyenne en prenant garde de bien convertir des unités, et donc de travailler en grande partie sur la base des détails mathématiques du problème au lieu de rapporter ces détails à des aspects du contexte. II ressort de la campagne définitive du cycle PISA 2012 que la difficulté de ces trois questions varie. La première question est moyennement difficile, alors que les deux dernières sont très difficiles.

La question 1 demande aux élèves de calculer un nombre moyen de personnes par jour. Comme le texte est simple et clair, il fait peu appel à la faculté de communication. La stratégie requise est d'une difficulté modérée, car elle consiste à trouver le nombre de jours entre les dates fournies, puis à l'utiliser pour calculer une moyenne. Cette solution en plusieurs étapes requiert un certain suivi, qui relève aussi de la faculté Conception de stratégies. La mathématisation est très facile, car les quantités mathématiques requises sont fournies directement dans la question (le nombre de personnes par jour). Il en va de même pour la représentation, car la question ne contient que du texte et des chiffres. Les élèves doivent utiliser des connaissances techniques : ils doivent savoir comment calculer une moyenne et le nombre de jours entre des dates, faire une division (à l'aide ou non d'une calculatrice, selon l'option retenue dans chaque pays) et arrondir le résultat. Cette question fait peu appel à la faculté de raisonnement et d'argumentation. Elle est d'une difficulté moyenne : 46 \% des élèves y ont répondu correctement (option C) lors de la campagne définitive du cycle PISA 2012. Les réponses incorrectes les plus souvent choisies sont l'option $\mathrm{E}$ (19\% des élèves), auquel cas les élèves se sont basés sur 27 jours, au lieu d'additionner 31 et 27 jours, et l'option A (12\% des élèves), auquel cas les élèves ont commis une erreur de valeur.

La question 2 est nettement plus difficile : $12 \%$ seulement des élèves y ont répondu correctement lors de la campagne définitive du cycle PISA 2012. Sa difficulté tient notamment au fait qu'il s'agit d'un item à réponse construite et non à choix multiple, de sorte que les élèves n'ont aucun indice sur les réponses possibles, mais de nombreux autres facteurs ajoutent à sa difficulté. Lors du cycle PISA 2012, quelque 61 \% des élèves y ont répondu de manière incorrecte, sans toutefois l'omettre.

La faculté de communication n'intervient guère, de surcroît uniquement sous l'aspect de la réception, comme dans la question 1. Côté expression, les élèves doivent simplement fournir une réponse sous forme de chiffre. La stratégie est nettement plus difficile, car les élèves doivent établir un plan sur la base de trois grandes composantes. Les élèves doivent calculer les temps d'ascension et de descente à partir des vitesses moyennes, puis calculer l'heure de départ en fonction de l'heure d'arrivée et du temps que prend la randonnée. La mathématisation requise est assez difficile, car elle consiste à comprendre, par exemple, que le temps consacré aux pauses-repas est compris et que la randonnée commence par l'ascension et se termine par la descente. La représentation est minimale, car il suffit aux élèves d'interpréter un texte. L'utilisation d'opérations et d'un langage symbolique, formel et technique est assez difficile : les calculs sont relativement simples (même si diviser par le nombre décimal de 1,5 kilomètre/heure peut être difficile), mais ils demandent une précision constante et la formule à employer pour déduire le temps de la vitesse et de la distance est implicitement ou explicitement requise. Le raisonnement et l'argumentation sont relativement simples également.

La question 3 est également assez difficile. Elle consiste à calculer la longueur moyenne de pas à partir de la distance et du nombre de pas, et implique la conversion d'unités. Lors du cycle PISA 2012, 11 \% des élèves ont obtenu un crédit 
complet parce qu'ils ont indiqué la réponse correcte $(40 \mathrm{~cm})$ et $4 \%$ ont obtenu un crédit partiel, parce qu'ils ont, par exemple, répondu « 0,4 » (laissant la longueur en mètres) ou « 4000 » (sans doute à cause d'une erreur de conversion entre le mètre et le centimètre). Lors de la campagne définitive du cycle PISA 2012, 62 \% des élèves y ont répondu de façon incorrecte, sans toutefois l'omettre. La faculté de communication intervient peu comme dans les questions précédentes, car le texte est relativement facile à comprendre et interpréter, et la question appelle une réponse sous forme de simple chiffre. La stratégie à utiliser pour répondre à la question 3 est similaire à celle décrite dans la question 1 - ces deux items consistent en effet à calculer une moyenne. Toutefois, si les deux questions consistent à calculer une moyenne, le raisonnement et l'argumentation sont nettement plus difficiles dans cette question que dans la première. Dans la question 1, les élèves doivent calculer un nombre de personnes par jour, sachant que le nombre de personnes est fourni et que le nombre de jours est facile à calculer. Dans la question 3, en revanche, ils doivent calculer la longueur moyenne de pas d'un individu à partir d'une distance totale et d'un nombre total de pas. Il faut un raisonnement plus poussé pour établir un lien entre ces quantités (entre la distance donnée et la longueur, par exemple). De même, la mathématisation est plus difficile dans cette troisième question, car les élèves doivent comprendre en quoi la longueur de pas, une variable du monde réel, est liée à des mesures globales. Apprécier le contexte, notamment savoir qu'un pas est susceptible d'être de l'ordre de 50 centimètres (et pas de 0.5 ou 500 centimètres) est utile pour juger de la plausibilité de la réponse. L'utilisation d'opérations et d'un langage symbolique, formel et technique est assez difficile, car les élèves doivent diviser un nombre peu élevé (9 kilomètres) par un nombre élevé (22 500 pas) et doivent connaître des facteurs de conversion. Cette question implique peu de représentation, car elle ne contient que du texte.

\section{PIZZAS}

L'item à réponse construite ouverte de l'unité PIZZAS (voir la figure 1.6) est présenté sous une forme simple, mais son contenu est riche : il illustre plusieurs éléments du cadre d'évaluation en mathématiques. II a été administré lors du premier essai de terrain de l'enquête PISA, en 1999, puis a été rendu public pour servir d'exemple. II illustre chaque version du cadre d'évaluation PISA en mathématiques depuis 2003. C'est l'un des items les plus difficiles de la batterie d'items utilisée lors de l'essai de terrain de 1999 : 11 \% seulement des élèves y ont répondu correctement.

\section{- Figure 1.6 - \\ Item de l'unité PIZZAS}

Une pizzeria propose deux pizzas rondes de la même épaisseur, de tailles différentes. La plus petite a un diamètre de $30 \mathrm{~cm}$ et coûte 30 zeds. La plus grande a un diamètre de $40 \mathrm{~cm}$ et coûte 40 zeds.

Laquelle des deux pizzas est la plus avantageuse par son prix? Indiquez votre raisonnement.

L'unité PIZZAS se situe dans un contexte personnel, vraisemblablement familier à de nombreux adolescents de 15 ans. Cet item se classe dans la catégorie Contextes personnels, car la question posée est de savoir quelle pizza est la plus avantageuse par son prix. II n'est guère difficile à lire, ce qui permet aux élèves de se concentrer presque entièrement sur les aspects mathématiques de la tâche.

Des termes courants dans la vie de tous les jours doivent être interprétés de façon mathématique ( rondes », " même épaisseur », " tailles différentes »). La variable de taille est définie sous forme mathématique via les diamètres des deux pizzas. Le coût est indiqué dans une monnaie fictive, le zed. La taille et le coût sont mis en correspondance par l'expression « avantageuse par son prix ».

Cet item relève de plusieurs branches en mathématiques. Il devrait en principe se classer dans la catégorie de contenus Espace et formes puisqu'il contient des éléments géométriques. Les pizzas peuvent être modélisées sous la forme de fins cylindres circulaires, ce qui nécessite le calcul de la surface d'un cercle. Cet item pourrait aussi se classer dans la catégorie de contenus Quantité à cause de la nécessité implicite de comparer des quantités de pizza par rapport à leur prix. Toutefois, toute la difficulté de l'item tient à la conceptualisation des relations entre les propriétés des pizzas et à la façon dont les propriétés pertinentes changent entre la petite et la grande pizza. Comme ces aspects sont au cœur du problème, cet item se classe dans la catégorie de contenus Variations et relations.

Cet item se classe dans la catégorie de processus Formulation. L'étape clé pour résoudre ce problème, difficile sur le plan cognitif, consiste à formuler un modèle mathématique qui décrit le concept que recèle l'expression " avantageuse par son prix ». Les élèves doivent comprendre que comme l'épaisseur des pizzas est en principe uniforme et qu'elle est identique dans les deux pizzas, ils doivent concentrer leur analyse sur la superficie circulaire des pizzas et non sur leur volume ou leur quantité. La relation entre la quantité de pizza et la quantité d'argent est à intégrer dans le concept 
de l'expression " avantageuse par son prix », soit le coût par unité de superficie. La superficie par coût unitaire est une autre variante possible. En mathématiques, le coût peut être calculé directement, puis comparé entre les deux pizzas : le coût du cercle plus grand est inférieur. Dans le monde réel, l'interprétation est que la grande pizza est meilleure marché.

Un autre raisonnement, qui montre plus clairement encore pourquoi cet item se classe dans la catégorie de contenus Variations et relations, serait de postuler (implicitement ou explicitement) que la superficie d'un cercle augmente en proportion du carré de son diamètre, soit de $(4 / 3)^{2}$, alors que le coût n'augmente que dans une proportion de (4/3). Comme $(4 / 3)^{2}$ est supérieur à (4/3), la grande pizza est meilleure marché.

Comme toute la difficulté de l'item consiste à formuler ses aspects de façon mathématique, il se classe dans la catégorie de processus Formuler des situations de façon mathématique. Des aspects des deux autres catégories de processus y sont également manifestes. Les élèves doivent employer à bon escient le modèle une fois qu'ils l'ont formulé, se livrer à un raisonnement approprié et utiliser des connaissances mathématiques en matière de surface et de proportions. Ils doivent ensuite interpréter le résultat pour le replacer dans le contexte de la question.

Pour résoudre cet item de l'unité PIZZAS, les élèves doivent exploiter leurs facultés mathématiques fondamentales à des degrés divers. La communication intervient relativement peu lorsqu'il s'agit de lire et d'interpréter l'énoncé qui est assez direct, mais intervient davantage lorsqu'il s'agit de présenter et d'expliquer la solution. La mathématisation est à la clé du problème : les élèves doivent en effet formuler un modèle pour décrire le concept « avantageuse par son prix ». Les élèves doivent créer une représentation des aspects pertinents du problème, notamment la représentation symbolique de la formule permettant de calculer la superficie et l'expression de taux qui représentent le concept « avantageuse par son prix », pour élaborer leur solution. Les raisonnements (pour décider que l'épaisseur peut être ignorée et justifier l'approche adoptée et les résultats obtenus) sont considérables et la conception de stratégies permettant de contrôler les calculs et les processus de modélisation est un aspect difficile dans ce problème. L'utilisation d'opérations et d'un langage symbolique, formel et technique s'impose, car les élèves doivent utiliser leurs connaissances en matière de concepts, de faits et de procédures pour aborder la géométrie des cercles et calculer les taux. L'utilisation d'outils mathématiques n'est guère difficile si les élèves savent utiliser correctement une calculatrice.

\section{Exemple de réponse à l'item de l'unité PIZZAS}

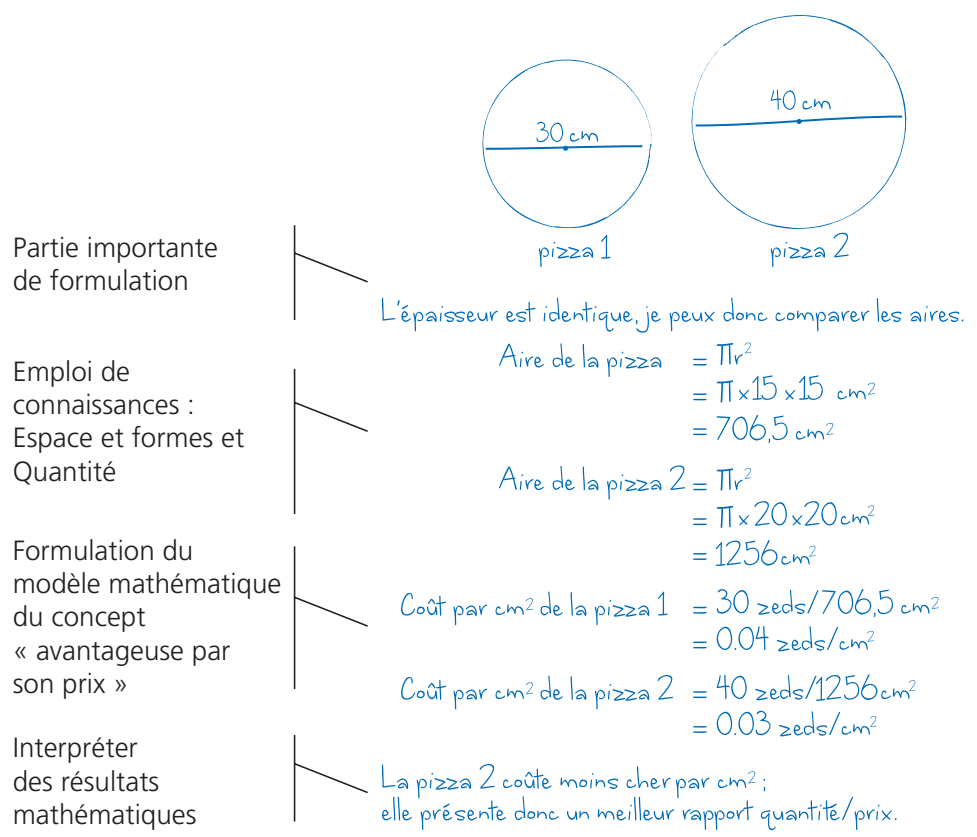




\section{DÉCHETS}

L'item DÉCHETS (voir la figure 1.8) illustre lui aussi certains aspects du cadre d'évaluation de la culture mathématique. Cet item à réponse construite a été administré lors de la campagne définitive du cycle PISA 2003 avant d'être rendu public. Le pourcentage de réponses correctes à cet item s'établit à $51 \%$, en moyenne, dans les pays de l'OCDE, ce qui le place au milieu du spectre de difficulté de la batterie d'items.

- Figure 1.8 -

Item de l'unité DÉCHETS

Pour un devoir portant sur l'environnement, des élèves ont recueilli des informations sur le temps de décomposition des différents types de déchets que les gens jettent :

\begin{tabular}{l|l}
\hline \multicolumn{1}{c|}{ Type de déchets } & Temps de décomposition \\
\hline Peau de banane & $1-3$ ans \\
\hline Pelure d'orange & $1-3$ ans \\
\hline Boîtes en carton & 0,5 année \\
\hline Chewing gum & $20-25$ ans \\
\hline Journaux & Quelques jours \\
\hline Gobelets en polystyrène & Plus de 100 ans \\
\hline
\end{tabular}

Un élève envisage de présenter ces résultats sous forme d'un diagramme en bâtons.

Donnez une raison pour laquelle le diagramme en bâtons ne conviendra pas pour présenter ces données.

Cet item se classe dans la catégorie Contextes scientifiques, car il porte sur un sujet scientifique (le temps de décomposition), et dans la catégorie de contenus Incertitude et données, dans la mesure où il présente des données à interpréter, même si des contenus Quantité interviennent implicitement, puisque les élèves doivent apprécier l'importance relative des temps indiqués. II se classe dans la catégorie de processus Interpréter, appliquer et évaluer des résultats mathématiques, car il demande essentiellement aux élèves d'évaluer l'adéquation d'un résultat mathématique (en l'occurrence un diagramme en bâtons fictif ou dessiné) pour décrire des données sur des éléments contextuels qui s'inspirent du monde réel. Cet item consiste à faire un raisonnement à propos des données fournies, à réfléchir sous l'angle mathématique à la relation entre les données et leur présentation, et à évaluer le résultat. Les élèves doivent comprendre qu'il ne serait pas judicieux de présenter ces données dans un diagramme en bâtons pour une des deux raisons suivantes : soit à cause de la très grande variance des temps de décomposition selon les déchets (qu'il n'est pas possible de représenter sous la forme d'un diagramme en bâtons standard), soit à cause de la variabilité des données dans certaines catégories (de sorte que sur un axe représentant le temps qui permettrait d'indiquer le temps de décomposition le plus long, les temps de décomposition les plus courts seraient invisibles). Les réponses telles que celles reproduites ci-dessous valent un crédit.

\section{RÉPONSE 1}

«Parce que ce serait difficile à faire dans un diagramme en bâtons puisqu'il y a 1-3,1-3, 0.5, etc. Ce serait difficile de le faire exactement. »

\section{RÉPONSE 2}

« Parce qu'il y a une grande différence entre le chiffre le plus élevé et le chiffre le moins élevé, ce serait donc difficile d'être précis avec 100 ans et quelques jours ».

La résolution de I'item DÉCHETS passe par l'activation des facultés mathématiques fondamentales suivantes. La communication intervient à cause de la nécessité de lire le texte et d'interpréter le tableau et, dans une plus grande mesure, de la nécessité de rédiger un raisonnement succinct. La mathématisation de la situation n'est guère difficile, dans la mesure où les élèves doivent identifier et extraire des caractéristiques mathématiques clés d'un diagramme en bâtons dans chaque catégorie de déchets. Les élèves doivent interpréter des données présentées sous forme de tableau, puis imaginer une représentation graphique, avant d'établir un lien entre les deux représentations, ce qui est tout l'enjeu de l'item. Le raisonnement requis est relativement facile, tout comme la conception de stratégies. L'utilisation 
d'opérations et d'un langage symbolique, formel et technique intervient, car les élèves doivent connaître des faits et des procédures pour imaginer ou dessiner un diagramme en bâtons et, surtout, comprendre l'échelle à employer pour imaginer l'ordonnée. L'utilisation d'outils mathématiques est selon toute vraisemblance inutile.

\section{CONCERT ROCK}

Un autre exemple d'item, tiré de l'unité CONCERT ROCK est présenté dans la figure 1.9. Cet item à choix multiple simple a été administré lors de l'essai de terrain précédant la campagne définitive du cycle PISA 2003. II a été rendu public par la suite pour donner un aperçu des épreuves. Dans la batterie d'items utilisée lors de cet essai de terrain, il se classe parmi ceux d'une difficulté modérée : quelque $28 \%$ des élèves y ont répondu correctement (option C). Cet item se classe dans la catégorie des Contextes sociétaux, car il concerne l'organisation d'un concert de rock, même s'il évoque une expérience personnelle, en l'occurrence celle de se trouver au milieu de la foule. II se classe dans la catégorie de contenus Quantité parce qu'il requiert un calcul, même s'il contient quelques éléments en rapport avec la catégorie Espace et formes.

- Figure 1.9 -

\section{Item de l'unité CONCERT ROCK}

Un terrain rectangulaire mesurant $100 \mathrm{~m}$ sur $50 \mathrm{~m}$ a été réservé pour le public d’un concert de rock.

Toutes les places ont été vendues et le terrain est plein de fans, tous debout.

Lequel des nombres ci-dessous est probablement la meilleure estimation du nombre total de personnes assistant au concert?
A. 2000
B. 5000
C. 20000
D. 50000
E. 100000

Cet item fait appel aux trois catégories de processus, mais surtout à celui qui consiste à formuler des situations de façon mathématique, car il demande aux élèves de donner un sens aux informations contextuelles qui leur sont fournies (la forme et les dimensions du terrain, et le fait que le concert affiche complet et que les fans sont debout). Les élèves doivent également identifier l'information manquante, qu'ils peuvent toutefois estimer sans trop de mal en se basant sur des hypothèses et des connaissances de la vie réelle. Ils doivent plus précisément concevoir un modèle pour déterminer l'espace requis par un individu ou un groupe de fans. En mathématiques, ils doivent employer des concepts, faits, procédures et raisonnements mathématiques pour établir un lien entre la superficie du terrain et l'espace occupé par un fan ou un groupe de fans, et établir les comparaisons qui s'imposent, puis interpréter, appliquer et évaluer des résultats mathématiques pour vérifier la plausibilité de leur solution ou comparer les options de réponse aux résultats mathématiques de leurs calculs.

Il y a également un autre modèle. Les élèves peuvent aussi imaginer des rangées uniformes de fans debout sur toute la superficie du terrain, puis estimer le nombre de fans en multipliant leur estimation du nombre de rangées par leur estimation du nombre de fans par rangée. Les élèves doués pour formuler des modèles mathématiques sont susceptibles d'apprécier l'efficacité de ce modèle, en dépit du contraste important qu'il présente avec le comportement de fans à un concert de rock. La réponse correcte vaut un crédit complet, quel que soit le modèle utilisé par les élèves.

Les facultés mathématiques fondamentales interviennent dans cet item de la façon suivante. Les facultés en communication sont relativement peu mises à contribution, car il suffit aux élèves de lire et de comprendre le texte. L'importance mathématique de termes tels que " mesurant » et " rectangulaire », la phrase " le terrain est plein » et le substantif " estimation » employé dans la consigne doivent être compris, puis interprétés. Des connaissances du monde réel peuvent être utiles pour ce faire. Cet item passe par un processus assez complexe de mathématisation, car il demande aux élèves de formuler des hypothèses sur l'espace qu'une personne occupe debout et de créer un modèle de base du type : «nombre de fans » $\mathrm{x}$ 《espace moyen $\mathrm{d}^{\prime}$ un fan $»=$ "superficie du terrain ». Les élèves doivent se représenter la situation mentalement ou la dessiner pour concevoir le modèle qui établit un lien entre l'espace occupé par un fan et la superficie du terrain. La conception de stratégies intervient à plusieurs stades du processus de résolution, notamment lorsque les élèves décident de la façon dont ils vont aborder le problème, imaginent le modèle qui pourrait se révéler 
utile pour estimer l'espace occupé par un fan lors d'un concert et comprennent qu'ils vont devoir en passer par quelques étapes de vérification et de validation. Les élèves peuvent choisir, à titre de stratégie de résolution, de postuler l'espace par personne, de le multiplier par le nombre de personnes fourni dans chaque option de réponse, puis de comparer le résultat aux conditions indiquées dans la question. Ils peuvent aussi faire l'inverse, en l'occurrence partir de la superficie fournie et calculer l'espace par personne qu'implique chaque option de réponse, puis identifier la réponse qui satisfait le mieux aux critères indiqués dans la question. L'utilisation d'opérations et d'un langage symbolique, formel et technique intervient une fois que les élèves appliquent la stratégie qu'ils ont adoptée, quelle qu'elle soit, pour interpréter et utiliser les dimensions fournies, et faire les calculs requis pour mettre en correspondance la superficie du terrain et l'espace occupé par un individu. Les facultés de raisonnement et d'argumentation interviennent, car les élèves doivent bien réfléchir à la relation entre le modèle qu'ils ont conçu, la solution qu'ils ont obtenue et le contexte réel, pour valider leur modèle et vérifier qu'ils ont choisi la réponse correcte. L'utilisation d'outils mathématiques est vraisemblablement superflue.

\section{MARCHE À PIED}

L'unité PISA MARCHE À PIED (voir la figure 1.10) montre une relation algébrique quelque peu illogique mais bien établie entre deux variables, sur la base de l'observation d'un grand nombre d'individus marchant d'un pas naturel, et pose aux élèves deux questions qui leur demandent $d^{\prime}$ exploiter leurs connaissances et compétences en algèbre. Dans la deuxième question, ils doivent également faire usage de leurs facultés de réflexion stratégique, de raisonnement et d'argumentation dans une mesure qui est difficile pour de nombreux adolescents de 15 ans. Ces deux items ont été administrés lors de la campagne définitive du cycle PISA 2003, puis ont illustré le cadre d'évaluation du cycle PISA 2009 et d'autres publications. Ils demandent tous deux aux élèves de se baser sur les informations qui leur sont fournies pour construire leur réponse. Ils se classent tous deux dans la catégorie de contenus Variations et relations, car ils portent sur des relations entre variables exprimées sous forme algébrique. Ils se classent dans la catégorie des Contextes personnels, car ils se rapportent à des aspects en rapport direct avec des perspectives et le vécu d'individus, et dans la catégorie de processus Employer des concepts, faits, procédures et raisonnements mathématiques, puisque les problèmes sont formulés en des termes qui possèdent déjà une structure mathématique et que leur résolution passe par une manipulation largement intramathématique d'objets et de concepts mathématiques.

\section{- Figure 1.10 -}

Items de l'unité MARCHE À PIED

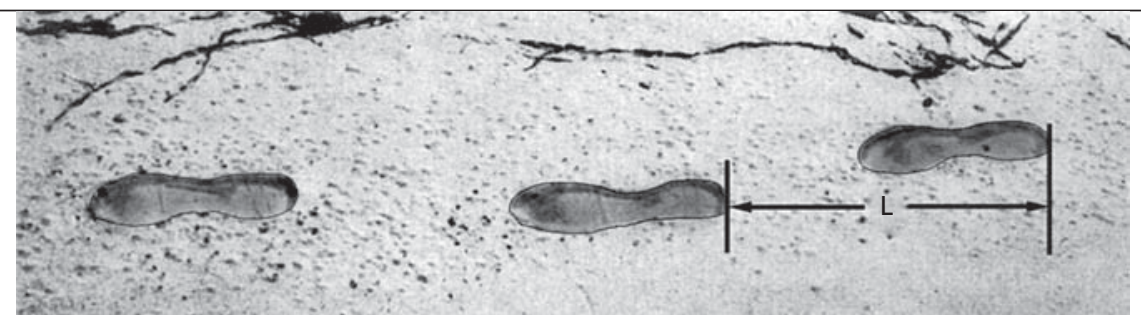

L'image montre les traces de pas d'un homme en train de marcher. La longueur de pas L est la distance entre l'arrière de deux traces de pas consécutives.

Pour les hommes, la formule $\frac{n}{L}=140$ donne un rapport approximatif entre $n$ et $L$ où :

$n=$ nombre de pas par minutes; et

$L=$ longueur de pas en mètres.

\section{QUESTION 1}

Si la formule s'applique à la façon de marcher d'Henri et qu'Henri fait 70 pas par minute, quelle est la longueur de pas d'Henri? Montrez vos calculs.

\section{QUESTION 2}

Bernard sait que la longueur de son pas est de 0,80 mètre. La formule s'applique à sa façon de marcher. Calculez la vitesse à laquelle marche Bernard en mètres par minute et en kilomètres par heure. Montrez vos calculs. 
Lors de la campagne définitive du cycle PISA 2003, 36 \% des élèves ont répondu correctement à la question 1, ce qui en fait un item plus difficile que $70 \%$ environ des items administrés lors de ce cycle. C'est surprenant car mathématiquement parlant, il ne leur demande que de remplacer la valeur $n=70$ dans la formule et de manipuler la formule algébriquement, de façon assez directe, pour obtenir la valeur de $L$. Cet item confirme ce qui s'observe dans de nombreux items PISA : lorsque les items s'inscrivent dans un contexte qui s'inspire du monde réel, les élèves éprouvent souvent des difficultés à utiliser efficacement leurs connaissances et compétences en mathématiques, et ce même si les composantes mathématiques sont présentées clairement dans la question.

Les facultés mathématiques fondamentales interviennent comme suit dans cet item. Les facultés de communication sont à exploiter pour lire et comprendre le stimulus, puis pour articuler la solution et montrer le cheminement. Cet item ne demande pas véritablement de mathématisation, car le modèle mathématique est fourni sous une forme familière pour de nombreux élèves âgés de 15 ans. Les facultés de représentation sont à exploiter dans une mesure significative, car le stimulus inclut un élément graphique, du texte et une expression algébrique que les élèves doivent mettre en correspondance. Les facultés de conception de stratégies interviennent très peu dans le processus de résolution, car la stratégie à adopter est formulée de manière très claire dans la question. Les facultés de raisonnement et d'argumentation ne jouent qu'un rôle minime parce qu'une nouvelle fois, cette tâche est formulée de manière claire et que tous les éléments requis sont évidents. L'utilisation d'opérations et d'un langage symbolique, formel et technique s'impose pour procéder à la substitution et manipuler l'expression pour faire en sorte que $L$ devienne le sujet de l'équation.

La question 2 est plus difficile : $20 \%$ seulement des élèves y ont répondu correctement. Elle se classe donc parmi les $10 \%$ d'items les plus difficiles qui aient été administrés lors du cycle PISA 2003. La conception de la stratégie est complexe, car les élèves ont un certain nombre d'étapes à franchir et doivent garder en ligne de mire le point final : comme la valeur de $L$ est connue, ils peuvent trouver la valeur de $n$ à partir de l'équation fournie ; ils doivent multiplier $n$ par $L$ pour obtenir la vitesse en mètres par minute, puis se livrer à un raisonnement proportionnel pour convertir cette vitesse en kilomètre par heure. Trois niveaux de crédit étaient prévus pour permettre de coder des réponses n'exposant que partiellement le raisonnement attendu pour la résolution du problème. La meilleure façon d'expliquer la différence de pourcentage de réponses correctes entre la question 2 et la question 1 est probablement de décrire les différents modes d'activation des facultés mathématiques fondamentales requis. Les élèves doivent utiliser leurs facultés de communication de manière comparable dans les deux questions lors de l'étape qui consiste à lire et comprendre la question, mais dans la question 2, ils doivent se servir du diagramme pour créer explicitement un lien entre un pas et la longueur de pas fournie - une relation qu'il est inutile d'établir dans la question 1. De plus, la présentation de la solution fait davantage appel à des facultés d'expression dans la question 2. Les élèves doivent passer par un processus de mathématisation puisqu'ils doivent concevoir un modèle proportionnel pour exprimer la vitesse de Bernard dans les unités requises pour résoudre le problème. Pour arriver à cette solution, les élèves doivent faire appel à des mécanismes de contrôle efficaces et soutenus dans le cadre d'un processus à étapes multiples ; les facultés de conception de stratégies interviennent donc à un niveau bien plus élevé que dans la question 1. La représentation intervient plus que dans la question 1, car les élèves doivent travailler plus activement avec la représentation algébrique qui leur est fournie. La mise en œuvre de la stratégie conçue et l'exploitation des représentations identifiées impliquent l'utilisation d'opérations et d'un langage symbolique, formel et technique, ce qui consiste à faire des manipulations algébriques, à appliquer des proportions et à faire des opérations arithmétiques pour procéder aux conversions requises. Les élèves doivent utiliser leurs facultés de raisonnement et d'argumentation dans toutes les étapes interdépendantes qu'ils doivent enchaîner pour parvenir à la solution. L'utilisation d'outils mathématiques intervient dans une mesure relativement faible si les élèves se servent bien de leur calculatrice.

\section{MENUISIER}

L'item PISA MENUISIER (voir la figure 1.11) a été administré lors des cycles PISA 2000 et 2003, avant d'être rendu public. Il illustre un format d'items à choix multiple dit " complexe » : les élèves doivent sélectionner une réponse parmi celles proposées dans plusieurs questions successives. Dans cet item, les élèves obtiennent un crédit complet s'ils répondent que tous les tracés, sauf le tracé $B$, peuvent être réalisés avec les planches fournies.

Cet item se classe dans la catégorie de contenus Espace et formes, car il porte sur des propriétés de formes, et dans la catégorie des Contextes professionnels, car il concerne un travail de menuiserie. II se classe dans la catégorie de processus Employer des concepts, faits, procédures et raisonnements mathématiques, car il consiste en grande partie à appliquer des connaissances en matière de procédures à des objets bien définis, même si les élèves doivent aussi dans une certaine mesure interpréter, appliquer et évaluer des résultats mathématiques, puisqu'ils doivent établir un lien entre les objets mathématiques représentés et l'élément contextuel - la contrainte imposée par la quantité de planches disponibles. 
C'est l'un des items les plus difficiles des épreuves du cycle PISA 2003 : un peu moins de $20 \%$ des élèves y ont répondu correctement. Pour le résoudre, les élèves peuvent utiliser leurs connaissances en géométrie et leurs facultés de raisonnement. Ils disposent de suffisamment d'informations pour calculer directement le périmètre exact des tracés $A, C$ et $\mathrm{D}$, qui est de 32 mètres. Toutefois, ils manquent d'informations pour le faire à propos du tracé $B$ et doivent donc asdopter une autre approche. Ils peuvent se livrer au raisonnement suivant : les segments « horizontaux » des quatre tracés sont équivalents, mais les segments obliques du tracé B sont plus longs que la somme des segments « verticaux » des autres tracés.

Cet item fait appel aux facultés de communication des élèves, en l'occurrence pour lire et comprendre la question, et pour établir un lien entre les informations fournies dans le texte et la représentation graphique des quatre tracés. L'item ne nécessite pas de mathématisation puisqu'il est présenté de façon explicitement mathématique. Des considérations qui s'inspirent du monde réel, telles que la longueur des planches disponibles et la géométrie des angles, ne posent pas de problème particulier. Les élèves doivent essentiellement utiliser leurs facultés de raisonnement et d'argumentation pour comprendre que le périmètre du tracé B est trop important et qu'ils connaissent la longueur totale des segments "verticaux » du tracé $\mathrm{A}$ (elle est similaire à celle des segments horizontaux et verticaux du tracé C), même si leur longueur spécifique leur est inconnue. En matière de conception de stratégies, ils doivent se rendre compte qu'ils peuvent estimer les périmètres, même en l'absence de données sur la longueur de certains segments. L'utilisation d'opérations et d'un langage symbolique, formel et technique intervient pour comprendre et manipuler le périmètre des formes présentées, y compris les propriétés des côtés, et additionner les longueurs des côtés. L'utilisation d'outils mathématiques est vraisemblablement superflue.

- Figure 1.11 .

Item de l'unité MENUISIER

Un menuisier dispose de 32 mètres de planches et souhaite s'en servir pour faire la bordure d'une plate-bande dans un jardin. Il envisage d'utiliser un des tracés suivants pour cette bordure :

A

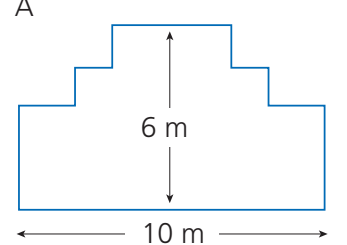

B

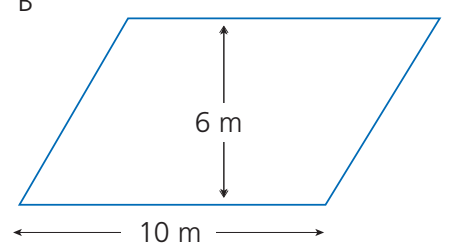

C

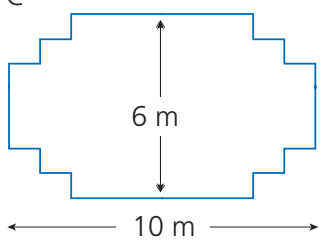

D

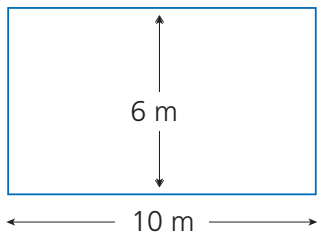

Indiquez, pour chacun des tracés, s'il peut être réalisé avec les 32 mètres de planches. Répondez en entourant « Oui » ou Non $»$.

\begin{tabular}{l|c}
\hline \multicolumn{1}{c|}{ Tracé de la bordure } & $\begin{array}{c}\text { En utilisant ce tracé, peut-on réaliser la plate-bande avec } \\
\mathbf{3 2} \text { mètres de planches ? }\end{array}$ \\
\hline Tracé A & Oui / Non \\
\hline Tracé B & Oui / Non \\
\hline Tracé C & Oui / Non \\
\hline Tracé D & Oui / Non \\
\hline
\end{tabular}




\section{Notes}

1. Le terme « construct » a volontairement été laissé en anglais, car il est d'usage courant dans la terminologie technique relative aux tests ; il renvoie à la dimension latente que cherche à mesurer une épreuve grâce aux données observables que constituent les réponses des élèves.

2. Dans certains pays, les « outils mathématiques » peuvent aussi désigner des procédures mathématiques établies, telles que les algorithmes. Dans le cadre PISA, les « outils mathématiques » désignent uniquement les outils physiques et numériques décrits dans cette section.

3. Les normes en vigueur ont été analysées dans deux groupes de pays, constitués d'une part de neuf pays membres de l'OCDE, à savoir I'Australie (Nouvelle-Galles-du-Sud), la Belgique (Communauté flamande), le Canada (Alberta), la Corée, la Finlande, I'Irlande, le Japon, la Nouvelle-Zélande et le Royaume-Uni, et, d'autre part, de six pays très performants, à savoir la Belgique (Communauté flamande), le Canada (Alberta), la Corée, la Finlande, Singapour et le Taipei chinois. L'une des exigences formulées dans le cadre de cette analyse était que les normes soient disponibles en anglais.

4. Ceux qui connaissent les cadres d'évaluation précédents constateront que la catégorie Incertitude s'appelle désormais Incertitude et données. Ce changement d'appellation vise à décrire la catégorie plus clairement et n'est pas le signe d'un remaniement fondamental de la catégorie.

5. Une épreuve informatisée a fait l'objet d'une expérience pilote en sciences lors du cycle PISA 2006 et en compréhension de l'écrit lors du cycle PISA 2009. 


\section{Références}

Bennett, R. (2003), Online Assessment and the Comparability of Score Meaning, Educational Testing Service, Princeton, New Jersey, www.ets.org/Media/Research/pdf/RM-03-05-Bennett.pdf.

Bennett, R.E., J. Braswell, A. Oranje, B. Sandene, B. Kaplan et F. Yan (2008), « Does it Matter if I Take My Mathematics Test on Computer? A Second Empirical Study of Mode Effects in NAEP ", Journal of Technology, Learning, and Assessment, vol. 9, nº 6.

Common Core State Standards Initiative (2010), Common Core State Standards for Mathematics, Common Core State Standards Initiative, Washington, DC, http://www.corestandards.org/assets/CCSSI_Math\%20Standards.pdf.

Devlin, K. (1994), Mathematics : The Science of Patterns: The Search for Order in Life, Mind and the Universe, W.H. Freeman Scientific American Library, New York.

Hoyles, C., A. Wolf, S. Molyneux-Hodgson et P. Kent (2002), Mathematical Skills in the Workplace: Final Report to the Science Technology and Mathematics Council, Project Report, Institute of Education, Université de Londres, Science, Technology and Mathematics Council, Londres, http://eprints.ioe.ac.uk/1565/1/Hoyles2002MathematicalSkills.pdf.

Mason, B., M. Patry et D. Berstein (2001), « An Examination of the Equivalence Between Non-adaptive Computer Based and Traditional Testing », Journal of Education Computing Research, vol. 1, n² 24, pp. 29-39.

Moore, D. (1997), « New Pedagogy and New Content: The Case of Statistics », International Statistical Review, vol. 2, n 65, pp. 123-137.

National Council of Teachers of Mathematics (2000), Principles and Standards for School Mathematics, NCTM, Reston, Virginie, http://www.nctm.org/standards/.

Niss, M. et T.H. Jensen (2002), Kompetencer og matematiklæring: Ideer og inspiration til udvikling af matematikundervisning i Danmark, Uddannelsesstyrelsens temahæfteserie, no 18, ministère de l'Éducation, Copenhague, http://pub.uvm.dk/2002/kom/.

Niss, M. (2003), « Mathematical Competencies and the Learning of Mathematics: The Danish KOM Project », in A. Gagatsis et S. Papastavridis (éd.), $3^{\text {rd }}$ Mediterranean Conference on Mathematics Education, The Hellenic Mathematical Society and Cyprus Mathematical Society, Athènes, pp. 115-124, http://w3.msi.vxu.se/users/hso/aaa_niss.pdf.

Niss, M., W. Blum et P. Galbraith (2007), "Introduction », in Blum, W., P. Galbraith, H.-W. Henn et M. Niss (éd.), Modelling and Applications in Mathematics Education (The 14th ICMI Study), Springer, New York, pp. 3-32.

Niss, M. et T. Højgaard (éd.) (2011), «Competencies and Mathematical Learning: Ideas and Inspiration for the Development of Mathematics Teaching and Learning in Denmark", ministère de l'éducation, rapport no 485, Université de Roskilde, Roskilde, https://pure.au.dk/ portal/files/41669781/THJ11_MN_KOM_in_english.pdf.

OCDE (2003), Cadre d'évaluation de PISA 2003 - Connaissances et compétences en mathématiques, lecture, science et résolution de problèmes, PISA, Éditions OCDE.

OCDE (2010), Pathways to Success: How Knowledge and Skills at Age 15 Shape Future Lives in Canada, PISA, Éditions OCDE, www.oecd. org/dataoecd/59/35/44574748.pdf.

Qualifications and Curriculum Authority (2007), « Mathematics: Programme of Study for Key Stage 3 and Attainment Targets », Qualifications and Curriculum Authority, Londres, http://media.education.gov.uk/assets/files/pdf/q/mathematics\%202007\%20programme\%20 of $\% 20$ study $\% 20$ for $\% 20 \mathrm{key} \% 20$ stage $\% 203 . p d f$.

Richardson, M., J.-A. Baird, J. Ridgway, M. Ripley, D. Shorrocks-Taylor et M. Swan (2002), "Challenging Minds? Students' perceptions of Computer-based World Class Tests of Problem Solving ", Computers in Human Behavior, vol. 18, nº 6, novembre, pp. 633-649.

Sandene, B., N. Horkay, R. Bennett, N. Allen, J. Braswell, B. Kaplan et A. Oranje (2005), Online Assessment in Mathematics and Writing: Reports From the NAEP Technology-Based Assessment Project, Research and Development Series (NCES 2005, 57), US Department of Education, National Center for Education Statistics, Washington, DC, US Government Printing Office, http://nces.ed.gov/ nationsreportcard/pdf/studies/2005457_1.pdf.

Stacey, K. et D. Wiliam (2013), "Technology and Assessment in Mathematics », in M.A. Clements (Ken), A. Bishop, C. Keitel, J. Kilpatrick et F. Leung (éd.), Third International Handbook of Mathematics Education, Springer, pp. 721-752.

Steen, L. (1990), On the Shoulders of Giants: New Approaches to Numeracy, National Academy Press Washington, DC.

Thomson, S. et K. Hillman (2010), Against the Odds: Influences on the Post-School Success of 'Low Performers', NCVER, Adélaïde, http://www.ncver.edu.au/publications/2285.html.

Turner, R. (2012), "Some Drivers of Test Item Difficulty in Mathematics », dossier présenté lors de la réunion annuelle de l'American Educational Research Association (AERA), 13-17 avril 2012, Vancouver, http://research.acer.edu.au/pisa/4/.

Turner, R. et R.J. Adams (2012), "Some Drivers of Test Item Difficulty in Mathematics: An Analysis of the Competency Rubric ", dossier présenté lors de la réunion annuelle de l'American Educational Research Association (AERA), 13-17 avril 2012, Vancouver, http:// research.acer.edu.au/pisa/7/.

Turner, R., J. Dossey, W. Blum et M. Niss (à paraître), "Using Mathematical Competencies to Predict Item Difficulty in PISA ", in M. Prenzel, M. Kobarg, K Schöps et S. Rönnebeck (éd.), Research on PISA: Research Outcomes of the PISA Research Conference 2009, Springer, New York, pp. 23-27.

Watson, J.M. et R. Callingham (2003), «Statistical Literacy: A Complex Hierarchical Construct », Statistics Education Research Journal, vol. 2, no 2, pp. 3-46. 


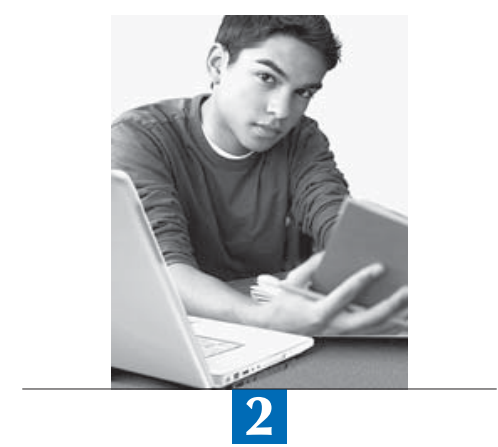

\section{Cadre d'évaluation \\ de la compréhension de l'écrit du cycle PISA 2012}

Ce chapitre présente le cadre conceptuel qui sous-tend les épreuves d'évaluation des compétences des élèves en compréhension de l'écrit lors du cycle PISA 2012. II expose la définition retenue dans I'enquête PISA des compétences en compréhension de l'écrit et examine les éléments de l'enquête restés inchangés entre les différents cycles, ainsi que le nouvel élément introduit lors du cycle PISA 2009 : la lecture et la compréhension des textes électroniques. II explique la façon dont les tâches de compréhension de l'écrit sur papier et de l'écrit électronique sont évaluées et analysées dans I'enquête PISA, et la façon dont les élèves naviguent entre les textes et mènent les tâches à bien. Des exemples d'items sur papier et sur support électronique jalonnent tout le chapitre pour illustrer la façon d'évaluer les compétences des élèves. 


\section{INTRODUCTION}

La compréhension de l'écrit a été le domaine majeur d'évaluation en 2000, lors du premier cycle PISA (PISA 2000), puis en 2009, lors du quatrième cycle PISA (PISA 2009). Lors du cinquième cycle PISA (PISA 2012), la compréhension de l'écrit est un domaine mineur d'évaluation, dont le cadre conceptuel reste inchangé par rapport à celui du cycle précédent, en l'occurrence le cycle PISA 2009 (OCDE, 2009). Le cadre adopté lors du cycle PISA 2009 se distingue par deux grandes innovations : I'inclusion de la compréhension de l'écrit électronique et le développement des constructs d'engagement dans la lecture et de métacognition.

Les compétences en lecture sont la clé de la découverte du monde de l'écrit, non seulement sur papier, mais également sur support électronique. Les textes électroniques constituent une part de plus en plus importante des lectures des élèves et des adultes. Dans tous les pays, l'usage d'Internet est étroitement lié au milieu socio-économique et au niveau de formation (Sweetes et Meates, 2004). Or, le fait d'avoir à utiliser un ordinateur ne se limite pas à des strates économiques et sociales particulières. L'informatique prend de l'importance au-delà du cadre professionnel, dans la vie personnelle, sociale et civique (Pew Internet et American Life Project, 2005).

La plupart des compétences requises pour lire sont similaires, qu'il s'agisse de textes imprimés ou de textes électroniques, mais la lecture de ces derniers impose aux lecteurs d'étoffer leur champ de compétences et leurs stratégies. Pour glaner des informations sur Internet, il faut être capable de passer en revue de gros volumes de données et d'en évaluer sur-lechamp la crédibilité. La réflexion critique est donc un aspect plus important que jamais de la compréhension de l'écrit (Halpern, 1989 ; Shetzer et Warschauer, 2000 ; Warschauer, 1999). Warschauer en arrive à la conclusion que pour réduire la "fracture numérique », il faut non seulement permettre aux individus d'accéder aux ressources en ligne, mais également améliorer leur faculté d'intégrer, d'évaluer et de communiquer l'information.

Ces nouvelles compétences en lecture requises par l'ère numérique expliquent l'ajout de textes électroniques. Cette version du cadre d'évaluation reconnaît donc pleinement qu'au XXle siècle, toute définition de la lecture se doit d'englober à la fois les textes sur papier et les textes sur support électronique. Le cycle PISA 2012 comprend également l'évaluation de la compréhension de l'écrit électronique. Les pays participants n'ont pas tous choisi de prendre part à l'évaluation de la compréhension de l'écrit électronique lors du cycle PISA 2009 ou 2012, de sorte que cette évaluation a été proposée à titre d'option internationale. Vingt-trois pays membres de l'OCDE et neuf pays et économies partenaires ont décidé d'administrer cette épreuve lors du cycle PISA 2012, soit une augmentation de plus de 50 \% par rapport au cycle PISA 2009.

L'évolution de notre conception de la compréhension de l'écrit depuis 2000 nous a déjà amenés à élargir notre définition, qui inclut des caractéristiques motivationnelles et comportementales, en plus des caractéristiques cognitives. À la lumière de recherches récentes, le cadre d'évaluation du cycle PISA 2009 a fait une plus large place à l'engagement dans la lecture et à la métacognition, éléments qui peuvent grandement aider les décideurs politiques à mieux cerner les facteurs qu'il est possible de développer, façonner et renforcer pour améliorer la compréhension de l'écrit. Comme la compréhension de l'écrit est un domaine mineur d'évaluation lors du cycle PISA 2012, l'engagement dans la lecture et la métacognition ne sont pas inclus.

Dans l'enquête PISA, l'évaluation de la compréhension de l'écrit chez les élèves qui approchent du terme de leur scolarité obligatoire doit donc se concentrer sur une série de compétences, notamment la capacité de trouver, sélectionner, interpréter et évaluer l'information dans tout l'éventail de textes qui se rencontrent en classe et en dehors du cadre scolaire.

Ce chapitre décrit le cadre conceptuel qui sous-tend l'évaluation des compétences des élèves en compréhension de l'écrit lors du cycle PISA 2012. La définition du domaine d'évaluation est la même que lors du cycle PISA 2009, où la compréhension de l'écrit était pour la deuxième fois le domaine majeur d'évaluation, à l'exception d'un nouvel élément : la lecture et la compréhension de textes électroniques. Ce chapitre explique la façon dont les tâches de lecture électronique sont évaluées et analysées dans I'enquête PISA, et la façon dont les élèves naviguent entre les textes et mènent les tâches à bien. Des exemples d'items sur papier et sur support électronique jalonnent tout le chapitre pour illustrer la façon d'évaluer les compétences des élèves.

\section{DÉFINITION DE LA COMPRÉHENSION DE L'ÉCRIT}

La définition de la lecture et de la compréhension de l'écrit a évolué au fil du temps, parallèlement aux évolutions sociales, économiques et culturelles. Le concept d'apprentissage, et plus particulièrement celui d'apprentissage tout au long de la vie, a élargi la notion de compréhension de l'écrit. La littératie n'est plus considérée comme une faculté 
qui ne s'acquiert que pendant l'enfance et les premières années de scolarisation. Elle est désormais définie comme un ensemble dynamique de connaissances, de compétences et de stratégies que les individus étoffent durant toute leur vie dans les diverses situations qui se présentent à eux, par leurs interactions avec leurs semblables et la communauté au sens large.

Les conceptions de la compréhension de l'écrit fondées sur les sciences cognitives mettent l'accent sur la nature interactive de la lecture des textes imprimés et la nature constructive de leur compréhension (Binkley et Linnakylä, 1997 ; Bruner, 1990 ; Dole et al., 1991) ; ceci est encore plus vrai lorsqu'il s'agit de textes électroniques (Fastrez, 2001 ; Legros et Crinon, 2002 ; Leu, 2007 ; Reinking, 1994). Face au texte, le lecteur construit du sens en utilisant ses connaissances antérieures et une série d'indices liés au texte et à la situation, qui ont souvent une valeur sociale ou culturelle. Pour construire du sens, le lecteur utilise divers processus, compétences et stratégies qui lui permettent d'alimenter, de contrôler et de maintenir sa compréhension. On peut s'attendre à ce que ces processus et ces stratégies soient variables en fonction des contextes et des objectifs que poursuivent les lecteurs lorsqu'ils interagissent avec divers types de textes continus et non continus sur papier et, surtout, avec des textes multiples sur support électronique.

La compréhension de l'écrit a été définie comme suit lors du cycle PISA 2012 :

Comprendre l'écrit, c'est non seulement comprendre et utiliser des textes écrits, mais aussi réfléchir à leur propos et s'y engager. Cette capacité devrait permettre à chacun de réaliser ses objectifs, de développer ses connaissances et son potentiel, et de prendre une part active dans la société.

\section{Comprendre l'écrit...}

L'expression «compréhension de l'écrit » a été utilisée de préférence à " lecture » parce qu'elle est susceptible de mieux traduire, pour un public de non-spécialistes, l'idée de ce que l'enquête va mesurer. Par lecture, on entend souvent un simple décodage, ou même le fait de lire à haute voix, alors que l'intention de cette enquête est de mesurer quelque chose de bien plus vaste et de bien plus profond. La compréhension de l'écrit fait appel à un large éventail de compétences cognitives, qui vont de la faculté élémentaire de décoder l'écrit à la connaissance du vocabulaire, de la grammaire et plus largement des structures et caractéristiques linguistiques et textuelles, en passant par la connaissance du monde.

Dans l'enquête PISA, I'expression « compréhension de l'écrit » désigne l'usage actif, réfléchi et fonctionnel de la lecture dans un éventail de situations et à des fins variées. Selon Holloway (1999), les compétences en lecture conditionnent la réussite scolaire des élèves dans le premier et le deuxième cycle de l'enseignement secondaire. L'enquête PISA cible une population très diverse d'élèves, dont certains iront à l'université, d'autres poursuivront leurs études dans le but de se préparer à entrer directement dans la vie active et d'autres encore commenceront à travailler dès la fin de l'obligation scolaire. Savoir lire n'est pas seulement essentiel pour réussir dans d'autres matières à l'école, c'est aussi une condition sine qua non pour réussir dans la plupart des domaines de la vie adulte (Cunningham et Stanovich, 1998 ; Smith et al., 2000). Quelles que soient les aspirations professionnelles ou scolaires des élèves, la compréhension de l'écrit est importante pour leur vie personnelle et pour leur participation active à la vie de la société et de l'économie.

Les compétences en compréhension de l'écrit ne sont pas seulement importantes pour les individus, elles le sont aussi pour l'économie dans son ensemble. Les décideurs politiques et autres en viennent à reconnaître que dans les sociétés modernes, le capital humain - l'ensemble de ce que les individus à la disposition d'une économie savent et sont capables de faire - est sans doute le capital le plus important. Des économistes ont élaboré, depuis des années déjà, des modèles qui montrent qu'en règle générale, le niveau de formation de la population d'un pays est une variable prédictive de son potentiel de croissance économique (Coulombe et al., 2004).

... c'est non seulement comprendre et utiliser [des textes écrits], mais aussi réfléchir à leur propos...

Le verbe " comprendre » est en rapport direct avec la notion de " compréhension de l'écrit », un aspect communément admis de la lecture. Le verbe « utiliser » fait référence à la notion d'application et de fonction - en l'occurrence faire quelque chose de ce qui est lu. Le verbe « réfléchir » vient s'ajouter aux verbes « comprendre » et « utiliser » pour insister sur le caractère interactif de la lecture : le lecteur se base sur ses propres pensées et ses propres expériences lorsqu'il aborde un texte. Chaque acte de lecture nécessite, à l'évidence, une certaine forme de réflexion sur la base d'informations extérieures au texte. Même aux stades les plus précoces, le lecteur fait appel à des connaissances symboliques pour décoder un texte et a besoin de connaître du vocabulaire pour lui donner un sens. À mesure que le lecteur emmagasine un ensemble d'informations, d'expériences et de croyances, il confronte constamment, quoique souvent de façon inconsciente, ce qu'il lit aux connaissances extérieures, et ajuste ainsi continuellement sa compréhension du texte. 
... et s'y engager...

Un individu qui comprend l'écrit possède les connaissances et compétences requises pour lire, mais au-delà, il valorise la lecture et l'utilise à diverses fins. C'est donc un objectif de l'éducation non seulement d'amener les élèves à un bon niveau de compétence en lecture, mais aussi de cultiver leur engagement dans la lecture. Dans ce contexte, la notion d'engagement recouvre la motivation à lire et se compose d'une série de caractéristiques affectives et comportementales, dont l'intérêt que le lecteur porte à la lecture, le plaisir qu'elle lui procure, le sentiment qu'il a d'exercer un certain contrôle sur ce qu'il lit, sa sensibilisation à la dimension sociale de la lecture, et ses différentes pratiques et habitudes de lecture.

\section{[des textes écrits]}

L'expression " textes écrits » désigne tous les textes cohérents dans lequel le langage est utilisé sous forme graphique, qu'ils soient imprimés ou électroniques. Le terme « texte » a été préféré au terme "information » employé dans plusieurs autres définitions de la lecture, car il est associé au langage écrit et renvoie plus directement à la lecture de textes " littéraires » ainsi que de textes d'information.

Sont exclus des textes visés les artefacts audio (tels que les enregistrements vocaux), les films, les émissions télévisées, les animations visuelles et les images sans légende. Y sont toutefois inclus les représentations visuelles, telles que les diagrammes, les images, les cartes, les tableaux, les graphiques et les bandes dessinées, accompagnées de textes (par exemple des légendes). Ces éléments peuvent exister en soi ou être intégrés dans des textes. Les textes sur support électronique se distinguent des textes sur papier à de nombreux égards : leur lisibilité physique, le volume de texte visible d'emblée par le lecteur, les connexions entre différents textes ou parties de texte au moyen des liens hypertextes et, fruit de toutes ces caractéristiques, la façon dont le lecteur aborde généralement les textes électroniques. Par comparaison avec les textes manuscrits et imprimés sur papier, les textes électroniques exigent beaucoup plus du lecteur qu'il construise son propre cheminement pour se livrer à une activité de lecture, quelle qu'elle soit.

... [afin] de réaliser ses objectifs, de développer ses connaissances et son potentiel, et de prendre une part active dans la société.

Par cette phrase, on entend désigner l'ensemble des situations où la compréhension de l'écrit joue un rôle : de la vie privée à la vie publique, de l'école au travail, au cours de toute une vie d'apprentissage et de citoyenneté active. «[...] réaliser ses objectifs, développer ses connaissances et son potentiel » renvoie à l'idée que la compréhension de l'écrit permet l'accomplissement des aspirations individuelles, qu'elles soient bien définies comme le fait d'obtenir un diplôme ou de trouver du travail, ou qu'elles soient moins précises et moins immédiates, comme enrichir sa vie personnelle, élargir ses horizons et pratiquer l'apprentissage tout au long de sa vie. L'expression " prendre une part active " a été retenue, car elle signifie que la compréhension de l'écrit permet aux gens non seulement d'apporter une contribution à la société, mais aussi de répondre à leurs propres besoins ; elle inclut l'engagement social, culturel et politique.

\section{ORGANISATION DU DOMAINE}

Dans cette section, nous expliquons comment se représente ce domaine, point essentiel s'il en est puisque son organisation conditionne la conception des épreuves et, en fin de compte, la nature des éléments qui pourront être recueillis, puis communiqués pour décrire les compétences des élèves ${ }^{1}$.

La compréhension de l'écrit est un domaine multidimensionnel. De nombreux éléments interviennent dans ce construct, mais tous ne peuvent être pris en considération et intégrés dans une évaluation telle que celle proposée dans le cadre de l'enquête PISA. Dans ce contexte, il faut donc sélectionner les éléments les plus importants pour concevoir les épreuves.

Les tâches PISA de compréhension de l'écrit sont élaborées autour de trois grandes caractéristiques pour assurer une large couverture du domaine d'évaluation :

- la situation, c'est-à-dire l'éventail des contextes dans lesquels la lecture intervient ou des usages qui en sont faits ;

- le texte, c'est-à-dire la nature de ce qui est lu ; et

- I'aspect, c'est-à-dire l'approche cognitive qui détermine comment le lecteur aborde un texte.

Dans l'enquête PISA, les textes et les aspects (mais pas les situations) sont manipulés pour faire varier le degré de difficulté des tâches.

La lecture est une activité complexe, dont les différents éléments ne sont pas cloisonnés et indépendants les uns des autres. La classification des textes et des tâches dans les catégories du cadre d'évaluation n'implique pas que ces catégories 
sont strictement délimitées ou que le matériel se répartit entre des cases tout à fait distinctes en fonction d'une structure théorique. Le cadre d'évaluation sert à garantir la couverture du domaine, à orienter le développement des épreuves et à définir les paramètres de compte rendu sur la base des caractéristiques saillantes de chaque tâche.

\section{Les situations}

Les variables PISA de situation sont adaptées du Cadre européen commun de référence pour les langues (CECR) du Conseil de l'Europe (Conseil de l'Europe, 1996). Les quatre variables de situation - la lecture à usage personnel, public, éducatif et professionnel - sont décrites ci-dessous.

La lecture à usage personnel renvoie à des textes lus pour satisfaire des intérêts personnels, qu'ils soient d'ordre pratique ou intellectuel. Sont également visés les textes qui servent à entretenir ou à développer des relations personnelles avec autrui. Cette catégorie inclut les lettres personnelles, les fictions, les biographies et les textes informatifs écrits pour être lus par curiosité personnelle, dans le cadre de loisirs ou de divertissement. Dans la catégorie des textes électroniques, les textes à usage personnel comprennent les messages électroniques personnels, les textes de messagerie instantanée et les blogs de type « journal ».

La lecture à usage public renvoie à la lecture en rapport avec des activités et des préoccupations en rapport avec la société au sens large. Sont notamment visés les documents officiels et les informations sur les événements publics. En règle générale, les textes de cette catégorie supposent des contacts plus ou moins anonymes avec autrui. Entrent également dans cette catégorie les blogs de type "forum », les sites web d'actualités et les informations publiques (imprimées et en ligne).

Le contenu des textes à usage éducatif est en général expressément conçu à des fins d'instruction. Les manuels imprimés et les logiciels didactiques interactifs sont des exemples typiques de matériel conçu pour ce type de lecture. La lecture à usage éducatif consiste d'ordinaire à acquérir des informations dans le cadre d'une activité plus large d'apprentissage. Souvent, ces textes ne sont pas choisis par leurs lecteurs, mais imposés par un enseignant. Les tâches associées à ce type de lecture relèvent généralement de la « lecture pour apprendre » (Sticht, 1975 ; Stiggins, 1982).

Bon nombre des jeunes âgés de 15 ans quitteront l'école pour entrer dans la vie active dans un délai d'un ou deux ans. Une activité typique de lecture à usage professionnel est celle qui est immédiatement utile pour accomplir une tâche. À titre d'exemple, citons la lecture des offres d'emploi dans un journal ou en ligne à la recherche d'un poste, ou encore la lecture de consignes données sur le lieu de travail. Les tâches de lecture de cette catégorie sont souvent associées à la «lecture pour agir » (Sticht, 1975 ; Stiggins, 1982).

Dans les épreuves PISA de compréhension de l'écrit, la dimension de situation définit les textes et les tâches qui y sont associées, et renvoie aux usages auxquels les auteurs destinent leurs textes et les circonstances dans lesquelles ils prévoient qu'ils seront lus. La variable de situation est donc spécifiée en fonction du lectorat et de l'usage prévus du texte, et pas uniquement sur la base du cadre dans lequel s'effectue l'activité de lecture. Ainsi, de nombreux textes sont utilisés en classe alors qu'ils ne sont pas spécifiquement conçus pour cet usage : il est fréquent par exemple que les élèves âgés de 15 ans lisent des extraits de textes littéraires dans leurs cours de langue maternelle ou de littérature, alors que leurs auteurs les ont (vraisemblablement) écrits pour le divertissement et le plaisir de leurs lecteurs. Au vu de leur objectif initial, ces textes sont donc classés dans I'enquête PISA dans la catégorie des lectures à usage personnel. Comme Hubbard (1989) l'a montré, certains types de lecture pour enfants sont associés à des contextes extrascolaires, par exemple le règlement $d^{\prime}$ un club ou le compte rendu d'un match, mais ils se retrouvent souvent aussi dans le cadre scolaire, de manière informelle. Ces textes sont classés dans la catégorie des lectures à usage public dans l'enquête PISA. À l'inverse, les manuels scolaires sont lus aussi bien à l'école qu'à la maison, mais les processus et les usages dont relève leur lecture sont similaires d'un endroit à l'autre, c'est pourquoi ils sont classés dans la catégorie des lectures à usage éducatif dans I'enquête PISA.

Il convient de souligner que ces quatre catégories d'usages de la lecture se chevauchent. En effet, un auteur peut avoir écrit un texte dans le but d'instruire et de divertir ses lecteurs (usages éducatif et personnel) ou dans le but de prodiguer des conseils d'ordre professionnel qui ont aussi valeur d'information générale (usages professionnel et public). Bien que la variable du contenu ne soit pas étudiée en tant que telle dans I'enquête PISA, on s'est efforcé d'y inclure des textes relevant de nombreuses situations différentes, afin de diversifier autant que possible les contenus des épreuves PISA de compréhension de l'écrit.

Le tableau 2.1 montre la répartition approximative du score par type de situation, pour les textes sur papier et pour les textes sur support électronique. Cette répartition ne sera finalisée qu'après analyse des données de la campagne définitive. 
Tableau 2.1

Répartition approximative du score en compréhension de l'écrit entre les types de situations

\begin{tabular}{l|c|c}
\hline \multirow{2}{*}{\multicolumn{1}{c|}{ Situation }} & \multicolumn{2}{|c}{ Pourcentage de points lors du cycle PISA 2012 } \\
\cline { 2 - 3 } & Épreuves papier-crayon & Épreuves électroniques \\
\hline Personnelle & 36 & 35 \\
\hline Éducative & 33 & 15 \\
\hline Professionnelle & 20 & 0 \\
\hline Publique & 11 & 50 \\
\hline Total & $\mathbf{1 0 0}$ & $\mathbf{1 0 0}$ \\
\hline
\end{tabular}

\section{Les textes}

La lecture suppose un contenu à lire. Dans une évaluation, ce contenu - un ou plusieurs textes associés à une tâche particulière - doit être cohérent en soi. En d'autres termes, le texte doit être signifiant en tant que tel : le lecteur doit pouvoir en comprendre le sens sans autre élément à l'appui². S'il est tout à fait clair que les types de textes sont innombrables et que toute évaluation doit en inclure un vaste éventail, il est difficile de déterminer s'il existe une classification idéale des types de textes. L'inclusion de textes électroniques rend l'exercice plus complexe encore. Depuis 2009, il existe quatre grands critères de classification des textes :

- Le support : textes sur papier et textes sur support électronique.

- L'environnement : contenus modifiables ou non modifiables par le lecteur.

- Le format de texte : continu, non continu, mixte ou multiple.

- Le type de texte : description, narration, information, argumentation, instructions ou transaction.

Le critère distinctif principal des textes est leur support - sur papier et sur support électronique. Viennent ensuite le format et le type, qui s'appliquent également à tous les textes, qu'ils soient sur papier ou sur support électronique. Enfin, l'environnement ne concerne que les textes électroniques.

\section{Support}

Le support - qui distingue les textes imprimés des textes électroniques - est un critère majeur de classification des textes depuis le cycle PISA 2009.

Les textes imprimés se présentent en général sur papier, que ce soit des feuilles volantes, des brochures, des magazines ou des livres. La nature même du texte sur papier encourage le lecteur à aborder le contenu du texte dans un ordre donné (sans toutefois le lui imposer). Les textes sur papier sont par nature figés, ou statiques. De plus, dans la vie de tous les jours comme dans le cadre d'épreuves d'évaluation, le volume ou la quantité de texte apparaît d'emblée au lecteur.

Les textes électroniques peuvent se définir par le support sur lequel ils s'affichent, écrans à cristaux liquides (LCD), écrans plasma, écrans à matrice active (Thin Film Transistor, TFT) et autres appareils électroniques. Dans I'enquête PISA, toutefois, le support électronique désigne les textes avec liens hypertextes, c'est-à-dire les textes dotés d'instruments de navigation qui permettent, voire imposent, une lecture non séquentielle. Chaque lecteur se construit un texte "sur mesure " à partir des liens qu'il suit. Ces textes sont par nature évolutifs et dynamiques. Dans les textes électroniques, seule une partie du contenu s'affiche en une fois et il est souvent impossible pour le lecteur de se faire une idée du volume réel du texte complet.

Les instruments de navigation aident le lecteur à parcourir les textes et à passer des uns aux autres. Relèvent de cette catégorie les icônes, les barres de défilement, les onglets, les menus, les liens hypertextes intégrés dans les textes, les fonctions de recherche textuelle et les instruments de représentation globale du contenu, tels que le plan des sites. Des instruments de navigation existent aussi dans les textes sur papier (les tables des matières, les index, les chapitres et les sections, les en-têtes et pieds de page, les numéros de page et les notes de bas de page), mais ils jouent un rôle particulièrement important dans les textes électroniques, et ce, pour au moins deux raisons. D'une part, la taille réduite de l'affichage impose le recours à des dispositifs qui permettent aux lecteurs de faire défiler la page de texte dans la fenêtre et de passer d'une page à l'autre, notamment les barres de défilement, les index, etc. D'autre part, la lecture de textes électroniques implique habituellement d'aborder des textes multiples, que le lecteur doit parfois sélectionner dans un volume potentiellement infini. Le lecteur doit maîtriser les fonctions de recherche, d'indexation et de navigation pour faire le lien entre les textes. 
Dans le cadre de l'évaluation PISA de la compréhension de l'écrit électronique, une série de structures et d'instruments de navigation ont été identifiés pour être systématiquement inclus dans les items, au motif qu'ils représentent une composante importante de la mesure des compétences en lecture sur support électronique : les barres de défilement, les onglets, des listes de liens hypertextes ${ }^{3}$ affichés sur une ligne, dans une colonne ou dans un menu déroulant, et les liens hypertextes intégrés dans le texte.

Les tâches sont plus ou moins difficiles selon le nombre et le type d'instruments de navigation à utiliser, et le nombre d'opérations à effectuer ou d'étapes à franchir. En règle générale, plus les opérations sont nombreuses et plus les instruments sont complexes, plus les items sont difficiles. La difficulté des tâches dépend aussi de la mesure dans laquelle les instruments de navigation présentés sont courants, clairs et «saillants ». Certaines tâches de compréhension de l'écrit électronique ne demandent guère, voire pas du tout, de navigation.

\section{Environnement}

La classification selon l'environnement s'applique exclusivement aux textes électroniques et aux environnements informatiques (comportant un ordinateur) dans I'enquête PISA. Deux grands types d'environnement électronique ont été retenus en vue de l'évaluation de la compréhension de l'écrit électronique : les environnements non modifiables et les environnements modifiables. Ce qui les différencie, c'est le fait que le lecteur puisse ou non influer sur leur contenu.

Dans l'environnement non modifiable, le lecteur est un destinataire passif : il ne peut influer sur les contenus. C'est essentiellement en quête d'informations que les internautes visitent ce type de site. Parmi les textes courants de cette catégorie, citons les pages d'accueil, les sites annonçant des événements, les sites publicitaires, les sites d'information des services publics, les sites en rapport avec l'éducation où les élèves peuvent s'informer, les sites d'actualité et les catalogues de bibliothèque en ligne.

Dans l'environnement modifiable, en revanche, le lecteur a la possibilité de modifier ou d'ajouter des éléments. Les individus visitent ces sites non seulement pour obtenir des informations, mais également pour communiquer. Les messageries, les blogs, les groupes de discussion, les forums et les formulaires en ligne sont autant d'exemples de textes s'inscrivant dans un environnement modifiable.

La classification selon l'environnement n'est pas stricte, à l'instar d'ailleurs des classifications basées sur de nombreuses autres variables du cadre d'évaluation. Quelques tâches requièrent l'utilisation combinée de textes modifiables et de textes non modifiables : ce sont les tâches dites mixtes. Le tableau 2.2 montre la répartition approximative du score entre les environnements.

Tableau 2.2

Répartition approximative du score en compréhension de l'écrit électronique entre les environnements

\begin{tabular}{l|c|}
\hline \multicolumn{1}{c|}{ Environnement } & Pourcentage de points dans les épreuves de compréhension de l'écrit électronique \\
\hline Non modifiable & 65 \\
\hline Modifiable & 27 \\
\hline Mixte & 8 \\
\hline Total & $\mathbf{1 0 0}$ \\
\hline
\end{tabular}

\section{Format des textes}

La distinction entre les textes continus et non continus est un critère important de classification des textes.

Les textes continus et non continus se rencontrent à la fois sur papier et sur support électronique. Les formats mixte et multiple se rencontrent sur les deux supports, mais en particulier sur support électronique. Ces quatre formats sont décrits en détail ci-dessous.

Les textes continus sont constitués de paragraphes, eux-mêmes constitués de phrases. Ils peuvent s'inscrire dans des structures plus vastes, telles que des sections, des chapitres et des livres (sur papier : les articles de journaux, les essais, les romans, les nouvelles, les comptes rendus et les lettres; sur support électronique : les comptes rendus, les blogs et les rapports en prose).

Les textes non continus ne sont pas structurés de la même manière que les textes continus. Leur lecture nécessite donc une approche différente. Les textes non continus s'organisent le plus souvent selon un format matriciel, où figurent des listes (Kirsch et Mosenthal, 1990) (listes, tableaux, graphiques, diagrammes, publicités, horaires, catalogues, index et formulaires). 
De nombreux textes, qu'ils soient sur papier ou sur support électronique, sont des ensembles cohérents en soi, qui se composent d'éléments relevant à la fois du format continu et du format non continu. Les composantes des textes mixtes bien construits (un texte en prose accompagné d'un tableau ou d'un graphique, par exemple) se conjuguent au moyen de liens qui ajoutent à la cohérence de l'ensemble, que ce soit ponctuellement ou globalement. Sur papier, les textes mixtes sont fréquents dans les magazines, les ouvrages de référence et les rapports : leurs auteurs emploient divers modes de présentation pour communiquer des informations. Sur support électronique, les pages web non modifiables correspondent généralement à des textes mixtes, puisqu'elles associent souvent des listes, de la prose et, souvent, des graphiques. Les textes électroniques modifiables, tels que les formulaires en ligne, les messages électroniques et les forums, combinent également des passages au format continu et non continu.

Les textes multiples désignent des textes conçus indépendamment les uns des autres, cohérents en soi, qui ont été clairement associés dans un but particulier ou simplement juxtaposés pour les besoins de l'évaluation. Les relations entre les textes n'apparaissent pas nécessairement d'emblée. Ces textes peuvent être complémentaires ou antagonistes. Par exemple, des sites web de tour-opérateurs différents peuvent proposer aux touristes les mêmes itinéraires ou au contraire des formules différentes. Les textes multiples peuvent se présenter sous le même format (continu, par exemple) ou sous deux formats différents (continu et non continu).

Tableau 2.3

Répartition approximative du score en compréhension de l'écrit entre les formats de texte

\begin{tabular}{|c|c|c|}
\hline \multirow{2}{*}{ Format de texte } & \multicolumn{2}{|c|}{ Pourcentage de points lors du cycle PISA 2012} \\
\hline & Épreuves papier-crayon & Épreuves électroniques \\
\hline Continu & 58 & 4 \\
\hline Non continu & 31 & $11^{*}$ \\
\hline Mixte & 9 & 4 \\
\hline Multiple & 2 & 81 \\
\hline Total & 100 & 100 \\
\hline
\end{tabular}

* Ce chiffre s'établit à $12 \%$ après arrondi (11.54), mais le total serait alors de 101 \%. L'utilisation dans le titre du terme « répartition approximative » couvre cet aspect.

\section{Types de textes}

Les textes sont également classifiés en fonction de leur type : description, narration, information, argumentation, instructions et transaction.

Les textes qui s'observent dans le monde réel résistent aux classifications : ils tendent à chevaucher une ou plusieurs catégories, car leurs auteurs ne les écrivent généralement pas avec les typologies présentes à l'esprit. Il est toutefois utile dans une évaluation comme l'enquête PISA de classer les textes par type (déterminé en fonction des caractéristiques prédominantes du texte) afin de garantir la représentativité de l'échantillon de textes retenu par rapport aux différents types de lecture.

La classification des textes utilisée dans I'enquête PISA, qui est décrite ci-dessous, s'inspire des travaux de Werlich (1976).

Les textes dits de description traitent des propriétés des objets dans l'espace. Les questions typiques auxquelles ils répondent sont «Qu'est-ce que... ?» (par exemple, la description d'un lieu particulier dans un carnet de voyage ou un journal, un catalogue, une carte géographique, un horaire de vol en ligne ou la description d'une caractéristique, d'une fonction ou d'un processus dans un guide technique).

Les textes dits de narration traitent des propriétés des objets dans le temps. Les questions typiques auxquelles ils répondent sont "Quand... ? » ou " Dans quel ordre... ? ». Ils répondent à d'autres questions importantes, comme celle de savoir pourquoi les personnages d'un récit agissent comme ils le font (par exemple, un roman, une nouvelle, une pièce de théâtre, une biographie, une bande dessinée, un article de presse relatant un événement). Dans les épreuves de compréhension de l'écrit sur papier, le pourcentage de textes de narration est légèrement plus élevé lors du cycle PISA 2012 (20 \% environ) que lors des cycles précédents (2000 et 2009) (15\% environ).

Les textes dits d'information présentent des informations sous la forme d'un ensemble de concepts ou de constructs mentaux, ou fournissent des éléments qui permettent d'analyser ces concepts ou constructs mentaux. Ces textes expliquent comment différents éléments s'associent pour former un tout porteur de sens et répondent souvent à la question « Comment... ?» 
(par exemple, une dissertation, un diagramme montrant un modèle de mémoire, un graphique montrant l'évolution démographique, une cartographie conceptuelle ou encore une entrée dans une encyclopédie en ligne).

Les textes dits d'argumentation présentent les relations entre des concepts ou des propositions. Ils répondent souvent à la question "Pourquoi... ?». Les textes persuasifs, qui développent des opinions et des points de vue, en constituent une sous-catégorie importante. Parmi les textes d'argumentation, citons à titre d'exemple le courrier des lecteurs d'un journal, une affiche publicitaire, les messages publiés sur un forum en ligne ou une critique de livre ou de film sur le web.

Les textes dits d'instructions donnent des consignes à suivre. Les consignes expliquent les comportements à adopter pour effectuer une tâche (par exemple, une recette, une série de schémas expliquant la procédure à suivre pour donner les premiers soins, ou encore l'aide en ligne d'un logiciel).

Les textes dits de transaction exposent des engagements à respecter, par exemple demander l'exécution d'une action, organiser une réunion ou prendre rendez-vous avec un ami. Avant la généralisation de la communication par messagerie électronique, les lettres représentaient une grande proportion de ces textes, les conversations téléphoniques étant leur pendant oral. Ce type de textes n'est pas repris dans la classification de Werlich (1976). Il a été utilisé pour la première fois dans le cadre d'évaluation du cycle PISA 2009, à cause de sa fréquence dans l'environnement électronique (par exemple, les courriers électroniques de tous les jours et les textos que s'échangent collègues ou amis pour prendre et confirmer un rendez-vous).

\section{Aspect}

Les caractéristiques et les instruments de navigation sont les attributs visibles ou matériels qui permettent aux lecteurs de trouver comment aborder des textes, les cerner et passer des uns aux autres, alors que les aspects se rapportent aux stratégies, approches ou intentions mentales que les lecteurs choisissent pour trouver comment aborder des textes, les cerner et passer des uns aux autres.

Cinq aspects ont présidé à l'élaboration des épreuves de compréhension de l'écrit :

- localiser et extraire l'information ;

- comprendre le sens global d'un texte;

- développer une interprétation ;

- réfléchir au contenu d'un texte et l'évaluer; et

- réfléchir à la forme d'un texte et l'évaluer.

Les épreuves PISA ne permettent pas d'inclure un nombre suffisant d'items pour pouvoir rendre compte de ces cinq aspects sur une sous-échelle distincte. Pour cette raison, ces cinq aspects sont regroupés en trois grandes catégories :

- localiser et extraire ;

- intégrer et interpréter; et

- réfléchir et évaluer.

Les tâches qui consistent à localiser et à extraire des informations, c'est-à-dire qui demandent aux élèves de se concentrer sur des fragments d'information distincts dans un texte, sont associées à l'échelle de compétence Localiser et extraire.

Les tâches qui consistent à comprendre le sens global d'un texte et à développer une interprétation demandent aux élèves de se concentrer sur les relations présentes dans un texte. Les tâches qui demandent aux élèves de considérer l'ensemble du texte sont celles qui consistent à comprendre le sens global d'un texte, et celles qui les amènent à considérer des relations entre des parties du texte sont celles qui consistent à développer une interprétation. Ces deux types de tâches sont regroupées dans l'échelle de compétence Intégrer et interpréter.

Les tâches associées aux deux derniers aspects, à savoir réfléchir sur le contenu d'un texte et réfléchir sur la forme d'un texte sont regroupées dans l'échelle de compétence Réfléchir et évaluer. Ces deux types de tâches demandent aux élèves de se baser sur des connaissances extérieures au texte et de les mettre en relation avec ce qu'ils lisent. Les tâches qui consistent à réfléchir sur le contenu d'un texte se rapportent à la substance des textes, alors que celles qui consistent à réfléchir sur la forme d'un texte se rapportent à la structure et aux caractéristiques formelles du texte.

La figure 2.1 montre la relation entre les cinq aspects retenus lors de la conception des épreuves et les trois grands aspects retenus pour la présentation des résultats. 


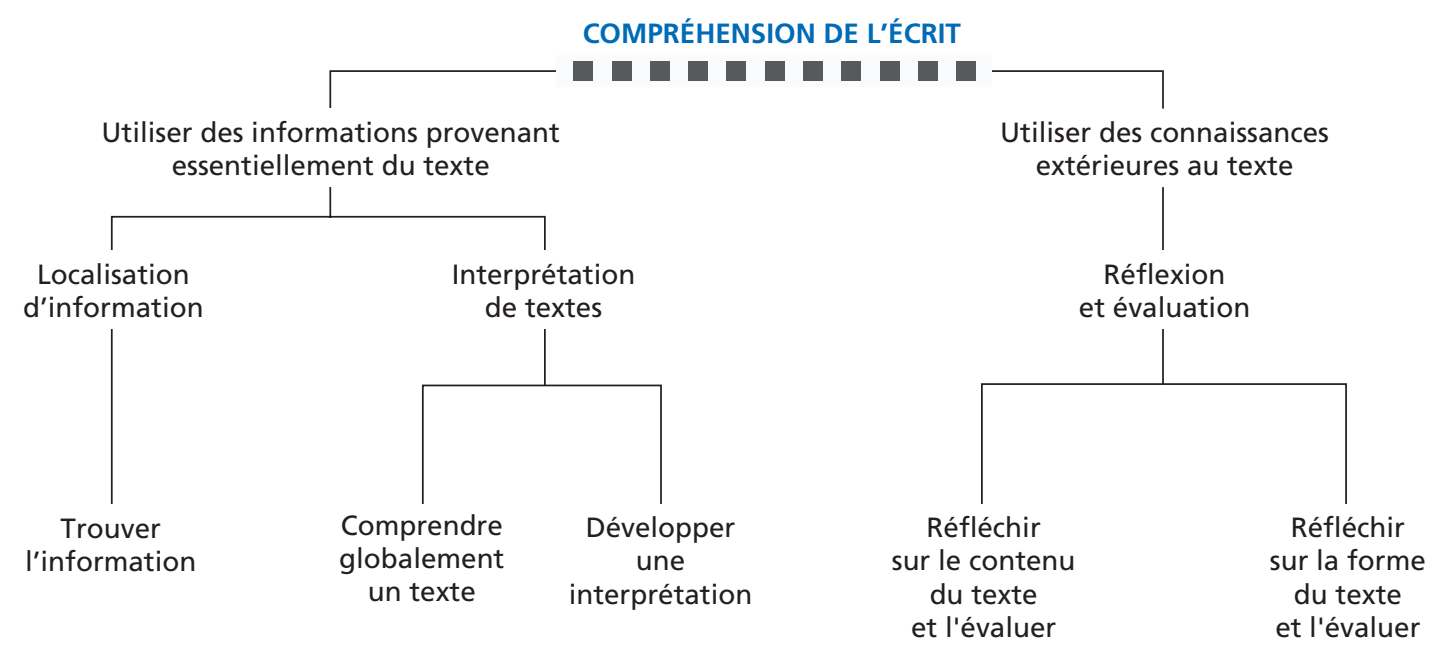

Les trois grandes catégories d'aspect sont décrites en détail, dans le cadre des épreuves papier-crayon et des épreuves électroniques.

\section{Localiser et extraire}

Localiser et extraire l'information consiste à repérer l'endroit où est donnée l'information et à parcourir le passage en question pour localiser, puis extraire un ou plusieurs fragments d'information. Ces tâches vont de la localisation des conditions d'embauche fixées par un employeur dans une offre d'emploi à l'identification d'un fait donné pour étayer ou infirmer l'assertion formulée par quelqu'un, en passant par l'identification d'un numéro de téléphone à plusieurs préfixes.

Extraire décrit le processus qui consiste à sélectionner l'information, et localiser, celui qui consiste à parvenir à l'endroit ad hoc, à trouver la source de l'information. Certains items demandent uniquement aux élèves d'extraire l'information, en particulier dans les épreuves papier-crayon, où l'information requise apparaît d'emblée et où les élèves ont uniquement à prélever un fragment d'information dans une source bien définie. En revanche, dans les épreuves électroniques, certains items exigent davantage : les élèves doivent, par exemple, cliquer sur un lien hypertexte intégré pour ouvrir une page web (dans un espace d'information très réduit) ou sur un élément dans une liste de résultats de recherche. Les deux processus interviennent toutefois dans la plupart des tâches des épreuves PISA. Le degré de difficulté des items dépend de plusieurs facteurs, dont le nombre de pages ou de liens à utiliser, le volume d'informations à traiter par page et, enfin, la spécificité et le caractère plus ou moins explicite des consignes données dans l'item.

\section{Intégrer et interpréter}

Le processus d'intégration et d'interprétation consiste à traiter un texte pour en découvrir le sens intrinsèque.

L'intégration consiste à démontrer que la cohérence d'un texte est bien comprise. Ce processus d'intégration consiste à mettre en relation divers fragments d'information pour construire du sens, que ce soit identifier des similitudes ou des différences, comparer des degrés ou comprendre des relations de cause à effet.

L'interprétation consiste à découvrir un sens implicite. C'est un processus qui amène le lecteur à identifier les hypothèses ou implications sous-jacentes dans l'ensemble ou une partie d'un texte.

Il faut passer par un processus d'interprétation et d'intégration pour comprendre le sens global d'un texte. Le lecteur doit considérer un texte dans son ensemble, sous une large perspective. Les élèves comprennent un texte s'ils parviennent à identifier le thème ou le message principal, ou à en établir l'intention ou l'usage.

Le processus d'interprétation et d'intégration se retrouve également dans celui qui consiste à développer une interprétation, un exercice qui demande aux élèves d'aller au-delà de leurs premières impressions générales pour comprendre ce qu'ils lisent de manière plus approfondie, plus spécifique ou plus complète. Dans les tâches d'intégration, les élèves doivent 
identifier des éléments pour étayer leur réponse et en dresser la liste, ou doivent comparer et confronter des informations, ce qui les amène à réunir plusieurs fragments d'information d'un texte. Les élèves doivent souvent inférer une relation ou une catégorie intentionnelle pour cerner des informations explicites ou implicites provenant d'une ou plusieurs sources. Les tâches d'interprétation peuvent demander aux élèves de faire une inférence sur la base d'un passage spécifique : par exemple, interpréter le sens d'un terme ou d'une phrase qui apporte une nuance particulière au texte. Ce processus de compréhension est également évalué par des tâches dans lesquelles les élèves doivent inférer l'intention de l'auteur ou identifier les éléments dont ils se servent pour faire cette inférence.

La relation entre le processus d'intégration et le processus d'interprétation peut dès lors être qualifiée d'étroite et $d^{\prime}$ interactive. L'intégration consiste à inférer une relation dans un texte (une forme d'interprétation), puis à réunir des éléments d'information pour en dégager une interprétation qui forme un nouveau tout intégré.

\section{Réfléchir et évaluer}

Le processus de réflexion et d'évaluation fait intervenir des connaissances, des idées ou positions extérieures au texte, dans la mesure où il demande au lecteur de mettre en relation les informations fournies dans le texte avec son propre cadre conceptuel et empirique de référence.

Les items de réflexion sont ceux qui demandent aux élèves de se baser sur leurs connaissances et expériences personnelles pour faire des comparaisons et élaborer des hypothèses. Quant aux items d'évaluation, ils les amènent à poser des jugements en se fondant sur des normes extérieures au texte.

Pour réfléchir sur le contenu d'un texte et l'évaluer, les élèves doivent établir un lien entre des informations du texte et des connaissances extérieures au texte. Ils doivent aussi juger de la pertinence des assertions avancées dans le texte en se basant sur leur propre connaissance du monde. Ils sont souvent amenés à élaborer leur propre point de vue, puis à le défendre. Pour ce faire, ils doivent être capables d'appréhender ce qui est énoncé et ce qui est sous-entendu dans le texte. Ils doivent ensuite confronter cette représentation mentale à leurs connaissances et leurs convictions, qu'elles leur viennent de leurs acquis ou d'informations trouvées dans d'autres textes. En outre, les élèves doivent trouver dans le texte des éléments qui étayent leur point de vue, puis confronter ces éléments à ceux d'autres sources d'information, ce qui leur demande de mobiliser des connaissances à la fois générales et spécifiques, ainsi que leur faculté de raisonnement abstrait.

Pour réfléchir sur la forme d'un texte et l'évaluer, les élèves doivent prendre de la distance par rapport au texte, le considérer en toute objectivité et juger de sa qualité et de sa pertinence. Les connaissances implicites sur la structure du texte, les styles typiques des différentes catégories de textes et des différents registres jouent un grand rôle dans ces tâches. Ces caractéristiques, fondamentales dans l'art de l'écriture, sont très importantes pour comprendre les normes inhérentes à ce type de tâches. Pour évaluer le talent d'un auteur à décrire certaines caractéristiques ou à persuader le lecteur, il faut non seulement pouvoir s'appuyer sur de solides connaissances, mais aussi avoir la faculté de détecter des nuances de langage.

Les modes d'évaluation peuvent prendre un tour légèrement différent dans les épreuves électroniques. L'homogénéité des formats électroniques (fenêtres, cadres, menus et liens hypertextes) tend à estomper les distinctions entre les types de textes. Ces caractéristiques des textes électroniques demandent au lecteur d'être plus attentif à l'origine des informations, à leur exactitude, à leur qualité et à leur crédibilité. L'évaluation est un aspect qui prend de plus en plus d'importance sachant que l'on a maintenant accès à un volume croissant d'informations dans des environnements en réseau.

Tout lecteur qui pose un jugement critique fait appel jusqu'à un certain point à sa propre expérience. En revanche, certaines formes de réflexion ne nécessitent pas d'étape d'évaluation (par exemple, comparer une expérience personnelle à une situation décrite dans un texte). L'évaluation peut donc être considérée comme une composante de la réflexion.

\section{Les aspects de la compréhension de l'écrit dans les épreuves papier-crayon et les épreuves électroniques}

Les trois grands aspects retenus dans l'évaluation PISA de la compréhension de l'écrit sont interdépendants, et non des entités totalement distinctes. Ils sont même à considérer comme semi-hiérarchiques dans le processus cognitif : il n'est pas possible d'interpréter ou d'intégrer des informations avant de les avoir localisées et extraites, pas plus qu'il n'est possible d'y réfléchir ou de les évaluer sans les avoir soumises au préalable à une certaine forme d'interprétation. Le cadre d'évaluation PISA décrit les aspects de la compréhension de l'écrit en faisant la distinction entre les différentes approches que le lecteur adopte selon les contextes et les objectifs, ce qui se reflète dans les épreuves dans la mesure où chaque tâche vise plus spécifiquement un aspect. 


\section{Tâches complexes de compréhension de l'écrit électronique : simuler la complexité de la lecture en situation réelle}

Ces trois aspects ne sont généralement pas totalement dissociables, que ce soit dans les items papier-crayon ou dans les items électroniques, mais il est possible de concevoir des tâches relativement simples qui relèvent clairement de l'un d'entre eux. Dans les tâches complexes, en revanche, les processus ne se définissent pas avec autant de précision. Les élèves doivent assimiler ce qui leur est demandé, puis ils doivent surmonter les problèmes d'interprétation, d'extrapolation et d'évaluation du texte qu'ils ont sous les yeux (par exemple, la page d'accueil d'un site web) pour trouver les informations pertinentes. Dans un exercice authentique de lecture électronique, le lecteur doit traiter ce qu'il a sous les yeux et l'extrapoler : localiser l'information, la synthétiser et la juger dans un processus intégré et récursif.

Tableau 2.4

Répartition approximative du score en compréhension de l'écrit entre les aspects

\begin{tabular}{l|c|c}
\hline \multirow{2}{*}{\multicolumn{1}{c|}{ Aspect }} & \multicolumn{2}{c}{ Pourcentage de points lors du cycle PISA 2012 } \\
\cline { 2 - 3 } & Épreuves papier-crayon & Épreuves électroniques \\
\hline Localiser et extraire & 22 & 19 \\
\hline Intégrer et interpréter & 56 & 23 \\
\hline Réfléchir et évaluer & 22 & 19 \\
\hline Complexe & 0 & $39^{*}$ \\
\hline Total & $\mathbf{1 0 0}$ & $\mathbf{1 0 0}$ \\
\hline
\end{tabular}

* Ce chiffre s'établit à 38 \% après arrondi (38.46), mais le total serait alors de $99 \%$. L'utilisation dans le titre du terme « répartition approximative » couvre cet aspect.

\section{Synthèse de la relation entre les tâches et les textes dans les épreuves papier-crayon et les épreuves électroniques}

Le tableau 2.5 résume quelques-unes des similitudes et différences fondamentales entre la lecture de textes sur papier et la lecture de textes électroniques. Dans de nombreux cas, les caractéristiques sont les mêmes dans les deux types d'épreuves, mais les descriptions fournies dans ce tableau mettent aussi en évidence des différences majeures.

Ce tableau montre aussi les similitudes et les différences entre ce que les épreuves PISA évaluent dans les deux types de support. Dans certains cas, les différences tiennent seulement à la plus ou moins grande importance donnée à telle ou telle caractéristique : les objectifs indiqués entre crochets sont ceux qui sont secondaires dans les épreuves PISA. Dans d'autres cas, au contraire, les différentes sont plus tranchées. Diverses caractéristiques se retrouvent dans les deux supports, mais elles ne sont pas nécessairement évaluées, ou ne peuvent l'être. Ces dimensions sont indiquées en bleu.

L'un des principes qui préside à la conception des cadres d'évaluation PISA et des épreuves qui les opérationnalisent est de représenter les domaines de la façon la plus " authentique » possible. II n’y a pas de méthode définie pour ce faire, et les décisions et les sélections sont jusqu'à un certain point arbitraires - même si elles se fondent sur le jugement d'experts internationaux spécialisés dans la lecture. La façon de décrire le domaine d'évaluation et de l'opérationnaliser est le fruit de considérations conceptuelles, empiriques et politiques. La description du champ d'application du domaine d'évaluation, faite ci-dessus, permet d'expliquer comment la construction des épreuves PISA cherche à refléter la substance de la compréhension de l'écrit. Ces épreuves permettront de réunir des données à partir desquelles il sera possible de rendre de compte du niveau de compétence des élèves âgés de 15 ans de manière approfondie, porteuse de sens et pertinente.

\section{ÉVALUER LA COMPRÉHENSION DE L'ÉCRIT}

Dans la section précédente, nous avons décrit le cadre conceptuel de la compréhension de l'écrit. Le moment est venu de transposer les concepts du cadre d'évaluation dans des tâches et des items qui permettront de recueillir des données sur le niveau de compétence des élèves en compréhension de l'écrit.

\section{Conception des tâches des épreuves papier-crayon}

La répartition des tâches entre les grandes variables du cadre d'évaluation, en l'occurrence les situations, les types et formats de texte, et les aspects, est décrite dans la section précédente. La présente section aborde quelques autres principes majeurs de la conception et de l'opérationnalisation de l'évaluation, à savoir les facteurs influant sur la difficulté des items et les moyens de faire varier le degré de difficulté, le choix des formats de réponse et plusieurs aspects du codage des réponses. 
Tableau 2.5

Similitudes et différences entre les épreuves papier-crayon et les épreuves électroniques, selon les caractéristiques retenues dans le cadre d'évaluation

\begin{tabular}{|c|c|c|}
\hline & Épreuves papier-crayon & Épreuves électroniques \\
\hline Situations & $\begin{array}{l}\text { Personnelle } \\
\text { Publique } \\
\text { Professionnelle } \\
\text { Éducative }\end{array}$ & $\begin{array}{l}\text { Personnelle } \\
\text { Publique } \\
\text { Professionnelle } \\
\text { Éducative }\end{array}$ \\
\hline Textes : Environnements & Non applicable & $\begin{array}{l}\text { Textes non modifiables } \\
\text { Textes modifiables } \\
\text { Textes mixtes }\end{array}$ \\
\hline Textes : Formats & $\begin{array}{l}\text { Continu } \\
\text { Non continu } \\
\text { [Mixte] } \\
\text { [Multiple] }\end{array}$ & $\begin{array}{l}\text { [Continu] } \\
\text { [Non continu] } \\
\text { [Mixte] } \\
\text { Multiple }\end{array}$ \\
\hline Textes : Types & $\begin{array}{l}\text { Argumentation } \\
\text { Description } \\
\text { Information } \\
\text { Narration } \\
\text { Instructions } \\
\text { Transaction }\end{array}$ & $\begin{array}{l}\text { Argumentation } \\
\text { Description } \\
\text { Information } \\
\text { Narration } \\
\text { Instructions } \\
\text { Transaction }\end{array}$ \\
\hline Aspects (1) & $\begin{array}{l}\text { Localiser et extraire } \\
\text { Rechercher } \\
\text { S'orienter et évoluer dans un espace concret d'information } \\
\text { Exemple : aller dans une bibliothèque, parcourir un catalogue, } \\
\text { trouver un livre } \\
\text { Utiliser les structures et les instruments de navigation } \\
\text { Exemple: table des matières, numéros de page et glossaire } \\
\text { Sélectionner l'information et organiser la lecture } \\
\text { - Faible degré de maîtrise de la part du lecteur } \\
\text { - Séquence unique de lecture linéaire }\end{array}$ & $\begin{array}{l}\text { Localiser et extraire } \\
\text { Rechercher } \\
\text { S'orienter et évoluer dans un espace abstrait d'information } \\
\text { Exemple : saisir I'URL d'un site, utiliser un moteur de } \\
\text { recherche } \\
\text { Utiliser les structures et les instruments de navigation } \\
\text { Exemple : utiliser les menus ou les liens hypertextes } \\
\text { Sélectionner l'information et organiser la lecture } \\
\text { - Grand degré de maîtrise de la part du lecteur } \\
\text { - Séquences multiples de lecture linéaire }\end{array}$ \\
\hline Aspects (2) & $\begin{array}{l}\text { Intégrer et interpréter } \\
\text { Intégrer à un niveau peu élevé d'exigence : } \\
\text { des parties plus longues visibles en même temps } \\
\text { (une ou deux pages) } \\
\text { Développer une interprétation } \\
\text { Comprendre le sens global d'un texte }\end{array}$ & $\begin{array}{l}\text { Intégrer et interpréter } \\
\text { Intégrer à un niveau élevé d'exigence: } \\
\text { des parties limitées visibles en même temps } \\
\text { (limitation en fonction de la taille de l'écran) } \\
\text { Développer une interprétation } \\
\text { Comprendre le sens global d'un texte }\end{array}$ \\
\hline Aspects (3) & $\begin{array}{l}\text { Réfléchir et évaluer } \\
\text { Pré-évaluer l'information } \\
\text { Exemple: utiliser une table des matières, écrémer des } \\
\text { passages, vérifier la crédibilité et l'à-propos } \\
\text { [Évaluer la crédibilité de la source } \\
\text { - Étape généralement moins importante, étant donné } \\
\text { le filtrage et la présélection durant le processus de publication] } \\
\text { Évaluer la plausibilité du contenu } \\
\text { Évaluer la cohérence et l'homogénéité } \\
\text { Faire des hypothèses } \\
\text { Réfléchir par rapport à son expérience personnelle }\end{array}$ & $\begin{array}{l}\text { Réfléchir et évaluer } \\
\text { Pré-évaluer l'information } \\
\text { Exemple : utiliser des menus, écrémer des pages web, } \\
\text { vérifier la crédibilité et l'à-propos } \\
\text { Évaluer la crédibilité de la source } \\
\text { - Étape généralement plus importante, étant donné } \\
\text { le manque de filtrage et de présélection dans un } \\
\text { environnement ouvert } \\
\text { Évaluer la plausibilité du contenu } \\
\text { Évaluer la cohérence et l'homogénéité } \\
\text { Faire des hypothèses } \\
\text { Réfléchir par rapport à son expérience personnelle }\end{array}$ \\
\hline Aspects (4) & $\begin{array}{l}\text { Complexe } \\
\text { L'éventail des sources à consulter est relativement indéfini } \\
\text { L'ordre dans lequel franchir les étapes n'est pas défini } \\
\text { Exemple : trouver, évaluer et intégrer des informations dans } \\
\text { plusieurs textes sur papier }\end{array}$ & $\begin{array}{l}\text { Complexe } \\
\text { L'éventail des sources à consulter est relativement indéfini } \\
\text { L'ordre dans lequel franchir les étapes n'est pas défini } \\
\text { Exemple : trouver, évaluer et intégrer des informations dans } \\
\text { plusieurs textes électroniques }\end{array}$ \\
\hline
\end{tabular}




\section{Facteurs influant sur la difficulté des items}

Le degré de difficulté de toute tâche de lecture dépend de l'action conjuguée de plusieurs facteurs : comme le montrent les travaux de Kirsch et Mosenthal (voir, par exemple, Kirsch, 2001 ; Kirsch et Mosenthal, 1990), il est possible de faire varier le degré de difficulté des items grâce au contrôle des variables d'aspect et de format suivantes.

Dans les tâches de localisation et d'extraction, la difficulté dépend du nombre d'éléments d'information à localiser, de I'importance des inférences à faire, du volume et de la visibilité des informations concurrentes, ainsi que de la longueur et de la complexité du texte.

Dans les tâches d'intégration et d'interprétation, la difficulté dépend du type d'interprétation à effectuer (faire une comparaison est plus facile qu'identifier un contraste, par exemple), du nombre d'éléments d'information à traiter, et du volume et de la visibilité des informations concurrentes. La nature du texte intervient aussi : plus le texte est long et complexe, et plus son sujet est abstrait et peu familier, plus la tâche s'avère difficile.

Dans les tâches de réflexion et d'évaluation, la difficulté dépend du type de réflexion et d'évaluation à effectuer (selon un degré croissant de difficulté, ces processus consistent à établir des liens, à expliquer et comparer, et enfin, à faire des hypothèses et à évaluer), de la nature des connaissances extérieures au texte auxquelles il faut faire appel (les tâches sont plus difficiles si les connaissances requises sont spécialisées et pointues, et non générales et courantes), de la longueur du texte et de son caractère plus ou moins abstrait, et enfin, du degré de compréhension requis pour mener les tâches à bien.

Dans les tâches associées à des textes continus, la difficulté dépend de la longueur du texte, de la " transparence » et du caractère explicite de sa structure, de la visibilité des relations entre les passages du texte et son thème général, de la présence ou non d'éléments textuels, tels que les titres et les paragraphes, et de marques de discours, tels que les mots de liaison.

Dans les tâches associées à des textes non continus, la difficulté dépend du volume d'informations dans le texte, du type de listes (les listes simples sont plus faciles à appréhender que les listes complexes), de la mesure dans laquelle les composants sont classés et explicitement structurés (par exemple, par le biais de titres de rubriques ou d'une mise en forme particulière), et de la localisation des informations requises (dans le corps du texte ou dans une autre partie, en note de bas de page, par exemple).

\section{Formats de réponse}

Le tableau 2.6 montre les modalités de codage des items : les items sont répartis selon leurs modalités de codage dans les trois aspects retenus dans les épreuves papier-crayon et dans les quatre aspects retenus dans les épreuves électroniques. Les items appelant une réponse construite ouverte ou une réponse construite courte nécessitent un jugement de la part du correcteur. Les items à choix multiple et à choix multiple complexe ne nécessitent aucun jugement de la part du correcteur. Quant aux items à réponse construite fermée, dans lesquels les élèves doivent générer une réponse, ils nécessitent une intervention minime du correcteur.

Dans les épreuves papier-crayon, la répartition des items entre les formats ne varie guère d'un cycle à l'autre. La batterie d'items retenue en vue du cycle PISA 2012 contient toutefois un pourcentage légèrement plus élevé que par le passé d'items ne nécessitant pas I'intervention d'un correcteur : I'intervention du correcteur n'est pas requise dans $58 \%$ d'items et l'est dans $42 \%$ des items (contre $55 \%$ et $45 \%$ respectivement lors des cycles précédents). Le ratio est le même dans les épreuves papier-crayon et dans les épreuves électroniques lors du cycle PISA 2012. 
Tableau 2.6

Répartition approximative du score en compréhension de l'écrit par modalités de codage dans chaque aspect de la compréhension de l'écrit

\begin{tabular}{l|c|c|c|c|c|c}
\hline & \multicolumn{3}{|c|}{ Épreuves papier-crayon } & \multicolumn{3}{c}{ Épreuves électroniques } \\
\hline \multicolumn{1}{c|}{ Aspect } & $\begin{array}{c}\text { Intervention requise } \\
\text { d'un correcteur }\end{array}$ & $\begin{array}{c}\text { Pas d'intervention } \\
\text { d'un correcteur }\end{array}$ & Total & $\begin{array}{c}\text { Intervention requise } \\
\text { d'un correcteur }\end{array}$ & $\begin{array}{c}\text { Pas d'intervention } \\
\text { d'un correcteur }\end{array}$ & \begin{tabular}{c} 
Total \\
\hline Localiser et extraire
\end{tabular} \\
\hline Intégrer et interpréter & 20 & 18 & 22 & 0 & 19 & 19 \\
\hline Réfléchir et évaluer & 18 & 36 & 56 & 0 & 23 & 23 \\
\hline Complexe & 0 & 4 & 22 & 15 & 4 & 19 \\
\hline Total & $\mathbf{4 2}$ & 0 & 0 & 27 & 12 & 38 \\
\hline
\end{tabular}

\section{Codage et attribution des crédits}

Des codes sont attribués aux réponses des élèves : dans les items à choix multiple, par le biais d'un processus plus ou moins automatisé d'enregistrement de l'option de réponse choisie par les élèves ; dans les items à réponse construite, c'est un correcteur expérimenté qui sélectionne le code qui traduit le mieux les réponses données par les élèves. Les codes sont ensuite convertis en scores. Le codage est un processus relativement simple dans les items à choix multiple et les items à réponse fermée : la réponse est correcte (code « 1 », crédit complet) ou ne l'est pas (code « 0 », pas de crédit). Les items à réponse construite se distinguent par un codage plus complexe : certaines réponses qui ne sont pas tout à fait correctes peuvent dénoter un niveau de compréhension de l'écrit supérieur à celui associé à des réponses incorrectes, auquel cas elles valent un crédit partiel.

\section{Conception des tâches des épreuves électroniques}

Cette section aborde plusieurs principes fondamentaux retenus lors de la conception de l'évaluation de la compréhension de l'écrit électronique et de son opérationnalisation : la relation entre le processus de navigation et le processus de traitement des textes, l'analyse des tâches en vue de faire varier le degré de difficulté des items et, enfin, les formats de réponse et certains aspects du codage. Cette section se termine par la description du mode de suivi de la progression des élèves dans les épreuves électroniques.

\section{Relation entre le processus de navigation et le processus de traitement des textes dans les épreuves de compréhension de l'écrit électronique}

Maîtriser quelques techniques et fonctions de navigation est une compétence fondamentale pour pouvoir lire des textes électroniques. Ces connaissances et compétences fondamentales sont à considérer comme des connaissances et compétences qui relèvent de la maîtrise des technologies de l'information et de la communication (TIC) et sont liées aux compétences associées à la compréhension de l'écrit. La lecture de l'écrit au sens traditionnel du terme, tout comme la faculté de naviguer dans l'environnement numérique, font partie intégrante de la compréhension de l'écrit. Dans les épreuves de compréhension de l'écrit électronique, les tâches impliquent toutes à des degrés variables un traitement mental pour prendre les décisions de navigation, d'une part, et pour appréhender les textes, d'autre part.

\section{Analyse des tâches de compréhension de l'écrit électronique}

Pour appréhender la complexité des étapes que le lecteur doit effectuer pour en arriver à la réponse explicitement demandée, les développeurs de tests ont utilisé un système d'analyse qui leur a permis de décrire les composantes de navigation et de traitement des textes de chaque tâche.

Toutes les tâches dont le degré de difficulté est modéré dans les épreuves électroniques peuvent être effectuées de différentes façons. Les développeurs de tests ont imaginé un cheminement optimal détaillé pour décrire et analyser les "sous-tâches ", dont chaque étape correspond à une action (le fait de cliquer sur un lien, de saisir un texte dans le navigateur, de sélectionner une option parmi celles proposées dans un item à choix multiple ou tout simplement de faire défiler une page à l'écran).

Chaque sous-tâche accomplie par une action est décrite selon différentes caractéristiques : la complexité de l'écrit, les instruments de navigation ou les caractéristiques des textes à utiliser, l'aspect de la compréhension de l'écrit et, enfin, l'action à effectuer. 


\section{EXEMPLES D'ITEMS PISA DE COMPRÉHENSION DE L'ÉCRIT ÉLECTRONIQUE OSONS PARLER}

ÉCRAN 1 A

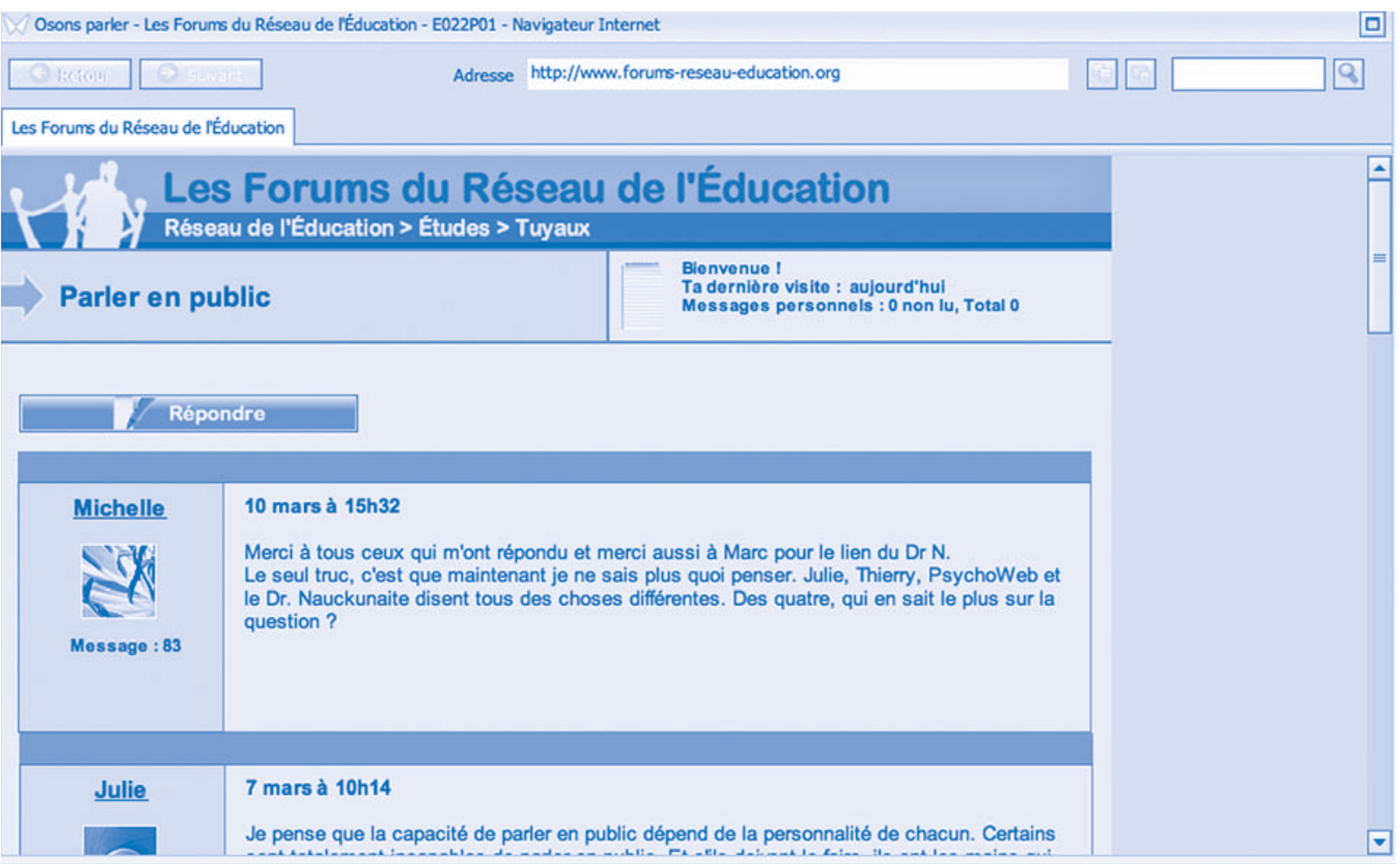

Cette unité simule un forum de discussion en ligne, dont le sujet est la difficulté à s'exprimer en public. La discussion a été lancée par Michelle : elle évoque la terreur qu'elle éprouve à l'idée de parler en public, devant sa classe, et demande de l'aide et des conseils dans un message qui se trouve au bas de l'écran du forum de discussion (voir l'écran 1E).

Cette discussion, qui se situe dans un contexte éducatif, est un exemple de contexte familier pour la plupart des élèves cibles de l'enquête PISA. En termes de format et de type de textes, I'unité OSONS PARLER propose des textes multiples, puisqu'ils sont écrits par des auteurs différents, qui se classent dans la catégorie de textes d'argumentation. L'unité OSONS PARLER présente une situation interactive, dans laquelle les auteurs se répondent directement. $C^{\prime}$ est une forme d'échange nouvelle, ou du moins très accélérée, qui prend de plus en plus d'importance dans le domaine de la communication. Dans ce type de textes multiples, la compréhension de chacune des interventions dépend en partie de la compréhension de l'ordre dans lequel elles s'affichent.

La page du forum de discussion est relativement longue : elle comporte huit interventions. Il faut faire défiler la page jusqu'en bas pour pouvoir lire la toute première intervention. Les écrans $1 \mathrm{~B}$ à $1 \mathrm{E}$ montrent ce qui s'affiche successivement à l'écran lorsque le lecteur fait défiler la page vers le bas. 


\section{ÉCRAN 1B}

Osons parler - Les Forums du Réseau de réducation - E022P01 - Navigateur Internet

\begin{tabular}{|c|c|c|c|}
\hline Q IREom & Adresse http:///mww.forums-reseau-education.org & $Q$ & \\
\hline \multicolumn{4}{|c|}{ Les Forums du Réseau de l'́́ducation } \\
\hline & & & $\Delta$ \\
\hline Message : 22 & $\begin{array}{l}7 \text { mars à } 10 \text { h14 } \\
\text { Je pense que la capacité de parler en public dépend de la personnalité de chacun. Certains } \\
\text { sont totalement incapables de parler en public. Et s'ils doivent le faire, ils ont les mains qui } \\
\text { tremblent et la voix qui chevrote. Par contre, d'autres personnes peuvent parler d'un sujet } \\
\text { avec aisance d'une façon qui rend le sujet intéressant pour le public. Ces personnes-là } \\
\text { donnent limpression d'être brillantes même si elles n'ont pas eu le temps de se préparer ! } \\
\text { En fait, ça ne sert à rien d'essayer de changer ce qu'on est. }\end{array}$ & & $\square$ \\
\hline $\begin{array}{c}\text { PsychoWeb } \\
\text { Messago: } 41\end{array}$ & $\begin{array}{l}28 \text { février à } 22 \mathrm{~h} 51 \\
\text { La façon d'aborder l'idée de parler en public dépend beaucoup de l'âge. C'est à trois ans qu'il } \\
\text { est le plus facile de s'exprimer en public. A cet âge, on parle tout le temps, en employant } \\
\text { des mots de son invention. On joue avec le langage sans se soucier du vocabulaire. Les } \\
\text { émotions s'expriment aussi très facilement - personne ne rit, ne pleure ou ne montre son } \\
\text { désespoir d'une manière plus expressive qu'un gamin de l'école maternelle. Pourquoi est-on } \\
\text { aussi franc à cet âge? Parce qu'on ne porte pas de jugement sur soi, qu'on n'a pas de } \\
\text { regard sur soi et qu'on ne porte pas le poids d'expériences douloureuses. C'est à l'école } \\
\text { secondaire qu'on se rend subitement compte qu'on est incapable de parler quand on est } \\
\text { appelé à s'exprimer devant toute la classe. }\end{array}$ & & \\
\hline & & & \\
\hline
\end{tabular}

\section{ÉCRAN $1 \mathrm{C}$}

Osons parler - Les Forums du Réseau de léducation - E022P01 - Navigateur Internet

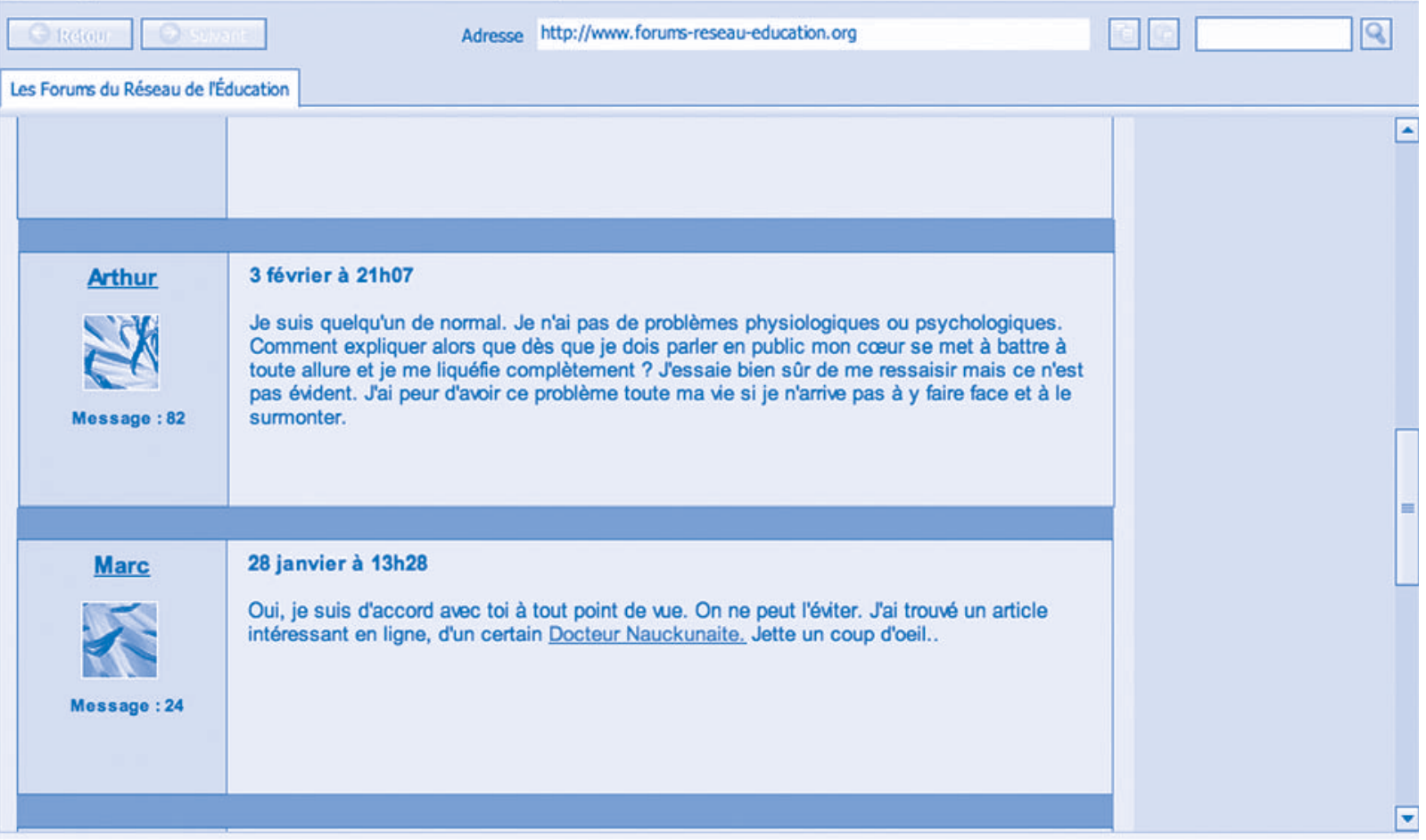




\section{ÉCRAN 1D}

Osons parler - Les Forums du Réseau de lÉducation - E022P01 - Navigateur Internet

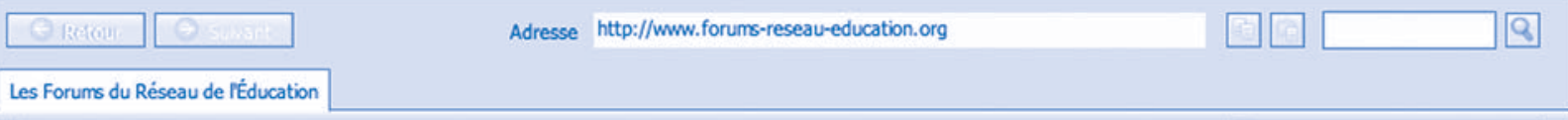

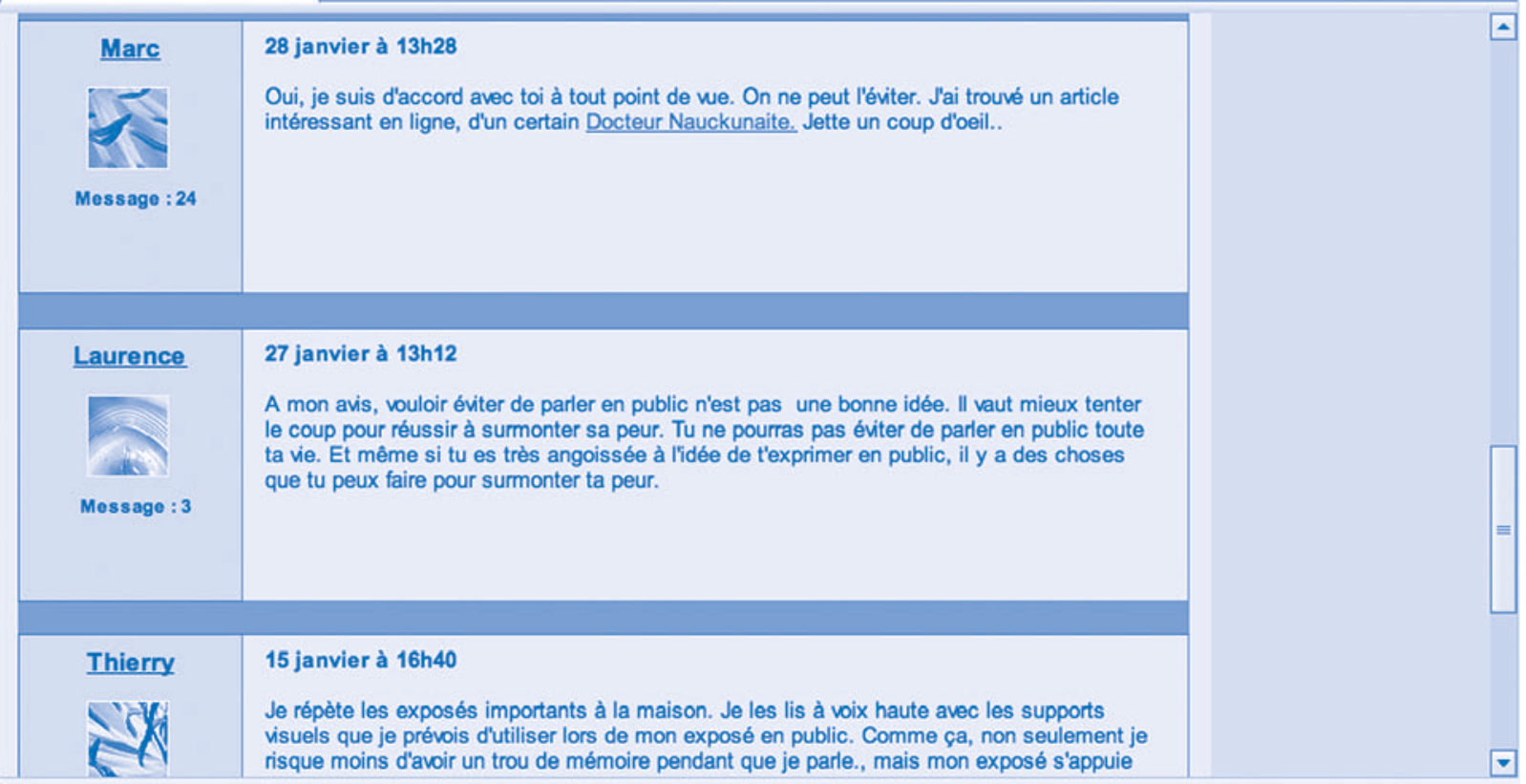

\section{ÉCRAN 1E}

Osons parler - Les Forums du Réseau de l'Éducation - E022P01 - Navigateur Internet

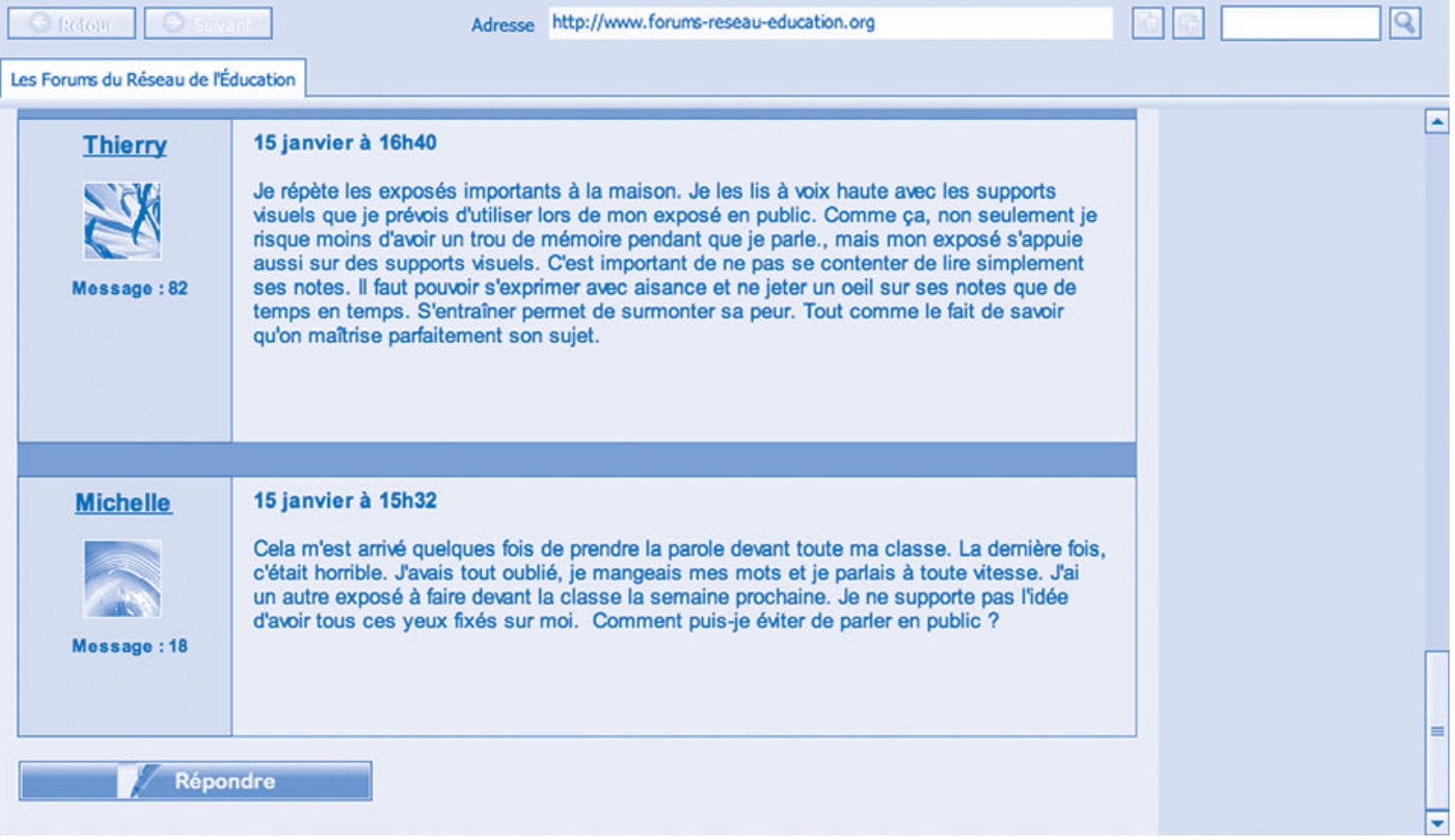


Le stimulus de cette unité ne comporte que la première page, et un seul autre élément, auquel le lecteur peut accéder en cliquant sur un lien intégré dans l'une des interventions qui évoque des « conseils d'expert ». Pour pouvoir lire les conseils du docteur Nauckunaite, il faut également faire défiler le deuxième écran (voir les écrans 2A et 2B).

\section{ÉCRAN 2A}

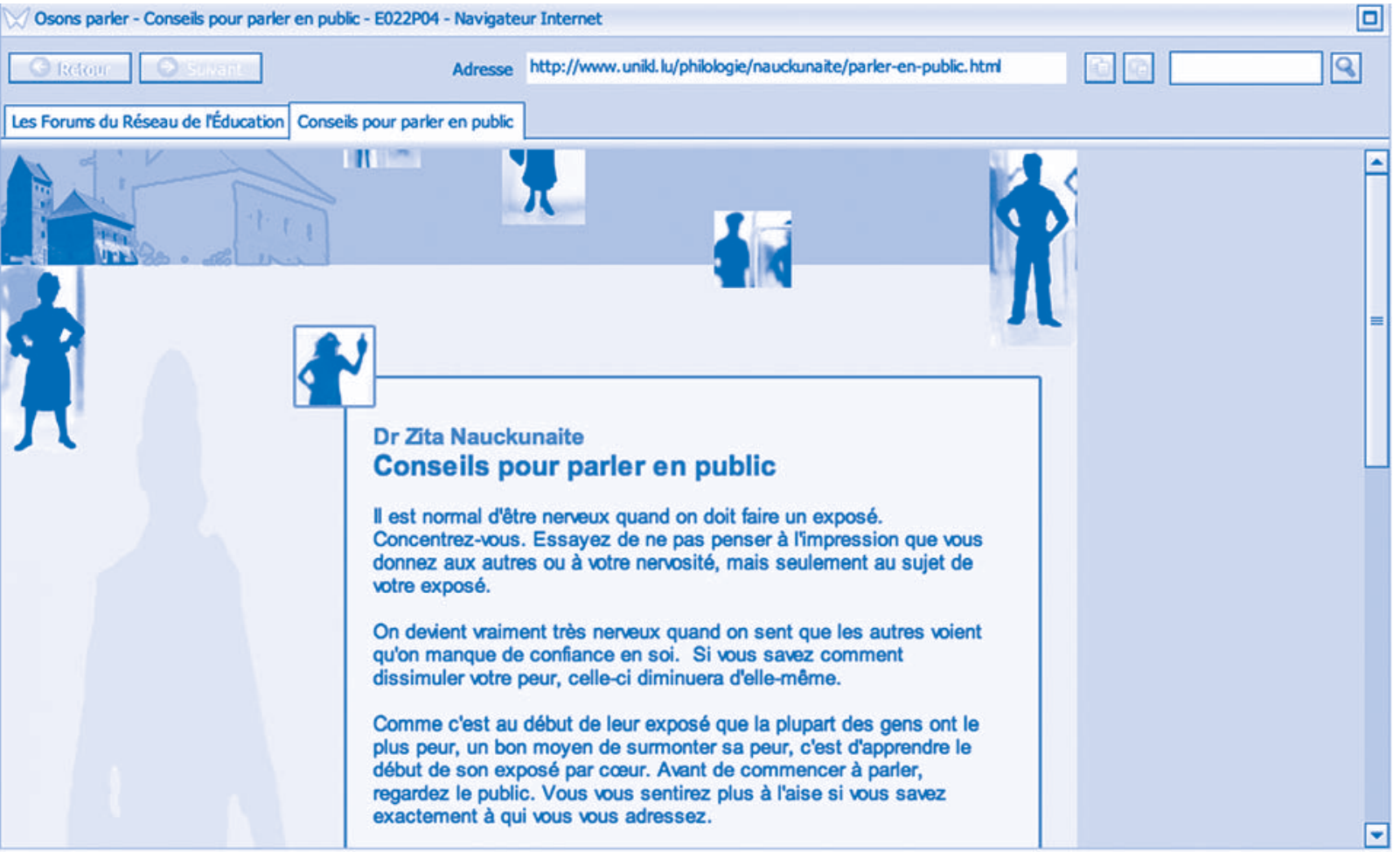

\section{ÉCRAN 2B}

\begin{tabular}{|c|c|c|}
\hline \multicolumn{2}{|c|}{ Wosons parler - Conseils pour parler en public - E022P04 - Navigateur Internet } & \multirow[t]{2}{*}{ 回 } \\
\hline 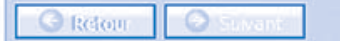 & Adresse http://wmw. unild. lu/philologie/nauckunaite/parler-en-public. html & \\
\hline Les forums du Réseau de l'Éducation & Conseils pour parler en public & \\
\hline & $\begin{array}{l}\text { On devient vaiment très nerveux quand on sent que les autres vient } \\
\text { qu'on manque de confiance en soi. Si vous savez comment } \\
\text { dissimuler votre peur, celle-ci diminuera d'elle-même. } \\
\text { Comme c'est au début de leur exposé que la plupart des gens ont le } \\
\text { plus peur, un bon moyen de surmonter sa peur, c'est d'apprendre le } \\
\text { début de son exposé par coeur. Avant de commencer à parler, } \\
\text { regardez le public. Vous vous sentirez plus à l'aise si vous savez } \\
\text { exactement à qui vous vous adressez. } \\
\text { Si vous sentez que la peur vous saisit pendant votre exposé, essayez } \\
\text { de ne pas fixer une personne précise dans le public. regardez plutốt } \\
\text { le milieu de l'assemblée. Si vous utilisez cette technique, ceux qui } \\
\text { sont assis devant et derrière auront l'impression que vous êtes en fait } \\
\text { en train de les regarder. Articulez chaque mot de manière claire. Rien } \\
\text { n'est plus apaisant que le son clair et posé de sa propre voix. } \\
\text { Extrait de } \\
\text { L'art oratoire, Dr Z Nauckunaite, Faculté de Philologie, } \\
\text { Université de pédagogie de Vilnius, Lithuanie }\end{array}$ & \begin{tabular}{|l}
$\Delta$ \\
$\square$
\end{tabular} \\
\hline
\end{tabular}


Cette unité de compréhension de l'écrit électronique, qui a été administrée lors de l'essai de terrain du cycle PISA 2009, comporte plusieurs tâches qui demandent aux élèves de comprendre l'organisation du site web, d'identifier les idées principales de différentes interventions et de prendre conscience des divergences de vues. La dernière tâche dirige les élèves vers la dernière intervention (la première qui s'affiche sur la page du forum de discussion) dans laquelle Michelle demande en quelque sorte un conseil final après avoir lu toutes les interventions selon le scénario imaginaire. Cette tâche est présentée ci-dessous.

\section{EXERCICE - OSONS PARLER}

Lisez le message que Michelle a écrit le 10 mars. Cliquez sur le bouton « Répondre » pour lui écrire un message. Répondez à sa question de savoir qui de tous ceux qui ont écrit en sait le plus sur la question selon vous. Expliquez pour quelle raison.

Cliquez sur le bouton « Envoyer la réponse » pour ajouter votre réponse sur le forum.

Cette tâche demande aux élèves d'accéder à plusieurs fragments d'information, puis de les intégrer. Dans sa deuxième intervention, Michelle invite le lecteur à comparer quatre petits textes (écrits par Julie, Thierry, PsychoWeb et le docteur Nauckunaite). Pour mener cette tâche à bien, les élèves doivent évaluer les interventions et juger soit de leur crédibilité sur la base du statut professionnel de leur auteur, soit de leur qualité intrinsèque et du pouvoir persuasif de leurs arguments. Il s'agit d'un item complexe, car il s'appuie sur les trois aspects : localiser et extraire, intégrer et interpréter, et réfléchir et évaluer.

Ce qui rend la tâche difficile est que les élèves doivent démontrer qu'ils sont capables de manipuler la structure formelle et les conventions de navigation dans un environnement électronique dont le contenu est modifiable : faire défiler les pages, cliquer sur un lien intégré dans le texte, puis cliquer sur un autre type de lien (un bouton) pour écrire leur réponse. Le fait de cliquer sur le bouton « Répondre » affiche l'écran suivant, où une réponse peut être saisie dans un cadre prévu à cet effet.

\section{ÉCRAN 3}

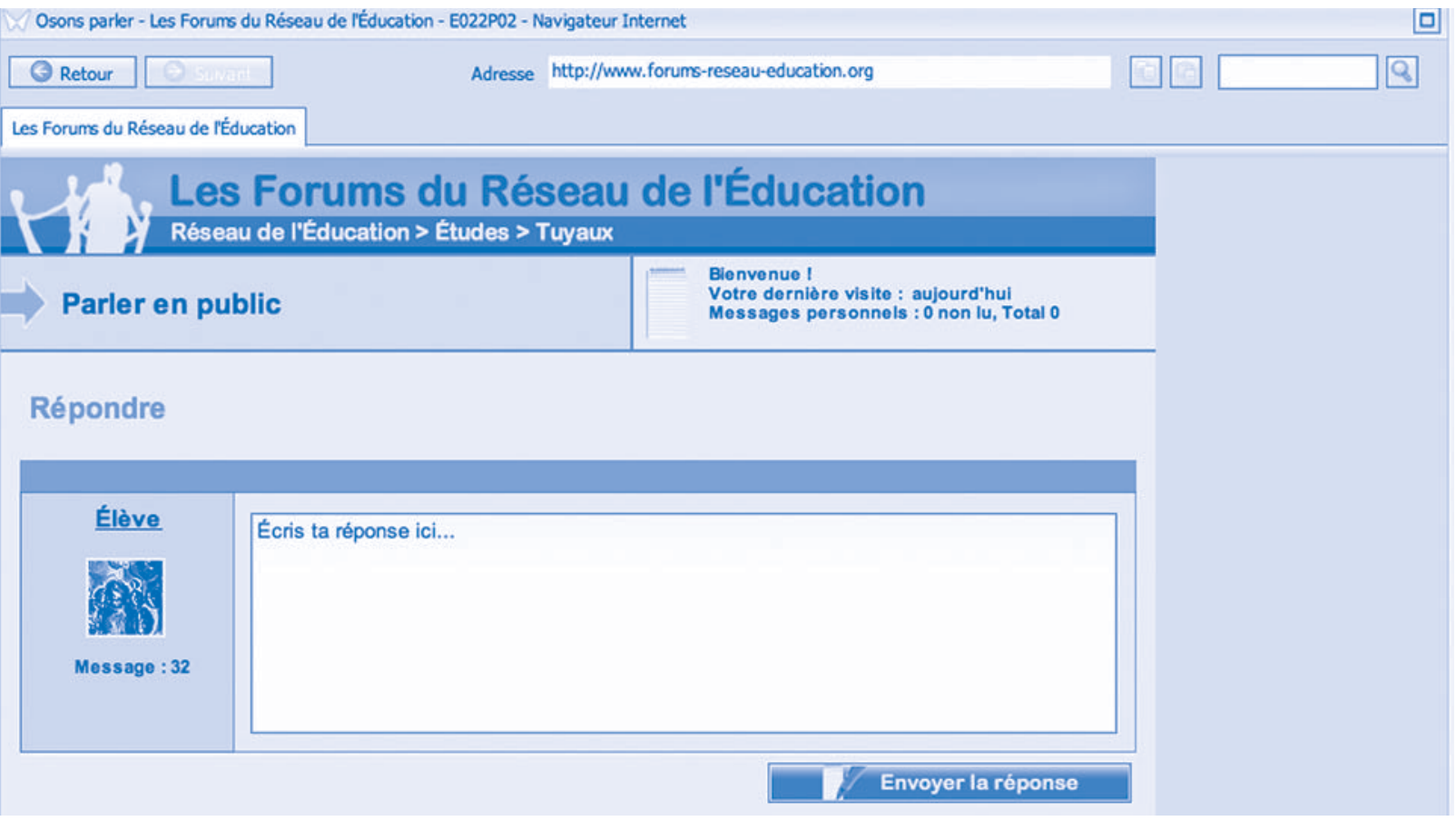

Le codage de cet item de l'essai de terrain du cycle PISA 2009 se base sur la réponse textuelle que les élèves saisissent dans le cadre de réponse. (Il y a lieu de souligner que le crédit complet peut être accordé même si les élèves ne cliquent pas sur le bouton «Envoyer la réponse », un détail ajouté dans un souci d'authenticité.) Toutefois, lors du développement de cet item, les variables de traitement du texte et du processus de navigation ont été délibérément manipulées pour que l'item apporte une contribution optimale à la description du volet informatique de l'évaluation. Le tableau 2.7 propose une version simplifiée de l'analyse de cet item de l'unité OSONS PARLER, en termes de traitement des textes et de navigation. 
Tableau 2.7

\section{Analyse d'un item de l'unité OSONS PARLER de l'évaluation de la compréhension de l'écrit électronique}

\begin{tabular}{|c|c|c|c|c|}
\hline Étape & $\begin{array}{l}\text { Première page/ } \\
\text { Traitement des textes/ } \\
\text { Degré de complexité des textes }\end{array}$ & $\begin{array}{l}\text { Instruments } \\
\text { de navigation à utiliser/ } \\
\text { Caractéristiques textuelles }\end{array}$ & $\begin{array}{l}\text { Aspect/Description } \\
\text { du traitement des textes }\end{array}$ & Action \\
\hline 1 & $\begin{array}{l}\text { Écran } 1 \mathrm{~A} \\
\text { Un petit texte d'argumentation } \\
\text { Degré de complexité : moyen }\end{array}$ & Barre de défilement & $\begin{array}{l}\text { Interpréter : comprendre le sens global } \\
\text { de la question posée par Michelle dans son } \\
\text { message du } 10 \text { mars. } \\
\text { Localiser : inférer que les interventions } \\
\text { des quatre participants mentionnés dans } \\
\text { le message de Michelle sont accessibles } \\
\text { moyennant l'utilisation de la barre } \\
\text { de défilement, le nom de la première } \\
\text { participante ("Julie») apparaissant déjà } \\
\text { à l'écran. }\end{array}$ & $\begin{array}{l}\text { Faire défiler la page } \\
\text { vers le bas }\end{array}$ \\
\hline 2 & $\begin{array}{l}\text { Écran 1B } \\
\text { Deux petits textes d'argumentation } \\
\text { Degré de complexité : moyen }\end{array}$ & Barre de défilement & $\begin{array}{l}\text { Extraire : établir un lien avec deux noms } \\
\text { dans le message de Michelle (« Julie » } \\
\text { et «Psychoweb »). } \\
\text { Interpréter : comprendre le sens global } \\
\text { de l'intervention de Julie et de PsychoWeb. } \\
\text { Localiser : inférer que certaines interventions } \\
\text { sont accessibles moyennant I'utilisation } \\
\text { de la barre de défilement. }\end{array}$ & $\begin{array}{l}\text { Faire défiler la page } \\
\text { vers le bas }\end{array}$ \\
\hline 3 & $\begin{array}{l}\text { Écran } 1 \mathrm{C} \\
\text { Deux termes mis en } \\
\text { évidence dans un petit texte } \\
\text { d'argumentation } \\
\text { Degré de complexité : faible }\end{array}$ & Lien intégré & $\begin{array}{l}\text { Localiser et extraire : localiser le lien du } \\
\text { Dr Nauckunaite intégré dans I'intervention } \\
\text { de Marc. }\end{array}$ & $\begin{array}{l}\text { Cliquer sur un } \\
\text { lien intégré dans } \\
\text { l'intervention de Marc }\end{array}$ \\
\hline 4 & $\begin{array}{l}\text { Écran } 2 \mathrm{~A} \\
\text { Texte formel comportant } \\
\text { des éléments d'information } \\
\text { et des instructions } \\
\text { Degré de complexité : moyen } \\
\text { à élevé }\end{array}$ & Barre de défilement & $\begin{array}{l}\text { Interpréter : comprendre le sens global } \\
\text { de la première partie de l'intervention } \\
\text { du Dr Nauckunaite. } \\
\text { Localiser : inférer que l'intervention } \\
\text { se poursuit au-delà de ce qui s'affiche } \\
\text { à l'écran. }\end{array}$ & $\begin{array}{l}\text { Faire défiler la page } \\
\text { vers le bas }\end{array}$ \\
\hline 5 & $\begin{array}{l}\text { Écran } 2 \mathrm{~B} \\
\text { Texte formel comportant } \\
\text { des éléments d'information } \\
\text { et des instructions } \\
\text { Degré de complexité : moyen } \\
\text { à élevé }\end{array}$ & Bouton « Retour» & $\begin{array}{l}\text { Interpréter : comprendre globalement } \\
\text { les idées principales de la deuxième partie } \\
\text { de l'intervention du Dr Nauckunaite. } \\
\text { Localiser : revenir à la page du forum } \\
\text { de discussion à l'aide du bouton "Retour » } \\
\text { (instruction de navigation explicitement } \\
\text { fournie dans la tâche). }\end{array}$ & $\begin{array}{l}\text { Cliquer sur le bouton } \\
\text { "Retour» }\end{array}$ \\
\hline 6 & $\begin{array}{l}\text { Écrans } 1 \mathrm{~A} \text { à } 1 \mathrm{E} \\
\text { Huit petits textes d'argumentation } \\
\text { (écrémage) } \\
\text { Écran } 1 \mathrm{E} \\
\text { Un des deux petits textes } \\
\text { d'argumentation } \\
\text { Degré de complexité : moyen }\end{array}$ & Barre de défilement & $\begin{array}{l}\text { Localiser : inférer que l'utilisation } \\
\text { de la barre de défilement est nécessaire } \\
\text { pour localiser la dernière intervention } \\
\text { mentionnée dans le message de Michelle. } \\
\text { Extraire : établir un lien avec le nom dans } \\
\text { le message de Michelle (« Tobias»). } \\
\text { Interpréter : comprendre globalement les } \\
\text { idées principales de l'intervention de Tobias. }\end{array}$ & $\begin{array}{l}\text { Faire défiler la page } \\
\text { vers le bas }\end{array}$ \\
\hline 7 & $\begin{array}{l}\text { Écran } 1 \mathrm{E} \\
\text { Bouton « Répondre » } \\
\text { Degré de complexité : très faible }\end{array}$ & Bouton « Répondre » & $\begin{array}{l}\text { Localiser : accéder à la page permettant } \\
\text { de répondre à Michelle. }\end{array}$ & $\begin{array}{l}\text { Cliquer sur le bouton } \\
\text { "Répondre " }\end{array}$ \\
\hline 8 & $\begin{array}{l}\text { Écran } 3 \\
\text { Encadré et bouton « Répondre » } \\
\text { [Remémoration des trois petits } \\
\text { textes d'argumentation des } \\
\text { écrans } 1 \mathrm{~A}, 1 \mathrm{~B} \text { et } 1 \mathrm{C} \text {, et du texte } \\
\text { formel comportant des éléments } \\
\text { d'information et des instructions } \\
\text { des écrans } 2 \mathrm{~A} \text { et } 2 \mathrm{~B}] \\
\text { Degré de complexité : très élevé }\end{array}$ & Aucun & $\begin{array}{l}\text { Réfléchir et évaluer : sélectionner le texte } \\
\text { qui fait le plus autorité, sur la base de } \\
\text { connaissances extérieures et d'informations } \\
\text { contenues dans les trois petits textes } \\
d^{\prime} \text { argumentation et le texte plus long } \\
\text { d'information et d'instructions. }\end{array}$ & $\begin{array}{l}\text { Saisir une réponse } \\
\text { textuelle }\end{array}$ \\
\hline $\begin{array}{c}9 \\
\text { (Facultative) }\end{array}$ & $\begin{array}{l}\text { Écran } 3 \\
\text { Bouton « Envoyer la réponse» }\end{array}$ & $\begin{array}{l}\text { Bouton } \\
\text { "Envoyer la réponse » }\end{array}$ & Non applicable & $\begin{array}{l}\text { Cliquer sur le bouton } \\
\text { "Envoyer la réponse» }\end{array}$ \\
\hline
\end{tabular}


Dans cette tâche, neuf étapes distinctes sont décrites (la dernière étant facultative). Toutefois, le même résultat peut être atteint si les étapes ne sont pas franchies dans cet ordre, à l'exception de la huitième étape. Ainsi, l'étape $n^{\circ} 1$ peut être suivie de l'étape $\mathrm{n}^{\circ}$ 3, ou le processus peut commencer par l'étape $\mathrm{n}^{\circ} 7$ (mais en cliquant sur le bouton "Répondre » affiché sur l'écran 1A, puis sur le bouton « Retour » pour revenir à la page d'accueil du forum). Il existe de nombreuses autres combinaisons possibles. Cette tâche montre bien que les lecteurs de textes électroniques se construisent jusqu'à un certain point leurs propres textes dans la mesure où ils choisissent l'ordre dans lequel ils consultent les pages et traitent l'information, et ce, même si le nombre de pages interconnectées est relativement limité. Pour mener à bien l'étape $n^{\circ} 8$ et obtenir le crédit complet, les élèves doivent posséder de bonnes compétences de navigation dans les textes électroniques (étapes $\mathrm{n}^{\circ} 1$ à 7 ) et d'excellentes compétences en matière de traitement des textes, car ils doivent traiter, intégrer et évaluer des textes multiples, dont un au moins est relativement difficile (voir les étapes $\mathrm{n}^{\circ} 4$ et 5 ).

\section{Contrôle de I'administration des tâches dans les épreuves de compréhension de l'écrit électronique}

Comme les captures d'écran de I'unité OSONS PARLER le montrent, I'interface des unités électroniques se divise en deux parties : la tâche, c'est-à-dire la question ou les consignes, s'affiche dans la partie inférieure de l'écran, et le navigateur, où se situe le stimulus, dans la partie supérieure de l'écran. La tâche reste affichée pendant la durée de l'item, mais les élèves peuvent naviguer entre les pages web ou les applications situées dans la partie « navigateur » de l'écran.

Dans les épreuves de compréhension de l'écrit électronique, les unités et les items sont administrés dans un ordre précis qui ne peut être modifié. En d'autres termes, les élèves ne peuvent revenir à une unité ou à un item une fois qu'ils sont passés à l'unité ou à l'item suivant. La conception des épreuves en termes d'affichage présente une autre caractéristique : la partie de la page qui s'affiche dans le navigateur au début de chaque item est fixe, c'est-à-dire que chaque élève voit la même page s'afficher au début de chaque item, et ce, indépendamment de l'étape à laquelle il a terminé l'item précédent. Ces deux caractéristiques contribuent à l'indépendance des items les uns par rapport aux autres.

\section{PRÉSENTATION DES RÉSULTATS EN COMPRÉHENSION DE L'ÉCRIT SUR PAPIER ET DE L'ÉCRIT ÉLECTRONIQUE}

\section{Compréhension de l'écrit sur papier}

Les résultats de I'enquête PISA sont présentés sur des échelles de compétence qui se prêtent à une interprétation pertinente pour l'action publique. Comme la compréhension de l'écrit est un domaine mineur d'évaluation dans le cadre du cycle PISA 2012, un nombre plus limité d'items ont été administrés aux élèves dont le niveau de compétence sera ensuite rapporté sur une seule échelle globale de compréhension de l'écrit.

Pour rendre compte de l'accroissement de la complexité et de la difficulté des items du cycle PISA 2012, I'échelle globale de compréhension de l'écrit sur papier, qui se base sur celle du cycle PISA 2009, est divisée en sept niveaux. La figure 2.2 décrit les sept niveaux de compétence en compréhension de l'écrit sur papier. Le niveau 6 de compétence est le plus élevé qui soit décrit (le niveau 5 était le niveau le plus élevé avant les épreuves du cycle PISA 2009). Le niveau 1b est le niveau de compétence le moins élevé (depuis les épreuves du cycle PISA 2009, le niveau 1 correspond maintenant au niveau $1 \mathrm{a}$ et un nouveau niveau, le niveau $1 \mathrm{~b}$, a été ajouté pour décrire le profil des élèves classés précédemment sous le niveau 1). Ces niveaux de compétence permettent aux pays d'en savoir davantage sur les types de tâches que les élèves très performants ou très peu performants sont capables de mener à bien. Les niveaux 2, 3, 4 et 5 restent inchangés par rapport au cycle PISA 2000. 
Description succincte des sept niveaux de compétence en compréhension de l'écrit sur papier lors du cycle PISA 2012

\begin{tabular}{|c|c|c|c|}
\hline Niveau & $\begin{array}{c}\text { Score } \\
\text { minimum }\end{array}$ & $\begin{array}{l}\text { Pourcentage } \\
\text { d'élèves } \\
\text { capables de } \\
\text { mener à bien les } \\
\text { tâches associées } \\
\text { au niveau } \\
\text { considéré, voire } \\
\text { à un niveau } \\
\text { supérieur } \\
\text { (moyenne de } \\
\text { l'OCDE) }\end{array}$ & Caractéristiques des tâches \\
\hline 6 & 698 & $0.8 \%$ & $\begin{array}{l}\text { Les tâches de ce niveau demandent généralement aux élèves de faire de nombreuses inférences, } \\
\text { de se livrer à des comparaisons et d'opposer des contrastes, dans le détail et avec précision. Pour y } \\
\text { répondre correctement, les élèves doivent bien comprendre un ou plusieurs textes en profondeur, } \\
\text { voire intégrer des informations provenant de plusieurs textes. Dans certaines tâches, les élèves sont } \\
\text { confrontés à des idées qui ne leur sont pas familières, en présence d'informations concurrentes } \\
\text { saillantes, ou doivent produire des catégories abstraites à des fins d'interprétation. Dans certaines } \\
\text { tâches de réflexion et d'évaluation, les élèves doivent formuler des hypothèses ou se livrer à une } \\
\text { évaluation critique à propos d'un texte complexe sur un thème qui ne leur est pas familier, compte } \\
\text { tenu de nombreux critères ou perspectives, en s'appuyant sur leur compréhension approfondie de } \\
\text { notions extérieures au texte. À ce niveau, les tâches de localisation et d'extraction présentent deux } \\
\text { caractéristiques : les élèves doivent faire des analyses d'une grande précision et doivent être très } \\
\text { attentifs à des détails peu visibles dans les textes. }\end{array}$ \\
\hline 5 & 626 & $7.6 \%$ & $\begin{array}{l}\text { À ce niveau, les tâches de localisation et d'extraction demandent aux élèves de localiser et } \\
\text { d'organiser plusieurs fragments d'information profondément enfouis et d'identifier les informations } \\
\text { pertinentes par déduction. Les tâches de réflexion passent par un processus d'évaluation critique } \\
\text { ou la formulation d'hypothèses sur la base de connaissances spécialisées. Pour mener à bien les } \\
\text { tâches d'interprétation et de réflexion, les élèves doivent comprendre en profondeur des textes } \\
\text { dont le fond ou la forme ne leur est pas familier. Quel que soit leur aspect, les tâches de ce niveau } \\
\text { demandent aux élèves d'aborder des concepts qui sont contraires aux attentes. }\end{array}$ \\
\hline 4 & 553 & $28.3 \%$ & $\begin{array}{l}\text { À ce niveau, les tâches de localisation et d'extraction demandent aux élèves de localiser et } \\
\text { d'organiser plusieurs fragments d'information enfouis. Pour mener à bien certaines tâches, les } \\
\text { élèves doivent interpréter le sens de nuances de langage dans un passage du texte tout en } \\
\text { tenant compte du texte dans sa globalité. D'autres tâches d'interprétation leur demandent de } \\
\text { comprendre et d'appliquer des catégories dans un contexte qui ne leur est pas familier. Dans les } \\
\text { tâches de réflexion, les élèves doivent formuler des hypothèses à propos d'un texte ou évaluer } \\
\text { celui-ci de manière critique en s'appuyant sur des connaissances formelles ou publiques. Les } \\
\text { élèves doivent montrer qu'ils comprennent bien des textes longs et complexes, dont le fond ou } \\
\text { la forme ne leur est pas nécessairement familier. }\end{array}$ \\
\hline 3 & 480 & $57.2 \%$ & $\begin{array}{l}\text { À ce niveau, les tâches demandent aux élèves de localiser plusieurs fragments d'information dans } \\
\text { le respect de nombreux critères et, dans certains cas, de reconnaître la relation qui existe entre eux. } \\
\text { Pour mener à bien certaines tâches d'interprétation, les élèves doivent intégrer plusieurs passages } \\
\text { d'un texte pour identifier une idée maîtresse, comprendre une relation ou découvrir le sens d'un } \\
\text { terme ou d'une phrase. Ils doivent tenir compte de nombreuses caractéristiques lorsqu'ils font } \\
\text { des comparaisons, opposent des contrastes ou établissent des classements. Dans de nombreuses } \\
\text { tâches, les informations pertinentes n'apparaissent pas d'emblée, les informations concurrentes sont } \\
\text { multiples ou les idées sont contraires aux attentes ou formulées de manière négative. Pour mener } \\
\text { à bien certaines tâches de réflexion, ils doivent bien comprendre des textes en rapport avec des } \\
\text { connaissances familières. Dans d'autres tâches, les élèves n'ont pas à comprendre les textes d'une } \\
\text { manière aussi approfondie, mais ils doivent se baser sur des connaissances moins courantes. }\end{array}$ \\
\hline 2 & 407 & $81.2 \%$ & $\begin{array}{l}\text { À ce niveau, certaines tâches demandent aux élèves de localiser un ou plusieurs fragments } \\
\text { d'information, que ce soit par déduction ou dans le respect de plusieurs critères. Dans d'autres } \\
\text { tâches, les élèves doivent identifier le thème d'un texte, comprendre des relations ou découvrir } \\
\text { le sens d'un passage limité d'un texte où les informations pertinentes ne sont pas saillantes, ce } \\
\text { qui leur impose de faire des inférences de niveau inférieur. Dans les tâches de ce niveau, les } \\
\text { élèves peuvent avoir à faire des comparaisons ou à opposer des contrastes en se basant sur un } \\
\text { aspect du texte. À ce niveau, les tâches de réflexion demandent généralement aux élèves de } \\
\text { faire une comparaison ou d'établir des liens entre le texte et des connaissances extérieures au } \\
\text { texte, sur la base d'expériences et d'attitudes personnelles. }\end{array}$ \\
\hline
\end{tabular}


- Figure 2.2 - (suite)

Description succincte des sept niveaux de compétence en compréhension de l'écrit sur papier lors du cycle PISA 2012

\begin{tabular}{l|l|l}
\hline $1 \mathbf{1 a}$ & $94.3 \%$ & $\begin{array}{l}\text { À ce niveau, les tâches demandent aux élèves de localiser un ou plusieurs fragments d'information } \\
\text { explicites, d'identifier l'idée principale d'un texte portant sur un thème familier ou de reconnaître } \\
\text { l'intention de son auteur, ou d'établir un lien simple entre des informations présentes dans le texte } \\
\text { et des connaissances courantes. Dans ces tâches, les informations pertinentes sont saillantes et il } \\
\text { n'y a guère, voire pas du tout, d'informations concurrentes. Les élèves sont explicitement orientés } \\
\text { vers les éléments pertinents de la tâche et du texte. }\end{array}$ \\
\hline $\mathbf{1 6}$ & $98.9 \%$ & $\begin{array}{l}\text { À ce niveau, les tâches demandent aux élèves de localiser un seul fragment d'information qui est } \\
\text { explicite et saillant dans des textes courts à la syntaxe simple dont le contexte et le type leur sont } \\
\text { familiers (une narration ou une liste simple, par exemple). Les textes sont conçus pour aider les } \\
\text { élèves : les informations y sont répétées ou accompagnées d'images ou de symboles familiers. Les } \\
\text { informations concurrentes y sont de surcroît peu nombreuses. Dans les tâches d'interprétation, } \\
\text { les élèves peuvent avoir à établir des liens simples entre des fragments d'information proches les } \\
\text { uns des autres. }\end{array}$ \\
\hline
\end{tabular}

\section{Compréhension de l'écrit électronique}

Dans les pays qui ont choisi d'administrer les épreuves de compréhension de l'écrit électronique, une autre échelle de compétence, basée sur les tâches de compréhension de l'écrit électronique, a été élaborée depuis le cycle PISA 2009. C'est le point de départ d'une nouvelle ligne de tendance. Étant donné le nombre relativement limité d'items lors du cycle PISA 2012 (et du cycle PISA 2009), le spectre de difficulté des tâches de compréhension de l'écrit électronique permet uniquement de décrire quatre niveaux de compétence, à savoir les niveaux 2, 3, 4 et 5. La figure 2.3 décrit ces quatre niveaux de compétence en compréhension de l'écrit électronique. Les élèves qui se situent au niveau 2 de l'échelle de compétence sont susceptibles de mener à bien des tâches associées à ce niveau de l'échelle, mais pas celles associées à un niveau supérieur de l'échelle. Les élèves qui se situent au niveau 4 de l'échelle de compétence sont susceptibles de mener à bien des tâches associées à ce niveau de l'échelle ainsi que celles associées aux niveaux inférieurs de l'échelle.

- Figure 2.3 -

Description succincte des quatre niveaux de compétence en compréhension de l'écrit électronique lors du cycle PISA 2012

\begin{tabular}{|c|c|c|c|}
\hline Niveau & $\begin{array}{c}\text { Score } \\
\text { minimum }\end{array}$ & $\begin{array}{l}\text { Pourcentage } \\
\text { d'élèves } \\
\text { capables de } \\
\text { mener à bien } \\
\text { les tâches } \\
\text { associées au } \\
\text { niveau considéré, } \\
\text { voire à un } \\
\text { niveau supérieur } \\
\text { (moyenne de } \\
\text { l'OCDE) }\end{array}$ & Caractéristiques des tâches \\
\hline $\begin{array}{c}5 \\
\text { ou } \\
\text { au-delà }\end{array}$ & 626 & $7.8 \%$ & $\begin{array}{l}\text { Les tâches de ce niveau demandent généralement aux élèves de localiser des informations dans } \\
\text { un contexte qui ne leur est pas familier, de les analyser et de les évaluer de façon critique. Pour } \\
\text { y répondre, ils doivent mettre au point des critères pour évaluer le texte. Ensuite, ils doivent } \\
\text { parfois naviguer entre plusieurs sites sans orientations explicites et étudier dans le détail des } \\
\text { textes présentés sous divers formats. }\end{array}$ \\
\hline 4 & 553 & $30.3 \%$ & $\begin{array}{l}\text { Les tâches de ce niveau demandent aux élèves d'évaluer des informations de plusieurs } \\
\text { sources, de naviguer entre plusieurs sites dont les textes se présentent sous plusieurs formats } \\
\text { et de générer des critères d'évaluation dans un contexte familier, personnel ou pratique. Dans } \\
\text { d'autres tâches, ils peuvent avoir à interpréter des informations complexes en fonction de } \\
\text { critères bien définis dans un contexte scientifique ou technique. }\end{array}$ \\
\hline 3 & 480 & $60.7 \%$ & $\begin{array}{l}\text { Les tâches de ce niveau demandent aux élèves d'intégrer des informations, que ce soit en } \\
\text { naviguant entre plusieurs sites pour localiser des informations bien définies ou en générant } \\
\text { des catégories simples lorsque l'objet de la question n'est pas explicite. } \\
\text { Quant aux tâches d'évaluation, elles leur demandent de traiter uniquement des informations } \\
\text { directement accessibles ou une partie seulement des informations disponibles. }\end{array}$ \\
\hline 2 & 407 & $83.1 \%$ & $\begin{array}{l}\text { Les tâches de ce niveau demandent généralement aux élèves de localiser et d'interpréter } \\
\text { des informations bien définies, d'ordinaire dans des contextes familiers. Les élèves peuvent } \\
\text { avoir à naviguer entre des sites dont le nombre est limité et à utiliser des instruments de } \\
\text { navigation tels que des menus déroulants, en présence d'orientations explicites ou sur la base } \\
\text { d'inférences de niveau inférieur. Ils peuvent avoir à intégrer des informations sous différents } \\
\text { formats et à identifier des exemples qui se classent dans des catégories bien définies. }\end{array}$ \\
\hline
\end{tabular}




\section{SYNTHÈSE}

L'une des vocations premières de l'enquête PISA est d'éclairer les décideurs politiques sur l'évolution des tendances au fil du temps. Depuis le cycle PISA 2009, la construction d'une échelle et de sous-échelles de compétence exclusivement basées sur les épreuves de compréhension de l'écrit sur papier ont contribué à repérer des tendances et à les analyser. Un groupe distinct d'échelles a été élaboré pour rendre compte des résultats des épreuves de compréhension de l'écrit électronique et, le cas échéant, des résultats combinés des épreuves sur papier et des épreuves électroniques. Cela permettra d'établir un nouveau point de référence pour suivre l'évolution des tendances au fil des cycles PISA. Le cadre d'évaluation PISA de compréhension de l'écrit prévoit un grand éventail d'options de compte rendu pour constituer une large base de données et, ainsi, éclairer les décideurs politiques, les chercheurs et les professionnels de l'éducation.

Le cadre dévaluation de la compréhension de l'écrit du cycle PISA 2012 reste inchangé par rapport à celui du cycle PISA 2009. La conception PISA de la compréhension de l'écrit implique d'aller au-delà de la simple mesure de la capacité des élèves à décoder et à comprendre des informations littérales, pour évaluer dans quelle mesure ils sont capables de comprendre l'écrit, de l'utiliser, de réfléchir et de s'engager dans les textes, tant pour accomplir des objectifs personnels que pour participer pleinement à la vie de la société.

\section{EXEMPLES D'ITEMS PISA EN COMPRÉHENSION DE L'ÉCRIT SUR PAPIER BIBLIOTHĖQUE}

- Figure 2.4 -

Items de l'unité BIBLIOTHÈQUE

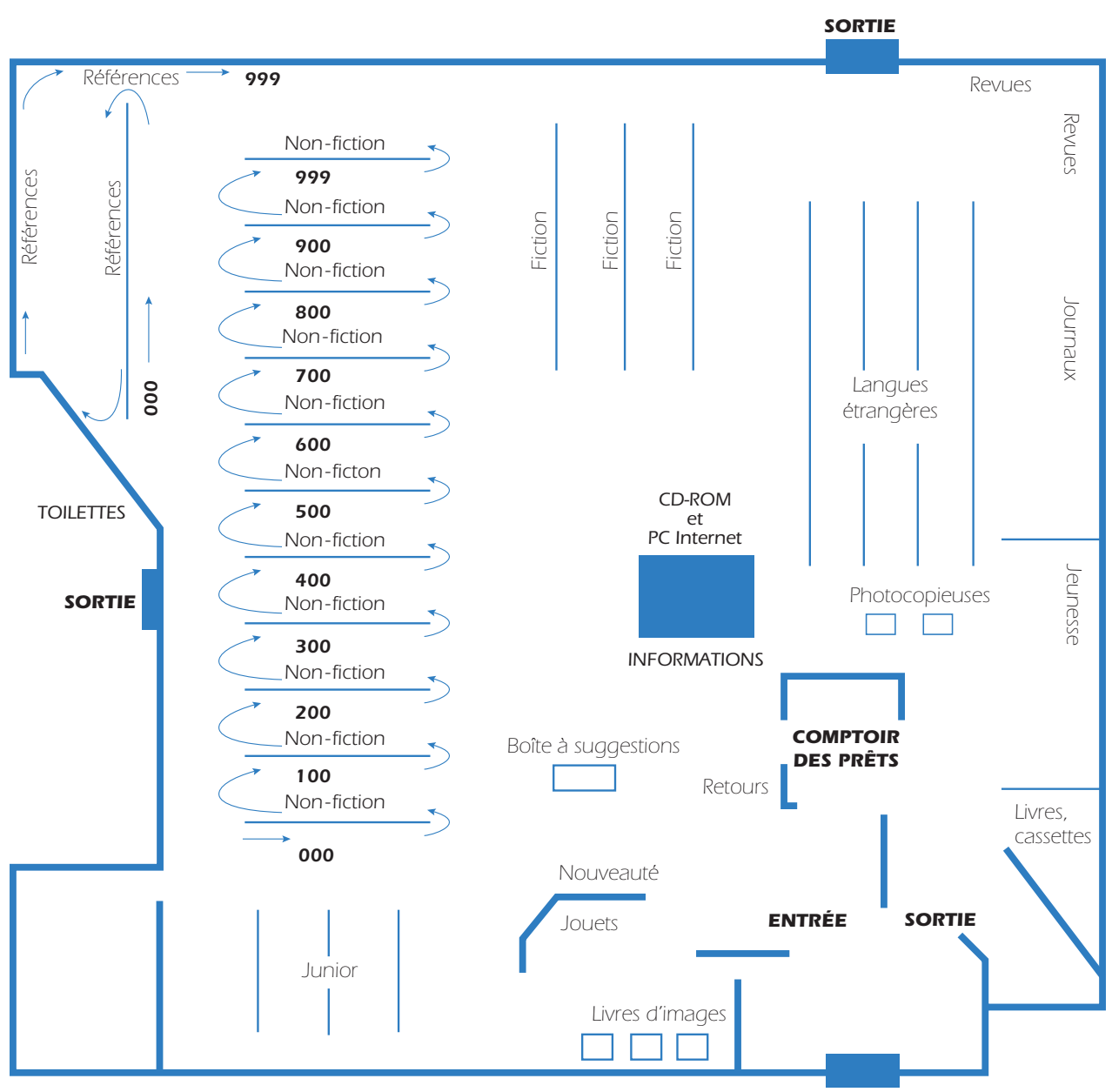


Le plan de bibliothèque qui constitue la base de cette unité est un exemple de texte non continu comme on peut en lire tous les jours au travail, à la maison, dans des contextes publics, éducatifs, etc. Le contexte de cet exemple est public car le plan se rapporte aux activités d'une communauté (une bibliothèque) et suppose donc un contact anonyme avec le lecteur. En ce qui concerne le type de texte, il entre dans la catégorie des descriptions puisque l'information proposée se réfère aux propriétés des objets dans l'espace et à leurs interrelations.

\section{QUESTION 1}

L'école vous demande de lire un roman en anglais. Entourez sur le plan la section de la bibliothèque où vous avez le plus de chances de trouver un ouvrage de ce type pour l'emprunter.

Caractéristiques conceptuelles

- Situation : Publique

- Support : Sur papier

- Format de texte : Non continu

- Type de texte : Description

- Aspect : Accéder à l'information et la localiser : Localiser l'information

- Objectif de la question : Localiser une information qui correspond à un facteur en recourant à une inférence de niveau inférieur

- Format d'item : Réponse courte

\section{Crédit complet}

Code 1 : Entoure les mots « Langues étrangères » ou les lignes (rayonnages) autour de ces mots.
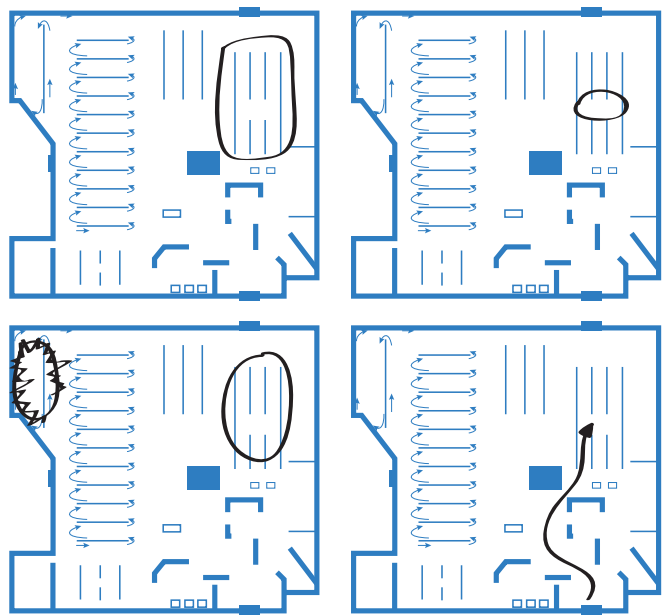

\section{Pas de crédit}

Code 0 : Autres réponses, y compris les cas où la partie entourée inclut complètement toute autre caractéristique de la carte.
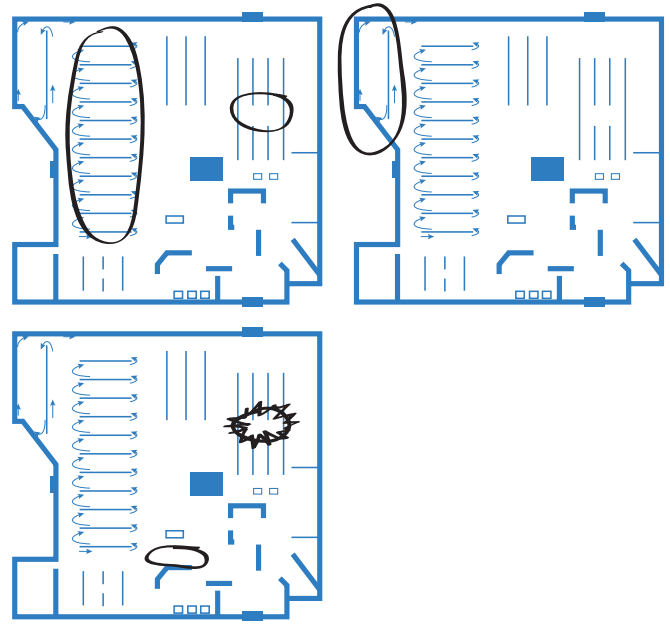
Code 9 : Omission.

Cet item à réponse courte exige du lecteur qu'il recherche, localise et sélectionne les informations pertinentes dans l'espace d'information. Dans ce cas précis, il s'agit d'un plan. Les informations nécessaires proviennent d'une seule source, et non de plusieurs, ce qui constitue un facteur susceptible de réduire la difficulté de l'exercice. Par ailleurs, la correspondance entre les mots figurant dans la tâche et la légende du plan n'est pas littérale : le lecteur doit faire une déduction pour classer «Anglais » dans la catégorie des «Langues étrangères ». (Une note de traduction et d'adaptation indiquait que dans les versions nationales de cet item, la langue mentionnée devait être une langue étrangère communément enseignée dans les établissements scolaires.) Cet item est plutôt facile : plus de 80 \% des élèves ayant participé à l'essai de terrain ont été capables d'identifier la bonne section dans la bibliothèque. Comme le montrent les exemples de crédit complet présentés dans les consignes de correction, les élèves pouvaient indiquer leur réponse de différentes façons. Bien que la question spécifie qu'il faut entourer la réponse, le format de la réponse ne constitue pas un critère déterminant dans l'attribution du crédit ; le point déterminant est en effet l'adéquation ou non entre la réponse et l'objectif de la question - « localiser une information qui correspond à un facteur en recourant à une inférence de niveau inférieur ».

\section{QUESTION 2A}

\section{Où se trouvent les « Nouveautés»? \\ A. Dans le rayon Fiction. \\ B. Dans le rayon Non-fiction. \\ C. Près de l'entrée. \\ D. Près du comptoir Informations.}

La réponse correcte est $C$ : Près de l'entrée. Cette question n'est posée qu'à titre d'information, et la réponse ne sera pas utilisée pour le calcul du score de l'élève. Elle est prise en compte dans l'évaluation de la réponse fournie à la question suivante (2B).

\section{QUESTION 2B}

Expliquez quelle est probablement la raison qui a fait choisir cet endroit pour placer les « Nouveautés».

Caractéristiques conceptuelles

- Situation : Publique

- Support : Sur papier

- Format de texte : Non continu

- Type de texte : Description

- Aspect : Réfléchir et évaluer : Réfléchir sur le contenu d'un texte et l'évaluer

- Objectif de la question : Émettre une hypothèse sur la localisation d'un élément dans un plan sur base des connaissances et de l'expérience personnelles

- Format d'item : Réponse construite ouverte

\section{Crédit complet}

Code 2 : La réponse à la question de la partie A est correcte. L'élève donne une explication en accord avec la réponse "Près de l'entrée ".

- Les gens peuvent les voir dès qu'ils entrent.

- Elles sont loin des autres livres et les gens les trouveront facilement.

- Pour que les gens les voient en premier lieu. [Implique que l'élève a remarqué que les Nouveautés se trouvent près de l'entrée.]

- Pour qu'elles soient très visibles.

- Elles sont très visibles au lieu d'être cachées quelque part dans les rayons, où il faudrait aller les chercher.

- On passe près d'elles quand va au rayon Fiction. 
OU : La réponse à la question de la partie A est correcte. L'élève donne une explication montrant qu'il comprend la position des Nouveautés par rapport à une section de la bibliothèque autre que l'entrée.

- Cela permet aux enfants de jouer pendant que les parents y jettent un coup d'œil. [A vu que les Nouveautés se trouvent près du rayon Jouets.]

- Quand les gens viennent restituer des livres, ils voient les nouveaux.

\section{Crédit partiel}

Code 1 : La réponse à la question de la partie $\mathrm{A}$ est incorrecte. L'élève fournit une réponse cohérente avec la réponse à la question précédente.

- [Réponse à la question de la partie A : Dans le rayon «Fiction ».] Parce que c'est la partie de la bibliothèque que la plupart des gens utilisent, de sorte qu'ils remarqueront les nouveaux livres.

- [Réponse à la question de la partie A : Près du comptoir « Information ».] Comme elles sont près du comptoir d'information, le bibliothécaire peut répondre à des questions à leur propos.

\section{Pas de crédit}

Code 0 : Réponse vague ou insuffisante que la réponse à la question de la partie A soit correcte ou non.

- Parce que c'est la meilleure place.

- Elles sont aussi près de l'entrée. [Indique où se trouvent les Nouveautés, sans donner d'explication.]

- Les Nouveautés sont près de la boîte à suggestions. [Indique où se trouvent les Nouveautés, sans donner d'explication.]

OU : Réponse manifestant une compréhension incorrecte du document, ou réponse peu plausible ou hors de propos (que la réponse à la question de la partie A soit correcte ou non).

- Pour que les gens les remarquent quand ils vont lire les journaux. [Incorrect. Implique que les Nouveautés sont près des journaux.]

- Parce qu'il n'y a pas d'autre endroit où les mettre. [Peu plausible.]

- Certaines personnes aiment lire les nouveautés. [Hors de propos.]

- [Réponse à la question de la partie A : Dans le rayon « Fiction ».] Pour qu'elles soient faciles à trouver. [Réponse hors de propos par rapport à la réponse de la partie A.]

Code 9 : Omission.

Les consignes de correction relatives à cette tâche sont relativement compliquées. On pose deux questions aux élèves, une à choix multiple et une avec réponse construite, mais seule la seconde est codée directement. Étant donné que cette tâche contribue à la détermination de l'échelle Réfléchir et évaluer, la question à choix multiple ne permet pas d'obtenir un crédit par elle-même puisqu'elle implique surtout une collecte d'information. Toutefois, elle est prise en compte dans le codage de la seconde question, la question à réponse construite.

Pour générer un crédit complet, la réponse implique à la fois une lecture précise du plan (localisation des Nouveaux livres près de l'entrée) et une hypothèse portant sur la raison de la localisation des Nouveaux livres à cet endroit. Pour formuler cette hypothèse, les lecteurs doivent se fonder sur leur propre expérience ou leurs connaissances, plus spécifiquement sur le mode de fonctionnement des bibliothèques et leur utilisation par les personnes qui les fréquentent. Dans les épreuves PISA, toutes les connaissances extérieures aux textes sont celles que les élèves de 15 ans ont en principe acquises.

Les élèves ne reçoivent qu'un crédit partiel s'ils ne parviennent pas à localiser les Nouveaux livres correctement sur le plan tout en donnant une hypothèse plausible portant sur la raison de la localisation des Nouveaux livres à un endroit spécifique. Tout comme les réponses donnant lieu à un crédit complet, ce type de réponse remplit l'objectif de la question qui est de réfléchir sur un contenu.

Cet item était facile puisque plus de quatre cinquième des élèves ont obtenu un crédit complet à l'essai de terrain. 


\section{AVIS AFFICHÉ AU SUPERMARCHÉ}

Cet avis destiné à un affichage public est un texte très court dont l'objet relève de la vie courante : prévenir les consommateurs de l'existence d'un risque concernant un produit et leur expliquer la marche à suivre pour retourner le produit et en obtenir le remboursement. Le format du stimulus est conforme aux normes internationales des avis de rappel de produits, mais il est possible que de nombreux élèves n'aient jamais vu de tels avis. Toutefois, le contenu de I'avis est clair et tient en peu de mots. Le produit en cause (biscuits fourrés au citron) a été choisi, car il est familier et susceptible $d$ 'intéresser les élèves. Comme indiqué ci-dessus, les items très faciles sont conçus sur la base de stimuli simples au contenu familier. Cette approche permet non seulement d'alléger le poids cognitif des items, mais aussi de présenter des textes qui ne risquent pas d'intimider les élèves dont le niveau de compétence est peu élevé en lecture, car ceux-ci peuvent très vite se décourager et abandonner jusqu'à l'idée d'essayer de lire un texte s'il leur apparait trop ardu ou trop long. En termes de format, le stimulus de cette unité se classe dans la catégorie des textes non continus, car il consiste en une liste descriptive de caractéristiques. Cet avis est un texte d'instruction, puisqu'il explique la marche à suivre aux consommateurs qui auraient acheté le produit en cause.

- Figure 2.5 -

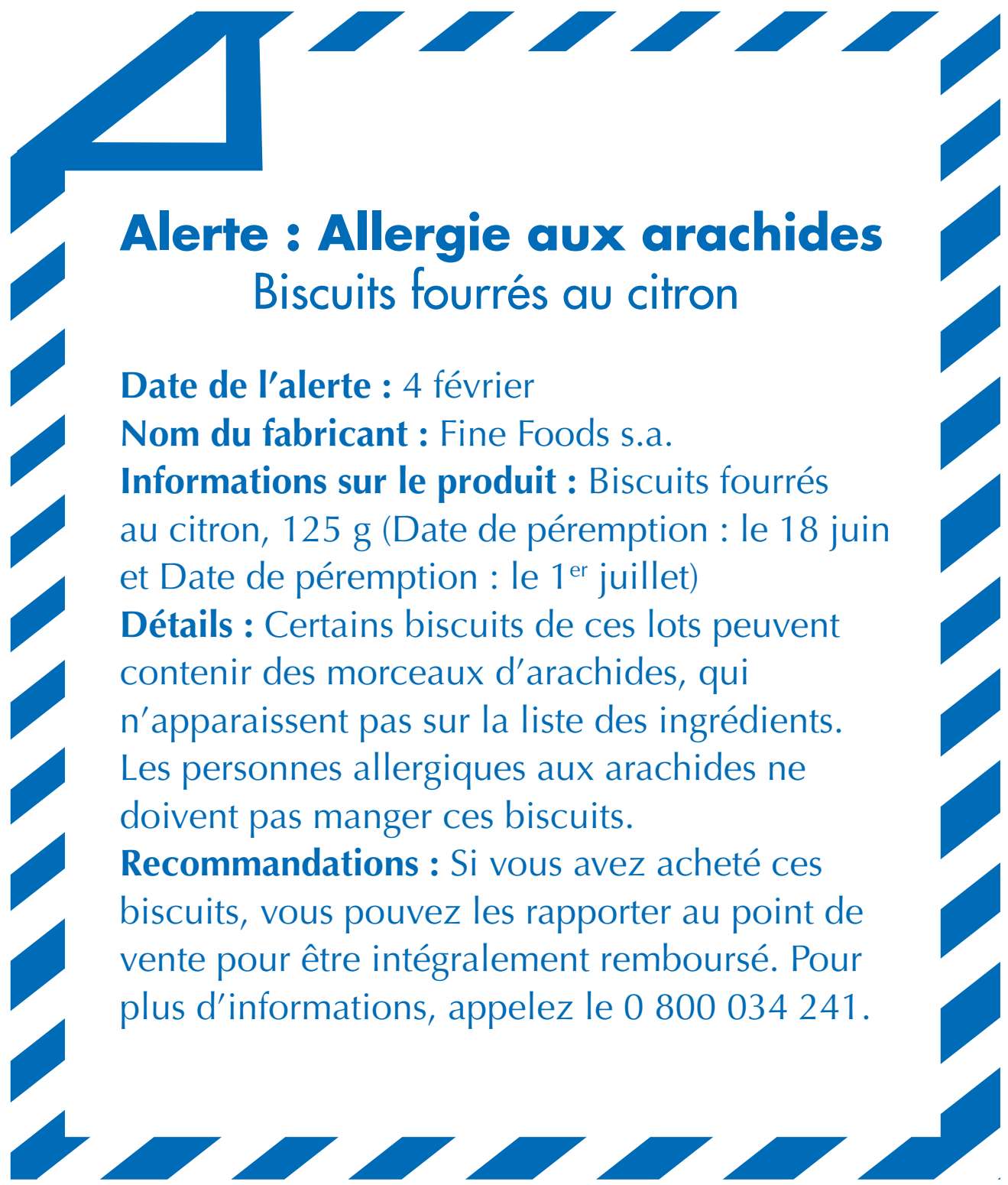




\section{QUESTION 1}

Quel est le but de cet avis?

A. Faire de la publicité pour les Biscuits fourrés au citron.

B. Indiquer quand les biscuits ont été fabriqués.

C. Mettre en garde au sujet des biscuits.

D. Expliquer où acheter les Biscuits fourrés au citron.

Caractéristiques conceptuelles

- Situation : Publique

- Support : Sur papier

- Format de texte : Non continu

- Type de texte : Instruction

- Aspect : Intégrer et interpréter : Comprendre le sens global d'un texte

- Objectif de la question : Identifier l'idée maîtresse d'un texte court en associant deux informations situées I'une en dessous de l'autre

- Format d'item : Choix multiple

\section{Crédit complet}

Code 1 : C. Mettre en garde au sujet des biscuits.

\section{Pas de crédit}

Code 0 : Autres réponses.

Code 9 : Omission.

Pour répondre correctement à cette question, les élèves doivent d'abord comprendre le sens global du texte afin d'en déduire l'objectif général. Plus spécifiquement, pour rejeter les distracteurs $A$ et $D$, les élèves doivent découvrir que, bien qu'il concerne un produit spécifique, ce texte n'est pas une publicité, mais une mise en garde. L'item était facile. Cette facilité s'explique partiellement par sa brièveté.

\section{QUESTION 2}

Quel est le nom de l'entreprise qui a produit ces biscuits?

Caractéristiques conceptuelles

- Situation : Publique

- Support : Sur papier

- Format de texte : Non continu

- Type de texte : Instruction

- Aspect : Accéder à l'information et la localiser : Localiser l'information

- Objectif de la question : Localiser une correspondance synonymique dans un texte court

- Format d'item : Réponse construite fermée

\section{Crédit complet}

Code 1 : Fine Foods s.a.

\section{Pas de crédit}

Code 0 : Autres réponses.

Code 9 : Omission. 
Pour répondre correctement à cette question, les élèves doivent localiser une information explicitement mentionnée dans le texte et établir une correspondance synonymique entre l'énoncé de la question et le texte ("fabricant » et " entreprise qui a produit »). La tâche est d'autant plus facile que le texte est très court et que l'information à localiser se situe au début du texte. II s'agit d'un item à réponse construite fermée, car seule une réponse (à quelques variantes près : « Fine Foods » et " Fine Foods s.a. ») donne droit à un crédit complet.

\section{QUESTION 3 \\ Que feriez-vous si vous aviez acheté ces biscuits?}

Pourquoi feriez-vous cela?

Utilisez les informations données dans le texte pour appuyer votre réponse.

Caractéristiques conceptuelles

- Situation : Publique

- Support : Sur papier

- Format de texte : Non continu

- Type de texte : Instruction

- Aspect : Réfléchir et évaluer : Réfléchir sur le contenu d'un texte et l'évaluer

- Objectif de la question : Émettre une hypothèse sur une décision personnelle en réponse à l'information contenue dans un texte

- Format d'item : Réponse construite ouverte

\section{Crédit complet}

Code $1: 3 \mathrm{~A}$ : Fournit une réponse en cohérence avec la compréhension du fait que les biscuits peuvent être rapportés et remboursés. Peut faire référence au fait de manger ou non les biscuits, de les rapporter ou de s'en débarrasser d'une manière ou d'une autre ET 3B : Donne une explication en cohérence avec le texte et la réponse à la question 3A. Doit être cohérente avec l'idée que la présence d'arachides comporte un risque potentiel.

- (3A) : Je demande à être remboursé.

(3B)

C'est ce qu'on me dit de faire.

Je suis allergique aux arachides.

Ils se sont trompés.

Il y a peut-être un (autre) problème.

Je n'aime pas les arachides.

- $(3 \mathrm{~A})$ : Je les jetterais.

(3B)

Je suis allergique aux arachides.

Il y a peut-être un problème.

- (3A) : Je les mangerais.

(3B)

Les arachides ne vont pas me faire de mal.

Je ne suis pas allergique aux arachides.

J'aime les arachides.

- (3A) : Je les donnerais à ma copine de classe.

(3B)

Elle n'est pas allergique aux arachides.

- (3A) : Rien.

(3B)

Je ne suis pas allergique aux arachides.

Je n'ai pas le courage de retourner au magasin. 
- 3A : Cite ou paraphrase un passage approprié du texte sans explication complémentaire (signifiant ainsi que le texte indique ce qu'il faut faire et qu'aucune autre explication n'est nécessaire).

3B : Pas de réponse.

- (3A) : Rapporter le produit au point de vente pour être intégralement remboursé. Pour plus d'informations, appelez le 0800034241.

(3B) : (pas de réponse).

- (3A) : Rapporter le produit au point de vente pour être intégralement remboursé.

(3B) : (pas de réponse).

- (3A) : Appeler le 0800034241 pour plus d'informations.

(3B) : (pas de réponse).

- (3A) : Appeler le numéro pour plus de d'informations.

(3B) : (pas de réponse).

- 3A : Pas de réponse ET 3B : fournit une explication au fait de ne rien faire. Doit être cohérente avec l'idée que la présence d'arachides comporte un risque potentiel.

- (3A) : (pas de réponse).

(3B) : Je ne suis pas allergique aux arachides.

- (3A) : (pas de réponse).

(3B) : Je n'ai pas le courage de retourner au magasin.

\section{Pas de crédit}

Code 0 : Donne une réponse insuffisante ou vague.

- $(3 \mathrm{~A})$ : Je ne sais pas.

(3B) : Ils pourraient contenir des arachides.

- (3A) : Je les mange.

- (3B) : Il pourrait y avoir des arachides.

Donne une réponse témoignant d'une mauvaise compréhension du texte ou une réponse non plausible ou hors de propos.

- (3A) : (pas de réponse).

(3B) : Je vérifie s'il y a des cacahuètes dedans.

- $(3 \mathrm{~A})$ : Je les mange.

(3B) : Ils ont l'air de pouvoir être mangés.

- (3A) : Je les donne à quelqu'un.

(3B) : Cela n'a pas d'importance.

- (3A) : (pas de réponse).

(3B) : Je suis allergique aux arachides.

- $(3 \mathrm{~A})$ : (pas de réponse).

(3B) : Les arachides peuvent être dangereuses.

- (3A) : Je les jette.

(3B) : La date de péremption est dépassée.

Code 9 : Omission.

Cette question demande aux élèves d'émettre des hypothèses sur leur réaction potentielle par rapport aux informations présentes dans le texte. Comme la question nécessite un jugement fondé sur des préférences individuelles, ou des comportements potentiels, on la classe dans la catégorie réfléchir et évaluer. Les consignes de correction indiquent qu'une variété de réponses peut bénéficier d'un crédit complet, pour autant qu'elles soient cohérentes avec deux idées maîtresses du texte : d'abord, qu'il est possible de ramener les biscuits, ensuite, que les biscuits peuvent être dangereux. Cet item est facile, plus de quatre cinquième des participants à l'essai de terrain ont obtenu un crédit complet. La facilité de l'item s'explique partiellement par le faible niveau de réflexion à appliquer : il ne faut pas de connaissances spécialisées pour expliquer une préférence personnelle sur une action à entreprendre concernant un sujet aussi familier que la nourriture. 


\section{QUESTION 4}

Pourquoi l'avis mentionne-t-il des dates de péremption?

Caractéristiques conceptuelles

- Situation : Publique

- Support : Sur papier

- Format de texte : Non continu

- Type de texte : Instruction

- Aspect : Intégrer et interpréter : Développer une interprétation

- Objectif de la question : Identifier l'objectif d'un élément conventionnel inclus dans un texte court

- Format d'item : Réponse construite ouverte

\section{Crédit complet}

Code 1 : Fait référence au fait que les dates de péremption identifient les lots de biscuits concernés.

- Pour identifier le(s) lot(s).

- Comme ça, on sait dans quels paquets il y a des arachides.

\section{Pas de crédit}

Code 0 : Fait référence au moment auquel consommer les biscuits.

- Parce que c'est quand on les mange.

- Pour nous dire quand manger les biscuits.

- Comme ça, on ne les garde pas trop longtemps.

- Pour nous dire quand ils arrivent à expiration.

- Donne une réponse insuffisante ou vague.

- C'est la date.

- Donne une réponse témoignant d’une mauvaise compréhension du texte ou une réponse non plausible ou hors de propos.

- Pour qu'on sache quand l'avis n'est plus valable.

Code 9 : Omission.

Moins d'un tiers des élèves ont répondu correctement à cette question. Au vu de la brièveté et de la simplicité du texte, ce résultat démontre que la difficulté d'un item n'est pas toujours liée aux seules caractéristiques d'un texte. Cette question impose aux élèves d'identifier l'objectif d'une partie spécifique du texte, les "dates de péremption ». Dans le cas de cet item, la difficulté provient du fait que les élèves doivent se concentrer sur l'objectif de l'élément dans ce texte en particulier. Les élèves qui répondent en donnant l'objectif habituel de cet élément (communiquer aux consommateurs la date ultime à laquelle le produit doit être utilisé) n'obtiennent pas de crédit pour cet item. La réponse donnant lieu à un crédit complet est contraire aux attentes, une caractéristique typique d'un item difficile.

\section{DESTINATION BUENOS AIRES}

«Destination Buenos Aires» est un extrait de Vol de Nuit, un roman écrit par Antoine de Saint-Exupéry en 1931. Le seul ajout par rapport au texte original est la note de bas de page explicative sur la " Patagonie », car les élèves auraient certainement eu des connaissances variées de ce lieu. Elle a été insérée uniquement pour la publication du texte dans l'épreuve PISA. L'explication apporte un contexte qui peut aider les élèves à mieux comprendre le texte. L'extrait se situe sur un aéroport à Buenos Aires et consiste en un portrait distant de Rivière, un homme chargé de lourdes responsabilités professionnelles. Bien que le roman ait été écrit en 1931, les thèmes qu'il aborde restent familiers car liés à l'humanité. 


\section{Items de l'unité DESTINATION BUENOS AIRES}

Ainsi les trois avions postaux de la Patagonie*, du Chili et du Paraguay revenaient du Sud, de l'Ouest et du Nord vers Buenos Aires. On y attendait leur chargement pour donner le départ, vers minuit, à l'avion d'Europe.

Trois pilotes, chacun à l'arrière d'un capot lourd comme un chaland, perdus dans la nuit, méditaient leur vol, et, vers la ville immense, descendraient lentement de leur ciel d'orage ou de paix, comme d'étranges paysans descendent de leur montagne.

Rivière, responsable du réseau entier, se promenait de long en large sur le terrain d'atterrissage de Buenos Aires. Il demeurait silencieux car, jusqu'à l'arrivée des trois avions, cette journée pour lui, restait redoutable. Minute par minute, à mesure que les télégrammes lui parvenaient, Rivière avait conscience d'arracher quelque chose au sort, de réduire la part d'inconnu et de tirer ses équipages hors de la nuit, jusqu'au rivage.

Un manœuvre aborda Rivière pour lui communiquer un message du poste radio :

Le courrier du Chili signale qu'il aperçoit les lumières de Buenos Aires.

Bien.

Bientôt Rivière entendrait cet avion : la nuit en livrait un déjà, ainsi qu'une mer, pleine de flux et de reflux et de mystères, livre à la plage le trésor qu'elle a si longtemps ballotté. Et plus tard on recevrait d'elle les deux autres.

Alors cette journée serait liquidée. Alors les équipes usées iraient dormir, remplacées par les équipes fraîches. Mais Rivière n'aurait point de repos : le courrier d'Europe, à son tour, le chargerait d'inquiétudes. Il en serait toujours ainsi. Toujours.

Antoine de Saint-Exupéry. Vol de Nuit. C Éditions Gallimard *Région du sud du Chili et de l'Argentine.

\section{QUESTION 1}

Comment Rivière se sent-il dans son travail ? Servez-vous du texte pour justifier votre réponse.

Caractéristiques conceptuelles

- Situation : Personnelle

- Support : Sur papier

- Format de texte : Continu

- Type de texte : Narration

- Aspect : Intégrer et interpréter : Développer une interprétation

- Objectif de la question : Établir un lien entre des informations dans un texte narratif et en tirer une généralisation sur I'état d'esprit d'un personnage tout en étayant cette généralisation

- Format d'item : Réponse construite ouverte

\section{Crédit complet}

Code 2 : Décrit la manière dont Rivière se sent dans son travail en faisant référence au stress, à l'endurance, au fait de se sentir surchargé ou au sens du devoir, ET donne une explication qui se réfère à un passage pertinent du texte. Peut faire référence au texte de manière générale, ou peut paraphraser le texte ou citer directement celui-ci. La citation doit correspondre à l'émotion mentionnée.

- Il est dépassé, on comprend à la dernière ligne qu'il ne se repose jamais.

- Il est stressé : «Cette journée pour lui, restait redoutable. »

- Il est accablé. Il se fait du souci toute la journée pour ces trois avions, et ensuite il doit s'inquiéter pour l'avion d'Europe !

- Il est résigné. Le dernier « toujours » nous révèle qu'il pense que les choses ne changeront jamais.

- Il s'engage à fond dans son travail. Il ne parvient pas à se détendre avant d'être sûr que chacun est sain et sauf. [Se réfère au texte de manière générale.]

\section{Crédit partiel}


Code 1 : Décrit la manière dont Rivière se sent dans son travail en faisant référence au stress, à l'endurance, au fait de se sentir surchargé ou au sens du devoir, sans donner d'explication qui se réfère au texte.

- Il se sent vraiment responsable de ce qui s'y passe.

- Il est stressé.

\section{Pas de crédit}

Code 0 : Donne une réponse insuffisante ou vague. Donne une réponse témoignant d'une mauvaise compréhension du texte ou une réponse non plausible ou hors de propos.

- Il aime son boulot parce qu'il dirige beaucoup de choses. [Pas étayé par le texte.]

- Il trouve que c'est cool parce qu'il peut regarder les avions. [Pas étayé par le texte.]

Code 9 : Omission.

Les consignes de correction de cet item montrent que deux types de réponse donnent droit à un crédit. Pour obtenir un crédit complet, il faut répondre précisément à la question et donner une explication en utilisant le texte. Un crédit partiel a été accordé aux élèves qui ont répondu précisément à la question mais n'ont pas pu expliquer leur réponse. Le code du crédit partiel indique qu'une réponse incomplète est supérieure à une mauvaise réponse. Lors de l'essai de terrain, moins de la moitié des élèves ont obtenu un crédit complet pour cet item, mais un quart supplémentaire a reçu un crédit partiel. En d'autres termes, environ trois quarts des élèves ont bénéficié d'un crédit (complet ou partiel) pour cet item. Cette question se classe dans la catégorie intégrer et interpréter, car bien que les élèves doivent produire une réponse qui n'est pas mentionnée explicitement dans le texte, toutes les informations nécessaires pour répondre à la question sont présentes dans le texte.

\section{QUESTION 2}

« Destination Buenos Aires » a été écrit en 1931. Pensez-vous que Rivière aurait des inquiétudes semblables aujourd'hui ? Justifiez votre réponse.

Caractéristiques conceptuelles

- Situation : Personnelle

- Support : Sur papier

- Format de texte : Continu

- Type de texte : Narration

- Aspect : Réfléchir et évaluer : Réfléchir sur le contenu d'un texte et l'évaluer

- Objectif de la question : Émettre une hypothèse sur l'impact qu'un changement dans le contexte du récit peut avoir sur un personnage

- Format d'item : Réponse construite ouverte

\section{Crédit complet}

Code 1 : Répond « Oui » OU «Non» (y compris de manière implicite), donne une comparaison circonstanciée en termes d'époque ET justifie sa réponse. Cette comparaison peut se fonder sur des aspects matériels, tels que le progrès technologique, I'amélioration de la sécurité, OU sur des aspects psychologiques, tels que l'anxiété. La réponse doit témoigner d'une lecture correcte du texte.

- De nos jours, les pilotes (les avions) disposent d'outils très sophistiqués pour s'orienter, ce qui résout la difficulté technique quand la météo est mauvaise.

- Non, à présent les avions sont équipés de radars et de systèmes de pilotage automatique qui peuvent aider les pilotes à se sortir de situations dangereuses.

- Oui, prendre l'avion reste dangereux, comme pour tout autre moyen de transport. Le risque d'un accident ou d'une panne de moteur n'est jamais exclu.

- Actuellement les nouvelles technologies et les progrès techniques sont considérables, tant au sol que dans les appareils. 
- Oui, il y a toujours un risque d'accident.

- Non, avant il n'y avait pas la crainte d'une attaque terroriste.

\section{Pas de crédit}

Code 0 : Donne une réponse insuffisante ou vague.

- Non, les craintes ne sont plus les mêmes aujourd'hui.

- Oui, on a réalisé pas mal de progrès.

- Dans un sens oui, mais dans le contexte actuel. [Vague.]

- Avec les années, les gens auraient changé cela. [Vague.]

Donne une réponse témoignant d'une mauvaise compréhension du texte ou une réponse non plausible ou hors de propos.

- Non, on ne voyage plus de nuit actuellement. [Incorrect, ce n'est pas ce qui se passe dans le monde actuel.]

- Non, parce qu'aujourd'hui les pilotes sont beaucoup mieux entraînés. [Hors de propos.]

- Non, Rivière est vraiment content de son travail, mais maintenant on peut avoir peur des terroristes. [Mauvaise lecture du texte.]

Code 9 : Omission.

Cet item était assez difficile. Un peu plus de la moitié des élèves ont répondu correctement. Pour cet item, les élèves doivent réfléchir au contexte propre à la rédaction de ce texte et le comparer à leur propre contexte. L'objectif de la question est de favoriser la réflexion. Dès lors, pour autant que les réponses correspondent à une lecture précise du texte et expriment une position plausible par rapport au contexte actuel, elles peuvent être variées et bénéficier tout de même d'un crédit complet, quelle que soit la position adoptée.

\section{QUESTION 3}

Qu'arrive-t-il au personnage principal de ce texte?

A. Il a une surprise désagréable.

B. Il décide de changer de travail.

C. Il attend que quelque chose se passe.

D. Il apprend à écouter les autres.

Caractéristiques conceptuelles

- Situation : Personnelle

- Support : Sur papier

- Format de texte : Continu

- Type de texte : Narration

- Aspect : Intégrer et interpréter : Comprendre le sens global d'un texte

- Objectif de la question : Identifier l'action principale dans un texte narratif

- Format d'item : Choix multiple

\section{Crédit complet}

Code 1 : C. II attend que quelque chose se passe.

\section{Pas de crédit}

Code 0 : Autres réponses.

Code 9 : Omission.

Cet item était facile. Environ trois quarts des élèves ont donné une réponse correcte. Cet item exige des élèves qu'ils 
montrent une vaste compréhension du texte en identifiant son idée maîtresse. Ils doivent établir des liens entre divers éléments du texte et généraliser pour percevoir l'action générale. Cet item est facile car l'idée maîtresse du texte est traitée implicitement et renforcée dans tout le texte.

\section{QUESTION 4}

D'après l'avant-dernier paragraphe (« Bientôt... »), en quoi la nuit et la mer sont-elles semblables?

A. Toutes deux cachent ce qui se trouve en elles.

B. Toutes deux sont bruyantes.

C. Toutes deux ont été domptées par l'homme.

D. Toutes deux sont dangereuses pour l'homme.

E. Toutes deux sont silencieuses.

Caractéristiques conceptuelles

- Situation : Personnelle

- Support : Sur papier

- Format de texte : Continu

- Type de texte : Narration

- Aspect : Intégrer et interpréter : Développer une interprétation

- Objectif de la question : Comprendre l'objet d'une comparaison dans une métaphore

- Format d'item : Choix multiple

\section{Crédit complet}

Code 1 : A. Toutes deux cachent ce qui se trouve en elles.

\section{Pas de crédit}

Code 0 : Autres réponses.

Code 9 : Omission.

Cet item demande aux élèves d'interpréter une métaphore, bien qu'on ait délibérément évité le mot " métaphore » dans l'énoncé. En effet, il est fort probable que des élèves ayant différents parcours éducatifs aient une connaissance variée de ce type de terme métalinguistique et cette connaissance métalinguistique ne fait pas partie de la description des compétences de compréhension de l'écrit prévues par PISA. Toutefois, la capacité d'interpréter un langage figuratif est considérée comme un facteur important de l'interprétation des textes, et plus spécifiquement des textes littéraires. On sait qu'il n'est pas aisé dans le cadre d'une évaluation internationale des compétences de compréhension de l'écrit d'appréhender cette capacité compte tenu des diverses langues et cultures des participants. Dans cet item, le langage figuratif concerne des mots (" mer » et «nuit ») que l'on peut considérer comme universellement familiers et qui possèdent une connotation similaire dans toutes les cultures dans le contexte fourni par le récit. Les résultats de l'essai de terrain indiquent que l'item présentait de solides qualités du point de vue psychométrique et il a donné des performances similaires quel que soit le pays ou la langue. Cet item démontre donc qu'il est possible de réussir à construire un item centré sur les caractéristiques littéraires d'un texte, tel que le langage figuratif, pour une évaluation internationale. Cette question montre aussi que, alors que la plupart des items à choix multiples de PISA offrent quatre réponses possibles, il peut arriver qu'il y en ait davantage. Cet item était assez difficile, avec moins de deux tiers de réponses correctes. 


\section{Notes}

1. Cette section porte sur la lecture de textes imprimés et électroniques, sauf mention contraire.

2. Cela n'exclut pas d'associer à une tâche plusieurs textes, mais chacun des textes doit être cohérent en soi.

3. Fruit d'une technologie remontant aux années 80 , le lien hypertexte permet de relier entre elles des unités d'information dans des documents électroniques volumineux (Conklin, 1987 ; Koved et Shneiderman, 1986 ; Lachman, 1989 ; Weyer, 1982). Le lien hypertexte, ou hyperlien, est un élément d'information (un mot, une phrase, une image ou une icône) en relation logique avec un autre élément $d^{\prime}$ information (habituellement une page). Les liens hypertextes permettent de créer des documents constitués d'un grand nombre de pages, dans une structure en réseau. 


\section{Références}

Binkley, M. et P. Linnakylâ (1997), "Teaching Reading in the United States and Finland », in M. Binkley, K. Rust et T. Williams (éd.), Reading Literacy in an International Perspective, ministère fédéral de l'Éducation, États-Unis, Washington DC.

Bruner, J. (1990), Acts of Meaning, Harvard University Press, Cambridge, Massachusetts.

Conklin, J. (1987), " Hypertext: An Introduction and Survey », Computer, vol. 20, pp. 17-41.

Conseil de l'Europe (1996), Modern Languages: Learning, Teaching, Assessment. A Common European Framework of Reference, CC LANG (95), 5, Rév. IV, Conseil de I'Europe, Strasbourg.

Coulombe, S., J-F. Tremblay et S. Marchand (2004), Literacy Scores, Human Capital, and Growth Across Fourteen OECD Countries, Statistique Canada, Ottawa.

Cunningham, A.E. et K.E. Stanovich (1998), « Early Reading Acquisition and its Relation to Reading Experience and Ability 10 Years Later », Developmental Psychology, vol. 33, pp. 934-945.

Dole, J., G. Duffy, L. Roehler et D. Pearson (1991), "Moving from the Old to the New: Research on Reading Comprehension Instruction », Review of Educational Research, vol. 16(2), pp. 239-264.

Fastrez, P. (2001), "Characteristic(s) of Hypermedia and how they Relate to Knowledge ", Education Media International, vol. 38, pp. 101-110.

Halpern, D.F. (1989), Thought and Knowledge: An Introduction to Critical Thinking, Lawrence Erlbaum Associates, Hillsdale, New Jersey.

Holloway, J.H. (1999), « Improving the Reading Skills of Adolescents », Educational Leadership, vol. 57(2), pp. 80-82.

Hubbard, R. (1989), "Notes from the Underground: Unofficial Literacy in one Sixth Grade », Anthropology and Education Quarterly, vol. 20, pp. 291-307.

Kirsch, I. (2001), The International Adult Literacy Survey: Understanding What Was Measured, Educational Testing Service, Princeton, New Jersey.

Kirsch, I. et P.B. Mosenthal (1990), "Exploring Document Literacy: Variables Underlying the Performance of Young Adults », Reading Research Quarterly, vol. 25(1), pp. 5-30.

Koved, L. et B Shneiderman (1986), « Embedded Menus: Selecting Items in Context », Communications of the ACM, vol. 29(4), pp. 312-318.

Lachman, R. (1989), «Comprehension Aids for Online Reading of Expository Text », Human Factors, vol. 31, pp. 1-15.

Legros, D. et J. Crinon (éd.) (2002), Psychologie des apprentissages et multimédia, Armand Colin, Paris.

Leu, D. (2007), Expanding the Reading Literacy Framework of PISA 2009 to Include Online Reading Comprehension, manuscrit non publié.

OCDE, (2009), Le cadre d'évaluation de PISA 2009 : Les compétences clés en compréhension de l'écrit, en mathématiques et en sciences, PISA, Éditions OCDE.

Pew Internet et American Life Project (2005), Internet: The Mainstreaming of Online Life, Trends 2005, Washington DC.

Reinking, D. (1994), «Electronic Literacy », Perspectives in Reading Research, vol. 4.

Shetzer, H. et M. Warschauer (2000), «An Electronic Literacy Approach to Network-based Language Teaching », in M. Warschauer et R. Kem (éd.), Network-based Language Teaching: Concepts and Practice, Cambridge University Press, New York, pp. 171-185.

Smith, M.C., L. Mikulecky, M.W. Kibby et M.J. Dreher (2000), "What will be the Demands of Literacy in the Workplace in the Next Millennium?», Reading Research Quarterly, vol. 35(3), pp. 378-383.

Sticht, T.G. (éd.) (1975), Reading for Working: A Functional Literacy Anthology, Human Resources Research Organization, Alexandria, Victoria

Stiggins, R.J. (1982), « An Analysis of the Dimensions of Job-related Reading », Reading World, vol. 82, pp. 237-247.

Sweets, R. et A. Meates (2004), ICT and Low Achievers: What does PISA Tell Us?, ministère hongrois de l'Éducation et OCDE, Budapest et Paris.

Warschauer, M. (1999), Electronic Literacies: Language Culture and Power in Online Education, Lawrence Erlbaum Associates, Mahwah, New Jersey.

Werlich, E. (1976), A Text Grammar of English, Quelle und Meyer, Heidelberg.

Weyer, S.A. (1982), « The Design of a Dynamic Book for Information Search », International Journal of Man-Machine Studies, vol. 17, pp. 87-107. 



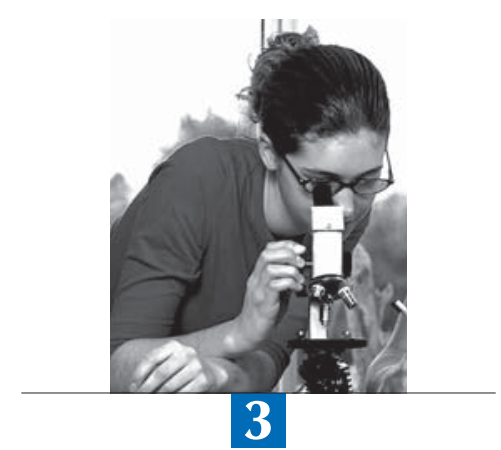

\section{Cadre d'évaluation de la culture scientifique du cycle PISA 2012}

Ce chapitre présente les fondements théoriques du cadre d'évaluation de la culture scientifique dans le cadre de l'enquête PISA 2012. II commence par définir la notion de culture scientifique, puis analyse l'organisation de ce domaine d'évaluation dans l'enquête PISA, avant de décrire le contexte des items. Le présent chapitre décrit, en outre, les connaissances et compétences au cœur de l'évaluation : identifier des questions d'ordre scientifique, expliquer des phénomènes de manière scientifique et utiliser des faits scientifiques. II étudie ensuite la façon dont les connaissances et les attitudes sont également intégrées dans la définition PISA de la culture scientifique. Enfin, il présente tout au long de l'analyse des exemples d'items afin d'illustrer la classification, le format et la structure de l'évaluation PISA de la culture scientifique. 


\section{INTRODUCTION}

Ce cadre décrit et illustre la définition de la culture scientifique retenue dans I'enquête PISA et définit le contexte des items. Les sciences sont un domaine mineur d'évaluation du cycle PISA 2012. La définition du domaine est inchangée depuis celle du cycle PISA 2006, dont les sciences étaient pour la première fois le domaine majeur d'évaluation (OCDE, 2006 ; Bybee et McCrae, 2009), en dehors de quelques modifications terminologiques introduites dans le souci d'aligner la terminologie de l'enquête PISA sur celle employée dans l'initiative DeSeCo de l'OCDE (OCDE, 2003).

Dans ce cadre d'évaluation, l'expression « culture scientifique » renvoie à une compétence globale, constituée de trois compétences scientifiques spécifiques. La notion de compétence $n$ 'implique pas uniquement des savoirs et savoir-faire (OCDE, 2003), elle inclut aussi la capacité de mobiliser des ressources cognitives et non cognitives dans tout contexte donné. Dans la description des dimensions cognitives des compétences scientifiques spécifiques, correspondant à l'évaluation PISA de sciences du cycle actuel, il est fait référence aux connaissances et compétences scientifiques des élèves, mais les sous-échelles créées en sciences lors du cycle PISA 2006 (OCDE, 2006) restent dites de compétences scientifiques.

Comme les sciences sont un domaine mineur d'évaluation dans ce cycle, le questionnaire « Élève » ne comportera pas d'items sur les attitudes générales des élèves à l'égard de la science; de la même façon, les épreuves n'incluront pas de questions sur les attitudes en plus des items cognitifs, comme ce fut le cas lors du cycle PISA 2006. Dans cette version du cadre d'évaluation de sciences révisée en vue du cycle PISA 2012, de la même manière que pour le cycle PISA 2009, la section «Évaluation PISA de la culture scientifique » a été modifiée pour intégrer ces changements, la description des échelles de compétence a été actualisée et des items du cycle PISA 2006 qui ont été rendus publics sont inclus à titre d'exemple.

Une bonne compréhension des sciences et des technologies est essentielle pour un jeune qui se prépare à vivre dans une société moderne. C'est ce qui permet aux individus de participer pleinement à la vie d'une société où les sciences et les technologies tiennent une grande place. Cette compréhension leur permet aussi de prendre une part active dans les débats sur l'action publique, lorsque des thématiques en rapport avec les sciences et les technologies ont un impact dans leur vie. Comprendre les sciences et les technologies contribue significativement à la vie personnelle, sociale, professionnelle et culturelle de tous.

Dans la vie de tous les jours, très nombreuses sont les occasions où les individus sont confrontés à des situations, à des problèmes ou à des débats qu'ils ne peuvent véritablement comprendre ou résoudre sans une certaine maîtrise des sciences ou des technologies. Des questions d'ordre scientifique ou technologique se présentent chaque jour à eux, dans leur vie personnelle, dans celle de leur communauté et de leur pays, et même à l'échelle mondiale. Dans ce contexte, les dirigeants nationaux devraient être amenés à se demander dans quelle mesure les citoyens de leur pays sont préparés à y faire face. La question de savoir comment les jeunes réagissent face à des questions scientifiques au sortir de l'école est essentielle à cet égard. Sonder les jeunes de 15 ans peut fournir, de manière précoce, des indications sur la façon dont ils se comporteront plus tard dans les diverses situations qui impliquent les sciences et les technologies.

Il semble donc judicieux de fonder une évaluation internationale des compétences des jeunes de 15 ans sur la question " quels sont les savoir-faire, les connaissances et les valeurs qu'un citoyen doit posséder pour faire face à des situations impliquant les sciences et les technologies ? ». Répondre à cette question revient à déterminer sur quelles bases il convient d'évaluer les connaissances, les valeurs et les compétences des élèves par rapport à ce dont ils auront besoin à l'avenir. Au cœur de cette réponse se trouvent les compétences sur lesquelles repose la définition de la culture scientifique de l'enquête PISA. II s'agit de déterminer dans quelle mesure les élèves sont capables de mettre en œuvre les compétences suivantes :

- identifier des questions d'ordre scientifique ;

- expliquer des phénomènes de manière scientifique ; et

- utiliser des faits scientifiques.

Pour pouvoir mettre ces compétences en œuvre lorsqu'ils abordent des questions scientifiques, les élèves doivent, d'une part, posséder des savoirs et des savoir-faire d'ordre cognitif et, d'autre part, avoir certaines valeurs, motivations et attitudes.

L'idée de cerner les savoirs, les valeurs et les savoir-faire des citoyens dans des situations impliquant les sciences et les technologies peut paraître simple et directe. S'atteler à cette tâche conduit à envisager le vaste champ de la compréhension des concepts scientifiques, ce qui n'implique pas la maîtrise de toutes les connaissances scientifiques. 
Ce cadre conceptuel s'articule autour de ce dont les citoyens ont besoin. Quels sont les savoirs les plus pertinents pour les individus en tant que citoyens? La réponse à cette question fait bien sûr intervenir la compréhension de concepts fondamentaux dans diverses disciplines scientifiques, sans oublier toutefois que ces savoirs doivent pouvoir être utilisés dans des situations que les individus sont amenés à rencontrer au cours de leur vie. Par ailleurs, il arrive souvent que les situations auxquelles les individus sont confrontés nécessitent une certaine compréhension de la science en tant que discipline, c'est-à-dire en tant que processus destiné à produire des connaissances et à proposer des explications relatives au monde naturel ${ }^{1}$. Enfin, les individus doivent être conscients de la complémentarité entre sciences et technologies, et de l'influence omniprésente que les technologies fondées sur la science exercent sur la nature de la vie contemporaine.

Quelles valeurs importantes les citoyens doivent-ils conférer à la science et à la technologie ? Le rôle important que jouent dans la société la science et les applications technologiques de la science, la contribution qu'elles lui apportent et la place considérable qu'elles tiennent dans les contextes personnel, social et global des individus sont autant d'éléments de réponse. Il semble raisonnable d'attendre des citoyens qu'ils s'intéressent à la science, qu'ils valorisent la démarche scientifique, et qu'ils agissent de manière responsable à l'égard de l'environnement et des ressources naturelles.

Quels sont les savoir-faire scientifiques qu'il est important pour les individus de pouvoir mettre en ouvre ? II arrive souvent que les individus aient à tirer des conclusions probantes des faits et des informations qui leur sont communiqués, à évaluer des conclusions tirées par d'autres sur la base des éléments donnés à l'appui, et à faire la distinction entre des opinions personnelles et des affirmations étayées par des faits. Les faits sont souvent d'ordre scientifique, certes, mais la science a un rôle plus général à jouer, dans la mesure où son propos est d'établir un principe de rationalité dans des démarches permettant de mettre des idées et des théories à l'épreuve des faits. Cela n'exclut pas pour autant la créativité et l'imagination, qui ont toujours joué un rôle essentiel dans les progrès accomplis par l'homme pour mieux comprendre le monde.

Les citoyens peuvent-ils faire la distinction entre des arguments scientifiques et des arguments dénués de fondements scientifiques? L’homme de la rue n'est généralement pas amené à se prononcer sur la valeur de grandes théories ou d'avancées scientifiques potentielles, mais il a assurément à prendre des décisions sur la base de faits, qu'il s'agisse d'arguments publicitaires, de preuves juridiques ou d'informations relatives à sa santé ou à des problèmes liés à l'environnement et aux ressources naturelles. Une personne instruite doit être capable de faire la distinction entre les questions auxquelles les chercheurs peuvent ou ne peuvent pas répondre, et les problèmes auxquels les technologies fondées sur la science peuvent ou ne peuvent pas apporter de solution.

\section{DÉFINITION DE LA CULTURE SCIENTIFIQUE}

La pensée moderne à propos des résultats souhaitables de l'enseignement des sciences met l'accent sur les savoirs scientifiques (y compris la connaissance de la démarche scientifique) et sur l'appréciation de la contribution des sciences à la société. Cela demande une compréhension globale non seulement des grands concepts scientifiques et des explications données par la science, mais aussi du potentiel et des limites de la science dans le monde - ce qui implique l'adoption d'une approche réfléchie et critique à l'égard de la science (Millar et Osborne, 1998).

Ces objectifs déterminent l'orientation de l'enseignement des sciences pour l'ensemble des individus (Fensham, 1985). L'enquête PISA se doit d'évaluer un vaste ensemble de compétences, compte tenu de divers aspects : utilité personnelle, responsabilité sociale, et valeur intrinsèque et extrinsèque des connaissances scientifiques.

Ce qui précède définit une caractéristique centrale de l'évaluation PISA de la culture scientifique : cette évaluation doit porter sur des compétences scientifiques qui témoignent des savoirs, valeurs et savoir-faire que les jeunes de 15 ans sont capables d'appliquer dans des contextes raisonnables et appropriés, tirés de la sphère personnelle, sociale et globale. Cette perspective se démarque d'une approche fondée uniquement sur les programmes scolaires et les différentes disciplines scientifiques, car elle inclut des problèmes situés dans des contextes éducatifs et professionnels, et tient compte du rôle essentiel des connaissances, des méthodes, des attitudes et des valeurs qui définissent ces disciplines (Bybee, 1997a ; Fensham, 2000 ; Gräber et Bolte, 1997 ; Mayer, 2002 ; Roberts, 1983 ; UNESCO, 1993).

L'enquête PISA vise à évaluer à la fois des aspects cognitifs et des aspects affectifs de la culture scientifique des élèves. Par aspects cognitifs, on entend les connaissances scientifiques des élèves et leur capacité à les utiliser efficacement lorsqu'ils mettent en œuvre des processus cognitifs qui sont spécifiques à la science et aux investigations scientifiques dans des situations personnelles, sociales ou globales. L'enquête PISA cible des problèmes que les connaissances scientifiques peuvent contribuer à résoudre et qui amèneront un jour ou l'autre les élèves à prendre des décisions. C'est en se fondant sur leur culture scientifique que les élèves réagissent à ce type de problèmes : ils doivent comprendre les 
notions scientifiques qui y interviennent, être capables de trouver les informations pertinentes, de les évaluer et de les interpréter, et pouvoir identifier les aspects scientifiques et technologiques des situations qui leur sont soumises (Koballa et al., 1997 ; Law, 2002). Outre ces aspects cognitifs, I'enquête PISA s'intéresse aussi à des aspects non cognitifs, c'està-dire à la façon affective dont les élèves réagissent face à un problème. Les réponses des élèves au problème posé montrent aussi l'intérêt qu'ils y portent, les valeurs qu'ils défendent et leur motivation à agir (Schibeci, 1984).

\section{Encadré 3.1. Connaissances scientifiques: La définition de l'enquête PISA}

L'expression " connaissances scientifiques » est utilisée tout au long de ce cadre d'évaluation pour désigner d'une manière générique à la fois les connaissances en sciences et les connaissances à propos de la science. Les connaissances en sciences font référence à la connaissance du monde naturel tel qu'il se définit à travers les grandes disciplines scientifiques (physique, chimie, biologie, sciences de la Terre et de l'univers, et applications technologiques de la science). Les connaissances à propos de la science font référence à la connaissance des moyens utilisés par la science (démarche scientifique) et à ses objectifs (explications scientifiques).

L'évaluation PISA de la culture scientifique porte sur un continuum de connaissances scientifiques et sur les capacités cognitives associées à la démarche scientifique, intègre de multiples dimensions et traite des relations entre la science et la technologie. Il s'agit donc d'évaluer la culture scientifique des élèves sous l'angle de leur faculté à utiliser leurs connaissances scientifiques (Bybee, 1997b ; Fensham, 2000 ; Law, 2002 ; Mayer et Kumano, 2002).

\section{Encadré 3.2. Culture scientifique : La définition de l'enquête PISA}

Dans I'enquête PISA, la culture scientifique d'un individu est définie comme suit :

- les connaissances scientifiques de l'individu et sa capacité d'utiliser ces connaissances pour identifier les questions auxquelles la science peut apporter une réponse, pour acquérir de nouvelles connaissances, pour expliquer des phénomènes scientifiques et pour tirer des conclusions fondées sur des faits à propos de questions à caractère scientifique ;

- la compréhension des traits caractéristiques de la science en tant que forme de recherche et de connaissances humaines ;

- la conscience du rôle de la science et de la technologie dans la constitution de notre environnement matériel, intellectuel et culturel; et

- la volonté de s'engager en qualité de citoyen réfléchi à propos de problèmes à caractère scientifique et touchant à des notions relatives à la science.

\section{Explication de la définition}

Les considérations suivantes clarifient la définition de la culture scientifique adoptée pour les besoins de l'enquête PISA.

L'expression «culture scientifique » a été préférée au terme "sciences » pour souligner que l'évaluation PISA accorde plus d'importance à l'application des connaissances scientifiques dans des situations tirées de la vie courante qu'à la simple restitution des acquis scolaires traditionnels. L'utilisation fonctionnelle de connaissances scientifiques exige de l'individu l'application de processus qui sont spécifiques à la science et à la recherche scientifique (les compétences scientifiques), et dépend de l'importance, de l'intérêt et de la valeur qu'il accorde aux questions scientifiques et de sa motivation à agir en la matière. La capacité des élèves à utiliser ces compétences scientifiques est déterminée non seulement par leurs connaissances en sciences, mais aussi par leur compréhension de la science en tant que moyen d'acquérir des connaissances (c'est-à-dire leurs connaissances à propos de la science). La définition de la culture scientifique proposée prend aussi en compte le fait que la capacité des individus à mener à bien ces processus dépend de leurs attitudes à l'égard de la science et de leur volonté de s'engager dans des thématiques à caractère scientifique. 


\section{Connaissances scientifiques de l'individu et sa capacité d'utiliser ces connaissances pour identifier les questions auxquelles la science peut apporter une réponse, pour acquérir de nouvelles connaissances, pour expliquer des phénomènes scientifiques et pour tirer des conclusions fondées sur des faits}

Dans cette définition de la culture scientifique, le terme « connaissances » désigne bien plus que la capacité de restituer des informations, des faits ou des noms. II inclut les connaissances en sciences (connaissance du monde naturel) et les connaissances à propos de la science. Les premières renvoient à la compréhension de théories et de concepts scientifiques fondamentaux, et les secondes, à la compréhension de la nature de la science en tant qu'activité humaine, ainsi que du potentiel et des limites des connaissances scientifiques. Les questions qui doivent être identifiées sont celles auxquelles il est possible de répondre par la recherche scientifique, ce qui implique à nouveau des connaissances à propos de la science tout autant que des connaissances en sciences, c'est-à-dire la maîtrise des concepts abordés. II est important de souligner que les individus doivent souvent acquérir de nouvelles connaissances, non pas en se livrant à leurs propres recherches scientifiques, mais en puisant dans des sources d'information (bibliothèques et sites Internet, par exemple). «Tirer des conclusions fondées sur des faits » signifie que l'individu doit connaître, sélectionner et évaluer des informations ou des données, tout en étant conscient que les informations disponibles sont souvent insuffisantes pour tirer des conclusions définitives, ce qui lui impose de faire des conjectures de manière prudente et réfléchie à partir des informations dont il dispose.

\section{Traits caractéristiques de la science en tant que forme de recherche et de connaissance humaines}

Tel que défini ici, être scientifiquement cultivé implique que les élèves puissent comprendre dans une certaine mesure comment les chercheurs s'y prennent pour obtenir des données et proposer des explications, et qu'ils reconnaissent les caractéristiques principales de la démarche scientifique ainsi que les types de réponse qu'il est raisonnable d'attendre de la science. Ainsi, les scientifiques se basent sur des observations et des expériences pour recueillir des données à propos d'objets, d'organismes et de phénomènes du monde naturel. Ils se servent ensuite de ces données pour proposer des explications qui viennent enrichir le savoir et qui peuvent être exploitées dans diverses formes de l'activité humaine. La collecte des données - qui est inspirée par des idées et des concepts (parfois énoncés sous la forme d'hypothèses), et qui doit se justifier en termes de pertinence, d'adéquation contextuelle et d'exactitude - et leur utilisation, le caractère provisoire des conclusions avancées, l'ouverture à l'analyse critique, la formulation d'arguments logiques, la nécessité d'établir des liens entre les connaissances actuelles et celles léguées par l'histoire, et l'obligation de rendre compte des méthodes et procédures appliquées pour recueillir des éléments de preuve, sont autant de caractéristiques fondamentales de la science.

\section{Rôle de la science et de la technologie dans la constitution de notre environnement matériel, intellectuel et culturel}

L'idée maîtresse de ce point est que la science est une entreprise humaine, qui influence notre société et notre vie personnelle. Il en va de même pour le progrès technologique (Fleming, 1989). Malgré leurs différences d'objectifs, de processus et de produits, la science et la technologie sont étroitement associées et sont complémentaires à maints égards. La définition de la culture scientifique proposée ici intègre donc la nature des sciences et des technologies, et leur complémentarité. En tant qu'individus, nous prenons des décisions qui influencent l'orientation de la science et de la technologie au travers de l'action publique. La science et la technologie jouent un rôle paradoxal dans la société : elles permettent de répondre à des questions et de résoudre des problèmes, mais peuvent également être à l'origine de nouvelles questions et de nouveaux problèmes.

\section{Volonté de s'engager en qualité de citoyen réfléchi à propos de problèmes à caractère scientifique et touchant à des notions relatives à la science}

La « volonté de s'engager à propos de problèmes à caractère scientifique » va plus loin que le fait de prendre acte de la situation et d'agir de la manière requise. Elle implique aussi que l'individu porte un intérêt permanent aux questions scientifiques actuelles et à venir, qu'il se forge une opinion à leur sujet et qu'il s'y engage. La mention « en qualité de citoyen réfléchi » renvoie aux diverses attitudes que les individus adoptent à l'égard de la science et aux valeurs qu'ils lui confèrent. L'énoncé complet esquisse le profil d'une personne qui s'intéresse à des thèmes scientifiques, qui réfléchit à des questions d'ordre scientifique, qui se préoccupe des problèmes liés aux technologies, aux ressources naturelles et à l'environnement, et qui prend conscience de l'importance de la science dans une perspective à la fois personnelle et sociale. 
Pour posséder des compétences scientifiques, il faut nécessairement des compétences en compréhension de l'écrit et en mathématiques (Norris et Phillips, 2003). Par exemple, l'élève doit faire appel à sa culture mathématique dans des situations où il faut interpréter des données et à ses compétences en compréhension de l'écrit pour appréhender la terminologie scientifique. Ces chevauchements entre l'évaluation de la culture scientifique et celle des autres domaines PISA ne peuvent être évités. Toutefois, il faut faire en sorte qu'au cœur de chaque tâche d'évaluation, il y ait des aspects relevant sans ambiguïté des compétences scientifiques.

\section{ORGANISATION DU DOMAINE}

La définition du domaine de la culture scientifique proposée ici fait référence à un continuum où les individus sont, à des degrés divers, scientifiquement cultivés ; il ne s'agit pas de distinguer les individus scientifiquement cultivés, et ceux qui ne le sont pas (Bybee, 1997a ; 1997b). Par exemple, un élève dont la culture scientifique est peu développée sera à même de restituer des connaissances scientifiques factuelles simples et d'utiliser des connaissances scientifiques courantes pour tirer ou évaluer des conclusions. Un élève dont la culture scientifique est plus développée se montrera capable de créer ou d'utiliser des modèles conceptuels pour faire des prévisions ou fournir des explications, d'analyser des recherches scientifiques, de proposer des données à titre de preuves, d'évaluer des explications différentes du même phénomène et de communiquer ses conclusions avec précision.

La définition de la culture scientifique retenue pour les besoins de l'évaluation PISA couvre quatre aspects interdépendants (voir la figure 3.1) :

- le contexte : reconnaître des situations de la vie courante en rapport avec la science et la technologie ;

- les connaissances : comprendre le monde naturel sur la base d'un savoir scientifique qui inclut à la fois des connaissances sur des concepts relatifs au monde naturel et des connaissances à propos de la science elle-même ;

- les compétences : faire montre de compétences scientifiques, telles qu'identifier des questions scientifiques, expliquer des phénomènes de manière scientifique et utiliser des faits scientifiques ; et

- les attitudes : porter de l'intérêt à la science, accorder de l'importance à la démarche scientifique, être motivé à l'idée d'agir de manière responsable à l'égard, par exemple, de l'environnement et des ressources naturelles.

Les sections suivantes reviennent sur ces aspects interdépendants de façon plus détaillée. En mettant ces aspects en évidence, le cadre PISA de la culture scientifique fait porter l'évaluation sur les résultats de l'enseignement des sciences. Ce cadre conceptuel d'évaluation a été conçu autour des questions suivantes :

- Quels sont les contextes à considérer comme appropriés pour l'évaluation d'élèves de 15 ans ?

- Quelles sont les compétences que l'on peut raisonnablement attendre des élèves de 15 ans?

- Quelles sont les connaissances que l'on peut raisonnablement attendre des élèves de 15 ans ?

- Quelles sont les attitudes que I'on peut raisonnablement attendre des élèves de 15 ans ?

- Figure 3.1

Cadre PISA d'évaluation de la culture scientifique

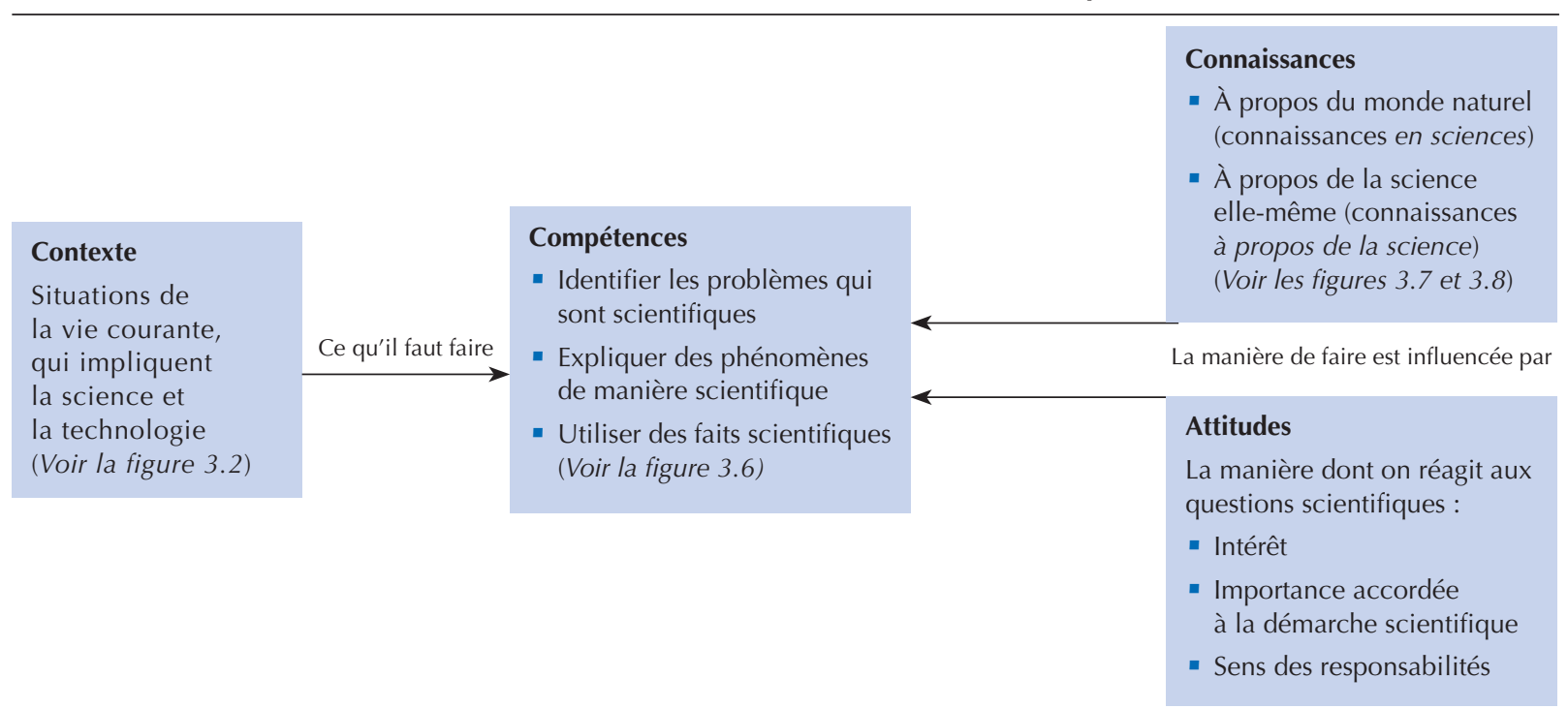




\section{Situations et contextes}

L'engagement à l'égard des sciences dans diverses situations est un aspect important de l'évaluation de la culture scientifique. Le choix des méthodes et des représentations dépend souvent des situations dans lesquelles les questions sont présentées.

La situation est la partie du monde de l'élève dans laquelle les tâches s'inscrivent. Les items se situent dans des situations de la vie en général et ne se limitent pas à la vie à l'école. Les épreuves PISA de sciences privilégient les items s'inscrivant dans des situations qui concernent l'individu, sa famille et ses semblables (situation personnelle), la collectivité (situation sociale) et la vie dans le monde en général (situation globale). Il convient d'ajouter, pour certains sujets, la situation historique qui permet d'évaluer la compréhension des progrès de la science.

L'enquête PISA évalue des connaissances scientifiques importantes en rapport avec le contenu des cours de sciences des pays participants, sans toutefois s'imposer la contrainte de ne cibler que les contenus formant le dénominateur commun de ces programmes nationaux. Les épreuves demandent aux élèves de montrer qu'ils sont capables d'appliquer des connaissances et compétences scientifiques dans des situations qui reflètent le monde réel, en accord avec les priorités PISA, ce qui implique l'exploitation de connaissances choisies sur le monde naturel et sur la science ellemême, ainsi que l'évaluation des attitudes des élèves à l'égard de thématiques scientifiques.

La figure 3.2 dresse la liste des principaux thèmes scientifiques repris dans les contextes personnels, sociaux et globaux des items d'évaluation. D'autres contextes (par exemple, historiques ou technologiques) et d'autres champs d'application sont également prévus. Ils couvrent les thèmes suivants : "Santé », "Ressources naturelles », "Environnement ", «Risques» et, enfin, "Frontières de la science et de la technologie ». Ce sont des domaines dans lesquels la science présente un intérêt particulier pour les individus et les communautés, car elle contribue au maintien et à l'amélioration de la qualité de vie, et à l'élaboration des politiques publiques.

L'évaluation PISA de sciences ne porte pas sur les contextes, mais sur des connaissances, compétences et attitudes qui sont présentées dans ces contextes ou qui s'y rapportent. Il importe donc de choisir les contextes sachant que le but de l'enquête est d'évaluer des connaissances, des compétences et des attitudes que les élèves ont acquises au terme de leur scolarité obligatoire.

Les items PISA sont regroupés en unités, basées sur un stimulus commun qui décrit leur contexte. Les contextes des items ont été choisis pour leur pertinence par rapport aux centres d'intérêt et à la vie des élèves. Les items ont été élaborés compte tenu des différences linguistiques et culturelles entre les pays participants.

- Figure 3.2 .

\section{Contextes des items PISA de sciences}

\begin{tabular}{|c|c|c|c|}
\hline & \begin{tabular}{|c} 
Personnel \\
(l'individu, sa famille et ses semblables)
\end{tabular} & $\begin{array}{c}\text { Social } \\
\text { (la collectivité) }\end{array}$ & $\begin{array}{c}\text { Global } \\
\text { (la vie dans le monde) }\end{array}$ \\
\hline Santé & $\begin{array}{l}\text { Préservation de la santé, accidents } \\
\text { et nutrition }\end{array}$ & $\begin{array}{l}\text { Prévention des maladies, transmission } \\
\text { des maladies, choix alimentaires, } \\
\text { santé publique }\end{array}$ & $\begin{array}{l}\text { Épidémies et propagation } \\
\text { de maladies infectieuses }\end{array}$ \\
\hline Ressources naturelles & $\begin{array}{l}\text { Consommation personnelle } \\
\text { de matériaux et d'énergie }\end{array}$ & $\begin{array}{l}\text { Survie des populations humaines, } \\
\text { qualité de vie, sécurité, production } \\
\text { et distribution d'aliments, } \\
\text { approvisionnement en énergie }\end{array}$ & $\begin{array}{l}\text { Énergies renouvelables et non } \\
\text { renouvelables, systèmes naturels, } \\
\text { croissance démographique, } \\
\text { exploitation durable des espèces }\end{array}$ \\
\hline Environnement & $\begin{array}{l}\text { Comportement respectueux } \\
\text { de l'environnement, utilisation des } \\
\text { matériaux, élimination des déchets }\end{array}$ & $\begin{array}{l}\text { Démographie, gestion des déchets, } \\
\text { impact sur l'environnement, } \\
\text { météorologie locale }\end{array}$ & $\begin{array}{l}\text { Biodiversité, durabilité } \\
\text { environnementale, contrôle } \\
\text { de la pollution, épuisement } \\
\text { et régénération des sols }\end{array}$ \\
\hline Risques & $\begin{array}{l}\text { Risques naturels et dus à l'homme, } \\
\text { choix en matière de logement }\end{array}$ & $\begin{array}{l}\text { Changements brutaux (séismes, } \\
\text { conditions météorologiques extrêmes), } \\
\text { changements lents et progressifs } \\
\text { (érosion côtière et sédimentation), } \\
\text { évaluation des risques }\end{array}$ & $\begin{array}{l}\text { Changement climatique, } \\
\text { impact des guerres modernes }\end{array}$ \\
\hline $\begin{array}{l}\text { Frontières de la science } \\
\text { et de la technologie }\end{array}$ & $\begin{array}{l}\text { Intérêt pour les explications } \\
\text { scientifiques des phénomènes naturels, } \\
\text { hobbies à caractère scientifique, sports } \\
\text { et loisirs en rapport avec la science, } \\
\text { musique et technologies utilisées } \\
\text { à titre individuel }\end{array}$ & $\begin{array}{l}\text { Matériaux, appareils et procédés } \\
\text { nouveaux, modification génétique, } \\
\text { technologie de l'armement, transports }\end{array}$ & $\begin{array}{l}\text { Extinction d'espèces, exploration } \\
\text { spatiale, origine et structure } \\
\text { de l'univers }\end{array}$ \\
\hline
\end{tabular}




\section{Exemples d'items PISA de sciences}

Cette section propose trois exemples parmi les unités de sciences utilisées lors du cycle PISA 2006. Ces exemples sont cités tout au long de ce chapitre pour illustrer la diversité des contextes, des compétences scientifiques, des domaines scientifiques et des formats des items PISA. De plus, les items sont accompagnés de leurs consignes de correction (voir la description des niveaux de compétence à la figure 3.10).

\section{PLUIES ACIDES}

Dans cet exemple, le stimulus est constitué d'une photo de statues de l'Acropole d'Athènes, accompagnée d'un petit texte qui explique que les statues originales ont été placées à l'intérieur du musée de l'Acropole, car elles étaient rongées par les pluies acides. Cette unité relève du domaine des "Risques » et s'inscrit dans des contextes personnel et social.

- Figure 3.3

Items de l'unité PLUIE ACIDE

La photo ci-dessous montre des statues appelées cariatides, qui ont été érigées sur l'Acropole d'Athènes il y a plus de 2500 ans. Les statues sont sculptées dans du marbre (un type de roche). Le marbre est composé de carbonate de calcium.

En 1980, les statues originales, qui étaient rongées par les pluies acides, ont été transportées à l'intérieur du musée de l'Acropole et remplacées par des copies.

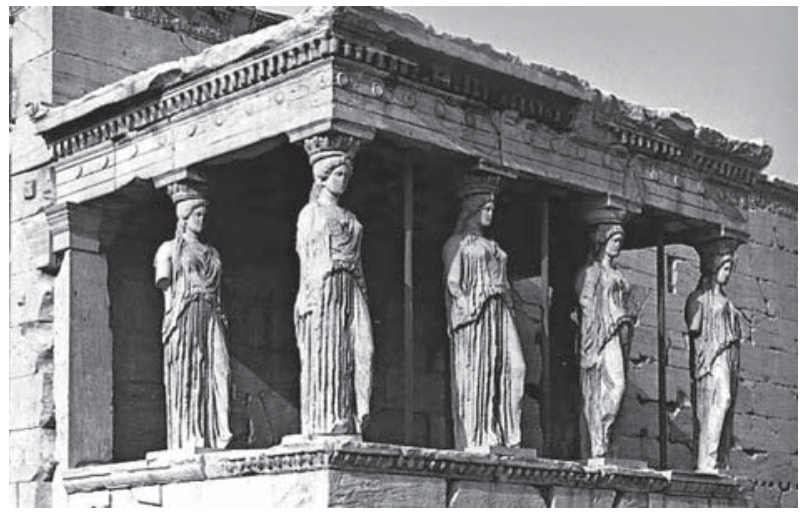

\section{QUESTION 1}

Les pluies ordinaires sont légèrement acides parce qu'elles ont absorbé du dioxyde de carbone présent dans l'air. Les pluies acides sont plus acides que les pluies ordinaires parce qu'elles ont absorbé, en plus, d’autres gaz, comme les oxydes de soufre et les oxydes d'azote.

D'où proviennent ces oxydes de soufre et oxydes d'azote présents dans l'air?

\section{Crédit complet (Niveau $3: 506$ points)}

La réponse mentionne n'importe laquelle des sources suivantes : les gaz d'échappement des voitures, les émissions de gaz des usines, la combustion de pétrole, de charbon et autres combustibles fissibles, les gaz émis par les volcans et autres sources analogues.

\section{Crédit partiel}

La réponse inclut une source de pollution correcte ainsi qu'une autre source incorrecte.

OU La réponse fait référence à la "pollution », mais ne mentionne pas une source de pollution qui est une cause significative des pluies acides.

On peut simuler l'effet des pluies acides sur le marbre en plaçant des éclats de marbre dans du vinaigre pendant une nuit. Le vinaigre et les pluies acides ont à peu près le même niveau d'acidité. Lorsqu'on place un éclat de marbre dans du vinaigre, des bulles de gaz se forment. On peut déterminer la masse de l'éclat de marbre sec, avant et après I'expérience. 


\section{QUESTION 2}

Un éclat de marbre a une masse de $2.0 \mathrm{grammes} a v a n t$ d'être plongé dans du vinaigre pendant une nuit. Le lendemain, on retire et on sèche l'éclat. Quelle sera la masse de l'éclat de marbre séché?

A. Moins de 2.0 grammes

B. Exactement $2.0 \mathrm{grammes}$

C. Entre 2.0 et $2.4 \mathrm{grammes}$

D. Plus de 2.4 grammes

Crédit complet (Niveau $2: 460$ points)

A. Moins de $2.0 \mathrm{grammes}$

QUESTION 3

Les élèves qui ont réalisé cette expérience ont également placé des éclats de marbre dans de l'eau pure (distillée) pendant une nuit.

Expliquez pourquoi les élèves ont inclus cette étape dans leur expérience.

\section{Crédit complet (Niveau $6: 717$ points)}

Pour pouvoir comparer avec le test du vinaigre et du marbre, et montrer que l'acide (le vinaigre) est nécessaire pour produire la réaction.

\section{Crédit partiel (Niveau $3: 513$ points)}

Pour pouvoir comparer avec le test du vinaigre et du marbre, mais la réponse n'établit pas clairement que cela permet de montrer que l'acide (le vinaigre) est nécessaire pour produire la réaction.

\section{L'EFFET DE SERRE}

Cette unité porte sur l'élévation de la température moyenne de l'atmosphère de la Terre. Le stimulus est constitué d'un texte bref qui explique l'expression « effet de serre » et de graphiques montrant l'évolution de la température moyenne de l'atmosphère et des émissions de dioxyde de carbone au fil du temps.

Cette unité s'inscrit dans le champ d'application « Environnement » et dans un contexte global.

- Figure 3.4 .

\section{Items de l'unité EFFET DE SERRE}

Lisez les textes suivants et répondez aux questions qui les accompagnent.

\section{L'EFFET DE SERRE : RÉALITÉ OU FICTION ?}

Les êtres vivants ont besoin d'énergie pour survivre. L'énergie qui alimente la vie sur Terre provient du Soleil, qui dégage de l'énergie dans l'espace, tant il est brûlant. Une infime proportion de cette énergie atteint la Terre.

L'atmosphère terrestre agit comme une couche de protection autour de la surface de la planète, empêchant les variations de température qui existeraient dans un monde sans air.

La plus grande partie de l'énergie venant du Soleil traverse l'atmosphère terrestre. La Terre absorbe une partie de cette énergie, et une autre partie est réfléchie et renvoyée par la surface de la Terre. Une partie de cette énergie réfléchie par la Terre est absorbée par l'atmosphère.

En conséquence, la température moyenne au-dessus de la surface terrestre est plus élevée qu'elle ne le serait s'il n'y avait pas d'atmosphère. L'atmosphère terrestre a le même effet qu'une serre, d'où l'expression " effet de serre".

L'effet de serre se serait intensifié au cours du XXe siècle.

C'est un fait que la température moyenne de l'atmosphère de la Terre a augmenté. Les journaux et les magazines attribuent souvent à l'augmentation des émissions de dioxyde de carbone la principale responsabilité du réchauffement intervenu au $X X^{e}$ siècle.

André, un étudiant, s'intéresse au rapport possible entre la température moyenne de l'atmosphère terrestre et l'émission de dioxyde de carbone sur Terre. 
Dans une bibliothèque, il découvre les deux graphiques suivants.

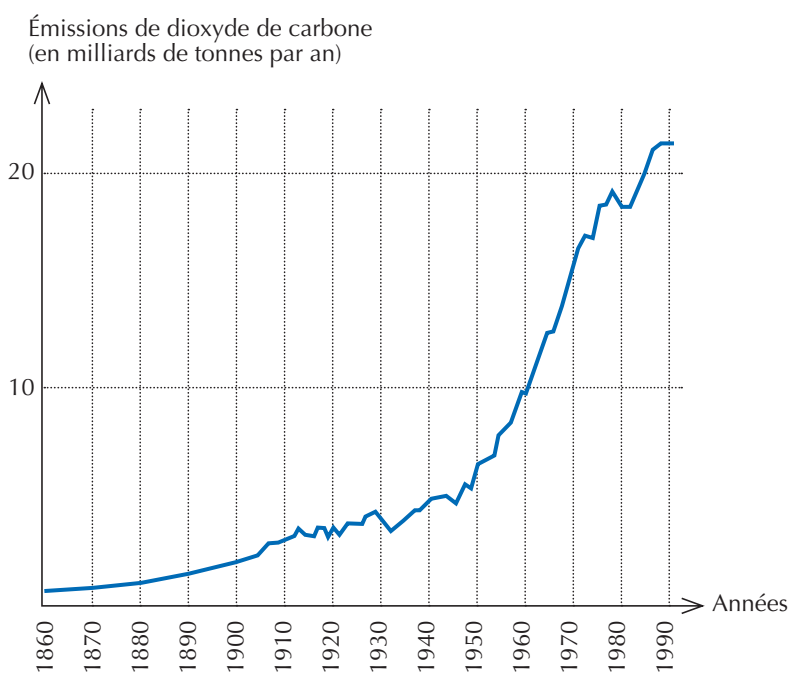

Température moyenne

de l'atmosphère terrestre $\left({ }^{\circ} \mathrm{C}\right)$

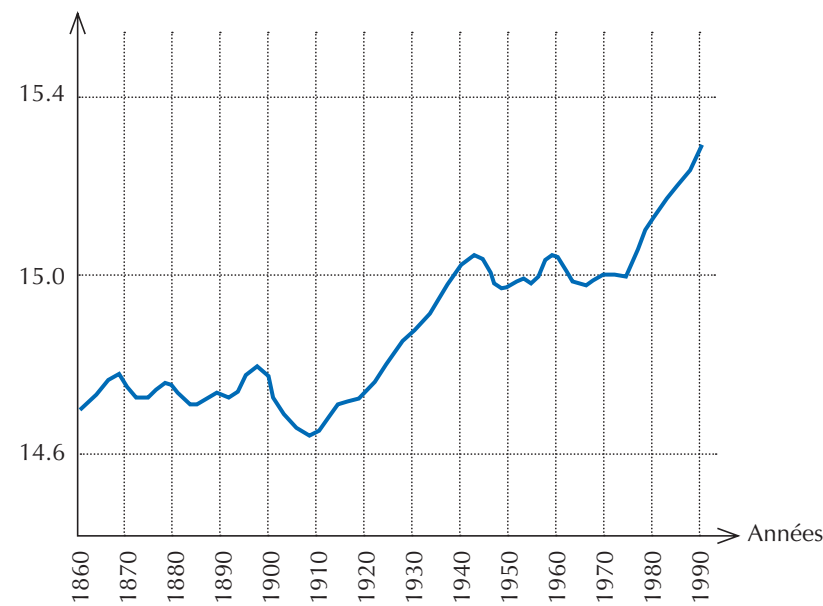

André conclut, à partir de ces deux graphiques, qu'il est certain que la hausse de la température moyenne de l'atmosphère de la Terre est due à l'augmentation des émissions de dioxyde de carbone.

\section{QUESTION 1}

Qu'est-ce qui, dans ces graphiques, confirme la conclusion d'André?

\section{Crédit complet (Niveau $3: 529$ points)}

Fait référence au fait que tant la température (moyenne) que les émissions de dioxyde de carbone ont augmenté.

OU Fait référence (en termes généraux) à un rapport positif entre la température et les émissions de dioxyde de carbone.

\section{QUESTION 2}

Jeanne, une autre élève, n'est pas d'accord avec la conclusion d'André. Elle compare les deux graphiques et dit que certaines parties de ceux-ci ne confirment pas sa conclusion.

Donnez un exemple, en citant une partie de ces graphiques qui ne confirme pas la conclusion d'André. Expliquez votre réponse. 


\section{Crédit complet (Niveau $5: 659$ points)}

Fait référence à une partie spécifique du graphique dans laquelle les courbes ne sont pas toutes deux ascendantes ou descendantes, et fournit une explication en rapport avec le phénomène constaté.

\section{Crédit partiel (Niveau $4: 568$ points)}

Cite une période correcte sans fournir d'explication.

OU Ne mentionne qu'une année particulière (pas une période), avec une justification acceptable.

OU Donne un exemple qui ne confirme pas la conclusion d'André, mais fait une erreur en citant la période.

OU Fait référence à la différence entre les deux courbes, sans mentionner de période spécifique.

OU Fait référence à une irrégularité dans un des graphiques.

$\mathbf{O U}$ Indique une divergence entre les graphiques, mais l'explication est très faible.

\section{QUESTION 3}

André maintient sa conclusion: le réchauffement de l'atmosphère est dûà l'augmentation des émissions de dioxyde de carbone. Mais Jeanne pense que sa conclusion est prématurée. Elle dit : « Avant d'accepter cette conclusion, tu dois t'assurer que d'autres facteurs qui pourraient avoir une influence sur l'effet de serre sont constants ».

Citez un des facteurs auxquels Jeanne fait allusion.

\section{Crédit complet (Niveau $6: 709$ points)}

Cite un facteur qui fait référence à l'énergie/au rayonnement solaire.

OU Cite un facteur qui fait référence à une composante naturelle ou à un agent polluant potentiel.

\section{EXERCICE PHYSIQUE}

Cette unité concerne l'effet de l'exercice physique sur la santé.

- Figure 3.5 -

\section{Items de l'unité EXERCICE PHYSIQUE}

Pratiqué régulièrement, mais avec modération, l'exercice physique est bon pour la santé.
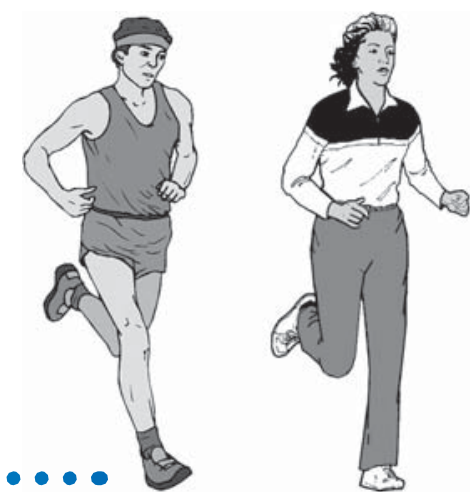

\section{QUESTION 1}

Quels sont les avantages d'un exercice physique régulier? Entourez « Oui » ou « Non» pour chacune des affirmations.

\begin{tabular}{l|l}
\hline S'agit-il d'un avantage de l' exercice physique rêgulier? & Oui ou Non? \\
\hline L' exercice physique prévient les maladies du coeur et les troubles de la circulation. & Oui / Non \\
\hline L' exercice physique conduit à un régime alimentaire sain. & Oui / Non \\
\hline L' exercice physique aide à éviter l'excès de poids. & Oui / Non \\
\hline
\end{tabular}




\section{Crédit complet (Niveau $3: 545$ points)}

Les trois réponses sont correctes. Dans I'ordre : Oui, Non, Oui.

QUESTION 2

Que se passe-t-il lors d'un exercice musculaire? Entourez «Oui» ou « Non » pour chacune des affirmations.

\begin{tabular}{l|l}
\hline Ceci se produit-il lors d'un exercice musculaire? & Oui ou Non? \\
\hline Le sang circule davantage dans les muscles. & Oui / Non \\
\hline Des graisses se forment dans les muscles. & Oui / Non \\
\hline
\end{tabular}

Crédit complet (Niveau $1: 386$ points)

Les deux réponses sont correctes. Dans l'ordre : Oui, Non.

QUESTION 3

Pourquoi doit-on respirer plus fort quand on fait un exercice physique que quand notre corps est au repos?

\section{Crédit complet (Niveau $4: 583$ points)}

Pour faire baisser le niveau de dioxyde de carbone, qui tend à s'élever, ET pour fournir au corps davantage d'oxygène.

\section{Crédit partiel}

Pour faire baisser le niveau de dioxyde de carbone, qui tend à s'élever, OU pour fournir au corps davantage d'oxygène, mais pas pour les deux raisons.

\section{Compétences scientifiques}

L'évaluation PISA de la culture scientifique donne la priorité aux compétences présentées dans la figure 3.6 : la capacité d'identifier des questions à caractère scientifique, de décrire, d'expliquer ou de prévoir des phénomènes sur la base de connaissances scientifiques, d'interpréter des faits et des conclusions et, enfin, d'utiliser des faits scientifiques ayant valeur de preuve pour prendre des décisions et communiquer. Ces compétences impliquent des connaissances scientifiques, à la fois des connaissances en sciences et des connaissances à propos de la science, en tant que forme de savoir et forme d'investigation.

- Figure 3.6 -

\section{Compétences scientifiques PISA}

Identifier des questions d'ordre scientifique

- Reconnaître les questions auxquelles l'on peut apporter une réponse par une investigation scientifique

- Identifier les mots-clés permettant d'effectuer une recherche d'informations scientifiques

- Reconnaître les caractéristiques principales d'une investigation scientifique

Expliquer des phénomènes de manière scientifique

- Appliquer des connaissances en sciences dans une situation donnée

- Décrire ou expliquer des phénomènes de manière scientifique, et prévoir leurs changements

- Identifier les descriptions, explications ou prévisions appropriées

\section{Utiliser des faits scientifiques}

- Interpréter des données scientifiques pour tirer et communiquer des conclusions

- Identifier les hypothèses, les éléments de preuve et les raisonnements qui sous-tendent des conclusions

- Réfléchir aux implications sociétales des progrès scientifiques et technologiques

Certains processus cognitifs sont particulièrement pertinents pour la culture scientifique. Parmi ceux qui relèvent des compétences scientifiques, citons le raisonnement inductif (raisonner à partir d'éléments particuliers pour aboutir à des principes généraux), le raisonnement déductif (raisonner du général au particulier), la réflexion critique et intégrée, la transposition de représentations (par exemple, reporter des données dans des tableaux ou représenter des tableaux sous forme de graphiques), l'élaboration d'explications ou d'arguments sur la base de données et leur communication, la modélisation et, enfin, I'utilisation des processus, connaissances et compétences mathématiques. 
La priorité accordée dans I'enquête PISA aux compétences scientifiques présentées dans la figure 3.6 se justifie par leur importance dans la démarche d'investigation scientifique. Ces compétences se fondent sur la logique, le raisonnement et I'analyse critique. Les compétences retenues sont décrites de manière plus détaillée ci-après, et leur mode d'évaluation est expliqué sur la base des unités fournies à titre d'exemple dans la section précédente.

\section{Identifier des questions d'ordre scientifique}

Il est essentiel de distinguer les questions et les contenus d'ordre scientifique de ceux qui sont sans rapport avec la science. Aspect primordial s'il en est, les questions scientifiques doivent conduire à des réponses fondées sur des faits scientifiques. La compétence Identifier des questions d'ordre scientifique suppose la capacité de reconnaître les questions qui peuvent faire l'objet de recherches scientifiques dans une situation donnée et d'identifier les mots-clés permettant de rechercher des informations scientifiques sur un sujet précis. Il faut aussi pouvoir déterminer les caractéristiques essentielles d'une démarche d'investigation scientifique, par exemple identifier les données à comparer, les paramètres à contrôler ou à faire varier, les informations supplémentaires à intégrer ou les procédures à utiliser pour recueillir des données pertinentes.

Pour être à même d'identifier des questions d'ordre scientifique, les élèves doivent posséder des connaissances à propos de la science elle-même et doivent aussi s'appuyer, à des degrés divers, sur leurs connaissances en science. Dans la question $\mathrm{n}^{\circ} 3$ de l'unité PLUIES ACIDES, il est par exemple demandé aux élèves de réfléchir au contrôle d'une expérience scientifique. Les élèves doivent comparer la réaction d'un acide (le vinaigre) à des réactions possibles avec de l'eau distillée pour être sûrs que l'acide est bien à l'origine de la réaction.

\section{Expliquer des phénomènes de manière scientifique}

La compétence Expliquer des phénomènes de manière scientifique consiste à appliquer des connaissances en sciences dans une situation donnée. Les élèves doivent pouvoir décrire ou interpréter des phénomènes, prévoir des changements et, dans certains cas, reconnaître ou identifier les descriptions, explications ou prévisions pertinentes. La question $\mathrm{n}^{\circ} 1$ de I'unité PLUIES ACIDES est un exemple d'item demandant aux élèves d'expliquer un phénomène de manière scientifique, en I'occurrence l'origine des oxydes de soufre et oxydes d'azote présents dans l'air. Autres exemples, la question $n^{\circ} 3$ de l'unité $L^{\prime} E F F E T$ DE SERRE, dans laquelle les élèves doivent identifier les facteurs qui expliquent l'élévation de la température moyenne de la Terre, et la question $n^{\circ} 3$ de l'unité EXERCICE PHYSIQUE, dans laquelle ils doivent appliquer leurs connaissances sur le système respiratoire humain.

\section{Utiliser des faits scientifiques}

La compétence Utiliser des faits scientifiques consiste à accéder à des informations scientifiques et à produire des arguments et des conclusions sur la base de faits scientifiques (Kuhn, 1992 ; Osborne et al., 2001). Les items peuvent impliquer soit des connaissances en sciences, soit des connaissances à propos de la science, soit les deux. Dans la question $\mathrm{n}^{\circ} 2$ de l'unité PLUIES ACIDES, par exemple, les élèves doivent tirer des informations données une conclusion concernant les effets du vinaigre sur le marbre, un modèle simple de l'effet des pluies acides sur le marbre. Autres exemples: les questions $\mathrm{n}^{\circ} 1$ et 2 de l'unité $L^{\prime} E F F E T D E S E R R E$, dans lesquelles les élèves doivent interpréter des éléments de preuves fournis dans deux graphiques.

La compétence Utiliser des faits scientifiques peut aussi nécessiter pour les élèves de choisir une conclusion parmi plusieurs en se fondant sur les éléments de preuve disponibles, d'expliquer les raisons pour lesquelles une conclusion donnée est valide ou non en fonction de la démarche mise en œuvre pour tirer cette conclusion à partir des données fournies, ou encore d'identifier les hypothèses qui sont à la base de telle ou telle conclusion scientifique. Réfléchir aux implications que peuvent avoir pour la société des progrès scientifiques ou technologiques relève également de cette compétence.

Les élèves doivent également être capables de communiquer à un public déterminé leurs observations et leurs décisions, en s'exprimant dans leurs propres termes ou en utilisant d'autres moyens appropriés : texte, schéma ou autre forme de représentation. En résumé, ils doivent pouvoir présenter de manière claire et logique les liens qui existent entre les faits et les conclusions ou décisions.

\section{Connaissances scientifiques}

Comme indiqué auparavant (voir l'encadré 3.1), l'expression « connaissances scientifiques » désigne à la fois les connaissances en sciences (connaissances sur le monde naturel) et les connaissances à propos de la science elle-même. 


\section{Connaissances en sciences}

Étant donné que les épreuves PISA ne permettent d'évaluer qu'un échantillon des connaissances en sciences, il importe de définir des critères clairs pour guider la sélection des connaissances à retenir. Par ailleurs, I'objectif de l'enquête PISA est de déterminer dans quelle mesure les élèves sont capables d'appliquer ces connaissances dans des contextes qui sont pertinents dans leur vie. Les connaissances à évaluer sont donc choisies dans les grandes disciplines scientifiques (physique, chimie, biologie, sciences de la Terre et de l'univers, et technologie) selon les critères suivants :

- la pertinence par rapport à des situations de la vie réelle : les connaissances scientifiques n'ont pas toutes le même degré d'utilité dans la vie des individus ;

- les connaissances retenues doivent représenter des concepts scientifiques fondamentaux, d'une utilité durable ; et

- les connaissances choisies doivent être en adéquation avec le niveau de développement des jeunes de 15 ans.

La figure 3.7 présente les connaissances en sciences retenues sur la base de ces critères et les illustre par des exemples. Les élèves doivent posséder ces connaissances pour comprendre le monde naturel et donner du sens à des expériences qui se situent dans des contextes personnel, social et global. On parle dans ce cadre d'évaluation de "systèmes » et non de "sciences » pour décrire les domaines majeurs et pour traduire l'idée que les citoyens doivent appliquer leur compréhension des concepts de sciences de la matière et de la vie, de sciences de la Terre et de l'univers et de technologie dans des contextes qui interagissent de façon plus ou moins forte.

\section{- Figure 3.7 .}

\section{Catégories PISA de connaissances en sciences}

Systèmes physiques

- Structure de la matière (exemples : modèle de particules et liaisons intramoléculaires)

- Propriétés de la matière (exemples : changements d'état, conductivité thermique et électrique)

- Changements chimiques de la matière (exemples : réactions, transfert d'énergie, acides et bases)

- Forces et mouvement (exemples : vitesse, friction)

- Énergie et transformation de l'énergie (exemples : conservation, dissipation, réactions chimiques)

- Interactions entre l'énergie et la matière (exemples : ondes lumineuses et radioélectriques, ondes sonores et sismiques)

\section{Systèmes vivants}

- Cellules (exemples : structures et fonctions, ADN, et faune et flore)

- Être humain (exemples : santé, nutrition, sous-systèmes [digestion, respiration, circulation et excrétion, et interactions entre ces sous-systèmes], maladies, reproduction)

- Populations (exemples : espèces, évolution, biodiversité, variation génétique)

- Écosystèmes (exemples : chaînes alimentaires, flux de matières et d'énergie)

- Biosphère (exemples : conservation des écosystèmes et durabilité)

\section{Systèmes de la Terre et de l'univers}

- Structures des systèmes terrestres (exemples : lithosphère, atmosphère et hydrosphère)

- Énergie des systèmes terrestres (exemples : sources d'énergie, climat mondial)

- Changements dans les systèmes terrestres (exemples : tectonique des plaques, cycles géochimiques, forces constructives et destructives)

- Histoire de la Terre (exemples : fossiles, origine et évolution de la Terre)

- Place de la Terre dans I'univers (exemples : gravité, systèmes solaires)

\section{Systèmes technologiques}

- Rôle des applications technologiques de la science (exemples : résolution de problèmes, contribution à la satisfaction des besoins et des attentes de I'Homme, conception et mise en œuvre des recherches)

- Relations entre la science et la technologie (exemple : contribution de la technologie aux progrès scientifiques)

- Concepts (exemples : optimisation, compromis et arbitrages, coûts, risques et bénéfices)

- Principes importants (exemples : critères, contraintes, innovation, invention, résolution de problèmes)

Les exemples fournis dans la figure 3.7 ont pour but d'illustrer ces diverses catégories ; on n'a pas tenté de proposer une liste exhaustive de toutes les connaissances susceptibles d'être mises en relation avec chacune des catégories.

Dans I'unité PLUIES ACIDES, la question n² 2 évalue les connaissances en sciences des élèves dans la catégorie «Systèmes physiques ». 
La question $n^{\circ} 3$ de l'unité L'EFFET DE SERRE évalue les connaissances des élèves dans la catégorie "Systèmes de la Terre et de l'univers », et les questions n 1, 2 et 3 de l'unité EXERCICE PHYSIQUE, leurs connaissances dans la catégorie «Systèmes vivants ».

\section{Connaissances à propos de la science}

La figure 3.8 montre les catégories de connaissances à propos de la science et les illustre par des exemples. La première catégorie, "Démarche scientifique », porte sur le processus qui est au centre de l'investigation scientifique et sur ses différentes composantes. Vient ensuite la catégorie «Explications scientifiques », très proche de la première, puisque les explications scientifiques sont le résultat d'une démarche scientifique. On peut considérer la démarche scientifique comme l'ensemble des moyens utilisés par la science (comment les chercheurs obtiennent leurs données) et les explications comme ses objectifs (comment les chercheurs utilisent leurs données). Les exemples fournis dans la figure 3.8 illustrent le contenu de chaque catégorie, sans toutefois tenter de dresser la liste exhaustive de toutes les connaissances susceptibles d'être mises en relation avec ces catégories.

- Figure 3.8 -

Catégories PISA de connaissances à propos de la science

Démarche scientifique

- Point de départ (exemples : curiosité et questions scientifiques)

- Objectif (exemples : produire des éléments de preuve qui aident à répondre à des questions scientifiques ; les hypothèses, théories et modèles actuels guident les recherches)

- Expériences (exemples : choix du type d'investigation scientifique en fonction de la nature de la question, conception)

- Types de données (exemples : données quantitatives [mesures] et données qualitatives [observations])

- Mesure (exemples : incertitude inhérente, reproductibilité, variations, précision des appareils et des procédures)

- Caractéristiques des résultats (exemples : résultats empiriques, provisoires ou susceptibles d'être mis à l'épreuve, d'être falsifiés ou de se corriger les uns les autres)

\section{Explications scientifiques}

- Types d'explications (exemples : hypothèses, théories, lois, modèles)

- Origine (exemples : représentation des données, rôle des connaissances existantes et des faits nouveaux, créativité et imagination, raisonnement logique)

- Principes à respecter (exemples : cohérence logique, recours aux faits comme éléments de preuve, connaissances historiques et contemporaines)

- Produits (exemples : créer de nouvelles connaissances, de nouvelles méthodes et de nouvelles technologies, susciter de nouvelles questions et recherches)

La question $n^{\circ} 3$ de l'unité PLUIES ACIDES est un exemple d'item sur les connaissances à propos de la science dans la catégorie "Démarche scientifique » : les élèves doivent identifier les objectifs possibles du contrôle d'une investigation (compétence : Identifier des questions d'ordre scientifique).

Les questions $n^{\circ} 1$ et 2 de l'unité L'EFFET DE SERRE sont des items qui concernent les connaissances à propos de la science, et se classent dans la catégorie "Explications scientifiques ». Dans la question $n^{\circ} 1$, les élèves doivent interpréter des données fournies dans deux graphiques et expliquer en quoi ces deux graphiques soutiennent la thèse d'une élévation de la température moyenne de la Terre due à l'accroissement des émissions de dioxyde de carbone. Dans la question $n^{\circ} 2$, ils doivent utiliser des données fournies dans les mêmes graphiques pour étayer une conclusion différente.

\section{Attitudes envers la science}

Les attitudes des individus jouent un rôle important dans l'intérêt qu'ils accordent à la science et à la technologie, en général, et à des questions qui les touchent plus personnellement, en particulier, ainsi que dans leurs réactions sur ces sujets. Un des objectifs de l'enseignement des sciences est de développer chez les élèves des attitudes qui les incitent à s'intéresser aux questions scientifiques et ensuite à acquérir des connaissances scientifiques et technologiques pour les appliquer à leur bénéfice personnel et au bénéfice de la société et du monde.

La priorité accordée aux attitudes dans I'enquête PISA repose sur I'hypothèse selon laquelle la culture scientifique d'une personne dépend aussi de ses attitudes, de ses convictions, de ses inclinations, de sa perception de sa propre efficacité, de ses valeurs et de ses actions finales. Cette thèse se fonde sur la structure conceptuelle proposée par Klopfer (1976) concernant le domaine affectif dans l'enseignement des sciences, ainsi que sur une analyse de la littérature à propos des attitudes (par exemple, Gardner, 1975, 1984 ; Gauld et Hukins, 1980 ; Blosser, 1984 ; Laforgia, 1988 ; Osborne 
et al., 2003 ; Schibeci, 1984) et sur des recherches relatives aux attitudes des élèves envers l'environnement (par exemple, Bogner et Wiseman, 1999 ; Eagles et Demare, 1999 ; Weaver, 2002 ; Rickinson, 2001).

Lors du cycle PISA 2006, dont les sciences étaient le domaine majeur d'évaluation, les attitudes et valeurs des élèves ont été analysées, d'une part dans le questionnaire «Élève » et, d'autre part, au travers d'items contextualisés posés dans de nombreuses unités immédiatement après les items cognitifs (OCDE, 2006). Ces questions contextualisées portaient sur des thèmes abordés dans les items cognitifs. Lors du cycle PISA 2012, dont les sciences sont un domaine mineur d'évaluation, les épreuves ne contiendront cette fois pas d'items d'attitude contextualisés.

\section{ÉVALUATION PISA DE LA CULTURE SCIENTIFIQUE \\ Caractéristiques des épreuves}

Comme le veut la définition de la culture scientifique retenue dans l'enquête PISA, les items cognitifs demandent aux élèves d'utiliser des compétences scientifiques (voir la figure 3.6) dans divers contextes (voir la figure 3.2). Cela implique aussi d'appliquer des connaissances scientifiques (voir les figures 3.7 et 3.8).

Variante de la figure 3.1, la figure 3.9 présente les composantes fondamentales du cadre PISA d'évaluation de la culture scientifique d'une manière qui facilite la mise en relation du cadre avec la structure et le contenu des unités de test. Cette figure 3.9 peut être utilisée comme outil de synthèse, pour planifier les exercices d'évaluation, et comme outil d'analyse, pour étudier les résultats des exercices d'évaluation standard. L'élaboration des unités d'évaluation peut se baser sur les contextes qui servent de stimulus ou sur les compétences scientifiques ou connaissances scientifiques requises pour pouvoir répondre aux questions.

- Figure 3.9 -

\section{Outil d'élaboration et d'analyse des unités et des items d'évaluation}

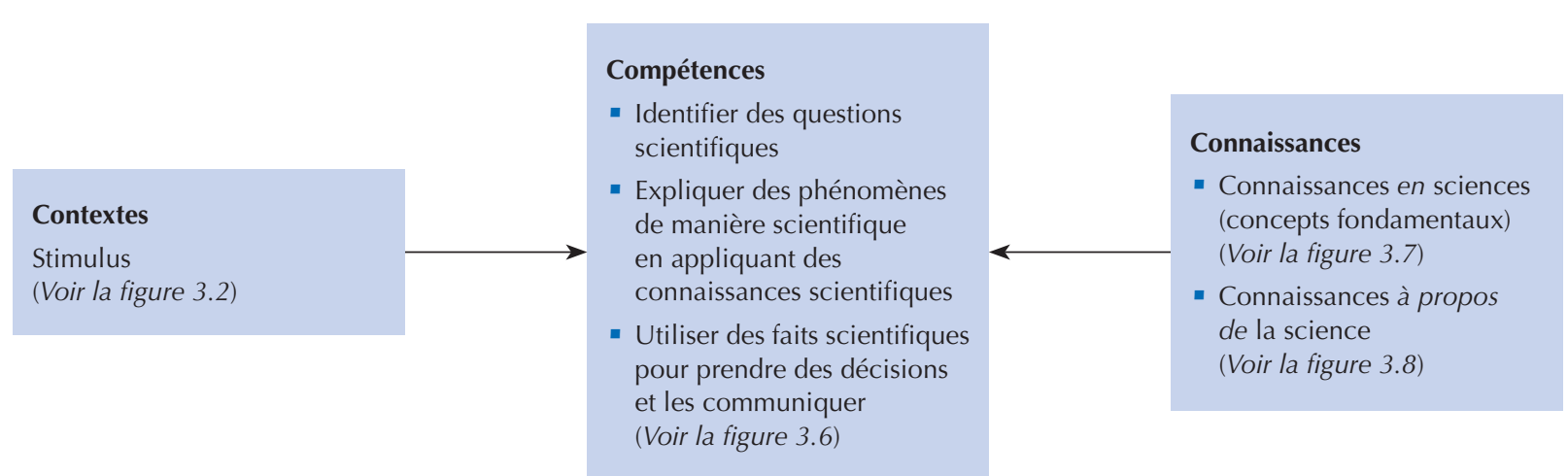

Une unité est constituée d'un stimulus, qui décrit le contexte des items, et d'une série d'items de divers types dont la correction est indépendante. De nombreux types différents de stimulus sont utilisés, souvent en même temps, pour décrire le contexte, notamment des extraits de textes, des photos, des tableaux, des graphiques et des diagrammes. Les trois unités proposées à titre d'exemple dans ce chapitre illustrent bien la diversité des stimuli. L'unité L'EFFET DE SERRE comporte un stimulus assez long constitué d'un texte d'une page et demie et de deux graphiques. Au contraire, le stimulus de l'unité EXERCICE PHYSIQUE est atypique dans la mesure où il est bref et repose sur un effet de suggestion visuelle.

Cette structure sous forme d'unités a été retenue dans I'enquête PISA, car elle facilite I'utilisation de contextes aussi pertinents que possible, et qui reflètent la complexité des situations de la vie réelle, tout en permettant d'exploiter de manière optimale le temps de passation. Poser plusieurs questions différentes dans la même situation au lieu de poser des questions isolées dans un grand nombre de situations différentes présente l'avantage de réduire le temps qu'il faut aux élèves pour comprendre le sujet de chaque question. Toutefois, il faut veiller à ce que les scores attribués aux divers items d'une même unité soient indépendants les uns des autres. De surcroît, comme cette approche réduit le nombre de contextes différents, il importe de proposer un éventail approprié de situations pour minimiser le biais résultant de leur sélection. 
Les unités du cycle PISA 20012 comportent jusqu'à quatre items cognitifs conçus pour évaluer les compétences scientifiques des élèves. Chaque item fait intervenir principalement une des compétences scientifiques retenues et un champ de connaissances : soit des connaissances en sciences, soit des connaissances à propos de la science. Dans la plupart des cas, plus d'une compétence et plus d'une catégorie de connaissances sont évaluées au sein d'une unité (dans des items différents).

Quatre formats d'items sont utilisés pour évaluer les compétences et les connaissances identifiées dans ce cadre : des items à choix multiple simple, des items à réponse construite fermée, des items à choix multiple complexe et des items à réponse construite ouverte. Un tiers environ des items sont des items à choix multiple simple, comme la question $n^{\circ} 2$ de I'unité PLUIES ACIDES, où les élèves doivent sélectionner leur réponse parmi les quatre qui leur sont proposées. Un autre tiers des items sont des items à réponse construite fermée ou des items à choix multiple complexe. Les questions $\mathrm{n}^{\circ} 1$ et 2 de l'unité EXERCICE PHYSIQUE sont des items à choix multiple complexe où les élèves doivent répondre à une série de questions par l'affirmative ou la négative. Le dernier tiers d'items sont des items à réponse construite ouverte, comme les autres items des unités PLUIES ACIDES et EXERCICE PHYSIQUE, et les trois items de l'unité L'EFFET DE SERRE. Ils demandent aux élèves des réponses assez longues, sous forme rédigée ou sous forme de schéma.

La majorité des items sont codés de manière dichotomique (réponse correcte ou non), mais certains items à choix multiple complexe ou à réponse ouverte peuvent faire l'objet d'un crédit partiel, si les élèves ont répondu en partie correctement à la question. Des consignes de codage détaillées sont fournies pour classer les réponses des élèves en trois catégories (les codes "Crédit complet », "Crédit partiel » et " Pas de crédit »), suivant la capacité à répondre dont ils ont fait preuve. Le code "Crédit complet» est attribué aux réponses qui dénotent un niveau de compréhension du sujet jugé approprié pour des élèves de 15 ans ayant une culture scientifique solide. Le code "Crédit partiel » est attribué aux réponses moins sophistiquées ou moins correctes. Enfin, le code "Pas de crédit » est attribué aux réponses totalement erronées, hors sujet ou manquantes. La question $n^{\circ} 3$ de l'unité PLUIES ACIDES et la question $n^{\circ} 2$ de l'unité L'EFFET DE SERRE sont des items dont le codage prévoit un crédit partiel.

Pour comprendre les items de sciences et y répondre, les élèves doivent avoir un certain niveau en lecture, ce qui pose la question du niveau d'exigence requis en compréhension de l'écrit. Les stimuli et les items sont formulés dans un langage aussi clair, simple et concis que possible, tout en transmettant le contenu voulu de manière appropriée. Le nombre de concepts introduits dans chacun des paragraphes est limité et les items qui nécessitent un niveau trop élevé de compétence en compréhension de l'écrit ou en mathématiques sont écartés.

\section{Structure de l'évaluation en sciences}

Les épreuves PISA doivent proposer un équilibre correct entre les items évaluant les compétences et ceux évaluant les connaissances scientifiques. Le tableau 3.1 montre la répartition des points de score entre les catégories des deux composantes (les connaissances en sciences et les connaissances à propos de la science), en pourcentage du nombre total de points de score, lors du cycle PISA 2006 (dont les sciences étaient le domaine majeur d'évaluation) et lors du cycle PISA 2012.

Tableau 3.1

Répartition approximative des points de score en sciences entre les catégories de connaissances scientifiques

\begin{tabular}{l|c|c}
\hline \multirow{2}{*}{ Connaissances en sciences } & \multicolumn{2}{|c}{ Pourcentage de points de score } \\
\cline { 2 - 3 } & PISA 2006 & PISA 2012 \\
\hline Systèmes physiques & 17 & 13 \\
\hline Systèmes vivants & 20 & 16 \\
\hline Système de la Terre et de l'univers & 10 & 12 \\
\hline Systèmes technologiques & 8 & 9 \\
\hline Sous-total & 55 & 50 \\
\hline
\end{tabular}

Connaissances à propos de la science

\begin{tabular}{l|r|r}
\hline Démarche scientifique & 23 & 23 \\
\hline Explications scientifiques & 22 & 27 \\
\hline Sous-total & 45 & 50 \\
\hline Total & $\mathbf{1 0 0}$ & $\mathbf{1 0 0}$ \\
\hline
\end{tabular}


Le tableau 3.2 montre la répartition correspondante pour les compétences scientifiques.

Tableau 3.2

Répartition approximative des points de score en sciences entre les compétences scientifiques

\begin{tabular}{l|c|c}
\hline \multirow{2}{*}{ Compétences scientifiques } & \multicolumn{2}{|c}{ Pourcentage de points de score } \\
\cline { 2 - 3 } & PISA 2006 & PISA 2012 \\
\hline Identifier des questions d'ordre scientifique & 22 & 23 \\
\hline Expliquer des phénomènes de manière scientifique & 46 & 41 \\
\hline Utiliser des faits scientifiques & 32 & 37 \\
\hline Total & $\mathbf{1 0 0}$ & $\mathbf{1 0 0}$ \\
\hline
\end{tabular}

Les items sont répartis par contexte personnel, social et global selon des proportions approximatives de $25 \% ; 50 \% ; 25 \%$. Ils se répartissent de manière assez équivalente entre les champs d’application présentés à la figure 3.2.

Le tableau 3.3 montre la répartition des points de score entre les types d'items.

Tableau 3.3

Répartition approximative des points de score en sciences entre les types d'item

\begin{tabular}{l|c|c}
\hline \multirow{2}{*}{ Types d'item } & \multicolumn{2}{|c}{ Pourcentage de points de score } \\
\cline { 2 - 3 } & PISA 2006 & PISA 2012 \\
\hline Items à choix multiple simple & 35 & 32 \\
\hline Items à choix multiple complexe & 27 & 34 \\
\hline Items à réponse construite fermée & 4 & 2 \\
\hline Items à réponse construite ouverte & 34 & 32 \\
\hline Total & $\mathbf{1 0 0}$ & $\mathbf{1 0 0}$ \\
\hline
\end{tabular}

\section{PRÉSENTATION DU NIVEAU DE COMPÉTENCE EN SCIENCES}

Les résultats aux épreuves PISA sont rapportés sur une échelle construite sur la base du modèle de Rasch décrit par Adams, Wilson et Wang (1997). Une échelle de compétence a été élaborée dans chaque domaine d'évaluation (compréhension de l'écrit, mathématiques et sciences). Le score moyen de ces échelles est fixé à 500 points et leur écart type, à 100, sur la base des pays de l'OCDE. En d'autres termes, le score de deux tiers environ des élèves des pays de l'OCDE se situe par construction entre 400 et 600 points.

Lors du cycle PISA 2006, premier cycle dont les sciences étaient le domaine majeur d'évaluation, six niveaux de compétence ont été définis sur l'échelle de culture scientifique. Les mêmes niveaux de compétence seront utilisés pour rendre compte des résultats aux épreuves de sciences lors du cycle PISA 2012. Chaque niveau de compétence correspond à des compétences que les élèves doivent posséder pour atteindre ce niveau. La figure 3.10 décrit les connaissances et compétences scientifiques que les élèves possèdent à chaque niveau, le niveau 6 étant le plus élevé. Elle indique également le score et le niveau des items des trois unités tirées des épreuves du cycle PISA 2006 qui sont utilisées à titre d’exemple tout au long de ce chapitre.

Les compétences des élèves qui se sont classés sous le niveau 1 lors du cycle PISA 2006 (environ $5.2 \%$ des élèves des pays de l'OCDE) n'ont pu être décrites de manière suffisamment fiable, à cause du nombre insuffisant d'items dans cette partie de l'échelle de culture scientifique. Le niveau 2 de l'échelle de compétence est considéré comme le "seuil » de culture scientifique, c'est-à-dire le niveau à partir duquel les élèves commencent à montrer qu'ils possèdent les compétences et connaissances scientifiques leur permettant de faire face aux situations de la vie courante en rapport avec les sciences et la technologie.

Les facteurs qui déterminent le degré de difficulté des items de sciences sont les suivants :

- la complexité générale du contexte ;

- la mesure dans laquelle la terminologie, les idées et les processus scientifiques sont familiers aux élèves ;

- la longueur du cheminement logique pour répondre aux questions - c'est-à-dire le nombre d'étapes à franchir pour parvenir à une réponse adéquate et le degré d'interdépendance de chaque étape par rapport aux étapes précédentes ;

- la mesure dans laquelle des idées ou des concepts scientifiques abstraits sont requis dans la réponse ; et

- le niveau de raisonnement, de compréhension et de généralisation requis pour poser des jugements, tirer des conclusions et donner des explications. 
- Figure 3.10 =

Description succincte des six niveaux de compétence de l'échelle PISA de culture scientifique

\begin{tabular}{|c|c|c|c|}
\hline Niveau & $\begin{array}{c}\text { Score } \\
\text { (limite } \\
\text { inférieure) }\end{array}$ & $\begin{array}{l}\text { Exemples d'items } \\
\text { pour chaque niveau }\end{array}$ & Compétences caractéristiques de chaque niveau \\
\hline 6 & 707.9 & $\begin{array}{l}\text { Q3 PLUIES ACIDES } \\
\text { Crédit complet } \\
\text { (717 points) } \\
\text { Q3 L'EFFET DE SERRE } \\
\text { (709 points) }\end{array}$ & $\begin{array}{l}\text { Les élèves situés au niveau } 6 \text { sont capables d'identifier, d'expliquer et d'appliquer } \\
\text { des connaissances en sciences et des connaissances à propos des sciences dans } \\
\text { un éventail de situations complexes inspirées de la vie réelle. Ils sont en mesure } \\
\text { d'établir des liens entre différentes sources d'information et explications, et d'y } \\
\text { puiser des éléments pertinents pour justifier des décisions. Ils sont clairement et } \\
\text { systématiquement capables de se livrer à des réflexions et à des raisonnements } \\
\text { scientifiques approfondis, et d'utiliser leur compréhension scientifique pour étayer } \\
\text { des solutions dans des situations scientifiques et technologiques qui ne leur sont } \\
\text { pas familières. Les élèves de ce niveau parviennent à exploiter leurs connaissances } \\
\text { scientifiques pour développer des arguments en faveur de conseils ou de décisions } \\
\text { dans des situations personnelles, sociales ou globales. }\end{array}$ \\
\hline 5 & 633.3 & $\begin{array}{l}\text { Q2 L'EFFET DE SERRE } \\
\text { Crédit complet } \\
\text { (659 points) }\end{array}$ & $\begin{array}{l}\text { Les élèves situés au niveau } 5 \text { sont capables d'identifier les aspects scientifiques de } \\
\text { nombreuses situations complexes inspirées de la vie réelle et de leur appliquer à la } \\
\text { fois des concepts scientifiques et des connaissances à propos de la science. Ils sont } \\
\text { en mesure de comparer, de sélectionner et d'évaluer les faits scientifiques requis } \\
\text { pour répondre à ces situations. Les élèves de ce niveau possèdent des facultés } \\
\text { bien développées de recherche et sont capables d'établir des liens de manière } \\
\text { appropriée entre des connaissances, et de cerner des situations de manière } \\
\text { critique. Ils sont capables d'élaborer des explications sur la base des faits et des } \\
\text { arguments qui découlent de leurs analyses critiques. }\end{array}$ \\
\hline 4 & 558.7 & $\begin{array}{l}\text { Q3 EXERCICE PHYSIQUE } \\
\text { (583 points) } \\
\text { Q2 L'EFFET DE SERRE } \\
\text { Crédit partiel (568 points) }\end{array}$ & $\begin{array}{l}\text { Les élèves situés au niveau } 4 \text { sont capables de travailler de manière efficace sur } \\
\text { des situations ou des problèmes qui impliquent des phénomènes explicites et qui } \\
\text { leur demandent de faire des inférences à propos du rôle des sciences ou de la } \\
\text { technologie. Ils parviennent à sélectionner des explications issues de différentes } \\
\text { disciplines scientifiques ou technologiques, puis à les intégrer et à les associer } \\
\text { directement à des aspects de situations de la vie réelle. Les élèves de ce niveau } \\
\text { sont capables de réfléchir à leurs actes et de communiquer leurs décisions en se } \\
\text { basant sur des connaissances et des faits scientifiques. }\end{array}$ \\
\hline 3 & 484.1 & $\begin{array}{l}\text { Q1 EXERCICE PHYSIQUE } \\
\text { (545 points) } \\
\text { Q1 L'EFFET DE SERRE } \\
\text { (529 points) } \\
\text { Q3 PLUIES ACIDES } \\
\text { Crédit partiel (513 points) } \\
\text { Q1 PLUIES ACIDES } \\
\text { (506 points) }\end{array}$ & $\begin{array}{l}\text { Les élèves situés au niveau } 3 \text { sont capables d'identifier des questions scientifiques } \\
\text { décrites clairement, dans des contextes variés. Ils sont en mesure de sélectionner } \\
\text { des faits et des connaissances pour expliquer des phénomènes, et d'appliquer des } \\
\text { stratégies de recherche ou des modèles simples. Ils sont capables d'interpréter, } \\
\text { d'utiliser et d'appliquer directement des concepts scientifiques issus de disciplines } \\
\text { différentes. Ils peuvent élaborer des arguments succincts sur la base de faits et } \\
\text { prendre des décisions en s'appuyant sur leurs connaissances scientifiques. }\end{array}$ \\
\hline 2 & 409.5 & $\begin{array}{l}\text { Q2 PLUIES ACIDES } \\
\text { (460 points) }\end{array}$ & $\begin{array}{l}\text { Les élèves situés au niveau } 2 \text { possèdent les connaissances scientifiques requises } \\
\text { pour fournir des explications plausibles dans des contextes familiers ou tirer } \\
\text { des conclusions à partir de recherches simples. Ils sont en mesure de se livrer } \\
\text { à des raisonnements directs et d'interpréter de manière littérale les résultats } \\
d^{\prime} \text { 'une recherche scientifique ou d'un problème de technologie. }\end{array}$ \\
\hline 1 & 334.9 & $\begin{array}{l}\text { Q2 EXERCICE PHYSIQUE } \\
\text { (386 points) }\end{array}$ & $\begin{array}{l}\text { Les élèves situés au niveau } 1 \text { ont des connaissances scientifiques tellement } \\
\text { limitées qu'ils peuvent uniquement les appliquer dans un petit nombre de } \\
\text { situations familières. Ils peuvent fournir des explications scientifiques qui vont } \\
\text { de soi et découlent explicitement des faits donnés. }\end{array}$ \\
\hline
\end{tabular}

La question $\mathrm{n}^{\circ} 3$ de l'unité L'EFFET DE SERRE est un exemple d'item difficile, qui se situe au niveau 6 de l'échelle PISA de culture scientifique. Elle combine des aspects de deux compétences, en l'occurrence identifier des questions d'ordre scientifique et expliquer des phénomènes de manière scientifique. Comme première étape pour résoudre ce problème, les élèves doivent identifier la variation et les variables mesurées, et être suffisamment au fait des méthodes de recherche pour savoir que d'autres facteurs interviennent. De plus, ils doivent reconnaître le scénario et en identifier les composantes majeures, ce qui consiste à identifier un certain nombre de concepts abstraits et leurs relations pour déterminer quels sont les « autres » facteurs susceptibles d'influer sur la relation entre la température de la Terre et la 
quantité de gaz carbonique dans l'atmosphère. Ainsi, pour répondre correctement à cet item, un élève doit comprendre la nécessité de contrôler des facteurs autres que les variables mesurées qui ont changé et posséder des connaissances suffisantes sur les "Systèmes de la Terre » pour identifier au moins un des facteurs à contrôler. Cette question est classée dans la catégorie Expliquer des phénomènes de manière scientifique, car elle nécessite avant tout des connaissances suffisantes dans la catégorie des « systèmes de la Terre ».

La question $\mathrm{n}^{\circ} 1$ de l'unité EXERCICE PHYSIQUE est un exemple d'item facile, qui se situe au niveau 1 de l'échelle PISA de culture scientifique, en dessous du niveau seuil de compétence. Pour obtenir le crédit, les élèves doivent se remémorer des connaissances à propos du fonctionnement des muscles et de la formation de graisse dans le corps, en particulier le fait que l'exercice physique accroît la circulation du sang et empêche la formation de graisse. Ces connaissances permettent aux élèves d'accepter la première proposition de cet item à choix multiple complexe et de rejeter la seconde. Ils ne doivent ni analyser de contexte, ni explorer ou établir de relations, et les connaissances auxquelles cet item fait appel sont couramment répandues.

Les résultats du cycle PISA 2006 ont également été rapportés sur trois sous-échelles correspondant aux trois compétences scientifiques retenues. Ces sous-échelles sont divisées en six niveaux de compétence comme l'échelle principale, mais leurs descriptions sont spécifiques. De plus, les résultats ont été comparés entre les pays sur la base des connaissances à propos de la science et des trois grandes catégories de connaissances en sciences (systèmes physiques; systèmes vivants; systèmes de la Terre et de l'univers).

Les analyses découlant de ces types de comparaisons peuvent être signifiantes, certes, mais la prudence est de rigueur lors de la mise en relation de la performance générale avec les compétences et connaissances, car les données sont issues de deux façons de classer les items qui ne sont pas indépendantes. Tous les items classés dans la compétence Identifier des questions d'ordre scientifique sont aussi des items portant sur des connaissances à propos de la science, et tous les items classés dans la compétence Expliquer des items de manière scientifique sont des items portant sur des connaissances en sciences (OCDE, 2009, p. 44).

\section{SYNTHÈSE}

La définition de la culture scientifique retenue dans l'enquête PISA se fonde sur cette question : quels sont les savoirs, les valeurs et les savoir-faire que les élèves de 15 ans doivent posséder pour être prêts à vivre dans la société contemporaine? Au cœur de cette définition et de l'évaluation de la culture scientifique se trouvent les compétences caractéristiques de la science et de la démarche scientifique : identifier des questions d'ordre scientifique, expliquer des phénomènes de manière scientifique et utiliser des faits scientifiques. La capacité des élèves à mettre en œuvre ces compétences dépend de leurs connaissances scientifiques - à la fois leurs connaissances sur le monde naturel (chimie, biologie, sciences de la Terre et de l'univers, technologie) et leurs connaissances à propos de la science (démarche scientifique et explications scientifiques) -, et de leurs attitudes à l'égard des questions scientifiques.

Ce cadre décrit et illustre les compétences et connaissances scientifiques et les attitudes que sous-tend la définition PISA de la culture scientifique (voir la figure 3.11), et présente le format et la structure des épreuves de sciences du cycle PISA 2012.

- Figure 3.11 -

\section{Composantes principales de la définition PISA de la culture scientifique}

\section{Compétences \\ - Identifier des questions d'ordre scientifique \\ - Expliquer des phénomènes de manière scientifique \\ - Utiliser des faits scientifiques}

Connaissances
- Connaissances en sciences
- Systèmes physiques
- Systèmes vivants
- Systèmes de la Terre et de I'univers
- Systèmes technologiques
- Connaissances à propos de la science
- Démarche scientifique
- Explications scientifiques

\section{Attitudes}

- Intérêt pour la science

- Valeur accordée à la démarche scientifique

- Responsabilité à l'égard des ressources et de l'environnement 
Les items PISA de sciences sont regroupés dans des unités qui commencent toutes par un stimulus qui décrit le contexte dans lequel ils s'inscrivent. La priorité est accordée aux situations dans lesquelles des applications scientifiques revêtent une importance particulière pour améliorer la qualité de vie des individus et des communautés. Les épreuves contiennent une combinaison d'items à choix multiple et d'items à réponse construite, dont certains se prêtent à un codage avec crédit partiel. Les épreuves du cycle PISA 2012 ne contiennent pas d'items d'attitude, contrairement à celles du cycle PISA 2006.

Les résultats aux épreuves de sciences du cycle PISA 2012 seront rapportés sur une seule échelle de compétence dont la moyenne est fixée à 500 points et l'écart type, à 100 points, selon les six niveaux de compétence définis pour la première fois à l'occasion du cycle PISA 2006, pour lequel les sciences étaient le domaine majeur d'évaluation. Le niveau 6 de l'échelle de compétence est le plus élevé et le niveau 2 est considéré comme le "seuil » de culture scientifique. Les élèves qui ne parviennent pas à se hisser au niveau 2 ne possèdent pas les connaissances et compétences scientifiques requises pour leur permettre de prendre une part active dans des situations de la vie en rapport avec la science et la technologie. 


\section{Note}

1. Dans le présent cadre d'évaluation, l'expression « monde naturel » renvoie aussi aux changements induits par l'activité de l'Homme, dont le «monde matériel » conçu et façonné par les technologies. 


\section{Références}

Adams, R.J., M. Wilson et W.C. Wang (1997), "The Multidimensional Random Coefficients Multinomial Logit Model », Applied Psychological Measurement, $\mathrm{n}^{\circ} 21$, pp. 1-23.

Blosser, P. (1984), Attitude Research in Science Education, ERIC Clearinghouse for Science, Mathematics and Environmental Education, Columbus, Ohio.

Bogner, F. et M. Wiseman (1999), «Toward Measuring Adolescent Environmental Perception », European Psychologist, vol. 4, n 3 , pp. 139-151.

Bybee, R. (1997a), «Towards an Understanding of Scientific Literacy », in W. Gräber et C. Bolte (éd.), Scientific Literacy: An International Symposium, Institute for Science Education (IPN), Université de Kiel, Kiel, Allemagne.

Bybee, R. (1997b), Achieving Scientific Literacy: From Purposes to Practices, Heinemann, Portsmouth.

Bybee, R.W. et B.J. McCrae (éd.) (2009), PISA Science 2006, Implications for Science Teachers and Teaching, NSTA Press, Arlington, Virginie.

Eagles, P.F.J. et R. Demare (1999), «Factors Influencing Children's Environmental Attitudes », The Journal of Environmental Education, vol. 30, n 4, pp. 33-37.

Fensham, P.J. (1985), «Science for All: A Reflective Essay », Journal of Curriculum Studies, vol. 17, n 4, pp. 415-435.

Fensham, P.J. (2000), "Time to Change Drivers for Scientific Literacy ", Canadian Journal of Science, Mathematics, and Technology Education, vol. 2, pp. 9-24.

Fleming, R. (1989), « Literacy for a Technological Age », Science Education, vol. 73, n 4.

Gardner, P.L. (1975), «Attitudes to Science. A Review », Studies in Science Education, vol. 2, pp. 1-41.

Gardner, P.L. (1984), "Students' Interest in Science and Technology: An International Overview », in M. Lehrke, L. Hoffmann et P.L. Gardner (éd.), Interests in Science and Technology Education, Institute for Science Education (IPN), Université de Kiel, Kiel, Allemagne, pp. 15-34

Gauld, C. et A.A. Hukins (1980), « Scientific Attitudes: A Review », Studies in Science Education, vol. 7, pp. 129-161.

Gräber, W. et C. Bolte (éd.) (1997), Scientific Literacy: An International Symposium, Institute for Science Education (IPN), Université de Kiel, Kiel, Allemagne.

Koballa, T., A. Kemp, et R. Evans (1997), «The Spectrum of Scientific Literacy », The Science Teacher, vol. 64, n 7, pp. 27-31.

Kuhn, D. (1992), «Thinking as Argument », Harvard Educational Review, vol. 62, n² 2.

LaForgia, J. (1988), «The Affective Domain Related to Science Education and its Evaluation », Science Education, vol. 72, n 4, pp. 407-421.

Law, N. (2002), "Scientific Literacy: Charting the Terrains of a Multifaceted Enterprise », Canadian Journal of Science, Mathematics, and Technology Education, vol. 2, pp. 151-176.

Mayer, V.J. (éd.) (2002), Global Science Literacy, Kluwer Academic Publishers, Dordrecht, Pays-Bas.

Mayer, V.J. et Y. Kumano (2002), "The Philosophy of Science and Global Science Literacy », in V.J. Mayer (éd.), Global Science Literacy, Kluwer Academic Publishers, Dordrecht, Pays-Bas.

Millar, R. et J. Osborne (1998), Beyond 2000: Science Education for the Future, King's College London, School of Education, Londres.

Norris, S. et L. Phillips (2003), " How Literacy in its Fundamental Sense is Central to Scientific Literacy », Science Education, vol. $87, \mathrm{n}^{\circ} 2$.

OCDE (2003), « Définition et sélection des compétences clés : Fondements théoriques et conceptuels (DeSeCo) », synthèse du rapport final Key Competencies for a Successful Life and a Well-Functioning Society, Éditions OCDE.

OCDE (2006), Compétences en sciences, lecture et mathématiques : Le cadre d'évaluation de PISA 2006, Éditions OCDE.

OCDE (2009), PISA 2006 Technical Report, Éditions OCDE.

Osborne, J., S. Erduran, S. Simon et M. Monk (2001), "Enhancing the Quality of Argumentation in School Science ", School Science Review, vol. 82, n³01.

Osborne, J., S. Simon et S. Collins (2003), "Attitudes Towards Science: A Review of the Literature and its Implications ", International Journal of Science Education, vol. 25, n 9, pp. 1049-1079. 
Rickinson, M. (2001), "Learners and Learning in Environmental Education: A Critical Review of the Evidence », Environmental Education Research, vol. 7, n³, pp. 207-208.

Roberts, D. (1983), Scientific Literacy: Towards Balance in Setting Goals for School Science Programs, Conseil des sciences du Canada, Ottawa.

Schibeci, R.A. (1984), «Attitudes to Science: An Update », Studies in Science Education, vol. 11, pp. 26-59.

UNESCO (1993), Forum international sur la culture scientifique et technologique pour tous, Rapport final, UNESCO, Paris.

Weaver, A. (2002), «Determinants of Environmental Attitudes: A Five-Country Comparison », International Journal of Sociology, vol. 32, $\mathrm{n}^{\circ} 1$, pp. $77-108$. 


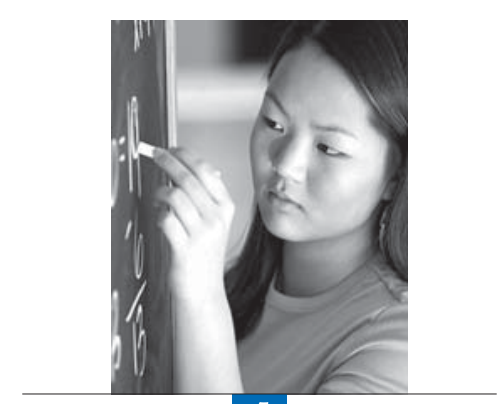

4

\section{Cadre d'évaluation \\ de la résolution de problèmes du cycle PISA 2012}

Ce chapitre présente le cadre qui sous-tend l'épreuve informatisée d'évaluation des compétences individuelles en résolution de problèmes lors du cycle PISA 2012. II en expose les fondements théoriques, décrit les recherches retenues pour l'étayer et définit les compétences en résolution de problèmes. La définition du domaine d'évaluation est analysée de façon approfondie, tout comme les trois éléments les plus importants de l'évaluation, à savoir le contexte des problèmes, la nature de leur situation et les processus cognitifs intervenant dans leur résolution.

La structure générale de l'évaluation et son administration informatisée sont décrites, tout comme l'interface de l'épreuve et les formats de réponse retenus. La répartition des items en fonction des contextes des problèmes, de la nature de leur situation et des processus cognitifs intervenant dans leur résolution est indiquée. Ce chapitre explique en particulier pourquoi l'épreuve inclut des problèmes qui demandent aux élèves d'entrer en interaction avec la situation pour découvrir des informations qui ne leur sont pas explicitement dévoilées. Enfin, des exemples d'items sont proposés. Ils sont commentés et la façon dont les données sur le cheminement et les réponses des élèves (enregistrées grâce à l'informatisation de l'épreuve) interviennent dans le codage est décrite. 


\section{INTRODUCTION}

Inculquer des compétences en résolution de problèmes est un objectif central des programmes de cours de nombreux pays. Acquérir des compétences d'un niveau plus élevé en résolution de problèmes permet de continuer à apprendre, de participer réellement à la vie de la société et de mener des activités personnelles. Les citoyens doivent être capables d'appliquer ce qu'ils ont appris quand ils se trouvent face à des situations nouvelles. L'analyse des points forts des individus en résolution de problèmes permet de mieux cerner leur capacité à mener un raisonnement de base et à adopter d'autres démarches cognitives face aux défis que la vie leur réserve (Lesh et Zawojewski, 2007).

\section{Contexte de l'évaluation lors du cycle PISA 2012}

La résolution de problèmes a été incluse à titre de domaine d'évaluation lors du cycle PISA 2003. Voici quelques-unes des conclusions qui ont été tirées à l'issue de cette évaluation (OCDE, 2005) :

- Les élèves qui réussissent à résoudre des problèmes relativement complexes sont $70 \%$ dans certains pays, mais moins de $5 \%$ dans d'autres.

- Dans la plupart des pays, plus de $10 \%$ des élèves sont incapables de résoudre des problèmes élémentaires.

- En moyenne, dans les pays de l'OCDE, la moitié des élèves sont incapables de résoudre des problèmes plus difficiles que des problèmes élémentaires.

- La façon dont le niveau de compétence des élèves en résolution de problèmes varie au sein même des pays est sensiblement différente d'un pays à l'autre.

- La façon dont le niveau de compétence des élèves varie au sein même des pays entre la résolution de problèmes et les autres domaines d'évaluation (les mathématiques, la compréhension de l'écrit et les sciences) est sensiblement différente d'un pays à l'autre.

Depuis la rédaction du cadre d'évaluation en résolution de problèmes du cycle PISA 2003 (OCDE, 2003a), de nombreuses recherches ont été menées sur plusieurs thématiques, dont la résolution de problèmes complexes, le transfert des acquis, l'évaluation informatisée des compétences en résolution de problèmes et l'évaluation à grande échelle des compétences en résolution de problèmes (voir, par exemple, Blech et Funke, 2005 ; Funke et Frensch, 2007 ; Greiff et Funke, 2008 ; Klieme, 2004 ; Klieme et al., 2005 ; Leutner et al., 2004 ; Mayer, 2002 ; Mayer et Wittrock, 2006 ; O'Neil, 2002 ; Osman, 2010 ; Reeff et al., 2006 ; Wirth et Klieme, 2004). Ces recherches ont permis de mieux comprendre les facultés en résolution de problèmes et de mieux les évaluer.

Par ailleurs, les progrès accomplis dans l'élaboration des logiciels et la généralisation des réseaux informatiques ont permis d'améliorer l'efficience et l'efficacité des évaluations, en offrant notamment la possibilité de soumettre aux élèves des problèmes dynamiques et interactifs, de les intéresser davantage et de recueillir plus d'informations sur leur cheminement lors de la résolution de problèmes. Concernant ce dernier point, ajoutons que l'administration informatisée de tâches permet de recueillir des données à propos de la nature, de la fréquence, de la durée et de l'enchaînement des actes posés par les élèves lorsqu'ils répondent aux items.

Dans ce contexte, il est donc indiqué d'inclure une nouvelle fois la résolution de problèmes parmi les domaines d'évaluation PISA et de concevoir à cet effet un nouveau cadre d'évaluation et de choisir des méthodologies d'évaluation qui permettent de recueillir des données en temps réel sur les facultés des élèves. C'est une épreuve informatisée qui a été retenue pour évaluer les compétences des élèves en résolution de problèmes lors du cycle PISA 2012, dont l'une des caractéristiques majeures est de cibler la façon dont les élèves entrent en interaction avec les problèmes.

L'évaluation des compétences en résolution de problèmes retenue lors du cycle PISA 2012 porte sur les compétences individuelles. Les compétences collaboratives - c'est-à-dire les compétences requises pour résoudre des problèmes en tant que membre d'un groupe - sont essentielles dans la vie professionnelle, car les travailleurs sont souvent membres d'une équipe composée de plusieurs spécialistes en poste dans des lieux différents. Toutefois, ces compétences n'ont pas été retenues lors du cycle PISA 2012 à cause des grandes difficultés qu'implique l'administration de tâches collectives dans une enquête internationale à grande échelle comme le programme PISA (Reeff et al., 2006) et du temps requis pour concevoir une plate-forme informatisée d'administration.

II ressort de nombreuses recherches que la résolution de problèmes dépend de stratégies et de connaissances dans des matières spécifiques (voir, par exemple, Mayer, 1992 ; Funke et Frensch, 2007). L'épreuve administrée lors du cycle PISA 2012 est conçue pour éviter autant que possible que des connaissances spécifiques soient requises afin de concentrer l'évaluation sur les processus cognitifs fondamentaux de la résolution de problèmes. Cette caractéristique 
différencie les tâches de résolution de problèmes des tâches administrées dans les autres domaines d'évaluation PISA (la compréhension de l'écrit, les mathématiques et les sciences) qui font appel à des connaissances dans ces matières.

II ressort également de recherches récentes que l'épreuve de résolution de problèmes doit privilégier les problèmes authentiques, relativement complexes, en particulier ceux qui impliquent des interactions directes de la part des répondants pour leur permettre de découvrir des informations. À titre d'exemple, citons les problèmes qui se posent souvent lors de la première utilisation d'appareils tels que des télécommandes, du matériel numérique personnel (les téléphones mobiles, par exemple), de l'électroménager ou des distributeurs automatiques. Ou encore ceux qui se posent dans des situations ayant pour cadre l'entraînement sportif, l'élevage, la culture de plantes et les interactions sociales. Des compétences en résolution de problèmes sont requises pour faire face à ce type de situations, et des éléments montrent que des compétences autres que les facultés classiques de raisonnement interviennent également (voir, par exemple, Klieme, 2004). C'est la première fois que des problèmes « interactifs » sont inclus dans une évaluation internationale à grande échelle, grâce à l'informatisation de l'administration de l'épreuve.

Un enseignement de qualité permet d'inculquer des compétences en résolution de problèmes. Les méthodes pédagogiques progressives, par exemple celles qui visent l'apprentissage par la résolution de problèmes ou le questionnement, et la réalisation de travaux individuels et collectifs, peuvent être utilisées pour améliorer les facultés de compréhension des élèves et les préparer à appliquer leurs connaissances dans des situations inédites. L'enseignement de qualité promeut l'autorégulation de l'apprentissage et la métacognition, tout en développant les processus cognitifs en rapport avec la résolution de problèmes. II prépare les élèves à raisonner à bon escient dans des situations qui ne leur sont pas familières et à combler leurs lacunes cognitives par l'observation, l'exploration et l'interaction avec des systèmes qui leur sont inconnus. L'épreuve informatisée de résolution de problèmes conçue en vue du cycle PISA 2012 vise à déterminer dans quelle mesure les élèves sont préparés à relever les défis inconnus que l'avenir leur réserve, sachant que les connaissances qui leur sont enseignées maintenant ne leur suffiront pas.

\section{La résolution de problèmes dans le programme de l'OCDE d'évaluation des compétences des adultes}

Le programme de I'OCDE d'évaluation des compétences des adultes a pour objet de mesurer, dans des environnements à forte composante technologique, les compétences en compréhension de l'écrit, en littératie, en numératie et en résolution de problèmes. Les épreuves sont administrées en face à face à des répondants âgés de 15 à 65 ans dont l'échantillon est prélevé dans les ménages. Le premier cycle a eu lieu en 2011, et ses résultats seront publiés en 2013.

L'évaluation des compétences en résolution de problèmes " dans des environnements à forte composante technologique » ${ }^{1}$ se distingue de l'approche retenue lors du cycle PISA 2012 à deux égards. La première différence réside dans le fait qu'elle porte sur des problèmes à forte composante informatique, par exemple localiser et évaluer des informations sur Internet ou sur le site de réseaux sociaux, naviguer sur le web entre des pages non familières et déterminer quelles sont les informations pertinentes pour mener à bien une tâche et écarter celles qui ne le sont pas.

L'autre différence majeure tient au fait que pour résoudre les problèmes, les répondants doivent utiliser une ou plusieurs applications logicielles (arborescence de fichiers, navigateur, tableur, etc.). Dans l'enquête PISA, les technologies de l'information et de la communication (TIC) font partie intégrante de l'épreuve de résolution de problèmes, mais sont exclues de la définition du domaine d'évaluation. Des compétences élémentaires en informatique (savoir utiliser le clavier et la souris) suffisent pour passer l'épreuve informatisée de résolution de problèmes du PISA. Les logiciels courants sont des auxiliaires précieux pour résoudre des problèmes dans des environnements à forte composante informatique ; un niveau élevé de compétence en informatique est essentiel dans l'ère numérique d'aujourd'hui. Toutefois, I'épreuve PISA se concentre sur les processus cognitifs fondamentaux qui sont essentiels pour réussir à résoudre des problèmes, avec ou sans recours à l'informatique.

\section{DÉFINITION DE LA NOTION DE COMPÉTENCE EN RÉSOLUTION DE PROBLĖMES}

L'épreuve de résolution de problèmes du cycle PISA 2012 a pour but d'évaluer les compétences individuelles en résolution de problèmes. Avant de définir ce que l'on entend par « compétences en résolution de problèmes » dans ce contexte, il est important d'expliquer ce que les chercheurs entendent par « problème » et par « résolution de problèmes ».

\section{Définition de la notion de problème}

Un problème se pose lorsqu'un individu s'est fixé un objectif, mais qu'il ne sait pas comment l'atteindre (Duncker, 1945). Cette définition est développée à la figure 4.1. La situation initiale (les données) correspond à ce que l'individu 
connaît du problème au début et les opérateurs, aux actions qu'il peut envisager pour atteindre la situation cible (les résultats) avec l'aide des outils qu'il a à sa disposition. Des obstacles (par exemple, le manque de connaissances ou de stratégies évidentes) l'empêchent d'atteindre son objectif. Il doit donc les surmonter, ce qui implique non seulement la cognition, mais également la motivation et l'affect (Funke, 2010).

- Figure 4.1 -

Problème

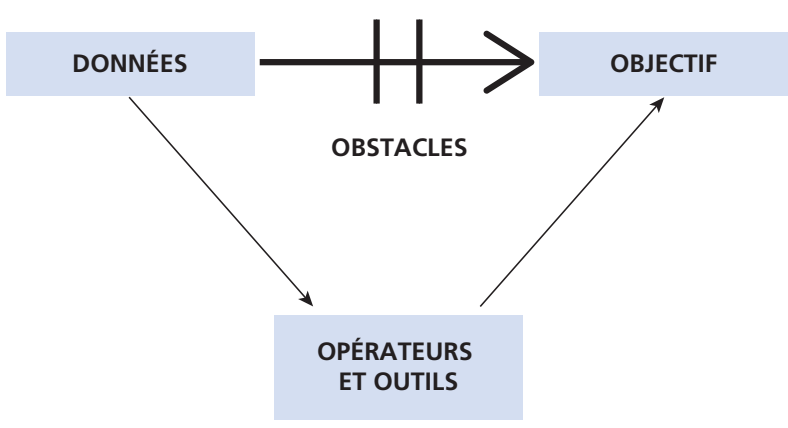

Source : Frensch et Funke, 1995.

Prenons à titre d'exemple un problème simple : trouver l'itinéraire le plus rapide entre deux villes à l'aide d'une carte routière où les temps de trajet sont estimés et d'une calculatrice. La situation initiale correspond aux informations fournies - la carte où les temps de trajet sont estimés - et l'objectif, la réponse voulue - l'itinéraire le plus rapide. Les actions envisageables (les opérateurs) sont : choisir un itinéraire possible et calculer le temps de trajet y correspondant, puis le comparer au temps de trajet d'autres itinéraires. Un outil (en l'espèce, une calculatrice) est fourni pour faciliter le calcul des temps de trajet.

\section{Définition de la notion de résolution de problèmes}

En cohérence avec la définition de la notion de problème, Mayer (1990) définit la résolution de problèmes comme le traitement cognitif visant à aller d'une situation initiale vers une situation cible en l'absence de solution évidente. Cette définition recueille un large consensus parmi les chercheurs (voir, par exemple, Klieme, 2004 ; Mayer et Wittrock, 2006 ; Reeff et al., 2006).

\section{Définition de la notion de compétence en résolution de problèmes}

Dans le cadre du cycle PISA 2012, la définition des compétences en résolution de problèmes se fonde sur les définitions communément admises de la notion de «problème » et de « résolution de problèmes » :

Les compétences en résolution de problèmes renvoient à la capacité d'un individu de s'engager dans un traitement cognitif pour comprendre et résoudre des problèmes, en l'absence de méthode de solution évidente, ce qui inclut sa volonté de s'engager dans de telles situations pour exploiter tout son potentiel de citoyen constructif et réfléchi.

Sans surprise, la première phrase de la définition est très proche de celle de la définition retenue lors du cycle PISA 2003. Toutefois, la définition de $2003^{2}$ n'évoque que la dimension cognitive et souligne la nature transdisciplinaire de l'évaluation dans sa deuxième partie, alors que la définition de 2012 retient la dimension de l'affect, dans le droit fil de la définition de la notion de compétence adoptée par l'OCDE (OCDE, 2003a).

Ce qui distingue l'épreuve de 2012 de celle de 2003 n'est pas tant la définition des compétences en résolution de problèmes, c'est surtout le mode d'administration (l'épreuve est informatisée en 2012) et l'inclusion de problèmes que les répondants ne peuvent résoudre sans entrer en interaction avec les problèmes.

Les paragraphes suivants expliquent les différents passages de la définition de la notion de résolution de problèmes retenue lors du cycle PISA 2012 pour clarifier sa signification par rapport à l'évaluation.

Les compétences en résolution de problèmes...

Par compétences, on entend bien plus que la simple restitution d'acquis cumulés, à savoir la mobilisation par l'individu de ses savoir-faire cognitifs et pratiques, de sa créativité et d'autres ressources psychosociales telles que les attitudes, la 
motivation et les valeurs (OCDE, 2003b). L'épreuve de résolution de problèmes administrée lors du cycle PISA 2012 est conçue pour évaluer non pas la capacité des élèves à restituer des connaissances propres à des matières données, mais bien les compétences cognitives qu'ils exploitent pour résoudre des problèmes qui ne leur sont pas familiers, qu'ils sont susceptibles de rencontrer dans la vie ${ }^{3}$ et qui ne se cantonnent pas aux matières scolaires traditionnelles.

Les connaissances antérieures sont importantes pour résoudre des problèmes. Toutefois, au nombre des compétences en résolution de problèmes figure la faculté d'acquérir de nouvelles connaissances et de les exploiter, ou d'utiliser des connaissances antérieures d'une façon inédite pour résoudre des problèmes inédits (qui sortent de la routine).

\section{... renvoient à la capacité d'un individu de s'engager dans un traitement cognitif...}

La résolution de problèmes est un processus interne qui a lieu dans le système cognitif des individus. Ce processus ne peut s'appréhender qu'indirectement, au travers des actes posés par les individus et de leurs résultats. II amène notamment les individus à représenter et manipuler divers types de connaissances dans leur système cognitif (Mayer et Wittrock, 2006). Les réponses des élèves aux items - leurs stratégies d'exploration, les représentations qu'ils utilisent pour modéliser les problèmes, leurs réponses numériques et autres, ou la façon dont ils expliquent leur cheminement vers la résolution du problème - sont utilisées pour tirer des conclusions sur les processus cognitifs utilisés.

Le raisonnement créatif (divergent) et le raisonnement critique sont des composantes importantes des compétences en résolution de problèmes (Mayer, 1992). Le raisonnement créatif est une activité cognitive qui permet de trouver des solutions à des problèmes inédits. Le raisonnement critique va de pair avec le raisonnement créatif et permet d'évaluer des solutions possibles. L'épreuve administrée lors du cycle PISA 2012 cible ces deux composantes.

\section{... pour comprendre et résoudre des problèmes...}

Dans quelle mesure les individus parviennent-ils à relever les défis d'un problème et à s'approcher de sa solution ? En plus des réponses explicites aux items, l'épreuve vise à mesurer le cheminement accompli par les élèves sur la voie de la résolution du problème et à cerner les stratégies qu'ils adoptent pour ce faire. Le cas échéant, le déploiement de ces stratégies est suivi sur la base de données comportementales recueillies grâce à l'informatisation de l'épreuve : la nature, la fréquence, la longueur et l'ordre des interactions avec le système peuvent être enregistrées, puis utilisées lors du codage des réponses ou de l'analyse de la performance des élèves.

Pour résoudre un problème, I'individu doit commencer par admettre qu'un problème existe et comprendre la nature de la situation. Il doit ensuite cerner le ou les problèmes à résoudre, puis élaborer une solution et la mettre en œuvre, tout en suivant et en évaluant ses progrès pendant le processus.

Les problèmes qui se posent dans la vie réelle n'ont bien souvent pas qu'une solution unique ou exacte. De plus, les problèmes peuvent évoluer au cours du processus de résolution, que ce soit sous l'effet de leur nature dynamique ou de leurs interactions avec le répondant. Ces caractéristiques complexes ont été prises en compte lors de l'élaboration des tâches dans le but de concilier deux impératifs : la nécessité de proposer des situations authentiques et la faisabilité de l'épreuve.

... en l'absence de méthode de solution évidente...

En principe, le cheminement vers la solution ne doit pas apparaître d'emblée aux élèves. Des obstacles ou des informations manquantes vont jalonner leur parcours. L'évaluation ne porte sur des problèmes de routine (c'est-à-dire qu'une solution déjà apprise est de toute évidence applicable), mais sur des problèmes qui ne le sont pas : les élèves doivent activement explorer et comprendre les problèmes, et soit concevoir une nouvelle stratégie, soit appliquer une stratégie qu'ils connaissent dans un contexte différent pour aboutir à la solution.

La nature du problème - le fait qu'il soit de routine ou non - dépend de la mesure dans laquelle il est familier aux individus. Ce qui pose "problème » à un individu peut être évident pour un autre individu qui a déjà été amené à résoudre ce genre de cas. Dans cet esprit, nous avons pris soin de choisir des problèmes qui ne sont pas de routine pour la grande majorité des adolescents de 15 ans.

Les contextes ou les objectifs ne sont pas nécessairement peu familiers aux individus ; ce qui compte, c'est que les problèmes soient inédits ou que les moyens de les résoudre n'apparaissent pas d'emblée. Les individus doivent parfois explorer la situation du problème ou entrer en interaction avec cette situation avant de tenter de résoudre le problème. Les interactions directes sont possibles grâce à l'informatisation de l'épreuve lors du cycle PISA 2012. 
... ce qui inclut sa volonté de s'engager dans de telles situations...

La résolution de problèmes est un processus personnel et dirigé, ce qui signifie que l'individu est guidé par ses objectifs personnels (Mayer et Wittrock, 2006). Les connaissances et compétences de l'individu contribuent à déterminer la facilité ou la difficulté avec laquelle il franchit les obstacles pour aboutir à la solution. Toutefois, l'activation de ces connaissances et compétences subit l'influence de facteurs en rapport avec la motivation et l'affect, tels que les convictions (la confiance en soi), l'intérêt et la capacité perçue de résoudre un problème (Mayer, 1998).

De plus, le contexte du problème (la mesure dans laquelle il est familier et compris), les ressources externes à la disposition de l'individu (l'accès à des outils, par exemple) et l'environnement dans lequel l'individu évolue (dans le cadre d'un examen, par exemple) influent sur la façon dont l'individu aborde le problème et s'y engage.

Les facteurs en rapport avec l'affect et la motivation ne sont pas évalués lors de l'épreuve cognitive de résolution de problèmes, mais le questionnaire « Élève » contient une série d'items permettant de mesurer la persévérance et l'ouverture à la résolution de problèmes en général. De plus, ce questionnaire comprend des questions qui offrent la possibilité de recueillir des informations sur les stratégies de résolution de problèmes des élèves (par exemple, s'adresser à quelqu'un qui s'y connaît, consulter les instructions, manquer de concentration, abandonner) lorsqu'ils sont confrontés à des situations problématiques.

\section{... pour exploiter tout son potentiel de citoyen constructif et réfléchi.}

La compétence est un facteur important qui contribue à aider les individus à façonner le monde et pas seulement à y faire face : «...des compétences clés peuvent être bénéfiques à la fois pour les individus et les sociétés " (Rychen et Salganik, 2003). Les individus doivent " gérer leur vie de manière sensée et responsable en maîtrisant leurs conditions de vie et de travail » (ibid.). Ils doivent être capables de résoudre des problèmes pour exploiter tout leur potentiel de citoyens constructifs, engagés et réfléchis.

\section{Nature de l'évaluation}

L'épreuve de résolution de problèmes du cycle PISA 2012 ne contient pas de problèmes dont la résolution passe par I'utilisation de connaissances spécialisées, ni de problèmes qui pourraient raisonnablement être inclus dans l'un des trois grands domaines d'évaluation PISA. Les items se concentrent sur des situations de la vie courante, qui s'inscrivent dans un large éventail de contextes pour minimiser le biais des connaissances antérieures.

La mobilisation de connaissances antérieures ne suffit pas pour résoudre des problèmes qui se posent dans de nombreuses situations de la vie courante. Au lieu d'appliquer directement des connaissances acquises, les élèves doivent réorganiser des connaissances antérieures et les combiner à des connaissances nouvelles grâce à leurs facultés de raisonnement. Ils doivent combler les lacunes dans leurs connaissances au travers de l'observation et de l'exploration de la situation du problème. Cela passe souvent par des interactions avec un nouveau système, à la découverte des règles à appliquer pour résoudre le problème. L'épreuve de résolution de problèmes du cycle PISA 2012 donne la priorité à ce type de problèmes, ce qui s'est révélé réalisable grâce à son informatisation.

\section{ORGANISATION DU DOMAINE}

La façon dont le domaine est représenté et organisé détermine la conception de l'épreuve et, en fin de compte, les éléments qui peuvent être recueillis et présentés à propos du niveau de compétence des élèves. De nombreux aspects différents constituent le construct ${ }^{4}$, mais tous ne peuvent être pris en considération dans une enquête telle que l'étude PISA. Les aspects les plus importants doivent être identifiés pour permettre la conception d'une épreuve dont les items s'étendent sur un spectre de difficulté approprié et couvrent bien le domaine d'évaluation.

Les éléments clés du domaine dévaluation retenus pour constituer l'épreuve de résolution de problèmes du cycle PISA 2012 sont les suivants :

- Le contexte du problème : le problème implique-t-il ou non une application technologique et se situe-t-il dans un contexte personnel ou social?

- La nature de la situation du problème : le problème est-il interactif ou statique?

- Les processus de résolution de problèmes : les processus cognitifs à mener pour résoudre un problème.

Les items ont été conçus pour évaluer dans quelle mesure les élèves réussissent à mener à bien les divers processus de résolution de problèmes dans les deux types de situations, dans un large éventail de contextes. Ces éléments clés sont décrits et illustrés dans les sections suivantes. 


\section{Le contexte des problèmes}

La mesure dans laquelle le contexte d'un problème est compris par un individu et lui est familier influe sur sa difficulté à résoudre ce problème. Deux dimensions ont été identifiées pour que les items s'inscrivent dans un éventail de contextes authentiques qui intéressent les adolescents de 15 ans : la technologie (ou non) et sa situation (personnelle ou sociale).

Les problèmes qui se situent dans un contexte technologique se basent sur la fonctionnalité d'une application technologique, par exemple un téléphone mobile, une télécommande ou un distributeur automatique de billets. Il n'est pas nécessaire de connaître le fonctionnement interne de ces appareils : les élèves sont amenés à explorer et à comprendre la fonctionnalité d'un appareil, que ce soit pour se préparer à l'utiliser ou trouver la cause à l'origine de son dysfonctionnement. Les situations qui posent d'autres types de problèmes, par exemple établir un itinéraire, déterminer un emploi du temps ou prendre des décisions, se situent dans un contexte non technologique.

Par contextes personnels, on entend ceux qui se rapportent essentiellement à l'individu, à sa famille et à ses pairs. Les contextes dits sociaux sont ceux qui ont trait à la communauté ou à la société en général (y compris dans le monde du travail ou dans le cadre de l'enseignement supérieur). À titre d'illustration, un item qui consisterait à régler I'heure sur une montre à affichage numérique se classerait dans la catégorie des contextes technologiques et personnels, alors qu'un item qui consisterait à organiser l'emploi du temps d'une équipe de basket se classerait dans la catégorie des contextes non technologiques et sociaux. D'autres exemples sont fournis en fin de chapitre : la première unité de la section, qui traite du fonctionnement d'un lecteur MP3, s'inscrit dans un contexte technologique et personnel, tandis que la deuxième, qui porte sur le plan de table lors d'un repas d'anniversaire, s'inscrit dans un contexte non technologique et social.

\section{Nature de la situation du problème}

La façon de présenter le problème a des implications importantes sur la façon dont il peut être résolu. L'élément crucial est la mesure dans laquelle les informations sur le problème qui sont fournies dès le départ sont complètes. C'est le cas dans l'item consistant à trouver l'itinéraire le plus rapide qui est évoqué auparavant (voir la section "Définition de la notion de problème »). Dans l'enquête PISA, ces problèmes sont dits statiques. L'unité REPAS D'ANNIVERSAIRE, décrite dans la section «Exemples d'items PISA de résolution de problèmes ", pose elle aussi un problème statique.

Par contraste, les problèmes peuvent se situer dans des situations interactives, ce qui veut dire que les élèves peuvent explorer la situation afin de découvrir des informations pertinentes supplémentaires ${ }^{5}$. La navigation en temps réel à l'aide $d^{\prime}$ un système de géolocalisation qui avertit des bouchons automatiquement ou à la demande est un exemple de situation interactive, tout comme I'unité LECTEUR MP3 décrite dans la section "Exemples d'items PISA de résolution de problèmes ».

Les situations interactives de problèmes peuvent être simulées sur ordinateur lors d'une épreuve. L'inclusion de situations interactives dans l'épreuve informatisée de résolution de problèmes du cycle PISA 2012 permet de soumettre aux élèves des scénarios s'inspirant de la vie réelle qui sont plus authentiques que ceux qui leur auraient été soumis lors d'une épreuve papier-crayon. Les problèmes dans lesquels les élèves contrôlent et explorent un environnement simulé constituent une caractéristique distinctive de l'évaluation.

Une série de problèmes qui s'inscrivent dans des situations statiques est également incluse dans l'épreuve. Ces problèmes étaient généralement soumis dans des épreuves papier-crayon. Toutefois, l'épreuve informatisée offre de nombreux avantages, notamment celui de permettre la présentation d'un éventail plus large de scénarios impliquant des éléments multimédias (avec animation, par exemple), de donner accès à des applications en ligne et d'utiliser un grand nombre de formats de réponse qui se prêtent à un codage automatique.

De plus, certaines études donnent à penser que I'acquisition de connaissances durant l'exploration d'un environnement interactif et la façon dont ces connaissances sont appliquées sont des compétences distinctes de celles généralement associées à la résolution de problèmes statiques (voir Klieme, 2004 ; Wirth et Klieme, 2004 ; Leutner et Wirth, 2005). La combinaison de problèmes statiques et interactifs dans l'épreuve du cycle PISA 2012 permet donc d'évaluer plus largement les compétences en résolution de problèmes que via une simple épreuve papier-crayon.

\section{Problèmes en situation interactive}

Les problèmes en situation interactive se posent souvent avec des applications technologiques, par exemple des distributeurs automatiques de billets, des appareils de climatisation ou des téléphones mobiles que l'on utilise pour la première fois, en particulier lorsque leur mode d'emploi n'est pas clair ou n'est pas disponible. Comprendre comment 
utiliser ces applications est un problème que tout le monde rencontre au quotidien. Dans ce type de situations, il est fréquent que des informations pertinentes ne soient pas disponibles d'emblée. Par exemple, le résultat de l'exécution d'une commande (appuyer sur un bouton de télécommande, par exemple) n'est pas nécessairement connu et ne peut être déduit : il faut l'inférer au travers d'une interaction avec le scénario, en l'occurrence appuyer sur ce bouton et formuler une hypothèse à propos de sa fonction compte tenu du résultat. En règle générale, l'exploration ou l'expérimentation sont indispensables en vue d'acquérir les connaissances requises pour utiliser l'application technologique. Le fait de chercher la cause à l'origine de la panne ou du dysfonctionnement d'une application est un autre scénario courant. Dans ce cas, il faut se livrer à un processus d'expérimentation pour recueillir des données sur les circonstances dans lesquelles I'application ne fonctionne pas.

Les situations interactives peuvent être dynamiques, ce qui signifie qu'elles évoluent sous l'effet de facteurs sans rapport avec les individus qui résolvent les problèmes (c'est-à-dire sans leur intervention) ${ }^{6}$. Un distributeur automatique de billets peut, par exemple, revenir au point de départ si l'utilisateur n'appuie sur aucun bouton pendant 20 secondes. Il faut observer un tel comportement autonome pour le comprendre et, donc, en tenir compte pour atteindre l'objectif voulu, en l'espèce acheter un billet.

\section{Problèmes en situation statique}

Les situations statiques peuvent poser des problèmes bien définis ou mal définis. Dans un problème bien défini, tel que celui de l'itinéraire le plus rapide (voir la section « Définition de la notion de problème »), l'état initial, l'état cible et les opérateurs autorisés sont clairement spécifiés (Mayer et Wittrock, 2006). Le problème ne s'inscrit pas dans une situation dynamique (celle-ci ne change pas d'elle-même durant le processus de résolution), toutes les informations pertinentes sont dévoilées dès le début et l'objectif est unique.

Parmi les autres problèmes bien définis, citons à titre d'exemple les problèmes classiques de puzzles logiques, tels que la Tour de Hanoï et les bidons d'eau (voir, par exemple, Robertson, 2001), les problèmes de prise de décision dans lesquels les individus doivent comprendre une situation impliquant un certain nombre de contraintes et d'options bien définies pour prendre une décision qui respecte les contraintes (par exemple, choisir le bon antidouleur sur la base d'informations suffisantes concernant le patient et sa plainte, et compte tenu des antidouleurs disponibles), et les problèmes de programmation, par exemple construire une maison ou créer un logiciel, lorsque les tâches à accomplir sont indiquées accompagnées de leur durée, et que les liens de dépendance entre elles sont précisés.

Mayer et Wittrock (2006) soulignent que « le matériel pédagogique met souvent l'accent sur des problèmes bien définis, alors que la plupart des problèmes qui se posent dans la vie réelle sont le plus souvent mal définis ». Ces problèmes mal définis, qui peuvent être soit statiques, soit interactifs, impliquent souvent d'atteindre plusieurs objectifs contradictoires, de sorte que des progrès sur la voie d'un objectif peuvent entraver des progrès sur la voie d'un ou de plusieurs autres objectifs. L'élaboration et le classement par ordre de priorité s'imposent pour trouver un équilibre entre les objectifs (Blech et Funke, 2010). À titre d'exemple, citons la recherche du « meilleur » itinéraire entre deux endroits - est-ce le plus court, le plus rapide, le plus direct, celui dont la durée varie le moins, etc. ? Autre exemple, la conception d'un véhicule qui serait puissant, bon marché, sûr et peu polluant, autant de caractéristiques qui pourraient être souhaitables.

\section{Processus de résolution de problèmes}

La conception des processus cognitifs qui interviennent dans la résolution de problèmes varie selon les auteurs, mais ceux-ci s'accordent à maints égards. Les processus identifiés ci-dessous sont dérivés des travaux sur la résolution de problèmes et le raisonnement de psychologues de la cognition (Baxter et Glaser, 1997 ; Bransford et al., 1999 ; Mayer et Wittrock, 1996, 2006 ; Vosniadou et Ortony, 1989), ainsi que des travaux précurseurs de Polya (1945). Par ailleurs, des travaux récents sur la résolution de problèmes complexes et dynamiques (Blech et Funke, 2005, 2010 ; Funke et Frensch, 2007 ; Greiff et Funke 2008 ; Klieme, 2004 ; Osman, 2010 ; Reeff et al., 2006 ; Wirth et Klieme, 2004) ont été pris en considération.

Il n'est pas postulé que les processus impliqués dans la résolution d’un problème donné sont séquentiels ou que tous les processus cités interviennent nécessairement dans la résolution d'un problème donné. Lorsque les individus abordent des problèmes authentiques inédits dans leur vie, qu'ils les structurent, qu'ils se les représentent et qu'ils les résolvent, ils peuvent s'orienter vers des solutions qui transcendent les limites des modèles linéaires, progressifs. La plupart des éléments dont nous disposons à propos du système de cognition confirment que le cerveau humain est capable de se livrer à des traitements d'informations parallèles (Lesh et Zawojewski, 2007). 
Dans le cadre de l'évaluation des compétences en résolution de problèmes du cycle PISA 2012, les processus suivants ont été identifiés :

- exploration et compréhension;

- représentation et formulation;

- planification et exécution; et

- suivi et réflexion.

Exploration et compréhension. Ce processus consiste à construire une représentation mentale de tous les fragments d'information présentés dans le problème. Il englobe les activités suivantes :

- explorer la situation du problème, c'est-à-dire l'observer, entrer en interaction avec elle et chercher des informations ainsi que des limites ou des obstacles; et

- comprendre les informations fournies et les informations découvertes durant les interactions avec la situation du problème, et comprendre les concepts pertinents.

Représentation et formulation. Ce processus consiste à construire une représentation mentale cohérente de la situation du problème (c'est-à-dire un modèle de la situation ou du problème). Pour ce faire, il faut sélectionner les informations pertinentes, les organiser mentalement et les combiner avec des connaissances antérieures pertinentes. II englobe les activités suivantes :

- représenter le problème via la conception de représentations graphiques, symboliques, verbales ou sous forme de tableaux, et naviguer entre les formats de représentations ; et

- formuler des hypothèses via l'identification des facteurs pertinents du problème et des relations entre eux, et via l'organisation et l'évaluation critique des informations.

Planification et exécution. Ce processus englobe les activités suivantes :

- planifier, c'est-à-dire fixer des objectifs, notamment clarifier l'objectif global et fixer des objectifs intermédiaires le cas échéant, et concevoir un plan ou une stratégie pour atteindre l'objectif fixé, notamment identifier les étapes à franchir pour y parvenir ; et

- exécuter le plan élaboré.

Suivi et réflexion. Ce processus englobe les activités suivantes :

- suivre les progrès sur la voie de l'objectif à chaque étape, notamment vérifier les résultats intermédiaires et finaux, détecter des événements imprévus et prendre des mesures correctives le cas échéant ; et

- réfléchir à des solutions sous divers angles, évaluer les hypothèses et d'autres solutions possibles de manière critique, identifier les informations manquantes, et clarifier et communiquer les progrès de façon adéquate.

\section{Facultés de raisonnement}

Tous les processus de résolution de problèmes dépendent d'une ou de plusieurs facultés de raisonnement. Les individus ont parfois à faire la distinction entre les faits et les opinions lorsqu'ils tentent de comprendre un problème, à identifier des relations entre variables lorsqu'ils formulent une solution, à identifier des causes et des effets lorsqu'ils sélectionnent une stratégie, et à organiser les informations de manière logique lorsqu'ils communiquent leurs résultats. Les facultés de raisonnement associées à ces processus sont au cœur de la résolution de problèmes. Elles sont importantes dans I'enquête PISA, car ce sont des compétences qui peuvent être inculquées et modélisées en classe (voir, par exemple, Adey et al., 2007 ; Klauer et Phye, 2008).

Les facultés de raisonnement qui interviennent dans la résolution de problèmes sont, par exemple, celles qui permettent de faire un raisonnement déductif, inductif, quantitatif, corrélationnel, analogique, combinatoire ou multidimensionnel. Ces facultés ne s'excluent pas les unes les autres et, dans la pratique, les individus en utilisent plusieurs lorsqu'ils recherchent des éléments et testent des cheminements vers la solution d'un problème avant de choisir une méthode plutôt qu'une autre pour le résoudre. Les facultés de raisonnement sont largement représentées dans les items, car la difficulté des items dépend de la complexité et du type de raisonnement à mener pour les résoudre. 


\section{ÉVALUATION DU NIVEAU DE COMPÉTENCE EN RÉSOLUTION DE PROBLÈMES Structure de l'épreuve}

L'épreuve informatisée de résolution de problèmes conçue en vue du cycle PISA 2012 dure 40 minutes. La batterie d'items de 80 minutes de test est divisée en quatre blocs d'items de 20 minutes. Dans les pays qui ont choisi de ne pas administrer les épreuves informatisées de mathématiques et de compréhension de l'écrit, les élèves répondront à deux des blocs, en fonction d'une rotation équilibrée. Dans les pays qui ont choisi d'administrer les épreuves informatisées de mathématiques et de compréhension de l'écrit, les élèves répondront à deux, un ou aucun des quatre blocs d'items, en fonction d'une rotation équilibrée distincte.

Comme le veut l'usage dans l'enquête PISA, les items sont regroupés en unités autour d'un stimulus commun qui décrit la situation du problème. Pour réduire le niveau de compétence requis en compréhension de l'écrit, les stimulus sont énoncés dans un langage aussi clair, simple et bref que possible (comme les tâches). Des animations, des images et des pictogrammes sont utilisés pour éviter de soumettre aux élèves des textes qui seraient trop longs. La difficulté en matière de calcul a également été réduite le plus possible, avec l'inclusion, le cas échéant, de totaux cumulés par exemple.

Il y a au total 16 unités, ce qui représente quelque 40 items répartis sur un spectre approprié de difficulté. Cette épreuve permettra d'identifier les forces et les faiblesses de groupes et de sous-groupes de la population concernant les processus cognitifs de résolution de problèmes.

\section{Fonctionnalité de l'informatisation de l'épreuve}

L'informatisation de l'épreuve de résolution de problèmes offre, entre autres avantages importants, la possibilité d'enregistrer des résultats intermédiaires et finaux, et de recueillir et d'analyser des données concernant les processus et les stratégies. Ce devrait être une contribution majeure du cycle PISA 2012 en matière d'évaluation du niveau de compétence en résolution de problèmes. Grâce à un référencement précis des items, des données concernant notamment la nature, la fréquence, la durée et l'enchaînement des actes des élèves pourront être recueillies.

L'épreuve ne demande que des compétences élémentaires en informatique, par exemple la capacité d'utiliser le clavier, la souris ou le pavé tactile, de cliquer sur des boutons, d'employer la commande " glisser-déposer », ou d'utiliser des menus déroulants et des hyperliens. Tout a été mis en œuvre pour faire en sorte que l'évaluation du niveau de compétence en résolution de problèmes soit le moins possible biaisée par la présentation des items et la nécessité d'utiliser l'informatique.

Les unités et les items qui les composent sont administrés dans un ordre déterminé, ce qui signifie que les élèves ne peuvent revenir à une unité ou à un item une fois qu'ils sont passés au suivant. À chaque fois que les élèves cliquent sur la flèche pour passer à l'unité ou à l'item suivant, une boîte de dialogue s'affiche pour les informer qu'ils ne pourront pas faire marche arrière. À ce moment, ils peuvent soit confirmer leur volonté de poursuivre, soit revenir à l'item qu'ils étaient sur le point de quitter.

L'interface de l'épreuve est la même dans tous les items (voir la figure 4.2) : le stimulus s'affiche dans la partie supérieure de l'écran et, séparée par un liseré, la question s'affiche dans la partie inférieure de l'écran. La division de l'écran en deux parties varie d'un item à l'autre, mais les élèves ne doivent jamais utiliser la barre de défilement pour afficher toutes les informations.

Dans le coin supérieur droit de l'écran, une barre indique le temps qu'il reste pour finir l'épreuve. Un autre indicateur de progression s'affiche dans le coin supérieur gauche : les items y sont groupés par unité et le numéro de l'item en cours est indiqué.

\section{Caractéristiques de l'épreuve et difficulté des items}

Dans l'ensemble, chaque item se concentre autant que possible sur un seul processus de résolution de problèmes. En conséquence, montrer que l'on reconnaît le problème suffit dans certains items et décrire la méthode à utiliser pour aboutir à la solution suffit dans certains autres. Dans beaucoup d'items toutefois, il est important de fournir la solution tout en adoptant une méthode efficace. Dans d'autres items, les élèves doivent évaluer les solutions proposées et choisir celle qui est la plus appropriée au problème posé. Inclure des items qui se concentrent sur un seul processus est approprié parce qu'en résolution de problèmes, les difficultés majeures pour la plupart des élèves tiennent à la représentation, à la planification et à l'autorégulation, alors que les cours donnent souvent la priorité à l'exécution (Mayer, 2003). 
Certains problèmes sont plus complexes en soi que d'autres (Funke et Frensch, 2007). De plus, un problème plus complexe signifie bien souvent qu'il est plus difficile à résoudre. Le tableau 4.1 résume les caractéristiques qui varient selon les items pour couvrir le spectre approprié de difficulté. Ces caractéristiques ne s'excluent pas mutuellement et constituent en quelque sorte un ensemble de quatre facteurs (Philpot et al., 2012) lors de I'analyse des problèmes administrés lors du cycle PISA 2012.

- Figure 4.2 -

L'interface de l'épreuve

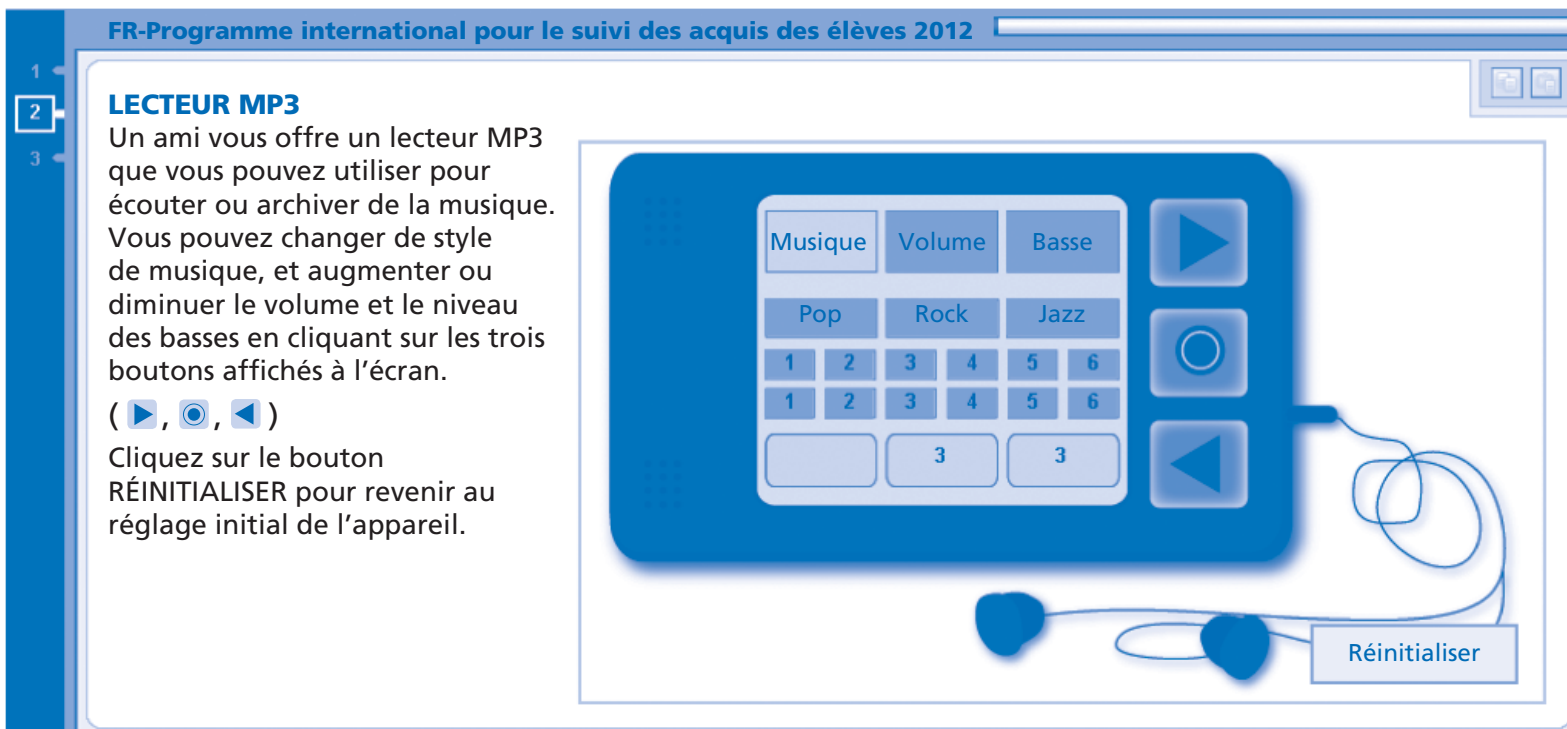

Question 1 : LECTEUR MP3 CP043Q03

Les réglages que vous avez choisis s'affichent dans les cases au bas du lecteur MP3. Déterminez si chacune des affirmations suivantes à propos du lecteur MP3 est vraie ou fausse.

Pour chaque affirmation, sélectionnez « Vrai » ou « Faux » pour indiquer votre réponse.

\begin{tabular}{l} 
Affirmation \\
II faut utiliser le bouton du milieu ( 0 ) pour changer de style de musique. \\
\hline II faut régler le volume avant de pouvoir régler le niveau des basses. \\
Une fois que l'on a augmenté le volume, on ne peut le diminuer que si l'on change \\
le style de musique que l'on écoute.
\end{tabular}

\section{Réponses : formats et codage}

Dans un tiers environ des items, les élèves doivent cocher une ou plusieurs cases, ou sélectionner une ou plusieurs options dans un menu déroulant pour y répondre. Il s'agit d'items à choix multiple simple, où une seule réponse est correcte, d'items à choix multiple complexe, où deux ou trois réponses distinctes doivent être sélectionnées, ainsi que des variantes de ces items, dans lesquels plusieurs réponses correctes doivent être sélectionnées dans une liste ou dans plusieurs menus déroulants. Tous ces items sont codés automatiquement.

Dans un peu plus de la moitié des items, les élèves doivent construire leur réponse d'une manière qui se prête à un codage automatique : ils doivent, par exemple, saisir un chiffre, faire glisser des formes, tracer des traits entre des points ou surligner la partie d'un diagramme.

Dans les autres items, les élèves doivent saisir leur réponse dans une zone de texte. Leurs réponses doivent être corrigées par des experts. Ces items sont utilisés lorsqu'il est jugé important de demander aux élèves d'expliquer le cheminement utilisé ou de justifier leur réponse.

Un système de codage en ligne a été créé pour faciliter le travail des correcteurs. Ce système, qui évite d'avoir à saisir des données, minimise le nettoyage des données et permet aux correcteurs de travailler où ils le veulent (pas nécessairement sur place). 
Les items peuvent valoir un crédit partiel le cas échéant, par exemple lorsque le crédit complet s'applique à plusieurs réponses correctes ou qu'une stratégie correcte est choisie, mais mal exécutée. Les comportements (les stratégies d'exploration, par exemple) qui démontrent de façon fiable le niveau de compétence en résolution de problèmes, audelà de la réponse aux items, sont enregistrés et interviennent dans le score.

Tableau 4.1

\section{Caractéristiques des tâches}

\begin{tabular}{|c|c|}
\hline Caractéristique & Impact sur la difficulté des tâches \\
\hline Volume d'informations & Plus il y a d'informations à prendre en considération, plus la tâche est susceptible d'être difficile. \\
\hline Représentation des informations & $\begin{array}{l}\text { Les représentations qui ne sont pas familières ou qui sont multiples (en particulier lorsqu'il y } \\
\text { a plusieurs façons différentes de représenter les informations fournies) tendent à accroître la } \\
\text { difficulté des tâches. }\end{array}$ \\
\hline Degré d'abstraction & $\begin{array}{l}\text { La mesure dans laquelle le scénario est abstrait ou concret influe sur le degré de difficulté d'une } \\
\text { tâche. Selon toute vraisemblance, plus un scénario est abstrait, plus une tâche est difficile. }\end{array}$ \\
\hline Degré de familiarité du contexte & $\begin{array}{l}\text { Si un contexte est familier aux élèves, ceux-ci auront l'impression qu'ils disposent des outils pour } \\
\text { résoudre le problème. }\end{array}$ \\
\hline Visibilité des informations & $\begin{array}{l}\text { Plus les élèves doivent découvrir des informations (l'effet d'opérations, le comportement } \\
\text { autonome, la présence d'obstacles imprévus), plus la tâche est susceptible d'être difficile. }\end{array}$ \\
\hline Complexité interne & $\begin{array}{l}\text { La complexité interne d'une tâche s'accroît avec l'augmentation du nombre de ses composantes } \\
\text { ou de ses éléments et de leur interdépendance (à cause de relations ou de contraintes). Les } \\
\text { tâches d'une plus grande complexité interne sont susceptibles d'être plus difficiles. }\end{array}$ \\
\hline Éloignement par rapport à l'objectif & Plus il y a d'étapes à franchir pour résoudre un problème, plus la tâche est susceptible d'être difficile. \\
\hline Facultés de raisonnement requises & $\begin{array}{l}\text { Le degré de difficulté de la tâche dépend de la complexité et du type de raisonnement à effectuer. } \\
\text { Les tâches qui requièrent un certain type de raisonnement (par exemple, le raisonnement } \\
\text { combinatoire) sont susceptibles d'être plus difficiles que les autres. }\end{array}$ \\
\hline
\end{tabular}

\section{Problèmes interactifs}

Les problèmes interactifs peuvent être basés sur des modèles formels sous-jacents dont les paramètres sont manipulés systématiquement pour entraîner la variation du degré de difficulté. Deux paradigmes sont utilisés : les équations différentielles linéaires et les automates finis.

Dans les situations de problème modélisées par des équations différentielles linéaires (aussi appelées équations structurelles linéaires) ${ }^{7}$, les individus doivent manipuler une ou plusieurs variables d'entrée (les commandes d’un climatiseur, par exemple) et constater les effets que cela a sur une ou plusieurs variables de sortie (la température ou I'humidité, par exemple). Les variables de sortie peuvent s'influencer les unes les autres de sorte que le système est dynamique. Parmi les situations pertinentes, citons celles en rapport avec les télécommandes, les thermostats, les mélanges de peintures et les écosystèmes.

Un automate fini est un système dont le nombre d'états, de signaux d'entrée et de signaux de sortie est fixe (Buchner et Funke, 1993) ${ }^{8}$. L'état suivant du système (et son signal de sortie) est uniquement déterminé par son état présent et le signal d'entrée spécifique. Dans les situations de problème basées sur ce modèle, les élèves doivent envoyer un signal d'entrée (généralement en actionnant des boutons) et déterminer l'effet que cela a sur l'état du système pour comprendre la structure du système et l'amener à l'état voulu. De nombreux appareils et contextes de la vie courante sont régis par les principes de l'automate fini, notamment les montres à affichage numérique, les téléphones mobiles, les fours à micro-ondes, les lecteurs MP3, les distributeurs automatiques de billets et les lave-linge.

Dans ce type de problèmes interactifs, tout l'enjeu est de faire ce qui suit (pour plus de précisions, voir Blech et Funke, 2005 ; Greiff et Funke, 2008) :

- Explorer : acquérir des connaissances sur la structure du système par le biais de l'exploration active ou dirigée (interaction). [Les stratégies d'exploration sont suivies et enregistrées grâce à l'informatisation de l'épreuve.]

- Identifier : formuler ou compléter la représentation du modèle mental du système élaboré durant l'exploration, sous forme graphique ou verbale. [La précision du modèle contribue à l'évaluation des connaissances causales qui ont été acquises.]

- Contrôler : c'est-à-dire appliquer concrètement les connaissances acquises - passer de l'état initial à l'état cible et (le cas échéant) perpétuer l'état cible dans le temps. Un modèle correct du système peut être fourni pour réduire la 
dépendance par rapport aux items précédents. [Le transfert de connaissances acquises est évalué par ce biais.]

- Expliquer : décrire les stratégies adoptées pour atteindre un objectif, expliquer le fonctionnement d'un système ou identifier les causes probablement à l'origine du dysfonctionnement d'un appareil.

Les élèves peuvent déjà avoir une idée plus ou moins précise des relations entre les variables d'un système s'ils sont familiarisés avec des systèmes similaires dans la vie réelle. Comme ces connaissances antérieures varient entre les élèves, un éventail de problèmes courants dans la vie réelle est utilisé pour atténuer cet effet lors de l'évaluation. De plus, quelques contextes moins courants, mais attrayants et ludiques sont inclus : les élèves ne peuvent inférer les relations que via la manipulation et l'observation des variables du système.

La difficulté des problèmes de ce type dépend largement de la complexité interne des modèles formels qui sous-tendent les situations. Le degré de difficulté des items peut être choisi via la manipulation systématique de cette complexité, qui est déterminée par le nombre de variables en cause et la mesure dans laquelle elles sont corrélées. Par exemple, un problème $n^{\prime}$ impliquant que quelques variables peut être très facile à résoudre si les effets entre les variables d'entrée et de sortie sont directs, mais extrêmement difficile si ces effets sont multiples et si les variables de sortie s'influencent mutuellement.

\section{Répartition des items}

La répartition du score entre les processus cognitifs de résolution de problèmes lors de la campagne définitive est indiquée dans le tableau 4.2. Les fourchettes recommandées par le groupe d'experts chargé de la résolution de problèmes sont indiquées entre parenthèses. Le coefficient le plus élevé est attribué au processus Planification et exécution, en raison de l'importance de la capacité à cheminer vers une solution pour mener une tâche à bien. Un coefficient inférieur à la moyenne est attribué au processus Suivi et réflexion, car il fait partie intégrante des trois autres processus et est, dès lors, également évalué (indirectement) dans les items qui se concentrent sur ces trois processus.

Tableau 4.2

Répartition approximative du score en résolution de problèmes entre les processus cognitifs

\begin{tabular}{c|c|c|c|c}
\hline $\begin{array}{c}\text { Exploration } \\
\text { et compréhension }\end{array}$ & $\begin{array}{c}\text { Représentation } \\
\text { et formulation }\end{array}$ & $\begin{array}{c}\text { Planification } \\
\text { et exécution }\end{array}$ & Suivi et réflexion & Total \\
\hline $\begin{array}{c}21.4 \% \\
(20-25 \%)\end{array}$ & $\begin{array}{c}23.2 \% \\
(20-25 \%)\end{array}$ & $\begin{array}{c}41.1 \% \\
(35-45 \%)\end{array}$ & $\begin{array}{c}14.3 \% \\
(10-20 \%)\end{array}$ & $100 \%$ \\
\hline
\end{tabular}

Le tableau 4.3 indique la répartition des items entre les deux autres grandes dimensions du domaine d'évaluation, en l'occurrence le contexte des problèmes et la nature de leur situation. Dans ce tableau également, les fourchettes recommandées par le groupe d'expert sont indiquées entre parenthèses. La priorité clairement accordée aux problèmes interactifs par rapport aux problèmes statiques (le ratio est de l'ordre de deux pour un) s'explique par la décision de concentrer l'épreuve sur ce type important de problèmes, avec l'avantage que grâce à l'informatique, il est possible de les inclure pour la première fois dans une évaluation internationale à grande échelle. Quant à la priorité accordée aux contextes technologiques par rapport aux contextes non technologiques, c'est une manière de reconnaître le rôle sans cesse croissant que jouent les applications technologiques dans la vie courante. De plus, ce type de contextes se prête bien à la simulation dans une épreuve informatisée.

Il a été conseillé de tendre à l'équilibre entre les contextes personnels et sociaux, sous réserve des contraintes qu'impose le respect des répartitions recommandées entre les grandes dimensions indiquées dans les tableaux 4.2 et 4.3. Les contextes personnels représentent 59 \% du score et les contextes sociaux, 41 \% du score lors de la campagne définitive.

Tableau 4.3

Répartition approximative du score en résolution de problèmes par type de situation et de contexte du problème*

\begin{tabular}{l|c|c|c}
\hline & Contexte technologique & Contexte non technologique & Total \\
\hline Situation statique & $11 \%$ & 20 & $31 \%$ \\
& $(10-15 \%)$ & $(25-35 \%)$ & $20 \%)$ \\
\hline Situation interactive & $45 \%$ & $25 \%$ & $(25-30 \%)$ \\
\hline Total & $(40-45 \%)$ & $45 \%$ & $(65-75 \%)$ \\
\hline
\end{tabular}

* La variation des totaux s'explique par les arrondis. 


\section{PRÉSENTATION DU NIVEAU DE COMPÉTENCE EN RÉSOLUTION DE PROBLÈMES}

Comme dans les autres domaines d'évaluation PISA, les résultats à l'épreuve de résolution de problèmes seront résumés sur une seule échelle de compétence combinée, dont la moyenne est fixée à 500 points et l'écart type, à 100 points.

Les tâches plus faciles se situent dans la partie inférieure de l'échelle, et les tâches plus difficiles, dans la partie supérieure de l'échelle. L'échelle est divisée en niveaux afin de montrer et résumer l'augmentation du degré de difficulté des items.

Six niveaux de compétence sont décrits pour montrer dans quelle mesure les savoir-faire des élèves en résolution de problèmes s'enrichissent et pour permettre la comparaison de la performance des élèves entre les pays et économies participants et au sein même de ceux-ci. Le nombre d'items est insuffisant pour rendre compte des résultats sur des sous-échelles.

Les compétences caractérisant la performance des élèves à chaque niveau de l'échelle seront décrites après analyse des savoirs et savoir-faire à mobiliser pour mener à bien les tâches correspondant à leur niveau et des caractéristiques de ces tâches (voir le tableau 4.1). Selon toute vraisemblance, ce sont les facultés suivantes qui caractériseront les élèves très performants :

- Capacité de planifier et exécuter des solutions qui impliquent de réfléchir à un certain nombre d'étapes à franchir et de respecter de nombreuses contraintes, d'utiliser des facultés aiguisées de raisonnement, de suivre les progrès dans la réalisation d'un objectif tout au long du processus de résolution et de changer de stratégie le cas échéant.

- Capacité de comprendre des fragments disparates d'information et d'établir des liens entre eux, même s'ils sont représentés sous des formes non familières.

- Capacité d'entrer systématiquement en interaction avec les problèmes et de découvrir à dessein des informations cachées.

Les élèves qui n'ont pas atteint un niveau de base de compétence se caractériseront vraisemblablement au mieux par les facultés suivantes :

- Capacité de planifier et exécuter des solutions impliquant un nombre limité d'étapes.

- Capacité de résoudre des problèmes comportant une ou deux variables et une seule, voire aucune contrainte.

- Capacité de formuler des principes simples et de découvrir des informations cachées lors d'une exploration désordonnée.

\section{SYNTHÈSE}

Avec le cycle PISA 2012, c'est la deuxième fois que la compétence en résolution de problèmes est évaluée. En 2003, une épreuve papier-crayon avait été administrée pour évaluer les compétences transdisciplinaires en résolution de problèmes. Comme l'épreuve administrée lors du cycle PISA 2012 est informatisée, il a été possible d'y inclure des items qui demandent aux élèves d'entrer en interaction avec les problèmes. De plus, les problèmes dont la résolution fait appel à des connaissances spécialisées en sont exclus pour privilégier l'évaluation des processus cognitifs fondamentaux de la résolution de problèmes.

Lors du cycle PISA 2012, les compétences en résolution de problèmes se définissent comme suit : « Les compétences en résolution de problèmes renvoient à la capacité d'un individu de s'engager dans un traitement cognitif pour comprendre et résoudre des problèmes, en l'absence de méthode de solution évidente, ce qui inclut sa volonté de s'engager dans de telles situations pour exploiter tout son potentiel de citoyen constructif et réfléchi. »

Les dimensions majeures qui ont été prises en considération lors de la conception des items sont le contexte du problème : technologique, non technologique, personnel ou social; la nature de la situation du problème : interactive ou statique ; et les processus de résolution de problèmes : exploration et compréhension, représentation et formulation, planification et exécution, et enfin, suivi et réflexion.

La nature de la situation dans laquelle le problème s'inscrit est dite statique si les informations dévoilées dès le début aux élèves sont complètes, et interactive si des interactions avec la situation du problème sont indispensables pour découvrir des informations permettant de résoudre le problème. Parmi les problèmes interactifs, citons à titre d'exemple ceux qui se posent souvent lorsqu'on utilise pour la première fois un appareil tel qu'un téléphone mobile ou un distributeur automatique de billets. Grâce à l'informatisation de l'épreuve, c'est la première fois que des problèmes interactifs ont pu être inclus dans une enquête internationale à grande échelle. 
Chaque item associé à son stimulus s'affiche sur un seul écran, et les élèves passent d'item en item dans un ordre déterminé, sans pouvoir revenir en arrière. Divers formats de réponse ont été utilisés, notamment des réponses à cocher ou à construire qui se prêtent à un codage automatique (comme les réponses " glisser-déposer », par exemple) et des réponses sous forme de texte libre à coder par des experts. Dans certains items, des données comportementales qui démontrent de façon fiable le niveau de compétence en résolution de problèmes sont enregistrées et interviennent dans le calcul du score. Des exemples d'items sont commentés dans la section suivante à titre d'illustration.

\section{EXEMPLES D'ITEMS PISA DE RÉSOLUTION DE PROBLÈMES}

Des items tirés de deux unités administrées lors de l'essai de terrain du cycle PISA 2012 sont décrits ci-dessous ${ }^{9}$. Chaque unité débute par une capture d'écran de son stimulus, accompagnée d'une description succincte de son contexte. Vient ensuite une capture d'écran de chaque item suivie de sa description.

\section{LECTEUR MP3}

- Figure 4.3 -

Lecteur MP3 : Stimulus

\section{LECTEUR MP3}

Un ami vous offre un lecteur MP3 que vous pouvez utiliser pour écouter ou archiver de la musique. Vous pouvez changer de style de musique, et augmenter ou diminuer le volume et le niveau des basses en cliquant sur les trois boutons affichés à l'écran.

\section{$(\nabla, 0,4)$}

Cliquez sur le bouton RÉINITIALISER pour revenir au réglage initial de l'appareil.

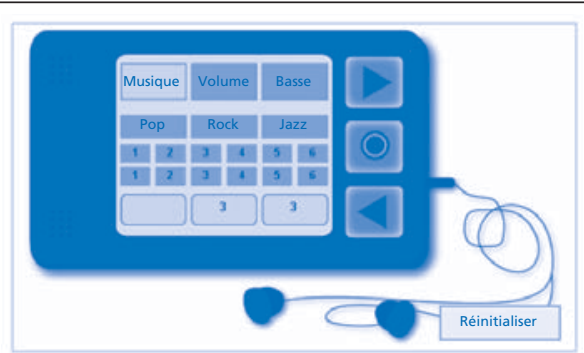

Dans I'unité LECTEUR MP3, les élèves apprennent qu'un ami leur a offert un lecteur MP3. Ils ne savent pas comment fonctionne un lecteur MP3 et doivent entrer en interaction avec l'appareil pour le découvrir, ce qui classe l'unité et les items qui la composent dans la catégorie des situations interactives. Comme l'unité consiste essentiellement à découvrir le mode d'emploi d'un appareil à usage individuel, I'unité et ses items se classent dans la catégorie de contextes technologiques et personnels.

- Figure 4.4 -

Lecteur MP3 : Item 1

\section{QUESTION 1}

Les réglages que vous avez choisis s'affichent dans les cases au bas du lecteur MP3. Déterminez si chacune des affirmations suivantes à propos du lecteur MP3 est vraie ou fausse.

Pour chaque affirmation, sélectionnez « Vrai » ou « Faux » pour indiquer votre réponse.

\begin{tabular}{lc} 
Affirmation & Vrai \\
\hline Il faut utiliser le bouton du milieu ( 0 ) pour changer de style de musique. \\
\hline Il faut régler le volume avant de pouvoir régler le niveau des basses. \\
\hline Une fois que l'on a augmenté le volume, on ne peut le diminuer que si l'on change le style de musique \\
que l'on écoute.
\end{tabular}

Dans le premier item de l'unité, une série d'affirmations sur le fonctionnement de l'appareil sont présentées aux élèves qui doivent dire si elles sont vraies ou fausses. Ces affirmations permettent aux élèves d'explorer l'appareil. Dans cet item, le processus de résolution de problèmes est celui qui consiste à explorer et comprendre. L'exploration est dirigée, mais pas limitée. Le bouton "Réinitialiser » permet aux élèves de revenir à tout moment au réglage initial de l'appareil et de recommencer leur exploration si besoin est, ce qu'ils peuvent faire autant de fois qu'ils le souhaitent. Lors de l'essai de terrain, cet item s'est révélé légèrement plus difficile que la moyenne : $38 \%$ des élèves ont obtenu un crédit complet ( Vrai », "Faux », «Faux »). Cela s'explique vraisemblablement par le fait que les trois réponses devaient être correctes et par la nécessité de découvrir des informations (aucune information n'est donnée d'emblée sur le fonctionnement du système de sorte que les élèves doivent se livrer à des interactions pour réunir tous les éléments le concernant). Le crédit partiel n'est pas applicable pour cet item. 


\section{QUESTION 2}

Réglez le lecteur MP3 sur : Rock, Volume 4, Basse 2.

Vous devez faire le moins de clics possible. Il n'y a pas de bouton RÉINITIALISER.

Le deuxième item de l'unité se classe dans la catégorie de processus Planification et exécution. Pour y répondre, les élèves doivent en effet élaborer un plan pour atteindre un objectif, puis l'exécuter. Cet item qui peut valoir un crédit partiel est intéressant, car les informations sur le cheminement des élèves enregistrées grâce à l'informatisation de l'épreuve interviennent dans le calcul du score. Dans cette tâche, il est demandé aux élèves de " faire le moins de clics possible ». De plus, les élèves n'ont pas la possibilité de cliquer sur le bouton " RÉINITIALISER » pour revenir au réglage initial de l'appareil. Le nombre de clics indique si les élèves ont atteint l'objectif d'une manière efficiente, soit 13 clics maximum, ce qui leur vaut un crédit complet. Un crédit partiel est attribué aux élèves qui ont atteint l'objectif d'une manière moins efficiente, soit plus de 13 clics. L'impératif d'efficacité ajoute à la difficulté de l'item, qui est légèrement plus difficile que la moyenne pour l'obtention d'un crédit complet, mais relativement facile pour l'obtention d'un crédit partiel. Lors de l'essai de terrain, 39 \% des élèves ont reçu un crédit complet et $33 \%$, un crédit partiel.

- Figure 4.6 -

Lecteur MP3 : Item 3

\section{QUESTION 3}

Voici quatre images de l'écran du lecteur MP3. Si le lecteur MP3 fonctionne correctement, il n'est en fait pas possible d'obtenir trois de ces écrans. L'écran restant représente, quant à lui, le lecteur MP3 quand il fonctionne correctement.

Quel est l'écran qui présente le lecteur MP3 fonctionnant correctement ?
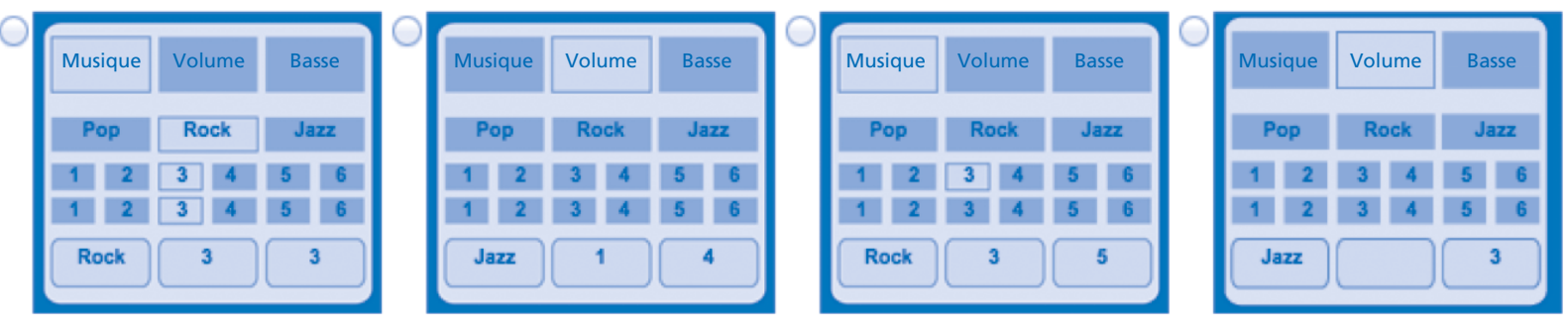

Le troisième item de l'unité se classe dans la catégorie de processus Représentation et formulation, car il demande aux élèves de se représenter mentalement la façon dont le système fonctionne globalement pour identifier laquelle des quatre options proposées indique un état possible de l'appareil. Ils peuvent réinitialiser l'appareil pour revenir au réglage initial, une possibilité qu'ils avaient dans le premier item, mais pas dans le deuxième. Ils peuvent donc entrer en interaction avec le système autant de fois que nécessaire, sans restriction. Le crédit partiel ne s'applique pas à cet item. L'essai de terrain a montré que le degré de difficulté de cet item était du même ordre que celui du premier item de I'unité : 39 \% des élèves y ont répondu correctement (option B).

- Figure 4.7 -

\section{Lecteur MP3 : Item 4}

\section{QUESTION 4}

Décrivez comment modifier la manière dont fonctionne le lecteur MP3, afin que l'on n'ait plus besoin du bouton inférieur $(4)$.

Il doit toujours être possible de changer de style de musique et d'augmenter ou de diminuer le volume et le niveau des basses.

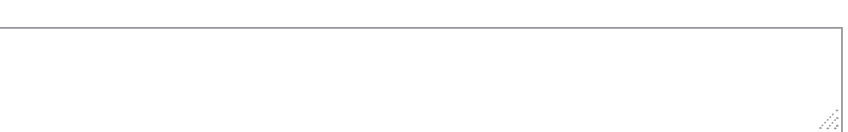


Le dernier item de l'unité se classe dans la catégorie de processus Suivi et réflexion. Il demande aux élèves de déterminer comment modifier le fonctionnement de l'appareil. Cet item est l'un des rares items à réponse construite ouverte et doit être codé par un expert. Le crédit complet s'applique aux réponses qui décrivent la manière dont le lecteur MP3 peut fonctionner avec un seul bouton. II n'y a pas qu'une seule réponse correcte : les élèves peuvent s'engager dans une réflexion créative pour trouver une solution, même si la solution la plus évidente est de modifier le fonctionnement du bouton du dessus pour qu'une fois dans la partie de droite de l'afficheur, I'utilisateur n'ait qu'un seul clic à faire pour revenir dans la partie de gauche de l'afficheur. Dans cette unité, c'est l'item de loin le plus difficile qui ait été administré lors de l'essai de terrain : $25 \%$ seulement des élèves y ont répondu correctement, ce qui s'explique assurément par le fait que cet item appelle une réponse construite et présente un certain degré d'abstraction. Les élèves doivent en effet imaginer un scénario hypothétique et le mettre en relation avec leur représentation mentale du mode actuel de fonctionnement de l'appareil pour décrire un autre mode de fonctionnement possible. Le crédit partiel n'est pas applicable à cet item.

\section{REPAS D'ANNIVERSAIRE}

- Figure 4.8 -

Repas d'anniversaire : Stimulus

\section{REPAS D'ANNIVERSAIRE}

C'est l'anniverssaire d'Alain et il organise un repas à cette occasion.

Sept autres personnes y participeront. Tous prendront place autour de la table pour le repas.

La disposition des invités autour de la table doit remplir les conditions suivantes:

- Alain et Ariane doivent être assis l'un à côté de l'autre.

- Boris et Barbara doivent être assis l'un à côté de l'autre.

- Charles doit être assis à côté de Delphine ou d'Émilie.

- Françoise doit être assise à côté de Delphine.

- Alain et Ariane ne doivent être assis ni à côté de Boris, ni à côté de Barbara.

- Boris ne doit pas être assis à côté de Charles ou Françoise.

- Delphine et Émilie ne doivent pas être assises côte à côte.

- Alain ne doit pas être assis à côté de Delphine ou d'Émilie.

- Ariane ne doit pas être assise à côté de Charles.

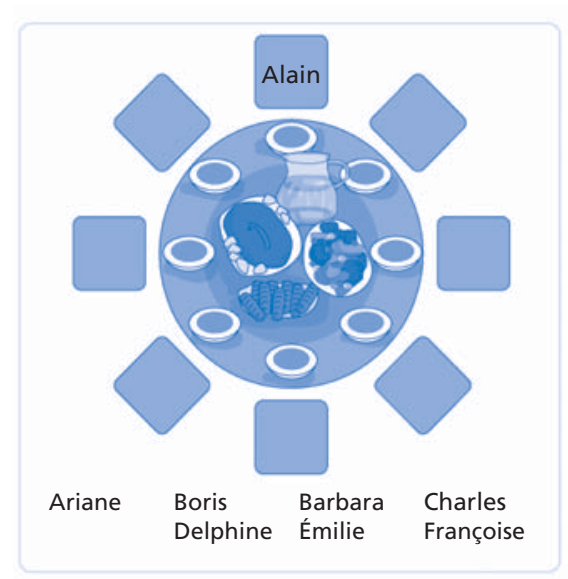

- Figure 4.9 -

Repas d'anniversaire : Item 1

\section{QUESTION 1}

Placez les invités autour de la table de façon à remplir toutes les conditions ci-dessus. Servez-vous de la fonction « glisserdéposer » pour installer les invités autour de la table.

Dans cette unité, c'est le seul item qui demande aux élèves d'utiliser la fonction " glisser-déposer », en l'occurrence pour placer des noms autour de la table dans le respect de neuf conditions. Cet item se classe dans la catégorie de processus Planification et exécution. Comme toutes les informations requises pour résoudre le problème sont fournies aux élèves dès le début, il se classe dans la catégorie des situations statiques. Cet item n'est statique qu'au sens de la définition de la nature de la situation dans laquelle s'inscrit le problème. L'informatisation de l'épreuve permet d'utiliser le format de réponse "glisser-déposer»: les élèves peuvent concevoir leur solution et la vérifier d'une façon nettement plus simple que dans une épreuve papier-crayon. Un crédit partiel s'applique à cet item. Les douze solutions qui respectent les neuf conditions valent un crédit complet (par exemple : «Alain - Ariane - Émilie - Boris -Barbara - Charles - Delphine - Françoise »). Un crédit partiel s'applique aux plans de table qui respectent huit conditions seulement sur les neuf indiquées. Par exemple, le plan de table «Alain - Ariane - Émilie - Boris - Barbara - Delphine - Françoise - Charles » ne satisfait pas à la condition "Charles doit être assis à côté de Delphine ou d'Émilie ». Lors de l'essai de terrain, 54 \% des élèves ont au moins obtenu le crédit partiel à cet item, et $43 \%$ des élèves, le crédit complet. La difficulté de l'item tient au grand nombre de conditions à respecter et aux facultés de raisonnement requises pour suivre et ajuster des solutions partielles en fonction des conditions pour aboutir à une solution complète. 


\section{Notes}

1. Dans l'enquête de l'OCDE sur les compétences des adultes, la résolution de problèmes dans des environnements à forte composante technologique se définit comme suit : "La résolution de problèmes dans des environnements à forte composante technologique implique l'utilisation de réseaux et d'applications des technologies de l'information et de la communication pour acquérir et évaluer des informations, communiquer avec autrui et effectuer des tâches concrètes » (Groupe d'experts en charge de la résolution de problèmes dans des environnements à forte composante technologique, PIAAC, 2009, p. 7).

2. «La résolution de problèmes renvoie à la capacité d'un individu de mettre en œuvre des processus cognitifs pour affronter et résoudre des problèmes posés dans des situations réelles, transdisciplinaires, dans des cas où le cheminement amenant à la solution n'est pas immédiatement évident et où les domaines de compétence ou les matières auxquels il peut être fait appel ne relèvent pas exclusivement d'un seul champ lié aux mathématiques, aux sciences ou à la compréhension de l'écrit »(OCDE, 2003a, p. 175).

3. Y compris dans la suite de leurs études ou dans leur vie professionnelle.

4. Le terme «construct » a volontairement été laissé en anglais, car il est d'usage courant dans la terminologie technique relative aux tests; il renvoie à la dimension latente que cherche à mesurer une épreuve grâce aux données observables que constituent les réponses des élèves.

5. L'adjectif « intransparent » est parfois utilisé pour caractériser les problèmes qui s'inscrivent dans une situation dont les informations ne sont pas disponibles dès le début (voir Funke et Frensch, 1995).

6. L'adjectif «dynamique » est utilisé par certains chercheurs pour caractériser tout système simulé avec lequel les individus peuvent entrer en interaction et dont ils peuvent recevoir des informations. Dans ce type de problèmes, les situations qui changent d'ellesmêmes sont parfois dites "à dynamique interne » (eigendynamic) (voir, par exemple, Blech et Funke, 2005).

7. Voir Greiff et Funke (2008), qui utilisent le terme MicroDYN pour décrire ces systèmes. Le terme de Dynamis a été utilisé auparavant (voir Blech et Funke, 2005).

8. L'automate fini a été mis en œuvre dans les évaluations sous le nom de MicroFin (voir http://www.psychologie.uni-heidelberg.de/ae/allg_en/forschun/probleml.html).

9. Les deux unités LECTEUR MP3 et REPAS D'ANNIVERSAIRE peuvent être consultées sur le site cbasq.acer.edu.au, à l'aide des données de connexion " public » et « access ». C'est le meilleur moyen d'apprécier la nature interactive de l'unité LECTEUR MP3. 


\section{Références}

Adey, P., B. Csapó, A. Demetriou, J. Hautamäki et M. Shayer (2007), « Can We be Intelligent about Intelligence? Why Education Needs the Concept of Plastic General Ability », Educational Research Review, n 2, pp. 75-97.

Baxter et R. Glaser (1997), An Approach to Analysing the Cognitive Complexity of Science Performance Assessments (Technical Report $\mathrm{n}^{\circ}$ 452), National Center for Research on Evaluation, Standards and Student Testing (CRESST), Los Angeles, Californie.

Blech, C. et J. Funke (2005), Dynamis Review: An Overview about Applications of the Dynamis Approach in Cognitive Psychology, Deutsches Institut für Erwachsenenbildung, Bonn, http://www.die-bonn.de/esprid/dokumente/doc-2005/blech05_01.pdf.

Blech, C. et J. Funke (2010), "You Cannot have Your Cake and Eat it, Too: How Induced Goal Conflicts Affect Complex Problem Solving », Open Psychology Journal, no 3, pp. 42-53.

Bransford, J.D., A.L. Brown et R.R. Cockling (éd.) (1999), How People Learn: Brain, Mind, Experience, and School, National Academy Press, Washington D.C.

Buchner, A. et J. Funke (1993), Finite-State Automata: Dynamic Task Environments in Problem-Solving Research, The Quarterly Journal of Experimental Psychology, vol. 46A, n 1, pp. 83-118.

Duncker, K. (1945), « On problem solving », Psychological Monographs, vol. 58, nº 3 (numéro complet : 270).

Frensch, P.A. et J. Funke (1995), " Definitions, Traditions, and a General Framework for Understanding Complex Problem Solving », in P.A. Frensch et J. Funke (éd.), Complex Problem Solving: The European Perspective, Hillsdale, NJ, Lawrence Erlbaum Associates, pp. 3-25.

Funke, J. (2010), « Complex Problem Solving: A Case for Complex Cognition? », Cognitive Processing, vol. 11, pp. 133-142.

Funke, J. et P.A. Frensch (2007), « Complex Problem Solving: The European Perspective - 10 Years After », in D.H. Jonassen (éd.), Learning to Solve Complex Scientific Problems, Lawrence Erlbaum, New York, New York, pp. 25-47.

Greiff, S. et J. Funke (2008), Indikatoren der Problemlöseleistung: Sinn und Unsinn verschiedener Berechnungsvorschriften. Bericht aus dem MicroDYN Projekt, Psychologisches Institut, Heidelberg.

Klauer, K. et G. Phye (2008), « Inductive Reasoning: A Training Approach », Review of Educational Research, vol. 78, n 1, pp. 85-123.

Klieme, E. (2004), « Assessment of Cross-Curricular Problem-Solving Competencies », in J.H. Moskowitz et M. Stephens (éd.), Comparing Learning Outcomes. International Assessments and Education Policy, Routledge Falmer, Londres, pp. 81-107.

Klieme, E., D. Leutner et J. Wirth (éd.) (2005), Problemlösekompetenz von Schülerinnen und Schülern: Diagnostische Ansätze, theoretische Grundlagen und empirische Befunde der deutschen PISA 2000 Studie, VS Verlag für Sozialwissenschaften, Wiesbaden.

Lesh, R. et J.S. Zawojewski (2007), " Problem Solving and Modelling », in F. Lester (éd.), The Handbook of Research on Mathematics Teaching and Learning ( $2^{\mathrm{e}}$ édition), National Council of Teachers of Mathematics, Reston, Virginie, et Information Age Publishing, Charlotte, Caroline-du-Nord (publication conjointe), pp. 763-804.

Leutner, D., E. Klieme, K. Meyer et J. Wirth (2004), « Problemlösen», in M. Prenzel et al. (Consortium PISA, Allemagne) (éd.), PISA 2003 : Der Bildungsstand der Jugendlichen in Deutschland - Ergebnisse des zweiten internationalen Vergleichs, Waxmann, Münster, pp. 147-175.

Leutner, D. et J. Wirth (2005), "What We Have Learned from PISA So Far: A German Educational Psychology Point of View », KEDI Journal of Educational Policy, vol. 2, n 2, pp. 39-56.

Mayer, R.E. (1990), "Problem Solving », in M.W. Eysenck (éd.), The Blackwell Dictionary of Cognitive Psychology, Basil Blackwell, Oxford, pp. 284-288.

Mayer, R.E. (1992), Thinking, Problem Solving, Cognition (2 édition), Freeman, New York, New York.

Mayer, R.E. (1998), « Cognitive, Metacognitive, and Motivational Aspects of Problem Solving », Instructional Science, vol. 26, pp. $49-63$.

Mayer, R.E. (2002), "A Taxonomy for Computer-Based Assessment of Problem Solving ", Computers in Human Behavior, vol. 18, pp. 623-632.

Mayer, R.E. (2003), Learning and Instruction, Merrill Prentice Hall, Upper Saddle River, New Jersey.

Mayer, R.E. et M.C. Wittrock (1996), « Problem-Solving Transfer », in R. Calfee et R. Berliner (éd.), Handbook of Educational Psychology, Macmillan, New York, pp. 47-62. 
Mayer, R.E. et M.C. Wittrock (2006), "Problem Solving », in P.A. Alexander et P.H. Winne (éd.), Handbook of Educational Psychology ( ${ }^{\mathrm{e}}$ édition), Lawrence Erlbaum Associates, Mahwah, New Jersey, chapitre 13.

OCDE (2003a), Cadre d'évaluation de PISA 2003 : Connaissances et compétences en mathématiques, lecture, science et résolution de problèmes, PISA, Éditions OCDE.

OCDE (2003b), The definition and Selection of Competencies (DeSeCo): Executive Summary of the Final Report, Éditions OCDE, www.oecd.org/dataoecd/47/61/35070367.pdf.

OCDE (2005), Résoudre des problèmes, un atout pour réussir - Premières évaluations des compétences transdisciplinaires issues de PISA 2003, PISA, Éditions OCDE.

O'Neil, H.F. (2002), "Perspectives on Computer-Based Assessment of Problem Solving », Computers in Human Behavior, vol. 18, pp. 605-607.

Osman, M. (2010), "Controlling Uncertainty: A Review of Human Behavior in Complex Dynamic Environments ", Psychological Bulletin, vol. 136, pp. 65-86.

Philpot, R., D. Ramalingam, J. Dossey et B. McCrae (2012), Dossier présenté lors du trentième congrès international de psychologie, Le Cap, du 22 au 27 juillet 2012.

PIAAC, Groupe d'experts en résolution de problèmes dans les environnements à forte composante technologique (2009), « PIAAC Problem Solving in Technology-Rich Environments: A Conceptual Framework », Documents de travail de l'OCDE sur l'éducation, $\mathrm{n}^{\circ} 36$, Éditions OCDE.

Polya, G. (1945), How to Solve It, Princeton University Press, Princeton, New Jersey.

Reeff, J.-P., A. Zabal et C. Blech (2006), The Assessment of Problem-Solving Competencies: A Draft Version of a General Framework, Deutsches Institut für Erwachsenenbildung, Bonn, www.die-bonn.de/esprid/dokumente/doc-2006/reeff06_01.pdf, consulté le 8 mai 2008.

Robertson, S.I. (2001), Problem Solving, Psychology Press, East Sussex.

Rychen D.S. et L.H. Salganik (éd.) (2003), Key Competencies for a Successful Life and a Well-Functioning Society, Hogrefe und Huber, Göttingen.

Vosniadou, S. et A. Ortony (1989), Similarity and Analogical Reasoning, Cambridge University Press, New York.

Wirth, J. et E. Klieme (2004), "Computer-Based Assessment of Problem Solving Competence ", Assessment in Education: Principles, Policy and Practice, vol. 10, nº 3, pp. 329-345. 


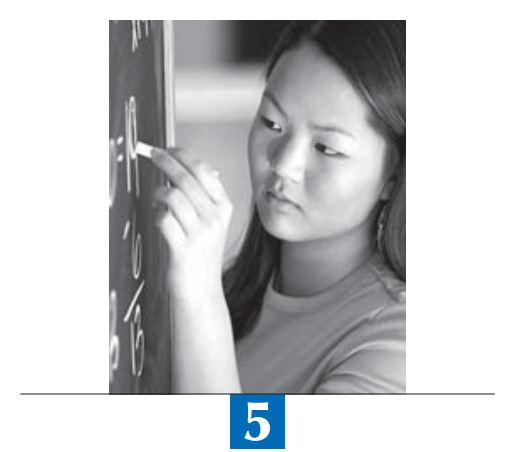

\section{Cadre d'évaluation de la culture financière du cycle PISA 2012}

Avec le cycle PISA 2012, c'est la première fois qu'une enquête internationale à grande échelle évalue la culture financière des adolescents. Ce cadre constitue une première étape sur la voie de l'élaboration d'une évaluation internationale de la culture financière : il décrit le canevas utilisé pour concevoir les items et structurer l'instrument, et propose un langage commun pour débattre de la culture financière. Ce cadre présente une définition opérationnelle de la culture financière et articule ce domaine autour des contenus, processus et contextes qui sont pertinents pour l'évaluer chez les élèves âgés de 15 ans. Les contenus décrits dans ce cadre sont l'argent et les transactions, la planification et la gestion des finances, le risque et le rendement, et le paysage financier, les processus qui y sont retenus sont identifier des informations financières, analyser des informations dans un contexte financier, évaluer des questions financières et appliquer des notions financières comprises et connues, dans des contextes scolaires et professionnels, ménagers et familiaux, individuels et sociétaux. Ces dimensions sont illustrées par 10 items. De plus, le cadre aborde les corrélations de la culture financière avec des compétences non cognitives, ainsi qu'avec la culture mathématique et la compréhension de l'écrit, tout en évaluant les expériences et les comportements des élèves par rapport aux matières financières. 


\section{INTRODUCTION}

\section{L'importance de la culture financière}

Depuis quelques années, les pays et économies développés ou émergents se préoccupent de plus en plus du niveau de culture financière de leurs citoyens, en raison de la contraction des systèmes publics et privés de soutien, de l'évolution démographique, dont le vieillissement de la population, et des développements importants intervenus sur les marchés financiers. Ils s'en préoccupent d'autant plus que la conjoncture économique et financière est difficile et qu'il est établi que le manque de culture financière compte parmi les facteurs qui amènent les citoyens à prendre des décisions financières en mauvaise connaissance de cause, ce qui est extrêmement lourd de conséquences (OCDE INFE, 2009 ; OCDE, 2009a ; voir également Gerardi et al., 2010, pour une analyse empirique de la culture financière et de la défaillance sur les prêts hypothécaires). Dans ce contexte, la culture financière est désormais considérée dans le monde comme un facteur important de développement et de stabilité économique et financière, comme en témoigne la récente adoption par le G20 des "Principes de haut niveau sur les stratégies nationales d'éducation financière " du Réseau international de l'OCDE sur l'éducation financière (G20, 2012 ; OCDE INFE, 2012).

Un certain nombre de tendances tangibles expliquent l'intérêt croissant dans le monde pour la culture financière, élevée au rang de compétence clé dans la vie. Ces tendances peuvent se résumer comme suit.

\section{Déplacement du risque}

Le risque s'est massivement déplacé des gouvernements et des employeurs vers les individus. De nombreux gouvernements ont réduit le financement public des prestations de retraite ou sont en passe de le faire, et certains d'entre eux revoient à la baisse les prestations de soins de santé. Les régimes de retraite à cotisations définies remplacent rapidement les régimes à prestations définies, déléguant ainsi aux travailleurs la responsabilité d'épargner pour garantir leur sécurité financière une fois retraités. Aux régimes traditionnels de retraite par répartition viennent s'ajouter de nouveaux régimes qui soumettent les individus au risque lié au rendement et à l'investissement. La plupart des enquêtes montrent qu'une majorité des travailleurs ne sont pas conscients des risques auxquels ils sont exposés et qu'ils manquent des connaissances et compétences requises pour bien gérer ces risques, même lorsqu'ils savent qu'ils existent (OCDE, 2008). De plus, les risques qui ont des implications financières se multiplient : les individus s'exposent, par exemple, aux risques liés à la longévité, au crédit, aux marchés financiers et à leur contribution personnelle au coût des soins de santé.

\section{Augmentation de la responsabilité individuelle}

Les individus doivent prendre de plus en plus de décisions financières à cause de l'évolution des marchés et de l'économie. L'augmentation de l'espérance de vie implique, par exemple, que les individus doivent se constituer une épargne qui couvrira une période de retraite nettement plus longue. Les citoyens assument également une plus grande part de responsabilité dans le financement de leurs soins de santé personnels et familiaux. De plus, avec l'accroissement des frais de scolarité, il est important que les parents programment avec soin leur investissement dans l'éducation de leurs enfants. Même lorsque les individus recourent aux services de conseillers ou d'intermédiaires financiers, ils doivent comprendre ce qui leur est proposé ou conseillé. Ils sont responsables du produit financier qu'ils décident d'acheter et devront assumer toutes les conséquences de leur choix. Ils doivent posséder une certaine culture financière pour prendre des décisions responsables en toute connaissance de cause.

\section{Élargissement de l'offre de produits et services financiers}

En outre, un nombre croissant de consommateurs ont accès dans tous les pays à un large éventail de produits et services financiers fournis par différents opérateurs via divers canaux. L'amélioration de l'inclusion financière dans les pays émergents, le progrès technologique et la dérégulation ont entraîné l'élargissement de l'accès à toutes sortes de produits financiers, des comptes courants aux crédits renouvelables, en passant par les transferts et les portefeuilles d'actions. Les produits gagnent aussi en complexité, et les individus doivent comparer un certain nombre de facteurs, tels que les frais, les taux d'intérêt applicables, la durée des contrats et le degré d'exposition au risque. Ils doivent aussi identifier des prestataires et des canaux appropriés dans le large éventail d'options, notamment les associations communautaires, les institutions financières traditionnelles, les banques en ligne et les opérateurs de téléphonie mobile.

\section{Accroissement de la demande de produits et services financiers}

L'évolution économique et technologique a entraîné l'amélioration de la connectivité mondiale et a conduit à d'énormes changements dans les communications et les transactions financières, ainsi que dans les interactions sociales et le comportement des consommateurs. Avec ces mutations, il est plus important que les individus soient capables d'entrer en interaction avec les prestataires de services financiers. Souvent, les consommateurs doivent avoir accès 
aux prestataires (les banques ainsi que d'autres opérateurs comme La Poste) pour recevoir des paiements et faire des transactions financières (salaires, virements, transactions en ligne), mais aussi pour faire des transactions directes avec des entreprises qui n'apprécient plus guère le liquide et les chèques. Les individus qui n'ont pas accès à ces services paient souvent plus cher pour les transactions en liquide, car ils utilisent des services financiers informels, comme ceux proposés par des prêteurs sur gages ou les encaisseurs de chèques (voir, par exemple, Kempson et al.,2005).

Toutes ces tendances ont transféré la responsabilité de décisions financières majeures aux individus. Parallèlement, elles ont élargi l'éventail d'options à la disposition de la majorité des individus (y compris les nouveaux consommateurs financiers) et ont accru la complexité à laquelle ils doivent faire face. Dans ce contexte, on attend des individus qu'ils aient suffisamment de culture financière pour prendre les mesures qui s'imposent pour se protéger, eux et leurs proches, et assurer leur bien-être financier.

\section{Avantages à attendre de l'éducation financière et de l'amélioration du niveau de culture financière}

II ressort d'éléments empiriques que les adultes qui ont reçu une éducation financière sont par la suite plus susceptibles que d'autres d'épargner en prévision de leur retraite, tant dans les économies développées que dans les économies émergentes (Bernheim et al., 2001 ; Cole et al.,2011; Lusardi, 2009). Ces éléments suggèrent l'existence d'une relation causale entre l'éducation financière et ces résultats, et donnent à penser que l'amélioration du niveau de culture financière peut entraîner un changement positif de comportement.

D'autres études, en grande partie menées dans des pays développés, en particulier aux États-Unis, ont identifié un certain nombre d'avantages potentiels de la culture financière. De plus en plus d'éléments montrent que les individus possédant un niveau plus élevé de culture financière sont plus à même de gérer leur argent et sont plus susceptibles d'investir sur les marchés boursiers, de tirer un meilleur rendement de leur portefeuille et de choisir des fonds communs de placement dont les commissions sont moins élevées (Hastings et Tejeda-Ashton, 2008 ; Hilgert et al., 2003 ; Lusardi et Mitchell, 2008, 2011 ; Stango et Zinman, 2009 ; van Rooij et al., 2011 ; Yoong, 2011). De surcroît, les individus qui possèdent de meilleures connaissances financières sont plus susceptibles d'accumuler davantage de richesses (Lusardi et Mitchell, 2011).

Il est établi que l'élévation du niveau de culture financière est en corrélation non seulement avec la constitution d'actifs, mais aussi avec l'endettement et sa gestion : les individus dont le niveau de culture financière est plus élevé optent pour des emprunts immobiliers moins chers et évitent de payer des taux d'intérêt élevés et des frais supplémentaires (Gerardi et al., 2010 ; Lusardi et Tufano, 2009a, 2009b ; Moore, 2003).

La culture financière procure non seulement des avantages aux individus, mais elle contribue aussi à la stabilité économique et financière à plusieurs égards. Les consommateurs qui possèdent une certaine culture financière peuvent prendre des décisions en meilleure connaissance de cause et exigent des services de qualité supérieure, ce qui attise la concurrence et dope l'innovation sur le marché. Ils sont aussi moins susceptibles de réagir de façon imprévisible aux conditions commerciales et de faire des réclamations sans fondements, et plus susceptibles de prendre les mesures qui conviennent pour gérer les risques qui leur ont été délégués. Tous ces facteurs se conjuguent pour améliorer l'efficience du secteur des services financiers et réduire la nécessité d'une réglementation et d'une surveillance coûteuses des marchés financiers. Ils peuvent aussi contribuer à réduire le budget que les pouvoirs publics consacrent à aider ceux qui ont pris de mauvaises décisions financières ou qui n'en ont pas prises du tout.

\section{Activités de I'OCDE en matière d'éducation financière}

En 2002, I'OCDE a lancé un vaste projet d'éducation financière en réponse à l'inquiétude grandissante des gouvernements au sujet des conséquences possibles de niveaux peu élevés de culture financière. Ce projet est géré par le Comité des assurances et des pensions privées (CAPP) et le Comité des marchés financiers (CMF) de l'OCDE, en coordination avec d'autres instances concernées, notamment le Comité des politiques de l'éducation (CPE). C'est une approche holistique qui a été retenue concernant les questions de consommation financière dans le cadre de ce projet qui consiste à montrer en quoi l'éducation financière peut contribuer à promouvoir la culture financière parallèlement à l'amélioration de l'accès aux services financiers et à l'adoption de cadres adéquats en matière de régulation et de protection des consommateurs.

L'une des premières étapes du projet d'éducation financière a été franchie avec l'adoption du texte Recommendation on Principles and Good Practices for Financial Education and Awareness par le Conseil de l'OCDE (OCDE, 2005a). En plus de ces recommandations, la publication Improving Financial Literacy: Analysis of Issues and Policies explique pourquoi 
il est important de se concentrer sur l'éducation financière et propose un premier aperçu international des travaux menés en la matière dans divers pays (OCDE, 2005b). Cet ouvrage présente également des principes et de bonnes pratiques pour éclairer les responsables politiques et d'autres parties prenantes désireux d'améliorer le niveau de culture financière dans leur pays. II est complété par un centre mondial d'information sur l'éducation financière, I'International Gateway for Financial Education (www.financial-education.org) de l'OCDE, qui compile les données, les ressources, les études et les actualités concernant l'éducation financière et les programmes en la matière dans le monde.

Consciente de la nature de plus en plus internationale des questions d'éducation et de culture financières, l'OCDE a créé en 2008 le Réseau international sur l'éducation financière (International Network on Financial Education, INFE) pour regrouper l'expérience et l'expertise d'économies développées et émergentes, et en tirer parti. À ce jour, plus de 220 institutions publiques de plus d'une centaine de pays ont rallié I'INFE. Les membres du réseau se rencontrent deux fois par an pour évoquer les derniers développements dans leur pays et recueillir des données, élaborer des études comparatives et analytiques, des méthodologies, des bonnes pratiques, des instruments au service de l'action publique et des orientations concrètes dans des domaines de première importance. L'OCDE et son réseau ont déclaré prioritaires les programmes d'éducation financière à l'école et l'évaluation internationale de la culture financière. Des sous-groupes d'experts ont été créés pour lancer la collecte de données ciblées et mener les travaux de recherche.

\section{Éducation financière des jeunes en milieu scolaire}

La thématique de l'éducation financière des jeunes, plus précisément en milieu scolaire, n'est pas neuve. Comme nous I'avons vu, la culture financière est de plus en plus considérée comme une compétence essentielle dans la vie. En 2005 déjà, l'OCDE préconisait dans ses recommandations que l'éducation financière commence à l'école et que les individus soient éduqués aux matières financières dès le plus jeune âge (OCDE, 2005a). Deux raisons majeures sous-tendent ces recommandations : I'importance de cibler la jeunesse et l'efficience de l'éducation financière en milieu scolaire. L'OCDE et I'INFE ont également élaboré des lignes directrices sur l'éducation financière qui ont reçu le soutien des ministres des Finances de la Coopération économique Asie-Pacifique (APEC) en août 2012 et qui devaient être publiées à la fin de l'année $2012^{1}$.

\section{Cibler la jeunesse}

Les générations plus jeunes sont non seulement susceptibles d'être confrontées à la complexité croissante des produits, des services et des marchés financiers, mais elles sont aussi plus susceptibles que leurs parents de s'exposer à des risques financiers plus importants à l'âge adulte. Elles sont plus susceptibles, par exemple, d'avoir à assumer davantage de responsabilité dans la planification de leur épargne et de leurs investissements en vue de leur retraite, ainsi que dans le financement de leurs soins de santé ; et elles auront affaire à des produits financiers plus diversifiés et plus complexes.

En raison de l'évolution des marchés et des systèmes de protection sociale (en particulier des régimes de retraites), les générations actuelles ne sont pas susceptibles de pouvoir apprendre des générations passées. Elles devront se baser sur leurs propres connaissances ou, au vu de la complexité des nouveaux systèmes, suivre de façon avisée les conseils financiers prodigués par des professionnels. Le manque d'exposition précoce des individus à l'éducation financière et la méconnaissance des avantages à poursuivre leur éducation financière peuvent compromettre gravement les efforts consentis pour améliorer les connaissances financières sur le lieu de travail ou dans d'autres cadres. Dans ce contexte, il est important d'offrir aux individus la possibilité de jeter les bases de leur culture financière quand ils sont jeunes.

L'éducation financière à l'école permet non seulement de préparer les jeunes à vivre leur vie d'adulte, mais également de les aider à résoudre des problèmes financiers qu'ils rencontrent dès à présent. Les enfants consomment souvent des services financiers dès leur plus jeune âge. Il n'est pas rare de voir des enfants être titulaires d'un compte bancaire qui leur permet de faire des transactions en ligne ou utiliser leur téléphone mobile pour effectuer des paiements, et ce même avant l'adolescence. Des compétences financières leur seraient de toute évidence utiles dans ce cadre. Les jeunes encore scolarisés peuvent avoir à prendre des décisions dans divers domaines, par exemple choisir une police d'assurance pour leur véhicule ou des produits d'épargne, ou gérer leur découvert bancaire.

Dans de nombreux pays, les jeunes qui ont entre 15 et 18 ans environ doivent prendre (avec leurs parents) l'une des décisions financières les plus importantes de leur vie : ils doivent en effet décider de poursuivre ou non des études. L'écart salarial entre les travailleurs selon qu'ils sont ou non diplômés de l'enseignement tertiaire s'est creusé dans de nombreuses économies. Parallèlement, le coût des études à charge des étudiants et de leur famille a augmenté, ce qui les oblige souvent à se tourner vers un crédit (OCDE, 2011). Selon les chiffres publiés en mars 2010 au Royaume-Uni, la moitié des étudiants britanniques devraient terminer leurs études endettés de plus de 15000 GBP (Smithers, 2010). 
Il est important que les individus possèdent une certaine culture financière avant qu'ils ne s'engagent dans des contrats et des transactions financières de grande ampleur. Dispenser dès le plus jeune âge des programmes d'éducation financière de qualité est essentiel pour inculquer aux individus de bonnes connaissances financières et leur apprendre à adopter un bon comportement en la matière, ce qui leur sera utile par la suite (Ministerial Council for Education, Early Childhood Development and Youth Affairs [Australie], 2011).

\section{L'efficience de I'éducation financière en milieu scolaire}

Des études donnent à penser qu'il existe un lien entre la culture financière des individus et le niveau de formation et le statut économique de leurs parents. Les individus qui possèdent une plus grande culture financière sont plus nombreux à être nés de parents très instruits qui utilisent un large éventail de produits financiers (Lusardi et al.,2010). Pour parvenir à l'égalité des chances, il est important de dispenser une éducation financière à ceux qui n'y auraient pas accès autrement. Les établissements d'enseignement sont en bonne position pour améliorer la culture financière dans tous les groupes de la population et réduire les écarts de culture financière (y compris entre les générations).

Conscients de l'importance de la culture financière pour les jeunes et du potentiel unique des programmes scolaires permettant d'améliorer les connaissances et les compétences des générations futures, de plus en plus de pays s'attèlent à l'élaboration de programmes d'éducation financière. Ces programmes s'adressent à tous les jeunes en général ou sont dispensés par les établissements d'enseignement. Certains sont dispensés à l'échelle nationale, régionale ou locale, voire à titre expérimental. Selon une enquête sur les programmes d'éducation financière financée par la Commission européenne (Habschick et al., 2007), la plupart des programmes s'adressent aux enfants et aux jeunes. Il ressort par ailleurs d'un large inventaire lancé par le sous-groupe de l'INFE en charge de l'éducation financière en milieu scolaire que des programmes d'éducation financière sont dispensés en milieu scolaire dans 21 des 32 pays/territoires à l'étude (OCDE, à paraître).

\section{La nécessité de recueillir des données}

Les responsables politiques, les professionnels de l'éducation et les chercheurs ont besoin de données de qualité sur le niveau de culture financière leur permettant d'identifier les priorités et de mesurer l'évolution au fil du temps dans le but d'éclairer leurs stratégies d'éducation financière et de mettre sur pied des programmes d'éducation financière en milieu scolaire.

Plusieurs pays ont mené des enquêtes nationales pour évaluer la culture financière de leur population adulte, tandis que I'OCDE a récemment testé un questionnaire permettant de déterminer le niveau de culture financière des adultes dans le cadre d'un projet international (Atkinson et Messy, 2012 ; OCDE INFE, 2011). Toutefois, il n'y a à ce jour que peu $d^{\prime}$ initiatives visant à évaluer la culture financière des individus de moins de 18 ans, et aucune n'est comparable entre les pays. C'est une grave lacune dans la mesure où les jeunes deviendront vite des adultes qui auront à prendre des décisions financières majeures toujours plus complexes. II est indispensable de disposer de données sur leur capacité à relever ces défis pour enrichir nos connaissances et déterminer dans quelle mesure les jeunes sont préparés à évoluer dans des environnements économiques en constante mutation.

L'évaluation probante de la culture financière des jeunes fournira à l'échelle nationale des informations indiquant si l'approche actuelle en matière d'éducation financière est efficace. Elle pourra en particulier contribuer à identifier des lacunes à combler par les établissements d'enseignement ou au travers de l'organisation d'activités extrascolaires pour former les jeunes, de manière appropriée et équitable, à prendre des décisions financières à l'âge adulte. Elle pourra également servir de référence pour évaluer l'efficacité des programmes scolaires et autres, et les modifier à l'avenir le cas échéant.

Une étude internationale a d'autres avantages pour les responsables politiques et autres parties prenantes. Comparer le niveau de culture financière entre les pays permet de découvrir les pays où ce niveau est le plus élevé et de commencer à identifier tant les bonnes pratiques que les stratégies nationales qui se révèlent particulièrement efficaces. Il sera également possible d'identifier des problèmes communs et d'étudier la possibilité de trouver des solutions internationales pour les résoudre.

Dans ce contexte, la collecte de données fiables et comparables à l'échelle internationale sur la culture financière des effectifs scolarisés fournira aux responsables politiques, aux professionnels de l'éducation, aux concepteurs des programmes de cours, aux chercheurs et autres :

- des informations sur les lacunes des jeunes pour concevoir des politiques et des programmes d'éducation financière plus ciblés ;

- des informations pour déterminer si le fait de dispenser une éducation financière en milieu scolaire est en corrélation avec une élévation du niveau de culture financière ; 
- un moyen de comparer les stratégies d'éducation financière entre les pays ;

- la possibilité d'explorer de bonnes pratiques via l'analyse du classement des pays en fonction de leur niveau de culture financière ; et

- la possibilité de comparer les données dans le temps, pour évaluer l'impact des initiatives d'éducation financière dans les établissements d'enseignement et identifier des pistes pour les améliorer en permanence.

L'évaluation internationale de la culture financière présente d'autres avantages. L'élaboration d'un cadre d'évaluation de la culture financière applicable à tous les pays permet de proposer aux autorités nationales des lignes directrices détaillées sur la portée et la définition opérationnelle de la culture financière sans qu'elles aient à financer des études à l'échelle de leur pays. Comme le souligne l'article " Financial Literacy and Education Research Priorities ", le manque de données dans la littérature sur la culture financière s'explique notamment par le fait que les chercheurs ne s'accordent pas sur la façon de définir et d'évaluer l'efficacité des programmes. Les chercheurs doivent s'accorder sur une définition sans équivoque de ce que signifie posséder une « culture financière » (Schuchardt et al., 2009).

\section{L'évaluation de la culture financière dans l'enquête PISA}

Avec le cycle PISA 2012, c'est la première fois qu'une enquête internationale à grande échelle évalue la culture financière des jeunes. L'enquête PISA cherche à déterminer dans quelle mesure les adolescents qui approchent du terme de leur scolarité obligatoire sont préparés à relever les défis que l'avenir leur réserve, en particulier leur capacité à utiliser leurs savoirs et savoir-faire, en recueillant et en analysant des données cognitives et autres sur les jeunes âgés de 15 ans dans de nombreux pays et économies. Elle permet donc de fournir un riche ensemble de données comparatives que les responsables politiques et autres parties prenantes peuvent utiliser pour prendre des décisions éclairées. Les données sur la culture financière qui sont comparables entre les pays offrent la possibilité de répondre à des questions telles que "Dans quelle mesure les jeunes sont-ils préparés à évoluer dans de nouveaux systèmes financiers qui se mondialisent et qui deviennent de plus en plus complexes ? » ou «Qui sont les jeunes qui possèdent la plus grande culture financière? ».

Comme dans les domaines majeurs d'évaluation PISA, à savoir la compréhension de l'écrit, la culture mathématique et la culture scientifique, la priorité des épreuves PISA de culture financière est de déterminer dans quelle mesure les élèves âgés de 15 ans sont capables d'appliquer leurs savoirs et savoir-faire en la matière. Et comme dans les autres domaines PISA, la culture financière est évaluée au travers de l'administration d'épreuves conçues pour fournir des données valides, fiables et exploitables.

La première étape de l'élaboration d'une évaluation conforme à ces trois grands critères consiste à rédiger un cadre d'évaluation. La rédaction d'un cadre d'évaluation présente avant tout l'avantage d'améliorer la précision de la mesure, car elle permet de concevoir un plan en fonction duquel élaborer chaque item et créer des épreuves à administrer pour couvrir le domaine d'évaluation. Autre avantage, le cadre d'évaluation propose un langage commun, ce qui permet de mieux comprendre ce qui est évalué. Il a également le mérite de promouvoir l'analyse des savoirs et savoir-faire associés à la culture financière, ce qui permet d'étayer la ou les échelles de culture financière qui seront utilisées pour interpréter les résultats.

L'élaboration des cadres d'évaluation PISA s'inscrit dans un processus au cours duquel les six étapes suivantes s'enchaînent :

- I'élaboration d'une définition opérationnelle du domaine d'évaluation et la description des hypothèses qui soustendent cette définition ;

- I'identification d'une série de caractéristiques majeures à prendre en considération lors de la conception des épreuves qui seront administrées à l'échelle internationale ;

- I'opérationnalisation des caractéristiques majeures qui seront utilisées pour concevoir les épreuves, sur la base de définitions de la littérature et d'expériences dans l'administration d'évaluations à grande échelle ;

- I'analyse de la façon d'organiser les tâches pour rendre compte aux responsables politiques et aux chercheurs de la performance des élèves âgés de 15 ans dans chaque domaine d'évaluation ;

- la validation des variables et l'évaluation de la contribution de chacune d'entre elles à la compréhension de la difficulté des tâches dans les pays participants ; et

- la préparation de l'échelle de compétence permettant de décrire les résultats. 


\section{DÉFINITION DE LA CULTURE FINANCIÈRE}

Lors de l'élaboration d'une définition opérationnelle de la culture financière qui puisse être utilisée pour préparer une évaluation internationale de la culture financière, le groupe d'experts a étudié les définitions retenues dans les autres domaines d'évaluation PISA et les aspects s'articulant autour de l'éducation financière.

Dans l'enquête PISA, la notion de « culture » renvoie à la capacité des élèves à appliquer des savoirs et des savoir-faire dans les domaines d'évaluation et à analyser, raisonner et communiquer lorsqu'ils énoncent, résolvent et interprètent des problèmes dans divers contextes. L'enquête PISA est prospective, dans la mesure où elle cherche à déterminer dans quelle mesure les adolescents sont capables d'appliquer leurs connaissances et compétences pour relever des défis qui se présentent au quotidien, et non à évaluer simplement s'ils maîtrisent telle ou telle matière du programme de cours (OCDE, 2010a).

Dans sa publication Recommendation on Principles and Good Practices for Financial Education and Awareness, I'OCDE définit l'éducation financière comme « le processus par lequel des consommateurs/investisseurs améliorent leur connaissance des produits, concepts et risques financiers, et acquièrent, au moyen d'une information, d'une instruction ou d'un avis objectif, les compétences et la confiance nécessaires pour devenir plus sensibles aux risques et opportunités en matière financière, faire des choix raisonnés, savoir où trouver une assistance et prendre d'autres initiatives efficaces pour améliorer leur bien-être financier »(OCDE, 2005a).

Le groupe d'experts en charge de l'éducation financière a recueilli un consensus au sujet des termes « connaissance » et " compétences», et de la notion d'utilisation des connaissances et des compétences (« initiatives efficaces »), qui sont des éléments majeurs de cette définition. II reconnaît toutefois que la définition de l'éducation financière décrit un processus - l'éducation - plutôt qu'un résultat. Or, le cadre d'évaluation requiert une définition qui inclut le résultat de ce processus en termes de compétence ou de « culture».

La définition opérationnelle de la culture financière retenue lors du cycle PISA 2012 est la suivante :

La culture financière renvoie à la connaissance et à la compréhension des concepts et risques financiers ainsi qu'aux compétences, à la motivation et à la confiance nécessaires pour utiliser cette connaissance et cette compréhension pour prendre des décisions fondées dans un large éventail de contextes financiers, pour améliorer le bien-être financier des individus et de la société, et pour participer activement à la vie économique.

Cette définition se divise en deux parties, comme celle des autres domaines PISA. La première partie renvoie au type de raisonnement et de comportement qui caractérise le domaine et la seconde, aux finalités qui sous-tendent le développement de cette culture particulière.

Les paragraphes suivants expliquent chaque élément de la définition de la culture financière retenue lors du cycle PISA 2012 pour en clarifier le sens par rapport à l'évaluation.

\section{La culture financière...}

Le terme culture est considéré ici au sens d'enrichir des connaissances, des compétences et des stratégies, ce que les individus font tout au long de leur vie, et non dans le sens d'une quantité fixe, d'une ligne de démarcation qui séparerait les individus cultivés des individus incultes. La culture implique plus que la simple reproduction de connaissances acquises, même si l'évaluation des connaissances financières antérieures est un élément important des épreuves. Elle implique également une mobilisation de compétences cognitives et pratiques, ainsi que d'autres ressources, telles que les attitudes, la motivation et les valeurs. Lors du cycle PISA 2012, l'évaluation de la culture financière porte sur un éventail de connaissances et de compétences associées au développement de la capacité à répondre à des exigences financières de la vie quotidienne dans la société contemporaine.

\section{... renvoie à la connaissance et la compréhension des concepts et risques financiers...}

La culture financière dépend donc de la connaissance et de la compréhension d'éléments fondamentaux du monde de la finance, dont des concepts financiers majeurs et l'objet et les caractéristiques principales des produits financiers. Cela inclut également les risques qui peuvent menacer le bien-être financier, ainsi que les polices d'assurance et les retraites. Selon toute vraisemblance, les adolescents de 15 ans commencent à acquérir ces connaissances et à découvrir le monde de la finance dans lequel eux-mêmes et leur famille évoluent, et les principaux risques auxquels ils s'exposent. Tous les adolescents de cet âge sont susceptibles d'avoir fait des achats pour le ménage ou pour eux-mêmes ; certains auront déjà pris part à des discussions en famille sur l'argent et sur des besoins qu'il est indispensable ou possible financièrement 
de satisfaire ; et un nombre non négligeable d'entre eux auront déjà commencé à gagner de l'argent et à en épargner. Certains élèves ont déjà découvert des produits et des engagements financiers via leur compte bancaire ou un contrat avec un opérateur de téléphonie mobile. S'ils ne l'ont pas déjà fait, ils devront bien vite maîtriser des concepts tels que I'intérêt, l'inflation ou la valeur de l'argent, car c'est important pour leur bien-être financier.

... ainsi qu'aux compétences...

Parmi ces compétences, citons les processus cognitifs génériques qui consistent à accéder à l'information, à comparer et à confronter, à extrapoler et à évaluer - dans un contexte financier. En relèvent les compétences élémentaires en mathématiques, par exemple la faculté de calculer un pourcentage ou de convertir un montant dans une autre devise, ainsi que les compétences en compréhension de l'écrit, par exemple la faculté de lire et d'interpréter des textes publicitaires ou des contrats.

\section{... à la motivation et à la confiance...}

La culture financière renvoie non seulement à la connaissance, à la compréhension et aux compétences nécessaires pour traiter des questions financières, mais également à des attributs non cognitifs : la motivation à l'idée de chercher des informations et des conseils pour s'engager dans des activités financières, la confiance en ses capacités d’y parvenir et la faculté de gérer les facteurs émotionnels et psychologiques qui interviennent dans la prise de décisions financières. Ces attributs sont considérés comme un objectif de l'éducation financière et sont essentiels pour inculquer des savoirs et des savoir-faire dans les matières financières.

... nécessaires pour utiliser cette connaissance et cette compréhension pour prendre des décisions fondées...

Dans l'enquête PISA, la priorité va à la capacité de se baser sur des notions comprises et d'activer et d'appliquer des connaissances dans des situations de la vie courante, et non à la reproduction des acquis. Dans l'évaluation de la culture financière, cela signifie mesurer la capacité des adolescents à transférer et appliquer ce qu'ils ont appris à propos des finances personnelles pour prendre des décisions fondées. L'expression « décisions fondées » désigne des décisions responsables prises en toute connaissance de cause pour répondre à tel ou tel besoin.

\section{... dans un large éventail de contextes financiers...}

Des décisions financières fondées s'appliquent dans un large éventail de contextes financiers en rapport non seulement avec ce que vivent les adolescents, mais également avec les étapes qu'ils sont susceptibles de franchir dans un avenir proche, en tant qu'adultes. Les jeunes sont susceptibles de prendre des décisions relativement simples, par exemple choisir leur opérateur de téléphonie mobile ou la façon d'utiliser leur argent de poche, mais ils seront très vite amenés à prendre des décisions majeures au sujet de leurs études ou de leur orientation professionnelle, décisions qui ont des implications financières à long terme.

\section{... pour améliorer le bien-être financier des individus et de la société...}

Dans I'enquête PISA, la culture financière se conçoit essentiellement comme une culture financière personnelle, qui se distingue de la culture économique où interviennent des concepts plus vastes, tels que la théorie de l'offre et de la demande, les structures commerciales, etc. La culture financière renvoie à la façon dont les individus comprennent, gèrent et programment leurs affaires financières et celles de leur ménage - souvent leur famille. Il est établi, cependant, que le fait que les individus comprennent, gèrent et programment bien leurs affaires financières a un impact sur la société, dans la mesure où cela contribue à la stabilité, à la productivité et au développement à l'échelle nationale, voire mondiale.

\section{... et pour participer activement à la vie économique.}

Comme la définition des autres domaines d'évaluation PISA, celle de la culture financière souligne l'importance du rôle des individus en tant que membres réfléchis et engagés de la société. Les individus qui possèdent une grande culture financière sont mieux armés pour prendre des décisions dans leur intérêt, ainsi que pour critiquer et contribuer de façon constructive au monde économique dans lequel ils vivent.

\section{ORGANISATION DU DOMAINE}

La façon dont le domaine se conçoit et s'organise détermine la structure de l'évaluation, dont l'élaboration des items, et, en fin de compte, les éléments qui peuvent être recueillis et présentés au sujet de la performance des élèves. La culture financière comporte de nombreux aspects qui ne peuvent pas tous être pris en considération et manipulés dans une évaluation telle que celle proposée dans l'enquête PISA. Dans ce contexte, il convient de sélectionner les aspects les 
plus appropriés pour concevoir une évaluation qui contienne des tâches dont le degré de difficulté varie et qui couvre largement le domaine défini.

L'analyse des approches et principes retenus dans des enquêtes antérieures à grande échelle, en particulier dans I'enquête PISA, montre que ce sont les contenus, les processus et les contextes qui priment la plupart du temps, car ces aspects spécifient ce qui est évalué. Les contenus, les processus et les contextes peuvent être considérés comme trois perspectives différentes au sujet du domaine d'évaluation, comme le montre la figure 5.1 .

- Figure 5.1 -

Modèle conceptuel d'organisation du domaine d'évaluation

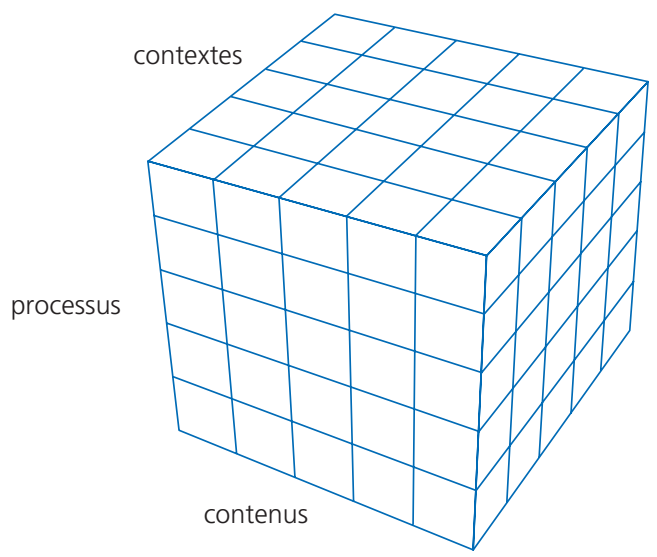

Les contenus renvoient à ce qu'il est essentiel de connaître et comprendre dans le domaine d'évaluation.

Les processus décrivent les approches ou stratégies mentales à adopter pour aborder les tâches et les mener à bien.

Les contextes désignent les situations dans lesquelles les savoirs et les savoir-faire sont appliqués. Les situations vont de la sphère personnelle à la sphère globale.

L'identification et la pondération des différents éléments ou catégories sous chaque perspective, puis la conception d'une série de tâches qui reflètent ces catégories sont autant d'étapes qui permettent de garantir la validité des épreuves et la couverture appropriée du domaine d'évaluation. Les trois perspectives sont également utiles pour réfléchir à la manière de rendre compte de la performance des élèves.

La section suivante décrit les trois perspectives et leur répartition entre les catégories du cadre d'évaluation. Elle présente les types de tâches que les élèves peuvent être amenés à effectuer. Des exemples d'items tirés des épreuves administrées lors de l'essai de terrain du cycle PISA 2012 sont proposés pour illustrer les perspectives et leurs catégories. Ces items n'ont pas été administrés lors de la campagne définitive, mais ils sont représentatifs de ceux qui l'ont été : seuls des items non publiés ont été administrés pour protéger l'intégrité des données recueillies pour évaluer la performance des élèves.

\section{Contenus}

Les contenus associés à la culture financière renvoient à ce que les élèves doivent connaître et comprendre pour mener à bien une tâche particulière. L'analyse des contenus retenus dans les cadres de culture financière d'un grand nombre de pays (I'Afrique du Sud, I'Angleterre, I'Australie, le Brésil, l'Écosse, les États-Unis, I'Irlande du Nord, le Japon, la Malaisie, la Nouvelle-Zélande et les Pays-Bas) révèle l'existence d'un consensus au sujet des contenus qui sont associés à la culture financière (OCDE, à paraître). Les données analysées montrent en particulier que les contenus des programmes d'éducation financière dispensés en milieu scolaire sont relativement similaires - même s'il existe des différences culturelles - et qu'il est possible d'identifier une série de thématiques souvent retenues dans ces cadres. Ces thématiques sont les quatre contenus de la culture financière dans l'enquête PISA : I'argent et les transactions, la planification et la gestion des finances, le risque et le rendement, et le paysage financier.

\section{L'argent et les transactions}

Cette catégorie inclut le fait de connaître les différentes formes et finalités de l'argent, et de savoir effectuer des transactions monétaires simples, par exemple faire un paiement ou une dépense, connaître la valeur de l'argent, les 
cartes bancaires, les chèques, les comptes bancaires et les devises. Dans les tâches associées à cette catégorie, les élèves sont, par exemple, amenés à montrer :

- Qu'ils connaissent les différentes formes et finalités de l'argent, ce qui consiste à :

- reconnaître les billets et les pièces de monnaie ;

- comprendre que l'argent sert dans les échanges de biens et services ;

- identifier différentes façons de payer des articles, en personne ou en ligne ;

- savoir qu'il existe différentes façons de recevoir de l'argent d'autrui et de transférer de l'argent entre personnes ou organisations; et

- comprendre que l'argent peut s'emprunter ou se prêter, et connaître les raisons pour lesquelles des intérêts se paient ou se perçoivent.

- Qu'ils sont capables de gérer et de suivre des transactions et qu'ils sont confiants en leur capacité d’y parvenir, ce qui consiste à :

- utiliser du liquide, des cartes bancaires et d'autres moyens de paiement pour faire des achats ;

- utiliser des distributeurs automatiques pour retirer du liquide ou obtenir le solde d'un compte ;

- calculer correctement le montant à rendre ;

- identifier parmi deux articles de taille différente lequel est le meilleur marché, en tenant compte des spécificités de situations et besoins individuels; et

- vérifier des transactions reprises sur un extrait de compte et relever toute irrégularité.

L'item suivant, qui est tiré de l'unité $A \cup M A R C H E ́$, illustre les tâches qui demandent aux élèves d'appliquer le concept de la valeur de l'argent. Dans cette question, comme dans de nombreuses autres, le zed est une devise fictive. Les items PISA font souvent référence à des situations qui ont pour cadre un pays fictif, la Zedlande, dont la monnaie est le zed. Ce stratagème (dont les élèves sont informés au début des épreuves) est appliqué pour améliorer la comparabilité entre les pays.

\section{$1^{\text {er }}$ exemple d'item PISA de culture financière - AU MARCHÉ}

- Figure 5.2 .

\section{Item de l'unité $A U$ MARCHÉ}

Vous pouvez acheter des tomates au kilo ou à la cagette.

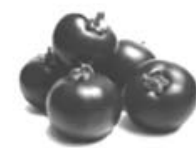

2,75 zeds le $\mathrm{kg}$

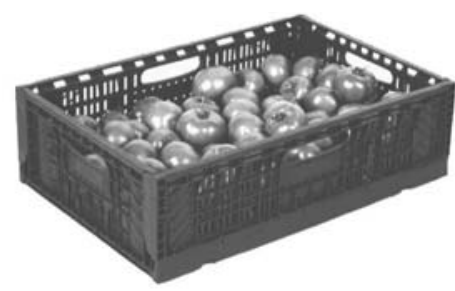

22 zeds la cagette de $10 \mathrm{~kg}$

\section{QUESTION}

La cagette de tomates coûte moins cher que les tomates en vrac.

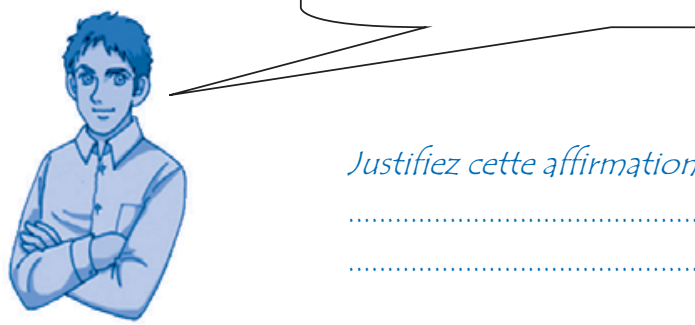


Dans un contexte de la vie courante, en l'occurrence faire les courses, cet item aborde la notion de la valeur de l'argent. Les items concernant les achats se classent généralement dans la catégorie de contenus L'argent et les transactions. Pour obtenir un crédit à cet item, les élèves doivent montrer qu'ils ont comparé les deux prix des tomates sur la base d'un critère commun. Parmi les réponses qui valent un crédit, citons à titre d'exemple les suivantes :

- Les tomates coûtent 2,75 zeds/kg en vrac, mais seulement 2,2 zeds/kg en cagette.

- Parce que $10 \mathrm{~kg}$ de tomates en vrac coûteraient 27,50 zeds.

- On achète plus de tomates par zed en cagette.

Lors de l'essai de terrain, trois quarts des élèves ont réussi à analyser les informations et à expliquer que le prix au kilo des tomates en cagette est inférieur au prix au kilo des tomates en vrac.

\section{La planification et la gestion des finances}

Il faut planifier et gérer ses revenus et sa richesse à court et long terme. Cette catégorie de contenu inclut ce qui suit :

- Connaître et pouvoir suivre ses revenus et ses dépenses :

- identifier divers types de revenus (prestations, salaires, commissions, avantages, salaire horaire, revenus nets et bruts); et

- établir un budget pour planifier ses dépenses régulières et son épargne.

- Connaître et pouvoir utiliser ses revenus et d'autres ressources à court et long terme pour garantir son bien-être financier :

- comprendre comment manipuler divers éléments d'un budget, par exemple identifier des priorités si les revenus sont inférieurs aux dépenses prévues ou trouver des solutions pour réduire ses dépenses ou accroître ses revenus pour augmenter son épargne ;

- évaluer l'impact de différents programmes de dépenses et être capable d'identifier des priorités en matière de dépense à court et long terme ;

- planifier des dépenses futures, par exemple calculer combien il faut épargner chaque mois pour financer un achat donné ;

- comprendre les raisons de contracter un emprunt et la façon dont les dépenses peuvent être étalées dans le temps au travers de l'emprunt ou de l'épargne ;

- Comprendre l'idée de se constituer un patrimoine, l'impact de l'intérêt composé sur l'épargne, et les avantages et inconvénients des produits d'investissement ;

- comprendre les avantages de l'épargne pour réaliser des objectifs à long terme ou anticiper des changements de situation (par exemple prendre son indépendance) ; et

- comprendre l'impact de la fiscalité et des prestations sociales sur la planification et la gestion des finances.

L'exemple suivant est tiré de l'unité PRIORITÉS. II illustre les tâches de la catégorie La planification et la gestion des finances dans un contexte pertinent pour les adolescents de 15 ans qui pensent à la vie qu'ils mèneront dans un avenir proche.

$2^{\mathrm{e}}$ exemple d'item PISA de culture financière - PRIORITÉS

- Figure 5.3 =

Item de l'unité PRIORITÉS

Claire et ses amis louent une maison.

Ils travaillent tous depuis deux mois.

Ils n'ont pas d'économies.

Leur salaire est mensuel et ils viennent de le recevoir.

Ils ont rédigé cette liste de tâches à faire.

À faire

- S'abonner à la télévision par câble

- Payer le loyer

- Acheter des meubles de jardin 
QUESTION

Parmi les tâches de la liste, lesquelles doivent être traitées à temps par Claire et ses amis?

Pour chaque tâche, entourez « Oui » ou « Non ».

\begin{tabular}{l|l}
\multicolumn{1}{c|}{ Tâche } & Cette tâche doit-elle être traitée à temps par Claire et ses amis ? \\
\hline S'abonner à la télé par câble. & Oui / Non \\
\hline Payer le loyer. & Oui / Non \\
\hline Acheter des meubles de jardin. & Oui / Non \\
\hline
\end{tabular}

Cet item de l'unité PRIORITÉS demande aux élèves d'évaluer les priorités dans les dépenses de la maison dans le cadre d'un budget et de faire la distinction entre des souhaits et des besoins. C'est la raison pour laquelle il se classe dans la catégorie La planification et la gestion des finances. Lors de l'essai de terrain, plus de trois quarts des élèves ont obtenu le crédit complet (soit, dans l'ordre, les réponses « Non », « Oui » et « Non ») : sur les trois tâches, seule celle qui consiste à payer le loyer doit être traitée à temps par Claire et ses amis.

Un autre exemple d'item de la catégorie La planification et la gestion des finances est proposé ci-après. Il s'agit du 9e exemple d'item, tiré de l'unité PARTIR EN VACANCES, qui demande aux élèves de planifier leurs économies et leurs dépenses pour financer une dépense à l'avenir.

\section{Le risque et le rendement}

Le contenu Le risque et le rendement est un contenu majeur de la culture financière. Il englobe la capacité d'identifier des moyens de gérer, compenser et couvrir des risques, et de comprendre le potentiel de perte ou de gain financier dans divers contextes financiers. II existe deux types de risques très importants dans ce domaine. Le premier a trait aux pertes financières qu'un individu ne peut assumer, à cause de coûts considérables ou répétés, par exemple, et le second, au risque inhérent aux produits financiers (les crédits à taux d'intérêt variable, par exemple) ou aux produits d'investissement.

Cette catégorie inclut les savoirs et savoir-faire suivants :

- Comprendre que certains processus (comme l'épargne) et produits financiers (y compris d'assurance) peuvent être utilisés pour gérer et réduire divers risques (selon les besoins et la situation) :

- savoir comment évaluer si une assurance peut être utile.

- Connaître les façons de gérer le risque, par exemple les avantages de la diversification et le danger des défauts de paiement de facture ou de crédit, et appliquer les connaissances en la matière pour prendre des décisions concernant :

- la limitation du risque au capital personnel ;

- les divers types d'investissement et d'épargne, notamment les produits financiers et d'assurance formels le cas échéant; et

- les divers types de crédit, y compris le crédit formel ou informel, garanti ou non garanti, rotatif ou à durée fixe, et à taux d'intérêt variable ou non.

- Connaître et gérer les risques et avantages associés à des événements de la vie, à l'économie et à d'autres facteurs externes, notamment l'impact potentiel :

- de la perte ou du vol d'objets personnels, du licenciement, de la naissance ou de l'adoption d'un enfant, ou de la dégradation de l'état de santé ;

- de la fluctuation des taux d'intérêt et de change ; et

- d'autres variations sur le marché.

- Connaître les risques et avantages associés à des substituts de produits financiers, par exemple :

- épargner du liquide, acheter des biens immobiliers, du bétail ou de l'or ; et

- emprunter à des prêteurs informels.

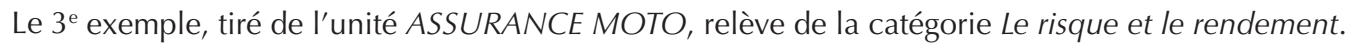


$3^{e}$ exemple d'item PISA de culture financière - ASSURANCE MOTO

- Figure 5.4 แ

Item de l'unité ASSURANCE MOTO

L'année dernière, la moto de Stéphane était assurée auprès de la compagnie d'assurance PANASSURE.

La police d'assurance couvrait les dommages causés à la moto en cas d'accident et le vol du véhicule.

\section{QUESTION}

Cette année, Stéphane souhaite renouveler son assurance après de PANASSURE, mais plusieurs facteurs ont changé depuis l'année dernière.

En quoi les facteurs présentés dans le tableau vont-ils vraisemblablement affecter le tarif de l'assurance moto de stéphane cette année?

Pour chaque facteur, entourez « Augmentation du tarif », « Réduction du tarif » ou « Sans effet sur le tarif ».

\begin{tabular}{|c|c|}
\hline Facteur & $\begin{array}{l}\text { En quoi ce facteur va-t-il vraisemblablement affecter } \\
\text { I'assurance de Stéphane? }\end{array}$ \\
\hline $\begin{array}{l}\text { Stéphane a remplacé son ancienne moto par une moto plus } \\
\text { puissante. }\end{array}$ & Augmentation du tarif / Réduction du tarif / Sans effet sur le tarif \\
\hline Stéphane a repeint sa moto d'une couleur différente. & Augmentation du tarif / Réduction du tarif / Sans effet sur le tarif \\
\hline $\begin{array}{l}\text { Stéphane a été responsable de deux accidents de la route } \\
\text { l'année dernière. }\end{array}$ & Augmentation du tarif / Réduction du tarif / Sans effet sur le tarif \\
\hline
\end{tabular}

Cet item de l'unité ASSURANCE MOTO relève de la catégorie de contenus Le risque et le rendement, car l'assurance est un produit spécifiquement conçu pour protéger les individus contre les risques et les pertes financières qu'ils seraient autrement incapables de supporter. Pour répondre à cette question, les élèves doivent s'appuyer sur leur compréhension d'une notion, en l'occurrence que plus le risque auquel ils s'exposent est élevé en fonction de critères mesurables, plus I'assurance qui couvre ce risque leur coûtera cher. Lors de l'essai de terrain, la moitié des élèves ont obtenu un crédit complet : le premier et le troisième facteur entraînent une augmentation du tarif de l'assurance, mais le deuxième facteur est sans effet.

Le $6^{e}$ exemple, qui est tiré de l'unité ACTIONS, relève lui aussi de la catégorie de contenus Le risque et le rendement. Pour répondre à cet item, les élèves doivent être familiarisés avec le fonctionnement d'un produit qui peut être risqué.

\section{Le paysage financier}

Ce contenu renvoie à la nature et aux caractéristiques du monde de la finance. Relève de cette catégorie le fait de connaître les droits et devoirs des consommateurs sur les places financières et dans l'environnement financier en général ainsi que les grandes implications des contrats financiers. Les sources $d^{\prime}$ information et la réglementation sont également des thématiques qui s'inscrivent dans cette catégorie. Au sens le plus large, connaître le paysage financier, c'est aussi comprendre les conséquences de changements dans la conjoncture économique et dans l'action des pouvoirs publics, par exemple la variation des taux d'intérêt, l'inflation, la fiscalité et les prestations sociales. Pour mener à bien les tâches associées à cette catégorie, les élèves doivent :

- Connaître les droits et devoirs des uns et des autres, et appliquer leurs connaissances en la matière pour :

- comprendre que les vendeurs et les acheteurs ont des droits, et qu'ils peuvent notamment demander réparation ;

- comprendre que les vendeurs et les acheteurs ont des responsabilités, notamment :

. que les consommateurs et les investisseurs doivent fournir des informations exactes lorsqu'ils sollicitent des produits financiers ;

. que les opérateurs doivent divulguer toutes les informations factuelles; et

- que les consommateurs et les investisseurs doivent être conscients des implications que peut avoir le non-respect des droits et devoirs dans le chef de l'une des deux parties.

- reconnaître l'importance de la documentation officielle fournie lors de l'achat de produits ou services financiers, et la nécessité d'en comprendre le contenu. 
- Connaître et comprendre l'environnement financier, notamment :

- identifier les opérateurs dignes de confiance ainsi que les produits et services protégés par la réglementation ou la législation relative à la protection des consommateurs ;

- savoir à qui s'adresser pour obtenir des conseils lors du choix de produits financiers ou pour obtenir de l'aide sur des sujets financiers; et

- avoir connaissance des délits financiers, tels que l'usurpation d'identité et l'escroquerie, et savoir prendre les précautions appropriées.

- Connaître et comprendre l'impact de décisions financières, y compris sur autrui :

- comprendre que les individus ont le choix en matière de dépense et d'épargne, et que tout acte a des conséquences pour les individus et la société ; et

- reconnaître que des habitudes, des décisions et des actes financiers ont un impact à l'échelle individuelle, communautaire, nationale et internationale.

- Connaître l'influence de facteurs économiques et de facteurs externes :

- être conscients de la conjoncture économique et comprendre l'impact de la réorientation de l'action publique, par exemple d'une réforme concernant le financement de la formation postscolaire ;

- comprendre en quoi la capacité de s'enrichir ou d'accéder au crédit dépend de facteurs économiques tels que les taux d'intérêt, l'inflation et la solvabilité ; et

- comprendre qu'un certain nombre de facteurs externes, tels que la publicité et la pression des pairs, peuvent influer sur les choix financiers des individus.

Le 4 exemple d'item, qui est tiré de l'unité ERREUR BANCAIRE, porte sur le paysage financier, car il traite de la criminalité financière.

$4^{e}$ exemple d'item PISA de culture financière - ERREUR BANCAIRE

- Figure 5.5 -

Item de l'unité ERREUR BANCAIRE

David est client chez ZedBanque. II reçoit ce courrier électronique.

Cher client,

Une erreur s'est produite sur le serveur de la ZedBanque et vos identifiants de connexion à votre compte en ligne ont été perdus.

Vous ne pouvez donc plus accéder à votre compte en ligne.

Par ailleurs, votre compte n'est plus sécurisé.

Veuillez cliquer sur le lien ci-dessous et suivre les instructions pour restaurer l'accès à votre compte. Pour cela, vous devrez fournir les identifiants de votre compte en ligne

https://ZedBank.com

\section{QUESTION}

Parmi les conseils suivants, lesquels sont pertinents pour David?

Pour chaque conseil, entourez « Oui » ou « Non ».

\begin{tabular}{l|c}
\hline $\begin{array}{l}\text { Répondre au courrier électronique en communiquant les identifiants } \\
\text { de son compte en ligne. }\end{array}$ & Oui / Non \\
\hline $\begin{array}{l}\text { Contacter sa banque pour demander davantage d'informations } \\
\text { concernant ce courrier électronique. }\end{array}$ & Oui / Non \\
\hline $\begin{array}{l}\text { Si le lien est identique à l'adresse du site Internet de sa banque, } \\
\text { cliquer sur le lien et suivre les instructions. }\end{array}$ & Oui / Non \\
\hline
\end{tabular}


Les services bancaires en ligne s'inscrivent dans le cadre plus vaste du paysage financier dans lequel les élèves sont susceptibles d'évoluer, aujourd'hui ou dans un avenir proche. Dans cet environnement, ils sont susceptibles d'être confrontés à des tentatives de fraude financière. L'item ERREUR BANCAIRE permet de déterminer dans quelle mesure ils connaissent les mesures de précaution à prendre. Dans cette question, les élèves sont invités à réfléchir à la réaction appropriée en présence d'une escroquerie financière via message électronique. Ils doivent évaluer les options qui leur sont proposées et comprendre que le deuxième conseil est le seul qui puisse être considéré comme pertinent. Lors de l'essai de terrain, un peu plus de $40 \%$ des élèves ont obtenu un crédit complet pour cet item (soit, dans l'ordre, les réponses «Non», «Oui » et « Non»).

\section{Processus}

Les catégories de processus portent sur les processus cognitifs. Elles servent à décrire la capacité des élèves à reconnaître et appliquer des concepts pertinents, et à comprendre, analyser, raisonner, évaluer et proposer des solutions. Dans la conception PISA de la culture financière, quatre catégories de processus sont définis : identifier des informations financières, analyser des informations dans un contexte financier, évaluer des questions financières et appliquer des notions financières comprises et connues. Les verbes employés ici ressemblent jusqu'à un certain point à la taxonomie des objectifs pédagogiques de Bloom (Bloom, 1956), mais une distinction importante est à souligner : les processus du construct de la culture financière ne sont pas opérationnalisés comme une hiérarchie de compétences. Au contraire, ils sont considérés comme des approches cognitives fondamentales parallèles, qui font toutes partie de l'arsenal des individus « cultivés ». L'ordre dans lequel les processus sont présentés est celui dans lequel s'enchaînent typiquement les raisonnements et les actes, et ne correspond pas à une gradation de la difficulté. Dans le même esprit, il est établi que dans les matières financières, les raisonnements, les décisions et les actes dépendent le plus souvent d'un enchaînement récursif et interactif des processus décrits dans cette section. Dans l'évaluation, chaque tâche est associée au processus dont on estime qu'il intervient le plus.

\section{Identifier des informations financières}

Les individus s'engagent dans ce processus lorsqu'ils cherchent des informations financières, y accèdent et identifient ou reconnaissent leur pertinence. Lors du cycle PISA 2012, les informations sont présentées sous la forme de textes imprimés, notamment des contrats, des publicités, des graphiques, des tableaux, des formulaires ou des instructions. Parmi les tâches typiques de ce processus, citons celles qui demandent aux élèves d'identifier les caractéristiques d'une facture ou de repérer le solde sur un extrait de compte. Dans les tâches plus difficiles, les élèves peuvent avoir à parcourir un contrat rédigé dans un langage juridique complexe pour y repérer des informations qui expliquent les conséquences d'un défaut de paiement des échéances d'un crédit. Cette catégorie de processus est également celle à laquelle font appel les tâches qui consistent à reconnaître la terminologie financière, par exemple comprendre que le terme « inflation » désigne l'augmentation des prix au fil du temps.

Le $5^{\mathrm{e}}$ exemple d'item, tiré de l'unité BULLETIN DE SALAIRE, illustre les tâches qui consistent à identifier et interpréter des informations financières.

$5^{e}$ exemple d'item PISA de culture financière - BULLETIN DE SALAIRE

- Figure 5.6 -

Item de l'unité BULLETIN DE SALAIRE

Chaque mois, Juliette perçoit son salaire sur son compte bancaire.

Voici le bulletin de salaire de Juliette pour le mois de juillet.

BULLETIN DE SALAIRE : Juliette Cassel

\begin{tabular}{l|r|r}
\hline Poste : Responsable & \multicolumn{1}{|c}{$\boldsymbol{1}^{\mathrm{er}}$ juillet -31 juillet } \\
\hline Salaire brut & 2800 zeds & \\
\hline Déductions & 300 zeds & \\
\hline Salaire net & 2500 zeds & \\
\hline Salaire brut cumulé cette année & 19600 zeds & \\
\hline
\end{tabular}


QUESTION

Quelle somme l'employeur de Juliette a-t-il versé sur son compte bancaire le 31 juillet?

\section{A. 300 zeds \\ B. 2500 zeds \\ C. 2800 zeds \\ D. 19600 zeds}

Les élèves doivent identifier des informations financières indiquées sur un simple bulletin de salaire. Lors de l'essai de terrain, un peu plus de la moitié d'entre eux répondu correctement à cet item (2 500 zeds).

\section{Analyser des informations dans un contexte financier}

Ce processus englobe un large éventail d'activités cognitives dans des contextes financiers, notamment interpréter, comparer et confronter, résumer et faire des extrapolations à partir des informations fournies. Il consiste essentiellement à reconnaître quelque chose qui n'est pas explicite : identifier des implications ou des hypothèses sous-jacentes au sujet d'une question qui s'inscrit dans un contexte financier. Les tâches relevant de cette catégorie peuvent par exemple demander aux élèves de comparer les conditions proposées dans différents contrats de téléphonie mobile ou de déterminer si une publicité pour un prêt est susceptible de passer sous silence certaines conditions. Le $6^{\mathrm{e}}$ exemple cidessous, tiré de l'unité ACTIONS, illustre les items relevant de cette catégorie de processus.

\section{$6^{e}$ exemple d'item PISA de culture financière - ACTIONS}

- Figure 5.7 -

\section{Item de l'unité ACTIONS}

Ce graphique montre l'évolution du prix d'une action Rich Rock sur une période de 12 mois.

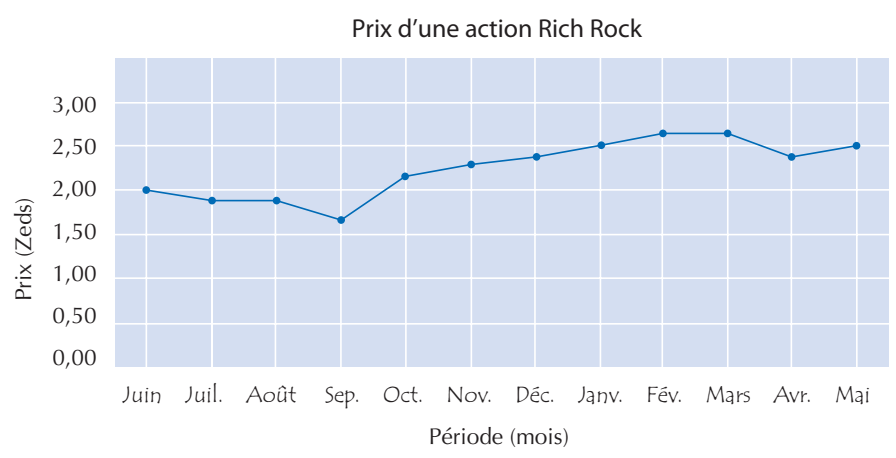

\section{QUESTION}

Parmi ces affirmations concernant le graphique, lesquelles sont vraies?

Pour chaque affirmation, entourez « Vrai » ou « Faux ».

\begin{tabular}{c|c}
\hline \multicolumn{1}{c|}{ Affirmation } & Cette affirmation est-elle vraie ou fausse ? \\
\hline Le meilleur mois pour acheter des actions était le mois de septembre. & Vrai / Faux \\
\hline Le prix de l'action a augmenté d'environ $50 \%$ sur toute l'année. & Vrai / Faux \\
\hline
\end{tabular}

Cette question se présente en deux parties. Pour y répondre, les élèves doivent analyser des informations dans un contexte financier : ils doivent étudier les informations fournies dans la courbe d'un graphique au sujet d'un produit d'investissement. Le graphique montre l'évolution du cours d'une action durant un an. La première partie de la question permet d'évaluer dans quelle mesure les élèves comprennent que les actions s'achètent quand leur cours est peu élevé (en l'occurrence en septembre) et la seconde, de déterminer si les élèves réussissent à identifier l'augmentation du cours de l'action et à 
calculer le taux de croissance dans le temps. Un peu plus de la moitié des élèves ont répondu correctement à cet item lors de l'essai de terrain (soit, dans l'ordre, les réponses « Vrai » et « Faux »).

\section{Évaluer des questions financières}

Ce processus consiste essentiellement à reconnaître ou à élaborer des explications ou des justifications financières, grâce à l'application de notions connues et comprises dans des contextes spécifiques. Il englobe diverses activités, notamment expliquer, évaluer et généraliser. Le raisonnement critique intervient dans ce processus lorsque les élèves doivent utiliser leurs connaissances et raisonner de manière logique et plausible pour dégager du sens d'un problème financier et s'en faire une idée. Les informations requises pour résoudre ce type de problèmes peuvent être fournies en partie dans le stimulus, mais les élèves doivent établir un lien entre ces informations et des notions qu'ils comprennent et qu'ils connaissent par ailleurs. Dans I'enquête PISA, toutes les informations requises pour comprendre un problème sont censées être connues des élèves de âgés de 15 ans - qu'ils les aient acquises par l'expérience directe ou qu'ils puissent facilement se les imaginer ou les comprendre. On peut ainsi supposer que les adolescents de 15 ans sont capables d'identifier par expérience des besoins qu'il n'est pas essentiel de satisfaire (acheter une nouvelle chaîne stéréo, par exemple). Une tâche basée sur ce type de scénario consisterait, par exemple, à demander aux élèves quels sont les facteurs à prendre en considération pour décider de faire un achat ou de le reporter dans des conditions financières particulières, selon les avantages financiers des deux options.

L'exemple d'item suivant, qui est tiré de l'unité $A U M A R C H E ́$, se base sur le même stimulus que le $1^{\text {er }}$ exemple d'item. Les élèves doivent évaluer des informations sur la base de connaissances antérieures en rapport avec la vie courante.

\section{$7^{e}$ exemple d'item PISA de culture financière - AU MARCHÉ}

- Figure 5.8 -

Item de l'unité $A U$ MARCHÉ

\section{QUESTION \\ Acheter une cagette de tomates peut parfois être une mauvaise décision financière pour certaines personnes. \\ Expliquez......}

Pour répondre à cette question, les élèves doivent comprendre qu'acheter des produits en grande quantité peut être du gaspillage ou que $c^{\prime}$ est un achat inabordable à court terme pour certaines personnes. Ils doivent évaluer la question financière dans la situation présentée. Ils obtiennent un crédit complet s'ils peuvent expliquer qu'acheter des tomates en grande quantité à un prix inférieur n'est pas nécessairement une bonne décision sur le plan financier. Les réponses ci-dessous, qui évoquent la notion de gaspillage, valent par exemple un crédit complet :

- Les tomates risquent de pourrir avant qu'on les consomme toutes.

- Parce qu'on n’a pas nécessairement besoin de $10 \mathrm{~kg}$ de tomates.

Les réponses qui évoquent la situation de personnes qui ne peuvent se permettre d'acheter de grandes quantités telles que celles-ci-dessous valent également un crédit complet :

- Cela coûterait 22 zeds (au lieu de 2,75 ou de 5,50 zeds pour 1 ou 2 kg), et tout le monde n'a pas nécessairement tout cet argent à dépenser.

- Pour payer cette cagette de tomates, certains devraient se passer d'autre chose dont ils ont pourtant besoin.

Lors de l'essai de terrain, plus de 80 \% des élèves ont obtenu un crédit à cet item, que ce soit en évoquant le risque de gaspillage ou le caractère inabordable de l'achat.

Le $8^{\text {e }}$ exemple, qui est tiré de l'unité NOUVELLE OFFRE, illustre les tâches plus difficiles de la catégorie Évaluer des questions financières. 
$8^{e}$ exemple d'item PISA de culture financière - NOUVELLE OFFRE

- Figure 5.9 -

Item de l'unité NOUVELLE OFFRE

$\mathrm{M}^{\text {me }}$ Jourdan a souscrit un prêt de 8000 zeds auprès de Zedfinance Premier. Le taux d'intérêt annuel est de $15 \%$. Elle rembourse 150 zeds chaque mois.

Au bout d'un an, $\mathrm{M}^{\mathrm{me}}$ Jourdan doit toujours rembourser 7400 zeds.

Un autre organisme financier, appelé Crédit Zedplus, propose à $\mathrm{M}^{\text {me }}$ Jourdan un prêt de 10000 zeds à un taux d'intérêt annuel de $13 \%$. Elle rembourserait aussi 150 zeds chaque mois.

QUESTION

Quelle est l'une des conséquences financières négatives possible pour Mme Jourdan si elle accepte l'offre de Crédit Zedplus?

Cet item, tiré de l'unité NOUVELLE OFFRE, demande aux élèves de réfléchir aux conséquences d'un changement de conditions dans le cadre d'un prêt et de les évaluer - un contexte moins susceptible d'être familier aux adolescents de 15 ans que celui de l'unité $A U$ MARCHÉ. Dans cet item, toutes les informations requises sont fournies dans la question, mais les élèves doivent identifier les informations pertinentes et réfléchir aux conséquences d'un acte particulier pour obtenir un crédit complet. Plusieurs types de réponse valent un crédit complet. Lors de l'essai de terrain, les réponses correctes les plus fréquentes sont celles qui évoquent l'augmentation de la dette de $M^{\text {me }}$ Jourdan. Parmi les autres réponses correctes, citons celles qui font référence aux conditions spécifiques du prêt, par exemple le fait que les intérêts à rembourser seront supérieurs (sur toute la durée du prêt), que la longueur du prêt augmente ou que des frais ne sont pas à exclure lors d'un changement de société de prêt.

À peine plus de $40 \%$ des élèves ont obtenu un crédit complet à cet item lors de l'essai de terrain.

Le $2^{\mathrm{e}}$ exemple d'item, tiré de l'unité PRIORITÉS, illustre les tâches de la catégorie Évaluer des questions financières : les élèves doivent en effet se livrer à un raisonnement plausible dans un contexte financier pour déterminer lesquelles des tâches inscrites sur une liste ne souffrent aucun retard.

\section{Appliquer des notions financières comprises et connues}

Le quatrième processus s'inspire d'un passage de la définition de la culture financière : « $[\ldots]$ pour utiliser cette connaissance et cette compréhension [des notions financières] ». Ce processus consiste à agir efficacement dans un contexte financier grâce à la mobilisation de connaissances concernant les contextes et produits financiers et à la compréhension de concepts financiers. Il intervient dans les tâches qui demandent aux élèves de faire des calculs et de résoudre des problèmes, souvent compte tenu de plusieurs conditions, par exemple de calculer les intérêts à verser pour un prêt de plus de deux ans. Ce processus est également à l'œuvre dans les tâches dans lesquelles les élèves doivent comprendre la pertinence de connaissances antérieures dans un contexte spécifique, par exemple réaliser que le pouvoir d'achat varie à la hausse ou à la baisse au fil du temps en fonction du taux d'évolution des prix, auquel cas ils doivent appliquer leurs connaissances de l'inflation.

L'exemple suivant, tiré de l'unité PARTIR EN VACANCES, s'inscrit dans la catégorie de processus Appliquer des notions financières comprises et connues.

$9^{e}$ exemple d'item PISA de culture financière - PARTIR EN VACANCES

- Figure 5.10 घ

Item de l'unité PARTIR EN VACANCES

Natacha travaille dans un restaurant 3 soirs par semaine.

Elle travaille 4 heures chaque soir et gagne 10 zeds de l'heure.

Natacha gagne également 80 zeds de pourboire chaque semaine.

Natacha économise exactement la moitié de la somme totale qu'elle gagne chaque semaine. 


\section{QUESTION}

Natacha veut économiser 600 zeds pour partir en vacances.

Combien de semaines faudra-t-il à Natacha pour économiser 600 zeds ?.......

Dans cette tâche, les élèves doivent analyser une série de conditions et de contraintes, et prévoir le financement de dépenses futures - en l'occurrence déterminer pendant combien de temps Natacha doit économiser de l'argent pour partir en vacances si elle épargne un montant fixe par semaine. La réponse correcte est « 6 semaines ». Moins de la moitié des élèves ont répondu correctement à cet item lors de l'essai de terrain.

\section{Contextes}

Tant l'élaboration du cadre d'évaluation que la conception et la sélection des items par rapport à ce cadre requièrent qu'une grande attention soit accordée à l'éventail de contextes dans lesquels la culture financière s'exerce. Les décisions à propos de matières financières dépendent souvent des contextes ou situations dans lesquels elles sont présentées. Le fait de situer les tâches dans divers contextes permet d'éveiller l'intérêt du plus grand nombre possible d'élèves et de refléter l'éventail de situations que les individus rencontrent au $\mathrm{XXI}^{\mathrm{e}}$ siècle.

Certaines situations sont plus familières que d'autres pour les adolescents de 15 ans. Dans l'enquête PISA, les tâches ont pour cadre des situations de la vie courante, notamment, mais pas uniquement dans le cadre scolaire. Les situations peuvent se rapporter à la vie de l'individu, de sa famille ou de ses semblables, à sa communauté, voire au monde.

Le groupe d'experts chargé de la culture financière a commencé par analyser les contextes retenus dans le cadre d'évaluation du Programme pour l'évaluation internationale des compétences des adultes (Programme for the International Assessment of Adult Competencies, PIAAC), à savoir l'éducation et le travail, la vie privée et familiale, les loisirs et le divertissement et, enfin, la communauté et la citoyenneté (OCDE, 2009b). Dans le cadre de la culture financière, les loisirs et le divertissement ont été remplacés par les contextes individuels pour refléter le fait que de nombreuses interactions financières des jeunes sont en rapport avec le fait qu'ils sont des consommateurs : ils utilisent un téléphone mobile et un ordinateur, ils se rendent dans des infrastructures de loisirs ou financent leurs dépenses en matière de divertissement. Il a été décidé de remplacer la communauté et la citoyenneté par les situations sociétales. Les contextes en rapport avec la communauté et la citoyenneté dénotent une perspective plus large que la perspective individuelle, mais il a été jugé que le terme « communauté » ne reflétait pas une perspective assez vaste. Les contextes sociétaux impliquent au contraire les situations nationales et mondiales ainsi que les situations plus locales, ce qui est plus approprié à la portée potentielle de la culture financière. Les contextes retenus dans l'évaluation PISA de la culture financière sont donc les suivants : les contextes scolaires et professionnels, ménagers et familiaux, individuels et sociétaux.

\section{Contextes scolaires et professionnels}

Les contextes scolaires et professionnels sont très importants pour les adolescents. Les contextes scolaires sont de toute évidence pertinents pour la population cible de l'enquête PISA, puisque les échantillons sont prélevés parmi les effectifs scolarisés. Bon nombre des élèves âgés de 15 ans poursuivront leurs études ou leur formation pendant un certain temps. Toutefois, de nombreux autres quitteront l'école pour entrer dans la vie active d'ici un an ou deux. De plus, beaucoup d'élèves âgés de 15 ans travaillent occasionnellement en dehors de leurs heures de cours. Les contextes professionnels sont donc pertinents pour les élèves PISA, aujourd'hui déjà et à moyen terme également. La quasi-totalité des jeunes de 15 ans commenceront alors à réfléchir à des matières financières en rapport avec les études ou le travail, que ce soit pour décider comment dépenser leur épargne, envisager les possibilités qui s'offrent à eux pour poursuivre leurs études ou plutôt entrer dans le monde du travail.

Les tâches typiques de cette catégorie de contextes consistent à comprendre un bulletin de salaire, à faire des économies pour financer des études tertiaires, à analyser les avantages et les risques d'un prêt d'études, et à participer à un programme d'épargne dans le cadre professionnel.

Le $5^{\mathrm{e}}$ exemple tiré de I'unité BULLETIN DE SALAIRE et le $9^{\mathrm{e}}$ exemple tiré de l'unité PARTIR EN VACANCES illustrent les items relevant de la catégorie Contextes scolaires et professionnels. Ils se situent plus précisément dans un contexte professionnel, car ils demandent aux élèves d'aborder des problèmes financiers en rapport avec des revenus du travail. Les élèves doivent identifier des informations sur un bulletin de salaire dans le premier item et se baser sur des revenus pour élaborer un plan d'épargne dans le second. 


\title{
Contextes ménagers et familiaux
}

Les contextes ménagers et familiaux concernent des sujets financiers en rapport avec la gestion d'un ménage. Selon toute vraisemblance, les adolescents de 15 ans vivent tous en famille, mais cette catégorie de contextes ne se limite pas aux ménages constitués sur la base de relations familiales et englobe les colocations auxquelles les jeunes ont souvent recours lorsqu'ils quittent le toit familial. Les tâches qui s'inscrivent dans ce contexte consistent, par exemple, à acheter des articles ménagers ou des produits d'épicerie, à tenir les comptes du ménage et à organiser des événements familiaux. Les décisions relatives à l'établissement d'un budget ou à la hiérarchisation de priorités peuvent également s'inscrire dans cette catégorie de contextes.

Les deux items de l'unité $A U$ MARCHÉ, le $1^{\text {er }}$ et le $7^{\mathrm{e}}$ exemples, se classent dans la catégorie Contextes ménagers et familiaux, car les achats sur lesquels ils portent sont typiques de ceux faits par les ménages. Le $2^{e}$ exemple d'item, qui est tiré de l'unité PRIORITÉS, s'inscrit également dans cette catégorie : il se situe dans le cadre d'une colocation, et les priorités retenues affectent le ménage (constitué en ce cas d'amis et non de membres d'une même famille).

\section{Contextes individuels}

Les contextes individuels sont importants dans le cadre des finances personnelles, car parmi les décisions prises par les individus, nombreuses sont celles qui concernent totalement leur avantage personnel. Qui plus est, les individus doivent supporter de nombreux risques et responsabilités. Parmi les décisions relevant de cette catégorie de contextes, citons celles qui consistent à choisir des produits et services personnels (habillement, coiffure, etc.), à acheter des biens de consommation (des appareils électroniques ou des équipements de sport), ou à prendre des engagements (s'abonner aux matches d'une saison ou s'inscrire dans une salle de sport). Ces décisions portent sur des besoins personnels essentiels ainsi que sur des loisirs. Les individus peuvent subir des influences sociales ou familiales lorsqu'ils prennent des décisions, mais c'est eux qui doivent assumer la responsabilité de leurs décisions lorsqu'il s'agit d'ouvrir un compte bancaire ou de contracter un emprunt. Les contextes individuels incluent donc les tâches en rapport avec les conditions contractuelles d'actes tels que l'ouverture d'un compte en banque, l'achat de biens de consommation, le financement d'activités de loisirs ou le choix de services financiers pertinents souvent associés à des aspects plus larges de la consommation, comme le crédit et l'assurance.

Le $10^{\mathrm{e}}$ exemple d'item, tiré de l'unité NOUVELLE CARTE BANCAIRE, illustre les items qui s'inscrivent dans la catégorie Contextes individuels.

\section{$10^{\mathrm{e}}$ exemple d'item PISA de culture financière - NOUVELLE CARTE BANCAIRE}

- Figure 5.11 -

\section{Item de l'unité NOUVELLE CARTE BANCAIRE}

\begin{abstract}
Lisa vit en Zedlande. Elle vient de recevoir
\end{abstract} cette nouvelle carte bancaire

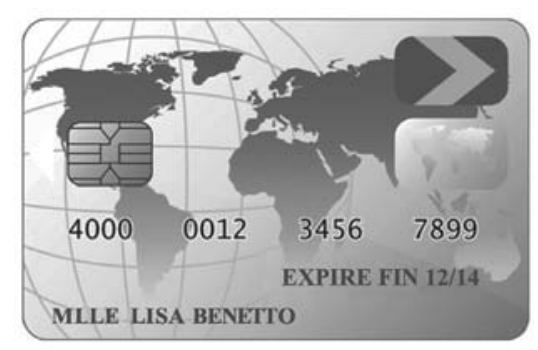

\section{QUESTION}

Le lendemain, Lisa reçoit le code confidentiel de sa carte bancaire.

Que doit faire Lisa avec son code confidentiel?

A. L'écrire sur un papier et le garder dans son portefeuille.

B. Le partager avec ses amis.

C. L'écrire au dos de sa carte bancaire.

D. Le mémoriser.

Cette tâche permet de déterminer dans quelle mesure les élèves comprennent une responsabilité individuelle, en l'occurrence celle de la préservation de la sécurité lors de l'accès aux services bancaires en ligne et de leur utilisation. 
Les élèves doivent évaluer laquelle des options proposées est la meilleure approche à adopter lors de l'utilisation d'une carte bancaire. Lors de I'essai de terrain, plus de $90 \%$ des élèves ont choisi l'option correcte (soit l'option D, « Le mémoriser »).

D'autres items parmi les exemples proposés ci-dessus relèvent de la catégorie des contextes individuels : le $3^{\text {e }}$ exemple

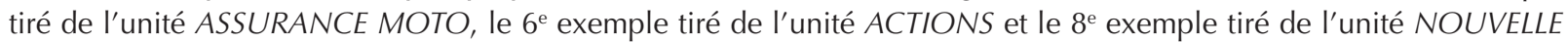
OFFRE. Ces items portent tous sur des décisions qui ont un impact sur l'individu (renouveler une police d'assurance, acheter des actions et refinancer un emprunt).

\section{Contextes sociétaux}

L'environnement dans lequel les jeunes évoluent se caractérise par le changement, la complexité et l'interdépendance. La mondialisation crée de nouvelles formes d'interdépendance, où les actes dépendent de facteurs économiques et ont des conséquences qui vont bien au-delà de l'individu et de la communauté locale. Le domaine d'évaluation de la culture financière cible les finances personnelles, mais l'inclusion de contextes sociétaux est un moyen de reconnaître que le bien-être financier individuel ne peut être totalement isolé du reste de la société. Le bien-être financier individuel affecte la communauté locale, nationale et même mondiale, et en subit l'influence. Les items qui se situent dans ces contextes portent sur diverses matières, par exemple le fait d'être informé sur les droits et devoirs des consommateurs, de comprendre l'objet de la fiscalité locale et nationale ou les intérêts commerciaux, et de tenir compte du rôle du pouvoir d'achat. Ils s'étendent aussi à des choix financiers, par exemple la décision de faire un don à des organisations sans but lucratif et à des œuvres de bienfaisance.

Le 4 exemple d'item proposé ci-dessus, qui est tiré de l'unité ERREUR BANCAIRE, se classe dans la catégorie Contextes sociétaux, car il porte sur un comportement frauduleux visant la société.

\section{Facteurs non cognitifs}

Dans l'enquête PISA, la définition opérationnelle de la culture financière inclut des facteurs non cognitifs en rapport avec la motivation et la confiance, des attitudes qui, d'après certains, ont un impact sur les comportements en matière de gestion financière (Johnson et Staten, 2010). Dans I'enquête PISA, les attitudes et les comportements relatifs aux matières financières sont considérés en soi comme des aspects de la culture financière. Les attitudes et les comportements sont également intéressants du point de vue de leurs interactions avec les éléments cognitifs de la culture financière. Les informations recueillies au sujet des attitudes et des comportements financiers des adolescents de 15 ans devraient en outre permettre de constituer une base de données de référence pour mener des études longitudinales de la culture financière des adultes, y compris de leurs comportements financiers.

Le groupe d'experts en charge de la culture financière a identifié quatre facteurs non cognitifs à inclure dans le cadre d'évaluation : l'accès à l'éducation et à l'information, l'accès à l'argent et aux produits financiers, les attitudes à l'égard des matières financières et la confiance en soi dans ce domaine et, enfin, les comportements en matière de dépense et d'épargne ${ }^{2}$.

\section{L'accès à l'éducation et à l'information}

Les élèves peuvent avoir diverses sources d'information financière, notamment leurs amis, leurs parents ou d'autres membres de leur famille. Il est utile de savoir lesquelles de ces sources ils utilisent le plus souvent et de déterminer si des niveaux plus élevés de culture financière sont associés à certaines sources. Les responsables politiques peuvent également se baser sur ces informations pour évaluer l'efficacité avec laquelle les messages sur des questions financières sont communiqués et pour choisir la cible de nouvelles interventions.

L'éducation et la formation suivies par les élèves varient entre les pays et au sein même de ceux-ci. Les informations sur l'existence d'une corrélation entre les niveaux de culture financière et l'offre en matière d'éducation financière en milieu scolaire et à l'extérieur devraient être particulièrement utiles pour élaborer les programmes d'éducation financière et, ainsi, améliorer la culture financière.

\section{L'accès à l'argent et aux produits financiers}

Les élèves qui ont une plus grande expérience personnelle des questions financières sont vraisemblablement plus susceptibles d'obtenir de meilleurs résultats aux épreuves cognitives. Ceux qui prennent régulièrement des décisions relatives à la gestion de leur argent sont susceptibles d'en savoir plus sur les questions financières, même s'ils n'ont pas suivi de cours spécifiques, que ceux qui ne prennent pas ce type de décisions. Les élèves peuvent avoir acquis cette 
expérience en gagnant de l'argent ou en utilisant le système bancaire ou des produits financiers tels que les cartes de crédit ou de débit. Il est une question majeure pour l'action publique dans ce domaine : «Dans quelle mesure la culture financière des jeunes dépend-elle des expériences qu'ils vivent en la matière dans la vie réelle?».

\section{Les attitudes à l'égard des matières financières et la confiance en soi dans ce domaine}

Les attitudes sont considérées comme des composantes importantes de la culture financière. De plus, les préférences individuelles sont des facteurs déterminants du comportement financier et peuvent se conjuguer à la culture financière. Des études de psychologie comportementale pourraient fournir des pistes intéressantes en matière de culture financière et permettre aux responsables politiques d'améliorer l'efficience des programmes en meilleure connaissance de cause. Parmi les domaines qu'il serait utile d'approfondir, citons la tolérance au risque - soit la volonté d'accepter la possibilité d'une perte dans l'espoir d'obtenir un gain plus élevé (Barsky et al., 1997 ; Holt et Laury, 2002) - et la sensibilité au temps - soit la volonté de renoncer à une gratification immédiate dans l'espoir d'obtenir un gain plus élevé à l'avenir (Barsky et al., 1997; Holt et Laury, 2002).

\section{Les comportements en matière de dépense et d'épargne}

Les items des épreuves cognitives servent à évaluer la capacité des élèves à prendre des décisions de dépense et d'épargne, certes, mais il est également utile de mieux cerner leurs comportements en matière de dépense et d'épargne dans la vie réelle. L'évaluation PISA de la culture financière permettra de recueillir des éléments sur la relation entre le niveau de culture financière et le comportement financier via l'analyse de la relation entre les comportements déclarés par les jeunes âgés de 15 ans et leurs résultats aux épreuves cognitives de culture financière.

\section{ÉVALUER LA CULTURE FINANCIÈRE}

La section précédente a décrit le cadre conceptuel d'évaluation de la culture financière. Les concepts retenus dans le cadre conceptuel doivent être représentés dans les tâches et les items pour recueillir des éléments à propos de la compétence des élèves en la matière. Cette section présente la conception des épreuves, la répartition des tâches entre les variables du cadre d'évaluation et le choix des formats de réponse. Elle est suivie d'un exposé succinct sur I'impact des connaissances et compétences dans d'autres domaines sur la culture financière et leurs implications pour les épreuves. Enfin, la méthode de collecte de données sur les expériences et comportements financiers est décrite.

Le cadre conceptuel sert à cartographier le domaine d'évaluation non seulement pour le cycle PISA 2012, mais également à des fins plus générales. Il présente la définition et les variables majeures retenues pour concevoir les épreuves. Les idées fondamentales qui le sous-tendent sont développées dans des sous-sections et illustrées par des exemples dans la section précédente. Ces développements ne sont pas à considérer comme des descriptifs des tâches incluses dans les épreuves administrées lors du cycle PISA 2012, car il n'est pas possible de couvrir tous les détails faute de temps (seule une heure est allouée à l'évaluation de la culture financière). En principe, d'autres aspects du domaine d'évaluation seront inclus dans des items lors des prochains cycles d'évaluation.

\section{La structure des épreuves}

Les épreuves papier-crayon du cycle PISA 2012 contiennent deux heures de test constituées de blocs de 30 minutes de test relevant d'un ou de plusieurs domaines cognitifs. La culture financière comporte deux blocs (soit 60 minutes de test) lors du cycle PISA 2012. L'analyse des taux de réponse enregistrés lors de l'essai de terrain a permis d'établir que la grande majorité des élèves réussissent à répondre à 20 items de culture financière en 30 minutes. En conséquence, 40 des 75 tâches de culture financière administrées lors de l'essai de terrain ont été retenues lors de la campagne définitive.

Les carnets de test de la campagne définitive du cycle PISA 2012 qui incluent les deux blocs d'items de culture financière incluent aussi un bloc d'items de mathématiques et un bloc d'items de compréhension de l'écrit. Pour atténuer les effets éventuels du classement des blocs dans les carnets, quatre carnets contenant des blocs de culture financière ont été créés, les blocs d'items de culture financière, de mathématiques et de compréhension de l'écrit apparaissant dans un ordre différent.

Comme dans les autres domaines d'évaluation PISA, les items de culture financière sont regroupés par unité d'un ou deux items autour d'un stimulus commun. La batterie d'items comporte des stimulus d'ordre financier sous divers formats, notamment en prose et sous forme de diagrammes, de tableaux, de graphiques et d'illustrations.

Les épreuves sont constituées d'un large éventail d'items dont le degré de difficulté varie fortement, ce qui permettra d'évaluer et de décrire les points forts et les points faibles des élèves et des sous-groupes d'élèves. 


\section{Réponses : formats et codage}

Dans les épreuves papier-crayon, certains items PISA appellent soit des réponses courtes, soit des réponses d'une ou deux phrases, alors que d'autres demandent simplement aux élèves d'entourer une réponse ou de cocher une case. Les décisions à propos des formats de réponse se basent sur ce qui est jugé approprié compte tenu du type d'informations à recueillir, ainsi que sur des considérations techniques et pragmatiques. Dans les épreuves de culture financière ainsi que dans les autres épreuves PISA, deux grands types d'items sont utilisés : les items à réponse construite et les items à choix multiple.

Les items à réponse construite demandent aux élèves de produire leur réponse. Leur réponse peut se résumer à un mot ou à un chiffre, mais elle peut aussi être plus longue : quelques phrases ou un calcul développé. Les items à réponse construite qui appellent une réponse plus élaborée sont idéaux pour recueillir des données à propos de la capacité des élèves à expliquer leurs décisions, ou du processus de leur analyse. Le 9e exemple, qui est tiré de l'unité PARTIR EN $V A C A N C E S$, illustre les items à réponse construite $d^{\prime}$ un seul chiffre, où les réponses correctes sont très peu nombreuses. Le $1^{\mathrm{er}}$ et le $7^{\mathrm{e}}$ exemples, tous deux tirés de l'unité $A U$ MARCHÉ, et le $8^{\mathrm{e}}$ exemple, tiré de l'unité NOUVELLE OFFRE, illustrent les items qui appellent une réponse plus élaborée, pour lesquels de nombreuses réponses différentes peuvent valoir un crédit complet.

Dans le codage de l'item tiré de l'unité NOUVELLE OFFRE, par exemple, quatre types de réponse valant un crédit complet sont identifiés :

(i) Les réponses qui font référence au fait que $M^{\text {me }}$ Jourdan va accroître son endettement si elle opte pour la nouvelle offre :

- Elle devra plus d'argent.

- Elle sera dans l'incapacité de maîtriser ses dépenses.

- Elle va s'enfoncer dans les dettes.

(ii) Les réponses qui font référence au fait que $M^{\text {me }}$ Jourdan aura plus d'intérêts à payer :

- $13 \%$ sur 10 000, c'est plus que $15 \%$ sur 8000.

(iii) Les réponses qui font référence à l'allongement de $\mathrm{I}^{\prime}$ endettement de $M^{\mathrm{me}}$ Jourdan :

- Ce sera plus long à rembourser, car l'emprunt est plus élevé et les remboursements sont identiques.

(iv) Les réponses qui font référence à la possibilité que $\mathrm{M}^{\mathrm{me}}$ Jourdan ait des frais à payer si elle renonce à son premier prêt auprès de Zedfinance Premier :

- Elle pourrait avoir des frais à payer si elle rembourse le prêt à Zedfinance plus vite que prévu.

Les items à choix multiple sont le deuxième type d'items en ce qui concerne le format de réponse et le codage. Pour répondre à ces items, les élèves choisissent une ou plusieurs des options qui leur sont proposées. Les items à choix multiple simple sont les plus courants : les élèves doivent choisir une option parmi celles qui leur sont proposées (au nombre de quatre, en général), comme dans le $5^{\mathrm{e}}$ et le $10^{\mathrm{e}}$ exemples, tirés respectivement de l'unité BULLETIN DE SALAIRE et de I'unité NOUVELLE CARTE BANCAIRE. Autre type d'items, les items à choix multiple complexe, dans lesquels les élèves doivent répondre à une série de questions «Vrai » ou «Faux ». Le $2^{\mathrm{e}}$ exemple, tiré de l'unité PRIORITÉS, illustre ce type d'items. Dans cet item, les élèves doivent répondre correctement à trois questions indépendantes les unes des autres pour obtenir un crédit. Le $3^{\text {e }}$ exemple, tiré de l'unité ASSURANCE MOTO, se présente sous un format similaire dans le sens où les élèves doivent répondre à trois questions indépendantes pour obtenir un crédit, mais en choisissant à chaque fois l'une des trois options : "Augmentation du tarif », "Réduction du tarif » et "Sans effet sur le tarif ». Ce type d'items est généralement considéré comme le plus approprié dans les tâches qui consistent à identifier et reconnaître des informations, mais il est également utile pour déterminer dans quelle mesure les élèves comprennent des concepts d'ordre supérieur qu'ils ne peuvent pas nécessairement exprimer facilement par eux-mêmes.

Bien que certains formats d'items se prêtent à des types spécifiques de questions, il faut veiller à ce que le format de l'item n'affecte pas l'interprétation des résultats. Des recherches donnent à penser que des groupes différents (les filles et les garçons, les élèves de pays différents, etc.) ne réagissent pas de la même façon aux divers formats d'items. Plusieurs études effectuées à propos des effets des formats de réponse sur la base des données PISA fournissent des arguments convaincants à l'appui d'un mélange d'items à choix multiple et d'items à réponse construite. Dans leur étude comparée de l'évaluation PISA de la compréhension de l'écrit et de I'IEA Reading Literacy Study (IEARLS), Lafontaine et Monseur (2006) ont établi que le format de réponse avait un impact sensible sur la performance selon le sexe. Une autre étude 
a établi la variation du degré de difficulté des items de compréhension de l'écrit entre les formats (Grisay et Monseur, 2007). Ce constat s'explique peut-être par le fait que des formats d'items sont plus familiers aux élèves dans certains pays que dans d'autres. En résumé, les épreuves PISA de culture financière sont constituées d'items de formats différents pour réduire le risque que le format d'item influe sur la performance des élèves. Cette influence serait extrinsèque à l'objet de l'évaluation, en l'espèce la culture financière.

S'agissant de la répartition des items entre les formats, le problème des ressources doit être pris en considération, tout comme les questions d'équité évoquées dans les paragraphes ci-dessus. Tous les items, sauf les items à réponse construite les plus simples, sont codés par des correcteurs spécialisés qui doivent être formés et encadrés. Les items à choix multiple et à réponse construite "fermée » très brève mobilisent moins de ressources, car ils ne requièrent pas I'intervention d'un correcteur.

Les pourcentages d'items à choix multiple et à réponse construite sont calculés compte tenu de toutes ces considérations. La majorité des items constituant les épreuves administrées lors de la campagne définitive du cycle PISA 2012 ne requièrent pas l'intervention d'un correcteur.

La plupart des items font l'objet d'un codage dichotomique (crédit complet ou pas de crédit), mais certains se prêtent à un codage qui prévoit aussi un crédit partiel. Le crédit partiel permet de corriger les items de manière plus nuancée. Certaines réponses, même si elles sont incomplètes, sont en effet meilleures que d'autres. Si des réponses incomplètes à un item dénotent un niveau plus élevé de culture financière que des réponses imprécises ou incorrectes, elles peuvent valoir un crédit partiel. Ces items à crédit partiel donnent lieu à l'attribution d'un score supérieur à 1.

\section{Répartition du score}

Cette section présente la répartition du score entre les catégories des trois grandes dimensions du cadre d'évaluation que nous avons décrites ci-dessus. Il est plus judicieux de parler en termes de «score » que d' « items », en raison de l'inclusion de plusieurs items à crédit partiel. Les répartitions sont indiquées sous forme de plages pour montrer la pondération relative des catégories.

Les items PISA de culture financière se classent tous dans une seule catégorie de contenu, de processus et de contexte, mais il est fréquent que des éléments appartenant à diverses catégories se retrouvent dans la même tâche, puisque I'enquête PISA propose des problèmes et des situations qui s'inspirent de la vie réelle. Dans ce cas, les items sont classés dans la catégorie dont on estime qu'elle est la plus représentative de la tâche.

La répartition du score entre les quatre catégories de contenus est indiquée dans le tableau 5.1.

Tableau 5.1

Répartition approximative du score en culture financière entre les catégories de contenus

\begin{tabular}{c|c|c|c|c}
\hline $\begin{array}{c}\text { L'argent } \\
\text { et les transactions }\end{array}$ & $\begin{array}{c}\text { La planification et } \\
\text { la gestion des finances }\end{array}$ & $\begin{array}{c}\text { Le risque } \\
\text { et le rendement }\end{array}$ & Le paysage financier & Total \\
\hline $30 \%-40 \%$ & $25 \%-35 \%$ & $15 \%-25 \%$ & $10 \%-20 \%$ & $100 \%$ \\
\hline
\end{tabular}

Cette répartition montre que la catégorie L'argent et les transactions est jugée la plus immédiatement pertinente pour les adolescents de 15 ans.

Le tableau 5.2 montre la répartition du score entre les quatre catégories de processus.

Tableau 5.2

Répartition approximative du score en culture financière entre les catégories de processus

\begin{tabular}{c|c|c|c|c}
\hline $\begin{array}{c}\text { Identifier } \\
\text { des informations } \\
\text { financières }\end{array}$ & $\begin{array}{c}\text { Analyser } \\
\text { des informations dans } \\
\text { un contexte financier }\end{array}$ & $\begin{array}{c}\text { Évaluer des questions } \\
\text { financières }\end{array}$ & $\begin{array}{c}\text { Appliquer des notions } \\
\text { financières comprises } \\
\text { et connues }\end{array}$ & Total \\
\hline $15 \%-25 \%$ & $15 \%-25 \%$ & $25 \%-35 \%$ & $25 \%-35 \%$ & $100 \%$ \\
\hline
\end{tabular}

Cette répartition montre que les catégories Évaluer des questions financières et Appliquer des notions financières comprises et connues sont plus importantes.

Le tableau 5.3 montre la répartition du score entre les quatre catégories de contextes. 
Tableau 5.3

Répartition approximative du score en culture financière entre les catégories de contextes

\begin{tabular}{c|c|c|c|c}
\hline $\begin{array}{c}\text { Contextes scolaires } \\
\text { et professionnels }\end{array}$ & $\begin{array}{c}\text { Contextes ménagers } \\
\text { et familiaux }\end{array}$ & Contextes individuels & Contextes sociétaux & Total \\
\hline $10 \%-20 \%$ & $30 \%-40 \%$ & $35 \%-45 \%$ & $5 \%-15 \%$ & $100 \%$ \\
\hline
\end{tabular}

Comme l'évaluation PISA cherche à mesurer la culture financière individuelle des adolescents de 15 ans, la pondération favorable aux contextes individuels ainsi qu'aux intérêts financiers ménagers et familiaux est logique. Les contextes scolaires, professionnels et sociétaux sont moins bien représentés, mais ils sont inclus car ils constituent des éléments importants de l'expérience financière.

\section{L'impact des connaissances et compétences dans d'autres domaines sur la culture financière}

\section{Numératie}

Un certain niveau de numératie (ou de culture mathématique) est indispensable à la culture financière. Huston (2010) affirme que «si un individu éprouve des difficultés en arithmétique, cela aura un impact sur sa culture financière. Toutefois, des outils (une calculatrice, par exemple) peuvent compenser ces déficiences; les informations en rapport direct avec la gestion des finances personnelles sont plus appropriées que les compétences en calcul pour évaluer la culture financière ». C'est la raison pour laquelle les épreuves de culture financière incluent souvent des items présentant un élément mathématique, même si ce n'est pas l'aspect essentiel de l'évaluation. Lusardi et al. (2010) signalent à propos de la National Longitudinal Survey of Youth, administrée aux États-Unis en 1997, que trois questions de culture financière ont permis de bien distinguer les répondants très performants des répondants peu performants. Deux des trois questions, en l'occurrence sur les taux d'intérêt et l'inflation, demandent des compétences élémentaires en mathématiques. Certaines compétences en mathématiques, notamment avoir le sens des nombres, être familiarisé avec de nombreuses représentations des nombres, posséder des compétences en calcul mental, des facultés d'estimation et la capacité d'évaluer la plausibilité des résultats, font partie intégrante de certains aspects de la culture financière.

Par ailleurs, les contenus des mathématiques et de la culture financière ne se chevauchent pas du tout à maints égards. La définition de la culture mathématique retenue à l'occasion du cycle PISA 2012 vise quatre catégories de contenus : les variations et les relations, l'espace et les formes, la quantité et l'incertitude et les données. De ces quatre contenus, seul celui de la quantité chevauche les contenus de l'évaluation PISA de la culture financière. Contrairement au contenu de culture mathématique l'incertitude et les données, où les élèves sont amenés à utiliser des probabilités et des statistiques, le contenu de culture financière le risque et le rendement leur demande de comprendre les caractéristiques d'une situation ou d'un produit qui dénotent un risque ou un rendement. C'est une appréciation non numérique de l'impact que l'aléatoire peut avoir sur le bien-être financier et une prise de conscience de la nécessité de protéger les produits et actions du risque de perte. Dans les épreuves de culture financière, les compétences en matière de quantité évoquées cidessus peuvent être appliquées dans des problèmes qui requièrent plus de connaissances financières que ceux proposés dans les épreuves de culture mathématique. De même, les connaissances dans les matières financières et la capacité d'appliquer ces connaissances et de se livrer à un raisonnement dans des contextes financiers (en l'absence de contenu mathématique spécifique) caractérisent les quatre contenus de culture financière, à savoir l'argent et les transactions, la planification et la gestion des finances, le risque et le rendement et le paysage financier. La figure 5.12 montre la relation entre les contenus de culture mathématique et de culture financière dans l'enquête PISA.

Concrètement, il y a peu d'items qui se classent dans la partie du diagramme où les deux cercles se chevauchent. Dans l'évaluation de la culture financière, I'arithmétique élémentaire est en principe le seul domaine de culture mathématique à intervenir : les quatre opérations (addition, soustraction, multiplication et division) sur la base de nombres entiers ou décimaux et de pourcentages courants. Cet aspect arithmétique est une composante intrinsèque de la culture financière, qui permet d'exploiter les connaissances dans les matières financières. Le $1^{\text {er }}$ exemple d'item, tiré de I'unité $A \cup M A R C H E$, illustre les items qui mobilisent des compétences arithmétiques : I'opération mathématique (une division par dix) est très élémentaire, à la portée de la plupart des adolescents de 15 ans. L'utilisation de formules financières (qui requiert des compétences en algèbre) n'est pas jugée appropriée. La dépendance des tâches au calcul est minimisée; les tâches sont conçues pour ne pas impliquer de calculs nombreux ou répétitifs. Les élèves peuvent utiliser la calculatrice d'usage en classe et dans les épreuves de culture mathématique pour répondre aux épreuves de culture financière, mais la réussite de celles-ci ne dépendent pas de l'utilisation d'une calculatrice. 


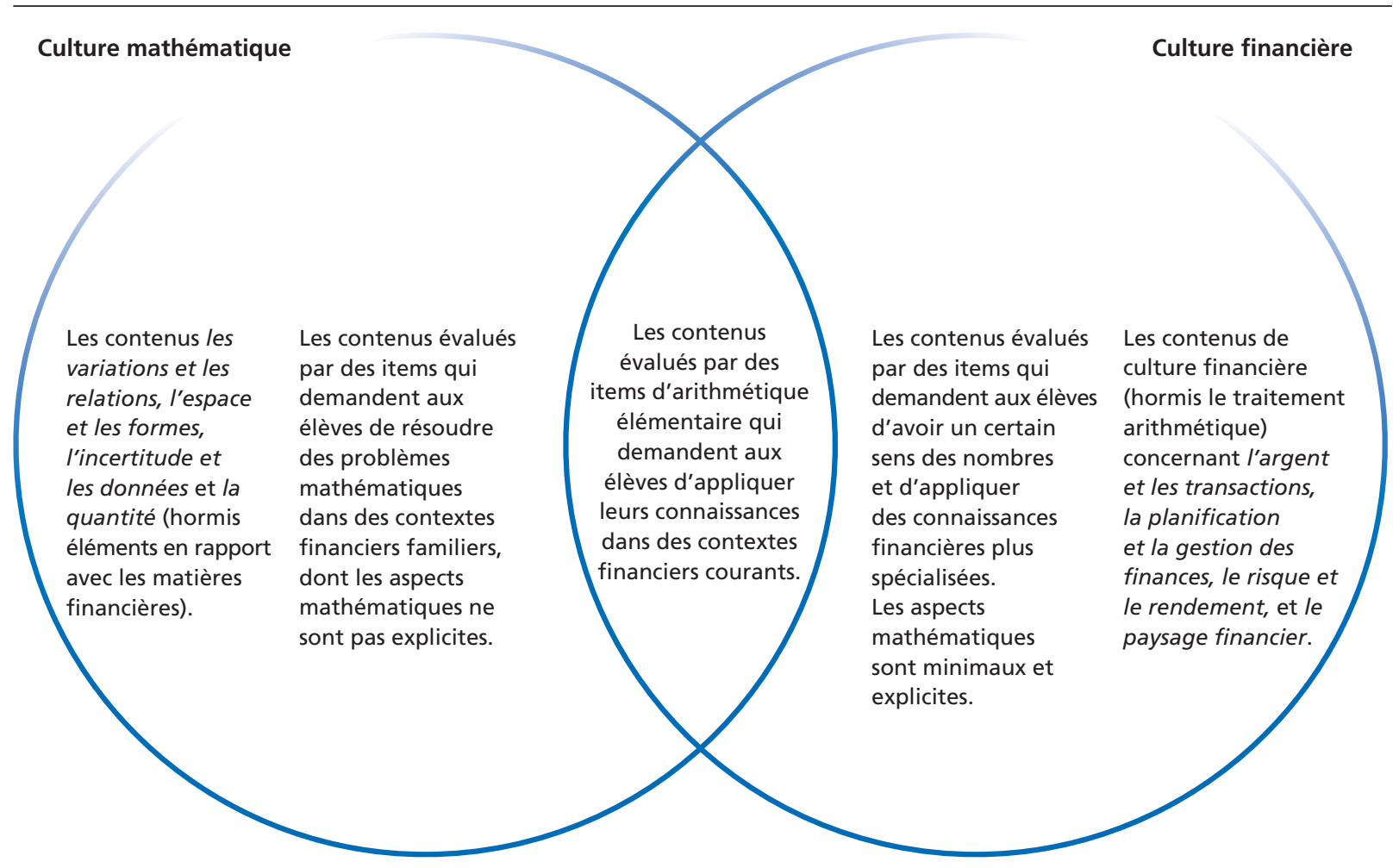

\section{Compréhension de l'écrit et vocabulaire}

Nous partons de l'hypothèse que tous les élèves soumis aux épreuves de culture financière possèdent un certain niveau en compréhension de l'écrit, même si nous savons, à la lumière des cycles PISA précédents, que leur niveau de compétence en la matière varie fortement entre les pays et au sein même de ceux-ci (OCDE, 2010b). Pour minimiser le niveau requis en compréhension de l'écrit, les stimulus et les énoncés sont formulés de la façon la plus claire, la plus simple et la plus brève possible. Dans certains cas, toutefois, les stimulus sont formulés à dessein dans un langage complexe ou assez technique, car la capacité de lire et d'interpréter le langage de documents financiers ou pseudofinanciers est considérée comme faisant partie de la culture financière. Dans le $4^{\mathrm{e}}$ exemple d'item, tiré de l'unité ERREUR BANCAIRE, les élèves doivent lire attentivement le courrier électronique pour comprendre qu'il est possible qu'il puisse s'agir d'un courrier malveillant.

La terminologie financière très technique n'est pas de mise. Le groupe d'experts en charge de la culture financière a donné des conseils sur les termes que les adolescents de 15 ans sont susceptibles de comprendre. Certains de ces termes sont parfois l'élément central des items. Le $5^{e}$ exemple d'item, tiré de l'unité BULLETIN DE SALAIRE, permet d'évaluer si les élèves sont capables de lire un simple bulletin de salaire et de reconnaître les termes "brut » et " net » (ou d'en déduire le sens).

\section{Recueillir des données à propos des expériences et des comportements dans le domaine financier}

Des informations concernant des facteurs non cognitifs en rapport avec la culture financière sont recueillies via un bref questionnaire soumis à l'issue des épreuves cognitives de culture financière. Les items portent sur des aspects liés à trois des quatre grandes catégories de contenu retenues par le groupe d'experts : l'accès à l'information et à l'éducation, l'accès à l'argent et aux produits financiers et les comportements en matière d'achat et d'épargne. Le questionnaire comporte un petit nombre de questions qui explorent l'étendue et la nature de l'intérêt que les élèves portent aux matières financières et leurs expériences dans ce domaine.

Ces questions incluses dans ce bref questionnaire s'inspirent de celles administrées lors d'enquêtes nationales sur la culture financière. D'autres informations pertinentes pour comprendre la répartition des élèves entre les niveaux de 
culture financière sont recueillies via le questionnaire contextuel "Élève ». Les données sur le milieu familial des élèves (en particulier le niveau socio-économique) et sur leur parcours scolaire peuvent être utiles pour comprendre leur performance en culture financière. De plus, le questionnaire «Établissement ", que tous les chefs d'établissement figurant dans l'échantillon PISA sont invités à remplir, inclut des questions sur l'accès des élèves à l'éducation financière ainsi que sur l'accès des enseignants à des activités de formation continue en rapport avec l'éducation financière.

\section{PRÉSENTATION DES RÉSULTATS EN CULTURE FINANCIÈRE}

Les résultats des épreuves de culture financière PISA sont enregistrés dans une base de données distincte de la base de données principale. Dans cette base de données figurent les résultats des élèves aux épreuves de culture financière, de culture mathématique et de compréhension de l'écrit, des données comportementales tirées de leurs réponses au questionnaire sur la culture financière et, enfin, des données provenant des réponses au questionnaire «Élève » et au questionnaire «Établissement».

De la sorte, il est possible de rendre compte du niveau de culture financière en tant que tel et en combinaison avec la performance en mathématiques et en compréhension de l'écrit, ainsi qu'en combinaison avec plusieurs variables contextuelles, notamment le milieu socio-économique et l'ascendance allochtone. Ces résultats alimenteront la poursuite des travaux dans le cadre du projet de l'OCDE sur l'éducation financière.

Les données cognitives sur la culture financière sont mises à l'échelle selon une méthode similaire à celle qui s'applique aux autres données PISA. La technique de modélisation utilisée pour la mise à l'échelle est décrite en détail dans le rapport technique sur le cycle PISA 2006, PISA 2006 Technical Report (OCDE, 2009c).

Chaque item se situe à un endroit précis de l'échelle PISA de culture financière en fonction de sa difficulté, comme chaque élève se situe à un point précis de cette échelle en fonction de l'estimation de sa performance.

Comme dans les autres domaines d'évaluation PISA, la difficulté relative des tâches est estimée sur la base du pourcentage d'élèves qui parviennent à y répondre correctement. La performance relative des élèves soumis à une épreuve est estimée en fonction du pourcentage d'items auxquels ils répondent correctement. Une échelle continue montrant la relation entre la difficulté des items et la performance des élèves sera élaborée.

L'échelle de compétence est divisée en niveaux sur la base d'une série de principes statistiques. Ces niveaux sont ensuite décrits en fonction des tâches qui y sont associées pour montrer le type de connaissances et de compétences requises pour les mener à bien. L'échelle et ses niveaux décrits constituent ce que l'on appelle une échelle de compétence.

Le calibrage du degré de difficulté des items permet d'estimer le niveau de culture financière que les items dénotent. Le fait de situer les élèves sur la même échelle permet de décrire leur niveau de culture financière. L'échelle de culture financière aide à interpréter le score des élèves.

Comme le veut l'usage dans I'enquête PISA, la moyenne de l'échelle de compétence est fixée à 500 points, et son écart type, à 100 points (sur la base des 13 pays de l'OCDE participants). Eu égard au nombre d'items (40) constituant les épreuves de culture financière administrées lors du cycle PISA 2012, quatre niveaux de compétence seront décrits - une première étape sur la voie de la description du développement de la culture financière -, ce qui permettra de comparer la performance des élèves entre les pays et économies participants, et au sein même de ceux-ci. L'administration facultative des épreuves de culture financière à I'occasion du cycle PISA 2012 donnera la possibilité de recueillir des données essentielles, tant pour l'enquête PISA que pour le projet de l'OCDE sur l'éducation financière. 


\section{Notes}

1. La déclaration conjointe du Conseil des ministres des Finances de I'APEC de 2012 est accessible à I'adresse suivante : www.apec.org/Meeting-Papers/Ministerial-Statements/Finance/2012_finance.aspx [en anglais].

2. Les attitudes à l'égard des matières financières et la confiance en soi dans ce domaine ne sont pas couvertes dans l'évaluation de la culture financière du cycle PISA 2012. 


\section{Références}

Atkinson, A. et F.-A. Messy (2012), « Measuring Financial Literacy: Results of the OECD / International Network on Financial Education (INFE) Pilot Study ", Documents de travail de l'OCDE sur la finance, l'assurance, et les pensions privées, vol. 15, Éditions OCDE.

Barsky, R.B., F.T. Juster, M.S. Kimball et M.D. Shapiro (1997), "Preference Parameters and Behavioural Heterogeneity: An Experimental Approach in the Health and Retirement Study ", Quarterly Journal of Economics, n 11, pp. 537-539.

Bernheim, D., D. Garrett et D. Maki (2001), «Education and Saving: The Long-Term Effects of High School Financial Curriculum Mandates », Journal of Public Economics, n 85, pp. 435-565.

Bloom, B.S. (éd.) (1956), Taxonomy of Educational Objectives: The Classification of Educational Goals, David McKay, New York.

Cole, S., T. Sampson et B. Zia (2011), « Prices or Knowledge? What Drives Demand for Financial Services in Emerging Markets? », The Journal of Finance, vol. 66, n 6 , pp. 1933-1967.

G20 (2012), G20 Leaders Declaration, Los Cabos, Mexique, http://www.g20.org/images/stories/docs/g20/conclu/G20_Leaders_ Declaration_2012.pdf.

Gerardi, K., L. Goette et S. Meier (2010), «Financial Literacy and Subprime Mortgage Delinquency: Evidence from a Survey Matched to Administrative Data », Federal Reserve Bank of Atlanta, Working Paper Series, n² 2010-10.

Grisay, A. et C. Monseur (2007), " Measuring the Equivalence of Item Difficulty in the Various Versions of an International Test », Studies in Educational Evaluation, vol. 33, n 1, pp. 69-86.

Habschick, M., B. Seidl et J. Evers (2007), Survey of Financial Literacy Schemes in the EU27, Hambourg.

Hastings, J. et L. Tejeda-Ashton (2008), «Financial Literacy, Information, and Demand Elasticity: Survey and Experimental Evidence from Mexico », NBER Working Paper, $\mathrm{n}^{\circ}$ 14538, Cambridge, Massachusetts.

Hilgert, M.A., J.M. Hogarth et S.G.Beverly (2003), " Household Financial Management: The Connection Between Knowledge and Behavior », Federal Reserve Bulletin, vol. 89, n 7, pp. 309-322.

Holt, C. et S. Laury (2002), «Risk Aversion and Incentive Effects », American Economic Review, vol. 92, n 5, pp. 1644-1655.

Huston, S.J. (2010), « Measuring Financial Literacy », The Journal of Consumer Affairs, vol. 44, n² 2, pp. 296-316.

Johnson, C. et M. Staten (2010), Do Inter-Temporal Preferences Trump Financial Education Courses in Driving Borrowing and Payment Behaviour?, article présenté lors de la 1 ère conférence annuelle sur la prise de décisions financières par les consommateurs, 27-29 juin, 2010, Boulder, Colorado.

Kempson, E., S. Collard et N. Moore (2005), Measuring Financial Capability: An Exploratory Study, Financial Services Authority, Londres.

Lafontaine, D. et C. Monseur (2006), Impact of Test Characteristics on Gender Equity Indicators in the Assessment of Reading Comprehension, Université de Liège.

Lusardi, A. (2009), " U.S. Household Savings Behavior: The Role of Financial Literacy, Information and Financial Education Programs », in C. Foote, L. Goette et S. Meier (éd.), Policymaking Insights from Behavioral Economics (pp. 109-149), Federal Reserve Bank of Boston.

Lusardi, A. et O.S. Mitchell (2008), "Planning and Financial Literacy: How Do Women Fare? ", American Economic Review, vol. 98, $\mathrm{n}^{\circ}$ 2, pp. 413-417.

Lusardi, A. et O.S. Mitchell (2011), "Financial Literacy and Planning: Implications for Retirement Wellbeing », in A. Lusardi et O.S. Mitchell (éd.), Financial Literacy: Implications for Retirement Security and the Financial Marketplace, Oxford University Press.

Lusardi, A., O.S.Mitchell et V. Curto (2010), "Financial Literacy among the Young », The Journal of Consumer Affairs, vol. 44, n 2 , pp. 358-380.

Lusardi, A. et P. Tufano (2009a), " Debt Literacy, Financial Experiences, and Overindebtedness », NBER Working Paper, n 14808 , Cambridge, Massachusetts.

Lusardi, A. et P. Tufano (2009b), "Teach Workers about the Perils of Debt », Harvard Business Review (novembre), pp. 22-24.

Ministerial Council for Education Early Childhood Development and Youth Affairs (2011), National Consumer and Financial Literacy Framework, MCEETYA Consumer and Financial Literacy Working Party, Melbourne.

Moore, D. (2003), Survey of Financial Literacy in Washington State: Knowledge, Behavior, Attitudes, and Experiences, Social and Economic Sciences Research Center, Washington State University.

OCDE (2005a), Recommendation on Principles and Good Practices for Financial Education, Éditions OCDE.

OCDE (2005b), Pour une meilleure éducation financière : Enjeux et initiatives, Éditions OCDE. 
OCDE (2008), Améliorer l'éducation financière et la sensibilisation aux questions d'assurance et de pensions privées, Éditions OCDE.

OCDE (2009a), Financial Literacy and Consumer Protection: Overlooked Aspects of the Crisis, Éditions OCDE.

OCDE (2009b), "PIAAC Literacy: A Conceptual Framework », Documents de travail de l'OCDE sur l'éducation, n 34, Éditions OCDE.

OCDE (2009c), PISA 2006 Technical Report, Éditions OCDE.

OCDE (2010a), Le cadre d'évaluation de PISA 2009 : Les compétences clés en compréhension de l'écrit, en mathématiques et en sciences, Éditions OCDE.

OCDE (2010b), Résultats du PISA 2009 : Savoirs et savoir-faire des élèves : Performance des élèves en compréhension de l'écrit, en mathématiques et en sciences (Volume I), Éditions OCDE.

OCDE (2011), Regards sur l'éducation 2011 : Les indicateurs de l'OCDE, Éditions OCDE.

OCDE (à paraître), Financial Education in Schools: Challenges, Case Studies and Policy Guidance, Éditions OCDE.

OCDE INFE (2009), Financial Education and the Crisis: Policy Paper and Guidance, Éditions OCDE.

OCDE INFE (2011), Measuring Financial Literacy: Core Questionnaire in Measuring Financial Literacy: Questionnaire and Guidance Notes for conducting an Internationally Comparable Survey of Financial literacy, Éditions OCDE.

OCDE INFE (2012), OECD/INFE High-Level Principles on National Strategies for Financial Education, Éditions OCDE.

Schuchardt, J., S.D. Hanna, T.K. Hira, A.C. Lyons, L. Palmer et J.J. Xiao (2009), « Financial Literacy and Education Research Priorities », Journal of Financial Counseling and Planning, vol. 20, n 1, pp. 84-95.

Smithers, R. (2010), "University Students Expect to Graduate with Debts in Excess of £15,000 », The Guardian, 18 mars 2010 , http://www.guardian.co.uk/money/2010/mar/18/university-students-graduate-mouting-debts.

Stango, V. et J. Zinman (2009), «Exponential Growth Bias and Household Finance », Journal of Finance, vol. 64, n 6, pp. $2807-2849$.

Van Rooij, M.A., A. Lusardi et R. Alessie (2011), "Financial Literacy and Stock Market Participation », Journal of Financial Economics, vol. $101, \mathrm{n}^{\circ} 2$, pp. $449-472$.

Yoong, J. (2011), "Financial Illiteracy and Stock Market Participation: Evidence from the RAND American Life Panel », in A. Lusardi et O.S. Mitchell (éd.), Financial Literacy: Implications for Retirement Security and the Financial Marketplace, Oxford University Press. 


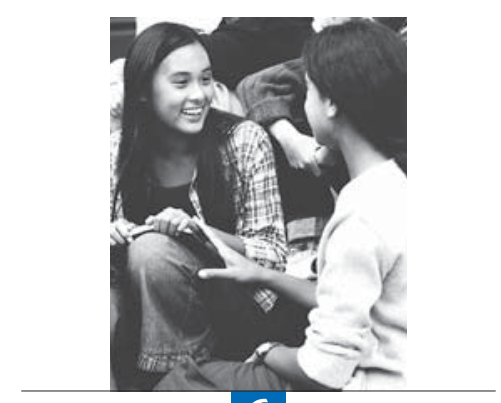

6

\section{Cadre conceptuel \\ des questionnaires contextuels du cycle PISA 2012}

Le cadre conceptuel des questionnaires contextuels du cycle PISA 2012 montre que les résultats PISA peuvent constituer une base de données durable qui soit pertinente pour la politique de l'éducation et la recherche en pédagogie. II décrit les fondements à retenir pour que les questionnaires contextuels PISA restent probants à l'avenir. II formule des recommandations à propos de la structure et de l'analyse pour exploiter au mieux les points forts de l'enquête PISA et jeter des bases encore plus solides pour l'avenir. Enfin, il expose les fondements théoriques d'une grande rigueur scientifique des concepts, des échelles et des indices du cycle PISA 2012 qui seront élaborés à partir des différents questionnaires contextuels administrés dans les pays et les économies participants. Ce cadre passe en revue de manière détaillée de nouveaux indicateurs de résultat individuel - les stratégies, les convictions et la motivation - en rapport avec la culture mathématique, ainsi que des indicateurs innovants sur les possibilités d'apprentissage et la qualité de l'instruction au niveau des élèves, des établissements d'enseignement et des systèmes d'éducation. 


\section{INTRODUCTION}

Depuis sa création à la fin des années 90, le Programme international de l'OCDE pour le suivi des acquis des élèves (PISA) est connu pour sa contribution significative aux débats sur la politique de l'éducation des pays de l'OCDE et des pays et économies partenaires. La "littératie » et les compétences utiles dans la vie, qui sont évaluées à l'âge de 15 ans, reflètent le rendement cumulé de l'apprentissage dans le cadre scolaire et en dehors. La compréhension de l'écrit, la culture mathématique et la culture scientifique sont des atouts importants pour la réussite académique, scolaire, civique, sociale et privée des individus. Rapporté par système ou par pays, le niveau moyen de ces compétences est un indicateur probant du capital humain, déterminant pour la prospérité et le bien-être de la société. La répartition de ces compétences dans la population en fonction du sexe, de l'origine, du milieu socio-économique ou de la situation géographique contribue à expliquer la diversité et l'équité entre les pays et au sein même de ceux-ci. Les résultats aux épreuves cognitives PISA sont combinés à des informations sur la situation des élèves pour fournir des informations sur la diversité, l'équité et le niveau des compétences utiles dans la vie. Les décideurs, les chercheurs, les professionnels de l'éducation, les parents, les élèves et l'opinion au sens large peuvent comparer les résultats de leur pays à ceux d'autres pays en utilisant les données PISA comme valeurs de référence. Pour recueillir les informations contextuelles requises, les élèves sélectionnés lors de tous les cycles de l'enquête PISA sont questionnés à divers égards, notamment leur sexe, leur origine (autochtone ou allochtone), et le niveau de formation et la profession de leurs parents.

La littératie et les compétences utiles dans la vie sont en grande partie le fruit de l'éducation dans le milieu familial et le cadre scolaire. Pour décrire ces contextes d'apprentissage et mieux cerner leur impact sur la performance des élèves, I'enquête PISA demande aussi aux élèves, aux chefs d'établissement et, parfois, aux parents d'élèves de faire part de leur point de vue sur ces contextes. Ces informations supplémentaires nous aident à répondre à diverses questions. La culture pédagogique varie-t-elle entre les pays, soit, en d'autres termes, pouvons-nous identifier des profils pédagogiques spécifiques aux pays ? Des établissements d'enseignement différents accueillent-ils à l'échelle nationale des effectifs d'élèves différents, soit, en d'autres termes, leurs politiques et pratiques ciblent-elles leurs effectifs respectifs ? Les écarts de performance entre les élèves peuvent-ils s'expliquer par des différences dans leurs caractéristiques sociétales, leur famille, les ressources et les pratiques pédagogiques de leur établissement, et leur communauté ? Les réponses apportées à ces questions par l'enquête PISA ont fait grand bruit dans les débats sur la qualité, l'efficacité et l'efficience des systèmes d'éducation dans le monde. En une décennie, les cycles PISA 2000, 2003, 2006 et 2009 ont permis de créer l'une des bases de données les plus importantes du monde qui peut être utilisée afin de tirer des enseignements pour éclairer l'action publique et d'évaluer l'efficience de l'instruction. Les responsables politiques peuvent se servir des réponses qu'ils trouvent dans les résultats de l'enquête PISA pour réorienter leur action, redéfinir leurs objectifs, revoir les priorités de la réforme de l'enseignement, réaffecter les ressources, promouvoir de nouveaux concepts pédagogiques et de nouvelles stratégies organisationnelles et, en fin de compte, améliorer l'éducation. Comme l'enquête PISA réévalue les systèmes d'éducation tous les trois ans, elle éclaire les changements dans l'éducation et en suit l'évolution.

Comme l'enquête PISA en est à son cinquième cycle, le moment est venu de revoir le cadre conceptuel qui oriente la conception des questionnaires et la façon dont les informations sont utilisées pour analyser et rendre compte des données. Entre 2000 et 2009, par exemple, le questionnaire "Élève » a permis de recueillir des données sur plus de 70 indicateurs (échelles agrégées à partir des données du questionnaire) à propos des activités et des ressources familiales, du cadre d'apprentissage en milieu scolaire et à domicile, et des perceptions et des motivations des élèves. Toutefois, aucun de ces indicateurs n'a été administré lors des quatre cycles, et la majorité d'entre eux (plus de 55) ne I'ont été qu'une seule fois. Les cycles PISA ont donc jusqu'à un certain point été conçus comme une étude indépendante sur les contextes d'apprentissage axée sur un domaine majeur spécifique. Le contenu des questionnaires a été largement déterminé par des priorités ponctuelles plutôt que par des objectifs à long terme en matière de recherche et d'action publique. Ce principe de conception s'est incontestablement révélé utile pendant que l'enquête était en cours de développement et que de nouveaux domaines ou indicateurs continuaient d'apparaître.

Le cadre conceptuel et analytique de l'enquête continuera assurément d'évoluer en fonction des changements dans la société et dans l'éducation - par exemple, l'importance croissante des technologies de l'information et de la communication (TIC) en tant qu'instrument d'apprentissage et domaine de compétences transversales, ou la nécessité de combiner plusieurs cadres d'apprentissage (à l'école, pendant des activités extrascolaires, par e-learning et même l'éducation à domicile). L'enquête PISA en est toutefois arrivée à un certain degré de maturité. L'enquête PISA étant une source probante et stable de comparaisons internationales et d'informations tendancielles sur les systèmes d'éducation, la mise au point des prochains questionnaires requiert I'adoption d'une structure cohérente, qui concilie stabilité et innovation, tout en combinant thématiques générales et thématiques spécifiques aux domaines d'évaluation. Ce document décrit la structure qui a été utilisée pour concevoir les questionnaires contextuels du cycle PISA 2012. 
Ce chapitre se divise en cinq grandes sections :

- La première section ( "L'objectif général de l'enquête PISA et sa pertinence pour l'action publique ») décrit l'objectif général de l'enquête et ses objectifs spécifiques à l'action publique, et explique les types d'informations contextuelles nécessaires pour réaliser ses objectifs et les raisons de leur utilité. L'enquête PISA doit s'intéresser à divers facteurs en rapport avec les élèves et leur famille, avec les établissements d'enseignement et avec le système d'éducation puisqu'elle procède à un suivi comparatif, cherche à comprendre l'efficacité de la politique de l'éducation et des pratiques pédagogiques, et alimente les recherches sur l'action publique. Les questions relatives à l'égalité des chances appellent une attention toute particulière dans la politique de l'éducation.

- La deuxième section («La base de connaissances générales : la recherche sur l'efficience de l'éducation ») décrit la base de connaissances générales que l'enquête PISA peut utiliser pour remplir ses missions. Elle commence par présenter la structure qui permet d'organiser les constructs et les indicateurs à différents niveaux. La taxinomie s'inspire de la littérature sur l'efficience de l'éducation, qui est décrite de façon détaillée pour justifier la sélection des intrants, processus et extrants de niveau Système, Établissement et Élève. Des conclusions d'ordre général sur l'efficience et la productivité de l'éducation y sont également présentées.

- La troisième section (« Les conditions d'apprentissage au service de la culture mathématique ») étudie les environnements d'apprentissage en rapport avec les mathématiques, domaine majeur d'évaluation du cycle PISA 2012. Les questionnaires sont conçus pour permettre l'inclusion d'autres indicateurs de résultats, par exemple les attitudes et les stratégies, la qualité de l'instruction en mathématiques - y compris la possibilité que les élèves aient été exposés aux types de problèmes retenus dans les épreuves PISA (notamment les possibilités d'apprentissage) - et l'ajout de variables de niveau Système et Établissement.

- La quatrième section («Conception des questionnaires du cycle PISA 2012 ») décrit les questionnaires administrés lors du cycle PISA 2012, qui reposent sur cette base de connaissance et dont le contenu est déterminé par les objectifs de I'enquête PISA dans le domaine de l'action publique. En premier lieu, elle présente la structure globale des constructs et indicateurs qui sera appliquée lors des prochains cycles PISA. En deuxième lieu, elle revisite les indicateurs utilisés lors du cycle PISA 2003, le premier à avoir les mathématiques comme domaine majeur d'évaluation, dont la pertinence et la qualité sont établies pour tirer parti des travaux antérieurs et décrire des tendances. Enfin, elle évoque des questions de mise en œuvre inédites dans I'enquête PISA, en I'occurrence au sujet de I'adoption de la rotation des items dans le questionnaire "Élève », qui permettra d'étoffer l'instrument. Certaines questions sont soumises à tous les élèves, comme lors des cycles précédents, et d'autres le sont uniquement à des sous-groupes d'élèves. Quant au questionnaire "Établissement », son administration informatisée améliorera la convivialité de l'instrument et son taux de réponse.

- La cinquième section ( "Garantir la validité des instruments PISA, leur pouvoir explicatif et leur pertinence pour l'action publique ») étudie des principes fondamentaux de la structure et de l'analyse qui pourraient contribuer à préserver le caractère novateur de l'enquête PISA et la validité de ses mesures à l'avenir : les techniques de modélisation statistique utilisées pour définir et identifier des effets, garantir l'équivalence et la validité des mesures entre les cultures, l'échantillonnage d'établissements d'enseignement déjà échantillonnés lors du cycle PISA 2003 pour suivre I'évolution à l'échelle des établissements depuis le cycle PISA 2003 et l'utilisation d'études de suivi ou d'extensions longitudinales pour quantifier la valeur ajoutée de l'éducation.

Les questionnaires communs et les questionnaires proposés à titre d'option internationale lors du cycle PISA 2012 sont repris à I'annexe $\mathrm{A}$.

\section{L'OBJECTIF GÉNÉRAL DE L'ENQUÊTE PISA ET SA PERTINENCE POUR L'ACTION PUBLIQUE Objectifs majeurs de l'enquête PISA}

L'enquête PISA poursuit un éventail d'objectifs parallèles. Les points de vue des différentes parties prenantes dans les pays et économies participants se répartissent entre les domaines suivants :

- L'enquête PISA est un dispositif de suivi qui fournit des informations comparatives fiables sur les systèmes d'éducation, dont elle décrit les structures, le fonctionnement et la productivité (c'est-à-dire leur « rendement »). Les données PISA couvrent la performance des élèves, leur motivation et leur parcours scolaire jusque dans l'enseignement secondaire, les caractéristiques des établissements, la gouvernance scolaire et les questions en rapport avec l'équité (par exemple, la variation de la performance selon le sexe ou le milieu socio-économique).

- L'enquête PISA est une étude internationale qui contribue à enrichir notre base de connaissances sur l'efficience de l'éducation. Elle permet d'observer les modèles de relations entre les intrants, les processus et les résultats de 
l'éducation. Elle offre la possibilité de décomposer la variation de la performance des élèves et de chiffrer la part de cette variation qui est imputable à des facteurs spécifiques aux élèves, aux établissements d'enseignement et au système d'éducation. Chaque cycle de l'enquête PISA fournit de surcroît des données sur de nombreux facteurs - en rapport avec ces trois niveaux - censés, selon des recherches antérieures, influer sur la performance en compréhension de l'écrit, en mathématiques et en sciences. L'enquête PISA décrit ces facteurs et estime leur impact direct ou indirect sur la performance des élèves et d'autres résultats. Le prélèvement de grands échantillons représentatifs et l'utilisation de modèles statistiques sophistiqués permettent de généraliser les résultats tant dans les pays qu'entre eux.

- L'enquête PISA est une source de données qui permet d'étudier les contextes d'apprentissage en général (par exemple, les interactions de l'éducation dans le milieu familial, scolaire et extrascolaire, et leur impact sur le développement des compétences utiles dans la vie), et les variables en rapport avec l'éducation dans des contextes économiques et sociologiques (par exemple, la relation entre la démographie, la richesse, la croissance économique et les ressources humaines). La base de connaissances est étoffée par des données tendancielles sur les mathématiques, et couvrira 12 années dès que les résultats du cycle PISA 2012 y seront inclus.

L'enquête PISA propose trois types de "produits " pertinents pour I'action publique $: i$ ) des indicateurs de suivi du fonctionnement, de la productivité et de l'équité des systèmes d'éducation ; ii) des informations sur les facteurs déterminants de l'efficience de l'éducation; et iii) une base de données fiable et durable se prêtant aux comparaisons qui permet aux chercheurs du monde entier d'approfondir leurs études sur des questions de pédagogie ainsi que sur des questions en rapport avec l'action publique.

\section{Suivi comparatif des systèmes}

L'un des objectifs majeurs de l'enquête PISA est de suivre les systèmes d'éducation à plusieurs égards : la performance des élèves (en termes de littératie et, plus généralement, de compétences utiles dans la vie), certains de leurs résultats non cognitifs (leur motivation et leur bien-être), leur parcours scolaire et certaines de leurs caractéristiques contextuelles (leur appartenance ethnique et leur milieu culturel et socio-économique), ainsi que des caractéristiques propres aux établissements d'enseignement et au système d'éducation (notamment les politiques d'évaluation et de responsabilisation, la sélection et l'affectation des élèves, l'implication des parents, la coopération entre les membres du personnel et les possibilités d'apprentissage). L'enquête PISA fournit également des indicateurs qui révèlent les relations entre ces facteurs, notamment le gradient social qui mesure l'intensité de la relation entre le milieu socio-économique et la performance, ou la relation entre les intrants et les extrants de l'éducation. Parmi les usages faits des données PISA, le plus important consiste à élaborer des indicateurs sur les intrants, les processus et les extrants, ainsi que des indicateurs relationnels, qui alimentent le programme de l'OCDE sur les indicateurs de l'éducation qui, à son tour, alimente le débat public, façonne la politique de l'éducation et éclaire la prise de décision.

L'enquête PISA s'inscrit dans une perspective internationale. Elle est conçue pour doubler les efforts consentis à l'échelle nationale et régionale en vue de recueillir des informations détaillées sur les élèves, les établissements d'enseignement et les systèmes d'éducation. Elle complète ces sources en fournissant des valeurs de référence internationales et des exemples de ce qui se fait dans le monde entier. À l'heure de la mondialisation économique et sociale, ces exemples peuvent donner de nouvelles idées et de nouveaux éléments pour orienter la politique de l'éducation à l'échelle nationale, régionale ou locale.

Ce système de suivi doit sa pertinence par rapport à l'action publique à plusieurs principes : i) la définition et l'opérationnalisation d'indicateurs de résultats cognitifs et non cognitifs qui permettent de choisir et de hiérarchiser les priorités de l'éducation dans les pays participants; ii) I'analyse et la présentation de facteurs sur lesquels l'action publique et les pratiques pédagogiques peuvent influer (les facteurs dits " malléables ») ; et iii) l'élaboration de valeurs de référence internationales, qui permettent aux responsables politiques de tirer des enseignements de ce que font d'autres pays. Les indicateurs sont en général sélectionnés à la lumière des demandes des responsables politiques. Les responsables de la politique de l'éducation doivent s'attacher au fonctionnement du système d'éducation (notamment à des caractéristiques telles que les ressources allouées aux établissements d'enseignement), à sa productivité (par exemple, les résultats bruts des élèves) et, aspect important s'il en est, à son équité (dont la répartition des ressources).

Pour présenter des indicateurs pertinents pour l'action publique, il faut non seulement évaluer la performance des élèves, en mathématiques par exemple, mais aussi analyser les données recueillies via les questionnaires "Élève ", «Établissement » et « Parents » qui couvrent un large éventail d'attributs en rapport avec des contextes, des processus et des résultats non cognitifs. Ce document présente les arguments théoriques et les considérations propres à l'action publique dont il est tenu compte pour sélectionner les constructs et définir les questions et les échelles. 
Avant l'enquête PISA, un certain nombre d'études quantitatives et qualitatives sur l'éducation ont permis de recueillir des éléments sur l'historique, le fonctionnement et, jusqu'à un certain point, sur l'efficience des systèmes d'éducation. L'enquête PISA est toutefois unique en son genre, car elle combine les atouts suivants :

- Elle fournit des données rigoureuses et une série intégrée d'indicateurs qui permettent de suivre les systèmes d'éducation.

- Elle se concentre à dessein sur le rendement, c'est-à-dire la performance des élèves à l'approche de la fin de la scolarité obligatoire. Elle cherche également à décrire des attributs malléables des systèmes d'éducation susceptibles de réorienter l'action publique, la mise en œuvre d'interventions et l'introduction d'améliorations.

- Elle fournit des indicateurs tendanciels, qui permettent de décrire l'évolution du niveau de performance des pays au fil du temps, élabore des variables sur des contextes, des processus et des résultats non cognitifs, et examine les relations entre ces divers constructs. Plus les cycles PISA se succèdent, plus des enseignements peuvent être tirés de l'examen de la stabilité ou de l'évolution des intrants, des processus et des extrants de l'éducation, et de la variation de leurs relations dans le temps.

De nombreux exemples d'indicateurs dérivés des données contextuelles de l'enquête PISA se trouvent dans les éditions récentes du rapport de I'OCDE Regards sur l'éducation (OCDE, 2007a, 2008, 2009a, 2011, 2012a), notamment :

- Les relations entre I'ascendance allochtone ou le statut socio-économique, d'un côté, et la performance des élèves, de l'autre (2007, indicateur A6 ; 2011 et 2012, indicateur A5).

- Le profil des élèves les plus performants en sciences, notamment leurs attitudes et leurs motivations à l'égard des sciences (2009, indicateurs A4 et A5).

- Les relations entre le plaisir de la lecture et la performance en compréhension de l'écrit (2007, indicateur A5).

- Les relations entre les ressources et les résultats de l'éducation (2007 et 2008, indicateur B7), en particulier compte tenu de la taille des classes (2008, indicateur D2).

- La comparaison du rendement de l'éducation entre la filière générale et la filière professionnelle (2007 et 2008 , indicateur (1).

- L'utilisation des évaluations et des examens dans les systèmes d'éducation (2008, indicateur D5).

- La qualité des établissements selon les parents (2008, indicateur A6),

- Les relations entre le milieu des élèves et leur accès à l'enseignement supérieur (ou leur motivation en la matière) (2007, indicateurs A4 et A7;2008, indicateurs A3 et A7).

L'un des défis les plus importants à relever concernant l'élaboration du cadre conceptuel des questionnaires contextuels consiste à garantir que les indicateurs sont comparables entre les cycles, tout en continuant à introduire de nouveaux indicateurs. Après une décennie de cycles PISA et d'évaluation internationale de la performance des élèves, le moment est venu de structurer et d'ordonner tous les constructs et instruments qui peuvent être utilisés soit ponctuellement, soit durablement à titre de source de données tendancielles. La pertinence de l'enquête PISA à l'avenir en dépend assurément.

\section{Comprendre les modèles d'efficience dans les systèmes d'éducation}

Des indicateurs attireront l'attention de l'opinion sur des aspects fructueux ou moins fructueux du système d'éducation, ou sur des objectifs qui ont été atteints ou qui ne l'ont pas encore été. L'objectif principal est donc d'orienter la hiérarchisation des priorités et la prise de décisions. Les responsables politiques veulent non seulement qu'on leur explique les points forts et les points faibles des élèves, et les conditions d'enseignement et de scolarisation dans leur pays, mais également comprendre pourquoi les élèves atteignent tel ou tel niveau de compétence. Les instruments contextuels PISA sont conçus pour contribuer à répondre à leurs questions. Les questionnaires PISA doivent donc couvrir les intrants et les processus les plus importants de l'apprentissage des élèves au niveau individuel, ainsi qu'à l'échelle des établissements d'enseignement et du système d'éducation. Les modèles statistiques contribuent, sur la base de ces données multiniveau, à mieux comprendre la complexité des interactions entre ces intrants et processus et les résultats des élèves. Lorsque les coûts et ressources sont connus, l'enquête PISA aide également à évaluer l'efficience, c'est-à-dire le retour sur investissement.

Durant la première décennie de l'enquête PISA, chaque cycle s'est concentré sur des variables en rapport avec le domaine majeur d'évaluation. En compréhension de l'écrit, par exemple, les expériences de lecture, l'intérêt pour la lecture, l'enseignement en classe, etc. ont été privilégiés en 2000 et en 2009. Le cycle PISA 2003 a accordé la priorité aux attitudes à l'égard des mathématiques et aux activités en cours de mathématiques, alors que le cycle PISA 2006 
a exploré les diverses pédagogies en sciences et les attitudes et les motivations des élèves en la matière. L'indice du climat de discipline en classe et l'indice du soutien apporté par les enseignants en classe ont été mesurés en langue de l'évaluation en 2000 et en 2009, et en mathématiques en 2003. Les données ont été opérationnalisées et analysées en fonction des domaines d'évaluation, mais les constructs, les relations théoriques avec les résultats des élèves et l'interprétation des données ont été définis compte tenu de théories générales sur l'efficience de l'éducation.

Ces données propres à chaque domaine d'évaluation ont permis de mieux cerner certains aspects, très importants pour la politique de l'éducation et les pratiques pédagogiques. L'OCDE clôture chaque cycle PISA par la rédaction d'un rapport initial (OCDE, 2001 ; OCDE, 2004 ; OCDE, 2007 ; OCDE, 2010). L'OCDE publie également à l'occasion de chaque cycle une série de rapports thématiques, c'est-à-dire des analyses approfondies des données PISA sur un large éventail de sujets concernant les élèves, les établissements d'enseignement et le système d'éducation (parmi les rapports récents, citons Untapped Skills: Realising the Potential of Immigrant Students, OCDE, 2012b ; Public and Private Schools: How Management and Funding Relate to their Socio-economic Profile, OCDE, 2012c ; Lisons-leur une histoire! Le facteur parental dans l'éducation, OCDE, 2012d). Depuis 2012, I'OCDE publie également PISA à la loupe, une série de notes de synthèse mensuelles sur la politique de l'éducation qui décrivent brièvement un sujet pertinent pour l'action publique'. L'OCDE vient aussi de lancer la série Strong Performers and Successful Reformers in Education pour prodiguer des conseils taillés sur mesure aux pays qui s'adressent à l'OCDE ou pour montrer la réussite du système d'éducation très performant d'un pays afin de permettre à d'autres pays de s'en inspirer².

Au-delà des publications de l'OCDE, voici quelques exemples intéressants tirés de la première conférence PISA qui a eu lieu en septembre 200933. Certains exemples sont basés sur des analyses internationales, alors que d'autres reposent sur les données PISA d'un seul pays :

- L'élévation du niveau d'engagement des élèves à l'égard de la lecture permet d'améliorer l'équité (Baye et al., 2009).

- Les moyens affectés aux établissements d'enseignement et la mesure dans laquelle ces derniers encouragent les élèves à exploiter tout leur potentiel sont des variables prédictives probantes de la performance en mathématiques en Corée (Kaplan, 2009a).

- Le temps d'instruction est en forte corrélation avec la performance en mathématiques dans les établissements d'enseignement suisses, sauf dans ceux où les exigences sont élevées (Angelone et al., 2009).

- Pour favoriser le développement des compétences des élèves en sciences et leur intérêt pour la science, polariser les cours sur des thématiques scientifiques semble être plus judicieux qu'élargir la gamme des activités scientifiques (Kobarg et al., 2009).

- La mesure dans laquelle les établissements d'enseignement prédisposent à l'exercice d'une profession scientifique est en forte corrélation avec la volonté des élèves d'exercer une telle profession (Lie et Kjaernsli, 2009).

- Les élèves japonais s'intéressent à l'apprentissage inquisitif, mais les professeurs de sciences ne tiennent pas compte de cet intérêt dans l'enseignement secondaire. Ce constat pourrait aider à expliquer pourquoi les élèves japonais accusent des faibles niveaux d'attitudes positives à l'égard de la science (Yasushi, 2009).

L'analyse des données PISA peut largement contribuer à enrichir la base de connaissances sur la politique de l'éducation et les pratiques pédagogiques, mais certaines limites doivent être prises en considération. La plus importante réside dans le fait que l'enquête PISA est une étude de rendement qui évalue les connaissances et compétences cumulées depuis la petite enfance jusqu'à l'âge de 15 ans, au fil des différents niveaux d'enseignement. L'enquête PISA ne cherche pas à déterminer ce que les élèves ont appris dans l'établissement d'enseignement qu'ils fréquentent au moment de l'administration des épreuves. Pour ce faire, il faudrait commencer par évaluer la performance des élèves au moment de leur entrée dans cet établissement et la comparer avec leur performance actuelle. Cela permettrait d'évaluer la progression de la performance associée à l'expérience d'apprentissage vécue dans cet établissement. Or, nous ne disposons pas d'indicateurs sur la performance des élèves avant l'âge de 15 ans, l'âge cible de l'enquête PISA. L'enquête PISA ne permet pas non plus - du moins en fonction de la structure en usage depuis plus de 10 ans - de juger de la qualité des enseignants et de son impact sur la performance des élèves. En effet, elle est administrée à des échantillons de sujets prélevés de façon aléatoire parmi les élèves âgés de 15 ans dans chaque établissement d'enseignement et non à des classes entières, ce qui empêche de recueillir des données sur les stratégies pédagogiques à l'échelle des classes. Enfin, les élèves viennent de commencer à fréquenter leur établissement d'enseignement dans un pays sur cinq parmi ceux qui ont participé au cycle PISA 2006 : il est donc pratiquement impossible de tirer des conclusions directes sur l'effet des établissements d'enseignement dans ces pays. 
Comme Baker (2009) le souligne, I'historique de l'élaboration des politiques à la lumière d'études comparatives internationales montre que des conclusions hâtives ont parfois été tirées à cause d'hypothèses trop simples sur les causes à l'origine des écarts de performance à l'échelle des systèmes. Par ailleurs, des économétriciens ont étudié un certain nombre d'aspects de la productivité de l'éducation, mais l'essentiel de leurs travaux est de nature descriptive et ne permet pas de faire des inférences causales (voir Hanushek et Woessmann, 2010).

Il est extrêmement difficile de dégager des inférences causales, par exemple conclure que telle ou telle politique d'éducation ou pratique pédagogique a un impact direct ou indirect sur la performance des élèves, de données issues d'observations et d'évaluations comme celles proposées dans l'enquête PISA (Gustafsson, 2007 ; Kaplan, 2009 b). Si des liens étaient établis entre la performance élevée des élèves et les données publiques sur les évaluations des établissements d'enseignement (dans le cadre de la politique sur les établissements, par exemple), la structure de l'enquête ne permettrait pas de les interpréter sous forme de relations causales. Cela s'explique par le fait que certains facteurs potentiellement importants, tels que la performance antérieure des élèves, ne peuvent être recueillis dans I'enquête PISA. En conséquence, il est impossible d'inclure ces facteurs potentiellement importants dans les analyses, ni de les contrôler de façon statistique. L'enquête PISA ne permet donc pas d'affirmer si cette politique n'est appliquée que dans les établissements d'enseignement très performants ou si elle entraîne effectivement une augmentation de la performance des élèves. Le problème fondamental réside dans le fait qu'en l'absence d'affectation aléatoire à un traitement (une politique ou une pratique), il n'est en général pas possible de déterminer si des facteurs non observés influent sur la sélection du traitement ou sur le résultat à l'étude. La possibilité de faire des inférences causales dépend donc de la volonté des chercheurs de formuler des hypothèses supplémentaires, qu'il leur est souvent impossible de tester. Certains chercheurs peuvent essayer de comparer des variables de contrôle observées entre les élèves témoins et les élèves traités pour écarter les élèves qui ne s'y conforment pas, mais le problème reste entier : il est possible que la sélection soit guidée par des variables non observées. En tout état de cause, il est essentiel que les chercheurs formulent clairement toutes les hypothèses qu'ils ont faites et, idéalement, qu'ils évaluent dans quelle mesure leurs inférences causales sont sensibles aux biais de ces hypothèses. (Voir la section "Garantir la validité des instruments PISA, leur pouvoir explicatif et leur pertinence pour l'action publique » pour un exposé analytique détaillé des méthodes et structures visant à remédier à certains de ces problèmes.)

La valeur du programme repose en grande partie sur les interactions constantes entre l'étude de suivi qu'est l'enquête PISA et des recherches plus rigoureuses sur l'efficience effectuées par ailleurs. Les résultats corrélationnels et exploratoires de l'enquête PISA peuvent ensuite être mis à l'épreuve dans des études longitudinales ou expérimentales, ou dans des analyses d'interventions. Des facteurs déclarés pertinents pour l'efficience de l'éducation dans la littérature sont très susceptibles de faire l'objet d'un suivi dans I'enquête PISA et d'être inclus dans la série d'indicateurs de I'OCDE sur l'éducation (voir la section «La base de connaissances générales : les recherches sur l'efficience de l'éducation »).

\section{Créer une base de données durable au service des recherches pertinentes pour I'action publique}

Les données PISA n'intéressent pas que les professionnels de l'éducation, les responsables politiques et les chercheurs en pédagogie. Elles sont de plus en plus utilisées par les économistes et les chercheurs en sciences sociales qui étudient des thématiques plus vastes, par exemple l'impact du capital humain sur la croissance économique (Hanushek et Woessmann, 2009) ou les variables prédictives de la réussite de l'intégration de familles de migrants (Stanat et Christensen, 2006).

Élargir la portée de l'enquête PISA pour en faire une base de données au service de recherches pertinentes pour l'action publique passe par une opérationnalisation très sophistiquée de constructs généraux tels que le milieu socio-économique des élèves et leur ascendance allochtone. Cela implique également la collecte d'autres informations (sur les valeurs civiques ou la santé, par exemple). Lors de la conceptualisation du contenu des questionnaires PISA, il faut tenir compte du fait que la base de données générée par l'enquête PISA doit répondre à des besoins - tant en matière de recherche que d'élaboration des politiques - qui vont au-delà du système d'indicateurs d'usage jusqu'ici.

À long terme, I'un des avantages majeurs de la base de données PISA sera de fournir des informations tendancielles. La production de l'éducation est difficile à comprendre à partir de données transversales, car il est pratiquement impossible d'inclure toutes les variables pertinentes - y compris les données sur les performances antérieures des élèves. Toutefois, l'évolution de la performance à l'échelle des pays (voir Gustafsson, 2007 ; Hanushek et Woessmann, 2010), voire des établissements d'enseignement (voir la section «Extension de l'échantillon pour étudier la variation au niveau Établissement ») et des élèves (voir la section «Extensions longitudinales de niveau Élève ») peut être interprétée et 
expliquée de façon probante si l'évolution des intrants et des processus est connue. Pour y parvenir, l'enquête PISA doit définir une série de variables qui resteront constantes pendant plusieurs cycles à l'avenir (voir la section « Répartition des contenus entre les cycles PISA : une structure globale de variables générales, les variables spécifiques au domaine d'évaluation et les variables d'extensions thématiques »).

\section{Priorité à l'équité}

L'équité se définit comme la répartition égale des ressources pédagogiques, des possibilités d'apprentissage et des résultats de l'éducation entre tous les individus scolarisés, quel que soit leur profil (OCDE, 2005a, p. 14). Étudier la variation des résultats cognitifs et non cognitifs entre les établissements d'enseignement et entre les élèves au sein des établissements est une première étape sur la voie de l'analyse de l'équité. L'écart entre les sujets très performants et les sujets peu performants est également intéressant. Il est possible de comparer des groupes d'élèves en fonction de leur établissement d'enseignement ou de leurs caractéristiques familiales. Ces variables ont souvent trait à la situation géographique (les régions ou le milieu rural ou urbain), aux effectifs des établissements d'enseignement publics ou privés, au milieu socioéconomique, au sexe, à l'ascendance allochtone et à la langue maternelle. Des écarts de performance ténus entre des groupes d'élèves ainsi constitués peuvent dénoter une plus grande équité. L'équité peut aussi s'évaluer en fonction de l'accès de ces groupes à l'éducation, aux ressources pédagogiques et aux possibilités d'apprentissage. Les politiques en faveur de l'amélioration du niveau d'équité peuvent consister soit à proposer une répartition égale des ressources, soit à fournir un soutien supplémentaire aux groupes défavorisés. Enfin, une notion " méritocratique » de la justice admet que la diversité existe parmi les élèves, mais veut que des différences d'accès aux possibilités d'apprentissage ou dans la répartition des résultats de l'éducation ne soient pas dues à l'origine sociale (Cleary, 1968). L'enquête PISA fournit des données qui aident à évaluer les systèmes d'éducation en fonction de ces différents critères d'équité.

Les données du cycle PISA 2012 permettent non seulement d'évaluer le degré d'équité, mais également d'analyser des caractéristiques des systèmes d'éducation et des établissements d'enseignement en rapport avec l'équité, par exemple les politiques d'éducation. À l'échelle des systèmes, cela peut consister à comparer les systèmes en fonction de pratiques en matière d'orientation des élèves et du financement et de la gestion des établissements d'enseignement. Les politiques qui promeuvent l'équité peuvent consister à répartir les ressources de manière uniforme, à fournir un soutien ciblé aux établissements d'enseignement dont les effectifs sont défavorisés ou encore à réglementer les cours de soutien, à encadrer l'apprentissage de la langue d'enseignement ou à proposer d'autres activités extrascolaires. À l'échelle des établissements d'enseignement, les pratiques de regroupement par aptitudes et celles qui visent à aider les élèves ayant des besoins spécifiques d'éducation semblent pertinentes. La pédagogie différenciée et un environnement d'apprentissage structuré et propice peuvent avoir un impact sur l'équité à l'échelle des classes. De plus, la prévalence de ces caractéristiques peut être comparée entre les systèmes plus ou moins équitables.

L'enquête PISA inclut des variables d'équité jugées essentielles depuis son premier cycle, en 2000. II est donc possible de suivre l'évolution du degré d'équité et d'identifier les changements qui y sont associés dans les politiques d'éducation et les processus agrégés. L'évolution de l'équité peut donc être comparée entre les pays et entre des groupes au sein même de ceux-ci sur une période de plus de 10 ans.

\section{LA BASE DE CONNAISSANCES GÉNÉRALES : LA RECHERCHE SUR L'EFFICIENCE DE L'ÉDUCATION}

\section{Un certain nombre de résultats de l'éducation et de variables prédictives}

L'école intègre les jeunes issus de milieux différents dans la société en leur permettant de participer à des activités cognitives et culturelles, de se livrer à des interactions sociales et de prendre part à la vie civique en général. Les établissements d'enseignement remplissent de nombreuses fonctions dans les sociétés modernes, notamment :

- instruire les jeunes et les préparer à réussir dans la vie, y compris dans leurs études et leur carrière professionnelle ;

- délivrer des diplômes et autres certificats pour guider les élèves dans la voie qui leur convient, que ce soit en termes d'études ou de carrière professionnelle, compte tenu de leur profil de compétence et de leurs objectifs personnels ; et

- cultiver le cour et l'esprit des élèves, car l'école est un lieu de règles et de normes - dont certaines sont explicites, et $\mathrm{d}^{\prime}$ autres informelles.

Dans I'enquête PISA, la littératie se définit comme la capacité des élèves à utiliser différents types de textes et d'outils mathématiques, et à se livrer à un raisonnement scientifique lorsqu'ils abordent, interprètent et résolvent des problèmes, et qu'ils prennent des décisions dans des situations de la vie réelle. Dans les sociétés modernes de la connaissance, la littératie est I'un des objectifs et des résultats les plus importants de l'éducation. L'enquête PISA cherche aussi à 
évaluer des compétences cognitives plus transversales, en rapport par exemple avec les stratégies d'apprentissage, le raisonnement et la résolution de problèmes. La réussite à l'école - et dans la vie - dépend également de l'engagement des élèves, de leurs valeurs et de leurs convictions, et de la mesure dans laquelle ils respectent et comprennent l'autre, ils sont motivés à l'idée d'apprendre et de collaborer, et ils sont capables de gérer leur comportement d'apprentissage. Ces constructs peuvent être considérés comme des conditions essentielles à l'apprentissage cognitif, mais aussi, en soi, comme des objectifs de l'éducation, comme l'explique la publication de l'OCDE Defining and Selecting Key Competencies (DeSeCo) (Rychen et Salganik, 2003). C'est la raison pour laquelle l'enquête PISA s'intéresse à des résultats non cognitifs tels que les attitudes, les convictions, la motivation, les attentes et le comportement en matière d'apprentissage (autorégulation, stratégies et temps investi). L'absentéisme est une autre variable non cognitive qui suscite beaucoup d'intérêt, car elle constitue un indicateur (négatif) important de la mesure dans laquelle les élèves utilisent les possibilités d'apprentissage qui leur sont offertes et est un facteur prédictif probant de l'abandon scolaire et des comportements déviants (Kearney, 2008 ; Lee et Burkam, 2003). Ces résultats non cognitifs s'évaluent essentiellement sur la base du questionnaire « Élève », mais également sur la base du questionnaire «Établissement ». Des questionnaires proposés à titre d'option internationale, par exemple ceux sur le parcours scolaire et sur la maîtrise des technologies de l'information et de la communication, étendent ces indicateurs à des domaines qui revêtent un intérêt particulier pour un certain nombre de pays.

Une longue section des questionnaires "Élève » et "Établissement », ainsi que des questionnaires proposés à titre d'option internationale, concerne des facteurs contextuels en rapport avec des résultats cognitifs et non cognitifs. Ces éléments sont utilisés pour évaluer l'efficience et pour définir des indicateurs. Ces facteurs font référence dans l'ensemble à des intrants ou à des processus. Les facteurs d'intrant concernent essentiellement le profil social et personnel des individus. Des caractéristiques structurelles telles que la taille des établissements d'enseignement et leur financement sont également considérées comme des intrants. Côté processus, l'apprentissage et l'enseignement sont considérés comme des processus majeurs, dont les aspects qualitatifs et quantitatifs sont évalués via plusieurs variables. De plus, les activités professionnelles des enseignants et des chefs d'établissement, et les politiques et pratiques scolaires, sont classées parmi les facteurs de processus.

Un problème particulier se pose dans les enquêtes internationales : les facteurs contextuels influant sur l'apprentissage des élèves sont à l'œuvre à quatre niveaux différents, soit les élèves et leur famille, leur classe, leur établissement d'enseignement et leur pays. Le processus de production se déroule comme suit :

- Des facteurs contextuels et structurels (intrants) via des interactions, des activités et des politiques (processus) jusqu'aux résultats cognitifs et non cognitifs : la performance des élèves en mathématiques, par exemple, dépend selon toute vraisemblance des activités au cours de mathématiques, qui dépendent à leur tour de la compétence de l'enseignant, de la taille de la classe, et des applications technologiques et autres ressources disponibles.

- Des facteurs d'ordre supérieur (système, établissement d'enseignement) à des facteurs propres à la classe et à des processus et des résultats propres aux élèves : la qualité de la direction pédagogique et de la collaboration entre enseignants dépend en partie du pouvoir de décision confié à l'établissement d'enseignement ou à un niveau administratif supérieur, ce qui façonne les stratégies pédagogiques et, donc, les activités d'apprentissage des élèves.

La structure fondamentale du modèle Intrant-Processus-Extrant a été élaborée dans les années 60 pour le compte de I'International Association for the Evaluation of Educational Achievement (IEA) (Purves, 1987). Une version plus récente de ce modèle, présentée dans le tableau 6.1, reprend ces dimensions. La première colonne affiche les quatre niveaux : Élève, Classe, Établissement d'enseignement et Pays. Les trois phases de production sont indiquées dans les autres colonnes, «Intrants », « Processus » et " Extrants ». Chaque cellule propose plusieurs constructs à titre d'exemple. Le modèle est relativement complet : il inclut la plupart des facteurs analysés dans I'enquête PISA et la quasi-totalité des constructs évoqués dans le présent cadre conceptuel. II s'agit d'une sélection parmi les nombreux constructs abordés dans la littérature sur l'efficience de l'éducation, mais le tableau reste trop fourni sous l'angle de la recherche. Le nombre de constructs qui pourront être pris en considération dépend du temps disponible pour le questionnaire et du système retenu en matière de rotation des items.

Côté intrants, certains facteurs sont relativement stables et difficiles à modifier, alors que d'autres peuvent être façonnés par des activités de développement scolaire ou des réorientations de l'action publique. Les processus sont généralement plus malléables, du moins indirectement (au travers de la formation initiale des enseignants et de leur développement professionnel). Les extrants sont le fruit des intrants et des processus. Toutefois, les limites entre les trois types de variables sont assez floues. Les extrants d'un cadre d'apprentissage deviennent les intrants d'un autre cadre, tandis que certains processus (les stratégies d'apprentissage) peuvent tout à fait être traités comme des intrants ou des extrants, selon des considérations théoriques, la nature des recherches ou des raisons pratiques. 
Une fois inclus dans un modèle dynamique sur l'efficience de l'éducation (voir Creemers et Kyriakides, 2008), les extrants deviennent des intrants au service du développement ultérieur. L'anxiété en mathématiques peut, par exemple, être un extrant et un intrant de l'éducation, qui a un impact sur les devoirs à domicile. De plus, les intrants peuvent s'influencer les uns les autres. Ainsi, dans de nombreux systèmes d'éducation, le niveau socio-économique des effectifs des établissements d'enseignement est en corrélation avec le financement des établissements, avec l'implication des parents dans la vie des établissements et même avec la qualité des enseignants. Tous ces facteurs se conjuguent pour offrir d'autres cadres d'enseignement et d'apprentissage (de meilleure qualité), et attirer des élèves issus de milieux favorisés (ou, plutôt, dont les parents sont plus favorisés), de sorte que la stratification sociale, les ressources et la qualité des processus se mêlent et sont difficiles à isoler.

Pour comprendre ces relations et effets correctement, et élaborer des modèles analytiques sophistiqués et adéquats, nous devons nous référer à la littérature sur l'éducation. Deux domaines de recherche sont particulièrement importants : les études sur l'efficience de l'éducation en général (voir ci-dessous) et sur l'enseignement et l'apprentissage en particulier (voir la section « Les conditions d'apprentissage au service de la culture mathématique »).

Le tableau 6.1 décrit une grande série de facteurs aux différents niveaux qui constituent le système d'éducation. Leurs interactions sont complexes et difficiles à comprendre, et chaque niveau contribue dans une grande mesure à façonner la politique d'éducation et ses résultats probables.

Tableau 6.1

Taxinomie bidimensionnelle des résultats de l'éducation et de leurs variables prédictives

\begin{tabular}{|c|c|c|c|}
\hline & Intrants & Processus & Extrants \\
\hline Élèves & $\begin{array}{l}\text { Sexe, année d'études, } \\
\text { statut socio-économique. } \\
\text { Parcours scolaire, notes. } \\
\text { Ascendance allochtone, } \\
\text { environnement et soutien } \\
\text { familiaux. } \\
\text { Expérience, attitudes et } \\
\text { compétences en informatique. } \\
\text { Ouverture, styles de résolution } \\
\text { de problèmes. }\end{array}$ & $\begin{array}{l}\text { Assiduité/absentéisme. } \\
\text { Activités extrascolaires (participation } \\
\text { à des activités extrascolaires, par exemple). } \\
\text { Motivation, engagement. } \\
\text { Stratégies d'apprentissage et de } \\
\text { raisonnement, stratégies lors de tests. } \\
\text { Temps d'apprentissage (y compris } \\
\text { temps consacré aux devoirs et aux cours } \\
\text { particuliers). }\end{array}$ & $\begin{array}{l}\text { Performance en mathématiques. } \\
\text { Attitudes, motivations } \\
\text { et convictions à l'égard des } \\
\text { mathématiques. } \\
\text { Attitudes et comportements } \\
\text { à l'égard de l'école en général } \\
\text { (engagement, absentéisme, etc.). } \\
\text { Motivation à l'idée d'apprendre, } \\
\text { attentes scolaires. }\end{array}$ \\
\hline Classes & $\begin{array}{l}\text { Taille des classes, milieu socio- } \\
\text { économique et composition } \\
\text { ethnique des effectifs. } \\
\text { Formation initiale et continue, } \\
\text { et expertise des enseignants. }\end{array}$ & $\begin{array}{l}\text { Qualité de l'instruction : structure, soutien, } \\
\text { défis. } \\
\text { Possibilité d'apprendre : programme } \\
\text { de cours, tâches à accomplir, activités } \\
\text { en rapport avec les mathématiques. } \\
\text { Temps d'enseignement, pratiques de } \\
\text { regroupement, évaluation et feed-back. }\end{array}$ & $\begin{array}{l}\text { Variables agrégées au niveau } \\
\text { Élève. }\end{array}$ \\
\hline $\begin{array}{l}\text { Établissements } \\
\text { d'enseignement }\end{array}$ & $\begin{array}{l}\text { Milieu socio-économique } \\
\text { et composition ethnique } \\
\text { des effectifs. } \\
\text { Milieu socio-économique } \\
\text { de la communauté. } \\
\text { Financement public ou privé } \\
\text { des établissements. } \\
\text { Taille des établissements. } \\
\text { Implications des parents. }\end{array}$ & $\begin{array}{l}\text { Importance de la performance, } \\
\text { normes partagées, leadership, moral } \\
\text { des enseignants, coopération entre les } \\
\text { enseignants, développement professionnel. } \\
\text { Politiques d'admission et de recrutement, } \\
\text { orientation des élèves, offre de cours, } \\
\text { programme de cours, évaluation. } \\
\text { Relations entre élèves et enseignants, } \\
\text { environnement favorable. }\end{array}$ & $\begin{array}{l}\text { Variables agrégées au niveau } \\
\text { Élève. } \\
\text { Passage de classe, redoublement } \\
\text { et taux d'obtention d'un diplôme. } \\
\text { Assiduité. }\end{array}$ \\
\hline $\begin{array}{l}\text { Pays } \\
\text { (systèmes) }\end{array}$ & $\begin{array}{l}\text { Richesse, (in-)égalité sociale. } \\
\text { Politiques en matière de } \\
\text { diversité. }\end{array}$ & $\begin{array}{l}\text { Financement des établissements } \\
\text { d'enseignement, politiques de } \\
\text { différenciation et d'orientation des élèves, } \\
\text { politiques de développement professionnel } \\
\text { des enseignants, soutien aux élèves ayant } \\
\text { des besoins spécifiques d'éducation ou } \\
\text { appartenant à un groupe linguistique } \\
\text { minoritaire, politiques de recrutement et } \\
\text { de certification. } \\
\text { Politiques de responsabilisation et } \\
\text { d'évaluation, instances décisionnelles. }\end{array}$ & $\begin{array}{l}\text { Variables agrégées au niveau } \\
\text { Élève. } \\
\text { Taux moyen d'obtention d'un } \\
\text { diplôme. }\end{array}$ \\
\hline
\end{tabular}


La recherche sur l'efficience de l'éducation vise essentiellement à identifier « les facteurs en rapport avec l'enseignement, le programme de cours et le cadre d'apprentissage, tels que la classe, l'établissement d'enseignement et les niveaux supérieurs, qui peuvent expliquer, directement ou indirectement, les écarts de performance entre les élèves, compte tenu de leurs caractéristiques, par exemple leurs aptitudes, leur milieu socio-économique et leur performance antérieure » (Creemers et Kyriakides, 2008, p. 12). Toutefois, les pratiques ne présentent pas la même efficacité pour tous les élèves d’un établissement d'enseignement, ni pour tous les systèmes d'éducation, contextes locaux ou établissements d'enseignement. De plus, différentes conclusions peuvent être tirées en fonction du type de résultat ciblé (Kyriakides et Tsangaridou, 2004). Dans ce contexte, les études récentes sur l'efficience de l'éducation tiennent également compte des interactions entre les intrants et analysent les différentiels d'efficience et les pratiques différenciées. De nombreuses études ont été menées dans ce domaine (voir, par exemple, Creemers et Kyriakides, 2008 ; Scheerens, 2000 ; Teddlie et Reynolds, 2000). Les sections suivantes résument les résultats obtenus pour chaque cellule de la taxinomie présentée dans le tableau 6.1. Par ailleurs, des façons d'opérationnaliser les grands constructs et les considérations à retenir en matière de conception seront présentées à la fin de chaque section.

\section{Intrants et processus à l'échelle des systèmes}

Dans la plupart des pays, les décisions en rapport avec la politique d'éducation et l'affectation des ressources sont du ressort d'une entité fédérale ou nationale. Dans les systèmes plus centralisés, l'instance suprême de décision est souvent le ministère de l'Éducation, alors que dans les systèmes moins centralisés, le pouvoir de décision peut être partagé avec les autorités des entités fédérées ou avec des agences régionales, au niveau des districts. Les facteurs systémiques sont classés parmi les intrants, les processus et les extrants dans cette taxinomie, mais leur rôle dans les modèles analytiques sera très vraisemblablement celui de variables modératrices ; en d'autres termes, les facteurs systémiques ont un impact sur les relations entre les intrants, les processus et les extrants au niveau inférieur.

Au niveau du système, les intrants de base concernent les ressources matérielles et leur répartition au sein des pays. Selon certaines études, le revenu par habitant est une variable prédictive probante de la performance des élèves tant entre les pays qu'au sein même de ceux-ci (Baker et al., 2002). La richesse permet de mobiliser des ressources au service de la performance des élèves (Baumert et al., 2005). Parallèlement, les résultats scolaires peuvent aussi influer sur la prospérité économique des pays. Les inégalités sociales sont en corrélation avec des inégalités dans l'éducation, à cause de la variation des ressources familiales et des conditions d'apprentissage. De plus, certains pays prévoient un financement similaire par élève, alors que d'autres accordent un soutien spécifique aux élèves qui vivent en zone rurale, qui sont défavorisés, qui sont issus de l'immigration ou qui souffrent de difficultés d'apprentissage ou de troubles physiques. Les orientations d'un pays concernant l'investissement dans l'éducation peuvent avoir des implications à la fois sur le niveau de performance ainsi que sur l'équité des résultats et de l'accès aux carrières. Il est un autre aspect important en rapport étroit avec les inégalités sociales : les politiques en matière d'immigration et de diversité (Stanat et Christensen, 2006). Les élèves issus de l'immigration accusent des résultats inférieurs dans plusieurs des matières principales dans de nombreux pays, alors qu'ils font jeu égal avec les autres élèves, voire les devancent dans d'autres pays (OCDE, 2012b). Les écarts de performance sont plus ténus dans les pays qui recrutent activement des immigrants très instruits. En revanche, des pourcentages élevés d'immigrants défavorisés peuvent poser des problèmes particuliers aux systèmes d'éducation. Des recherches montrent aussi que des politiques de soutien linguistique permettent de réduire les désavantages des élèves dont la langue maternelle n'est pas la langue d'enseignement.

Pour décrire les systèmes scolaires, il est important aussi d'étudier les politiques concernant le regroupement par aptitudes et la responsabilité de la gestion des établissements d'enseignement. Dans certains pays, les élèves sont répartis entre les établissements en fonction de filières d'enseignement (générale, professionnelle ou technique) selon leurs résultats scolaires et/ou leurs préférences. Dans ces systèmes différenciés, les élèves n’ont pas tous accès aux mêmes possibilités d'apprentissage. Ces pratiques reviennent régulièrement dans les débats sur la question de savoir si la différenciation prive de possibilités d'apprentissage des élèves dont le développement intellectuel est plus tardif ou qui sont issus de milieux socio-économiques moins favorisés (Levin, 1978). Les systèmes inclusifs, où tous les élèves suivent au moins un tronc commun minimal, peuvent être plus efficients en termes d'équité (OCDE, 2007b). Toutefois, même au sein des établissements d'enseignement, des classes peuvent être constituées d'effectifs hétérogènes ou regroupés par aptitudes. Les pays se distinguent non seulement en fonction de leur degré de différenciation, mais également en fonction de l'âge auquel les élèves sont répartis entre les établissements d'enseignement ou entre les filières, et de la possibilité de changer de filière. Enfin, point important s'il en est, les politiques d'admission et de passage de classe ont un impact sur les résultats aux épreuves PISA; en effet, la performance augmente si les élèves progressent plus vite entre les années d'études - toutes choses étant égales par ailleurs (Gustafsson, 2007). 
II ressort des cycles antérieurs de l'enquête PISA que le financement et la gestion des établissements d'enseignement sont le plus souvent publics, mais que dans une majorité des pays participants, il existe aussi un certain nombre d'établissements d'enseignement dont le financement et la gestion sont essentiellement du ressort d'organisations non gouvernementales, par exemple des associations confessionnelles ou des entreprises privées. Il existe aussi des établissements d'enseignement gérés par des organisations privées, mais essentiellement financés par les pouvoirs publics, ce que l'on appelle " les établissements d'enseignement privés subventionnés par l'État 》. Les deux types d'établissements d'enseignement s'inscrivent dans le même cadre réglementaire, mais les établissements privés jouissent généralement d'une plus grande latitude pour prendre des décisions en matière d'éducation. Ils représentent donc une forme de décentralisation de la prise de décision. Le choix entre les établissements publics et privés est considéré comme un mécanisme qui encourage les établissements à se concurrencer pour attirer les élèves, ce qui aurait pour effet d'améliorer le niveau de performance global du système d'éducation (Belfield et Levin, 2002). Toutefois, des résultats antérieurs de l'enquête PISA suggèrent que la performance moyenne plus élevée des établissements d'enseignement privés indépendants et subventionnés par l'État s'explique essentiellement par le fait que leurs élèves sont issus de milieux socio-économiques favorisés, ce qui a des conséquences en termes de composition des effectifs (OCDE, 2007b). Le pourcentage d'établissements d'enseignement privés et leurs caractéristiques peuvent donc influer sur l'équité, comme le degré de différenciation des élèves (OCDE, 2012c).

Depuis quelques années, de nombreux pays se demandent comment accroître la réactivité de leurs établissements d'enseignement et leur faculté d'adaptation aux besoins d'élèves, de groupes d'élèves et de communautés spécifiques. Beaucoup prétendent que lorsque la prise de décision est du ressort d'une instance centrale, le gouvernement national par exemple, le recrutement et la formation des enseignants et les programmes de cours sont trop rigides pour répondre aux besoins d'établissements d'enseignement dont les effectifs d'élèves sont différents. C'est la raison pour laquelle de nombreux pays ont entrepris de décentraliser leur système d'éducation et de déléguer le pouvoir de décision à des niveaux plus proches des élèves et des communautés, dans l'espoir d'améliorer la performance de leurs établissements d'enseignement (Hannaway et Carnoy, 1993). Toutefois, les études sur la relation entre cet aspect et les résultats scolaires n'ont pas permis de recueillir des éléments probants à l'appui d'une forte corrélation (voir, par exemple, Schmidt et McKnight, 1998). Certaines analyses secondaires des résultats des élèves à des épreuves internationales suggèrent que I'autonomie scolaire est bénéfique si elle va de pair avec une forme d'évaluation descendante ou l'administration d'épreuves centralisées (Woessmann, 2006).

Il peut être important de soumettre les établissements d'enseignement et les élèves à des évaluations pour garantir que les élèves atteignent tous des normes supérieures et sont tous préparés à poursuivre des études, en particulier dans les systèmes décentralisés (Carnoy et al., 2003). Plusieurs pays ont choisi cette voie, mais leurs approches en matière d'évaluation et de responsabilisation sont sensiblement différentes. Certains pays ont instauré un système périodique d'évaluation pour recueillir des données normalisées sur la performance des élèves et des établissements d'enseignement à l'échelle fédérale, nationale ou régionale. D'autres pays imposent ce type d'évaluations, mais laissent aux autorités régionales et locales le soin de concevoir et d'administrer les épreuves. D'autres encore ne procèdent pas à des évaluations systématiques. Par ailleurs, la responsabilisation s'étend aussi aux conséquences des résultats d'évaluation (Carnoy et al., 2003 ; Koretz, 2008). Dans certains pays, les tests et les enquêtes servent uniquement à informer l'opinion et les établissements d'enseignement. Les résultats aux tests peuvent pourtant être utilisés à d'autres fins, par exemple encourager l'augmentation de la performance ou identifier les points faibles des établissements et les aider à y remédier. Des systèmes différents peuvent avoir des conséquences différentes, que ce soit en termes d'incitations à apprendre, d'uniformité de l'instruction ou de résultats scolaires.

Outre ces variables systémiques plutôt descriptives, un certain nombre de politiques et pratiques plus spécifiques ont été proposées pour être incluses dans le modèle dynamique d'efficience de l'éducation (Creemers et Kyriakides, 2008). Ces politiques et pratiques visent à aider les établissements d'enseignement à créer un environnement d'apprentissage plus positif, à dispenser un enseignement aux normes qualitatives et quantitatives élevées, et à offrir aux élèves un large éventail de possibilités d'apprentissage. Peu d'études ont intégré ces processus systémiques. Le cycle PISA 2012 permet, dans une certaine mesure, d'analyser leur prévalence et leur corrélation avec la performance dans un échantillon important de pays. De plus, l'existence de ces politiques et la nature de leur mise en œuvre pourront être étudiées.

Les possibilités d'apprentissage dépendent des programmes de cours nationaux. Le programme de I'IEA Trends in Mathematics and Science Study (TIMSS) a identifié une variation sensible entre les programmes de cours tels que prévus et ceux appliqués dans les pays, en particulier en fonction du temps investi, de la cohérence et des priorités, ce qui explique en partie les écarts de performance observés entre les élèves, du moins dans des évaluations basées sur les programmes de cours telles que l'étude TIMSS (Schmidt et al., 2001 ; Schmidt et Houang, 2007 ; voir également la 
section «Possibilités d'apprentissage et qualité de l'instruction : évaluation de l'environnement d'apprentissage »). Les responsables politiques peuvent aussi soutenir et encourager la création de possibilités extrascolaires d'apprentissage, en plus de l'apprentissage formel. Ils peuvent, par exemple, lancer des concours de mathématiques ou fournir aux établissements d'enseignement les moyens et le soutien requis pour organiser des programmes extrascolaires. Par ailleurs, les pays peuvent choisir des orientations politiques différentes au sujet des cours payants et d'autres formes $d^{\prime}$ « éducation parallèle » (Baker et LeTendre, 2005).

La qualité de l'instruction peut être contrôlée à l'échelle du système au travers de la définition de normes d'enseignement, dont le respect est vérifié. Cela implique la formulation d'attentes explicites sur la base des recherches les plus récentes et présuppose de surcroît que les évaluations ne portent pas uniquement sur les extrants, mais également sur l'enseignement et I'instruction. Les responsables politiques peuvent concevoir des politiques et mobiliser des ressources au service de la formation continue pour contribuer à la professionnalisation du personnel des établissements d'enseignement. Par activités de développement professionnel, on entend les activités visant à développer les connaissances, les compétences, l'expertise et d'autres caractéristiques liées au corps enseignant (OCDE, 2009b, p. 49). Dans de nombreux pays, les enseignants sont dans l'obligation de consacrer un certain nombre de jours à des activités de développement professionnel. Toutefois, le niveau et les tendances de participation aux différents types d'activités de développement professionnel varient sensiblement entre les systèmes d'éducation. La participation peut être encouragée via l'octroi de primes ou de compléments de salaire et l'adaptation des emplois du temps. Toutefois, les résultats de l'Enquête internationale de I'OCDE sur les enseignants, I'enseignement et I'apprentissage (Teaching and Learning International Survey, TALIS) donnent à penser que l'effet de ces mesures est limité (OCDE, 2009b).

Enfin, des valeurs et des normes culturelles influent également sur le comportement des différentes parties prenantes. L'engagement des élèves et de leurs parents à l'égard de l'établissement d'enseignement et de l'apprentissage dépend de la mesure dans laquelle ils apprécient les valeurs en rapport avec l'éducation. Le statut global des enseignants est important, car il a un impact sur l'attractivité de la profession aux yeux des personnes qui l'envisagent. L'implication de la communauté dans la scolarisation peut améliorer la sensibilité via l'adaptation aux besoins des populations locales.

Concernant l'élaboration des variables systémiques, certaines, telles que la richesse, l'investissement dans l'éducation et l'équité de sa répartition, la composition des populations de migrants et les règles en matière de différenciation et d'admission, peuvent être dérivées de bases de données existantes, par exemple du système d'indicateurs de l'OCDE sur l'économie, le bien-être et l'éducation ${ }^{5}$. D'autres pourraient être dérivées d'un questionnaire sur le système à soumettre à la direction de l'administration qui traiterait, par exemple, des politiques en rapport avec le recrutement et le développement professionnel des enseignants, l'évaluation et la responsabilisation, le financement des établissements, le degré de centralisation ou de décentralisation, les normes et les activités extrascolaires, et le soutien aux élèves ayant des besoins spécifiques d'éducation ou appartenant à des minorités. Plusieurs indicateurs peuvent être dérivés des données PISA agrégées, par exemple l'indicateur relatif à la participation des établissements d'enseignement au processus de prise de décision calculé à partir du questionnaire "Établissement ». Des données sur les élèves, les enseignants et les parents peuvent agrégées à l'échelle nationale pour fournir des indicateurs culturels, par exemple la valeur accordée à l'éducation par rapport à d'autres aspects de la vie personnelle et sociale.

\section{Intrants et processus à l'échelle des établissements d'enseignement}

L'organisation des établissements d'enseignement est complexe et varie considérablement, non seulement entre les pays, mais également au sein même des pays, c'est-à-dire entre les entités fédérées, les provinces, les régions, les districts scolaires et les établissements d'enseignement. Parmi les intrants des établissements d'enseignement, ceux en rapport avec leur effectif d'élèves sont susceptibles d'influer sur les résultats scolaires. Les établissements d'enseignement qui accueillent des élèves issus de milieux socio-économiques plus favorisés tendent à créer des environnements propices à l'apprentissage académique où les attentes sont élevées. De plus, ces établissements peuvent attirer de meilleurs enseignants. Cela avantage même les élèves issus de milieux socio-économiques moins favorisés qui les fréquentent. Les intrants relatifs aux élèves dépendent vraisemblablement de la richesse de la communauté environnante, ce qui a des effets sur l'apprentissage des élèves. Les quartiers plus riches tendent à craindre moins que les autres les effets possibles de la criminalité et de la violence, et à disposer de davantage de ressources pour accompagner les établissements d'enseignement de façon informelle. Par ailleurs, les bons établissements d'enseignement peuvent attirer des parents engagés, ce qui a également un impact sur la communauté. Le pourcentage d'élèves dont la langue maternelle n'est pas la langue d'enseignement est une dimension quelque peu différente. La diversité linguistique est une richesse pour les établissements d'enseignement, mais elle a aussi un coût, dans la mesure où elle implique la mise en place de systèmes spécifiques de soutien et peut nécessiter l'intervention d’enseignants spécialisés pour remédier aux difficultés 
de compréhension et tenir compte de différences culturelles potentielles. Dans de nombreux pays, le milieu socioéconomique varie fortement entre les communautés urbaines, suburbaines et rurales.

Lorsque les établissements d'enseignement encouragent et organisent l'implication des parents, ceux-ci peuvent être plus efficaces pour aider leurs enfants à progresser et pour apporter leur soutien à l'établissement. De plus, les parents qui participent à des activités scolaires sont plus susceptibles de se porter volontaires pour aider les établissements, ce qui accroît les ressources disponibles. Les parents qui connaissent les attentes des établissements d'enseignement parce qu'ils sont familiarisés avec les programmes et les exigences des enseignants sont plus à même d'aider leurs enfants. Enfin, amener les parents à participer activement aux initiatives éducatives et scolaires permet de créer des réseaux sociaux qui leur offrent la possibilité de faire connaissance et de s'entraider. Ces réseaux sociaux entraînent vraisemblablement une augmentation de la performance globale via la constitution d'un « capital social » (Coleman, 1988).

On estime également que la taille des établissements d'enseignement est en corrélation avec la performance. Les établissements qui accueillent beaucoup d'élèves peuvent leur offrir un éventail plus diversifié d'enseignants, de cours, de matières à option et d'activités extrascolaires, ce qui permet aux élèves de choisir les cours qui leur conviennent le mieux sur le plan éducatif et qui les motivent le plus. Les établissements plus grands tendent toutefois à être plus impersonnels, et le soutien aux élèves peut y être moins individualisé. Selon certaines études, la performance et l'engagement des élèves âgés de 15 ans sont plus élevés et moins variables dans les établissements d'enseignement secondaire de plus petite taille (Coleman, 1988). La question de la taille optimale des établissements d'enseignement reste sans réponse. En fait, certaines études suggèrent que la taille des établissements a des effets qui varient selon les groupes d'élèves, par exemple ceux constitués en fonction du milieu socio-économique (Lee et Smith, 1997).

En plus de ces intrants, un certain nombre de processus sont des variables prédictives directes ou indirectes des résultats à l'échelle des établissements d'enseignement. La caractéristique malléable la plus importante des établissements d'enseignement est vraisemblablement le climat (ou la qualité de l'environnement) scolaire. Le climat scolaire dépend non seulement des normes et des valeurs, mais également de la qualité des relations et de l'ambiance générale. Les priorités académiques - un consensus général au sujet de la mission de l'établissement d'enseignement et de la valeur accordée à l'éducation - favorisent l'apprentissage. De plus, un environnement d'apprentissage discipliné optimise l'exploitation du temps d'apprentissage. En revanche, les environnements qui se caractérisent par un manque de discipline et de respect sont contre-productifs, tant pour les enseignants que pour les élèves, et éloignent les établissements d'enseignement de leur mission. Les environnements disciplinés peuvent être façonnés par l'adoption de règles cohérentes (pour remédier à des comportements inappropriés des élèves, I'absentéisme par exemple). Les établissements d'enseignement efficients sont capables de s'adapter aux besoins spécifiques des élèves et des enseignants. Ils organisent des cours de soutien ou de perfectionnement pour les élèves ayant des besoins spécifiques d'éducation, les élèves très doués et les élèves dont la langue maternelle n'est pas la langue d'enseignement. De plus, ils proposent des services d'orientation et d'aide aux devoirs. Plus généralement, la variété et la qualité des programmes extrascolaires peuvent aussi favoriser l'apprentissage des élèves et sont particulièrement pertinentes pour les résultats non cognitifs et les perspectives professionnelles des élèves.

Trois aspects de l'organisation des établissements d'enseignement sont pertinents pour améliorer l'environnement d'enseignement et d'apprentissage : la collaboration entre enseignants, la professionnalisation et la direction. La collaboration entre enseignants fournit à ceux-ci un soutien pratique et affectif, et contribue à leur professionnalisation. Elle se présente sous diverses formes : coordonner les pratiques, rendre les programmes de cours plus cohérents, et fournir un soutien et un feed-back mutuels. Dans de nombreux pays, il est relativement courant que les enseignants s'échangent du matériel pédagogique ou discutent des difficultés d'apprentissage de certains élèves. Il existe des formes plus sophistiquées de coopération, par exemple les activités collectives d'apprentissage, telles que les visites d'études, la transmission de feed-back, la participation à des activités de développement professionnel, I'organisation d'activités communes à plusieurs classes ou groupes d'âge (OCDE, 2009b). Ces pratiques sont particulièrement utiles pour transformer les établissements en structures d'apprentissage ainsi que pour fournir un feed-back constructif aux enseignants et un soutien à la participation à des activités de développement professionnel qui répondent à leurs besoins spécifiques. Les chefs d'établissement doivent s'acquitter de tâches administratives en rapport avec la réglementation et les budgets, gérer les infrastructures et s'occuper des relations publiques. Toutefois, leur mission consiste avant tout à s'assurer que l'instruction et l'apprentissage sont de grande qualité dans leur établissement. Depuis quelques décennies, le concept de direction pédagogique, qui met l'accent sur cet aspect, est abondamment traité dans la littérature (Blase et Blase, 1998 ; Hallinger et Heck, 1998 ; Heck et Hallinger, 2005 ; Krüger et al., 2007 ; Leithwood et Riehl, 2005 ; Witziers et al., 2003). Toutefois, les résultats empiriques à propos des relations entre la direction pédagogique et la performance des élèves ne sont pas cohérents, vraisemblablement parce que la direction pédagogique a un effet indirect qui subit I'influence d'autres processus à l'échelle de l'établissement et de la classe. 
Le volume d'instruction peut être déterminé par les mesures politiques et la réglementation sur les cours, les horaires, la gestion du temps d'enseignement, l'assiduité des élèves et l'implication des parents. Des directives sur l'enseignement, les procédures de regroupement et le comportement des enseignants peuvent contribuer à améliorer la qualité de I'enseignement. Les possibilités d'apprentissage dépendent fortement des programmes de cours et du choix du matériel pédagogique. Toutefois, la description formelle des opérations à mener au sein des établissements d'enseignement est souvent trompeuse, car la mise en œuvre varie énormément (Fullan, 1992 ; Fullan et Stiegelbauer, 1991). C'est pourquoi les questionnaires PISA tentent d'obtenir des informations sur les opérations directement auprès des participants, en particulier des chefs d'établissement.

Il ne suffit pas de présenter ces politiques et pratiques, il faut aussi en évaluer les effets. L'évaluation des établissements d'enseignement peut être utilisée pour identifier les forces et les faiblesses des opérations, étudier les pratiques et analyser leur impact sur les résultats. L'enquête TALIS montre que les pratiques d'évaluation varient fortement entre les pays, non seulement quant à leur fréquence, mais également quant à leurs sources d'information, à leurs priorités et à leurs conséquences (OCDE, 2009b). Les effets des évaluations des établissements d'enseignement sur les résultats n'ont fait l'objet que de peu d'études à ce jour (Creemers et Kyriakides, 2008).

Le degré de centralisation (par opposition à l'autonomie) de la gouvernance est une caractéristique quelque peu différente qui a un impact sur la prise de décision. Dans certains pays, le recrutement des enseignants, l'élaboration des programmes de cours et l'évaluation des élèves s'effectuent au niveau central. Dans d'autres pays, les établissements d'enseignement doivent simplement se conformer aux orientations qui leur sont fournies au sujet des opérations scolaires de routine. À l'autre extrême du spectre, les établissements d'enseignement sont très autonomes : ils choisissent leurs enseignants, influent sur leur rémunération et sélectionnent la façon dont ils évaluent la performance de leurs élèves. Depuis quelques années, l'autonomie scolaire est souvent citée comme un moyen de réduire la rigidité bureaucratique ; elle pourrait avoir un impact sur les résultats de l'apprentissage (Bottani et Favre, 2001 ; Chubb et Moe, 1990). Toutefois, aucune conclusion définitive n'a encore été tirée, et la décentralisation fait toujours débat.

Concernant l'élaboration des variables scolaires, la plupart sont dérivées des réponses des chefs d'établissement à des questions sur les ressources de leur établissement (ou le manque de ressources), les programmes de cours (les horaires, les filières, les cours de soutien et de perfectionnement, les activités extrascolaires), le climat scolaire (les attentes, le moral des enseignants et des élèves, l'implication des parents et les problèmes de comportement) et les activités professionnelles (la collaboration entre enseignants, les normes, le leadership et les procédures d'évaluation). L'ajout de données dérivées d'un questionnaire soumis aux enseignants et agrégées à l'échelle des établissements d'enseignement permettrait de brosser un tableau plus vaste et probablement plus probant de la situation à ces égards. Cette approche est applicable dans les pays où le cycle PISA 2012 est associé au prochain cycle de l'enquête TALIS (OCDE, 2009b). En plus des informations dérivées du questionnaire "Établissement » sur ces sujets, des données de niveau Élève, dérivées notamment du questionnaire facultatif sur la maîtrise des TIC, et des données de niveau Parents peuvent être agrégées pour élaborer des indicateurs sur la composition des effectifs d'élèves, le capital social, les ressources des établissements d'enseignement et le climat scolaire (par exemple les TIC disponibles, les relations entre enseignants et élèves, I'attitude des parents à l'égard de l'établissement et leur implication dans la vie de l'établissement). Enfin, le questionnaire sur le parcours scolaire inclut notamment des questions sur les services d'orientation proposés par les établissements d'enseignement.

Le questionnaire «Établissement » administré lors du cycle PISA 2012 permet également de recueillir des données qui vont au-delà de la performance et de la conduite des élèves directement évalués. Les chefs d'établissement ont, par exemple, été invités à répondre à des questions sur l'absentéisme des élèves, ainsi que sur les taux de passage de classe et d'obtention d'un diplôme. Ces résultats comportementaux « objectifs » sont vraisemblablement plus comparables, du moins dans un même système d'éducation, que la plupart des indicateurs dérivés des déclarations ou des descriptions d'attitudes faites par les répondants. De plus, ils représentent des résultats de niveau Établissement, tandis que la performance théorique des élèves peut en grande partie être estimée sur la base de variables de niveau Enseignant et Classe.

\section{Intrants et processus à l'échelle des classes}

La plupart des expériences d'apprentissage que vivent les élèves se déroulent en classe. C'est en classe que les élèves sont exposés aux contenus, au matériel pédagogique, aux stratégies pédagogiques, à la composition des effectifs et au climat qui y règne. Comme des recherches antérieures l'ont montré, des variables proximales - dont des caractéristiques et pratiques propres à la classe - sont en corrélation plus forte avec la performance des élèves que des variables plus distales, telles que des facteurs à l'échelle des établissements d'enseignement et des systèmes (voir, par exemple, Wang et al., 1993). 
Les intrants les plus importants à l'échelle de la classe sont le contexte et l'enseignant (Wayne et Youngs, 2003). Par contexte, on entend les caractéristiques des élèves et leur regroupement, et la taille de classe. Les considérations à propos du milieu socio-économique et de la langue maternelle des élèves sont similaires à l'échelle des classes et des établissements. Dans de nombreux cas, la composition des effectifs varie non seulement entre les établissements d'enseignement, mais également au sein même de ceux-ci, en particulier lorsque les élèves sont répartis entre les classes en fonction de leur niveau de performance. Cette forme de regroupement a pour principe de permettre aux enseignants d'améliorer l'enseignement en l'adaptant aux aptitudes de leurs élèves, mais de nombreux éléments montrent qu'elle peut creuser les écarts de performance entre les groupes (Gamoran, 1992 ; Oakes, 2005). Les formes plus souples de regroupement par aptitudes, par exemple celles qui se limitent à certaines tâches ou à certaines périodes, ou le regroupement hétérogène, peuvent toutefois être utiles pour appliquer des pratiques pédagogiques différenciées et collaboratives (Slavin, 1990).

L'évaluation et la notation des élèves au jour le jour est un aspect spécifique des pratiques en classe, qui semble relativement important dans le contexte des études telles que l'enquête PISA. Les élèves sont-ils soumis régulièrement à des tests normalisés ? Reçoivent-ils un feed-back des enseignants sous la forme de notes ou de commentaires écrits ou oraux sur leurs notes? Les établissements d'enseignement, les enseignants, les élèves et/ou leurs parents reçoivent-ils des informations sur la performance des élèves par rapport à des normes générales ? Comment utilisent-ils ces informations ? Les élèves s'entrainent-ils à passer des tests ? Comment ces pratiques évoluent-elles dans le temps dans les différents pays ? Comme le montrent des études sur les évaluations formatives et sommatives (Brookhart, 2009) ainsi que sur les conditions et les effets de tests dont les enjeux sont très importants (Borko et al., 2007 ; Koretz, 2008), les résultats des élèves à des épreuves normalisées telles que celles administrées dans l'enquête PISA peuvent varier selon les politiques et pratiques en matière d'évaluation.

On estime généralement que la taille des classes a un effet (négatif) sur la performance des élèves. Lorsque les effectifs sont moins nombreux par classe, les élèves ont davantage de possibilités de participer et il est plus facile pour les enseignants d'utiliser des pratiques pédagogiques ciblées sur des élèves ou des groupes d'élèves. De plus, les enseignants ont moins de copies à corriger, ce qui leur laisse plus de temps pour se concentrer sur le feed-back et concevoir des activités et des évaluations plus riches. Des recherches internationales montrent pourtant que la réduction des effectifs doit être conséquente pour avoir quelque impact et qu'elle est moins pertinente dans l'enseignement secondaire que dans l'enseignement primaire (Gustafsson, 2007). Enfin, les effets de la taille des classes semblent spécifiques aux cultures. Les tailles de classes sont relativement grandes dans de nombreux pays asiatiques, où la performance moyenne des élèves est pourtant élevée.

Les enseignants se caractérisent par leur formation, leur expérience et leur expertise. Une formation académique poussée, une spécialisation dans la matière enseignée et une expérience professionnelle sont autant de qualifications souhaitables et d'indicateurs de la qualité des enseignants dans certains modèles. Toutefois, les éléments à l'appui de leur corrélation avec la performance des élèves ne sont pas cohérents (voir, par exemple, Hanushek et Rivkin, 2007 ; Libman, 2009 ; Mullis et Martin, 2007 ; Zuzovsky, 2009). Des effets plus importants sont établis avec l'expertise des enseignants (voir, par exemple, Baumert et al., 2009 ; Hill, Rowan et Ball, 2005). Ces effets sont décrits comme issus de la combinaison des connaissances des enseignants en pédagogie générale et appliquée à leur matière, de leurs convictions, de leur motivation et de la capacité à s'autogérer (voir, par exemple, Bromme, 1997 ; Brunner et al., 2006 ; Shulman, 1987). Outre les intrants décrits ci-dessus, un certain nombre de processus à l'œuvre à l'échelle de la classe améliorent, selon certaines études, l'efficacité de l'éducation. Il s'agit en particulier du climat et de la qualité de I'instruction en classe. II ressort de certaines recherches que l'apprentissage des élèves est dans l'ensemble favorisé par un climat positif et respectueux, relativement peu perturbé et axé sur la performance des élèves (Creemers et Kyriakides, 2008 ; Harris et Chrispeels, 2006 ; Hopkins, 2005 ; Scheerens et Bosker, 1997).

Les aspects majeurs d'un climat positif en classe sont : des interactions propices entre enseignants et élèves, de bonnes relations entre enseignants et élèves, une priorité aux accomplissements et un environnement d'apprentissage qui se caractérise par des règles claires de discipline. La qualité de l'instruction est toutefois une dimension plus complexe. Des éléments suggèrent qu'il n'y a pas qu'une seule façon de bien enseigner. Des cours bien structurés et bien gérés au rythme étudié, un suivi rapproché, une présentation claire et un feed-back encourageant et instructif (les aspects clés de l'« instruction directe ») sont en corrélation positive avec la performance des élèves. Toutes ces composantes contribuent à créer un environnement propice à l'apprentissage en classe et optimisent l'exploitation du temps d'apprentissage. Toutefois, la motivation et les résultats non cognitifs des élèves augmentent sous l'effet d'autres caractéristiques qualitatives de l'instruction, tels que le climat en classe et les relations entre enseignants et élèves qui favorisent l'autonomie des élèves, la compétence et les rapports sociaux. De plus, pour améliorer la compréhension 
conceptuelle, l'instruction doit se baser sur des contenus exigeants (Brown, 1994). Par ailleurs, différents sous-groupes d'élèves peuvent bénéficier de pratiques différentes en matière d'instruction. Les enseignants doivent donc orchestrer les activités d'apprentissage pour répondre aux besoins spécifiques de leur classe. Klieme et al. (2009) ont condensé ces aspects dans un cadre de qualité de l'instruction à trois dimensions : i) une gestion claire et bien structurée de la classe ; ii) un climat propice et axé sur les élèves en classe ; et iii) une activation cognitive sur la base de contenus exigeants. Plusieurs études indépendantes sur les cours de mathématiques dans l'enseignement secondaire ont confirmé depuis lors cette structure triarchique de la qualité de la classe et corroborent dans une certaine mesure l'impact cognitif et motivationnel supposé (TIMSS-Video : Klieme et al., 2001 ; COACTIV : Baumert et al., 2009 ; Pythagoras : Lipowsky et al., 2009). Klieme et Rakoczy (2003, voir également Kunter et al., 2008) ont identifié des structures similaires dans des extensions nationales de l'enquête PISA. Toutefois, la qualité de l'instruction, en particulier l'activation cognitive, est dans une grande mesure propre aux matières. Des aspects spécifiques aux mathématiques sont décrits dans la section « Les conditions d'apprentissage au service de la culture mathématique ».

Concernant I'élaboration des variables, I'enquête PISA n'inclut pas de niveau Classe dans la mesure où les échantillons d'élèves âgés de 15 ans sont prélevés dans toutes les classes des établissements d'enseignement échantillonnés, si ce n'est dans les quelques pays qui ont étendu leur échantillon pour y inclure des composantes basées sur les années d'études. Cette conception ne va pas sans difficultés pour analyser des effets spécifiques aux classes et aux enseignants (Opdenakker et van Damme, 2000 ; Van Landeghem et al., 2005), et étudier des modèles bien spécifiés d'efficacité de l'éducation. De plus, elle renforce l'orientation de l'enquête PISA sur le rendement. L'enquête PISA peut toutefois rendre compte des intrants et des processus à l'échelle de la classe de façon descriptive, compte tenu de variables dont d'autres sources établissent le lien avec la performance des élèves et moyennant leur évaluation via les questionnaires "Élève ", «Établissement » et, le cas échéant, «Enseignant ». Les chefs d'établissement ont, par exemple, répondu à des questions au sujet de caractéristiques contextuelles des enseignants et de leurs orientations pédagogiques principales, tandis que les élèves ont été interrogés sur le contexte de leur classe et les pratiques qui y sont d'usage. Les échelles PISA de climat de discipline et de soutien de l'enseignant en cours de mathématiques sont des indicateurs de la structure et du soutien dans le modèle triarchique de la qualité de l'instruction. Le cycle PISA 2006 a inclus plusieurs indicateurs sur les pratiques pédagogiques novatrices en cours de sciences, et le cycle PISA 2012 en a fait de même en mathématiques (voir la section «Possibilités d'apprentissage et qualité de l'instruction : évaluation de l'environnement d'apprentissage »). Des informations détaillées sur les convictions des enseignants, entre constructivisme et transmission directe par exemple, et sur leurs activités de développement professionnel viendront assurément affiner le trait. À l'occasion du cycle PISA 2012, certains pays devraient y parvenir en établissant un lien entre l'enquête PISA 2012 et l'enquête TALIS sur les enseignants en 2013. Ces données agrégées à l'échelle des établissements d'enseignement pourront donc alimenter des analyses secondaires des résultats de l'enquête PISA.

\section{Intrants et processus à l'échelle des élèves}

La performance des individus à l'âge de 15 ans n'est pas seulement le fruit des effets cumulés de la scolarité, elle est également le fruit des expériences d'apprentissage vécues dans le milieu familial. Les parents plus instruits sont capables d'offrir de meilleures possibilités d'apprentissage à leurs enfants. Ils sont aussi capables de leur donner un meilleur accès à l'écrit, au voyage et à d'autres ressources qui excitent la curiosité de leurs enfants. Selon certaines études, le fait que les parents nourrissent de grandes ambitions au sujet de la scolarité de leurs enfants et s'intéressent à leur travail scolaire est en corrélation avec leur réussite scolaire ; il en va de même avec la participation des parents aux conférences scolaires et leur implication dans les devoirs (Alexander et al., 2007 ; Christenson, 2004 ; Hoover-Dempsey et Sandler, 1997 ; Ma, 1999 ; Sui-Chu et Willms, 1996 ; Wang et al., 1993).

L'un des objectifs de la collecte de données sur le milieu familial des élèves est de tenir compte de ses impacts sur I'apprentissage lors de l'estimation des effets à l'échelle des établissements d'enseignement. Outre le questionnaire « Élève », le questionnaire "Parents» proposé à titre d'option internationale permet de recueillir des informations sur plusieurs variables pertinentes, notamment les attentes des parents concernant la carrière de leurs enfants, leur implication dans l'établissement d'enseignement et les matières scolaires évoquées en famille. Ensemble, ces instruments permettent de procéder à la triangulation du soutien des parents et de leurs attentes académiques sous divers angles. Les données sur le matériel informatique que les élèves ont à leur disposition, sur l'usage qu'ils en font - y compris à domicile - et sur leurs attitudes et compétences en la matière qui sont recueillies via le questionnaire sur la maîtrise des TIC contribueront en particulier à expliquer les résultats aux épreuves cognitives informatisées.

Outre le milieu socio-économique et familial des élèves, leur contexte linguistique semble pertinent. Grandir dans un milieu bilingue peut favoriser la sensibilisation linguistique et faciliter l'apprentissage de langues étrangères (Hesse et al., 2008). Toutefois, la maîtrise imparfaite de la langue d'enseignement durant les premières années de scolarité peut 
aussi avoir un impact négatif sur l'apprentissage dans d'autres matières et nuire au parcours scolaire des élèves à long terme (Schmid, 2001 ; Stanat et Christensen, 2006). D'une part, les migrants et les minorités ethniques peuvent avoir à s'adapter à un contexte culturel qui ne leur est pas familier à l'école (Berry, 1980, 1990 ; Hovey et King, 1996 ; Liebkind, 1996) et s'exposer à la discrimination (Amiot et Bourhis, 2005 ; Perreault et Bourhis, 1999). D'autre part, ces groupes se distinguent souvent par de plus hautes aspirations et une plus grande motivation que leurs condisciples qui ne vivent pas dans des minorités ethniques (Kao et Tienda, 1998 ; Krahn et Taylor, 2005 ; Stanat, 2006 ; Stanat et Christensen, 2006). Au sujet des élèves issus de I'immigration, I'enquête PISA cherche à identifier les différents intrants, processus et extrants de l'éducation pour isoler les effets de la migration des effets socio-économiques et linguistiques. Elle cherche aussi à isoler les effets individuels des effets collectifs ou contextuels. Le cycle PISA 2012 tente d'accroître le pouvoir explicatif de l'étude en incluant des indicateurs d'acculturation et de proximité culturelle perçue ainsi que des informations détaillées sur le contexte linguistique via le questionnaire sur le parcours scolaire. Le questionnaire facultatif «Parents » fournit des informations supplémentaires sur l'ascendance allochtone, et le questionnaire facultatif sur le parcours scolaire, des informations supplémentaires sur le contexte linguistique familial.

Les processus généraux de niveau Élève concernent essentiellement le temps d'apprentissage. Les possibilités formelles $d^{\prime}$ apprentissage sont d'ordinaire obligatoires, mais les élèves peuvent ne pas en tirer pleinement parti, que ce soit parce qu'ils sèchent les cours, qu'ils arrivent en retard ou qu'ils ne sont pas attentifs en classe. L'absentéisme et le temps consacré aux tâches influent fortement sur la performance des élèves et sont en corrélation avec les taux d'abandon, de criminalité et de toxicomanie (voir, par exemple, Baker et al., 2001 ; Lee et Burkam, 2003 ; McCluskey et al., 2004 ; Wilmers et al., 2002). De plus, ces comportements sont également pertinents sous forme d'indicateur agrégé de l'environnement d'apprentissage en milieu scolaire. En revanche, les élèves peuvent allonger leur temps d'apprentissage et diversifier leurs possibilités d'apprentissage en se livrant à des activités d'apprentissage individuelles ou à des activités extrascolaires, par exemple les clubs académiques, les concours, le bénévolat et les clubs de discussion. Les élèves issus de milieux sociaux défavorisés semblent bénéficier particulièrement de la participation à ce type d'activités (voir, par exemple, McComb et Scott-Little, 2003). Dans certains pays, suivre des cours payants de soutien scolaire (comme ceux proposés dans les juku au Japon) est courant et constitue une partie importante du système d'éducation (Baker et LeTendre, 2005).

Les ambitions scolaires sont une variable prédictive probante du niveau de formation futur des élèves (voir, par exemple, Thiessen, 2007) ; elles varient entre les groupes ethniques en termes de qualité et de stabilité (voir, par exemple, Kao et Tienda, 1998 ; Mau et Heim Bikos, 2000). La motivation des élèves et leurs attitudes à l'égard de l'apprentissage sont des intrants importants de ce niveau, qui peuvent également être considérés comme des résultats non cognitifs. Comme les styles d'apprentissage des élèves, elles sont en grande partie spécifiques aux matières ; c'est la raison pour laquelle nous y reviendrons dans la section suivante «Les conditions d'apprentissage au service de la culture mathématique ». Des caractéristiques générales, indépendantes des matières, des élèves peuvent en revanche expliquer la performance des élèves, en particulier des compétences transversales telles que la résolution de problèmes. Le cycle PISA 2012 inclut un indicateur sur l'ouverture des élèves à l'apprentissage et à l'exploration, ainsi qu'une taxinomie des approches de résolution de problèmes et un indicateur sur les stratégies lors des tests.

Concernant l'élaboration des variables, contrairement aux caractéristiques spécifiques au milieu et au soutien familial qu'il est plus judicieux de dériver du questionnaire "Parents », la plupart des caractéristiques constituant le profil d'apprenant des élèves sont, comme par le passé, évaluées sur la base du questionnaire "Élève » (d'autant que le questionnaire "Parents » est une option internationale en 2012). Deux des options internationales proposées lors du cycle PISA 2012 fourniront des informations supplémentaires, en l'occurrence les questionnaires sur la maîtrise des TIC et sur le parcours scolaire. Les variables prédictives de niveau Élève les plus probantes de l'apprentissage (la capacité intellectuelle et les connaissances antérieures) ne sont toutefois pas faciles à évaluer dans I'enquête PISA. Toutefois, des extensions longitudinales de l'enquête PISA, qui ont été menées avec succès dans certains pays, ont réussi à utiliser les capacités intellectuelles et les connaissances antérieures pour estimer l'impact de variables scolaires. II y a lieu d'envisager la possibilité d'opter pour des extensions longitudinales si les responsables politiques veulent être bien informés des effets des établissements d'enseignement et de la scolarité (voir les conclusions dans la section « Garantir la validité des instruments PISA, leur pouvoir explicatif et leur pertinence pour l'action publique »).

\section{LES CONDITIONS D'APPRENTISSAGE AU SERVICE DE LA CULTURE MATHÉMATIQUE}

Les mathématiques sont le domaine majeur d'évaluation du cycle PISA 2012, ce qui a des implications claires pour l'élaboration des variables contextuelles propres aux élèves et au système d'éducation. Conformément à la taxinomie des variables de l'éducation de niveau Élève présentée au tableau 6.1, I'enquête PISA cherche à décrire des aspects 
importants de l'affect des élèves et leur expérience des mathématiques dans le cadre scolaire et en dehors (voir, par exemple, l'expérience d'approches différentes à l'égard de l'enseignement des mathématiques, les approches d'apprentissage préférées), leur motivation, et leur intérêt et leur engagement en la matière. Au niveau institutionnel (voir les variables de niveau Classe et Établissement dans le tableau 6.1), l'enquête PISA cherche à décrire des aspects importants de l'apprentissage et de l'instruction en mathématiques, et notamment à analyser la relation entre les stratégies d'apprentissage et d'enseignement et la performance des élèves, ainsi que la relation entre l'organisation des établissements d'enseignement, les structures et l'engagement actif des élèves dans I'apprentissage. Ces objectifs cadrent bien avec la "Stratégie de développement de l'enquête PISA à plus long terme ", un texte dans lequel le Comité directeur PISA a affirmé dès 2005 qu'il était " concevable de faire de l'efficience et de l'efficacité des processus éducatifs la thématique majeure du cycle PISA 2012 ", car cette analyse "se prêterait particulièrement bien aux mathématiques, le domaine d'évaluation le plus fortement corrélé aux programmes de cours de l'enquête PISA » ${ }^{6}$.

Dans les sections suivantes sont décrits les fondements de l'analyse des diverses facettes de la culture mathématique, dont les conditions essentielles à l'échelle des élèves et des systèmes. De plus, les considérations conceptuelles sont accompagnées de recommandations concernant la conception des questionnaires contextuels du cycle PISA 2012.

\section{La culture mathématique : Un défi en matière d'instruction et d'évaluation}

Comme la culture mathématique est l'une des principales conditions de réussite dans la société moderne de la connaissance à forte composante technologique, c'est l'une des priorités de la plupart des systèmes d'éducation. Les compétences en mathématiques ont été et restent un domaine essentiel de la quasi-totalité des évaluations cognitives à grande échelle, qu'elles soient nationales ou internationales. Dans le monde entier, les responsables politiques se soucient énormément de la qualité de l'instruction des mathématiques. Toutefois, la signification même d'instruction de qualité a beaucoup changé ces 20 dernières années (voir Schoenfeld, 2006). Ce mouvement de réforme a de multiples origines, du pragmatisme de Dewey à la notion de développement cognitif du psychologue russe Vygotsky, en passant par la "Reformpädagogik » (réforme pédagogique) allemande, l'épistémologie constructiviste moderne et la théorie de l'autorégulation de l'apprentissage. À partir de ces sources, des concepts non mécanistiques d'apprentissage et d'enseignement ont été inventés, et ont fini par s'infiltrer dans les pratiques pédagogiques des professeurs de mathématiques.

Le document "Curriculum and Evaluation Standards for School Mathematics », publié en 1989 aux États-Unis par une association d'enseignants, est sans doute le texte fondateur de la conception moderne de l'enseignement des mathématiques ; il y a été fait largement écho dans le monde entier. Ce document décrit " cinq objectifs fixés à tous les élèves : i) apprendre à valoriser les mathématiques; ii) devenir confiants en leur capacité de faire des mathématiques ; iii) devenir des acteurs de la résolution de problèmes mathématiques; iv) apprendre à communiquer de façon mathématique ; et $v$ ) apprendre à raisonner de façon mathématique » (NCTM, 1989, p. 5). Les normes du NCTM marquent un changement net dans la façon de concevoir l'enseignement des mathématiques, car les facultés générales de raisonnement mathématique viennent remplacer les objectifs d'apprentissage axés sur les contenus que l'on utilisait auparavant pour caractériser la finalité de l'éducation. L'enseignement des mathématiques vise désormais à aiguiser les facultés de raisonnement mathématique et à améliorer leur application dans des problèmes tirés du monde réel, bien audelà de ce qui se faisait auparavant, à savoir inculquer des connaissances à restituer et des compétences procédurales. Des recherches plus récentes ont développé et étoffé ces compétences au travers de travaux théoriques et d'études empiriques (Niss, 2003 ; Blomhoj et Jensen, 2007). Plus récemment encore, des avancées similaires ont été faites via la Common Core State Standards Initiative (CCSSI) (2010) pour les mathématiques aux États-Unis. La version préliminaire du document de la CCSSI contient non seulement des normes de contenus mathématiques, mais aussi des pratiques mathématiques qui se basent explicitement sur les normes du NCTM évoquées ci-dessus.

Le cadre PISA d'évaluation des mathématiques s'inspire de ces avancées. Depuis 10 ans, I'enquête PISA a compté parmi les acteurs majeurs de la promotion de l'approche basée sur la modélisation et le raisonnement à l'égard des mathématiques, qui bénéficie du soutien sans faille au niveau mondial des responsables politiques et des experts spécialisés dans I'enseignement des mathématiques. Le cadre PISA 2012 d'évaluation des mathématiques (voir le chapitre 1) ne reflète pas les disciplines traditionnelles des mathématiques telles qu'elles sont enseignées (l'algèbre, la géométrie, les statistiques, etc.), mais accorde la priorité aux "idées majeures » qui guident la compréhension conceptuelle et s'attache aux compétences mathématiques, bien au-delà des savoirs et savoir-faire techniques.

Lors de la conceptualisation de l'enquête PISA, I'idée fondamentale était d'évaluer le raisonnement mathématique en contexte. Dans la version préliminaire du cadre d'évaluation des mathématiques du cycle PISA 2012, la culture mathématique se définit comme " l'aptitude d'un individu à formuler, employer et interpréter des mathématiques 
dans un éventail de contextes, autrement dit sa capacité à se livrer à un raisonnement mathématique et à utiliser des concepts, procédures, faits et outils mathématiques pour décrire, expliquer et prévoir des phénomènes. Elle aide les individus à comprendre le rôle que les mathématiques jouent dans le monde et à se comporter en citoyens constructifs, engagés et réfléchis, c'est-à-dire capables de poser des jugements et de prendre des décisions en toute connaissance de cause » (voir le chapitre 1). Le concept n'est guère différent de celui qui a été élaboré, puis utilisé pour concevoir les épreuves PISA des cycles antérieurs, où les items étaient conçus pour évaluer «la mesure dans laquelle [les élèves] sont capables de mettre en œuvre leurs compétences mathématiques pour résoudre des problèmes de la vie courantes»(OCDE, 2004, p. 40). Le cadre du cycle PISA 2012 (voir le chapitre 1) base toujours l'évaluation des savoirfaire mathématiques des adolescents de 15 ans sur les phases du processus de modélisation mathématique et retient une série de compétences mathématiques fondamentales : communication, représentation, élaboration de stratégies, mathématisation, raisonnement et argumentation, utilisation d'opérations et d'un langage symbolique, formel et technique, et utilisation d'outils mathématiques. De plus, les items sont conçus en fonction de quatre grandes catégories de contenus mathématiques dans l'enseignement secondaire : la quantité, l'incertitude et les données, les variations et les relations, et l'espace et les formes. L'évaluation PISA de la culture mathématique continuera donc à moyen terme à aller bien au-delà des connaissances et compétences mathématiques purement techniques.

Les épreuves cognitives PISA couvrent donc trois des cinq objectifs de l'enseignement des mathématiques cités ci-dessus, à savoir la capacité des élèves à se livrer à un raisonnement mathématique, à résoudre des problèmes et à communiquer. Les deux autres objectifs, valoriser les mathématiques et avoir confiance en sa capacité à faire des mathématiques, sont évalués à titre de résultats non cognitifs dans le questionnaire "Élève », comme les stratégies en rapport avec les mathématiques. Ces constructs sont considérés comme des résultats de l'enseignement des mathématiques et aideront de surcroît à expliquer les écarts de performance dans les épreuves PISA de mathématiques. La section suivante en explique les fondements conceptuels et les indicateurs.

\section{Indicateurs de résultats en rapport avec la culture mathématique : Stratégies, convictions et motivation}

\section{Stratégies et métacognition}

Les professeurs de mathématiques veulent non seulement connaître la nature et le nombre des problèmes que les élèves sont capables de résoudre, mais également leur cheminement pour résoudre ces problèmes (les stratégies qu'ils appliquent pour étudier leurs leçons de mathématiques, se préparer à des tests ou encore aborder des problèmes). Les stratégies d'apprentissage des mathématiques et de résolution de problèmes sont des résultats importants de I'enseignement et une condition essentielle à la poursuite de l'apprentissage en mathématiques - au même titre que les stratégies d'apprentissage sur la base de textes en compréhension de l'écrit. Les élèves qui connaissent et maîtrisent des stratégies sont capables d'utiliser leur culture mathématique dans de nouveaux contextes et de nouvelles tâches.

En compréhension de l'écrit, I'enquête PISA a utilisé les autoévaluations des élèves au sujet de trois stratégies d'apprentissage, à savoir la mémorisation (apprendre des mots clés, réciter des contenus), l'élaboration (établir des liens entre des domaines connexes, réfléchir à d'autres solutions) et le contrôle (des stratégies métacognitives impliquant une planification, un suivi et une régulation). Ces échelles ont été adaptées aux mathématiques lors du cycle PISA 2003. L'échelle d'élaboration a été constituée sur la base d'items adaptés de l'évaluation de la compréhension de l'écrit, tels que «Quand j'apprends des mathématiques, j'essaie d'établir des relations entre ce travail et des choses apprises dans d'autres matières », ainsi que d'items spécifiques tels que "Quand je résous des problèmes de mathématiques, j'imagine souvent de nouvelles façons de trouver la réponse » et «Quand je résous un problème de mathématiques, je réfléchis souvent à la façon dont on pourrait appliquer la solution à d'autres cas intéressants ». Cette nouvelle version de l'échelle s'est révélée valide : c'est une variable prédictive probante de la performance en mathématiques dans les pays participants. En revanche, l'échelle de mémorisation n'a pas été retenue à titre d'échelle distincte valide, et des résultats mitigés ont été obtenus pour les stratégies de contrôle que les élèves ont été invités à évaluer (OCDE, 2005b, p. 297 ; Vieluf et al., 2009a, 2009b).

C'est à dessein que les items relatifs aux stratégies d'élaboration en mathématiques se situent dans le contexte de la résolution de problèmes. Des études comparatives internationales montrent en effet que l'enseignement des mathématiques est essentiellement défini en termes de résolution de problèmes dans le monde entier (Christiansen et Walther, 1986 ; Hiebert et al., 2003 ; Stigler et Hiebert, 1999). Lorsque les élèves travaillent seuls, en petits groupes ou tous ensemble, ils doivent souvent résoudre des problèmes ou effectuer des tâches de routine. Dans de nombreux pays, les enseignants se servent d'exemples de problèmes, même lorsqu'ils présentent un nouveau contenu, pour que leurs élèves découvrent de nouvelles méthodes de résolution de problèmes. Étudier ou apprendre en mathématiques signifie 
donc essentiellement résoudre des problèmes. Ce sont les stratégies de résolution de problèmes qui sont au cœur de la culture mathématique, et non les stratégies d'apprentissage comme en compréhension de l'écrit. Des études sur les aspects cognitifs de l'apprentissage des mathématiques les ont redéfinies en tant qu'instances de ce que I'on appelle couramment la métacognition (Desoete et Veenman, 2006 ; Garofalo et Lester, 1985 ; Schneider et Artelt, à paraître ; Schoenfeld, 1992). En général, la métacognition désigne le fait que l'individu connaît ses stratégies et processus cognitifs et y réfléchit, comme le définit Flavell dans un texte désormais de référence (1979).

Concernant l'élaboration des échelles, l'échelle de stratégies d'élaboration créée à l'occasion du cycle PISA 2003 (autoévaluation des stratégies d'apprentissage en mathématiques et de résolution de problèmes) a été conservée pour permettre des analyses de tendances. Toutefois, comme Schneider et Artelt (à paraître) le soulignent, les indicateurs de métacognition dérivés des évaluations que font les élèves de leurs stratégies et de leur usage ne sont guère probants. De plus, il est possible d'envisager à l'avenir l'utilisation d'indicateurs dérivés des fichiers journaux des épreuves cognitives informatisées ou d'indicateurs dérivés des connaissances métacognitives déclarées comme ceux élaborés sur la base de I'évaluation de la métacognition en compréhension de l'écrit lors du cycle PISA 2009.

\section{Motivation et intentions}

À l'heure où les formations en sciences, en technologie, en ingénierie et en mathématiques attirent peu les étudiants, surtout de sexe féminin - un problème qui se pose dans de nombreux pays -, accroître l'intérêt pour les mathématiques et la motivation à l'idée d'apprendre en mathématiques, et faire naître des attitudes générales plus positives à l'égard des mathématiques, sont des priorités pour les responsables politiques. De plus, les éléments à l'appui d'une relation positive entre des attitudes favorables à l'égard des mathématiques et la performance en mathématiques abondent (voir, par exemple, la méta-analyse de Ma et Kishor, 1997).

Les théories sur la motivation des élèves font généralement la distinction entre la motivation intrinsèque et la motivation extrinsèque. La motivation intrinsèque provient de la gratification inhérente à une tâche ou à une activité. Lors du cycle PISA 2003, ce type de motivation a fait l'objet d'une échelle d'intérêt et de plaisir des mathématiques (sur la base d'items tels que "Je m'intéresse aux choses que j'apprends en mathématiques »). La littérature suggère que la motivation intrinsèque a un impact sur l'engagement des élèves et le temps qu'ils consacrent aux tâches, leurs activités d'apprentissage, leur performance et leur choix de carrière, et qu'elle peut être forgée en classe (Kunter, 2005 ; Rakoczy et al., 2008 ; Ryan et Deci, 2000). Par contraste, la motivation extrinsèque provient de la gratification attendue. II est établi qu'elle est une variable prédictive probante du choix de cours et de carrière, et de la performance (Wigfield et al., 1998). Lors du cycle PISA 2003, une échelle de motivation instrumentale a été élaborée au sujet de l'apprentissage des mathématiques, à partir d'items tels que «Pour moi, cela vaut la peine d'apprendre les mathématiques, car cela améliore mes < perspectives, chances $>$ de carrière professionnelle ».

Des modèles plus sophistiqués de la maîtrise comportementale font la distinction entre des dispositions plus générales telles que la motivation intrinsèque et extrinsèque et les intentions. Les intentions sont plus proches du processus réel de prise de décision et sont plus pertinentes pour déterminer dans quelle mesure les élèves utilisent les mathématiques dans leur vie et les intègrent dans leurs projets d'avenir. Ces items sont vraisemblablement moins biaisés en raison de réponses spécifiques aux cultures ou de normes liées à des groupes de pairs. Les intentions à court terme peuvent être évaluées sur la base d'une échelle dérivée d'items tels que « Je vais travailler dur pour bien apprendre en mathématiques » (Lipnevich et al., à paraître). Les intentions à long terme ou les orientations à l'avenir peuvent être rapportées sur une version adaptée de l'échelle élaborée au sujet des sciences lors du cycle PISA 2006 sur la base d'items tels que "J'aimerais exercer une profession dans laquelle interviennent les mathématiques ». Cette échelle permettrait de déterminer dans quelle mesure les pays peuvent espérer amener des sujets très performants à opter pour des professions en rapport avec les mathématiques, et d'étudier les inégalités d'accès dans les professions en rapport avec les sciences, la technologie, I'ingénierie et les mathématiques.

Concernant I'élaboration des variables, ces quatre échelles, à savoir l'intérêt et le plaisir des mathématiques, la motivation instrumentale relative à l'apprentissage en mathématiques, les intentions à court terme et les intentions à long terme, suffiront pour répondre à des questions sur la motivation des élèves en mathématiques qui sont pertinentes pour l'action publique. L'inclusion de six échelles d'attitudes dans le questionnaire "Élève » et l'intégration de plusieurs items d'attitude dans les épreuves cognitives, comme lors du cycle PISA 2006, surchargeraient inutilement les élèves. Toutefois, des approches novatrices pourraient être adoptées à titre expérimental pour réduire les biais culturels et garantir la comparabilité des résultats entre les cultures (voir la section «Extension de l'échantillon pour étudier la variation au niveau Établissement [option internationale] »). 


\section{Image de soi et comportement prévu}

La façon dont les élèves se considèrent est une variable prédictive probante de leurs actes et de leurs décisions face à des tâches et des situations difficiles (Bandura, 1977). L'efficacité perçue se définit comme la perception d'un individu de sa capacité à mener à bien certaines tâches. Quant à la perception de soi, elle renvoie plus largement à la façon dont les individus évaluent leurs attributs. Selon des études sur l'enseignement des mathématiques, I'efficacité perçue en mathématiques est une variable prédictive probante des notes en mathématiques, de la performance en résolution de problèmes, de l'intérêt pour les mathématiques et - une fois encore - des choix de carrière (Britner et Pajares, 2006 ; Pajares et Miller 1997 ; Turner et al., 2004). Lors du cycle PISA 2003, deux indicateurs ont été élaborés : la perception de soi en mathématiques (sur la base d'items tels que "J'apprends vite en mathématiques ») et la perception des capacités personnelles à surmonter les difficultés en mathématiques (voir Lee, 2009 ; OCDE, 2005b). Les élèves ont plus précisément été invités à indiquer dans quelle mesure ils se sentent sûrs d'eux à l'idée d'effectuer huit tâches de mathématiques dont la difficulté varie : certaines se basent sur des procédures techniques directes (résoudre une équation du type : $3 x+5=17$ ) et d'autres requièrent une modélisation mathématique (calculer la consommation d'essence d'une voiture) (OCDE, 2005b, p. 292). La perception de soi en mathématiques et la perception des capacités personnelles à surmonter des difficultés en mathématiques se sont toutes deux révélées équivalentes entre les cultures et il est apparu qu'elles étaient des variables prédictives probantes de la performance des élèves (Vieluf et al., 2009a, 2009b).

La mesure dans laquelle les élèves s'estiment confiants dans leur capacité à mener à bien des tâches mathématiques et la mesure dans laquelle ils valorisent les mathématiques sont des variables très pertinentes pour prévoir et expliquer le comportement des élèves en mathématiques, en cours et dans leur choix de carrière. Un certain nombre de modèles Attentes-Valeur ont été proposés, tant en psychologie qu'en économie, pour intégrer les deux aspects de la prise de décision. L'un de ces modèles est celui de la théorie du comportement planifié d'Ajzen (1991), selon laquelle le comportement volitif est déterminé par des attitudes spécifiques et des normes subjectives (côté valeur), et par le contrôle comportemental perçu (côté attentes). (Pour une application aux mathématiques dans les recherches interculturelles, voir Lipnevich et al., à paraître.) Selon Ajzen (1991), la manipulation de ces variables prédictives permet d'accroître la probabilité qu'un individu ait l'intention de poser un acte, ce qui entraîne l'augmentation de la probabilité qu'il pose effectivement cet acte (voir la figure 6.1).

- Figure 6.1 -

\section{La théorie du comportement planifié}

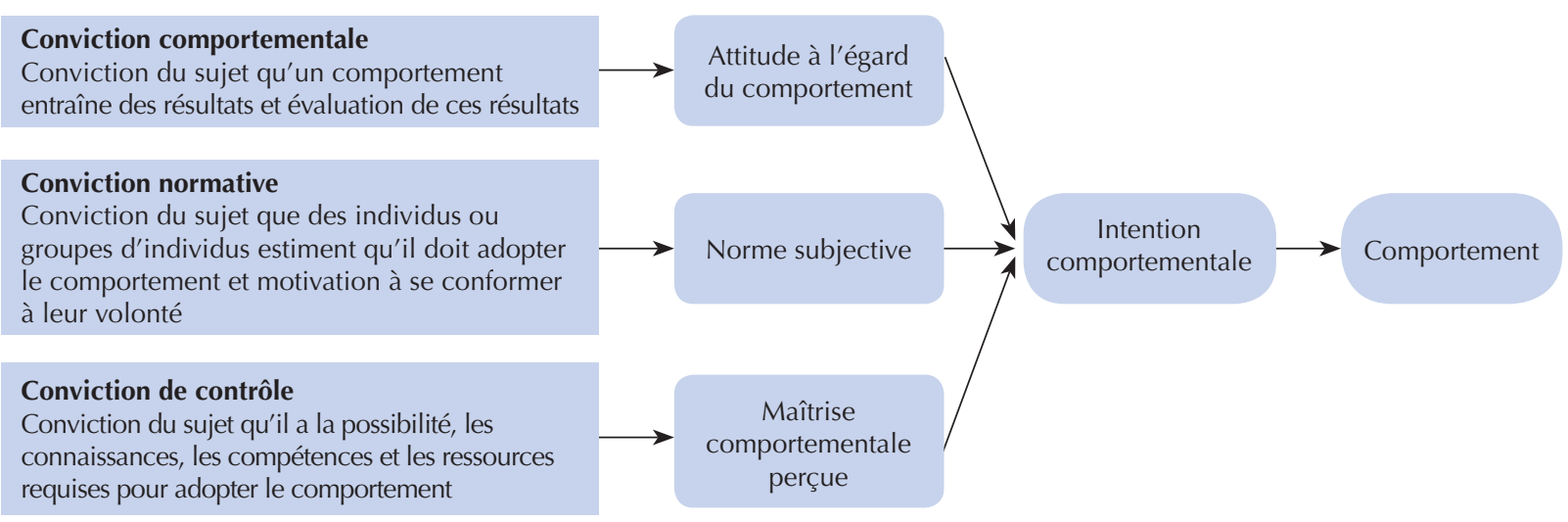

Dans ce contexte, les attitudes, la maîtrise comportementale perçue et les normes subjectives peuvent être des variables prédictives de la volonté des élèves de consacrer du temps à leurs devoirs de mathématiques, de poser des questions en classe ou de se livrer à des exercices de relaxation pour lutter contre leur anxiété et, donc, d'améliorer leur performance en mathématiques.

Concernant l'élaboration des variables, I'application du modèle complet d'Ajzen accroît sensiblement le pouvoir analytique de l'enquête pour expliquer les efforts des élèves en mathématiques, leur comportement en mathématiques et, indirectement, leurs résultats, si possible. Toutefois, en raison des coûts et des ressources à mobiliser pour évaluer toutes les facettes du modèle, cette extension n'est soumise qu'à des sous-groupes d'élèves, moyennant la rotation des items (voir la section « Rotation des items dans les extensions du questionnaire "Élève" et les instruments informatisés »). 
Dans le questionnaire "Élève ", les deux indicateurs d'image de soi en mathématiques sont utilisés et l'indicateur d'anxiété en mathématiques est conservé. Toutefois, de nouveaux items ont été expérimentés en raison de doutes sur la validité interculturelle et le pouvoir explicatif incrémentiel (Lee, 2009 ; Vieluf et al., 2009a, 2009b).

\section{Possibilités d'apprentissage et qualité de l'instruction : Évaluation de l'environnement d'apprentissage}

L'enquête PISA cherche à décrire les conditions d'apprentissage dans le domaine majeur d'évaluation du cycle pour deux raisons. En premier lieu, I'enquête PISA contribue à décrire et comparer les environnements d'apprentissage des élèves âgés de 15 ans dans les différents pays, sur la base de variables dont la littérature établit qu'elles sont des indicateurs probants de la qualité de l'instruction. En deuxième lieu, ces variables contribuent à expliquer les résultats des élèves - pas dans tous les pays, mais dans ceux où la plupart des élèves fréquentent un établissement échantillonné à deux reprises au moins - ce qui est le cas dans deux tiers des pays qui ont participé au cycle PISA 2006.

Les environnements d'apprentissage qui aident les élèves à développer leur culture mathématique - telle qu'elle se conçoit dans l'enquête PISA - sont décrits dans la littérature comme des cadres d'apprentissage en contexte (Schoenfeld, 2006), où l'enseignement est basé sur la discussion (Boaler et Greeno, 2000 ; Stein et al., 2008) et qui incluent la modélisation mathématique (Blum et Leiss, 2005) à titre d'activité essentielle. Dans ses travaux précurseurs, Schoenfeld $(1987,1992)$ a plaidé en faveur d'une culture mathématique qui permette un apprentissage éclairé, qui établisse des liens entre des concepts mathématiques et qui rapporte ces liens à la vie courante des élèves - par comparaison avec I'instruction classique qui vise la maîtrise de formules, d'algorithmes, de définitions et autres contenus techniques. La " conception moderne de l'enseignement efficace des mathématiques » (Stein et al., 2008) consiste notamment " à présenter aux élèves des problèmes mathématiques complexes plus réalistes, à les amener à se renvoyer la balle pour travailler sur ces problèmes, puis à partager les stratégies et les solutions des uns et des autres lors d'une discussion orchestrée par l'enseignant avec toute la classe» (p. 315).

Comme l'enquête PISA entend couvrir les aspects majeurs de l'environnement d'apprentissage des mathématiques dans ses questionnaires, cette conception moderne de l'enseignement des mathématiques est un défi. Les items sur les contenus ne suffisent pas pour décrire les processus d'enseignement et d'apprentissage propices au développement de la culture mathématique. Tout d'abord, parce que la culture mathématique telle qu'elle se conçoit dans l'enquête PISA renvoie à un rendement cumulé, qui dépend plus de compétences et de processus mathématiques fondamentaux et, donc, moins de contenus spécifiques qui auraient été enseignés. Ensuite, parce que la qualité de l'enseignement des mathématiques dépend moins des contenus que de la clarté et de la structure des cours, du soutien aux élèves et des difficultés cognitives (voir la section "Intrants et processus à l'échelle des classes »). C'est pourquoi l'enquête PISA a redéfini la notion de possibilité d'apprentissage, un construct important qui caractérise les environnements d'apprentissage dans d'autres études internationales sur les acquis des élèves. De plus, les données sur les possibilités d'apprentissage obtenues auprès des élèves peuvent être étudiées à l'échelle des établissements d'enseignement, grâce à la conception des instruments PISA.

\section{Possibilités d'apprentissage : couverture des catégories de contenus et des types de problèmes}

La notion de possibilité d'apprentissage a été introduire par John Carroll au début des années 60, initialement pour indiquer si les élèves avaient eu suffisamment de temps et reçu une instruction adéquate pour apprendre (Carroll, 1963 ; Abedi et al., 2006). Elle est depuis lors devenue un concept important dans les évaluations internationales des acquis des élèves (Husén, 1967 ; Schmidt et McKnight, 1995 ; Schmidt et al., 2001), et il est établi qu'elle est en forte corrélation avec la performance des élèves, en particulier dans les comparaisons entre les pays (Schmidt et Maier, 2009, pp. 552556). Parallèlement, la portée du construct s'est considérablement développée. Stevens (1993, pp. 233-234) a identifié les quatre types de variables les plus souvent cités dans la littérature au sujet des possibilités d’apprentissage :

- La couverture du contenu : ces variables permettent de déterminer si les élèves voient ou non le programme prévu dans une année d'études ou une matière.

- L'exposition au contenu : ces variables portent sur le temps prévu et consacré à l'instruction, et sur le caractère approfondi de l'enseignement dispensé.

- L'importance du contenu : ces variables montrent l'importance accordée aux thématiques du programme et identifient les élèves sélectionnés pour recevoir une instruction privilégiant les compétences d'ordre inférieur (mémorisation par cœur) ou d'ordre supérieur (résolution de problèmes).

- La qualité de l'instruction : ces variables montrent en quoi les pratiques pédagogiques en classe (la présentation des cours) influent sur la performance académique des élèves. 
Pour certains auteurs, les possibilités d'apprentissage sont devenues plus ou moins synonymes de qualité de l'instruction. Schmidt et Maier (2009) précisent toutefois dans leur analyse que les possibilités d'apprentissage sont un concept relativement simple : "Ce que les élèves apprennent à l'école dépend de ce qu'on leur enseigne » (p. 541), et ils se concentrent à dessein sur les possibilités d'apprentissage " au sens le plus strict, c'est-à-dire l'exposition des élèves au contenu» (p. 542).

Schmidt et Maier reconnaissent que les possibilités d'apprentissage sont un construct direct, mais qu'elles sont difficiles à mesurer. Pour expliquer les différences de matières vues, il est d'usage de demander aux enseignants et/ou aux élèves si des matières du programme prévu ont été enseignées ou non, parfois avec l'appui des journaux de classe (Rowan et al., 2004). De plus, il est demandé à des experts spécialisés dans les programmes de cours si certains éléments des programmes sont couverts dans des documents tels que les syllabus, les manuels, etc. (le programme prévu) et, dans l'affirmative, de quelle manière. Divers indicateurs ont été dérivés de ces données brutes. Dans de nombreux cas, les matières enseignées sont jugées à deux égards, en termes de thématique et de niveau, alors qu'à l'échelle des systèmes, ce sont des indicateurs de cohérence, de rigueur et de priorité qui ont été dérivés (Schmidt et Maier, 2009).

Concernant l'élaboration des variables, l'évaluation PISA des possibilités d'apprentissage s'écarte des approches adoptées dans d'autres études, car la culture mathématique n'est pas mesurée en termes de contenus, mais sur la base de sept facultés mathématiques fondamentales et de quatre catégories de contenu. Dans I'enquête PISA, l'opérationnalisation des variables relatives aux possibilités d'apprentissage repose essentiellement sur les appréciations des élèves.

Le cycle PISA 2012 vise à déterminer le niveau de possibilités d'apprentissage dans les pays (voire dans les établissements d'enseignement). Les élèves se voient présenter des tâches mathématiques élaborées avec soin - certaines faisant intervenir les facultés mathématiques et les catégories de contenu décrites dans le cadre PISA de mathématiques, d'autres ressemblant à des tâches plus traditionnelles requérant la reproduction de procédures et la restitution de connaissances. Après avoir répondu à ces items, les élèves sont invités à indiquer s'ils ont déjà abordé de telles tâches au cours de mathématiques ou lors d'évaluations précédentes et, dans l'affirmative, à quelle fréquence. Il est donc possible d'évaluer les possibilités d'apprentissage - à l'échelle des pays, voire des établissements d'enseignement - d'une façon qui permet de différencier les types de problèmes et de contenus.

De plus, les élèves sont invités à indiquer dans quelle mesure certains concepts mathématiques leur sont familiers. Cet indicateur, ainsi que l'échelle d'efficacité perçue qui est décrite dans la section « Indicateurs de résultats en rapport avec la culture mathématique : stratégies, convictions et motivation », peuvent être utilisés comme indicateurs proximaux des possibilités d'apprentissage.

\section{Temps d'apprentissage}

Après Carroll (1963), de nombreuses études ont montré que le temps d'apprentissage était une variable prédictive majeure des résultats des élèves dans de nombreuses matières, y compris en mathématiques. Le cycle PISA 2012 a utilisé un indicateur de 2003 pour permettre la réalisation d'analyses tendancielles. Les élèves ont indiqué le nombre moyen d'heures qu'ils consacrent par semaine à leurs devoirs, à des cours de soutien ou de perfectionnement, à des cours avec un professeur particulier, et à des cours en dehors de l'école. De plus, la durée et le nombre de cours de mathématiques par semaine ont été évalués via les questionnaires "Élève » et "Établissement ».

\section{Qualité de l'instruction}

Comme indiqué dans la section «Intrants et processus à l'échelle des classes », les études sur les cours de mathématiques dans l'enseignement secondaire suggèrent que les dimensions fondamentales de la qualité de l'instruction sont : i) la structure et la gestion efficiente de la classe ; ii) le soutien des enseignants et l'orientation des élèves ; et iii) la difficulté et l'activation cognitive. Deux de ces dimensions sont couvertes par des échelles élaborées lors du cycle PISA 2003, à savoir le climat de discipline et le soutien par l'enseignant en cours de mathématiques, à partir du questionnaire "Élève ».

Le rapport international sur le cycle PISA 2003 (OCDE, 2004) montre que le construct du climat de discipline en cours de mathématiques est en forte corrélation avec la culture mathématique, alors que d'autres variables - telles que la taille des classes, les activités proposées en mathématiques dans l'établissement d'enseignement et le fait de ne pas pratiquer le regroupement par aptitudes - n'ont pas d'effet sensible après contrôle du milieu socio-économique. Ces constats rejoignent les conclusions d'études antérieures et la théorie exposée dans la section "Intrants et processus à l'échelle des classes", selon laquelle une bonne structure et une gestion de classe efficiente sont des conditions essentielles à l'apprentissage des élèves. Toutefois, lors du cycle PISA 2003, la relation entre l'environnement d'apprentissage et des résultats non cognitifs tels que l'intérêt des élèves pour les mathématiques ou le plaisir que leur procurent les 
mathématiques n'a pas été analysée. Depuis lors, Vieluf et al. (2009a) ont établi l'existence d'une relation positive entre le soutien des enseignants et l'intérêt des élèves pour les mathématiques, même après contrôle du milieu socioéconomique.

La difficulté et l'activation cognitive sont des dimensions cruciales pour le développement de la culture mathématique, mais elles sont très difficiles à évaluer. Des concepts similaires, qui mettent en évidence l'importance de l'orchestration de l'enseignement des mathématiques d'une façon qui offre aux élèves de nombreuses possibilités de développer leurs compétences, sont évoqués sous un angle général dans la littérature sur l'enseignement des mathématiques (voir, par exemple, Blum et Leiss, 2007). Toutefois, aucun des indicateurs utilisés lors du cycle PISA 2003 ne permet de refléter cette dimension. Plusieurs approches ont été employées avec succès dans des études nationales et ont été expérimentées lors de l'essai de terrain du cycle PISA 2012 :

- Comme les devoirs sont une composante quasi universelle de l'enseignement des mathématiques, le traitement que les enseignants leur réservent en classe peut être utilisé comme indicateur de difficulté. Rakoczy et al. (2005) ont élaboré une échelle qui rend compte des processus relatifs aux devoirs sur la base d'items tels que "Le professeur de mathématiques tient à savoir comment nous avons résolu les problèmes de notre devoir » et "Lorsque nous en venons aux résultats des devoirs, nous tentons de comprendre les erreurs et de les corriger» (remarque : cette échelle ne porte pas sur les devoirs en tant que tels, ni sur le temps que les élèves y consacrent, mais sur la qualité du discours en classe à propos des résultats des devoirs).

- Les élèves peuvent aussi être interrogés sur le type d'items qu'ils rencontrent souvent en cours de mathématiques, par exemple : "Nous devons généralement réfléchir un moment pour résoudre les problèmes que nous donne le professeur de mathématiques " (échelle de tâches d'activation cognitive utilisée en Allemagne à l'occasion du cycle PISA 2003, Baumert et al., 2008).

\section{Pratiques pédagogiques et activités mathématiques des élèves}

Une version à orientation comportementale du modèle triarchique de la qualité de l'instruction a été adoptée dans I'enquête TALIS de I'OCDE : les enseignants ont été invités à indiquer à quelle fréquence ils utilisent 13 pratiques pédagogiques différentes :

- Des pratiques structurantes (cinq items), par exemple annoncer clairement les objectifs d'apprentissage, résumer les cours précédents, passer les devoirs en revue, contrôler le cahier d'exercice et poser des questions aux élèves pour vérifier qu'ils comprennent la leçon.

- Des pratiques axées sur les élèves (quatre items), par exemple faire travailler les élèves en petits groupes pour qu'ils trouvent ensemble une solution à un problème ou à un exercice, pratiquer le regroupement par aptitudes, amener les élèves à s'autoévaluer et à participer à la planification des cours.

- Des activités de renforcement (quatre items), par exemple faire travailler les élèves à des projets d'une semaine au moins et leur demander de concevoir un produit, d'écrire une rédaction et de se livrer à des débats contradictoires.

II ressort de l'analyse des résultats de la campagne définitive de l'enquête TALIS dans 23 pays que : $i$ ) les trois dimensions peuvent être différenciées entre les pays (c'est-à-dire que le modèle triarchique présente une certaine validité culturelle) ; ii) I'hypothèse selon laquelle les pratiques structurantes seraient associées à une meilleure discipline en classe (telle qu'elle est perçue par les enseignants) se confirme; et iii) la participation à des activités de développement professionnel et la capacité d'enseigner dans des classes très performantes entraînent une augmentation de la fréquence de ces pratiques. Les professeurs de mathématiques et de sciences déclarent utiliser moins que leurs collègues qui enseignent d'autres matières les pratiques axées sur les élèves et les activités de renforcement (OCDE, 2009b).

Concernant l'élaboration des variables, les échelles TALIS de pratiques pédagogiques ont été adaptées pour pouvoir être utilisées lors du cycle PISA 2012, moyennant un bref ajout au questionnaire « Enseignant » de l'enquête TALIS, les items relatifs aux activités renforcées étant placés dans le contexte des mathématiques, par exemple utiliser les mathématiques pour résoudre un problème de la vie courante, établir un lien entre les mathématiques et d'autres matières, découvrir des règles à partir de structures mathématiques, démontrer un théorème mathématique, discuter d'exemples et de contre-exemples, utiliser de nombreuses représentations graphiques dans un problème mathématique, et comparer diverses méthodes de résolution ou plusieurs solutions pour la même tâche.

Seuls certains pays PISA peuvent établir un lien avec l'enquête TALIS, mais tous les élèves peuvent être invités à indiquer à quelle fréquence ils participent à de telles activités. 


\section{Soutien à l'enseignement et à l'apprentissage des mathématiques à l'échelle du système et des établissements d'enseignement}

La plupart des variables de niveau Système et Établissement décrites dans les sections « Intrants et processus à l'échelle des systèmes » et "Intrants et processus à l'échelle des établissements d'enseignement » sont susceptibles d'être en corrélation avec la performance des élèves en mathématiques et/ou avec leurs attitudes à l'égard des mathématiques. Les indicateurs d'intrants et de processus spécifiques aux mathématiques peuvent être obtenus via l'agrégation des réponses des élèves aux items sur les possibilités d'apprentissage et la qualité de l'instruction (voir la section « Possibilités d'apprentissage et qualité de l'instruction : évaluation de l'environnement d'apprentissage ») à des niveaux supérieurs.

Toutefois, un certain nombre de variables spécifiques aux mathématiques à l'échelle des établissements d'enseignement ou du système façonnent directement les conditions d'enseignement et d'apprentissage des mathématiques. C'est pourquoi l'accent pourrait être mis sur les aspects suivants.

Réforme à l'échelle du système :

- initiatives, activités en vue d'améliorer le cadre scolaire et programmes de développement professionnel axés sur les mathématiques ;

- rôle des mathématiques dans l'admission des élèves, leur différenciation, leur évaluation et leur passage de classe, et l'évaluation des établissements d'enseignement; et

- temps d'instruction prévu par an en mathématiques.

Réforme à l'échelle des établissements d'enseignement :

- offre de cours en mathématiques (cours de soutien et de perfectionnement, tutorat) ;

- qualification des professeurs de mathématiques; et

- collaboration entre les professeurs de mathématiques.

Concernant l'élaboration des variables, les données peuvent être agrégées du niveau Établissement au niveau Système, car les chefs d'établissement sont interrogés sur toutes ces thématiques dans l'enquête PISA.

\section{CONCEPTION DES QUESTIONNAIRES DU CYCLE PISA 2012}

\section{Répartition des contenus entre les cycles PISA : Une structure globale pour les variables générales, les variables spécifiques au domaine d'évaluation et les variables d'extensions thématiques}

Parmi les facteurs pertinents pour comprendre la performance des élèves, leurs attitudes et leur comportement ainsi que le fonctionnement des systèmes d'éducation, certains sont directs (par exemple, les variables démographiques, le parcours scolaire antérieur, le temps d'enseignement et la taille des classes) et d'autres créés lors de précédents cycles PISA sont bien établis (le milieu socio-économique des élèves et leurs stratégies cognitives, ou encore le degré de participation des établissements d'enseignement à la prise de décision). Il est apparu que d'autres facteurs étaient moins faciles à cerner au travers de l'enquête PISA (par exemple, les politiques de responsabilisation à l'échelle des systèmes, les variables spécifiques aux enseignants, certains aspects de l'environnement d'apprentissage en classe ou les activités extrascolaires). Faire un choix parmi les nombreuses variables susceptibles d'être incluses est un processus complexe qui s'effectue sur la base des priorités fixées par les pays, ainsi que compte tenu de la littérature sur la pédagogie, comme indiqué dans les sections précédentes.

Dans la mesure où l'enquête PISA poursuit de nombreux objectifs et s'intéresse à de nombreux résultats de l'apprentissage des élèves, et que des informations sur les tendances sont nécessaires, les questionnaires contextuels doivent couvrir :

i) Des variables générales (lors de tous les cycles)

- Variables générales d'intrants :

- Intrants de niveau Élève (année d'études, sexe et milieu socio-économique = niveau de formation et profession des parents, richesse, patrimoine culturel et ressources pédagogiques de la famille, données sur l'ascendance $=$ ascendance autochtone ou allochtone, langue maternelle et âge d'arrivée dans le pays, soutien familial).

- Contextes et intrants de niveau Établissement (taille de la communauté, ressources et qualifications du personnel enseignant). 
- Variables générales de processus :

- Processus de niveau Établissement (prise de décision, politiques d'admission, politiques d'évaluation, développement professionnel, engagement et moral des enseignants, relations entre enseignants et élèves, implication des parents).

- Processus d'instruction (temps d'apprentissage, climat de discipline, soutien des enseignants).

- Variables générales de résultats :

- Résultats non cognitifs d'ordre général - Engagement à l'égard de l'apprentissage (comportement : absentéisme ; objectif personnel : attentes en matière d'éducation ; motivation : engagement à l'égard de l'apprentissage ; affect : sentiment d'appartenance).

ii) Des variables tendancielles spécifiques au domaine d'évaluation (uniquement le domaine majeur d'évaluation, soit tous les neuf ans)

- Variables de résultats non cognitifs spécifiques au domaine d'évaluation (stratégies et métacognition, convictions, image de soi et motivation).

- Variables de processus spécifiques au domaine d'évaluation (possibilités d'apprentissage, qualité de l'instruction, soutien à l'échelle de l'établissement d'enseignement et du système).

iii) Des variables d'extensions thématiques (extensions lors de cycles précis) :

- Options internationales (questionnaires sur le parcours scolaire et la maîtrise des TIC, et questionnaire « Parents » en 2012, par exemple).

- Variables contextuelles dans d'autres domaines (les expériences en matière de TIC pertinentes pour les épreuves informatisées de résolution de problèmes, par exemple).

- Variables descriptives et explicatives pertinentes pour des rapports spécifiques (les motivations et les intentions en rapport avec les mathématiques en 2012, par exemple).

- Variables malléables à l'échelle des établissements d'enseignement (les politiques de différenciation et la certification des enseignants, par exemple), spécialement choisies à des fins de description ou d'inférence causale.

iv) Des données systémiques, recueillies via le projet INES ou un questionnaire «Système d'éducation »

- Résultats des établissements d'enseignement (délivrance des diplômes, par exemple).

- Ressources financières et humaines investies dans l'éducation.

- Accessibilité de l'éducation et scolarisation.

- Environnement d'apprentissage et organisation des établissements d'enseignement.

Lors de précédents cycles PISA - en particulier en 2006 -, les questionnaires ont largement négligé des constructs sans rapport avec le domaine majeur d'évaluation. La série de variables générales i) s'est parfois limitée à des intrants de niveau Élève. Le questionnaire "Élève » et, dans une certaine mesure, le questionnaire "Établissement », se sont concentrés sur des processus et des résultats non cognitifs spécifiques au domaine d'évaluation. Cette approche n'est pas appropriée lorsqu'il s'agit d'évaluer des tendances.

Trouver le juste équilibre entre les variables i), ii), iii) et iv) est essentiel pour la structure globale des questionnaires PISA et la réussite à long terme de l'enquête PISA.

Pour rendre compte de façon valide et fiable de tendances à l'échelle des pays, il est important de garder une série constante de variables de cycle en cycle, à titre de variables majeures de compte rendu et de bases d'estimation du niveau de compétence. Ces variables de contexte et d'intrant ne doivent donc pas changer.

Les responsables politiques n'ont pas seulement besoin d'informations tendancielles sur la performance des élèves, car les systèmes d'éducation poursuivent d'autres objectifs. Ils requièrent des informations sur des résultats non cognitifs, comportementaux ou sans rapport avec le domaine d'évaluation, à savoir les aspirations professionnelles (la profession que les élèves prévoient d'exercer à l'âge de 30 ans), l'engagement à l'égard de l'établissement d'enseignement (le sentiment d'appartenance), l'absentéisme et la motivation pour l'apprentissage (les efforts et la persévérance) - des résultats qui ont tous été couverts dans de précédents cycles PISA. Jusqu'ici, les auteurs de l'enquête PISA n'étaient pas favorables à leur utilisation, car ils craignaient que leur comparabilité entre cultures soit insuffisante. Toutefois, comme les informations sur les tendances sont disponibles pour un nombre croissant de cycles, la priorité va maintenant à l'évolution des taux 
au sein des pays, plutôt qu'à la comparaison transversale des niveaux. Par exemple, l'éventuelle variation à la hausse ou à la baisse de l'engagement à l'égard de l'établissement d'enseignement est un indicateur pertinent au sein des pays. La validité interculturelle fait également l'objet d'une plus grande attention (voir la section «Validité interculturelle »).

Deux échelles de processus, le climat de discipline en classe et le soutien des enseignants en classe, sont respectivement en corrélation avec la performance des élèves et l'intérêt des élèves (voir Klieme et Rakoczy, 2003 ; Vieluf et al., $2009 a)$. Avec le temps d'apprentissage, ces échelles doivent être gardées lors des prochains cycles, à titre de variables générales de processus. Il convient toutefois de ne pas changer leur formulation lors de chaque cycle et de ne pas les associer au domaine majeur d'évaluation pour permettre l'application de modèles d'évolution.

Selon le domaine majeur d'évaluation des épreuves cognitives, des variables supplémentaires (des variables tendancielles spécifiques au domaine) seront prises en considération. Enfin, chaque cycle a ses propres priorités, que ce soit sous la forme d'options internationales ou d'extensions aux questionnaires obligatoires.

\section{Exploration des indicateurs du cycle PISA 2003}

Les aspects suivants de l'environnement d'apprentissage en mathématiques ont été analysés sur la base des résultats du cycle PISA 2003 :

- La taille de la classe (questionnaire «Élève »).

- Le climat de discipline en cours de mathématiques (questionnaire «Élève »).

- Le soutien de l'enseignant en cours de mathématiques (questionnaire «Élève »).

- Le temps consacré à l'apprentissage des mathématiques à l'école et à domicile (questionnaire « Élève »).

- La fréquence du regroupement par aptitudes en mathématiques (questionnaire "Établissement »).

- Les activités scolaires qui promeuvent l'engagement des élèves à l'égard des mathématiques, la participation à des concours de mathématiques, par exemple (questionnaire «Établissement»).

- Les caractéristiques concernant les professeurs de mathématiques, à savoir leur niveau de qualification, leur collégialité et les politiques concernant leur évaluation (questionnaire «Établissement »).

Plusieurs résultats en rapport avec les mathématiques ont également été évalués : les stratégies d'apprentissage, l'intérêt et le plaisir, la motivation instrumentale, l'image de soi et l'anxiété.

Pour fournir des informations tendancielles sur des résultats non cognitifs et des variables de processus et de contexte en rapport avec les mathématiques, le cycle PISA 2012 a gardé toutes les variables du cycle PISA 2003 qu'il était possible de retenir et a exclu les variables dont il est établi qu'elles présentent un biais interculturel ou n'expliquent pas de différences de résultats. Le tableau 6.2 donne un aperçu de tous les items des questionnaires «Élève » et «Établissement » du cycle PISA 2003. Dans la première rangée figurent les intrants individuels - variables démographiques et familiales - qui sont restés stables au fil des cycles ${ }^{7}$. Ces variables d'intrant servent à étudier la répartition des ressources et des résultats de l'éducation au sein des pays, et sont utilisées à titre de variables de contrôle dans les modèles analytiques.

Dans la deuxième rangée figurent les variables d'intrants de niveau Établissement, notamment leur statut privé ou public, leurs ressources financières, humaines et autres, et la taille et la composition de leur effectif d'élèves. Ces variables concernent des intrants à l'échelle des établissements d'enseignement, mais les décisions budgétaires, la modification des programmes de cours et les politiques de privatisation sont, en partie du moins, contrôlées au niveau du pays ou du système.

Les variables d'intrant de niveau Établissement à retenir ont été choisies sur la base des caractéristiques techniques décrites dans le rapport technique sur le cycle PISA 2003 (OCDE, 2005b) et compte tenu des analyses multiniveau du pouvoir prédictif (Vieluf et al., 2009b), de la validité interculturelle (Vieluf et al., 2009b) et d'une analyse de l'usage des informations obtenues via le questionnaire «Établissement » lors des précédents cycles PISA (Hersbach et Lietz, 2010).

Au niveau Élève, les résultats des analyses du pouvoir prédictif ont montré que la corrélation de la performance en mathématiques était relativement faible avec les préférences en matière d'apprentissage et extrêmement faible avec l'apprentissage collaboratif. Des analyses similaires réalisées sur la base des données du cycle PISA 2000 (Jehanghir et Glas, 2007) ont montré que la performance en compréhension de l'écrit n'était pas significativement corrélée avec l'apprentissage collaboratif et ne l'était que faiblement avec l'apprentissage compétitif. Ces deux échelles pourraient donc être supprimées. Parmi les trois échelles de stratégies, les stratégies de mémorisation spécifiques au domaine d'évaluation ne sont pas corrélées avec la performance en mathématiques. De plus, l'invariance interculturelle de cette 
échelle n'a pu être établie - même au niveau métrique. En revanche, les stratégies d'élaboration sont fortement corrélées à la performance et l'invariance interculturelle de leur échelle est acceptable (voir la section « Indicateurs de résultats en rapport avec la culture mathématiques : stratégies, convictions et motivation »).

Au niveau Établissement, des effets relativement importants ont été observés au sujet du sentiment d'appartenance, du temps consacré aux devoirs en mathématiques et du climat de discipline au cours de mathématiques, des processus qui sont des variables prédictives probantes de la performance en mathématiques et de l'intérêt pour les mathématiques. Ces variables ont été retenues; $c^{\prime}$ est le climat de discipline en classe dont le niveau d'invariance (scalaire) est le plus élevé, ce qui permet de comparer les scores moyens entre les pays.

Concernant l'efficacité perçue et la perception de soi, l'invariance scalaire des deux échelles permet d'utiliser ces variables à titre d'indicateurs de résultats non cognitifs en rapport avec les mathématiques, de suivre des tendances et de faire des analyses comparatives entre les pays. D'autres indicateurs ont toutefois été utilisés à titre expérimental pour améliorer la comparabilité interculturelle.

\section{Rotation des items dans les extensions du questionnaire " Élève " et les instruments informatisés}

Couvrir toutes les variables générales, spécifiques au domaine d'évaluation et en rapport avec des extensions thématiques serait excessif vu le temps et la place laissés aux questionnaires contextuels dans l'enquête PISA. Ce problème potentiel est particulièrement vrai pour le questionnaire "Élève », dont l'agrégation permet de générer des données à tous les niveaux de l'enquête (du niveau Élève au niveau Système). Le questionnaire "Établissement " pourrait aussi être compromis s'il était étendu, à cause du risque accru de non-réponse. Pour permettre au cycle PISA 2012 ainsi qu'aux prochains cycles de remplir de nombreux objectifs et éviter l'accroissement des données manquantes, de nouvelles méthodes s'imposent pour minimiser la charge à l'égard des répondants. Deux innovations techniques ont été introduites lors du cycle PISA 2012.

\section{Rotation}

Une rotation des items, similaire à celle appliquée aux épreuves cognitives, a été introduite dans le questionnaire "Élève » du cycle PISA 2012. Utilisée jusqu'ici dans les épreuves cognitives, la rotation permet de répartir les blocs d'items entre différents carnets. Un carnet spécifique est attribué à chaque élève, qui doit donc répondre à un nombre limité d'items cognitifs. Ensemble, les carnets couvrent la totalité des items dans les différents domaines d'évaluation. Les carnets sont attribués de façon aléatoire aux répondants dans chaque établissement d'enseignement, d'où le nom de rotation. Cette forme de rotation est courante dans les évaluations à grande échelle, qu'elles soient nationales ou internationales.

L'application d'une forme de rotation dans les questionnaires est relativement neuve dans les évaluations de l'éducation, alors que c'est une technique courante dans d'autres types d'enquête. Les implications de cette pratique pour i) les estimations de la compétence cognitive, ii) le rapport international et la présentation des tendances, iii) les analyses plus approfondies, iv) la documentation et la structure de la base de données internationale, et v) la logistique, ont déjà été étudiées (Berezner et Lietz, 2009). La rotation des items a été adoptée pour le questionnaire « Élève » du cycle PISA 2012 compte tenu de l'expérience acquise et d'analyses des données du cycle PISA 2006. On estime que cette approche permet d'augmenter la couverture du matériel d'un tiers.

La partie commune, sans rotation d'items, du questionnaire "Élève ", doit comprendre à tout le moins les variables générales d'intrants de niveau Élève (voir la section « Répartition des contenus entre les cycles PISA »). Cette approche répond à des exigences de méthodologie et de compte rendu, et se base sur des travaux théoriques et sur des analyses des données du cycle PISA 2006 (Berezner et Lietz, 2009). Le tableau 6.3 dresse la liste des variables reprises dans la partie commune. 
Tableau 6.2

Classification des questionnaires du cycle PISA 2003

\begin{tabular}{|c|c|c|}
\hline & Questionnaire " Élève » & Questionnaire « Établissement » \\
\hline $\begin{array}{l}\text { Antécédents } \\
\text { (Contexte personnel, } \\
\text { familial et scolaire des } \\
\text { élèves) }\end{array}$ & $\begin{array}{l}\text { Âge, Sexe, Ascendance allochtone } \\
\text { Langue maternelle } \\
\text { Profession et niveau de formation des } \\
\text { parents, richesse et patrimoine familial } \\
\text { (= } 4 \text { indices), Structure familiale } \\
\text { Parcours scolaire, Année d'études, } \\
\text { Filière d'enseignement, Raisons de l'assiduité, } \\
\text { Temps d'étude en dehors du cadre scolaire, } \\
\text { Niveau de formation prévu }\end{array}$ & Taille de la communauté \\
\hline $\begin{array}{l}\text { Intrants scolaires } \\
\text { (Contrôlés à l'échelle } \\
\text { du système ou des } \\
\text { établissements } \\
\text { d'enseignement, selon la } \\
\text { structure de gouvernance) }\end{array}$ & & $\begin{array}{l}\text { Effectifs scolarisés de sexe féminin } \\
\text { et masculin, Pourcentage d'élèves apprenant } \\
\text { une deuxième langue, } \\
\text { Statut public ou privé, Années d'études, } \\
\text { Horaires par filière } \\
\text { Nombre d'enseignants, Nombre d'ordinateurs } \\
\text { disponibles, Ressources financières, Ressources } \\
\text { scolaires (3 échelles) }\end{array}$ \\
\hline $\begin{array}{l}\text { Processus généraux } \\
\text { (Contrôlés à l'échelle } \\
\text { des établissements } \\
\text { d'enseignement et, } \\
\text { parfois, du système) }\end{array}$ & Relations entre élèves et enseignants & $\begin{array}{l}\text { Prise de décision et types d'autonomie, Politiques } \\
\text { d'admission, Politiques d'évaluation, Options } \\
\text { pour les élèves apprenant une deuxième langue } \\
\text { Moral des enseignants, Comportements négatifs } \\
\text { des enseignants, Participation des enseignants }\end{array}$ \\
\hline $\begin{array}{l}\text { Processus spécifiques } \\
\text { aux mathématiques } \\
\text { (Contrôlés à l'échelle } \\
\text { du système ou des } \\
\text { établissements } \\
\text { d'enseignement) }\end{array}$ & $\begin{array}{l}\text { Temps d'instruction, Temps consacré aux } \\
\text { devoirs de mathématiques } \\
\text { Taille des classes } \\
\text { Climat de discipline, Soutien de l'enseignant }\end{array}$ & $\begin{array}{l}\text { Regroupement par aptitudes en mathématiques } \\
\text { Activités en mathématiques } \\
\text { Professeurs de mathématiques : Qualification, } \\
\text { politiques d'évaluation ; collégialité des } \\
\text { professeurs de mathématiques }\end{array}$ \\
\hline $\begin{array}{l}\text { Résultats non cognitifs } \\
\text { généraux }\end{array}$ & $\begin{array}{l}\text { Attitudes à l'égard de l'établissement } \\
\text { d'enseignement, Absentéisme, } \\
\text { Sentiment d'appartenance à l'établissement } \\
\text { d'enseignement }\end{array}$ & $\begin{array}{l}\text { Comportements négatifs des élèves } \\
\text { Moral des élèves } \\
\text { Redoublement }\end{array}$ \\
\hline $\begin{array}{l}\text { Résultats non cognitifs } \\
\text { spécifiques aux } \\
\text { mathématiques }\end{array}$ & $\begin{array}{l}\text { Intérêt et plaisir des mathématiques, } \\
\text { Motivation instrumentale, } \\
\text { Efficacité perçue, Perception de soi, Anxiété, } \\
\text { Stratégies (contrôle, élaboration, mémorisation), } \\
\text { Préférences d'apprentissage : apprentissage } \\
\text { collaboratif vs apprentissage compétitif }\end{array}$ & \\
\hline
\end{tabular}

Remarques : Il avait été envisagé de supprimer les constructs soulignés et de retenir les constructs en gras lors du cycle PISA 2012. Aucune raison valable n'a motivé l'inclusion ou la suppression des constructs qui ne sont ni soulignés ni en gras. 
Tableau 6.3

Partie commune du questionnaire " Élève "

\begin{tabular}{|c|c|}
\hline $\begin{array}{l}\text { No de la } \\
\text { question }\end{array}$ & Description \\
\hline ST01 & Année d’études \\
\hline ST02 & Filière nationale d'enseignement \\
\hline STO3 & Âge de l'élève \\
\hline ST04 & Sexe de l'élève \\
\hline ST05 & Fréquentation du niveau <CITE $0>$ \\
\hline ST06 & Âge au niveau <CITE $1>$ \\
\hline ST07 & Redoublement \\
\hline ST08 & Absentéisme - Arrivée en retard à l'école \\
\hline ST09 & Absentéisme - Jours d'absence non justifiée \\
\hline ST115 & Absentéisme - Fréquence de cours séchés \\
\hline ST11 & Structure familiale \\
\hline ST12 & Profession de la mère (CITP) ; composante de I'indice SESC \\
\hline ST13 & Niveau de formation de la mère (CITE) durant la scolarité ; composante de l'indice SESC \\
\hline ST14 & Niveau de formation de la mère (CITE) après la scolarité ; composante de l'indice SESC \\
\hline ST15 & Situation actuelle de la mère au regard de l'emploi ; composante de l'indice SESC \\
\hline ST16 & Profession du père (CITP) ; composante de l'indice SESC \\
\hline ST17 & Niveau de formation du père (CITE) durant la scolarité ; composante de l'indice SESC \\
\hline ST18 & Niveau de formation du père (CITE) après la scolarité ; composante de l'indice SESC \\
\hline ST19 & Situation actuelle du père au regard de I'emploi ; composante de l'indice SESC \\
\hline ST20 & Situation au regard de l'immigration \\
\hline ST21 & Âge à l'arrivée dans le pays de l'évaluation \\
\hline ST25 & Langue parlée en famille \\
\hline ST26 & Équipement général du domicile et items nationaux sur la richesse ; composante de l'indice SESC \\
\hline ST27 & Nombre d'articles d'équipement à domicile ; composante de l'indice SESC \\
\hline ST28 & Bibliothèque familiale \\
\hline
\end{tabular}

En outre, la partie commune du questionnaire comprend des constructs nécessaires pour expliquer des différences entre des groupes minoritaires majeurs. Sous l'angle de l'action publique, il s'agit de variables qui peuvent contribuer à expliquer des écarts entre les élèves selon qu'ils sont ou non issus de l'immigration.

\section{Administration informatisée}

L'informatisation des questionnaires offre une plus grande souplesse, une plus grande capacité d'adaptation, et rend I'administration et le codage plus efficaces. Ainsi, les questions de filtre sont faciles à appliquer pour guider les répondants dans le questionnaire, les incohérences peuvent être contrôlées en ligne et les données ne doivent pas être saisies manuellement. À long terme, I'informatisation des questionnaires pourrait donc aussi améliorer I'administration des instruments.

Lors du cycle PISA 2012, le questionnaire «Établissement » a été administré de façon informatisée. Cette technologie pourrait être adaptée au questionnaire « Élève » et à des options internationales lors des prochains cycles. 
Tableau 6.4

Rotation $A$, B et $C$ du questionnaire "Élève "

\begin{tabular}{|c|c|c|c|c|c|}
\hline \multicolumn{2}{|r|}{ Rotation A } & \multicolumn{2}{|r|}{ Rotation B } & \multicolumn{2}{|r|}{ Rotation C } \\
\hline Q. $n^{\circ}$ & Description & Q. $n^{\circ}$ & Description & Q. $n^{\circ}$ & Description \\
\hline ST01-28 & Partie commune (voir le tableau 6.3) & ST01-28 & Partie commune (voir le tableau 6.3) & ST01-28 & Partie commune (voir le tableau 6.3) \\
\hline ST29 & $\begin{array}{l}\text { Motivation intrinsèque et instrumentale } \\
\text { en mathématiques }\end{array}$ & ST42 & $\begin{array}{l}\text { Perception de soi en mathématiques } \\
\text { (Q2, 4, 6, 7, 9); } \\
\text { Anxiété en mathématiques } \\
\text { (Q1, } 3,5,8,10)\end{array}$ & ST53 & $\begin{array}{l}\text { Stratégies d'apprentissage (Contrôle vs } \\
\text { Élaboration vs Mémorisation) }\end{array}$ \\
\hline ST35 & Normes subjectives & ST77 & $\begin{array}{l}\text { Soutien de l'enseignant en cours } \\
\text { de mathématiques }\end{array}$ & ST55 & $\begin{array}{l}\text { Participation à des cours en dehors } \\
\text { de l'école }\end{array}$ \\
\hline ST37 & Efficacité perçue en mathématiques & ST79 & $\begin{array}{l}\text { Comportement de l'enseignant: } \\
\text { - Instruction dirigée par l'enseignant } \\
\text { - Évaluation formative } \\
\text { - Orientation des élèves }\end{array}$ & ST57 & $\begin{array}{l}\text { Nombre total d'heures consacré aux } \\
\text { devoirs et leçons en dehors de l'école }\end{array}$ \\
\hline ST43 & $\begin{array}{l}\text { Contrôle perçu de la performance } \\
\text { en mathématiques }\end{array}$ & ST 80 & $\begin{array}{l}\text { Activation cognitive en cours } \\
\text { de mathématiques }\end{array}$ & ST61 & $\begin{array}{l}\text { Expérience des mathématiques } \\
\text { appliquées }(\mathrm{Q} 1,4,6,8) \text {; Expérience des } \\
\text { mathématiques fondamentales }(\mathrm{Q} 5,7,9\end{array}$ \\
\hline ST44 & Raisons de l'échec en mathématiques & ST81 & Climat de discipline & ST62 & $\begin{array}{l}\text { Mesure dans laquelle des concepts } \\
\text { mathématiques sont familiers }\end{array}$ \\
\hline ST46 & Éthique en mathématiques & ST82 & + Vignettes d'ancrage & ST69 & $\begin{array}{l}\text { Nombre de minutes par <période } \\
\text { de cours> }\end{array}$ \\
\hline ST48 & Intentions en mathématiques & ST83 & $\begin{array}{l}\text { Soutien du professeur de } \\
\text { mathématiques }\end{array}$ & ST70 & $\begin{array}{l}\text { Nombre de <périodes de cours }> \\
\text { par semaine }\end{array}$ \\
\hline ST49 & Comportement en mathématiques & ST84 & + Vignettes d'ancrage & ST71 & $\begin{array}{l}\text { Nombre total de <périodes de cours }> \\
\text { par semaine }\end{array}$ \\
\hline ST93 & Persévérance & ST85 & $\begin{array}{l}\text { Gestion de la classe par le professeur } \\
\text { de mathématiques }\end{array}$ & ST72 & Taille de classe \\
\hline ST94 & Ouverture à la résolution de problèmes & ST86 & Relations entre enseignants et élèves & ST73 & Expérience des problèmes lexicaux \\
\hline ST96 & $\begin{array}{l}\text { Stratégies de résolution de problèmes } \\
\text { (SJT) }\end{array}$ & ST87 & $\begin{array}{l}\text { Sentiment d'appartenance à } \\
\text { l'établissement d'enseignement }\end{array}$ & ST74 & Expérience des tâches procédurales \\
\hline ST101 & $\begin{array}{l}\text { Stratégies de résolution de problèmes } \\
\text { (SJT) }\end{array}$ & ST88 & $\begin{array}{l}\text { Attitudes à l'égard de l'établissement } \\
\text { d'enseignement : résultats de } \\
\text { l'apprentissage }\end{array}$ & ST75 & $\begin{array}{l}\text { Expérience du raisonnement } \\
\text { en mathématiques fondamentales }\end{array}$ \\
\hline ST104 & $\begin{array}{l}\text { Stratégies de résolution de problèmes } \\
\text { (SJT) }\end{array}$ & ST89 & $\begin{array}{l}\text { Attitudes à l'égard de l'établissement } \\
\text { d'enseignement : activités } \\
\text { d'apprentissage }\end{array}$ & ST76 & $\begin{array}{l}\text { Expérience du raisonnement } \\
\text { en mathématiques appliquées }\end{array}$ \\
\hline ST53 & $\begin{array}{l}\text { Stratégies d'apprentissage (Contrôle vs } \\
\text { Élaboration vs Mémorisation) }\end{array}$ & ST91 & Contrôle perçu de la réussite scolaire & ST42 & $\begin{array}{l}\text { Perception de soi en mathématiques } \\
(\mathrm{Q} 2,4,6,7,9) \\
\text { Anxiété en mathématiques } \\
(\mathrm{Q} 1,3,5,8,10)\end{array}$ \\
\hline ST55 & $\begin{array}{l}\text { Participation à des cours en dehors de } \\
\text { l'école }\end{array}$ & ST29 & $\begin{array}{l}\text { Motivation intrinsèque et instrumentale } \\
\text { en mathématiques }\end{array}$ & ST77 & $\begin{array}{l}\text { Soutien de l'enseignant en cours } \\
\text { de mathématiques }\end{array}$ \\
\hline ST57 & $\begin{array}{l}\text { Nombre total d'heures consacré aux } \\
\text { devoirs et leçons en dehors de l'école }\end{array}$ & ST35 & Normes subjectives & ST79 & $\begin{array}{l}\text { Comportement de l'enseignant: } \\
\text { - Instruction dirigée par l'enseignant } \\
\text { - Évaluation formative } \\
\text { - Orientation des élèves }\end{array}$ \\
\hline ST61 & $\begin{array}{l}\text { Expérience des mathématiques } \\
\text { appliquées }(\mathrm{Q} 1,4,6,8) \text {; Expérience } \\
\text { des mathématiques fondamentales } \\
(\mathrm{Q} 5,7,9)\end{array}$ & ST37 & Efficacité perçue en mathématiques & ST80 & $\begin{array}{l}\text { Activation cognitive en cours } \\
\text { de mathématiques }\end{array}$ \\
\hline ST62 & $\begin{array}{l}\text { Mesure dans laquelle des concepts } \\
\text { mathématiques sont familiers }\end{array}$ & ST43 & $\begin{array}{l}\text { Contrôle perçu de la performance } \\
\text { en mathématiques }\end{array}$ & ST81 & Climat de discipline \\
\hline ST69 & $\begin{array}{l}\text { Nombre de minutes par < période } \\
\text { de cours> }\end{array}$ & ST44 & Raisons de l'échec en mathématiques & ST82 & + Vignettes d'ancrage \\
\hline ST70 & $\begin{array}{l}\text { Nombre de <périodes de cours }> \\
\text { par semaine }\end{array}$ & ST46 & Éthique en mathématiques & ST83 & $\begin{array}{l}\text { Soutien du professeur } \\
\text { de mathématiques }\end{array}$ \\
\hline ST71 & $\begin{array}{l}\text { Nombre total de <périodes de cours }> \\
\text { par semaine }\end{array}$ & ST48 & Intentions en mathématiques & ST84 & + Vignettes d'ancrage \\
\hline ST72 & Taille des classes & ST49 & Comportement en mathématiques & ST 85 & $\begin{array}{l}\text { Gestion de la classe par le professeur } \\
\text { de mathématiques }\end{array}$ \\
\hline ST73 & Expérience des problèmes lexicaux & ST93 & Persévérance & ST86 & Relations entre enseignants et élèves \\
\hline ST74 & Expérience des tâches procédurales & ST94 & Ouverture à la résolution de problèmes & ST87 & $\begin{array}{l}\text { Sentiment d'appartenance } \\
\text { à l'établissement d'enseignement }\end{array}$ \\
\hline ST75 & $\begin{array}{l}\text { Expérience du raisonnement } \\
\text { en mathématiques fondamentales }\end{array}$ & ST96 & $\begin{array}{l}\text { Stratégies de résolution de problèmes } \\
\text { (SJT) }\end{array}$ & ST 88 & $\begin{array}{l}\text { Attitudes à l'égard de l'établissement } \\
\text { d'enseignement : résultats } \\
\text { de l'apprentissage }\end{array}$ \\
\hline ST76 & $\begin{array}{l}\text { Expérience du raisonnement } \\
\text { en mathématiques appliquées }\end{array}$ & ST101 & $\begin{array}{l}\text { Stratégies de résolution de problèmes } \\
\text { (SJT) }\end{array}$ & ST89 & $\begin{array}{l}\text { Attitudes à l'égard de l'établissement } \\
\text { d'enseignement : activités } \\
\text { d'apprentissage }\end{array}$ \\
\hline & & ST104 & $\begin{array}{l}\text { Stratégies de résolution de problèmes } \\
\text { (SJT) }\end{array}$ & ST91 & Contrôle perçu de la réussite scolaire \\
\hline
\end{tabular}




\section{GARANTIR LA VALIDITÉ DES INSTRUMENTS PISA, LEUR POUVOIR EXPLICATIF ET LEUR PERTINENCE POUR L'ACTION PUBLIQUE}

Cette section présente plusieurs recommandations concernant la structure et le plan d'analyse de l'enquête PISA pour améliorer la validité et la comparabilité interculturelles, élaborer des indicateurs probants à tous les niveaux et accroître le pouvoir explicatif de l'enquête. Le perfectionnement de la structure de l'enquête sous l'angle de la méthodologie de la recherche permettra de dégager sur la base des données PISA des séries plus nombreuses et plus sophistiquées d'éclairages pertinents pour l'action publique.

La structure et les méthodes normalisées de compte rendu utilisées actuellement permettent, par exemple, de quantifier les cours de soutien proposés par les établissements d'enseignement (déclarés par les chefs d'établissement) et les cours suivis par les élèves en dehors du cadre scolaire (déclarés par les élèves et/ou leurs parents). Les responsables politiques peuvent toutefois se poser plusieurs questions à ce sujet. L'offre de cours de soutien varie-t-elle selon les établissements d'enseignement? Les élèves ont-ils autant accès à des cours de soutien selon qu'ils sont issus d'un milieu social favorisé ou défavorisé, qu'ils sont ou non issus de l'immigration ou qu'ils suivent telle ou telle filière d'enseignement (d'après le cadre d'échantillonnage PISA) ? Existe-t-il une discrimination positive en faveur des élèves de condition modeste ? Les cours de soutien privés compensent-ils le manque de cours de soutien en milieu scolaire? En quoi ces relations évoluentelles au fil du temps (entre les cycles PISA) ? Où se situe mon pays par rapport à d'autres pays dans ces classements ?

Une modélisation plus sophistiquée s'impose toutefois pour répondre aux questions suivantes, et les résultats obtenus doivent être interprétés avec prudence. Le travail rémunéré empêche-t-il les élèves de participer à des cours de soutien et/ ou à du tutorat ? La performance des élèves augmente-t-elle grâce à ces cours ? L'offre de cours de soutien entraîne-t-elle une augmentation de la performance moyenne à l'échelle des établissements d'enseignement, réduit-elle la variation de la performance entre les élèves inscrits dans le même établissement ou diminue-t-elle le gradient social (c'est-àdire l'intensité de la relation entre le milieu socio-économique et la performance) au sein même des établissements d'enseignement ? Il s'agit d'hypothèses à propos de l'impact d'un traitement (le travail rémunéré, le tutorat et les cours de soutien) sur une variable (la participation à ces activités et la performance des élèves). Il faut passer par des inférences causales pour tester ces hypothèses. La validité de ces inférences dépend des variables de contrôle qui peuvent expliquer la sélection, personnelle ou non, de ces traitements (voir la section "Comprendre les modèles d'efficience dans les systèmes d'éducation »). Des analyses devraient être menées pays par pays.

La participation à des cours de soutien (imposée aux élèves ou choisie par eux) dépend vraisemblablement des pressions ou des recommandations des enseignants et des parents, et est influencée par les notes des élèves, leur sexe, leur milieu socio-économique et leur filière d'enseignement. Pour contrôler ces conditions et couvrir les variables de traitement, tous les élèves devraient être interrogés : i) sur la nature et la durée des cours de soutien qu'ils ont suivis en mathématiques durant l'année scolaire en cours; ii) sur leurs notes à la fin de l'année scolaire précédente; et iii) sur la question de savoir si leurs enseignants et/ou leurs parents leur ont recommandé, à la fin de l'année scolaire précédente, de suivre des cours de soutien. L'inclusion de ces questions dans le questionnaire "Élève » est indispensable pour faire des inférences causales à cet égard. Des effets de traitements similaires pourraient être étudiés lors de prochains cycles.

À l'échelle des établissements d'enseignement, la politique en matière d'absentéisme et son impact sur l'absentéisme des élèves, leur sentiment d'appartenance à l'établissement d'enseignement et leur performance devraient faire l'objet d'analyses. II pourrait, par exemple, être demandé aux chefs d'établissement d'indiquer si les autorités en charge de l'éducation considéraient que l'absentéisme était un problème grave trois ans auparavant et de préciser le type de mesures mises en œuvre depuis lors.

\section{Comment modéliser les effets des variables contextuelles sur l'apprentissage et la performance des élèves}

Les précédents cycles PISA et des études sur l'efficacité de l'éducation ont analysé les effets des processus de niveau Établissement et Élève sur la performance et la motivation des élèves (par exemple, leur intérêt pour les mathématiques), après contrôle d'intrants tels que le sexe, le milieu socio-économique et l'ascendance allochtone (à l'échelle individuelle) et des mêmes intrants agrégés (à l'échelle des établissements d'enseignement). L'inclusion de ces variables de contrôle permet d'éviter de tirer des conclusions erronées de type "faux positif " à propos de relations entre deux variables qui s'expliquent en fait par une troisième variable. Dans de nombreux pays, la variation de la performance entre les élèves selon qu'ils sont ou non issus de l'immigration s'explique, par exemple, en grande partie par le fait que les élèves issus de l'immigration vivent souvent dans un milieu socio-économique défavorisé et ont, dès lors, moins de capital social et culturel. Ignorer la différence de milieu socio-économique reviendrait à 
surestimer l'impact de I'ascendance allochtone. De plus, I'enquête PISA utilise des techniques de modélisation multiniveau telles que la modélisation linéaire hiérarchique pour tenir compte de la structure hiérarchique des données relatives aux élèves selon leur établissement d'enseignement et aux établissements d'enseignement selon les pays. II est dès lors possible d'éviter de sous-estimer des erreurs types à cause du non-respect de l'hypothèse des observations indépendantes dans un échantillon, car les élèves d'un même établissement d'enseignement sont susceptibles d'avoir plusieurs caractéristiques en commun (voir, par exemple, Raudenbush et Bryk, 2002 ; Snijders et Bosker, 1999). Les modèles multiniveau permettent de surcroît l'analyse des effets à différents niveaux, c'est-à-dire les effets des variables $d^{\prime}$ intrant et de processus à l'échelle des établissements d'enseignement et des élèves.

Toutefois, les études sur l'efficience de l'éducation montrent que - après modélisation des relations entre les intrants, les processus et les extrants - trois observations supplémentaires issues de recherches antérieures devraient être prises en considération, à savoir que les effets sont souvent : i) non linéaires ; ii) atténués par d'autres variables ; et iii) qu'ils peuvent être indirects ou influencés par d'autres variables.

- Les effets non linéaires sont liés au fait qu' « augmentation » ne signifie pas nécessairement « amélioration » et qu’un moyen terme est parfois optimal. Monk suggère par exemple dans une étude (1994) que la relation entre la mesure dans laquelle l'enseignant maîtrise sa matière et la performance de ses élèves peut être curvilinéaire. D'une part, les enseignants sont de toute évidence incapables d'aider leurs élèves à comprendre des concepts qu'ils ne maîtrisent pas eux-mêmes et, d'autre part, les enseignants qui maîtrisent extrêmement bien leur discipline peuvent avoir des difficultés à transmettre leur savoir d'une façon que les élèves comprennent bien.

- Dans le droit fil de la théorie de l'efficience différentielle (voir, par exemple, Kyriakides et Tsangaridou, 2004), il est important d'admettre que des relations entre variables ne sont pas nécessairement similaires dans des sous-groupes différents. Par exemple, certains éléments montrent que des élèves issus de milieux sociaux différents peuvent bénéficier de pratiques pédagogiques différentes (voir, par exemple, Brophy, 1992 ; Walberg, 1986). En pédagogie, les théories actuelles, qui s'inspirent de la conception constructiviste de l'apprentissage, se basent sur l'hypothèse que l'apprentissage des élèves dépend dans une grande mesure de processus autorégulés, qui sont modérés par des facteurs propres à l'établissement d'enseignement, à la classe et à l'enseignant. La modélisation de ces différences passe par l'analyse de ces effets d'interaction et de modération.

- Enfin, il est raisonnable de postuler que les effets sur les résultats des élèves ne sont pas tous directs. Les politiques à l'échelle des établissements d'enseignement et des systèmes ont souvent des effets plus faibles sur les résultats des élèves que des variables contextuelles de niveau Élève et des processus à l'œuvre en classe (voir, par exemple, Wang et al., 1993). Ce constat peut en partie s'expliquer par le fait que les premières variables n'ont pas d'effets directs sur les élèves, mais qu'elles sont liées aux processus spécifiques à l'établissement d'enseignement et à la classe, qui ont à leur tour un effet sur la performance des élèves. De plus, des variables de niveau Établissement, telles que le climat, les normes et les valeurs ou les procédures prévues en cas de problèmes de comportement, peuvent avoir un effet direct sur des résultats non cognitifs (par exemple, la motivation pour l'apprentissage et les aspirations académiques) et le comportement des élèves (par exemple, I'absentéisme, la violence), alors que les effets de niveau Établissement sur la performance des élèves et d'autres résultats spécifiques aux matières (par exemple, l'intérêt et l'efficacité perçue) seront très vraisemblablement influencés par l'enseignement et I'apprentissage en classe.

Dans certains cas, les aspects i), ii) et iii) s'appliquent simultanément, par exemple lorsque les effets du climat en classe et des relations entre enseignants et élèves subissent l'influence de l'intérêt des élèves. Toutefois, l'effet de l'intérêt des élèves pour les mathématiques sur leur performance est plus important dans les établissements d'enseignement où les enseignants utilisent des pratiques pédagogiques novatrices d'activation cognitive que dans ceux où ils appliquent des pratiques plus traditionnelles. (Ce modèle est représenté dans la figure 6.2. Il s'inspire en partie des résultats d'une étude de l'enseignement des mathématiques dans deux pays par Klieme et al., 2009 et Lipowsky et al., 2009.) Un effet non linéaire peut être inclus s'il peut être établi que ni un climat trop laxiste, ni un climat trop strict n'est optimal pour améliorer l'intérêt des élèves ou leur performance. (Pour un exemple plus complexe basé sur l'analyse des données du cycle PISA 2003 dans un pays, voir Kaplan, 2009c et Kaplan et al., 2009.) 
- Figure 6.2 -

Exemple de modération

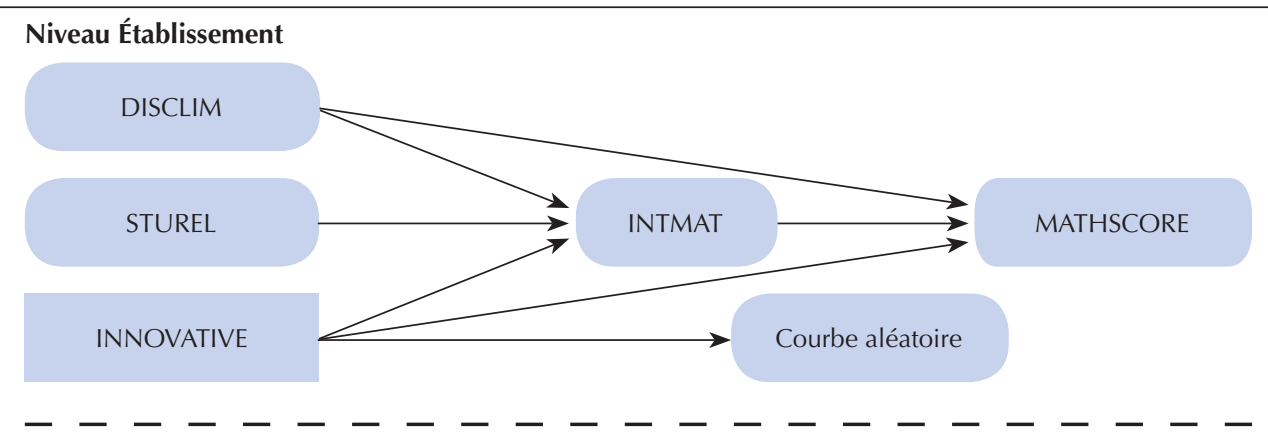

Niveau Élève

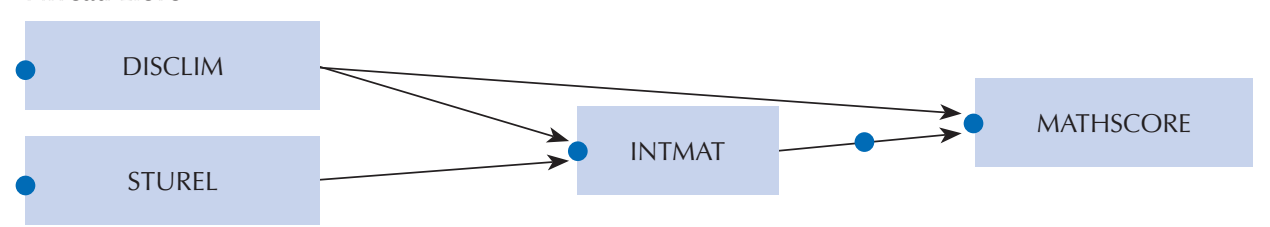

Remarques : DISCLIM : climat de discipline en cours de mathématiques; STUREL : relations entre enseignants et élèves ; INNOVATIVE : stratégies pédagogiques novatrices ; INTMAT : intérêt et plaisir des mathématiques; MATHSCORE : performance en mathématiques. Chaque point représente un paramètre estimé dans le modèle.

Les effets modérateurs, médiateurs et non linéaires peuvent être modélisés à l'aide de techniques de modélisation linéaire hiérarchique. Toutefois, la modélisation par équation structurelle se prête mieux à la modélisation des processus médiateurs (voir, par exemple, Kaplan, 2009c). Par comparaison avec la régression multiniveau, le principal avantage de la modélisation par équation structurelle réside dans le fait que les variables utilisées sont latentes et non observables, ce qui permet d'éviter les biais liés à l'erreur de mesure au niveau Élève (voir, par exemple, Muthén, 2002). Autre mérite, les mesures agrégées à l'échelle des établissements d'enseignement permettent de modéliser des effets contextuels (Lüdtke et al., 2008). L'utilisation de modèles par équation structurelle améliore donc la validité des conclusions à propos de relations entre différents aspects du système d'éducation. Les modèles multiniveau par équation structurelle offrent aussi la possibilité de combiner de manière plus souple les effets modérateurs, médiateurs et non linéaires à différents niveaux, et d'analyser les variables catégorielles latentes et observables. Ainsi, une modélisation par équation structurelle pourrait être utilisée pour déterminer si l'effet du climat de discipline en classe sur la performance des élèves est similaire dans tous les pays ou s'il est plus important dans les pays où les enseignants ont moins d'autorité. Les modèles basés sur des variables latentes peuvent être appliqués pour vérifier l'hypothèse selon laquelle c'est la combinaison de stratégies d'apprentissage différentes plutôt que l'adoption fréquente de telle ou telle stratégie d'apprentissage qui a un impact sur la performance des élèves. Des modèles mixtes permettent l'identification, dans les pays, de groupes d'élèves où les relations entre le climat de discipline et la performance varient (voir, par exemple, Muthén, 2002). Ces modèles pourraient mieux refléter la réalité complexe des processus d'éducation, de leurs tenants et aboutissants.

En fait, les modèles multiniveau par équation structurelle permettraient aux responsables politiques et aux professionnels de l'éducation de mieux comprendre les relations entre : $i$ ) les possibilités d'apprentissage à l'échelle des établissements d'enseignement; ii) l'exploitation individuelle de ces possibilités (qualité et quantité des activités d'apprentissage à l'échelle individuelle); et iii) les résultats cognitifs et motivationnels des élèves (voir, par exemple, les travaux de Kaplan et al., 2009, basés sur l'analyse des données du cycle PISA 2003 dans un pays).

En résumé, il n'est pas approprié d'adopter une approche basée sur une fonction de production à l'égard des résultats de l'éducation. L'action publique et des facteurs propres aux systèmes et aux établissements d'enseignement ont des effets sur l'apprentissage des élèves via les processus d'enseignement, d'apprentissage et de collaboration. Les chercheurs doivent tenir compte de ces corrélations lorsqu'ils modélisent les effets à l'échelle des systèmes ou des établissements d'enseignement, et qu'ils en tirent des conclusions pertinentes pour l'action publique. 


\section{Validité interculturelle}

L'objectif de l'enquête PISA est de comparer la performance des élèves et les facteurs qui la conditionnent dans un grand nombre de pays. Les études internationales permettent non seulement d'analyser les orientations politiques et d'autres intrants et processus systémiques et leurs relations avec la performance, mais également de déterminer si des conclusions de recherches pédagogiques sont transposables dans d'autres cultures. Les études internationales présentent toutefois des difficultés méthodologiques. II faut non seulement vérifier régulièrement la fiabilité et la validité, mais également explorer les problèmes d'équivalence et de biais, deux aspects en rapport avec le temps consacré aux tâches. Les biais de constructs proviennent de différences réelles dans les systèmes d'éducation, les normes culturelles et I'interprétation de divers phénomènes, mais ils peuvent aussi être imputables à des erreurs de traduction ou à des problèmes de méthodologie. Pour remédier à ce problème potentiel, I'enquête PISA fait appel à des experts dans tous les pays participants. Certaines des différences dans la signification des constructs sont éliminées via des adaptations nationales, mais une modélisation par équation structurelle s'impose pour évaluer le niveau d'équivalence interculturelle et, donc, la nature des éléments qui peuvent être comparés entre les pays (moyennes, profils et corrélations). Lors des précédents cycles PISA, c'est une analyse factorielle confirmatoire multigroupe qui a été utilisée pour déterminer si le degré d'adéquation du modèle était acceptable et vérifier l'invariance des saturations factorielles. Les résultats de ces analyses montrent que les échelles PISA sont relativement équivalentes dans l'ensemble des cultures et systèmes d'éducation. Les mêmes analyses ont été effectuées lors du cycle PISA 2012, tant pour les échelles existantes que pour les nouvelles échelles. Ces dernières n'ont été retenues lors de la campagne définitive que si les résultats d'analyse étaient satisfaisants ${ }^{8}$.

\section{Biais d'items}

Les items sont biaisés lorsqu'ils ne se comportent pas de la même façon que d'autres items mesurant la même variable dans des groupes particuliers. Ces biais peuvent être détectés grâce à des méthodes de fonctionnement différentiel d'items qui s'inspirent de la théorie de réponse à l'item. Lors des précédents cycles PISA, ces analyses ont été réalisées lors de l'essai de terrain et de la campagne définitive. Un rapport sur les items douteux a été envoyé aux représentants des pays, et les items qui posaient problème ont été révisés (lors de l'essai de terrain) ou exclus. La même stratégie a été appliquée lors du cycle PISA 2012.

\section{Biais de méthodes}

Dans ce type de biais, le manque de comparabilité est imputable aux méthodes utilisées, c'est-à-dire à l'échantillonnage, à l'administration ou aux instruments eux-mêmes. L'un des biais courants provient d'une caractéristique des items, en l'occurrence l'utilisation d'échelles dites de Likert - courante dans les questionnaires contextuels PISA. Ces échelles sont particulièrement sensibles aux différences de style de réponse. Or, il est établi que les styles de réponse dépendent de la culture. Ce problème n'est pas sans conséquence pour la comparaison des valeurs moyennes de résultats non cognitifs et pour l'analyse des relations entre les facteurs contextuels et la performance (Hui et Triandis, 1985 ; van de Vijver et al., 2008). Les styles de réponse peuvent être à l'origine de défauts d'équivalences qui peuvent être détectés via une analyse factorielle confirmatoire multiniveau ou une analyse du fonctionnement différentiel des items. Toutefois, des analyses supplémentaires peuvent s'imposer, car les styles de réponse ont souvent un impact similaire sur tous les items. Les différences de corrélation entre certaines échelles et la performance au niveau Élève entre les pays et au sein même de ceux-ci (voir, par exemple, van de Gaer et Adams, 2010) sont un problème connu dans l'enquête PISA. L'intérêt pour les mathématiques peut, par exemple, être en corrélation positive avec la performance en mathématiques au sein d'un pays, mais la corrélation entre les deux variables peut être fortement négative une fois comparée à l'échelle internationale. En d'autres termes, les élèves plus performants déclarent, logiquement, un intérêt plus élevé pour les mathématiques que d'autres élèves de leur pays. Dans le classement international, toutefois, des pays où la performance est plus élevée accusent des valeurs moyennes inférieures d'intérêt pour les mathématiques (par exemple, la Corée, la Finlande et le Japon), alors que des pays où la performance est moins élevée affichent des valeurs supérieures d'intérêt pour les mathématiques (par exemple, le Brésil, I’Indonésie et la Tunisie).

Une analyse plus détaillée des biais liés aux méthodes aidera à écarter les différences de scores moyens et de corrélations entre les pays qui sont dues à la variation des styles de réponse. Trois stratégies ont été utilisées lors du cycle PISA 2012 pour remédier à ce problème. En premier lieu, des analyses ont été réalisées pour déterminer si des ajustements statistiques permettraient de corriger le problème, du moins dans certaines échelles. L'adoption d'une approche de modélisation mixte (Rost et al., 1997) permet, par exemple, de grouper les pays en fonction de leurs similitudes en matière de styles de réponse. La deuxième stratégie consiste à déterminer s'il est possible de remédier au problème de validité interculturelle via l'inclusion de divers nouveaux types d'items et d'autres formes de manipulations d'items, telles que celle proposée par Buckley (2009). À titre d'exemple, citons I'inclusion d'items inversés, d'items à choix 
forcé et d'items basés sur des ancrages d'échelle différents, et le classement des items, qui permettent de manipuler I'usage des échelles de réponse et la position des items. De plus, on peut pratiquer l'alternance de formats d'items dans un questionnaire (par exemple, l'inclusion d'items basés sur une échelle de Likert à 3, 4 et 5 points dans un même questionnaire). Enfin, l'inclusion de vignettes et d'items de jugement contextuel, et la variation de la formulation des questions (par exemple, "par comparaison avec les autres élèves de votre classe » vs " dans votre pays », ou «par comparaison avec d'autres matières ») ont été analysées. D’autres procédures ont été proposées, notamment I'identification d'un groupe multilingue au sein d'un pays pour calibrer l'échelle de réponse. Comme certaines de ces méthodes requièrent la collecte de nouvelles données, elles n'ont pu être mises en œuvre que lors de l'essai de terrain.

\section{Extension de l'échantillon pour étudier la variation au niveau Établissement (option internationale)}

Le manque de données longitudinales à l'échelle des établissements d'enseignement est manifeste à la lumière de la causalité inversée (un phénomène que l'on appelle aussi déterminisme réciproque, effet simultané ou effet récursif, Scheerens et Bosker, 1997). La mise en œuvre de certaines interventions politiques (l'organisation de cours de soutien, par exemple) à cause du niveau peu élevé de performance relève, par exemple, de la causalité inversée. Dans ce cas, des données transversales peuvent donner lieu à des corrélations négatives entre la performance et des interventions politiques, même après contrôle de variables contextuelles de niveau Élève.

Scheerens et Bosker (1997) avancent l'idée que des études longitudinales à l'échelle des établissements d'enseignement permettraient de jeter des bases empiriques pour l'analyse d'effets de causalité inversée, mais ils n'en ont trouvé aucune. L'amélioration de la méthodologie des études sur l'efficacité de l'éducation (voir, par exemple, Creemers et Kyriakides, 2008) et la collecte de données d'évaluation des établissements d'enseignement par année d'études (y compris de données tendancielles au fil du temps) ont profondément modifié ce domaine de recherche. Les chercheurs sont conscients du phénomène et savent donc que les enquêtes transversales ne permettent que jusqu'à un certain point d'expliquer pourquoi des établissements d'enseignement obtiennent des résultats plus positifs que d'autres.

Le moyen de remédier à ce problème serait de réaliser des analyses longitudinales à l'échelle des établissements d'enseignement : il faudrait échantillonner les mêmes établissements lors de cycles PISA différents pour étudier les changements qui y sont intervenus (évolution des qualifications des enseignants, de la composition socio-économique des effectifs d'élèves, des attitudes et de la performance des élèves, et variation des corrélations au fil du temps).

\section{Extensions longitudinales de niveau Élève (recommandations pour les prochains cycles)}

L'inclusion d'une composante longitudinale dans l'enquête PISA permettrait l'exploration d'un certain nombre de thématiques intéressantes, pertinentes pour I'action publique. Elle offrirait en premier lieu la possibilité d'estimer des indicateurs à valeur ajoutée, par exemple à propos de l'effet de différentes variables sur les gains de performance. Les études transversales permettent d'étudier les relations entre des variables, et les études longitudinales, d'étudier leur causalité. L'application de modèles de changement latent ou l'analyse de corrélations avec décalage permettraient, par exemple, de déterminer si l'efficacité perçue a un impact sur la performance, si la performance a un impact sur l'efficacité perçue, ou si chaque variable a un impact sur l'autre, auquel cas l'effet est réciproque.

Certains pays ont d'ores et déjà mis en œuvre une extension longitudinale de l'enquête PISA à l'échelle nationale : I'Allemagne, I'Australie (Longitudinal Study of Australian Youth), le Canada (Enquête auprès des jeunes en transition), le Danemark, la République tchèque, la Suisse (Transitions from Education to Employment) et I'Uruguay. Ils ont utilisé deux approches différentes :

- En Australie, au Canada, au Danemark, en Suisse et en Uruguay, ces études ont porté sur la transition entre l'enseignement secondaire et post-secondaire et/ou le monde du travail (voir, par exemple, Andersen, 2005 ; Bertschy et al., 2008 ; Looker et Thiessen, 2008 ; Zoido et Gluszynski, 2009). Leurs résultats contribuent à la validation des épreuves PISA : ils montrent en effet que la performance en compréhension de l'écrit et en mathématiques est une variable prédictive probante du parcours scolaire (abandon, obtention d'un diplôme, études post-secondaires, études tertiaires, chômage, revenus, etc.). Ces études permettent d'examiner les effets de variables contextuelles et d'attitude sur des résultats ultérieurs. II ressort, par exemple, de l'enquête menée au Canada que l'engagement académique des élèves, leurs attentes scolaires et leurs activités extrascolaires, ainsi que le soutien éducatif de leurs pairs et le soutien de leurs parents, sont des variables prédictives probantes de la poursuite de leur parcours scolaire (Looker et Thiessen, 2008 ; Thiessen, 2007). Selon l'étude réalisée en Australie, la perception individuelle des relations entre enseignants et élèves et du moral des enseignants (Curtis et McMillan, 2008) est en corrélation avec les résultats ultérieurs des élèves. Si d'autres pays mettaient cette option en œuvre, il serait possible de déterminer si les effets observés au Canada et en Australie s'appliquent à d'autres pays, 
et si des caractéristiques systémiques (l'offre de programmes de « deuxième chance », par exemple) ont également un impact sur le parcours scolaire des élèves.

- Une autre approche consiste à étudier l'évolution de la performance des élèves en mathématiques durant leurs études secondaires et à analyser les corrélations entre les intrants et les processus de niveau Établissement et Élève, et la variation latente des compétences des élèves. Les études transversales permettent souvent d'observer des effets négatifs entre différentes variables de processus, tels que l'aide aux devoirs, les cours de soutien ou la coopération entre enseignants. Ces résultats peuvent en partie s'expliquer par le fait que ces indicateurs concernent les élèves en difficulté, à qui l'on donne plus de temps d'apprentissage. De même, dans les établissements d'enseignement en proie à de nombreux problèmes de discipline, les enseignants sont susceptibles de coopérer plus étroitement, car ils éprouvent un plus grand besoin de soutien. Les études longitudinales permettraient d'isoler l'impact de ces variables des impacts réels des programmes. Plus généralement, elles amélioreraient la validité des conclusions tirées des analyses concernant l'impact des politiques et des processus sur la performance des élèves, ce qui permettrait de constituer une base de connaissances plus probante pour éclairer les choix d'orientations politiques. Cette approche longitudinale a déjà été appliquée dans deux études allemandes, le Projet d'analyse du développement de la performance en mathématiques et PISA International Plus (PISA-I-Plus). II ressort par exemple de ces études que la valeur moyenne de l'indicateur de l'engouement des élèves de $7^{\mathrm{e}}$ année a un effet sensible sur l'engouement des enseignants en poste en $8^{\mathrm{e}}$ année, qui a à son tour un effet sensible sur l'engouement des élèves de $8^{\mathrm{e}}$ année, lequel subit l'influence de la perception de l'enthousiasme des enseignants (Frenzel et al., 2009). Il en ressort également que la gestion de la classe a un effet significatif sur la performance des élèves de $10^{\circ}$ année en mathématiques, qui est nettement plus élevée dans les établissements d'enseignement où de nombreux enseignants sont actifs et/ou attachés à la discipline que dans les établissements d'enseignement où de nombreux enseignants sont passifs (Prenzel et al., 2006).

Un autre exemple a récemment fait l'objet de débats aux États-Unis (Loveless, 2009) sur la question des élèves mal orientés (les effets négatifs de l'orientation d'élèves peu performants dans des filières exigeantes). Si les critiques formulées par Loveless (dans ce contexte spécifique) sont valables, il y aurait un effet négatif d'interaction entre le fait de suivre des cours spéciaux de physique et certains facteurs de risques (par exemple, l'ascendance allochtone ou un niveau peu élevé de performance en compréhension de l'écrit) sur la performance en mathématiques. Cette hypothèse ne peut être vérifiée comme il se doit via une étude transversale. Des extensions longitudinales contribueraient donc à apporter des réponses à des questions complexes en rapport avec l'action publique.

\section{SYNTHÈSE}

Ce cadre décrit les fondements des questionnaires contextuels administrés lors du cycle PISA 2012 dans le but de constituer une base de données durable pour éclairer les responsables de la politique de l'éducation et alimenter les études en matière pédagogique. Nous avons commencé par rappeler les objectifs généraux de l'enquête PISA et sa pertinence pour l'action publique. Nous avons ensuite passé en revue la littérature spécialisée par rapport à l'efficacité de l'éducation. Comme le domaine majeur d'évaluation du cycle PISA 2012 est la culture mathématique, des aspects spécifiques de l'enseignement et de l'apprentissage des mathématiques ont également été ciblés. L'essentiel de ce cadre conceptuel porte toutefois sur les fondements à retenir pour faire des questionnaires PISA des instruments durables. La structure choisie se prête à l'administration de questions générales récurrentes lors de chaque cycle et à l'administration de questions spécifiques au domaine majeur d'évaluation tous les quatre cycles pour décrire des tendances générales et spécifiques à chaque domaine. Cette structure permet aussi l'inclusion d'extensions thématiques et spécifiques, pour que l'enquête PISA puisse ajouter de nouveaux contenus ou thèmes intéressant son public. Ce cadre rappelle également des recommandations spécifiques concernant des contenus à inclure dans le cycle PISA 2012 sur la base des analyses des données PISA et d'autres études. Enfin, il se termine par des suggestions en matière de structure et d'analyse pour exploiter au mieux les points forts de l'enquête PISA et jeter des bases encore plus solides pour l'avenir. 


\section{Notes}

1. Pour plus d'informations, voir la page www.oecd.org/pisa/pisainfocus/.

2. Toutes ces publications sont disponibles à l'adresse : www.oecd.org/pisa/pisaproducts/pisain-depthreports.htm.

3. Les résumés et les dossiers sont disponibles à l'adresse : www.pisaresconf09.org/.

4. Pour établir un lien entre le présent cadre d'évaluation PISA et les travaux conceptuels antérieurs de l'enquête PISA, les sections "Intrants et processus à l'échelle des systèmes ", "Intrants et processus à l'échelle des établissements d'enseignement ", "Intrants et processus à l'échelle des classes » et «Intrants et processus à l'échelle des élèves » s'inspirent largement du cadre conceptuel des questionnaires du cycle PISA 2009, qui a été rédigé par Hank Levin sur la base des travaux approfondis de Jaap Scheerens. Les auteurs les remercient pour leurs travaux importants et remercient les autres experts pour leur contribution aux précédents cycles PISA.

5. Voir : www.oecd.org/document/7/0,2340,en_21571361_31938349_36043527_1_1_1_1,00.html.

6. Voir EDU/PISA/GB (2005)21, p. 17, paragraphe 67.

7. Les variables qui n'ont été utilisées qu'en 2003 ou qui ont été abandonnées par la suite sont celles en rapport avec la structure familiale, le parcours scolaire et les raisons de l'assiduité scolaire.

8. L'ensemble de ces informations seront publiées dans l'ouvrage PISA 2012 Technical Report (à paraître). 


\section{Références}

Abedi, J., M. Courtney, S. Leon, J. Kao et T. Azzam (2006), English Language Learners and Math Achievement: A Study of Opportunity to Learn and Language Accommodation (CSE Report 702, 2006), Universite de Californie, Center for the Study of Evaluation/National Center for Research on Evaluation, Standards, and Student Testing, Los Angeles, Californie.

Ajzen, I. (1991), "The Theory of Planned Behavior », Organizational Behavior and Human Decision Processes, vol. 50, pp. $179-211$.

Alexander, K.L., D.R. Entwisle et L.S. Olson (2007), "Lasting Consequences of the Summer Learning Gap », American Sociological Review, vol. 72, pp. 167-180.

Amiot, C. et R.Y. Bourhis (2005), " Discrimination between Dominant and Subordinate Groups: The Positive-Negative Asymmetry Effect and Normative Processes », British Journal of Social Psychology, vol. 44, pp. 289-308.

Andersen, D. (2005), « Four Years after Basic Compulsory Education 19-year-olds on Post Compulsory Education Choices and Paths », http://www.akf.dk/udgivelser_en/2005/4aarefter_grundskolen.

Angelone, D., U. Moser et E. Ramseier (2009), «Instruction Time and Performance: Analyses of the Importance of Instruction Time for Competencies in Science and Mathematics based on Swiss PISA 2006 Data », dossier présenté lors de la conférence PISA, Kiel, Allemagne, 14-16 septembre, http://www.pisaresconf09.org/user_uploads/files/context/room3/Angelone_Moser_Ramseier.pdf.

Baker, D.P. (2009), "The Invisible Hand of World Education Culture », in G. Sykes, B. Schneider et D.N. Plank (éd.), Handbook of Education Policy Research, Routledge, New York, pp. 958-968.

Baker, D.P., B. Goesling et G.K. LeTendre (2002), « Socioeconomic Status, School Quality, and National Economic Development: A Cross-National Analysis of the 'Heyneman-Loxley effect' on Mathematics and Science Achievement », Comparative Education Review, vol. 46, pp. 291-312.

Baker, D.P. et G.K. LeTendre (2005), National Differences, Global Similarities: World Culture and the Future of Schooling, Stanford University Press, Stanford, Californie.

Baker, M.L., J.N. Sigmon et M.E. Nugent (2001), "Truancy Reduction: Keeping Students in School», Juvenile Justice Bulletin, Office of Juvenile Justice and Delinquency Prevention, Washington DC.

Bandura, A. (1977), Social Learning Theory, Prentice Hall, Englewood Cliffs, New Jersey.

Baumert, J., C.H. Carstensen et T. Siegle (2005), « Wirtschaftliche, Soziale und Kulturelle Lebensverhältnisse und Regionale Disparitäten des Kompetenzerwerbs ", in PISA-Konsortium Deutschland (éd.), PISA 2003: Der zweite Vergleich der Länder Deutschland - Was wissen und können Jugendliche?, Waxmann Verlag, Münster.

Baumert, J., W. Blum, M. Brunner, T. Dubberke, A. Jordan, U. Klusmann, S. Krauss, M. Kunter, K. Löwen, M. Neubrand et Y.-M. Tsai (2008), Professionswissen von Lehrkräften, kognitiv aktivierender Mathematikunterricht und die Entwicklung von mathematischer Kompetenz (COACTIV) - Dokumentation der Erhebungsinstrumente, [Baumert et al. (2009), Materialien aus der Bildungsforschung, $\mathrm{n}^{\circ}$ 83], Max-Planck-Institut für Bildungsforschung, Berlin.

Baumert, J., M. Kunter, W. Blum, M. Brunner, T. Voss, A. Jordan, U. Klusmann, S. Krauss, M. Neubrand et Y.-M. Tsai (2009), " Teachers' Mathematical Knowledge, Cognitive Activation in the Classroom, and Student Progress ", American Educational Research Journal, vol. 47, pp. 133-180.

Baye, A., C. Monseur et D. Lafontaine (2009), "Institutional and Socioeconomic Factors Influencing the Reading Engagement », dossier présenté lors de la conférence PISA, Kiel, Allemagne, 14-16 septembre.

Belfield, C. et H. Levin (2002), «Education Privatization: Causes, Consequences, and Planning Implications », Institut international de la planification de l'éducation, UNESCO, Paris.

Berezner, A. et P. Lietz (2009), « Implication for Preferred Model of Rotation on Cognitive Proficiency Estimate Generation Reporting and Subsequent Analyses ", dossier présenté à la réunion du groupe d'experts en charge des questionnaires, Offenbach, Allemagne, 19-21 octobre.

Berry, J.W. (1980), «Comparative Studies of Acculturative Stress », International Migration Review, vol. 21, pp. 491-511.

Berry, J.W. (1990), «Psychology of Acculturation», in R.W. Brislin (éd.), Applied Cross-Cultural Psychology, Sage, Newbury Park, Californie, pp. 232-253.

Bertschy, K., E. Boni et T. Meyer (2008), "Young People in Transition from Education to Labor Market. Results of the Swiss youth panel survey TREE », mise à jour de 2007, Bâle.

Blase, J. et J. Blase (1998), Handbook of Instructional Leadership: How Really Good Principals Promote Teaching and Learning, Corwin Press, Thousand Oaks, Californie. 
Blomhoj, M. et T.H. Jensen (2007), "What's all the Fuss about Competencies? Experiences with Using a Competence Perspective on Mathematics Education to Develop the Teaching of Mathematical Modelling », in W. Blum, P.L. Galbraith, H-W. Henn et M. Niss (éd.), Modelling and Applications in Mathematics Education, 14 ICMI Study, Springer, New York, pp. 45-56.

Blum, W. et D. Leiss (2005), " How do Students and Teachers Deal with Mathematical Modelling Problems? The Example "Sugarloaf" », in ICTMA 12 Proceedings, Ellis Horwood, Chichester.

Blum, W. et D. Leiss (2007), «Investigating Quality Mathematics Teaching - the DISUM Project », in C. Bergsten et B. Grevholm (éd.), Developing and Researching Quality in Mathematics Teaching and Learning. Proceedings of MADIF 5, SMDF, Linköping, pp. 3-16.

Boaler, J. et J. Greeno (2000), «Identity, Agency and Knowing in Mathematical Worlds », in J. Bowler (éd.), Multiple Perspectives on Mathematics Teaching and Learning, Ablex Publishing, Westport, Connecticut.

Borko, H., K.L. Kuffner, S.C. Arnold, L. Creighton, B.M. Stecher, F. Martinez, D. Barnes et M.L. Gilbert (2007), Using Artifacts to Describe Instruction: Lessons Learned from Studying Reform-Oriented Instruction in Middle School Mathematics and Science, CSE Technical Report 705, National Center for Research on Evaluation, Standards, and Student Testing, Los Angeles, Californie.

Bottani, N. et B. Favre (éd.) (2001), « Open File: School Autonomy and Evaluation », Prospects, vol. 31.

Britner, S.L. et F. Pajares (2006), "Sources of Science Self-Efficacy Beliefs of Middle School Students », Journal of Research in Science Teaching, vol. 43, pp. 485-499.

Bromme, R. (1997), « Kompetenzen, Funktionen und unterrichtliches Handeln des Lehrers », in F.E. Weinert (éd.), « Reihe: Enzyklopädie der Psychologie », Pädagogische Psychologie, vol. 3, Psychologie des Unterrichts und der Schule, Hogrefe, Göttingen, pp. 177-212.

Brookhart, S.M. (2009), Exploring Formative Assessment, ASCD Publishers, Alexandria, Virginie.

Brophy, J. (1992) (éd.), Planning and Managing Learning Tasks and Activities: Advances in Research on Teaching, vol. 3, Greenwich, JAI Press, Connecticut.

Brown, A.L. (1994), "The Advancement of Learning ", Educational Researcher, vol. 23, nº 8, pp. 4-12.

Brunner, M., M. Kunter, S. Krauss, U. Klusmann, J. Baumert, W. Blum, M. Neubrand, T. Dubberke, A. Jordan, K. Löwen et Y.-M. Tsai (2006), « Die professionelle Kompetenz von Mathematiklehrkräften: Konzeptualisierung, Erfassung und Bedeutung für den Unterricht. Eine Zwischenbilanz des COACTIV-Projekts », in M. Prenzel et L. Allolio-Näcke (éd.), Untersuchungen zur Bildungsqualität von Schule. Abschlussbericht des DFG-Schwerpunktprogramms, Waxmann Verlag, Münster, pp. 54-82.

Buckley, J. (2009), Cross-National Response Styles in International Educational Assessments: Evidence from PISA 2006, Steinhardt School of Culture, Education, and Human Development, Université de New York.

Carnoy, M., R. Elmore et L. Siskin (éd.) (2003), The New Accountability: High Schools and High Stakes Testing, Routledge Falmer, New York.

Carroll, J.B. (1963), «A Model of School Learning », Teachers College Record, vol. 64, pp. 723-733.

Christenson, S.L. (2004), "The Family-School Partnership: An Opportunity to Promote the Learning Competence of all Students ", School Psychology Review, vol. 33, pp. 83-104.

Christiansen, B. et G. Walther (1986), "Task and Activity », in B. Christiansen, A.G. Howson et M. Otte (éd.), Perspectives on Mathematics Education, pp. 243-307.

Chubb, J. et T. Moe (1990), Politics, Markets, and American Schools, The Brookings Institution, Washington DC.

Cleary, T.A. (1968), "Test Bias: Prediction of Grades of Negro and White Students in Integrated Colleges », Journal of Educational Measurement, vol. 5, pp. 115-124.

Coleman, J. (1988), « Social Capital in the Creation of Human Capital », American Journal of Sociology, vol. 94, pp. 95-120.

Common Core State Standards Initiative (CCSSI) (2010), Common Core State Standards, http://corestandards.org/.

Creemers, B.P.M. et L. Kyriakides (2008), The Dynamics of Educational Effectiveness: A Contribution to Policy, Practice, and Theory in Contemporary Schools, Routledge, Londres.

Curtis, D.D. et J. McMillan (2008), School Non-completers: Profiles and Initial Destinations. Longitudinal Surveys of Australian Youth, Research Report 54, The Australian Council for Educational Research Ltd., Camberwell, Victoria.

Desoete, A. et M. Veenman (éd.) (2006), Metacognition in Mathematics Education, Nova Science, Haupauge, New York.

Flavell, J.H. (1979), "Metacognition and Cognitive Monitoring: A New Area of Cognitive-Developmental Inquiry ", American Psychologist, vol. 34, pp. 906-911.

Frenzel, A.C., T. Goetz, O. Lüdtke, R. Pekrun et R.E. Sutton (2009), "Emotional Transmission in the Classroom: Exploring the Relationship between Teacher and Student Enjoyment », Journal of Educational Psychology, vol. 101, pp. 705-716. 
Fullan, M. (1992), Successful School Improvement: The Implementation Perspective and Beyond, Open University Press, Milton Keynes.

Fullan, M. et S. Stiegelbauer (1991), The New Meaning of Educational Change, 2e édition, Teachers College Press, New York.

Gamoran, A. (1992), "The Variable Effects of High School Tracking », American Sociological Review, vol. 57, pp. 812-828.

Garofalo, J. et F.K. Lester (1985), "Metacognition, Cognitive Monitoring, and Mathematical Performance », Journal for Research in Mathematics Education, vol. 16, pp. 163-176.

Gustafsson, J.E. (2007), « Understanding Causal Influences on Educational Achievement through Analysis of Differences over Time within Countries », in T. Loveless (éd.), Lessons Learned:What International Assessments Tell Us about Math Achievement, The Brookings Institution, Washington DC, pp. 37-63.

Hallinger, P. et R.H. Heck (1998), "Exploring the Principal's Contribution to School Effectiveness: 1980 - 1995 », School Effectiveness and School Improvement, vol. 9, n², pp. 157-191.

Hannaway, J. et M. Carnoy (éd.) (1993), Decentralization and School Improvement: Can We Fulfill the Promise?, Jossey-Bass, San Francisco, Californie.

Hanushek, E.A. et S.G. Rivkin (2007), "Teacher Quality », in E.A. Hanushek et F. Welch (éd.), Handbook of the Economics of Education, Elsevier, Amsterdam, pp. 1051-1078.

Hanushek, E.A. et L. Woessmann (2009), « Do Better Schools Lead to More Growth? Cognitive Skills, Economic Outcomes, and Causation », NBER Working Paper $n^{\circ}$ 14633, National Bureau of Economic Research, Cambridge, Massachusetts.

Hanushek, E.A. et L. Woessmann (2010), "The Economics of International Differences in Educational Achievement », IZA Discussion Paper $n^{\circ}$ 4925, IZA, Bonn.

Harris, A. et J.H. Chrispeels (éd.) (2006), Improving Schools and Educational Systems: International Perspectives, Routledge, Londres.

Heck, R.H. et P. Hallinger (2005), "The Study of Educational Leadership and Management. Where does the Field Stand Today? ", Educational Management Administration and Leadership, vol. 33, n 2, pp. 229-244.

Hersbach, E. et P. Lietz (2010), « Towards a School Questionnaire in PISA 2012 », QEG(1006)3.doc, dossier présenté lors de la réunion du groupe d'experts en charge des questionnaires, Boston, Massachusetts, 21-23 juin.

Hesse, H.G., K. Göbel et J. Hartig (2008), « Sprachliche Kompetenzen von mehrsprachigen Jugendlichen und Jugendlichen nicht-deutscher Erstsprache», in DESI-Konsortium (éd.), Unterricht und Kompetenzerwerb in Deutsch und Englisch, Weinheim, Beltz, pp. 208-230.

Hiebert, J., R. Gallimore, H. Garnier, K.B. Givvin, H. Hollingsworth, J. Jacobs, A.M.-Y. Chui, D. Wearne, M. Smith, N. Kersting, A. Manaster, E. Tseng, W. Etterbeek, C. Manaster, P. Gonzales et J. Stigler (2003), Teaching Mathematics in Seven Countries: Results from the TIMSS 1999 Video Study, États-Unis, ministère fédéral de I'Éducation, National Center for Education Statistics, Washington DC.

Hill, H.C., B. Rowan et D.L. Ball (2005), "Effects of Teachers' Mathematical Knowledge for Teaching on Student Achievement », American Educational Research Journal, vol. 42, pp. 371-406.

Hoover-Dempsey, K. et H.M. Sandler (1997), "Why do Parents Become Involved in their Children's Education », Review of Educational Research, vol. 67, pp. 3-42.

Hopkins, D. (éd.) (2005), The Practice and Theory of School Improvement: International Handbook of Educational Change, Springer, Dordrecht.

Hovey, J.D. et C.A. King (1996), "Acculturative Stress, Depression, and Suicidal Ideation Among Immigrant and Second-Generation Latino Adolescents ", Journal of the American Academy of Child and Adolescent Psychiatry, vol. 35, pp. 1183-1192.

Hui, C.H. et H.C. Triandis (1985), " Measurement in Cross-Cultural Psychology: A Review and Comparison of Strategies », Journal of Cross-Cultural Psychology, vol. 16, n², pp. 131-152.

Husén, T. (1967), International Study of Achievement in Mathematics, vol. 2, Wiley, New York.

Jehanghir, K. et C. Glas (2007), "The Predictive Power of the Variables in the Student Questionnaires », dossier présenté à la réunion du groupe d'experts en charge des questionnaires, Arnhem, 8-9 juin.

Kao, G. et M. Tienda (1998), «Educational Aspirations of Minority Youth », American Journal of Education, vol. 106, n 3 , pp. 349-384.

Kaplan, D. (2009a), "Advances in Multilevel Latent Variable Models for PISA Data », dossier présenté lors de la conférence PISA, Kiel, Allemagne, 14-16 septembre, http://www.pisaresconf09.org/user_uploads/files/methodology/room 1/Kaplan.pdf.

Kaplan, D. (2009b), "Causal Inference in Non-Experimental Educational Policy Research », in G. Sykes, B. Schneider et D.N. Plank (éd.), Handbook on Education Policy Research, Taylor and Francis, New York, pp. 139-153.

Kaplan, D. (2009c), Structural Equation Modeling: Foundations and Extensions, 2e édition, Sage Publications, Newbury Park, Californie. 
Kaplan, D., J-S. Kim et S-Y. Kim (2009), " Multilevel Latent Variable Modeling: Current Research and Recent Developments », in R.E. Millsap (éd.), The SAGE Handbook of Quantitative Methods in Psychology, pp. 592-613.

Kearney, C.A. (2008), "School Absenteeism and School Refusal Behavior in Youth: A Contemporary Review ", Clinical Psychology Review, vol. 28, pp. 451-471.

Klieme, E., C. Pauli et K. Reusser (2009), « The Pythagoras Study: Investigating Effects of Teaching and Learning in Swiss and German Classrooms ", in T. Janik et T. Seidel (éd.), The Power of Video Studies in Investigating Teaching and Learning in the Classroom, Waxmann Verlag, Münster, pp. 137-160.

Klieme, E., G. Schümer et S. Knoll (2001), " Mathematikunterricht in der Sekundarstufe I: Aufgabenkultur und Unterrichtsgestaltung ", in Bundesministerium für Bildung und Forschung (BMBF) (éd.), TIMSS-Impulse für Schule und Unterricht. Forschungsbefunde, Reforminitiativen, Praxisberichte und Video-Dokumente, BMBF, Bonn, pp. 43-57.

Klieme, E. et K. Rakoczy (2003), « Unterrichtsqualität aus Schülerperspektive», in J. Baumert et al. (éd.), PISA 2000: Ein differenzierter Blick auf die Länder der Bundesrepublik Deutschland, Leske und Budrich, Opladen, pp. 333-359.

Kobarg, M., T. Seidel, M. Prenzel, B. McCrae et M. Walker (2009), "Patterns of Science Teaching and Learning in an International Comparison ", dossier présenté lors de la conférence PISA, Kiel, Allemagne, 14-16 septembre http://www.pisaresconf09.org/user_ uploads/files/context/room3/Kobarg_etal.pdf.

Koretz, D. (2008), What Educational Testing Really Tells Us, Harvard University Press, Cambridge, Massachusetts.

Krahn, H. et A. Taylor (2005), "Resilient Teenagers: Explaining the High Educational Aspirations of Visible Minority Immigrant Youth in Canada », Journal of International Migration and Integration, vol. 6, n 3/4, pp. 405-434.

Krüger, M.L., B. Witziers et P.J.C. Sleegers (2007), «The Impact of School Leadership on School Level Factors: Validation of a Causal Model ", School Effectiveness and School Improvement, vol. 18, n 1, pp. 1-20.

Kunter, M. (2005), Multiple Ziele im Mathematikunterricht, Waxmann, Münster.

Kunter, M., Y.-M. Tsai, U. Klusmann, M. Brunner, S. Krauss et J. Baumert (2008), " Students' and Mathematics Teachers' Perception of Teacher Enthusiasm and Instruction», Learning and Instruction, vol. 18, pp. 468-482.

Kyriakides, L. et N. Tsangaridou (2004), «School Effectiveness and Teacher Effectiveness in Physical Education », dossier présenté lors de la $85^{\mathrm{e}}$ conférence de l'American Educational Research Association, Chicago, Illinois.

Lee, J. (2009), « Universals and Specifics of Math Self-Concept, Math Self-Efficacy, and Math Anxiety across 41 PISA 2003 Participating Countries », Learning and Individual Differences, vol. 19, n³, pp. 355-365.

Lee, V.E. et D.T. Burkham (2003), "Dropping out of High-School: The Role of School-Organization and Structure », American Educational Research Journal, vol. 40, n², pp. 353-393.

Lee, V.E. et J.B. Smith (1997), " High School Size: Which Works Best and for Whom? », Educational Evaluation and Policy Analysis, vol. 19, pp. 205-227.

Leithwood, K. et C. Riehl (2005), "What we Know about Successful School Leadership », in W. Firestone et C. Riehl (éd.), A New Agenda: Directions for Research on Educational Leadership, Teachers College Press, New York, pp. 22-47.

Levin, H. (1978), "The Dilemma of Comprehensive Secondary School Reforms in Western Europe ", Comparative Education Review, vol. 22, pp. 434-451.

Libman, Z. (2009), "Teacher Licensing Examinations - True Progress or Illusion? », Studies in Educational Evaluation, vol. 35, n 1, pp. 7-15.

Lie, S. et M. Kjaernsli (2009), "Predictors for Students' Choice of Science Careers », dossier présenté lors de la conférence PISA, Kiel, Allemagne, 14-16 septembre, http://www.pisaresconf09.org/user_uploads/files/context/room3/Lie_Kjaernsli.pdf.

Liebkind, K. (1996), "Acculturation and Stress: Vietnamese Refugees in Finland », Journal of Cross-Cultural Psychology, vol. 27, pp. 161-180.

Lipnevich, A.A., C. MacCann, S. Krumm, J. Burrus et R.D. Roberts (à paraître), " Math Attitudes in Belarusian and US Middle School Students ", Journal of Educational Psychology.

Lipowsky, F., K. Rakoczy, C. Pauli, B. Drollinger-Vetter, E. Klieme et K. Reusser. (2009), « Quality of Geometry Instruction and its ShortTerm Impact on Students' Understanding of the Pythagorean Theorem », Learning and Instruction, vol. 19, pp. 527-537.

Looker, E.D. et V. Thiessen (2008), Le système de la seconde chance : Résultats des trois cycles de l'Enquête auprès des jeunes en transition, Direction de la politique sur I'apprentissage, Politique stratégique et de recherche, Québec, http://www.rhdcc.gc.ca/fra/ publications_ressources/politique_sur_apprentissage/sp-836-04-08f/sp_836_04_08f.pdf.

Loveless, T. (2009), " How Well are American Students Learning? With Sections on International Assessments, the Misplaced Math Student, and Urban Schools ", in The 2008 Brown Center Report on American Education, Brown Center on Education Policy, Brookings Institute, Washington DC. 
Lüdtke, O., H.W. Marsh, A. Robitzsch, U. Trautwein, T. Asparouhov et B. Muthén (2008), " The Multilevel Latent Covariate Model: A New, more Reliable Approach to Group-Level Effects in Contextual Studies », Psychological Methods, vol. 13, pp. 203-229.

Ma, X. (1999), "Dropping Out of Advanced Mathematics: The Effects of Parental Involvement », Teachers College Record, vol. 101, pp. 60-81.

Ma, X. et N. Kishor (1997), "Assessing the Relationship between Attitude toward Mathematics and Achievement in Mathematics: A Meta-Analysis », Journal for Research in Mathematics Education, vol. 28, n 1, pp. 26-47.

Mau, W-C. et L. Heim Bikos (2000), «Educational and Vocational Aspirations of Minority and Female Students: A Longitudinal Study », Journal of Counseling and Development, vol. 78, n² 2, pp. 186-194.

McCluskey, C.P., T.S. Bynum et J.W. Patchin (2004), « Reducing Chronic Absenteeism: An Assessment of an Early Truancy Initiative », Crime and Delinquency, vol. 50, $\mathrm{n}^{\circ} 2$, pp. 214.34.

McComb, E.M. et C. Scott-Little (2003), A Review of Research on Participant Outcomes in After-School Programs: Implications for School Counselors, ERIC Digest, ERIC Clearinghouse on Counseling and Student Services, ED482765, Greensboro, Caroline du Nord.

Monk, D.H. (1994), "Subject Matter Preparation of Secondary Mathematics and Science Teachers and Student Achievement ", Economics of Education Review, vol. 13, n² 2, pp. 125-145.

Mullis, I.V.S. et M.O. Martin (2007), " TIMSS in Perspective: Lessons Learned from IEA's Four Decades of International Mathematics Assessments ", in T. Loveless (éd.), Lessons Learned. What International Assessments Tell Us about Math Achievement, Brookings, Washington, pp. 9-36.

Muthén, B. (2002), « Beyond SEM: General Latent Variable Modeling », Behaviormetrika, vol. 29, pp. 81-117.

National Council of Teachers for Mathematics (NCTM) (1989), Curriculum and Evaluation Standards for School Mathematics, NCTM, Reston, Virginie.

Niss, M. (2003), "Mathematical Competencies and the Learning of Mathematics: The Danish KOM Project ", in A. Gagatsis et S. Papastavridis (éd.), $3^{\text {rd }}$ Mediterranean Conference on Mathematical Education, 3-5 janvier 2003, The Hellenic Mathematical Society, Athènes, Grèce, pp. 115-124.

Oakes, J. (2005), Keeping Track: How Schools Structure Inequality (2 éd.), Yale University, New Haven, Connecticut.

Opdenakker, M-C. et J. van Damme (2000), "Effects of Schools, Teaching Staff and Classes on Achievement and Well-Being in Secondary Education: Similarities and Differences Between School Outcomes », School Effectiveness and School Improvement, vol. 11, no 2, pp. 165-196.

OCDE (2001), Connaisssances et compétences : des atouts pour la vie - Premiers résultats de PISA 2000, PISA, Éditions OCDE.

OCDE (2004), Apprendre aujourd'hui, réussir demain - Premiers résultats de PISA 2003, PISA, Éditions OCDE.

OCDE (2005a), School Factors Related to Quality and Equity: Results from PISA 2000, PISA, Éditions OCDE.

OCDE (2005b), PISA 2003 Technical Report, PISA, Éditions OCDE.

OCDE (2007a), Regards sur l'éducation : Les indicateurs de l'OCDE, Éditions OCDE.

OCDE (2007b), PISA 2006 : Les compétences scientifiques : un atout pour l'avenir, volume l, Analyse des résultats, PISA, Éditions OCDE.

OCDE (2008), Regards sur l'éducation : Les indicateurs de l'OCDE, Éditions OCDE.

OCDE (2009a), Regards sur l'éducation : Les indicateurs de l'OCDE, Éditions OCDE.

OCDE (2009b), Creating Effective Teaching and Learning Environments: First Results from TALIS, Éditions OCDE.

OCDE (2010), Résultats du PISA 2009, Volumes I, II, II, IV, V et VI, PISA, Éditions OCDE.

OCDE (2011), Regards sur l'éducation : Les indicateurs de l'OCDE, Éditions OCDE.

OCDE (2012a), Regards sur l'éducation : Les indicateurs de l'OCDE, Éditions OCDE.

OCDE (2012b), Untapped Skills: Realising the Potential of Immigrant Students, PISA, Éditions OCDE.

OCDE (2012c), Public and Private Schools: How Management and Funding Relate to their Socio-Economic Profile, PISA, Éditions OCDE.

OCDE (2012d), Lisons-leur une histoire! Le facteur parental dans l'éducation, PISA, Éditions OCDE.

Pajares, F. et M.D. Miller (1997), " Mathematics Self-Efficacy and Mathematical Problem Solving: Implications of Using Different Forms of Assessment », The Journal of Experimental Education, vol. 65, pp. 213-228.

Perreault, S. et R.Y. Bourhis (1999), "Ethnocentrism, Social Identification and Discrimination », Personality and Social Psychology Bulletin, vol. 25, pp. 92-103. 
Prenzel, M., J. Baumert, W. Blum, R. Lehmann, D. Leutner, M. Neubrand, R. Pekrun, J. Rost et U. Schiefele (éd.) (2006), PISA 2003 : Untersuchungen zur Kompetenzentwicklung im Verlauf eines Schuljahres, Waxmann Verlag, Münster.

Purves, A.C. (1987), « The Evolution of the IEA: A Memoir », Comparative Education Review, vol. 31, no 1, pp. 10-28.

Rakoczy, K., A. Buff et F. Lipowsky (2005), «Teil 1: Befragungsinstrumente "_, in E. Klieme et al. (éd.), Dokumentation der Erhebungsund Auswertungsinstrumente zur schweizerisch-deutschen Videostudie Unterrichtsqualität, Lernverhalten und mathematisches Verständnis, GFPF, Francfort.

Rakoczy, K., E. Klieme et C. Pauli (2008), « Die Bedeutung der wahrgenommenen Unterstützung motivationsrelevanter Bedürfnisse und des Alltagsbezugs im Mathematikunterricht für die selbstbestimmte Motivation », Zeitschrift für Pädagogische Psychologie, vol. 22, $\mathrm{n}^{\circ} 1$, pp. 25-35.

Raudenbush, S.W. et A.S. Bryk (2002), Hierarchical Linear Models: Applications and Data Analysis Methods, Sage Publications, Thousand Oaks, Californie.

Rost, J., C.H. Carstensen et M. von Davier (1997), " Applying the Mixed Rasch Model to Personality Questionnaires », in J. Rost et R. Langeheine (éd.), Applications of Latent Trait and Latent Class Models in the Social Sciences, Waxmann, New York, pp. $324-332$.

Rowan, B., E. Camburn et R. Correnti (2004), « Using Teacher Logs to Measure the Enacted Curriculum in Large-Scale Surveys: Insights from the Study of Instructional Improvement », Elementary School Journal, vol. 105, pp. 75-102.

Ryan, R.M. et E.L. Deci (2000), «Intrinsic and Extrinsic Motivations: Classic Definitions and New Directions », Contemporary Educational Psychology, vol. 25, pp. 54-67.

Rychen, D.S. et L.H. Salganik (éd.) (2003), Key Competencies for a Successful Life and a Well-Functioning Society, Hogrefe and Huber Publishers, Göttingen.

Scheerens, J. (2000), «Improving School Effectiveness », série Principes de la planification de l'éducation, IIEP, vol. 68, UNESCO, Paris.

Scheerens, J. et R.J. Bosker (1997), The Foundations of Educational Effectiveness, Pergamon, Oxford.

Schmid, C.L. (2001), "Educational Achievement, Language-Minority Students, and the New Second Generation », Sociology of Education (hors série), pp. 71-87.

Schmidt, W.H. et C.C. McKnight (1998), "What Can We Really Learn from TIMSS? », Science, vol. 282, pp. 1831-1839.

Schmidt, W.H., C.C. McKnight, R.T. Houang, H.C. Wang, D.E. Wiley, L.S. Cogan et R.G. Wolfe (2001), Why Schools Matter: A CrossNational Comparison of Curriculum and Learning, Jossey Bass, San Francisco, Californie.

Schmidt, W.H. et R.T. Houang (2007), "Lack of Focus in the Mathematics Curriculum: Symptom or Cause ", in T. Loveless (éd.), Lessons Learned: What International Assessments Tell Us about Math Achievement, Brookings, Washington, pp. 65-84.

Schmidt, W.H. et A. Maier (2009), "Opportunity to Learn», in G. Sykes, B. Schneider et D.N. Plank (éd.), Handbook of Education Policy Research, Routledge, New York, pp. 541-559.

Schmidt, W.H. et C. McKnight (1995), "Surveying Educational Opportunity in Mathematics and Science: An International Perspective », Educational Evaluation and Policy Analysis, vol. 17, n 3, pp. 337-353.

Schneider, W. et C. Artelt (à paraître), "Metacognition and Mathematics Education », The International Journal on Mathematics Education, ZDM.

Schoenfeld, A.H. (éd.) (1987), Cognitive Science and Mathematics Education, Erlbaum, Hillsdale, New Jersey.

Schoenfeld, A.H. (1992), "Learning to Think Mathematically: Problem Solving, Metacognition, and Sense-Making in Mathematics », in D. Grouws (éd.), Handbook for Research on Mathematics Teaching and Learning, MacMillan, New York, pp. 334-370.

Schoenfeld, A.H. (2006), « Mathematics Teaching and Learning », in P.A. Alexander et I.H. Winne (éd.), Second Handbook of Educational Psychology, Routledge, Londres.

Shulman, L. (1987), « Knowledge and Teaching: Foundations of the New Reform », Harvard Educational Review, vol. 57, n 1, pp. 1-22.

Slavin, R.E. (1990), "Achievement Effects of Ability Grouping in Secondary Schools: A Best-Evidence Synthesis ", Review of Educational Research, vol. 60, n³, pp. 471-499.

Snijders, T. et R. Bosker (1999), Multilevel Analysis, Sage Publications, Londres.

Stanat, P. (2006), " Schulleistungen von Jugendlichen mit Migrationshintergrund: Die Rolle der Zusammensetzung der Schülerschaft ", in J. Baumert, P. Stanat et R. Watermann (éd.), Herkunftsbedingte Disparitäten im Bildungswesen: Differenzielle Bildungsprozesse und Probleme der Verteilungsgerechtigkeit; vertiefende Analysen im Rahmen von PISA 2000, VS Verl. für Sozialwiss, Wiesbaden, pp. 189-219.

Stanat, P. et G. Christensen, (2006), Where Immigrant Students Succeed - A Comparative Review of Performance and Engagement in PISA 2003, Éditions OCDE. 
Stein, M.K., R.A. Engle, M.S. Smith et E.K. Hughes (2008), " Orchestrating Productive Mathematical Discussions: Five Practices for Helping Teachers Move Beyond Show and Tell », Mathematical Thinking and Learning, vol. 10, pp. 315.

Stevens, F. (1993), « Applying an Opportunity-to-Learn Conceptual Framework to the Investigation of the Effects of Teaching Practices via Secondary Analyses of Multiple-Case-Study Summary Data », Journal of Negro Education, vol. 62, n 3, pp. 232-248.

Stigler, J.W. et J. Hiebert (1999), The Teaching Gap: Best Ideas from the World's Teachers for Improving Education in the Classroom, Free Press, New York.

Sui-Chu, H. et J.D. Willms (1996), «Effects of Parental Involvement on Eighth-Grade Achievement », Sociology of Education, vol. 69, pp. 126-141.

Teddlie, C. et D. Reynolds (éd.) (2000), The International Handbook of School Effectiveness Research, Routledge, New York.

Thiessen, V. (2007), The Impact of Factors on Trajectories that Lead to a High School Diploma and to Participation in Post Secondary Education, Ressources humaines et Développement des compétences, Canada, Québec.

Turner, S.L., J.C. Steward et R.T. Lapan (2004), « Family Factors Associated with Sixth Grade Adolescents' Math and Science Career Interests », Career Development Quarterly, vol. 53, n 1, pp. 41-52.

Van de Gaer, E. et R. Adams (2010), "The Modeling of Response Style Bias: An Answer to the Attitude-Achievement Paradox? », dossier présenté à la conférence annuelle de I'American Educational Research Association, Denver, Colorado, 30 avril-4 mai.

Van de Vijver, F.J.R., D.A. Van Hemert et Y.H. Poortinga (éd.) (2008), Multilevel Analysis of Individuals and Cultures, Erlbaum, New York.

Van Landeghem, G., B. De Fraine et J. Van Damme (2005), « The Consequence of Ignoring a Level of Nesting in Multilevel Analysis: A Comment », Multivariate Behavioral Multivariate Behavioral Research, vol. 40, pp. 423-434.

Vieluf, S., J. Lee et P. Kyllonen (2009a), "The Predictive Power of Variables from the PISA 2003 Student Questionnaire ", dossier présenté lors de la réunion du groupe d'experts en charge des questionnaires, Offenbach, Allemagne, 19-21 octobre.

Vieluf, S., J. Lee et P. Kyllonen (2009b), "The Cross-Cultural Validity of Variables from the PISA 2003 Student Questionnaire », dossier présenté lors de la réunion du groupe d'experts en charge des questionnaires, Offenbach, Allemagne, 19-21 octobre.

Walberg, H.J. (1986), "Syntheses of Research on Teaching », in M.C. Wittrock (éd.), Handbook of Research on Teaching (3e édition), Macmillan, New York, pp. 214-229.

Wang, M.C., G.D. Haertel et H.D. Walberg (1993), "Toward a Knowledge Base for School Learning ", Review of Educational Research, vol. $63, \mathrm{n}^{\circ}$ 3, pp. $249-294$.

Wayne, A. et P. Youngs (2003), "Teacher Characteristics and Student Achievement Gain: A Review », Review of Educational Research, vol. 73, pp. 89-122.

Wigfield, A., J.S. Eccles et D. Rodriguez (1998), "The Development of Children's Motivation in School Contexts », in A. Iran-Nejad et P.D. Pearson (éd.), Review of Research in Education, American Educational Research Association, vol. 23, Washington DC.

Wilmers, N., D. Enzmann, D. Schaefer, K. Herbers, W. Greve et P. Wetzels (2002), Jugendliche in Deutschland zur Jahrtausendwende: Gefährlich oder gefährdet? Ergebnisse wiederholter, repräsentativer Dunkelfelduntersuchungen zu Gewalt und Kriminalität im Leben junger Menschen 1998 - 2000, Nomos Verlagsgesellschaft, Baden-Baden, Allemagne.

Witziers, B., R.J. Bosker et M.L. Krüger (2003), «Educational Leadership and Student Achievement: The Elusive Search for an Association », Educational Administrative Quarterly, vol. 39, n 3, pp. 398-425.

Woessmann, L. (2006), "The Complementarity of Central Exams and School Autonomy: Economic Theory and International Evidence ", in E. Gori, D. Vidoni, E. Hanushek et C. Glenn (éd.), Institutional Models in Education: Legal Framework and Methodological Aspects for a New Approach to the Problem of School Governance, Wolf Legal Publishers, Nimègue, pp. 47-65.

Yasushi, O. (2009), "Comparison of Attitudes toward Science between Grade 9 and 10 Japanese Students by Using the PISA Questions and Its Implications on Science Teaching in Japan », dossier présenté lors de la réunion du groupe d'experts en charge des questionnaires, Offenbach, Allemagne, 19-21 octobre.

Zoido, P. et T. Gluszynski (2009), " Pathways to Success: How Knowledge and Skills at Age 15 Shape Future Life Outcome », dossier présenté à la conférence PISA, Kiel, Allemagne, 14-16 septembre.

Zuzovsky, R. (2009), "Teachers' Qualifications and Their Impact on Student Achievement: Findings from TIMSS 2003 Data for Israel », in M. von Davier et D. Hastedt (éd.), IERI Monograph Series Issues and Methodologies in Large-Scale Assessments, IER Institute, Hambourg/Princeton, Allemagne/États-Unis, vol. 2, pp. 37-62. 


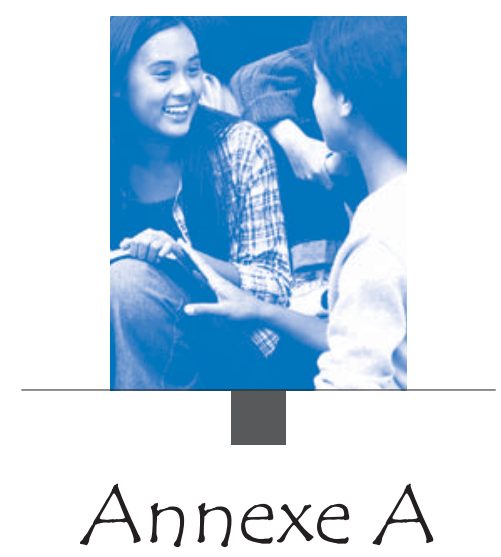

\section{QUESTIONNAIRES CONTEXTUELS}

L'annexe A reprend les questionnaires contextuels administrés lors du cycle PISA 2012. Ces questionnaires ont pour but de recueillir des informations sur les établissements d'enseignement, les élèves et leurs parents.

Le questionnaire "Établissement ", qui a été administré aux chefs d'établissement, porte sur : la structure et l'organisation de l'établissement d'enseignement ; ses élèves et ses enseignants ; ses ressources ; son enseignement, ses programmes de cours et ses pratiques d'évaluation ; son climat; ses stratégies et pratiques pédagogiques ; et ses programmes d'éducation financière. Une question supplémentaire est incluse dans le questionnaire «Établissement » informatisé.

Le questionnaire "Élève », qui a été administré aux élèves, porte sur : leurs caractéristiques et leur parcours scolaire ; le contexte et les ressources de leur famille; I'apprentissage des mathématiques; leur expérience de divers types de problèmes mathématiques à l'école ; leur expérience des mathématiques ; le climat dans leur classe et leur établissement d'enseignement ; et leur expérience en matière de résolution de problèmes.

Deux questionnaires facultatifs ont été administrés aux élèves : un questionnaire sur leur parcours scolaire qui concerne leurs antécédents scolaires, leurs ambitions professionnelles et leur apprentissage linguistique ; et un questionnaire sur leur accès aux technologies de l'information et de la communication (TIC), I'usage qu'ils en font et leurs attitudes à l'égard de l'informatique.

Un troisième questionnaire facultatif a été administré aux parents. Ce questionnaire porte sur : leur profil personnel ; le coût des services d'éducation ; leurs attitudes à l'égard de l'établissement d'enseignement de leur enfant ; leur implication dans la vie de l'établissement d'enseignement; le choix de l'établissement d'enseignement ; le soutien à l'apprentissage de leur enfant à la maison ; la place des mathématiques dans les perspectives professionnelles de leur enfant ; leurs attentes scolaires et professionnelles en mathématiques pour leur enfant; les résultats scolaires de leur enfant; les intérêts professionnels de leur enfant ; et leur ascendance autochtone ou allochtone. 


\section{QUESTIONNAIRE “ ÉTABLISSEMENT "}

Le questionnaire «Établissement » est administré au chef d'établissement. Il faut environ 30 minutes pour le remplir. II aborde différents aspects en rapport avec l'établissement d'enseignement :

- la structure et l'organisation de l'établissement ;

- les élèves et le corps enseignant ;

- les ressources de l'établissement ;

- I'enseignement, le programme des cours et les pratiques d'évaluation de l'établissement ;

- le climat de l'établissement ;

- les stratégies et pratiques de l'établissement ;

- I'éducation financière ; et

- une question supplémentaire pour le questionnaire «Établissement » informatisé.

Les termes techniques indiqués entre <crochets> sont adaptés au contexte national par le centre national de collecte de données des pays ou économies participants. Dans cette annexe, ces termes techniques sont expliqués sous les items.

\section{Structure et organisation de l'établissement}

Q1 Votre établissement est-il un établissement public ou privé ?

(SC01) (Ne cochez qu'une seule case.)

Un établissement public.

(Il s'agit dans ce cas d'un établissement relevant et dépendant directement ou indirectement d'instances publiques en charge de l'éducation, d'un organisme gouvernemental ou dont la direction est désignée/nommée par les pouvoirs publics.)

Un établissement privé.

(Il s'agit dans ce cas d'un établissement relevant et dépendant directement ou indirectement d'un organisme non gouvernemental, par exemple une congrégation religieuse, un syndicat, une entreprise ou toute autre institution privée.)

Au cours d'une année scolaire ordinaire, quel est le pourcentage approximatif de votre budget total qui provient des sources suivantes?

(Inscrivez un nombre par ligne. Si une des sources ne contribue pas à votre budget, inscrivez 0 (zéro) sur la ligne correspondante.)

\begin{tabular}{|c|c|c|}
\hline & & $\%$ \\
\hline a) & Des pouvoirs publics (qu'ils soient municipaux, départementaux, régionaux, nationaux ou fédéraux). & \\
\hline b) & Des droits d'inscription et frais scolaires payés par les parents. & \\
\hline c) & De dons, de legs, de parrainages, de collectes de fonds réalisées par les parents. & \\
\hline \multirow[t]{2}{*}{ d) } & Autres sources. & \\
\hline & Total & $100 \%$ \\
\hline
\end{tabular}

Q3 Comment peut-on le mieux définir la collectivité dans laquelle se situe votre établissement ?

(SC03) (Ne cochez qu'une seule case.)

\begin{tabular}{ll|l} 
Un village, hameau ou collectivité rurale (moins de 3000 habitants). & $\square$ \\
\hline Un bourg (de 3000 à 15000 habitants environ). & $\square$ \\
\hline Une petite ville (de 15000 à 100000 habitants environ). & $\square=$ \\
\hline Une ville (de 100000 à un million d'habitants environ). & $\square$. \\
\hline
\end{tabular}


Une grande agglomération de plus d'un million d'habitants.

Nous souhaiterions connaître les possibilités qui s'offrent aux parents lorsqu'ils choisissent une école pour leur enfant.

Parmi les affirmations suivantes, laquelle décrit le mieux les choix possibles dans la localité où se trouve votre établissement?

(Ne cochez qu'une seule case.)

Il y a dans cette localité deux autres établissements, ou davantage, qui sont en concurrence avec notre établissement pour le recrutement des élèves.

Il y a dans cette localité un autre établissement qui est en concurrence avec notre établissement pour le recrutement des élèves.

Il n’y a dans cette localité aucun autre établissement en concurrence avec notre établissement pour

le recrutement des élèves. de <grade modal du pays pour les élèves de 15 ans> ?

(Ne cochez qu'une seule case.)

\begin{tabular}{l|c}
\hline 15 élèves ou moins. & $\square_{1}$ \\
\hline De 16 à 20 élèves. & $\square_{2}$ \\
\hline De 21 à 25 élèves. & $\square_{3}$ \\
\hline De 26 à 30 élèves. & $\square_{4}$ \\
\hline De 31 à 35 élèves. & $\square_{5}$ \\
\hline De 36 à 40 élèves. & $\square_{6}$ \\
\hline De 41 à 45 élèves. & $\square_{7}$ \\
\hline De 46 à 50 élèves. & $\square_{8}$ \\
\hline Plus de 50 élèves. & $\square_{9}$ \\
\hline
\end{tabular}

Notes : < Grade modal du pays pour les élèves de $\mathbf{1 5}$ ans $>$ - Ce terme fait référence au nom de l'année d'études fréquentée par la majorité des élèves de 15 ans dans le pays ou l'économie participant.

$<$ Langue de l'évaluation $>$ - Ce terme désigne la langue dans laquelle le test de culture mathématique est administré.

\section{Élèves et enseignants de l'établissement}

Q6 Le <jour, mois 2012>, quel était le nombre total d'élèves inscrits dans votre établissement ?

(SC07) (Écrivez un nombre par ligne. Indiquez 0 (zéro) s'il n'y en a aucun.)

a) Nombre de garçons.

b) Nombre de filles.

Note : <Jour, mois 2012>> - La date à utiliser doit se situer un mois environ avant le début de la campagne définitive. 

consacre au moins $90 \%$ d'un emploi du temps complet à l'enseignement en salle de classe, et ce tout au long de l'année scolaire. Tous les autres enseignants doivent être considérés "à temps partiel ».

(Écrivez un nombre dans chaque espace disponible. Indiquez 0 (zéro) s'il n'y en a pas.)

\begin{tabular}{c|l|c|c}
\multicolumn{2}{l|}{} & $\begin{array}{c}\text { À temps } \\
\text { plein }\end{array}$ & $\begin{array}{c}\text { À temps } \\
\text { partiel }\end{array}$ \\
\hline a) & Enseignants au TOTAL. & & \\
\hline b) & $\begin{array}{l}\text { Enseignants ayant des titres donnant accès à la fonction d'enseignant qui sont pleinement } \\
\text { reconnus par <l'autorité de tutelle>. }\end{array}$ & & \\
\hline c) & Enseignants possédant un diplôme de <niveau CITE 5A>. & & \\
\hline
\end{tabular}

Notes : < Autorité de tutelle> - L'instance officielle habilitée à certifier qu'une personne a les titres requis pour exercer la profession d'enseignant dans un établissement d'enseignement.

$<$ Niveau CITE 5A > - Diplôme obtenu à l'issue d'un programme d'études tertiaires caractérisé par une base théorique importante, d'une durée habituellement équivalente à au moins trois années d'études à temps complet, donnant accès à des professions demandant un niveau élevé de qualification ou à des programmes de recherche de haut niveau.

Dans votre établissement, combien de <professeurs de mathématiques> y a-t-il dans chacune des catégories suivantes?

Comptez séparément les enseignants à temps partiel et à temps plein. Un enseignant est considéré "à temps plein » s'il consacre au moins $90 \%$ d'un emploi du temps complet à l'enseignement en salle de classe, et ce tout au long de l'année scolaire. Tous les autres enseignants doivent être considérés "à temps partiel ».

Ne prenez en compte que les membres du personnel qui ont enseigné ou enseigneront les mathématiques durant l'année scolaire en cours.

(Écrivez un nombre dans chaque espace disponible. Indiquez 0 (zéro) s'il n'y en a pas.)

\begin{tabular}{|c|c|c|c|}
\hline & & $\begin{array}{l}\text { À temps } \\
\text { plein }\end{array}$ & $\begin{array}{c}\text { À temps } \\
\text { partiel }\end{array}$ \\
\hline a) & Professeurs de mathématiques au TOTAL. & & \\
\hline b) & Professeurs de mathématiques possédant un diplôme $<$ de niveau CITE 5A $>$. & & \\
\hline c) & $\begin{array}{l}\text { Professeurs de mathématiques possédant un diplôme }<\text { de niveau CITE } 5 \text { A }><\text { avec une } \\
\text { spécialisation }>\text { en mathématiques. }\end{array}$ & & \\
\hline d) & Professeurs de mathématiques possédant un diplôme $<$ de niveau CITE 5A $>$ en $<$ pédagogie $>$. & & \\
\hline e) & $\begin{array}{l}\text { Professeurs de mathématiques possédant un diplôme }<\text { de niveau CITE } 5 B>\text {, mais n'ayant pas } \\
\text { de diplôme }<\text { de niveau CITE } 5 \mathrm{~A}>\text {. }\end{array}$ & & \\
\hline
\end{tabular}

Notes : Veuillez vous référer à la Question 7 pour une définition du $<$ niveau CITE 5A $>$.

$<$ Niveau CITE 5B > - Diplôme obtenu à l'issue d'un programme d'études tertiaires caractérisé par une orientation plus pratique/technique/ professionnelle et par une durée moins longue que les programmes CITE 5A. Ces programmes ont habituellement une durée équivalente à deux années d'études à temps complet au minimum, et préparent leurs étudiants à une profession spécifique.

La <spécialisation> correspond ici à la notion anglo-saxonne de " major » (la matière principale dans un cycle d'études universitaires menant à un diplôme de niveau CITE 5A). Une spécialisation en mathématiques est un cycle complet de mathématiques dans le cadre d'études sanctionnées par un diplôme de niveau CITE 5A. 


\section{Ressources de l'établissement}

L'objectif des trois questions ci-dessous est d'estimer le nombre d'élèves de <grade modal du pays pour les élèves de 15 ans> par ordinateur dans votre établissement.

Q9a Dans votre établissement, quel est le nombre total d'élèves de < grade modal du pays

(SC11) pour les élèves de 15 ans>?

Q9b Quel est le nombre approximatif d'ordinateurs qui sont mis à la disposition de ces élèves

(SC11) à des fins d'enseignement ?

Q9c Quel est le nombre approximatif de ces ordinateurs qui sont connectés à Internet/

(SC11) au réseau web mondial ?

Note : Veuillez vous référer à la Question 5 pour une définition du <grade modal du pays pour les élèves de 15 ans>.

Q10

(SC13)

Dans votre établissement, pour quelle proportion du travail scolaire, toutes matières confondues, les élèves de <grade modal du pays pour les élèves de 15 ans> sont-ils censés consulter Internet/ le réseau web mondial ?

(Cochez une case par ligne.)

\begin{tabular}{c|l|c|c|c|c|c}
\multicolumn{2}{l|}{} & $<\mathbf{1 0} \%$ & $\mathbf{1 0 - 2 5} \%$ & $\mathbf{2 6 - 5 0} \%$ & $\mathbf{5 1 - 7 5 \%}$ & $>\mathbf{7 5} \%$ \\
\hline a) & Travail pendant les heures de cours. & $\square_{1}$ & $\square_{2}$ & $\square_{3}$ & $\square_{4}$ & $\square_{5}$ \\
\hline b) & Devoirs à la maison. & $\square_{1}$ & $\square_{2}$ & $\square_{3}$ & $\square_{4}$ & $\square_{5}$ \\
\hline c) & Travaux ou recherches. & $\square_{1}$ & $\square_{2}$ & $\square_{3}$ & $\square_{4}$ & $\square_{5}$ \\
\hline
\end{tabular}

Note : Veuillez vous référer à la Question 5 pour une définition du < grade modal du pays pour les élèves de 15 ans>

Q11 L'enseignement que votre établissement est à même de dispenser est-il affecté par les problèmes suivants ?

(SC14) (Cochez une case par ligne.)

\begin{tabular}{|c|c|c|c|c|c|}
\hline & & Pas du tout & Très peu & $\begin{array}{l}\text { Dans une } \\
\text { certaine } \\
\text { mesure }\end{array}$ & Beaucoup \\
\hline a) & Manque de professeurs de sciences qualifiés. & $\square_{1}$ & $\square_{2}$ & $\square_{3}$ & $\square_{4}$ \\
\hline b) & Manque de professeurs de mathématiques qualifiés. & $\square_{1}$ & $\square_{2}$ & $\square_{3}$ & $\square_{4}$ \\
\hline c) & Manque de professeurs de <langue de l'évaluation > qualifiés. & $\square_{1}$ & $\square_{2}$ & $\square_{3}$ & $\square_{4}$ \\
\hline d) & Manque de professeurs qualifiés dans d'autres matières. & $\square_{1}$ & $\square_{2}$ & $\square_{3}$ & $\square_{4}$ \\
\hline e) & $\begin{array}{l}\text { Pénurie ou inadéquation en matière d'équipement des laboratoires } \\
\text { de sciences. }\end{array}$ & $\square_{1}$ & $\square_{2}$ & $\square_{3}$ & $\square_{4}$ \\
\hline f) & $\begin{array}{l}\text { Pénurie ou inadéquation en matière de matériel didactique } \\
\text { (par ex., manuels scolaires). }\end{array}$ & $\square_{1}$ & $\square_{2}$ & $\square_{3}$ & $\square_{4}$ \\
\hline g) & $\begin{array}{l}\text { Pénurie ou inadéquation en matière d'ordinateurs pour le travail } \\
\text { en classe. }\end{array}$ & $\square_{1}$ & $\square_{2}$ & $\square_{3}$ & $\square_{4}$ \\
\hline h) & Pénurie ou inadéquation en matière de connectivité à Internet. & $\square_{1}$ & $\square_{2}$ & $\square_{3}$ & $\square_{4}$ \\
\hline i) & Pénurie ou inadéquation en matière de logiciels didactiques. & $\square_{1}$ & $\square_{2}$ & $\square_{3}$ & $\square_{4}$ \\
\hline j) & $\begin{array}{l}\text { Pénurie ou inadéquation en matière de ressources } \\
\text { de la bibliothèque. }\end{array}$ & $\square_{1}$ & $\square_{2}$ & $\square_{3}$ & $\square_{4}$ \\
\hline k) & Manque ou inadéquation en matière de locaux et terrains scolaires. & $\square_{1}$ & $\square$ प & $\square_{3}$ & $\square$ प \\
\hline I) & $\begin{array}{l}\text { Manque ou inadéquation en matière d'installations de chauffage/ } \\
\text { d'air conditionné/d'éclairage. }\end{array}$ & $\square_{1}$ & $\square_{2}$ & $\square_{3}$ & $\square_{4}$ \\
\hline m) & $\begin{array}{l}\text { Manque ou inadéquation en matière de locaux destinés } \\
\text { à l'enseignement (par ex., les classes). }\end{array}$ & $\square_{1}$ & $\square_{2}$ & $\square_{3}$ & $\square_{4}$ \\
\hline
\end{tabular}

Note : Veuillez vous référer à la Question 5 pour une définition de la <langue de l'évaluation>. 


\section{Enseignement, programme des cours et pratiques d'évaluation de l'établissement}

\begin{tabular}{|c|c|c|c|c|}
\hline \multirow{5}{*}{$\begin{array}{c}\text { Q12 } \\
(\mathrm{SC} 15)\end{array}$} & \multicolumn{4}{|c|}{$\begin{array}{l}\text { Certains établissements organisent l'enseignement de manière différente en fonction des niveaux de compétence } \\
\text { des élèves en mathématiques et de leur intérêt pour cette matière. }\end{array}$} \\
\hline & \multirow{2}{*}{\multicolumn{4}{|c|}{$\begin{array}{l}\text { Lesquelles des options suivantes décrivent-elles les pratiques mises en ouvre dans les classes } \\
\text { de mathématiques des élèves de < grade modal du pays pour les élèves de } 15 \text { ans }>\text { dans votre } \\
\text { établissement? }\end{array}$}} \\
\hline & & & & \\
\hline & \multicolumn{4}{|l|}{ (Cochez une case par ligne.) } \\
\hline & & $\begin{array}{l}\text { Dans } \\
\text { toutes les } \\
\text { classes }\end{array}$ & $\begin{array}{l}\text { Dans } \\
\text { certaines } \\
\text { classes }\end{array}$ & $\begin{array}{l}\text { Dans } \\
\text { aucune } \\
\text { classe }\end{array}$ \\
\hline a) & $\begin{array}{l}\text { Les diverses classes de mathématiques voient les mêmes contenus, } \\
\text { mais à des niveaux de difficulté différents. }\end{array}$ & $\square_{1}$ & $\square_{2}$ & $\square_{3}$ \\
\hline b) & $\begin{array}{l}\text { Les diverses classes voient des contenus mathématiques différents ou des } \\
\text { ensembles différents de points de matière, dont le niveau de difficulté est plus } \\
\text { ou moins élevé. }\end{array}$ & $\square_{1}$ & $\square_{2}$ & $\square_{3}$ \\
\hline c) & $\begin{array}{l}\text { Les élèves sont organisés en différents groupes de niveau au sein de leur classe } \\
\text { de mathématiques. }\end{array}$ & $\square_{1}$ & $\square_{2}$ & $\square_{3}$ \\
\hline d) & $\begin{array}{l}\text { Dans les classes de mathématiques, les professeurs utilisent une méthode } \\
\text { pédagogique qui convient à des élèves dont le niveau de compétence est } \\
\text { hétérogène (en d'autres termes, les élèves ne sont pas placés dans des groupes } \\
\text { de niveau). }\end{array}$ & $\square_{1}$ & $\square_{2}$ & $\square_{3}$ \\
\hline
\end{tabular}
du pays pour les élèves de 15 ans> <cette année scolaire-ci> ?

(Cochez une case par ligne.)

\begin{tabular}{|c|c|c|c|}
\hline & & Oui & Non \\
\hline a) & Fanfare, orchestre ou chorale. & $\square_{1}$ & $\square_{2}$ \\
\hline b) & Pièce de théâtre ou comédie musicale. & $\square_{1}$ & $\square_{2}$ \\
\hline c) & Rédaction de l'album-souvenir annuel, du journal ou de la revue de l'établissement. & $\square_{1}$ & $\square_{2}$ \\
\hline d) & Bénévolat ou activités humanitaires, par exemple, <exemples nationaux>. & $\square_{1}$ & $\square_{2}$ \\
\hline e) & Club de mathématiques. & $\square_{1}$ & $\square_{2}$ \\
\hline f) & Compétitions de mathématiques, par exemple, <exemples nationaux>. & $\square_{1}$ & $\square_{2}$ \\
\hline g) & Club d'échecs. & $\square_{1}$ & $\square_{2}$ \\
\hline h) & Club axé sur l'informatique/les technologies de l'information et de la communication (TIC). & $\square_{1}$ & $\square_{2}$ \\
\hline i) & Club artistique ou activités artistiques. & $\square_{1}$ & $\square_{2}$ \\
\hline j) & Équipe sportive ou activités sportives. & $\square_{1}$ & $\square_{2}$ \\
\hline k) & $<$ ltem national $>$. & $\square_{1}$ & $\square_{2}$ \\
\hline
\end{tabular}


Dans votre établissement, les résultats des évaluations des élèves de <grade modal du pays pour les élèves de $\mathbf{1 5}$ ans> sont-ils utilisés aux fins suivantes ?

(Cochez une case par ligne.)

\begin{tabular}{|c|c|c|c|}
\hline & & Oui & Non \\
\hline a) & Informer les parents des progrès de leur enfant. & $\square_{1}$ & $\square_{2}$ \\
\hline b) & Prendre des décisions relatives au redoublement ou au passage de classe des élèves. & $\square_{1}$ & $\square_{2}$ \\
\hline c) & Regrouper les élèves à des fins didactiques. & $\square_{1}$ & $\square_{2}$ \\
\hline d) & $\begin{array}{l}\text { Comparer les performances de l'établissement à celles du < district }>\text { ou aux performances } \\
<\text { nationales }>\text {. }\end{array}$ & $\square$ & $\square_{2}$ \\
\hline e) & Suivre les progrès de l'établissement d’année en année. & $\square_{1}$ & $\square_{2}$ \\
\hline f) & Juger de l'efficacité des enseignants. & $\square_{1}$ & $\square_{2}$ \\
\hline g) & Identifier des aspects de l'enseignement ou des programmes qui pourraient être améliorés. & $\square_{1}$ & $\square_{2}$ \\
\hline h) & Comparer l'établissement à d'autres établissements. & $\square_{1}$ & $\square_{2}$ \\
\hline
\end{tabular}

Notes : Veuillez vous référer à la Question 5 pour une définition du < grade modal du pays pour les élèves de 15 ans $>$.

Les performances $<$ du district $>$ ou les performances $<$ nationales $>-$ Se réfère à la comparaison avec une entité administrative plus grande ; il peut s'agir du district, de la région, de la province et/ou du pays dans son ensemble.

Dans votre établissement, les données relatives aux résultats scolaires des élèves sont-elles utilisées dans le cadre d'un des <mécanismes de pilotage> suivants?

$P a r$ "données relatives aux résultats scolaires", on entend notamment des données agrégées (les moyennes de résultats d'examen ou de notes, ou bien les taux de réussite), calculées à l'échelle de l'établissement ou d'un grade donné.

(Cochez une case par ligne.)

a) Les données relatives aux résultats scolaires sont rendues publiques (par exemple, dans les médias).

b) Les données relatives aux résultats scolaires font l'objet d'un suivi au cours du temps par une instance administrative.

\begin{tabular}{|l|l|}
\hline Oui & Non \\
\hline$\square_{1}$ & $\square_{2}$ \\
\hline$\square_{1}$ & $\square_{2}$ \\
\hline
\end{tabular}

Note : L'expression <mécanismes de pilotage> traduit ici le terme anglais accountability procedures et fait référence à l'utilisation régulière de statistiques sur les résultats scolaires des élèves, agrégées au niveau de l'établissement, en vue de rendre compte aux parents ou à des autorités externes des aspects qualitatifs du fonctionnement de l'établissement.

Votre établissement propose-t-il des cours de mathématiques en plus de ceux dispensés selon I'horaire ordinaire?

(Ne cochez qu'une seule case.)

\begin{tabular}{|l|c|l}
\hline Oui. & $\square_{1}$ & Passez à la question suivante \\
\hline Non. & $\square_{2}$ & Passez à la Question 18 \\
\hline
\end{tabular}

Q17 Quel est l'objectif de ces cours de mathématiques supplémentaires?

(SC21) (Ne cochez qu'une seule case.)

\begin{tabular}{|l|c}
\hline Cours de <perfectionnement en mathématiques> uniquement. & $\square_{1}$ \\
\hline Cours de <soutien en mathématiques $>$ uniquement. & $\square_{2}$ \\
\hline À la fois cours de < perfectionnement > et cours de <soutien en mathématiques $>$. & $\square_{3}$ \\
\hline Pas de différenciation fondée sur le niveau de performance préalable des élèves. & $\square_{4}$
\end{tabular}

Notes : "Cours de <perfectionnement en mathématiques> 》- Cours de mathématiques proposé en dehors de l'horaire de cours ordinaire et destiné habituellement à améliorer les performances des élèves les plus brillants.

"Cours de <soutien en mathématiques> »-Cours de mathématiques proposé en dehors de l'horaire de cours ordinaire et destiné à faciliter la remise à niveau d'élèves qui accusent un retard par rapport à leurs camarades. 


\section{Climat de l'établissement}

Q18 Dans votre établissement, dans quelle mesure l'apprentissage des élèves est-il gêné par les facteurs suivants ?

\begin{tabular}{|c|c|c|c|c|c|}
\hline & & Pas du tout & Très peu & $\begin{array}{l}\text { Dans une } \\
\text { certaine } \\
\text { mesure }\end{array}$ & Beaucoup \\
\hline a) & L'absentéisme des élèves. & $\square_{1}$ & $\square_{2}$ & $\square_{3}$ & $\square_{4}$ \\
\hline b) & Les élèves sèchent des cours. & $\square_{1}$ & $\square_{2}$ & $\square_{3}$ & $\square_{4}$ \\
\hline c) & Les élèves arrivent en retard en classe. & $\square_{1}$ & $\square_{2}$ & $\square_{3}$ & $\square_{4}$ \\
\hline d) & $\begin{array}{l}\text { Les élèves ne se présentent pas aux activités scolaires obligatoires } \\
\text { (par ex., une journée sportive) ou aux excursions. }\end{array}$ & $\square_{1}$ & $\square_{2}$ & $\square_{3}$ & $\square_{4}$ \\
\hline e) & Le manque de respect des élèves envers les enseignants. & $\square_{1}$ & $\square_{2}$ & $\square_{3}$ & $\square_{4}$ \\
\hline f) & Les élèves perturbent les cours. & $\square_{1}$ & $\square_{2}$ & $\square_{3}$ & $\square_{4}$ \\
\hline g) & La consommation d'alcool ou de substances illicites par les élèves. & $\square_{1}$ & $\square_{2}$ & $\square_{3}$ & $\square_{4}$ \\
\hline h) & Les élèves menacent ou brutalisent d'autres élèves. & $\square_{1}$ & $\square_{2}$ & $\square_{3}$ & $\square_{4}$ \\
\hline i) & $\begin{array}{l}\text { Les élèves ne sont pas encouragés à donner la pleine mesure de leurs } \\
\text { capacités. }\end{array}$ & $\square_{1}$ & $\square_{2}$ & $\square_{3}$ & $\square_{4}$ \\
\hline j) & Les relations médiocres entre élèves et enseignants. & $\square_{1}$ & $\square_{2}$ & $\square_{3}$ & $\square_{4}$ \\
\hline k) & $\begin{array}{l}\text { Les enseignants doivent donner cours à des élèves de niveaux de } \\
\text { compétences hétérogènes dans une même classe. }\end{array}$ & $\square_{1}$ & $\square_{2}$ & $\square_{3}$ & $\square_{4}$ \\
\hline l) & $\begin{array}{l}\text { Les enseignants doivent donner cours à des élèves d'origines } \\
\text { ethniques différentes (c'est-à-dire de langue et de culture différentes) } \\
\text { dans une même classe. }\end{array}$ & $\square_{1}$ & $\square_{2}$ & $\square_{3}$ & $\square_{4}$ \\
\hline m) & Les enseignants ont un niveau d'attentes trop bas à l'égard des élèves. & $\square_{1}$ & $\square_{2}$ & $\square_{3}$ & $\square_{4}$ \\
\hline n) & Les enseignants ne satisfont pas aux besoins individuels des élèves. & $\square_{1}$ & $\square_{2}$ & $\square_{3}$ & $\square_{4}$ \\
\hline o) & L'absentéisme des enseignants. & $\square_{1}$ & $\square_{2}$ & $\square_{3}$ & $\square_{4}$ \\
\hline p) & La résistance au changement du personnel. & $\square_{1}$ & $\square_{2}$ & $\square_{3}$ & $\square_{4}$ \\
\hline q) & La sévérité excessive des enseignants à l'égard des élèves. & $\square_{1}$ & $\square_{2}$ & $\square_{3}$ & $\square_{4}$ \\
\hline r) & Les enseignants arrivent en retard au cours. & $\square_{1}$ & $\square_{2}$ & $\square_{3}$ & $\square_{4}$ \\
\hline s) & Les enseignants ne sont pas assez bien préparés pour leurs cours. & $\square_{1}$ & $\square_{2}$ & $\square_{3}$ & $\square_{4}$ \\
\hline
\end{tabular}
votre établissement sans <certificat ou titre de fin d'études secondaires qui permette à l'élève de s'orienter vers différentes voies, par exemple suivre une formation universitaire, technique ou professionnelle, un stage de formation ou entrer dans la vie active $>$ ?

Note : <'année scolaire précédente > - Cette expression fait référence à l'année scolaire précédente, qui ne correspond pas nécessairement à une année civile.

(Ne cochez qu'une seule case.)

Il existe une pression constante de la part de nombreux parents qui souhaitent que l'établissement se fixe des objectifs très ambitieux en termes de performance scolaire et qu'il s'assure que les élèves les atteignent.

La pression à laquelle est soumis l'établissement pour élever le niveau de performance scolaire des élèves émane d'une minorité de parents.

L'établissement $n^{\prime}$ est pas soumis à une réelle pression des parents pour élever le niveau de performance scolaire des élèves. 
Au cours de <l'année scolaire précédente $>$, quel est le pourcentage de parents d'élèves ayant pris part aux activités suivantes en rapport avec l'école?

(Inscrivez un nombre par ligne. Inscrivez 0 (zéro) si aucun parent n'a pris part à cette activité. Inscrivez 100 (cent) si tous les parents ont pris part à cette activité.)

\begin{tabular}{|c|c|c|}
\hline & & $\%$ \\
\hline a) & Discuter du comportement de leur enfant avec un professeur, sur leur initiative. & \\
\hline b) & Discuter du comportement de leur enfant avec un professeur, sur l'initiative de l'un des professeurs. & \\
\hline C) & Discuter des progrès de leur enfant avec un professeur, sur leur initiative. & \\
\hline d) & Discuter des progrès de leur enfant avec un professeur, sur l'initiative de l'un des professeurs. & \\
\hline e) & $\begin{array}{l}\text { Se porter volontaires pour des tâches manuelles, par exemple, travaux de menuiserie, de jardinage ou } \\
\text { d'entretien du bâtiment ou de la cour. }\end{array}$ & \\
\hline f) & Se porter volontaires pour des activités extrascolaires, par exemple, club de lecture, pièce de théâtre, sport, excursion. & \\
\hline g) & Se porter volontaires pour travailler à la bibliothèque ou à la médiathèque de l'établissement. & \\
\hline h) & Aider un professeur de l'établissement. & \\
\hline i) & Faire une conférence. & \\
\hline j) & $\begin{array}{l}\text { Participer à des activités liées à la <gestion> de l'établissement, par exemple, faire partie d'un comité } \\
\text { consultatif de parents d'élèves ou du comité de direction de l'établissement. }\end{array}$ & \\
\hline k) & Aider à rassembler des fonds pour l'établissement. & \\
\hline l) & Se porter volontaires pour travailler à la <cantine> de l'établissement.. & \\
\hline
\end{tabular}

Pensez au personnel enseignant de votre établissement. Dans quelle mesure êtes-vous d'accord avec les affirmations suivantes?

(Cochez une case par ligne.)

\begin{tabular}{c|l|c|c|c|c}
\multicolumn{2}{l|}{} & $\begin{array}{c}\text { Tout à fait } \\
\text { d'accord }\end{array}$ & D'accord & $\begin{array}{c}\text { Pas } \\
\text { d'accord }\end{array}$ & $\begin{array}{c}\text { Pas du tout } \\
\text { d'accord }\end{array}$ \\
\hline a) & Le moral des enseignants est très bon dans cet établissement. & $\square_{1}$ & $\square_{2}$ & $\square_{3}$ & $\square_{4}$ \\
\hline b) & Les enseignants travaillent avec enthousiasme. & $\square_{1}$ & $\square_{2}$ & $\square_{3}$ & $\square_{4}$ \\
\hline c) & Les enseignants sont fiers de cet établissement. & $\square_{1}$ & $\square_{2}$ & $\square_{3}$ \\
\hline d) & $\begin{array}{l}\text { Les enseignants attachent beaucoup d'importance aux performances } \\
\text { scolaires. }\end{array}$ & $\square_{1}$ & $\square_{2}$ & $\square_{3}$ \\
\hline
\end{tabular}


Dans quelle mesure êtes-vous d'accord avec les affirmations suivantes à propos des enseignants de votre établissement?

\begin{tabular}{c|l|c|c|c}
\hline a) & $\begin{array}{l}\text { Les professeurs de mathématiques essaient volontiers de nouvelles } \\
\text { méthodes et pratiques pédagogiques. }\end{array}$ & $\square_{1}$ & $\square_{2}$ & $\square_{3}$ \\
\hline b) & $\begin{array}{l}\text { Les professeurs de mathématiques préfèrent les méthodes } \\
\text { et les pratiques pédagogiques qui ont fait leurs preuves. }\end{array}$ & $\square_{1}$ & $\square_{2}$ & $\square_{3}$ \\
\hline
\end{tabular}

c) de l'idée que le niveau des résultats scolaires doit être maintenu aussi haut que possible.

Parmi les professeurs de mathématiques, il existe un consensus autour

d) de l'idée que le mieux est d'adapter les exigences scolaires aux niveaux et aux besoins des élèves.

\begin{tabular}{|l|l|l|l}
$\square_{1}$ & $\square_{2}$ & $\square_{3}$ & $\square_{4}$ \\
\hline$\square_{1}$ & $\square_{2}$ & $\square_{3}$ & $\square_{4}$ \\
\hline
\end{tabular}

Parmi les professeurs de mathématiques, il existe un consensus

aussi important que son acquisition des compétences et savoir-faire
a mathématiques.

Parmi les professeurs de mathématiques, il existe un consensus autour

de l'idée que le développement des compétences et savoir-faire

f) mathématiques chez les élèves est l'objectif primordial du cours de mathématiques.

\begin{tabular}{|l|l|l|l}
$\square_{1}$ & $\square_{2}$ & $\square_{3}$ & $\square_{4}$ \\
\hline$\square_{1}$ & $\square_{2}$ & $\square_{3}$ & $\square_{4}$ \\
\hline
\end{tabular}

(Cochez une case par ligne.)

\begin{tabular}{|c|c|c|c|}
\hline & & Oui & Non \\
\hline a) & Tests ou contrôles évaluant les performances des élèves. & $\square_{1}$ & $\square_{2}$ \\
\hline b) & $\begin{array}{l}\text { Évaluation mutuelle entre collègues (passer en revue des plans de cours, des instruments } \\
\text { d'évaluation, des leçons). }\end{array}$ & $\square_{1}$ & $\square_{2}$ \\
\hline c) & Observation des cours par le chef d'établissement ou par des enseignants chevronnés. & $\square_{1}$ & $\square_{2}$ \\
\hline d) & Observation des cours par des inspecteurs ou par d'autres personnes extérieures à l'établissement. & $\square_{1}$ & $\square_{2}$ \\
\hline
\end{tabular}

Dans quelle mesure une évaluation des enseignants ou un feedback sur eux a-t-il entraîné directement les conséquences suivantes?

\begin{tabular}{|c|c|c|c|c|c|}
\hline & & $\begin{array}{c}\text { Pas de } \\
\text { conséquence }\end{array}$ & $\begin{array}{c}\text { Légère } \\
\text { conséquence }\end{array}$ & $\begin{array}{l}\text { Conséquence } \\
\text { moyenne }\end{array}$ & $\begin{array}{l}\text { Conséquence } \\
\text { importante }\end{array}$ \\
\hline a) & Une évolution de salaire. & $\square_{1}$ & $\square_{2}$ & $\square_{3}$ & $\square_{4}$ \\
\hline b) & Une prime ou un autre avantage financier. & $\square_{1}$ & $\square_{2}$ & $\square_{3}$ & $\square_{4}$ \\
\hline c) & Des possibilités d'activités de formation continue. & $\square_{1}$ & $\square_{2}$ & $\square_{3}$ & $\square_{4}$ \\
\hline d) & $\begin{array}{l}\text { Une évolution de perspective d'avancement } \\
\text { professionnel. }\end{array}$ & $\square_{1}$ & $\square_{2}$ & $\square_{3}$ & $\square_{4}$ \\
\hline e) & Une reconnaissance publique de votre part. & $\square_{1}$ & $\square_{2}$ & $\square_{3}$ & $\square_{4}$ \\
\hline f) & $\begin{array}{l}\text { Une évolution des responsabilités professionnelles qui } \\
\text { rend le poste plus intéressant. }\end{array}$ & $\square_{1}$ & $\square_{2}$ & $\square_{3}$ & $\square_{4}$ \\
\hline g) & $\begin{array}{l}\text { Un rôle dans la prise d'initiatives destinées à faire } \\
\text { progresser l'établissement (par ex., au sein du groupe } \\
\text { d'élaboration du programme ou des objectifs de } \\
\text { l'établissement). }\end{array}$ & $\square_{1}$ & $\square_{2}$ & $\square_{3}$ & $\square_{4}$ \\
\hline
\end{tabular}




\section{Stratégies et pratiques pédagogiques}

Q28

(SC32)

Dans quelle mesure tient-on compte des facteurs suivants pour admettre un élève dans votre établissement ?

(Cochez une case par ligne.)

\begin{tabular}{|c|c|c|c|c|}
\hline & & Jamais & Parfois & Toujours \\
\hline a) & Dossier scolaire de l'élève avec ses résultats (y compris à des tests de placement). & $\square_{1}$ & $\square_{2}$ & $\square_{3}$ \\
\hline b) & Recommandation de l'établissement dont provient l'élève. & $\square_{1}$ & $\square_{2}$ & $\square_{3}$ \\
\hline c) & $\begin{array}{l}\text { Adhésion des parents à la " philosophie » pédagogique ou religieuse } \\
\text { de l'établissement. }\end{array}$ & $\square_{1}$ & $\square_{2}$ & $\square_{3}$ \\
\hline d) & Le fait que l'élève doive suivre un programme scolaire spécifique ou s’y intéresse. & $\square_{1}$ & $\square_{2}$ & $\square_{3}$ \\
\hline e) & $\begin{array}{l}\text { Priorité accordée aux membres de la famille d'un élève fréquentant ou ayant } \\
\text { fréquenté l'établissement. }\end{array}$ & $\square_{1}$ & $\square_{2}$ & $\square_{3}$ \\
\hline f) & Domicile dans une entité géographique déterminée. & $\square_{1}$ & $\square_{2}$ & $\square_{3}$ \\
\hline g) & Autres. & $\square_{1}$ & $\square_{2}$ & $\square_{3}$ \\
\hline
\end{tabular}

Quelles sont les instances qui ont une part importante de responsabilité dans la gestion des tâches suivantes concernant votre établissement?

(Cochez toutes les cases qui conviennent à chaque ligne.)

\begin{tabular}{|c|c|c|c|c|c|c|}
\hline & & $\begin{array}{l}\text { Le chef } \\
\text { d'établissement }\end{array}$ & Les enseignants & $\begin{array}{l}\text { Le }<\text { Conseil de } \\
\text { direction de } \\
\text { l'établissement }>\end{array}$ & $\begin{array}{l}\text { Les <autorités } \\
\text { régionales } \\
\text { ou locales } \\
\text { en charge de } \\
\text { l'éducation> }\end{array}$ & $\begin{array}{l}\text { Les autorités } \\
\text { nationales } \\
\text { en charge de } \\
\text { l'éducation }\end{array}$ \\
\hline a) & $\begin{array}{l}\text { Choisir les enseignants } \\
\text { à engager. }\end{array}$ & $\square_{1}$ & $\square_{2}$ & $\square_{3}$ & $\square_{4}$ & $\square_{5}$ \\
\hline b) & Congédier les enseignants. & $\square_{1}$ & $\square_{2}$ & $\square_{3}$ & $\square_{4}$ & $\square_{5}$ \\
\hline c) & $\begin{array}{l}\text { Déterminer le salaire } \\
\text { initial des enseignants. }\end{array}$ & $\square_{1}$ & $\square_{2}$ & $\square_{3}$ & $\square_{4}$ & $\square_{5}$ \\
\hline d) & $\begin{array}{l}\text { Déterminer les } \\
\text { augmentations de salaire } \\
\text { des enseignants. }\end{array}$ & $\square_{1}$ & $\square_{2}$ & $\square_{3}$ & $\square_{4}$ & $\square_{5}$ \\
\hline e) & $\begin{array}{l}\text { Établir le budget de } \\
\text { l'établissement. }\end{array}$ & $\square_{1}$ & $\square_{2}$ & $\square_{3}$ & $\square_{4}$ & $\square_{5}$ \\
\hline f) & $\begin{array}{l}\text { Décider de la ventilation } \\
\text { du budget dans } \\
\text { l'établissement. }\end{array}$ & $\square_{1}$ & $\square_{2}$ & $\square_{3}$ & $\square_{4}$ & $\square_{5}$ \\
\hline g) & $\begin{array}{l}\text { Définir le règlement } \\
\text { intérieur pour les élèves. }\end{array}$ & $\square_{1}$ & $\square_{2}$ & $\square_{3}$ & $\square_{4}$ & $\square_{5}$ \\
\hline h) & $\begin{array}{l}\text { Définir les politiques } \\
\text { d'évaluation des élèves. }\end{array}$ & $\square_{1}$ & $\square_{2}$ & $\square_{3}$ & $\square_{4}$ & $\square_{5}$ \\
\hline i) & $\begin{array}{l}\text { Décider de l'admission } \\
\text { des élèves dans } \\
\text { l'établissement. }\end{array}$ & $\square_{1}$ & $\square_{2}$ & $\square_{3}$ & $\square_{4}$ & $\square_{5}$ \\
\hline j) & $\begin{array}{l}\text { Choisir les manuels } \\
\text { à utiliser. }\end{array}$ & $\square_{1}$ & $\square_{2}$ & $\square_{3}$ & $\square_{4}$ & $\square_{5}$ \\
\hline k) & $\begin{array}{l}\text { Déterminer le contenu } \\
\text { des cours. }\end{array}$ & $\square_{1}$ & $\square_{2}$ & $\square_{3}$ & $\square_{4}$ & $\square_{5}$ \\
\hline l) & $\begin{array}{l}\text { Décider des cours } \\
\text { à proposer. }\end{array}$ & $\square_{1}$ & $\square_{2}$ & $\square_{3}$ & $\square_{4}$ & $\square_{5}$ \\
\hline \multicolumn{7}{|c|}{$\begin{array}{l}\text { Notes : <Conseil de direction de l'établissement }>\text { - Cette expression fait référence à l'organe qui est directement responsable de la gestion de } \\
\text { l'établissement. Ce conseil peut, dans certains cas, être entièrement externe à l'établissement ; dans d'autres, il comprend des représentants du } \\
\text { personnel et des élèves. }\end{array}$} \\
\hline \multicolumn{7}{|c|}{$\begin{array}{l}<\text { Autorités régionales ou locales en charge de l'éducation> - Il s'agit ici d'autorités qui ne sont pas des instances nationales et qui ne dirigent pas } \\
\text { directement l'établissement. }\end{array}$} \\
\hline
\end{tabular}


Vous trouverez ci-dessous des affirmations concernant votre gestion de l'établissement. Veuillez indiquer la fréquence à laquelle vous avez adopté les attitudes suivantes dans l'établissement lors de <l'année scolaire précédente>.

(Cochez une case par ligne.)

\begin{tabular}{|c|c|c|c|c|c|c|c|}
\hline & & $\begin{array}{l}\text { Ce n'est } \\
\text { pas arrivé }\end{array}$ & $\begin{array}{l}1 \text { à } 2 \text { fois } \\
\text { dans } \\
\text { I'année }\end{array}$ & $\begin{array}{l}3 \text { à } 4 \text { fois } \\
\text { dans } \\
\text { I'année }\end{array}$ & $\begin{array}{l}\text { Une fois } \\
\text { par mois }\end{array}$ & $\begin{array}{l}\text { Une } \\
\text { fois par } \\
\text { semaine }\end{array}$ & $\begin{array}{l}\text { Plus d'une } \\
\text { fois par } \\
\text { semaine }\end{array}$ \\
\hline a) & $\begin{array}{l}\text { Je cherche à promouvoir la réputation de } \\
\text { l'établissement au sein de la communauté locale. }\end{array}$ & $\square_{1}$ & $\square_{2}$ & $\square_{3}$ & $\square_{4}$ & $\square_{5}$ & $\square_{6}$ \\
\hline b) & $\begin{array}{l}\text { Je me sers des résultats des élèves pour élaborer } \\
\text { les objectifs pédagogiques de l'établissement. }\end{array}$ & $\square_{1}$ & $\square_{2}$ & $\square_{3}$ & $\square_{4}$ & $\square_{5}$ & $\square_{6}$ \\
\hline c) & $\begin{array}{l}\text { Je fais en sorte que les activités de formation } \\
\text { continue des enseignants soient en accord avec } \\
\text { les objectifs d'enseignement de l'établissement. }\end{array}$ & $\square_{1}$ & $\square_{2}$ & $\square_{3}$ & $\square_{4}$ & $\square$ $\square_{5}$ & $\square_{6}$ \\
\hline d) & $\begin{array}{l}\text { Je veille à ce que les enseignants travaillent } \\
\text { en accord avec les objectifs pédagogiques de } \\
\text { l'établissement. }\end{array}$ & $\square_{1}$ & $\square_{2}$ & $\square_{3}$ & $\square_{4}$ & $\square_{5}$ & $\square_{6}$ \\
\hline e) & $\begin{array}{l}\text { J'encourage les méthodes d'enseignement } \\
\text { fondées sur les résultats de recherches récentes } \\
\text { en éducation. }\end{array}$ & $\square_{1}$ & $\square_{2}$ & $\square_{3}$ & $\square_{4}$ & $\square_{5}$ & $\square_{6}$ \\
\hline f) & $\begin{array}{l}\text { Je complimente les enseignants dont les } \\
\text { élèves participent activement aux activités } \\
\text { d'apprentissage. }\end{array}$ & $\square_{1}$ & $\square 2$ & $\square_{3}$ & $\square 4$ & $\square_{5}$ & $\square_{6}$ \\
\hline g) & $\begin{array}{l}\text { Quand un professeur rencontre un problème } \\
\text { dans sa classe, je prends l'initiative d'en discuter } \\
\text { avec lui. }\end{array}$ & $\square_{1}$ & $\square_{2}$ & $\square_{3}$ & $\square_{4}$ & $\square_{5}$ & $\square_{6}$ \\
\hline h) & $\begin{array}{l}\text { J'attire l'attention des enseignants sur } \\
\text { l'importance du développement de l'esprit } \\
\text { critique et du sens social chez les élèves. }\end{array}$ & $\square_{1}$ & $\square_{2}$ & $\square_{3}$ & $\square_{4}$ & $\square_{5}$ & $\square_{6}$ \\
\hline i) & $\begin{array}{l}\text { Je suis attentif aux comportements qui risquent } \\
\text { de perturber le travail en classe. }\end{array}$ & $\square_{1}$ & $\square_{2}$ & $\square_{3}$ & $\square_{4}$ & $\square_{5}$ & $\square_{6}$ \\
\hline j) & $\begin{array}{l}\text { Je donne au personnel enseignant la possibilité } \\
\text { d'intervenir dans les décisions concernant } \\
\text { l'établissement. }\end{array}$ & $\square_{1}$ & $\square$ $\square_{2}$ & $\square_{3}$ & $\square_{4}$ & $\square$ $\square_{5}$ & $\square_{6}$ \\
\hline k) & $\begin{array}{l}\text { J'incite les enseignants à instaurer une culture } \\
\text { de l'établissement axée sur l'amélioration } \\
\text { continue. }\end{array}$ & $\square_{1}$ & $\square_{2}$ & $\square_{3}$ & $\square_{4}$ & $\square_{5}$ & $\square_{6}$ \\
\hline I) & $\begin{array}{l}\text { Je demande aux enseignants de participer à } \\
\text { l'évaluation des pratiques de gestion. }\end{array}$ & $\square_{1}$ & $\square_{2}$ & $\square_{3}$ & $\square_{4}$ & $\square_{5}$ & $\square_{6}$ \\
\hline m) & $\begin{array}{l}\text { Quand un professeur évoque un problème } \\
\text { en classe, nous le résolvons ensemble. }\end{array}$ & $\square_{1}$ & $\square_{2}$ & $\square_{3}$ & $\square_{4}$ & $\square_{5}$ & $\square_{6}$ \\
\hline n) & $\begin{array}{l}\text { Je discute des objectifs pédagogiques } \\
\text { de l'établissement avec les enseignants lors } \\
\text { de réunions pédagogiques. }\end{array}$ & $\square_{1}$ & $\square 2$ & $\square_{3}$ & $\square_{4}$ & $\square_{5}$ & $\square 6$ \\
\hline o) & $\begin{array}{l}\text { Je me réfère aux objectifs pédagogiques de } \\
\text { l'établissement lorsque je prends des décisions } \\
\text { relatives au programme avec les enseignants. }\end{array}$ & $\square_{1}$ & $\square_{2}$ & $\square_{3}$ & $\square_{4}$ & $\square_{5}$ & $\square_{6}$ \\
\hline p) & $\begin{array}{l}\text { Je discute des résultats scolaires en réunion } \\
\text { pédagogique pour identifier les lignes de force } \\
\text { et les faiblesses du programme. }\end{array}$ & $\square_{1}$ & $\square_{2}$ & $\square_{3}$ & $\square 4$ & $\square$ $\square_{5}$ & $\square_{6}$ \\
\hline q) & $\begin{array}{l}\text { Je dirige dans l'établissement des activités } \\
\text { de formation continue en rapport avec } \\
\text { l'enseignement ou j'y prends part. }\end{array}$ & $\square_{1}$ & $\square_{2}$ & $\square_{3}$ & $\square_{4}$ & $\square_{5}$ & $\square_{6}$ \\
\hline r) & $\begin{array}{l}\text { Lors des réunions pédagogiques, je prévois } \\
\text { du temps pour permettre aux enseignants } \\
\text { d'échanger des idées ou des informations } \\
\text { découlant d'activités de formation continue } \\
\text { dans l'établissement. }\end{array}$ & $\square_{1}$ & $\square_{2}$ & $\square_{3}$ & $\square_{4}$ & $\square_{5}$ & $\square_{6}$ \\
\hline s) & $\begin{array}{l}\text { Je vais régulièrement dans les classes pour } \\
\text { observer les cours de façon informelle (ces } \\
\text { observations informelles ne sont pas annoncées } \\
\text { à l'avance, elles durent au moins } 5 \text { minutes } \\
\text { et peuvent déboucher ou non sur un feedback } \\
\text { écrit ou sur une réunion officielle). }\end{array}$ & $\square_{1}$ & $\square_{2}$ & $\square_{3}$ & $\square_{4}$ & $\square_{5}$ & $\square_{6}$ \\
\hline t) & $\begin{array}{l}\text { J'examine le travail fourni par les élèves lorsque } \\
\text { j'évalue l'enseignement dispensé dans les classes. }\end{array}$ & $\square_{1}$ & $\square_{2}$ & $\square_{3}$ & $\square_{4}$ & $\square_{5}$ & $\square_{6}$ \\
\hline u) & J'évalue les résultats obtenus par les enseignants. & $\square_{1}$ & $\square_{2}$ & $\square_{3}$ & $\square$ प & $\square_{5}$ & $\square_{6}$ \\
\hline
\end{tabular}

Note : Veuillez vous référer à la Question 19 pour une définition de la <L'année scolaire précédente>. 
Au cours des trois derniers mois, quel est le pourcentage d'enseignants de votre établissement ayant participé à un programme de formation continue axé principalement sur les mathématiques ?

Par formation continue, on entend un programme officiel, destiné à améliorer les compétences ou les pratiques pédagogiques des enseignants. Ce programme peut être sanctionné par une certification reconnue ou non. La durée totale du programme doit être d'au moins une journée, et doit porter sur la pédagogie et l'enseignement des mathématiques.

\begin{tabular}{c|l|c}
\hline \multicolumn{2}{l}{} & $\%$ \\
\hline a) & Le personnel enseignant de l'établissement, toutes matières confondues. & \\
\hline b) & Les professeurs de mathématiques de l'établissement. & \\
\hline
\end{tabular}
mises en œuvre dans votre établissement?

\begin{tabular}{|c|c|c|c|}
\hline & & Oui & Non \\
\hline a) & Descriptif écrit du programme et des objectifs pédagogiques de l'établissement. & $\square_{1}$ & $\square_{2}$ \\
\hline b) & Descriptif écrit des normes requises en termes de performances des élèves. & & $\square_{2}$ \\
\hline c) & $\begin{array}{l}\text { Enregistrement systématique de données, parmi lesquelles les taux de présence des enseignants et } \\
\text { des élèves, les taux de réussite, les résultats aux évaluations et la formation continue des enseignants. }\end{array}$ & & \\
\hline d) & Évaluation interne/auto-évaluation. & $\square_{1}$ & $\square_{2}$ \\
\hline e) & Évaluation externe. & $\square_{1}$ & $\square_{2}$ \\
\hline f) & $\begin{array}{l}\text { Appel à un feedback écrit de la part des élèves (par ex., par rapport aux cours, aux enseignants } \\
\text { ou aux ressources). }\end{array}$ & & \\
\hline g) & Mentorat des enseignants. & $\square_{1}$ & $\square_{2}$ \\
\hline h) & $\begin{array}{l}\text { Projet d'amélioration de l'établissement en concertation avec un ou plusieurs experts pendant } \\
\text { six mois au moins. }\end{array}$ & $\square_{1}$ & $\square$ \\
\hline i) & $\begin{array}{l}\text { Mise en œuvre d'une politique normalisée d'enseignement des mathématiques (le programme } \\
\text { de l'établissement est mis en œuvre avec du matériel pédagogique commun ; formation et } \\
\text { formation continue du personnel enseignant). }\end{array}$ & $\square_{1}$ & $\square_{2}$ \\
\hline
\end{tabular}

\begin{tabular}{|c|c|c|c|}
\hline & & Oui & Non \\
\hline a) & $\begin{array}{l}\text { L'établissement a une politique quant à l'utilisation des ordinateurs pour l'enseignement } \\
\text { des mathématiques (par ex., durée d'utilisation des ordinateurs pendant les cours de } \\
\text { mathématiques, utilisation de logiciels spécifiques aux mathématiques). }\end{array}$ & $\square_{1}$ & $\square_{2}$ \\
\hline b) & $\begin{array}{l}\text { Le même manuel est utilisé dans tous les cours de mathématiques dispensés aux élèves de } \\
\text { <grade modal du pays pour les élèves de } 15 \text { ans> de l'établissement. }\end{array}$ & $\square_{1}$ & $\square_{2}$ \\
\hline c) & $\begin{array}{l}\text { Les professeurs de mathématiques de l'établissement suivent un programme normalisé spécifiant } \\
\text { la matière à enseigner chaque mois (au moins). }\end{array}$ & $\square_{1}$ & $\square_{2}$ \\
\hline
\end{tabular}

Note : Veuillez vous référer à la Question 5 pour une définition du < grade modal du pays pour les élèves de $\mathbf{1 5}$ ans $>$. 
Q34

Dans votre établissement, quelle est la probabilité qu'un élève de < grade modal du pays pour les élèves de 15 ans> soit transféré dans un autre établissement pour les raisons suivantes?

(Cochez une case par ligne.)

\begin{tabular}{c|l|c|c}
\multicolumn{2}{l|}{} & $\begin{array}{c}\text { Peu } \\
\text { probable }\end{array}$ & $\begin{array}{c}\text { Probable } \\
\text { probable }\end{array}$ \\
\hline a) & Performances scolaires insuffisantes. & $\square_{1}$ & $\square_{2}$ \\
\hline b) & Très bonnes performances scolaires. & $\square_{1}$ & $\square_{2}$ \\
\hline c) & Problèmes de comportement. & $\square_{1}$ & $\square_{2}$ \\
\hline d) & Besoins éducatifs spécifiques. & $\square_{1}$ & $\square_{3}$ \\
\hline e) & Requête des parents ou du tuteur. & $\square_{1}$ & $\square_{2}$ \\
\hline f) & Autres raisons. & $\square_{1}$ & $\square_{3}$ \\
\hline Note : Veuillez vous référer à la Question 5 pour une définition du <grade modal du pays pour les élèves de 15 ans>. & $\square_{3}$ \\
\hline
\end{tabular}

\section{Enseignement des matières financières à l'école}

Les cinq questions suivantes concernent l'enseignement des matières financières (y compris les finances personnelles) dispensé dans votre établissement. Cet enseignement vise le développement des connaissances des élèves, leur confiance en eux et leurs compétences dans des domaines tels que : l'argent et les revenus; la gestion d'un budget et sa planification à long terme ; l'épargne et les dépenses ; le crédit et l'endettement; les investissements et les assurances; les risques et les bénéfices possibles liés aux produits financiers; et enfin, le contexte financier (y compris les droits et devoirs des consommateurs, et la compréhension du système financier, économique et social à plus grande échelle).

Parmi les affirmations suivantes, laquelle décrit le mieux l'accès des élèves de < grade modal du pays pour les élèves de 15 ans> à des cours de matières financières dans votre établissement ?

(Ne cochez qu'une seule case.)

Il n'y a pas de cours de matières financières.

Il y a des cours de matières financières depuis moins de deux ans.

Il y a des cours de matières financières depuis deux ans ou plus.

Note : Veuillez vous référer à la Question 5 pour une définition du <grade modal du pays pour les élèves de 15 ans>

Q36 L'enseignement des matières financières est-il obligatoire dans votre établissement ?

(SC45) (Ne cochez qu'une seule case.)

\begin{tabular}{|l|l|}
\hline Oui. & $\square$ \\
\hline Non. & $\square_{2}$ \\
\hline
\end{tabular}


Parmi les affirmations suivantes, lesquelles correspondent à l'enseignement des matières financières dans votre établissement?

Pour chaque affirmation, veuillez indiquer le nombre d'heures consacrées à ce type d'enseignement pour les élèves de < grade modal du pays pour les élèves de 15 ans > lors de <l'année scolaire précédente>.

(Cochez une case par ligne.)

\begin{tabular}{|c|c|c|c|c|c|c|}
\hline & & Aucune & $\begin{array}{l}\text { De } 1 \text { à } \\
4 \text { heures } \\
\text { par an }\end{array}$ & $\begin{array}{l}\text { De } 5 \text { à } \\
19 \text { heures } \\
\text { par an }\end{array}$ & $\begin{array}{l}\text { De } 20 \text { à } \\
49 \text { heures } \\
\text { par an }\end{array}$ & $\begin{array}{l}50 \text { heures } \\
\text { ou plus } \\
\text { par an }\end{array}$ \\
\hline a) & $\begin{array}{l}\text { Les matières financières sont enseignées en tant que matière } \\
\text { à part entière. }\end{array}$ & $\square_{1}$ & $\square_{2}$ & $\square_{3}$ & $\square_{4}$ & $\square_{5}$ \\
\hline b) & $\begin{array}{l}\text { Les matières financières sont enseignées en tant que matière } \\
\text { pluridisciplinaire. }\end{array}$ & $\square_{1}$ & $\square_{2}$ & $\square_{3}$ & $\square_{4}$ & $\square_{5}$ \\
\hline c) & $\begin{array}{l}\text { Les matières financières sont enseignées dans le cadre } \\
\text { des < cours d'économie/de sciences économiques }>\text {. }\end{array}$ & $\square_{1}$ & $\square_{2}$ & $\square_{3}$ & $\square_{4}$ & $\square_{5}$ \\
\hline d) & $\begin{array}{l}\text { Les matières financières sont enseignées dans le cadre } \\
\text { des mathématiques. }\end{array}$ & $\square_{1}$ & $\square_{2}$ & $\square_{3}$ & $\square_{4}$ & $\square_{5}$ \\
\hline e) & $\begin{array}{l}\text { Les matières financières sont enseignées dans le cadre d'autres } \\
\text { cours de sciences sociales et humaines et/ou dans le cadre du } \\
\text { cours de langue et de littérature (comme l'histoire, la géographie, } \\
<\text { l'économie domestique }>\text { et <la citoyenneté }>\text { ). }\end{array}$ & $\square_{1}$ & $\square_{2}$ & $\square_{3}$ & $\square_{4}$ & $\square_{5}$ \\
\hline f) & C'est une activité optionnelle qui <ne fait pas partie du programme>. & $\square_{1}$ & $\square_{2}$ & $\square_{3}$ & $\square_{4}$ & $\square_{5}$ \\
\hline g) & $\begin{array}{l}\text { Les matières financières sont enseignées dans le cadre } \\
\text { des }<\text { cours de l'enseignant titulaire de la classe }>\text {. }\end{array}$ & $\square_{1}$ & $\square_{2}$ & $\square_{3}$ & $\square_{4}$ & $\square_{5}$ \\
\hline
\end{tabular}

Q38 Qui est chargé de l'enseignement des matières financières dans votre établissement ?

(SC52) (Cochez une case par ligne.)

\begin{tabular}{c|l|c|c}
\hline \multicolumn{2}{l|}{} & Oui & Non \\
\hline a) & Des enseignants. & $\square_{1}$ & $\square_{2}$ \\
\hline b) & $\begin{array}{l}\text { Des personnes issues d'institutions du secteur privé (par ex., des banques } \\
\text { commerciales ou des compagnies d'assurances). }\end{array}$ & $\square_{1}$ \\
\hline c) & $\begin{array}{l}\text { Des personnes issues d'institutions du secteur public (par ex., le <ministère des } \\
\text { Finances> ou la <Banque nationale>). }\end{array}$ & $\square_{1}$ & $\square_{2}$ \\
\hline d) & $\begin{array}{l}\text { Des personnes issues d'organisations non gouvernementales (par ex., } \\
\text { <exemples nationaux>). }\end{array}$ & $\square_{2}$ \\
\hline
\end{tabular}

Au cours des douze derniers mois, quel est le pourcentage d'enseignants de votre établissement ayant participé à un programme de formation continue axé principalement sur l'enseignement des matières financières?

(SC51) Par formation continue, on entend un programme officiel, destiné à améliorer les compétences ou les pratiques pédagogiques des enseignants. Ce programme peut être sanctionné par une certification reconnue ou non. La durée totale du programme doit être d'au moins une journée, et doit porter sur la pédagogie et l'enseignement des matières financières.

a) Les enseignants qui enseignent les matières financières dans votre établissement.

b) Le personnel enseignant de l'établissement, toutes matières confondues.

Question supplémentaire dans le cas d'une administration en ligne du questionnaire "Établissement » 


\section{QUESTIONNAIRE ÉLËVE}

Le questionnaire "Élève » est administré après les épreuves cognitives. Il faut environ 30 minutes pour le remplir. Les questions sur le milieu familial sont similaires à celles utilisées lors des cycles PISA précédents. Le questionnaire porte sur les points suivants :

- les caractéristiques de l'élève et son parcours scolaire ;

- le contexte et les ressources de sa famille ;

- I'apprentissage des mathématiques ;

- I'expérience de différents types de problèmes mathématiques vus à l'école ;

- I'expérience des mathématiques ;

- le climat dans sa classe et son établissement ; et

- l'expérience de la résolution de problèmes.

Les termes techniques indiqués entre <crochets > sont adaptés au contexte national par le centre national de collecte de données des pays ou économies participants. Dans cette annexe, ces termes techniques sont expliqués sous les items.

\section{Vos caractéristiques personnelles et votre parcours scolaire}

\begin{tabular}{l|l|l} 
Q1 & Quel < grade> fréquentez-vous ? \\
(ST01) & Grade & \\
\hline & Note : < Grade> - Ce terme fait référence au niveau administratif de scolarisation auquel l'élève est inscrit dans l'établissement d'enseignement. \\
Le nombre d'années de scolarité est la mesure habituelle du grade dans de nombreux pays.
\end{tabular}

Lequel de ces <programmes> suivez-vous ?

(Ne cochez qu'une seule case.)

\begin{tabular}{|l|c}
\hline <Programme $1>$. & $\square_{1}$ \\
\hline$<$ Programme $2>$. & $\square_{2}$ \\
\hline$<$ Programme $3>$. & $\square_{3}$ \\
\hline$<$ Programme $4 .$. & $\square_{4}$ \\
\hline$<$ Programme $5>$. & $\square_{5}$ \\
\hline$<$ Programme $>$. & $\square_{6}$
\end{tabular}

Q3 Quelle est votre date de naissance?

(ST03) (Veuillez indiquer le jour, le mois et l'année de votre naissance.)

$$
\text { Jour }
$$$$
\text { Mois }
$$

Année

\section{Q4 Êtes-vous une fille ou un garçon?}

\begin{tabular}{|l|c}
\hline Fille. & $\square_{1}$ \\
\hline Garçon. & $\square_{2}$ \\
\hline
\end{tabular}


Q5 Avez-vous fréquenté le <niveau CITE 0>?

(Ne cochez qu'une seule case.)

\begin{tabular}{l|c} 
Non. & $\square_{1}$ \\
\hline Oui, pendant une année scolaire, ou moins. & $\square_{2}$ \\
\hline Oui, pendant plus d'une année scolaire. & $\square_{3}$
\end{tabular}

Note : <Niveau CITE 0> - Enseignement préprimaire, défini comme le premier niveau de formation organisée, essentiellement destiné à familiariser de très jeunes enfants avec un environnement de type scolaire. Pour être prises en compte, les formations doivent être dispensées dans un établissement d'enseignement ou une institution, et destinées ordinairement à des enfants âgés de 3 à 6 ans.

\section{Q6 Quel âge aviez-vous lorsque vous êtes entré au <niveau CITE 1>?}

Note : < Niveau CITE 1> - Enseignement primaire, commençant entre l'âge de 5 et 7 ans selon les pays, généralement d'une durée allant de 4 ans (par ex. en Allemagne) à 6 ans (durée modale dans les pays membres de l'OCDE). Il est destiné à fournir aux élèves une solide formation de base (lecture, expression écrite, mathématiques) et une approche élémentaire dans d'autres matières.

\section{Q7 Vous est-il arrivé de redoubler un <grade> ?}

(ST07) (Cochez une case par ligne.)

\begin{tabular}{c|l|c|c|c}
\multicolumn{2}{l|}{} & Non, jamais & Oui, une fois & $\begin{array}{c}\text { Oui, deux fois } \\
\text { ou plus }\end{array}$ \\
\hline a) & Au $<$ niveau CITE 1 $>$. & $\square_{1}$ & $\square_{2}$ & $\square_{3}$ \\
\hline b) & Au <niveau CITE 2 $>$. & $\square_{1}$ & $\square_{2}$ & $\square_{3}$ \\
\hline c) & Au <niveau CITE 3 $>$. & $\square_{1}$ & $\square_{2}$ & $\square_{3}$ \\
\hline
\end{tabular}

Notes : Veuillez vous référer à la Question 1 pour une définition du $<$ grade $>$.

Veuillez vous référer à la Question 6 pour une définition du $<$ niveau CITE $1>$.

$<$ Niveau CITE 2> - Premier cycle de l'enseignement secondaire. Au début du niveau 2, les programmes commencent à être davantage structurés par matière. À ce niveau d'enseignement, les enseignants sont plus spécialisés et dispensent des cours dans leur domaine de spécialisation.

$<$ Niveau CITE 3> - Deuxième cycle de l'enseignement secondaire. Il s'agit du dernier cycle de l'enseignement secondaire dans la plupart des pays de I'OCDE. Par comparaison avec le niveau CITE 2, I'enseignement est souvent plus structuré par matière et les enseignants doivent souvent être plus qualifiés ou spécialisés.

$<$ Niveau CITE 3A $>$ - Prépare les élèves à l'accès direct au niveau 5A, et le $<$ niveau $\mathbf{C I T E} 3 \mathbf{3}>$, à leur accès direct au niveau CITE 5B. Le $<$ niveau $\mathbf{C I T E} 3 \mathbf{C}>$ ne prépare pas les élèves à l'accès direct au niveau CITE $5 \mathrm{~A}$ ou $5 \mathrm{~B}$ : les formations de ce niveau préparent les élèves à entrer directement dans la vie active ou à suivre une autre formation relevant du niveau CITE 3 ou une formation relevant du niveau CITE 4 .

Au cours des deux dernières semaines de classe complètes, combien de fois êtes-vous arrivé en retard ?

(Ne cochez qu'une seule case.)

\begin{tabular}{|l|c|}
\hline Aucune. & $\square_{1}$ \\
\hline Une ou deux fois. & $\square_{2}$ \\
\hline Trois ou quatre fois. & $\square_{3}$ \\
\hline Cinq fois ou plus. & $\square_{4}$
\end{tabular}

Au cours des deux dernières semaines de classe complètes, combien de fois avez-vous <séché> toute une journée de cours?

(Ne cochez qu'une seule case.)

Pas une seule fois.

Une ou deux fois.

Trois ou quatre fois.

Cinq fois ou plus.

$\square_{1}$
$\square_{2}$
$\square_{3}$
$\square_{4}$

Note : «<Sécher> toute une journée de cours » - Cette expression fait référence aux cas où un élève manque une journée de cours sans autorisation. 
Q10

Au cours des deux dernières semaines de classe complètes, combien de fois avez-vous <séché> certains cours?

(Ne cochez qu'une seule case.)

\begin{tabular}{|l|c}
\hline Pas une seule fois. & $\square_{1}$ \\
\hline Une ou deux fois. & $\square_{2}$ \\
\hline Trois ou quatre fois. & $\square_{3}$ \\
\hline Cinq fois ou plus. & $\square_{4}$ \\
\hline
\end{tabular}

Note : Veuillez vous référer à la Question 9 pour une définition de < sécher>.

\section{Votre famille et votre environnement à la maison}

\section{Q11 D'habitude, qui vit avec vous <à la maison>?}

(ST11) (Cochez une case par ligne.)

\begin{tabular}{c|l|c|c}
\hline \multicolumn{2}{l|}{} & Oui & Non \\
\hline a) & Votre mère (ou votre belle-mère ou votre mère adoptive). & $\square_{1}$ & $\square_{2}$ \\
\hline b) & Votre père (ou votre beau-père ou votre père adoptif). & $\square_{1}$ & $\square_{2}$ \\
\hline c) & Un ou des frères (y compris demi-frères). & $\square_{1}$ & $\square_{2}$ \\
\hline d) & Une ou des sœurs (y compris demi-sœurs). & $\square_{1}$ & $\square_{2}$ \\
\hline e) & Votre grand-mère, votre grand-père. & $\square_{1}$ & $\square_{2}$ \\
\hline f) & D'autres membres de votre famille (des cousins, etc.). & $\square_{1}$ & $\square_{2}$ \\
\hline
\end{tabular}

Écrivez le nom de son travail : les repas, elle dirige une équipe de vente.)

En une phrase, indiquez en quoi consiste ou consistait son travail :

\begin{tabular}{|l|c}
\hline <Niveau CITE 3A $>$. & $\square_{1}$ \\
\hline$<$ Niveau CITE 3B, 3C $>$ & $\square_{2}$ \\
\hline$<$ Niveau CITE 2 $>$. & $\square_{3}$ \\
\hline$<$ Niveau CITE 1 $>$. & $\square_{4}$ \\
\hline Elle n'a pas terminé le <niveau CITE 1 $>$. & $\square_{5}$ \\
\hline
\end{tabular}

Notes : Veuillez vous référer à la Question 6 pour une définition du <niveau CITE $1>$.

Veuillez vous référer à la Question 7 pour une définition du $<$ niveau CITE $2>$ et du $<$ niveau CITE $3>$.

$<$ Niveau de scolarité le plus élevé> - Cette expression doit être adaptée de façon à ce que la question posée porte sur la certification la plus haute obtenue par la mère au cours des étapes de scolarité correspondant aux niveaux CITE 1 à 3 . 


\begin{tabular}{|c|c|c|c|}
\hline a) & $<$ Niveau CITE 6>. & $\square_{1}$ & $\square_{2}$ \\
\hline b) & $<$ Niveau CITE 5A $>$. & $\square_{1}$ & $\square_{2}$ \\
\hline c) & $<$ Niveau CITE 5B $>$. & $\square_{1}$ & $\square_{2}$ \\
\hline d) & $<$ Niveau CITE 4>. & $\square_{1}$ & $\square_{2}$ \\
\hline
\end{tabular}

Notes : < Niveau CITE 6 > - Diplôme obtenu à l'issue d'un programme de recherche de niveau avancé, consacré à des études approfondies et à des activités de recherche originales, avec présentation d'une thèse ou d'une dissertation susceptible d'être publiée.

$<$ Niveau CITE 5A> - Diplôme obtenu à l'issue d'un programme d'études tertiaires, caractérisé par une base théorique importante, d'une durée habituellement équivalente à au moins trois années d'études à temps complet, donnant accès à des professions demandant un niveau élevé de qualification ou à des programmes de recherche de haut niveau.

$<$ Niveau CITE 5B $>$ - Diplôme obtenu à l'issue d'un programme d'études tertiaires, caractérisé par une orientation plus pratique/technique/ professionnelle et par une durée moins longue que les programmes de niveau CITE 5A. Ces programmes ont habituellement une durée équivalente à deux années d'études à temps complet au minimum, et ils préparent leurs étudiants à une profession spécifique.

$<$ Niveau CITE 4> - Diplôme obtenu à l'issue d'un programme qui se situe à la frontière entre l'enseignement secondaire supérieur et l'enseignement postsecondaire. En général, ces programmes ne sont pas significativement plus avancés que les programmes de niveau CITE 3 (voir la Question 7), et leur durée est l'équivalent de 6 mois à 2 ans à temps complet.

\section{Q15 Quelle activité exerce actuellement votre mère ?}

(ST15) (Ne cochez qu'une seule case.)

\begin{tabular}{|l|c}
\hline Travail <rémunéré> à temps plein. & $\square_{1}$ \\
\hline Travail <rémunéré> à temps partiel. & $\square_{2}$ \\
\hline Ne travaille pas, mais cherche un emploi. & $\square_{3}$ \\
\hline Autre (par exemple, femme au foyer, retraitée). & $\square_{4}$ \\
\hline
\end{tabular}

\section{Quelle est l'activité professionnelle principale de votre père ?}

(Par exemple, enseignant, aide-cuisinier, directeur des ventes.)

(S'il ne travaille pas actuellement, veuillez indiquer sa dernière activité professionnelle principale.)

Écrivez le nom de son travail : repas, il dirige une équipe de vente.)

En une phrase, indiquez en quoi consiste ou consistait son travail :

(Ne cochez qu'une seule case.)

\begin{tabular}{|l|c}
\hline$<$ Niveau CITE 3A $>$. & $\square_{1}$ \\
\hline$<$ Niveau CITE 3B, 3C $>$ & $\square_{2}$ \\
\hline$<$ Niveau CITE 2>. $>$ & $\square_{3}$ \\
\hline$<$ Niveau CITE 1 $>$. & $\square_{4}$ \\
\hline Il n'a pas terminé le <niveau CITE 1 >. & $\square_{5}$
\end{tabular}

Notes : Veuillez vous référer à la Question 6 pour une définition du <niveau CITE 1 $>$. Veuillez vous référer à la Question 7 pour une définition du $<$ niveau CITE 2> et du <niveau CITE 3>. Veuillez vous référer à la Question 13 pour une définition du <niveau de scolarité le plus élevé>. 
Votre père a-t-il obtenu un des diplômes suivants ?

Si vous hésitez sur les réponses à cette question, veuillez demander de l'aide à <la personne qui fait passer les tests>. (Cochez une case par ligne.)

\begin{tabular}{l|l|c|c}
\multicolumn{2}{l|}{} & Oui & Non \\
\hline a) & <Niveau CITE 6 $>$. & $\square_{1}$ & $\square_{2}$ \\
\hline b) & <Niveau CITE 5A $>$ & $\square_{1}$ & $\square_{2}$ \\
\hline c) & <Niveau CITE 5B $>$. & $\square_{1}$ & $\square_{2}$ \\
\hline d) & <Niveau CITE 4 $>$. & $\square_{1}$ & $\square_{2}$ \\
\hline
\end{tabular}

Note : Veuillez vous référer à la Question 14 pour une définition du $<$ niveau CITE $4>$ au $<$ niveau CITE $\mathbf{6}>$.

\section{Q19 Quelle activité exerce actuellement votre père ?}

(Ne cochez qu'une seule case.)

Travail <rémunéré> à temps plein.

Travail <rémunéré> à temps partiel.

Ne travaille pas, mais cherche un emploi.

Autre (par exemple, homme au foyer, retraité).

\section{Q20 Dans quel pays vos parents et vous-même êtes-vous nés ?}

(ST20) (Ne cochez qu'une case par colonne.)

\begin{tabular}{|c|c|c|c|}
\hline & Vous & Votre mère & Votre père \\
\hline$<$ Pays A $>$. & $\square_{1}$ & $\square_{1}$ & $\square_{1}$ \\
\hline$<$ Pays B $>$. & $\square_{2}$ & $\square_{2}$ & $\square_{2}$ \\
\hline$<$ Pays C>. & $\square_{3}$ & $\square_{3}$ & $\square_{3}$ \\
\hline$<$ Pays D>. & $\square_{4}$ & $\square_{4}$ & $\square_{4}$ \\
\hline$<\ldots$ etc. $>$. & $\square_{5}$ & $\square_{5}$ & $\square_{5}$ \\
\hline Autre pays. & $\square_{6}$ & $\square_{6}$ & $\square_{6}$ \\
\hline
\end{tabular}

Note : <Pays A> est généralement le pays dans lequel se fait l'évaluation. "Autre pays » est habituellement la dernière variable proposée.

Entre ces deux variables, les pays ou économies participants peuvent choisir plusieurs pays d'origine.

(Ne cochez qu'une seule case.)

\begin{tabular}{|l|c}
\hline$<$ Langue $1>$. & $\square_{1}$ \\
\hline$<$ Langue $2>$. & $\square_{2}$ \\
\hline$<$ Langue 3>. & $\square_{3}$ \\
\hline$<$ <..etc. $>$. & $\square_{4}$ \\
\hline Autre langue. & $\square_{5}$ \\
\hline
\end{tabular}




\section{Q23 À la maison, disposez-vous des choses suivantes?}

(ST26) (Cochez une case par ligne.)

\begin{tabular}{|c|c|c|c|}
\hline & & Oui & Non \\
\hline a) & Un bureau ou une table pour travailler. & $\square_{1}$ & $\square_{2}$ \\
\hline b) & Une chambre pour vous seul. & $\square_{1}$ & $\square_{2}$ \\
\hline c) & Un endroit calme pour travailler. & $\square_{1}$ & $\square_{2}$ \\
\hline d) & Un ordinateur dont vous pouvez vous servir pour votre travail scolaire. & $\square_{1}$ & $\square_{2}$ \\
\hline e) & Des logiciels éducatifs. & $\square_{1}$ & $\square_{2}$ \\
\hline f) & Une connexion à Internet. & $\square_{1}$ & $\square_{2}$ \\
\hline g) & De la littérature classique (par exemple, $<$ Victor Hugo $>$ ). & $\square_{1}$ & $\square_{2}$ \\
\hline h) & Des recueils de poésie. & $\square_{1}$ & $\square_{2}$ \\
\hline i) & Des œuvres d'art (par exemple, des tableaux). & $\square_{1}$ & $\square_{2}$ \\
\hline j) & Des livres utiles à votre travail scolaire. & $\square_{1}$ & $\square_{2}$ \\
\hline k) & Des < ouvrages techniques de référence $>$. & $\square_{1}$ & $\square_{2}$ \\
\hline l) & Un dictionnaire. & $\square_{1}$ & $\square_{2}$ \\
\hline m) & Un lave-vaisselle. & $\square_{1}$ & $\square_{2}$ \\
\hline n) & Un lecteur de <DVD $>$. & $\square_{1}$ & $\square_{2}$ \\
\hline o) & $<$ Item national d'aisance économique $1>$. & $\square_{1}$ & $\square_{2}$ \\
\hline p) & $<$ Item national d'aisance économique $2>$. & $\square_{1}$ & $\square_{2}$ \\
\hline q) & $<$ Item national d'aisance économique 3>. & $\square_{1}$ & $\square_{2}$ \\
\hline
\end{tabular}

\section{Q24 Y a-t-il chez vous les choses suivantes et, si oui, combien ?}

(ST27) (Cochez une case par ligne.)

\begin{tabular}{c|l|c|c|c|c}
\hline \multicolumn{2}{l|}{} & Aucun(e) & Un(e) & Deux & Trois ou plus \\
\hline a) & Téléphone portable. & $\square_{1}$ & $\square_{2}$ & $\square_{3}$ & $\square_{4}$ \\
\hline b) & Télévision. & $\square_{1}$ & $\square_{2}$ & $\square_{3}$ & $\square_{4}$ \\
\hline c) & Ordinateur. & $\square_{1}$ & $\square_{2}$ & $\square_{3}$ & $\square_{4}$ \\
\hline d) & Voiture. & $\square_{1}$ & $\square_{2}$ & $\square_{3}$ & $\square_{4}$ \\
\hline e) & Pièce équipée d'une baignoire ou d'une douche. & $\square_{1}$ & $\square_{2}$ & $\square_{3}$ & $\square_{4}$ \\
\hline
\end{tabular}

\section{Combien de livres y a-t-il chez vous?}

On compte environ 40 livres par mètre d'étagère. Ne tenez pas compte des magazines et des journaux, ni de vos manuels scolaires.

(Ne cochez qu'une seule case.)

0 à 10 livres.

11 à 25 livres.

26 à 100 livres.

101 à 200 livres.

201 à 500 livres.

Plus de 500 livres. 


\section{L'apprentissage des mathématiques}

Q26 Pensez aux mathématiques. Dans quelle mesure êtes-vous d'accord avec les affirmations suivantes ?

(ST29) (Cochez une case par ligne.)

\begin{tabular}{|c|c|c|c|c|c|}
\hline & & $\begin{array}{l}\text { Tout à fait } \\
\text { d'accord }\end{array}$ & D'accord & Pas d'accord & $\begin{array}{l}\text { Pas du tout } \\
\text { d'accord }\end{array}$ \\
\hline a) & J'aime bien lire des textes qui traitent de mathématiques. & $\square_{1}$ & $\square_{2}$ & $\square_{3}$ & $\square_{4}$ \\
\hline b) & $\begin{array}{l}\text { Cela vaut la peine de faire des efforts en mathématiques, } \\
\text { car cela m'aidera dans le métier que je veux faire plus tard. }\end{array}$ & $\square_{1}$ & $\square_{2}$ & $\square_{3}$ & $\square_{4}$ \\
\hline c) & J'attends mes cours de mathématiques avec impatience. & $\square_{1}$ & $\square_{2}$ & $\square_{3}$ & $\square_{4}$ \\
\hline d) & Je fais des mathématiques parce que cela me plaît. & $\square_{1}$ & $\square_{2}$ & $\square_{3}$ & $\square_{4}$ \\
\hline e) & $\begin{array}{l}\text { Pour moi, cela vaut la peine d'apprendre les } \\
\text { mathématiques, car cela améliore mes < perspectives, } \\
\text { chances }>\text { de carrière professionnelle. }\end{array}$ & $\square_{1}$ & $\square_{2}$ & $\square_{3}$ & $\square_{4}$ \\
\hline f) & Je m'intéresse aux choses que j’apprends en mathématiques. & $\square_{1}$ & $\square_{2}$ & $\square_{3}$ & $\square_{4}$ \\
\hline g) & $\begin{array}{l}\text { Les mathématiques sont une matière importante pour moi, } \\
\text { parce qu'elles sont nécessaires pour les études que je veux } \\
\text { faire plus tard. }\end{array}$ & $\square_{1}$ & $\square_{2}$ & $\square_{3}$ & $\square_{4}$ \\
\hline h) & $\begin{array}{l}\text { En mathématiques, je vais apprendre beaucoup de choses } \\
\text { qui m'aideront à trouver du travail. }\end{array}$ & $\square_{1}$ & $\square_{2}$ & $\square_{3}$ & $\square_{4}$ \\
\hline
\end{tabular}

Pensez à la manière dont les personnes importantes pour vous considèrent les mathématiques. Dans quelle mesure êtes-vous d'accord avec les affirmations suivantes ?

(Cochez une case par ligne.)

\begin{tabular}{|c|c|c|c|c|c|}
\hline & & $\begin{array}{l}\text { Tout à fait } \\
\text { d'accord }\end{array}$ & D'accord & Pas d'accord & $\begin{array}{l}\text { Pas du tout } \\
\text { d'accord }\end{array}$ \\
\hline a) & La plupart de mes amis ont de bons résultats en mathématiques. & $\square_{1}$ & $\square_{2}$ & $\square_{3}$ & $\square_{4}$ \\
\hline b) & $\begin{array}{l}\text { La plupart de mes amis travaillent beaucoup } \\
\text { en mathématiques. }\end{array}$ & $\square_{1}$ & & & \\
\hline C) & $\begin{array}{l}\text { Mes amis prennent plaisir à faire les contrôles } \\
\text { de mathématiques. }\end{array}$ & $\square_{1}$ & & $\square_{3}$ & $\square_{4}$ \\
\hline d) & $\begin{array}{l}\text { Mes parents pensent qu'il est important pour moi d'étudier } \\
\text { les mathématiques. }\end{array}$ & $\square_{1}$ & $\square_{2}$ & & $\square_{4}$ \\
\hline e) & $\begin{array}{l}\text { Mes parents pensent que les mathématiques sont importantes } \\
\text { pour ma carrière. }\end{array}$ & $\square_{1}$ & $\square_{2}$ & $\square_{3}$ & $\square_{4}$ \\
\hline f) & Mes parents aiment bien les mathématiques. & $\square_{1}$ & $\square_{2}$ & $\square_{3}$ & $\square_{4}$ \\
\hline
\end{tabular}

\section{sûr d'y arriver}

(Cochez une case par ligne.)

\begin{tabular}{|c|c|c|c|c|c|}
\hline a) & $\begin{array}{l}\text { Utiliser un }<\text { horaire de trains }>\text {, pour calculer combien } \\
\text { de temps prendrait le trajet d'un endroit à un autre. }\end{array}$ & $\square_{1}$ & $\square_{2}$ & $\square_{3}$ & $\square_{4}$ \\
\hline b) & $\begin{array}{l}\text { Calculer de combien diminuerait le prix d'un poste } \\
\text { de télévision après une réduction de } 30 \% \text {. }\end{array}$ & $\square_{1}$ & $\square_{2}$ & $\square_{3}$ & $\square_{4}$ \\
\hline c) & $\begin{array}{l}\text { Calculer combien de mètres carrés de dalles il faut pour } \\
\text { carreler un sol. }\end{array}$ & $\square_{1}$ & $\square_{2}$ & $\square_{3}$ & $\square_{4}$ \\
\hline d) & Comprendre les graphiques présentés dans les journaux. & $\square_{1}$ & $\square_{2}$ & $\square_{3}$ & $\square_{4}$ \\
\hline e) & Résoudre une équation du type : $3 x+5=17$. & $\square_{1}$ & $\square_{2}$ & $\square_{3}$ & $\square_{4}$ \\
\hline f) & $\begin{array}{l}\text { Calculer la distance réelle entre deux endroits sur une carte } \\
\text { à l'échelle } 1 / 10000 .\end{array}$ & $\square_{1}$ & $\square_{2}$ & $\square_{3}$ & $\square_{4}$ \\
\hline g) & Résoudre une équation du type : $2(x+3)=(x+3)(x-3)$. & $\square_{1}$ & $\square_{2}$ & $\square_{3}$ & $\square_{4}$ \\
\hline h) & Calculer la consommation d'essence d'une voiture. & $\square_{1}$ & $\square_{2}$ & $\square_{3}$ & $\square_{4}$ \\
\hline
\end{tabular}


Pensez à ce qui se passe quand vous étudiez les mathématiques. Dans quelle mesure êtes-vous d'accord avec les affirmations ci-dessous?

(Cochez une case par ligne.)

\begin{tabular}{c|l|c|c|c|c}
\multicolumn{1}{c|}{} & $\begin{array}{c}\text { Tout à fait } \\
\text { d'accord }\end{array}$ & D'accord & Pas d'accord & $\begin{array}{c}\text { Pas du tout } \\
\text { d'accord }\end{array}$ \\
\hline a) & $\begin{array}{l}\text { Je m'inquiète souvent en pensant que j'aurai des difficultés } \\
\text { en cours de mathématiques. }\end{array}$ & $\square_{1}$ & $\square_{2}$ & $\square_{3}$ & $\square_{4}$ \\
\hline b) & Je ne suis tout simplement pas bon en mathématiques. & $\square_{1}$ & $\square_{2}$ & $\square_{3}$ & $\square_{4}$ \\
\hline c) & Je suis très tendu quand j'ai un devoir de mathématiques à faire. & $\square_{1}$ & $\square_{2}$ & $\square_{3}$ & $\square_{4}$ \\
\hline d) & J'ai de bonnes <notes> en mathématiques. & $\square_{1}$ & $\square_{2}$ & $\square_{3}$ & $\square_{4}$ \\
\hline e) & $\begin{array}{l}\text { Je deviens très nerveux quand je travaille à des problèmes } \\
\text { de mathématiques. }\end{array}$ & $\square_{1}$ & $\square_{2}$ & $\square_{3}$ & $\square_{4}$ \\
\hline f) & J'apprends vite en mathématiques. & $\square_{1}$ & $\square_{2}$ & $\square_{3}$ & $\square_{4}$ \\
\hline g) & $\begin{array}{l}\text { J'ai toujours pensé que les mathématiques sont une des matières } \\
\text { où je suis le plus fort. }\end{array}$ & $\square_{1}$ & $\square_{2}$ & $\square_{3}$ & $\square_{4}$ \\
\hline h) & $\begin{array}{l}\text { Je me sens perdu quand j'essaie de résoudre un problème } \\
\text { de mathématiques. }\end{array}$ & $\square_{1}$ & $\square_{2}$ & $\square_{3}$ & $\square_{4}$ \\
\hline i) & $\begin{array}{l}\text { En cours de mathématiques, je comprends même les exercices } \\
\text { les plus difficiles. }\end{array}$ & $\square_{1}$ & $\square_{2}$ & $\square_{3}$ & $\square_{4}$ \\
\hline j) & $\begin{array}{l}\text { Je m'inquiète à l'idée d'avoir de mauvaises <notes> } \\
\text { en mathématiques. }\end{array}$ & $\square_{1}$ & $\square_{2}$ & $\square_{3}$ & $\square_{4}$ \\
\hline
\end{tabular}

Note : <Notes $>$ - Ce terme fait référence à l'évaluation normalisée de la performance des élèves par les enseignants, dans un cours ou une matière, et doit être adapté en fonction du contexte national.

Q30 Pensez à vos cours de mathématiques. Dans quelle mesure êtes-vous d'accord avec les affirmations suivantes ?

(Cochez une case par ligne.)

\begin{tabular}{c|l|c|c|c|c}
\multicolumn{2}{l|}{} & $\begin{array}{c}\text { Tout à fait } \\
\text { d'accord }\end{array}$ & D'accord & Pas d'accord & $\begin{array}{c}\text { Pas du tout } \\
\text { d'accord }\end{array}$ \\
\hline a) & Si je fais suffisamment d'efforts, je peux réussir en mathématiques. & $\square_{1}$ & $\square_{2}$ & $\square_{3}$ & $\square_{4}$ \\
\hline b) & Réussir ou échouer en mathématiques ne dépend que de moi. & $\square_{1}$ & $\square_{2}$ & $\square_{3}$ & $\square_{4}$ \\
\hline c) & $\begin{array}{l}\text { Des obligations familiales ou autres m'empêchent de consacrer } \\
\text { beaucoup de temps à mon travail en mathématiques. }\end{array}$ & $\square_{1}$ & $\square_{2}$ & $\square_{3}$ & $\square_{4}$ \\
\hline d) & $\begin{array}{l}\text { Je ferais plus d'efforts en mathématiques si j'avais d'autres } \\
\text { professeurs. }\end{array}$ & $\square_{1}$ & $\square_{2}$ & $\square_{3}$ & $\square_{4}$ \\
\hline e) & $\begin{array}{l}\text { Si je le voulais, je pourrais avoir de bons résultats } \\
\text { en mathématiques. }\end{array}$ & $\square_{1}$ & $\square_{2}$ & $\square_{3}$ & $\square_{4}$ \\
\hline f) & $\begin{array}{l}\text { Que j'étudie ou non pour mes examens, je n'ai pas de bons } \\
\text { résultats en mathématiques. }\end{array}$ & $\square_{1}$ & $\square_{2}$ & $\square_{3}$ & $\square_{4}$ \\
\hline
\end{tabular}

Supposez que vous vous trouvez dans la situation suivante :

Chaque semaine, votre professeur de mathématiques fait un petit contrôle. Ces derniers temps, vous avez obtenu de mauvais résultats à ces contrôles. Aujourd'hui, vous essayez de comprendre pourquoi.

Dans quelle mesure est-il probable que vous ayez les réactions suivantes dans cette situation ?

(Cochez une case par ligne.)

\begin{tabular}{c|l|c|c|c}
\multicolumn{1}{l|}{} & $\begin{array}{c}\text { Très } \\
\text { probable }\end{array}$ & Probable & $\begin{array}{c}\text { Peu } \\
\text { probable }\end{array}$ & $\begin{array}{c}\text { Très peu } \\
\text { probable }\end{array}$ \\
\hline a) & $\begin{array}{l}\text { Je ne suis pas très bon en résolution de problèmes } \\
\text { mathématiques. }\end{array}$ & $\square_{1}$ & $\square_{2}$ & $\square_{3}$ \\
\hline b) & Cette semaine, mon professeur n'a pas bien expliqué cette matière. & $\square_{1}$ & $\square_{2}$ & $\square_{3}$ \\
\hline c) & Cette semaine, j'ai mal répondu aux questions du contrôle & $\square_{1}$ & $\square_{2}$ & $\square_{3}$ \\
\hline d) & Parfois le contenu du cours est trop difficile. & $\square_{1}$ & $\square_{2}$ & $\square_{3}$ \\
\hline e) & Le professeur n'a pas réussi à intéresser les élèves à cette matière. & $\square_{1}$ & $\square_{2}$ & $\square_{3}$ \\
\hline f) & Parfois, je n'ai simplement pas de chance. & $\square_{1}$ & $\square_{2}$ & $\square_{3}$ \\
\hline
\end{tabular}


Pensez aux mathématiques que vous faites pour l'école. Dans quelle mesure êtes-vous d'accord avec les affirmations suivantes?

(Cochez une case par ligne.)

\begin{tabular}{|c|c|c|c|c|c|}
\hline & & $\begin{array}{l}\text { Tout à fait } \\
\text { d'accord }\end{array}$ & D'accord & Pas d'accord & $\begin{array}{l}\text { Pas du tout } \\
\text { d'accord }\end{array}$ \\
\hline a) & Je fais mes devoirs de mathématiques à temps. & $\square_{1}$ & $\square_{2}$ & $\square_{3}$ & $\square_{4}$ \\
\hline b) & Je travaille dur à mes devoirs de mathématiques. & $\square_{1}$ & $\square_{2}$ & $\square_{3}$ & $\square_{4}$ \\
\hline c) & Je prépare bien mes examens de mathématiques. & $\square_{1}$ & $\square_{2}$ & $\square_{3}$ & $\square_{4}$ \\
\hline d) & J'étudie beaucoup pour les contrôles de mathématiques. & $\square_{1}$ & $\square_{2}$ & $\square_{3}$ & $\square_{4}$ \\
\hline e) & $\begin{array}{l}\text { J'étudie jusqu'à ce que je comprenne la matière } \\
\text { en mathématiques. }\end{array}$ & $\square_{1}$ & $\square_{2}$ & $\square_{3}$ & $\square_{4}$ \\
\hline f) & Je suis attentif pendant les cours de mathématiques. & $\square_{1}$ & $\square_{2}$ & $\square_{3}$ & $\square_{4}$ \\
\hline g) & J'écoute pendant les cours de mathématiques. & $\square_{1}$ & $\square_{2}$ & $\square_{3}$ & $\square_{4}$ \\
\hline h) & $\begin{array}{l}\text { J'évite les distractions quand j'étudie mes leçons } \\
\text { de mathématiques. }\end{array}$ & $\square_{1}$ & $\square_{2}$ & $\square_{3}$ & $\square_{4}$ \\
\hline i) & Je travaille de façon organisée en mathématiques. & $\square_{1}$ & $\square_{2}$ & $\square_{3}$ & $\square_{4}$ \\
\hline
\end{tabular}

\section{Q33 Dans chaque paire d'affirmations, choisissez celle qui vous convient le mieux.}

(ST 48) (Ne cochez qu'une des deux cases suivantes.)

J'ai l'intention de suivre des cours supplémentaires en mathématiques quand j'aurai fini l'école.

a)

J'ai l'intention de suivre des cours supplémentaires en <langue de l'évaluation> quand j'aurai fini l'école.

J'ai décidé de $\mathrm{m}^{\prime}$ orienter vers des études $\mathrm{d}^{\prime}<$ enseignement postsecondaire > où les compétences

b) en mathématiques sont nécessaires.

J'ai décidé de $\mathrm{m}^{\prime}$ orienter vers des études $\mathrm{d}^{\prime}<$ enseignement postsecondaire> où les compétences en sciences sont nécessaires.

c)

J'ai envie de travailler plus en mathématiques que ce qui est demandé en classe.

J'ai envie de travailler plus en <langue de l'évaluation> que ce qui est demandé en classe.

d)

J'ai décidé de <suivre> autant de cours de mathématiques que possible pendant mes études.

J'ai décidé de <suivre> autant de cours de sciences que possible pendant mes études.

e)

J'ai l'intention de choisir une profession en rapport avec les mathématiques.

J'ai l'intention de choisir une profession en rapport avec les sciences.

Notes : <Langue de l'évaluation> - Désigne la langue d'enseignement dans laquelle l'évaluation PISA de la compréhension de l'écrit est administrée. Dans certains pays, la matière liée à l'enseignement de la <langue de l'évaluation> peut être répartie entre différents cours, par exemple des cours de langue et des cours de littérature. Si tel est le cas, le terme <langue de l'évaluation> doit être correctement adapté.

$<$ Sciences $>-$ Fait uniquement référence à la physique, à la chimie, aux sciences de la Terre et à la biologie, que ces disciplines scientifiques soient enseignées dans le programme national sous forme de matières distinctes ou d'un seul cours de sciences « intégré ». Le terme ne recouvre pas diverses disciplines liées aux sciences, comme l'ingénierie, la technologie, les mathématiques, la psychologie, l'économie, ni les aspects des sciences de la Terre qui sont parfois abordés dans les programmes de géographie. Dans de nombreux pays, cette expression a été adaptée au contexte national.

<Enseignement postsecondaire> - Cette expression fait référence au niveau d'enseignement universitaire ou tertiaire. Il y aura lieu d'adapter cette expression au contexte national.

L'expression « <suivre> des cours » fait référence aux élèves qui ont le choix de sélectionner des cours en option à l'école. Les pays ayant des programmes obligatoires, qui ne permettent pas de choix ou de cours en option, devront adapter ce terme à leur contexte national. 
Q34 Faites-vous souvent les choses suivantes à l'école et en dehors de l'école ?

(ST49) (Cochez une case par ligne.)

\begin{tabular}{|c|c|c|c|c|c|}
\hline & & $\begin{array}{c}\text { Toujours } \\
\text { ou presque } \\
\text { toujours }\end{array}$ & Souvent & Parfois & $\begin{array}{l}\text { Jamais ou } \\
\text { rarement }\end{array}$ \\
\hline a) & Je discute de problèmes de mathématiques avec mes amis. & $\square_{1}$ & $\square_{2}$ & $\square_{3}$ & $\square_{4}$ \\
\hline b) & J'aide mes amis en mathématiques. & $\square_{1}$ & $\square_{2}$ & $\square_{3}$ & $\square_{4}$ \\
\hline C) & $\begin{array}{l}\text { Je participe à des activités mathématiques <en dehors } \\
\text { du programme des cours }>\text {. }\end{array}$ & $\square_{1}$ & $\square_{2}$ & $\square_{3}$ & $\square_{4}$ \\
\hline d) & Je participe à des compétitions de mathématiques. & $\square_{1}$ & $\square_{2}$ & $\square_{3}$ & $\square_{4}$ \\
\hline e) & $\begin{array}{l}\text { Je fais plus de deux heures de mathématiques par jour } \\
\text { en dehors de l'école. }\end{array}$ & $\square_{1}$ & $\square_{2}$ & $\square_{3}$ & $\square_{4}$ \\
\hline f) & Je joue aux échecs. & $\square_{1}$ & $\square_{2}$ & $\square_{3}$ & $\square_{4}$ \\
\hline g) & Je fais de la programmation informatique. & $\square_{1}$ & $\square_{2}$ & $\square_{3}$ & $\square_{4}$ \\
\hline h) & Je suis membre d'un club de mathématiques. & $\square_{1}$ & $\square_{2}$ & $\square_{3}$ & $\square_{4}$ \\
\hline
\end{tabular}

Dans chaque groupe de trois affirmations, choisissez celle qui décrit le mieux votre méthode de travail en mathématiques.

(Ne cochez qu'une des trois cases suivantes.)

Quand j'étudie pour un contrôle de mathématiques, j'essaie de déterminer quels sont les points les plus importants à apprendre.

a) Quand j'étudie pour un contrôle de mathématiques, j'essaie de comprendre de nouveaux concepts en les mettant en relation avec des choses que je connais déjà.

Quand j'étudie pour un contrôle de mathématiques, j'essaie d'apprendre le plus de choses possible par cœur.

Quand j'étudie des mathématiques, j'essaie de déterminer quelles sont les notions que je n'ai pas encore bien comprises.

b)

Quand j'étudie des mathématiques, je réfléchis à de nouvelles méthodes pour trouver la réponse.

Quand j'étudie des mathématiques, je m’oblige à vérifier si j'ai bien retenu les points sur lesquels j'ai déjà travaillé.

Quand j'étudie des mathématiques, j'essaie de faire le lien avec ce que j'ai appris dans d'autres matières.

C)

Quand j'étudie des mathématiques, je commence par déterminer exactement ce que je dois apprendre.

Quand j'étudie des mathématiques, je refais certains problèmes si souvent que j'ai l'impression de pouvoir les résoudre les yeux fermés.

Pour bien retenir la méthode à suivre pour résoudre un problème de mathématiques, je revois les exemples encore et encore.

d)

Je pense à la manière d'utiliser dans la vie de tous les jours les concepts mathématiques que j'ai appris.

Quand je ne comprends pas quelque chose en mathématiques, je cherche toujours un complément

d'informations pour mieux cerner le problème. 
Habituellement, combien d'heures par semaine consacrez-vous à suivre des cours dans les matières suivantes <en dehors des heures de classe normales $>$ ?

Il s'agit uniquement ici de cours dans des matières que vous étudiez aussi à l'école, mais que vous suivez éventuellement en complément, en dehors des heures de cours normales. Ces cours peuvent être donnés dans votre établissement,

chez vous ou à un autre endroit.

(Cochez une case par ligne.)

\begin{tabular}{|c|c|c|c|c|c|c|}
\hline & & $\begin{array}{c}\text { Je ne suis } \\
\text { pas de cours } \\
\text { <en dehors } \\
\text { des heures } \\
\text { de classe } \\
\text { normales> } \\
\text { dans cette } \\
\text { matière }\end{array}$ & $\begin{array}{l}\text { Moins de } \\
2 \text { heures par } \\
\text { semaine }\end{array}$ & $\begin{array}{l}2 \text { heures ou } \\
\text { plus, mais } \\
\text { moins de } \\
4 \text { heures par } \\
\text { semaine }\end{array}$ & $\begin{array}{l}4 \text { heures ou } \\
\text { plus, mais } \\
\text { moins de } \\
6 \text { heures par } \\
\text { semaine }\end{array}$ & $\begin{array}{l}6 \text { heures } \\
\text { ou plus pa } \\
\text { semaine }\end{array}$ \\
\hline a) & $<$ Langue de l'évaluation $>$. & $\square_{1}$ & $\square_{2}$ & $\square_{3}$ & $\square_{4}$ & $\square_{5}$ \\
\hline b) & Mathématiques. & $\square_{1}$ & $\square_{2}$ & $\square_{3}$ & $\square_{4}$ & $\square_{5}$ \\
\hline c) & $<$ Sciences $>$. & $\square_{1}$ & $\square_{2}$ & $\square_{3}$ & $\square_{4}$ & $\square_{5}$ \\
\hline d) & Autres matières. & $\square_{1}$ & $\square_{2}$ & $\square_{3}$ & $\square_{4}$ & $\square_{5}$ \\
\hline
\end{tabular}

Notes : "Cours <en dehors des heures de classe normales > - Cours que les élèves suivent dans leurs matières scolaires en dehors de leur horaire normal. Ces cours peuvent leur être dispensés à l'école, à leur domicile ou ailleurs. Veuillez vous référer à la Question 33 pour une définition de la $<$ langue de l'évaluation $>$ et des $<$ sciences $>$. aux activités suivantes?

Tenez également compte du temps passé pendant les week-ends.

\begin{tabular}{c|l|c}
\multicolumn{2}{l}{} & $\begin{array}{c}\text { Heure(s) par } \\
\text { semaine }\end{array}$ \\
\hline a) & Devoirs et autres travaux scolaires imposés par vos professeurs. & \\
\hline b) & $\begin{array}{l}\text { Sur le temps que vous consacrez au point a), combien d'heures passez-vous avec une personne qui } \\
\text { supervise votre travail et vous aide si nécessaire (« travail scolaire dirigé »), à l'école ou ailleurs ? }\end{array}$ & \\
\hline c) & Travail avec un <professeur> particulier (payé ou non). & \\
\hline d) & Cours en dehors des heures de classe normales, organisés par une société commerciale et payés par vos parents. & \\
\hline e) & Étude avec l'un de vos parents ou un autre membre de la famille. & \\
\hline f) & $\begin{array}{l}\text { Travail sur l'ordinateur pour réviser la matière vue en classe et faire des exercices (par exemple, } \\
\text { apprendre du vocabulaire avec un logiciel éducatif). }\end{array}$ & \\
\hline
\end{tabular}

Note : Cours <en dehors des heures de classe normales $>$ - Cours que les élèves suivent dans leurs matières scolaires en dehors de leur horaire normal. Ces cours peuvent leur être dispensés à l'école, à leur domicile ou ailleurs.

Q38 À quelle fréquence avez-vous eu les types de tâches mathématiques suivantes à l'école ?

(ST61) (Cochez une case par ligne.)

\begin{tabular}{|c|c|c|c|c|c|}
\hline & & Fréquemment & Parfois & Rarement & Jamais \\
\hline a) & $\begin{array}{l}\text { Utiliser un <horaire de trains }>\text { pour calculer combien } \\
\text { de temps prendrait le trajet d'un endroit à un autre. }\end{array}$ & $\square_{1}$ & $\square_{2}$ & $\square_{3}$ & $\square_{4}$ \\
\hline b) & $\begin{array}{l}\text { Calculer l'augmentation du prix d'un ordinateur après } \\
\text { ajout de la taxe. }\end{array}$ & $\square_{1}$ & $\square_{2}$ & $\square_{3}$ & $\square_{4}$ \\
\hline c) & $\begin{array}{l}\text { Calculer combien de mètres carrés de dalles il faut pour } \\
\text { carreler un sol. }\end{array}$ & $\square_{1}$ & $\square_{2}$ & $\square_{3}$ & $\square_{4}$ \\
\hline d) & $\begin{array}{l}\text { Comprendre des tableaux scientifiques présentés dans } \\
\text { un article. }\end{array}$ & $\square_{1}$ & $\square_{2}$ & $\square_{3}$ & $\square_{4}$ \\
\hline e) & Résoudre une équation du type : $6 x^{2}+5=29$. & $\square_{1}$ & $\square_{2}$ & $\square_{3}$ & $\square_{4}$ \\
\hline f) & $\begin{array}{l}\text { Calculer la distance réelle entre deux endroits sur une } \\
\text { carte à l'échelle } 1 / 10000 .\end{array}$ & $\square_{1}$ & $\square_{2}$ & $\square_{3}$ & $\square_{4}$ \\
\hline g) & Résoudre une équation du type : $2(x+3)=(x+3)(x-3)$. & $\square_{1}$ & $\square_{2}$ & $\square_{3}$ & $\square_{4}$ \\
\hline h) & $\begin{array}{l}\text { Calculer la consommation hebdomadaire d'un appareil } \\
\text { électrique. }\end{array}$ & $\square_{1}$ & $\square_{2}$ & $\square_{3}$ & $\square_{4}$ \\
\hline i) & Résoudre une équation du type : $3 x+5=17$ & $\square_{1}$ & $\square_{2}$ & $\square_{3}$ & $\square_{4}$ \\
\hline
\end{tabular}


Q39 Pensez aux concepts mathématiques. Dans quelle mesure les termes suivants vous sont-ils familiers ?

(ST62) (Cochez une case par ligne.)

\begin{tabular}{l|l|c|c|c|c|c}
\multicolumn{2}{l|}{} & $\begin{array}{c}\text { Je n'en } \\
\text { ai jamais } \\
\text { entendu } \\
\text { parler }\end{array}$ & $\begin{array}{c}\text { J'en ai } \\
\text { entendu } \\
\text { parler une } \\
\text { fois ou deux }\end{array}$ & $\begin{array}{c}\text { J'en ai } \\
\text { entendu } \\
\text { parrer } \\
\text { quelques fois }\end{array}$ & $\begin{array}{c}\text { J'en ai } \\
\text { souvent } \\
\text { entendu } \\
\text { parler }\end{array}$ & $\begin{array}{c}\text { Je connais et } \\
\text { comprends } \\
\text { le concept }\end{array}$ \\
\hline a) & Fonction exponentielle. & $\square_{1}$ & $\square_{2}$ & $\square_{3}$ & $\square_{4}$ & $\square_{5}$ \\
\hline b) & Diviseur. & $\square_{1}$ & $\square_{2}$ & $\square_{3}$ & $\square_{4}$ & $\square_{5}$ \\
\hline c) & Fonction du second degré. & $\square_{1}$ & $\square_{2}$ & $\square_{3}$ & $\square_{4}$ & $\square_{5}$ \\
\hline d) & <Nombre propre>. & $\square_{1}$ & $\square_{2}$ & $\square_{3}$ & $\square_{4}$ & $\square_{5}$ \\
\hline e) & Équation linéaire. & $\square_{1}$ & $\square_{2}$ & $\square_{3}$ & $\square_{4}$ & $\square_{5}$ \\
\hline f) & Vecteurs. & $\square_{1}$ & $\square_{2}$ & $\square_{3}$ & $\square_{4}$ & $\square_{5}$ \\
\hline g) & Nombre complexe. & $\square_{1}$ & $\square_{2}$ & $\square_{3}$ & $\square_{4}$ & $\square_{5}$ \\
\hline h) & Nombre rationnel. & $\square_{1}$ & $\square_{2}$ & $\square_{3}$ & $\square_{4}$ & $\square_{5}$ \\
\hline i) & Radicaux. & $\square_{1}$ & $\square_{2}$ & $\square_{3}$ & $\square_{4}$ & $\square_{5}$ \\
\hline j) & $<$ Échelle subjonctive>. & $\square_{2}$ & $\square_{3}$ & $\square_{4}$ & $\square_{5}$ \\
\hline k) & Polygone. & $\square_{1}$ & $\square_{2}$ & $\square_{3}$ & $\square_{4}$ & $\square_{5}$ \\
\hline l) & $<$ Fraction déclarative>. & $\square_{1}$ & $\square_{2}$ & $\square_{3}$ & $\square_{4}$ & $\square_{5}$ \\
\hline m) & Figure isométrique. & $\square_{1}$ & $\square_{2}$ & $\square_{3}$ & $\square_{4}$ & $\square_{5}$ \\
\hline n) & Cosinus. & $\square_{1}$ & $\square_{2}$ & $\square_{3}$ & $\square_{4}$ & $\square_{5}$ \\
\hline o) & Moyenne arithmétique. & $\square_{2}$ & $\square_{3}$ & $\square_{4}$ & $\square_{5}$ \\
\hline p) & Probabilité. & & &
\end{tabular}

Q40 En moyenne, combien de minutes dure une <période de cours> dans les matières suivantes?

\begin{tabular}{c|l|l}
\hline a) & Nombre de minutes dans une $<$ période de cours $>$ de $<$ langue de l'évaluation $>$. & \\
\hline b) & Nombre de minutes dans une $<$ période de cours $>$ de $<$ mathématiques $>$. & \\
\hline c) & Nombre de minutes dans une $<$ période de cours $>$ de $<$ sciences $>$. & \\
\hline
\end{tabular}

Notes : $<$ Période de cours $>-$ Le temps que dure chaque cours durant une semaine de classe ordinaire.

Veuillez vous référer à la Question 33 pour une définition de la <langue de l'évaluation > et des $<$ sciences $>$.

Q41 Dans une semaine de cours ordinaire, combien de < périodes de cours> avez-vous dans les matières (ST70) suivantes?

\begin{tabular}{c|l|l}
\hline a) & Nombre de <périodes de cours $>$ de $<$ langue de l'évaluation $>$ par semaine. & \\
\hline b) & Nombre de $<$ périodes de cours $>$ de mathématiques par semaine. & \\
\hline c) & Nombre de < périodes de cours $>$ de $<$ sciences $>$ par semaine. & \\
\hline
\end{tabular}

Notes : Veuillez vous référer à la Question 40 pour une définition de la <période de cours $>$.

Veuillez vous référer à la Question 33 pour une définition de la <langue de l'évaluation $>$ et des $<$ sciences $>$.

Q42 Combien de < périodes de cours > avez-vous <en tout $>$ dans une semaine de cours ordinaire complète? (ST71) 
Q43 En moyenne, combien d'élèves sont présents dans votre classe de <langue de l'évaluation>?

(ST72)

Élèves.

Note : Veuillez vous référer à la Question 33 pour une définition de la <langue de l'évaluation>.

\section{Votre expérience de différents types de problèmes mathématiques vus à l'école}

L'encadré ci-dessous contient une série de problèmes. Pour chacun d'entre eux, il s'agit de comprendre l'énoncé et d'effectuer
les calculs appropriés. Les problèmes décrivent en général des situations pratiques, mais les chiffres, les personnes et les
lieux sont imaginaires. Vous disposez de toutes les informations nécessaires. Voici deux exemples.

Nous voulons savoir si vous avez eu affaire à ces types de problèmes à l'école.

Il est inutile de résoudre ces problèmes !

(Cochez une case par ligne.)

\begin{tabular}{c|l|c|c|c|c}
\multicolumn{2}{l|}{} & Fréquemment & Parfois & Rarement & Jamais \\
\hline a) & $\begin{array}{l}\text { Avez-vous vu ces types de problèmes en cours } \\
\text { de mathématiques? }\end{array}$ & $\square_{1}$ & $\square_{2}$ & $\square_{3}$ & $\square_{4}$ \\
\hline b) & $\begin{array}{l}\text { Avez-vous eu ces types de problèmes lors des évaluations } \\
\text { à l'école? }\end{array}$ & $\square_{1}$ & $\square_{2}$ & $\square_{3}$ & $\square_{4}$ \\
\hline
\end{tabular}

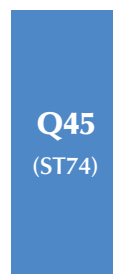

Voici des exemples d'un autre ensemble de compétences mathématiques.

1) Résoudre $2 x+3=7$.

Q45

2) Trouver le volume d'une boîte dont les côtés mesurent $3 \mathrm{~m}, 4 \mathrm{~m}$ et $5 \mathrm{~m}$.

NT74) Nous voulons savoir si vous avez eu affaire à ces types de problèmes à l'école.

Il est inutile de résoudre ces problèmes !

(Cochez une case par ligne.)

\begin{tabular}{c|l|c|c|c}
\hline a) & $\begin{array}{l}\text { Avez-vous vu ces types de problèmes en cours } \\
\text { de mathématiques? }\end{array}$ & $\square_{1}$ & $\square_{2}$ & $\square_{3}$ \\
\hline b) & $\begin{array}{l}\text { Avez-vous eu ces types de problèmes lors des évaluations } \\
\text { à l'école? }\end{array}$ & $\square_{1}$ & $\square_{2}$ & $\square_{3}$ \\
\hline
\end{tabular}


Pour les types de problèmes suivants, il s'agit d'utiliser vos connaissances en mathématiques pour tirer des conclusions. Aucune application pratique ne vous est fournie. Voici deux exemples.

1) Pour celui-ci, vous devez utiliser des théorèmes géométriques :

Déterminer la hauteur de la pyramide.

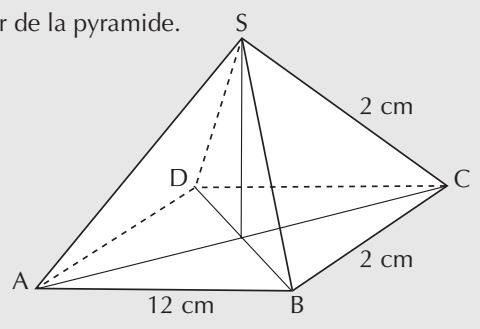

2) Pour celui-ci, vous devez savoir ce qu'est un nombre premier :

Si $n=$ tout nombre, $(n+1)^{2}$ peut-il être un nombre premier ?

Nous voulons savoir si vous avez eu affaire à ces types de problèmes à l'école.

Il est inutile de résoudre ces problèmes !

(Cochez une case par ligne.)

\begin{tabular}{c|l|c|c|c} 
a) & $\begin{array}{l}\text { Avez-vous vu ces types de problèmes en cours } \\
\text { de mathématiques ? }\end{array}$ & $\square_{1}$ & $\square_{2}$ & $\square_{3}$ \\
\hline b) & $\begin{array}{l}\text { Avez-vous eu ces types de problèmes lors } \\
\text { des évaluations à l'école ? }\end{array}$ & $\square_{1}$ & $\square_{2}$ & $\square_{3}$ \\
\hline
\end{tabular}

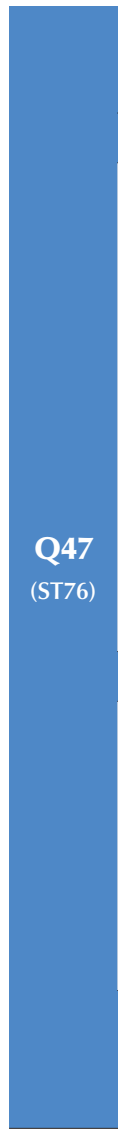

Pour ces types de problèmes, il s'agit d'appliquer des connaissances en mathématiques nécessaires pour résoudre un problème qui se présente dans la vie de tous les jours ou au travail. Les données et les informations portent sur des situations réelles. Voici deux exemples.

Exemple 1:

Lors d'une émission télévisée, un journaliste montre ce graphique et dit :

"Ce graphique montre qu'il y a eu une très forte augmentation du nombre de cambriolages entre 1998 et 1999. .

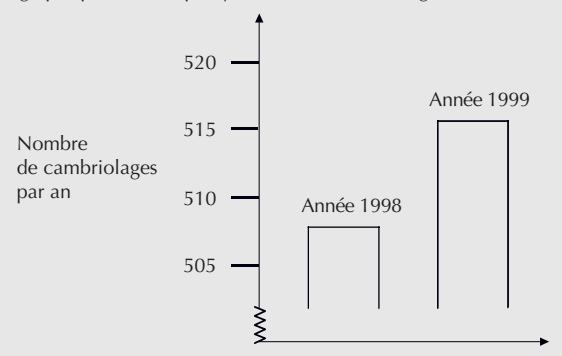

Considérez-vous que l'affirmation du journaliste est une interprétation correcte de ce graphique ?

ustifiez votre réponse par une explication.

Exemple 2:

Pendant longtemps, la relation entre la fréquence cardiaque maximum recommandée et l'âge de la personne a été décrite par la formule suivante:

Fréquence cardiaque maximum recommandée $=\mathbf{2 2 0}$ - âge.

Des recherches récentes ont montré que cette formule devait être légèrement modifiée. La nouvelle formule est :

Fréquence cardiaque maximum recommandée $=208-(0,7 \times$ âge $)$.

À partir de quel âge la fréquence cardiaque maximum recommandée commence-t-elle à augmenter, d'après la nouvelle formule? Montrez votre travail.

Nous voulons savoir si vous avez eu affaire à ces types de problèmes à l'école.

Il est inutile de résoudre ces problèmes !

(Cochez une case par ligne.)

\begin{tabular}{c|l|c|c|c} 
a) & $\begin{array}{l}\text { Avez-vous vu ces types de problèmes en cours } \\
\text { de mathématiques? }\end{array}$ & $\square_{1}$ & $\square_{2}$ & $\square_{3}$ \\
\hline b) & $\begin{array}{l}\text { Avez-vous eu ces types de problèmes lors } \\
\text { des évaluations à l'école? }\end{array}$ & $\square_{1}$ & $\square_{2}$ & $\square_{3}$ \\
\hline
\end{tabular}


Votre expérience en mathématiques

Q48 À quelle fréquence les situations suivantes se présentent-elles durant vos cours de mathématiques ?

(ST77) (Ne cochez qu'une seule case par ligne.)

\begin{tabular}{c|l|c|c|c|c}
\multicolumn{2}{l|}{} & $\begin{array}{c}\text { À chaque } \\
\text { cours }\end{array}$ & $\begin{array}{c}\text { À la plupart } \\
\text { des cours }\end{array}$ & $\begin{array}{c}\text { À quelques } \\
\text { cours }\end{array}$ & $\begin{array}{c}\text { Jamais } \\
\text { ou presque } \\
\text { jamais }\end{array}$ \\
\hline a) & Le professeur s'intéresse aux progrès de chaque élève. & $\square_{1}$ & $\square_{2}$ & $\square_{3}$ & $\square_{4}$ \\
\hline b) & $\begin{array}{l}\text { Le professeur apporte de l'aide supplémentaire quand } \\
\text { les élèves en ont besoin. }\end{array}$ & $\square_{1}$ & $\square_{2}$ & $\square_{3}$ & $\square_{4}$ \\
\hline c) & Le professeur aide les élèves dans leur apprentissage. & $\square_{1}$ & $\square_{2}$ & $\square_{3}$ & $\square_{4}$ \\
\hline d) & $\begin{array}{l}\text { Le professeur continue à expliquer jusqu'à ce que les élèves } \\
\text { aient compris. }\end{array}$ & $\square_{1}$ & $\square_{2}$ & $\square_{3}$ & $\square_{4}$ \\
\hline e) & $\begin{array}{l}\text { Le professeur donne aux élèves l'occasion d'exprimer } \\
\text { leurs opinions. }\end{array}$ & $\square_{1}$ & $\square_{2}$ & $\square_{3}$ & $\square_{4}$ \\
\hline
\end{tabular}

Q49 À quelle fréquence les situations suivantes se présentent-elles durant vos cours de mathématiques ?

(ST79) (Ne cochez qu'une seule case par ligne.)

\begin{tabular}{c|l|c|c|c|c}
\multicolumn{2}{l|}{} & $\begin{array}{c}\text { À chaque } \\
\text { cours }\end{array}$ & $\begin{array}{c}\text { À la plupart } \\
\text { des cours }\end{array}$ & $\begin{array}{c}\text { À quelques } \\
\text { cours }\end{array}$ & $\begin{array}{c}\text { Jamais } \\
\text { ou presque } \\
\text { jamais }\end{array}$ \\
\hline a) & Le professeur nous explique clairement les objectifs de la leçon. & $\square_{1}$ & $\square_{2}$ & $\square_{3}$ & $\square_{4}$ \\
\hline b) & $\begin{array}{l}\text { Le professeur demande à l'un de nous d'expliquer } \\
\text { sa réflexion ou son raisonnement en détail. }\end{array}$ & $\square_{1}$ & $\square_{2}$ & $\square_{3}$ & $\square_{4}$ \\
\hline c) & $\begin{array}{l}\text { Le professeur donne des travaux différents aux élèves qui } \\
\text { ont des difficultés d'apprentissage ou à ceux qui progressent } \\
\text { plus vite. }\end{array}$ & $\square_{1}$ & $\square_{2}$ & $\square_{3}$ & $\square_{4}$ \\
\hline d) & $\begin{array}{l}\text { Le professeur donne des travaux de recherche qui prennent } \\
\text { au moins une semaine de travail. }\end{array}$ & $\square_{1}$ & $\square_{2}$ & $\square_{3}$ & $\square_{4}$ \\
\hline e) & Le professeur me dit si je travaille bien en mathématiques. & $\square_{1}$ & $\square_{2}$ & $\square_{3}$ & $\square_{4}$ \\
\hline f) & $\begin{array}{l}\text { Le professeur nous pose des questions pour s'assurer que } \\
\text { nous avons compris le contenu enseigné. }\end{array}$ & $\square_{1}$ & $\square_{2}$ & $\square_{3}$ & $\square_{4}$ \\
\hline g) & $\begin{array}{l}\text { Le professeur nous fait travailler en petits groupes et nous } \\
\text { demande de résoudre des problèmes ensemble. }\end{array}$ & $\square_{1}$ & $\square_{2}$ & $\square_{3}$ & $\square_{4}$ \\
\hline h) & $\begin{array}{l}\text { Au début du cours, le professeur nous rappelle brièvement } \\
\text { le cours précédent. }\end{array}$ & $\square_{1}$ & $\square_{2}$ & $\square_{3}$ & $\square_{4}$ \\
\hline i) & $\begin{array}{l}\text { Le professeur nous demande de l'aider à planifier } \\
\text { des activités ou des sujets à aborder en classe. }\end{array}$ & $\square_{1}$ & $\square_{2}$ & $\square_{3}$ & $\square_{4}$ \\
\hline j) & $\begin{array}{l}\text { Le professeur me dit quels sont mes points forts et } \\
\text { mes points faibles en mathématiques. }\end{array}$ & $\square_{1}$ & $\square_{2}$ & $\square_{3}$ & $\square_{4}$ \\
\hline k) & $\begin{array}{l}\text { Le professeur nous explique ce qu'il attend de nous quand } \\
\text { nous avons une évaluation, un contrôle ou un devoir. }\end{array}$ & $\square_{1}$ & $\square_{2}$ & $\square_{3}$ & $\square_{4}$ \\
\hline I) & Le professeur nous dit ce que nous devons étudier. & $\square_{1}$ & $\square_{2}$ & $\square_{3}$ & $\square$ \\
\hline m) & $\begin{array}{l}\text { Le professeur me dit ce que je dois faire pour progresser } \\
\text { en mathématiques. }\end{array}$ & $\square_{1}$ & $\square_{2}$ & $\square_{3}$ & $\square_{4}$ \\
\hline
\end{tabular}


Pensez au professeur de mathématiques qui vous a donné votre dernier cours de mathématiques.

\begin{tabular}{c|l|c|c|c}
\multicolumn{1}{l|}{} & $\begin{array}{c}\text { Toujours } \\
\text { ou presque } \\
\text { toujours }\end{array}$ & Souvent & $\begin{array}{c}\text { Jamais } \\
\text { ou presque } \\
\text { jamais }\end{array}$ \\
\hline a) & $\begin{array}{l}\text { Le professeur pose des questions qui nous font réfléchir } \\
\text { au problème. }\end{array}$ & $\square_{1}$ & $\square_{2}$ & $\square_{3}$ \\
\hline b) & $\begin{array}{l}\text { Le professeur nous donne des problèmes qui nécessitent } \\
\text { une longue réflexion de notre part. }\end{array}$ & $\square_{1}$ & $\square_{2}$ & $\square_{3}$ \\
\hline c) & $\begin{array}{l}\text { Le professeur nous demande d'élaborer nos propres } \\
\text { procédures pour résoudre des problèmes complexes. }\end{array}$ & $\square_{1}$ & $\square_{2}$ & $\square_{3}$ \\
\hline d) & $\begin{array}{l}\text { Le professeur nous donne des problèmes pour lesquels } \\
\text { la méthode de résolution n'apparaît pas immédiatement. }\end{array}$ & $\square_{1}$ & $\square_{2}$ & $\square_{3}$ \\
\hline e) & $\begin{array}{l}\text { Le professeur nous donne des problèmes dans différents } \\
\text { contextes afin que nous vérifiions que nous avons compris } \\
\text { les concepts. }\end{array}$ & $\square_{1}$ & $\square_{2}$ & $\square_{3}$ \\
\hline f) & Le professeur nous aide à tirer les leçons de nos erreurs. & $\square_{1}$ & $\square_{2}$ & $\square_{3}$ \\
\hline g) & $\begin{array}{l}\text { Le professeur nous demande d'expliquer comment } \\
\text { nous avons résolu un problème. }\end{array}$ & $\square_{1}$ & $\square_{2}$ & $\square_{3}$ \\
\hline h) & $\begin{array}{l}\text { Le professeur nous présente des problèmes qui } \\
\text { nous amènent à appliquer dans de nouveaux contextes } \\
\text { ce que nous avons appris. }\end{array}$ & $\square_{1}$ & $\square_{2}$ & $\square_{4}$ \\
\hline i) & $\begin{array}{l}\text { Le professeur nous donne des problèmes qui peuvent être } \\
\text { résolus de différentes manières. }\end{array}$ & $\square_{1}$ & $\square_{3}$ & $\square_{4}$ \\
\hline
\end{tabular}

Q51 À quelle fréquence les situations suivantes se présentent-elles durant vos cours de mathématiques ?

(ST81) (Cochez une case par ligne.)

\begin{tabular}{c|l|c|c|c|c}
\multicolumn{2}{l|}{} & $\begin{array}{c}\text { À chaque } \\
\text { cours }\end{array}$ & $\begin{array}{c}\text { À la plupart } \\
\text { des cours }\end{array}$ & $\begin{array}{c}\text { À quelques } \\
\text { cours }\end{array}$ & $\begin{array}{c}\text { Jamais } \\
\text { ou presque } \\
\text { jamais }\end{array}$ \\
\hline a) & Les élèves n'écoutent pas ce que dit le professeur. & $\square_{1}$ & $\square_{2}$ & $\square_{3}$ & $\square_{4}$ \\
\hline b) & Il y a du bruit et de l'agitation. & $\square_{1}$ & $\square_{2}$ & $\square_{3}$ & $\square_{4}$ \\
\hline c) & $\begin{array}{l}\text { Le professeur doit attendre un long moment avant que les élèves } \\
\text { <se calment>. }\end{array}$ & $\square_{1}$ & $\square_{2}$ & $\square_{3}$ & $\square_{4}$ \\
\hline d) & Les élèves ne peuvent pas bien travailler. & $\square_{1}$ & $\square_{2}$ & $\square_{3}$ & $\square_{4}$ \\
\hline e) & $\begin{array}{l}\text { Les élèves ne commencent à travailler que bien après } \\
\text { le début du cours. }\end{array}$ & $\square_{1}$ & $\square_{2}$ & $\square_{3}$ & $\square$ \\
\hline
\end{tabular}

Des descriptions de trois professeurs de mathématiques vous sont proposées ci-dessous. Lisez chaque description et indiquez dans quelle mesure vous êtes d'accord avec l'affirmation qui conclut chaque description.

(Cochez une case par ligne.)

\begin{tabular}{|c|c|c|c|c|c|}
\hline & & $\begin{array}{l}\text { Tout à fait } \\
\text { d'accord }\end{array}$ & D'accord & Pas d'accord & $\begin{array}{l}\text { Pas du tout } \\
\text { d'accord }\end{array}$ \\
\hline a) & $\begin{array}{l}\mathrm{M}^{\text {me }}<\text { Nom }>\text { donne des devoirs de mathématiques un jour } \\
\text { sur deux. Elle remet toujours les réponses aux élèves avant } \\
\text { les examens. } \\
\mathbf{M}^{\text {me }}<\text { Nom> se soucie de l'apprentissage de ses élèves. }\end{array}$ & $\square_{1}$ & $\square_{2}$ & $\square_{3}$ & $\square_{4}$ \\
\hline b) & $\begin{array}{l}\text { M. }<\text { Nom }>\text { donne des devoirs de mathématiques une fois } \\
\text { par semaine. Il remet toujours les réponses aux élèves avant } \\
\text { les examens. } \\
\text { M. }<\text { Nom> se soucie de l'apprentissage de ses élèves. }\end{array}$ & $\square_{1}$ & $\square_{2}$ & $\square_{3}$ & $\square_{4}$ \\
\hline c) & $\begin{array}{l}\mathrm{M}^{\mathrm{me}}<\text { Nom> donne des devoirs de mathématiques une fois } \\
\text { par semaine. Elle ne rend jamais les réponses aux élèves } \\
\text { avant les examens. } \\
\mathbf{M}^{\text {me }}<\text { Nom> se soucie de l'apprentissage de ses élèves. }\end{array}$ & $\square_{1}$ & $\square_{2}$ & $\square_{3}$ & $\square_{4}$ \\
\hline
\end{tabular}


Pensez au professeur de mathématiques qui vous a donné votre dernier cours de mathématiques.

(Cochez une case par ligne.)

\begin{tabular}{c|l|c|c|c|c}
\multicolumn{2}{l|}{} & $\begin{array}{c}\text { Tout à fait } \\
\text { d'accord }\end{array}$ & D'accord & Pas d'accord & $\begin{array}{c}\text { Pas du tout } \\
\text { d'accord }\end{array}$ \\
\hline a) & Le professeur nous dit qu'il faut travailler dur. & $\square_{1}$ & $\square_{2}$ & $\square_{3}$ & $\square_{4}$ \\
\hline b) & $\begin{array}{l}\text { Le professeur apporte une aide supplémentaire quand c'est } \\
\text { nécessaire. }\end{array}$ & $\square_{1}$ & $\square_{2}$ & $\square_{3}$ & $\square_{4}$ \\
\hline c) & Le professeur nous aide dans notre apprentissage. & $\square_{1}$ & $\square_{2}$ & $\square_{3}$ & $\square_{4}$ \\
\hline d) & Le professeur nous donne l'occasion d'exprimer nos opinions. & $\square_{1}$ & $\square_{2}$ & $\square_{3}$ & $\square_{4}$ \\
\hline
\end{tabular}

Des descriptions de trois professeurs de mathématiques vous sont proposées ci-dessous. Lisez chaque description et indiquez dans quelle mesure vous êtes d'accord avec l'affirmation qui conclut chaque description.

(Cochez une case par ligne.)

(Cochez une case par ligne.)

\begin{tabular}{c|l|c|c|c|c}
\multicolumn{2}{l|}{} & $\begin{array}{c}\text { Tout à fait } \\
\text { d'accord }\end{array}$ & D'accord & Pas d'accord & $\begin{array}{c}\text { Pas du tout } \\
\text { d'accord }\end{array}$ \\
\hline a) & Le professeur sait se faire écouter par les élèves. & $\square_{1}$ & $\square_{2}$ & $\square_{3}$ & $\square_{4}$ \\
\hline b) & Le professeur maintient la discipline dans sa classe. & $\square_{1}$ & $\square_{2}$ & $\square_{3}$ & $\square_{4}$ \\
\hline c) & Le professeur commence ses cours à l'heure. & $\square_{1}$ & $\square_{2}$ & $\square_{3}$ & $\square_{4}$ \\
\hline d) & $\begin{array}{l}\text { Le professeur doit attendre longtemps avant que les élèves } \\
\text { <se calment>. }\end{array}$ & $\square_{1}$ & $\square_{2}$ & $\square_{3}$ & $\square_{4}$ \\
\hline
\end{tabular}

\section{Le climat dans votre classe et dans votre établissement}

Pensez aux professeurs de votre établissement. Dans quelle mesure êtes-vous d'accord avec les affirmations suivantes?

(Cochez une case par ligne.)

\begin{tabular}{|c|c|c|c|c|c|}
\hline & & d'accord & D decora & ras a decoro & d'accord \\
\hline a) & Les élèves s'entendent bien avec la plupart des professeurs. & $\square_{1}$ & $\square_{2}$ & $\square_{3}$ & $\square_{4}$ \\
\hline b) & $\begin{array}{l}\text { La plupart des professeurs s'intéressent au bien-être } \\
\text { de leurs élèves. }\end{array}$ & $\square_{1}$ & $\square_{2}$ & $\square_{3}$ & $\square_{4}$ \\
\hline c) & $\begin{array}{l}\text { La plupart de mes professeurs sont réellement à l'écoute } \\
\text { de ce que j'ai à dire. }\end{array}$ & $\square_{1}$ & $\square_{2}$ & $\square_{3}$ & $\square_{4}$ \\
\hline d) & $\begin{array}{l}\text { Si j'ai besoin d'aide supplémentaire, mes professeurs } \\
\text { me l'apporteront. }\end{array}$ & $\square_{1}$ & $\square_{2}$ & $\square_{3}$ & $\square_{4}$ \\
\hline e) & La plupart de mes professeurs me traitent avec justice. & $\square_{1}$ & $\square_{2}$ & $\square_{3}$ & $\square_{4}$ \\
\hline
\end{tabular}


Q57 Pensez à votre école. Dans quelle mesure êtes-vous d'accord avec les affirmations suivantes ?

(ST87) (Cochez une case par ligne.)

\begin{tabular}{c|l|c|c|c|c}
\multicolumn{2}{l|}{} & $\begin{array}{c}\text { Tout à fait } \\
\text { d'accord }\end{array}$ & D'accord & Pas d'accord & $\begin{array}{c}\text { Pas du tout } \\
\text { d'accord }\end{array}$ \\
\hline a) & Je me sens comme un étranger (ou hors du coup) à l'école. & $\square_{1}$ & $\square_{2}$ & $\square_{3}$ & $\square_{4}$ \\
\hline b) & Je me fais facilement des amis à l'école. & $\square_{1}$ & $\square_{2}$ & $\square_{3}$ & $\square_{4}$ \\
\hline c) & Je me sens chez moi à l'école. & $\square_{1}$ & $\square_{2}$ & $\square_{3}$ & $\square_{4}$ \\
\hline d) & Je me sens mal à l'aise, pas à ma place dans mon école. & $\square_{1}$ & $\square_{2}$ & $\square_{3}$ & $\square_{4}$ \\
\hline e) & Les autres élèves ont l'air de m'apprécier. & $\square_{1}$ & $\square_{2}$ & $\square_{3}$ & $\square_{4}$ \\
\hline f) & Je me sens seul à l'école. & $\square_{1}$ & $\square_{2}$ & $\square_{3}$ & $\square_{4}$ \\
\hline g) & Je me sens bien à l'école. & $\square_{1}$ & $\square_{2}$ & $\square_{3}$ & $\square_{4}$ \\
\hline h) & Tout se passe très bien dans mon école. & $\square_{1}$ & $\square_{2}$ & $\square_{3}$ & $\square_{4}$ \\
\hline i) & Je suis satisfait de mon école. & $\square_{1}$ & $\square_{2}$ & $\square_{3}$ & $\square_{4}$ \\
\hline
\end{tabular}

Q58 Pensez à ce que vous avez appris à l'école. Dans quelle mesure êtes-vous d'accord avec les affirmations suivantes ?

(ST88) (Cochez une case par ligne.)

\begin{tabular}{c|l|c|c|c|c}
\multicolumn{2}{l|}{} & $\begin{array}{c}\text { Tout à fait } \\
\text { d'accord }\end{array}$ & D'accord & Pas d'accord & $\begin{array}{c}\text { Pas du tout } \\
\text { d'accord }\end{array}$ \\
\hline a) & L'école n'a pas fait grand chose pour me préparer à la vie d'adulte. & $\square_{1}$ & $\square_{2}$ & $\square_{3}$ & $\square_{4}$ \\
\hline b) & L'école a été une perte de temps. & $\square_{1}$ & $\square_{2}$ & $\square_{3}$ & $\square_{4}$ \\
\hline c) & $\begin{array}{l}\text { L'école a contribué à me donner confiance en moi pour } \\
\text { prendre des décisions. }\end{array}$ & $\square_{1}$ & $\square_{2}$ & $\square_{3}$ \\
\hline d) & $\begin{array}{l}\text { L'école m'a appris des choses qui pourront m'être utiles dans } \\
\text { mon futur travail. }\end{array}$ & $\square_{1}$ & $\square_{2}$ & $\square_{3}$ & $\square_{4}$ \\
\hline
\end{tabular}

Q59 Pensez à votre école. Dans quelle mesure êtes-vous d'accord avec les affirmations suivantes ?

(ST89) (Cochez une case par ligne.)

\begin{tabular}{|c|c|c|c|c|c|}
\hline & & $\begin{array}{l}\text { Tout à fait } \\
\text { d'accord }\end{array}$ & D'accord & Pas d'accord & $\begin{array}{l}\text { Pas du tout } \\
\text { d'accord }\end{array}$ \\
\hline a) & Travailler dur à l'école m'aidera à trouver un travail intéressant. & $\square_{1}$ & $\square_{2}$ & $\square_{3}$ & $\square_{4}$ \\
\hline b) & $\begin{array}{l}\text { Travailler dur à l'école me donnera accès à un <établissement } \\
\text { de l'enseignement postsecondaire> de bon niveau. }\end{array}$ & $\square_{1}$ & $\square_{2}$ & $\square_{3}$ & $\square_{4}$ \\
\hline c) & J'aime bien avoir de bonnes $<$ notes $>$. & $\square_{1}$ & $\square_{2}$ & $\square_{3}$ & $\square_{4}$ \\
\hline d) & Il est important de travailler dur à l'école. & $\square_{1}$ & $\square_{2}$ & $\square_{3}$ & $\square_{4}$ \\
\hline
\end{tabular}

Q60 Pensez à votre école. Dans quelle mesure êtes-vous d'accord avec les affirmations suivantes ?

(ST91) (Ne cochez qu'une seule case par ligne.)

\begin{tabular}{|c|c|c|c|c|c|}
\hline & & $\begin{array}{l}\text { Tout à fait } \\
\text { d'accord }\end{array}$ & D'accord & Pas d'accord & $\begin{array}{l}\text { Pas du tout } \\
\text { d'accord }\end{array}$ \\
\hline a) & Si je fais suffisamment d'efforts, je peux réussir à l'école. & $\square_{1}$ & $\square_{2}$ & $\square_{3}$ & $\square_{4}$ \\
\hline b) & Réussir ou échouer à l'école ne dépend que de moi. & $\square_{1}$ & $\square_{2}$ & $\square_{3}$ & $\square_{4}$ \\
\hline c) & $\begin{array}{l}\text { Des obligations familiales ou autres m'empêchent de } \\
\text { consacrer beaucoup de temps à l'école. }\end{array}$ & $\square_{1}$ & $\square_{2}$ & $\square_{3}$ & $\square_{4}$ \\
\hline d) & Je ferais plus d'efforts à l'école si j'avais d'autres professeurs. & $\square_{1}$ & $\square_{2}$ & $\square_{3}$ & $\square_{4}$ \\
\hline e) & Si je le voulais, je pourrais avoir de bons résultats à l'école. & $\square_{1}$ & $\square_{2}$ & $\square_{3}$ & $\square_{4}$ \\
\hline f) & $\begin{array}{l}\text { Que j'étudie ou non pour mes examens, je n'ai pas de bons } \\
\text { résultats à l'école. }\end{array}$ & $\square_{1}$ & $\square_{2}$ & $\square_{3}$ & $\square_{4}$ \\
\hline
\end{tabular}




\section{Votre expérience en résolution de problèmes}

Q61 Dans quelle mesure chacune des affirmations suivantes vous correspond-elle bien ?

(ST93) (Cochez une case par ligne.)

\begin{tabular}{|c|c|c|c|c|c|c|}
\hline & & $\begin{array}{l}\text { Tout à fait } \\
\text { comme moi }\end{array}$ & $\begin{array}{l}\text { Presque tout } \\
\text { comme moi }\end{array}$ & $\begin{array}{l}\text { Un peu } \\
\text { comme moi }\end{array}$ & $\begin{array}{l}\text { Pas vraiment } \\
\text { comme moi }\end{array}$ & $\begin{array}{l}\text { Pas du tout } \\
\text { comme moi }\end{array}$ \\
\hline a) & $\begin{array}{l}\text { Face à un problème à résoudre, } \\
\text { j'abandonne facilement. }\end{array}$ & $\square_{1}$ & $\square_{2}$ & $\square_{3}$ & $\square_{4}$ & $\square_{5}$ \\
\hline b) & Je remets les problèmes difficiles à plus tard. & $\square_{1}$ & $\square_{2}$ & $\square_{3}$ & $\square_{4}$ & $\square_{5}$ \\
\hline c) & $\begin{array}{l}\text { Quand j'entame un exercice, } \\
\text { il m’intéresse jusqu'au bout. }\end{array}$ & $\square_{1}$ & $\square_{2}$ & $\square_{3}$ & $\square_{4}$ & $\square_{5}$ \\
\hline d) & $\begin{array}{l}\text { Je travaille sur mes exercices jusqu'à ce } \\
\text { que tout soit parfait. }\end{array}$ & $\square_{1}$ & $\square_{2}$ & $\square_{3}$ & $\square_{4}$ & $\square_{5}$ \\
\hline e) & $\begin{array}{l}\text { Quand j'ai un problème difficile à } \\
\text { résoudre, j'en fais plus que ce que l'on } \\
\text { attend de moi. }\end{array}$ & $\square_{1}$ & $\square_{2}$ & $\square_{3}$ & $\square_{4}$ & $\square_{5}$ \\
\hline
\end{tabular}

Q62 Dans quelle mesure chacune des affirmations suivantes vous correspond-elle bien?

(ST94) (Cochez une case par ligne.)

\begin{tabular}{c|l|c|c|c|c}
\multicolumn{2}{l|}{} & $\begin{array}{c}\text { Tout à fait } \\
\text { comme moi }\end{array}$ & $\begin{array}{c}\text { Presque tout } \\
\text { comme moi }\end{array}$ & $\begin{array}{c}\text { Un peu } \\
\text { comme moi }\end{array}$ & $\begin{array}{c}\text { Pas vraiment } \\
\text { comme moi }\end{array}$ \\
\hline $\begin{array}{c}\text { Pas du tout } \\
\text { comme moi }\end{array}$ \\
\hline a) & Je peux traiter beaucoup d'informations. & $\square_{1}$ & $\square_{2}$ & $\square_{3}$ \\
\hline b) & Je comprends vite. & $\square_{1}$ & $\square_{2}$ & $\square_{3}$ \\
\hline c) & Je cherche des explications. & $\square_{1}$ & $\square_{2}$ & $\square_{3}$ \\
\hline d) & J'établis facilement des liens entre des faits. & $\square_{1}$ & $\square_{2}$ & $\square_{3}$ \\
\hline e) & $\begin{array}{l}\text { J'aime bien résoudre des problèmes } \\
\text { complexes. }\end{array}$ & $\square_{1}$ & $\square_{2}$ & $\square_{5}$ \\
\hline
\end{tabular}

Supposez que cela fait plusieurs semaines que vous envoyez des SMS avec votre téléphone portable. Aujourd'hui, pourtant, vous n'y arrivez pas. Vous voulez tenter de résoudre le problème.

Que feriez-vous d'abord? Pour chaque proposition, cochez la case qui vous correspond le mieux. (Cochez une case par ligne.)

\begin{tabular}{|c|c|c|c|c|c|}
\hline & & $\begin{array}{l}\text { Je le ferais } \\
\text { certainement }\end{array}$ & $\begin{array}{l}\text { Je le ferais } \\
\text { probablement }\end{array}$ & $\begin{array}{c}\text { Je ne le ferais } \\
\text { probablement } \\
\text { pas }\end{array}$ & $\begin{array}{c}\text { Je ne le ferais } \\
\text { certainement } \\
\text { pas }\end{array}$ \\
\hline a) & J'appuie sur chaque touche pour voir ce qui ne va pas. & $\square_{1}$ & $\square_{2}$ & $\square_{3}$ & $\square_{4}$ \\
\hline b) & $\begin{array}{l}\text { Je réfléchis à l'origine possible du problème et à ce que } \\
\text { je peux faire pour le résoudre. }\end{array}$ & 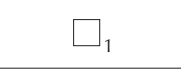 & 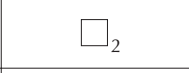 & $\square$ & 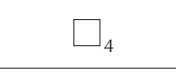 \\
\hline C) & Je lis le mode d'emploi. & $\square_{1}$ & $\square_{2}$ & $\square_{3}$ & $\square_{4}$ \\
\hline d) & Je demande de l'aide à un ami. & $\square_{1}$ & $\square_{2}$ & $\square_{3}$ & $\square_{4}$ \\
\hline
\end{tabular}

\begin{tabular}{c|l|c|c|c|c}
\multicolumn{2}{l|}{} & $\begin{array}{c}\text { Je le ferais } \\
\text { certainement }\end{array}$ & $\begin{array}{c}\text { Je le ferais } \\
\text { probablement }\end{array}$ & $\begin{array}{c}\text { Je ne le ferais } \\
\text { probablement } \\
\text { pas }\end{array}$ & $\begin{array}{c}\text { Je ne le ferais } \\
\text { certainement } \\
\text { pas }\end{array}$ \\
\hline a) & Je regarde si le chemin est indiqué dans la brochure du zoo. & $\square_{1}$ & $\square_{2}$ & $\square_{3}$ & $\square_{4}$ \\
\hline b) & Je cherche le meilleur itinéraire à l'aide d'une carte. & $\square_{1}$ & $\square_{2}$ & $\square_{3}$ & $\square_{4}$ \\
\hline c) & Je laisse mon frère chercher un itinéraire. & $\square_{1}$ & $\square_{2}$ & $\square_{3}$ & $\square_{4}$ \\
\hline d) & $\begin{array}{l}\text { Je sais à peu près où le zoo se trouve, je propose donc } \\
\text { qu'on se mette en route. }\end{array}$ & $\square_{1}$ & $\square_{2}$ & $\square_{3}$ & $\square_{4}$ \\
\hline
\end{tabular}


Supposez que vous arrivez à la gare. Il y a une billetterie automatique que vous n'avez jamais utilisée. Vous voulez acheter un billet.

Que feriez-vous ? Pour chaque proposition, cochez la case qui vous correspond le mieux.

(Ne cochez qu'une seule case par ligne.)

\begin{tabular}{c|l|c|c|c|c}
\multicolumn{2}{l|}{} & $\begin{array}{c}\text { Je le ferais } \\
\text { certainement }\end{array}$ & $\begin{array}{c}\text { Je le ferais } \\
\text { probablement }\end{array}$ & $\begin{array}{c}\text { Je ne le ferais } \\
\text { probablement } \\
\text { pas }\end{array}$ & $\begin{array}{c}\text { Je ne le ferais } \\
\text { certainement } \\
\text { pas }\end{array}$ \\
\hline a) & $\begin{array}{l}\text { Je regarde si elle ressemble aux autres billetteries } \\
\text { automatiques que j'ai déjà utilisées. }\end{array}$ & $\square_{1}$ & $\square_{2}$ & $\square_{3}$ & $\square_{4}$ \\
\hline b) & J'essaie toutes les touches pour voir ce qui se passe. & $\square_{1}$ & $\square_{2}$ & $\square_{3}$ & $\square_{4}$ \\
\hline c) & Je demande de l'aide à quelqu'un. & $\square_{1}$ & $\square_{2}$ & $\square_{3}$ & $\square_{4}$ \\
\hline d) & $\begin{array}{l}\text { J'essaie de trouver un guichet traditionnel pour y acheter } \\
\text { mon billet. }\end{array}$ & $\square_{1}$ & $\square_{2}$ & $\square_{3}$ & $\square_{4}$ \\
\hline
\end{tabular}




\section{OUESTIONNAIRE SUR LE PARCOURS SCOLAIRE}

Comme lors des cycles précédents, d'autres questionnaires ont été élaborés. Ces questionnaires sont proposés à titre d'options internationales aux pays et économies participants. Lors du cycle PISA 2012, ces questionnaires portent sur le parcours scolaire des élèves et sur leur maîtrise des TIC ; enfin, il est proposé d'administrer un questionnaire aux parents d'élève.

Le questionnaire sur le parcours scolaire porte sur les aspects suivants :

- le parcours scolaire ;

- la préparation à un futur métier ; et

- le soutien à I'apprentissage linguistique.

\section{Parcours scolaire}

Q1

(EC01)

(Ne cochez qu'une seule case.)

\begin{tabular}{|l|c}
\hline Non, jamais. & $\square_{1}$ \\
\hline Oui, une fois. & $\square_{2}$ \\
\hline Oui, deux fois ou plus. & $\square_{3}$
\end{tabular}

Note : Veuillez vous référer à la Question 6 du questionnaire « ́́lève » pour une définition du <niveau CITE $1>$.

Durant votre scolarité de <niveau CITE 2>, vous est-il arrivé de manquer l'école pendant deux mois de suite ou davantage ?

(Ne cochez qu'une seule case.)

\begin{tabular}{|l|c}
\hline Non, jamais. & $\square$ \\
\hline Oui, une fois. & $\square_{2}$ \\
\hline Oui, deux fois ou plus. & $\square_{3}$ \\
\hline
\end{tabular}

Note : Veuillez vous référer à la Question 7 du questionnaire « ́́lève » pour une définition du $<$ niveau CITE 2>. 


\section{Se préparer à son futur métier}

Q3

(EC03)

Avez-vous fait quelque chose parmi ce qui suit pour vous renseigner sur de futures études ou différents types de métiers?

(Cochez une case par ligne.)

\begin{tabular}{|c|c|c|c|}
\hline & & Oui & Non, jamais \\
\hline a) & J'ai fait un stage. & $\square_{1}$ & $\square_{2}$ \\
\hline b) & J'ai effectué un <stage d'observation ou des visites d'entreprises>. & $\square_{1}$ & $\square_{2}$ \\
\hline c) & Je me suis rendu à un <salon de l'emploi>. & $\square_{1}$ & $\square_{2}$ \\
\hline d) & J'ai parlé avec un <conseiller d'orientation> de mon école. & $\square_{1}$ & $\square_{2}$ \\
\hline e) & J'ai parlé avec un <conseiller d'orientation> externe. & $\square_{1}$ & $\square_{2}$ \\
\hline f) & J'ai répondu à un questionnaire afin de déterminer mes centres d'intérêts et mes aptitudes. & $\square_{1}$ & $\square_{2}$ \\
\hline g) & J'ai cherché des informations sur Internet à propos de différentes professions. & $\square_{1}$ & $\square_{2}$ \\
\hline h) & J'ai participé à une visite organisée dans un établissement de <niveau CITE 3-5>. & $\square_{1}$ & $\square_{2}$ \\
\hline i) & $\begin{array}{l}\text { J'ai cherché des informations sur Internet à propos des programmes d'études de }<\text { niveau } \\
\text { CITE } 3-5>\text {. }\end{array}$ & $\square_{1}$ & $\square_{2}$ \\
\hline j) & $<$ Item national $>$. & $\square_{1}$ & $\square_{2}$ \\
\hline
\end{tabular}

Notes : Une « visite dans un <établissement de niveau CITE 3-5> » est une visite organisée dans un collège, une université ou un autre établissement du deuxième cycle de l'enseignement secondaire ou de l'enseignement postsecondaire, dont l'objectif consiste à informer les étudiants potentiels sur les études supérieures en général et sur l'offre particulière existant au sein de l'établissement en question.

Veuillez vous référer à la Question 7 du questionnaire «Élève » pour une définition du $<$ niveau CITE 3>.

Veuillez vous référer à la Question 14 du questionnaire «Élève » pour une définition du $<$ niveau CITE $4>$ et du $<$ niveau CITE $5>$

Q4 Parmi les compétences suivantes, lesquelles avez-vous acquises?

(EC04) (Cochez toutes les cases qui conviennent.)

\begin{tabular}{|c|c|c|c|c|}
\hline & & Oui, à l'école & $\begin{array}{l}\text { Oui, en } \\
\text { dehors de } \\
\text { l'école }\end{array}$ & Non, jamais \\
\hline a) & Trouver des informations sur les métiers qui m'intéressent. & $\square_{1}$ & $\square_{2}$ & $\square_{3}$ \\
\hline b) & Chercher un travail. & $\square_{1}$ & $\square_{2}$ & $\square_{3}$ \\
\hline c) & Rédiger mon <curriculum vitae $(\mathrm{CV})>$ ou un profil de mes qualifications. & $\square_{1}$ & $\square_{2}$ & $\square_{3}$ \\
\hline d) & Préparer un entretien d'embauche. & $\square_{1}$ & $\square_{2}$ & $\square_{3}$ \\
\hline e) & $\begin{array}{l}\text { Trouver des informations sur les programmes de < niveau CITE 3-5> qui } \\
\text { m'intéressent. }\end{array}$ & $\square_{1}$ & $\square_{2}$ & $\square_{3}$ \\
\hline f) & $\begin{array}{l}\text { Trouver des informations sur le financement des études (par ex., prêt } \\
\text { étudiant ou bourse d'études). }\end{array}$ & $\square_{1}$ & $\square_{2}$ & $\square_{3}$ \\
\hline
\end{tabular}

Notes : Veuillez vous référer à la Question 7 du questionnaire "Élève » pour une définition du $<$ niveau CITE $3>$.

Veuillez vous référer à la Question 14 du questionnaire « Élève » pour une définition du <niveau CITE 4> et du <niveau CITE $5>$. 


\section{Soutien à l'apprentissage linguistique}

\section{Q5 Quelle est la première langue que vous avez apprise à la maison ?}

(EC05) (Ne cochez qu'une seule case.)

\begin{tabular}{|l|l|l}
\hline $\begin{array}{l}\text { La première langue que j'ai apprise à la maison est <la langue de l'évaluation> } \\
\text { ou <autre(s) langue(s) nationale(s) officielle(s) ou dialecte(s)>. }\end{array}$ & $\square_{1}$ & $\begin{array}{l}\text { Vous avez fini de remplir } \\
\text { ce questionnaire. } \\
\text { Merci de votre participation. }\end{array}$ \\
\hline $\begin{array}{l}\text { J'ai appris <la langue de l'évaluation> ou <autre(s) langue(s) nationale(s) } \\
\text { officielle(s) ou dialecte(s)> en même temps qu'une autre langue à la maison. }\end{array}$ & $\square_{2}$ & Allez à la Question 6. \\
\hline $\begin{array}{l}\text { La première langue que j'ai apprise à la maison est une autre langue que } \\
\text { <la langue de l'évaluation> ou que < autre(s) langue(s) nationale(s) } \\
\text { officielle(s) ou dialecte(s)>. }\end{array}$ & $\square_{3}$ & Allez à la Question 6. \\
\hline
\end{tabular}

Notes : < Langue de l'évaluation> - Ce terme désigne la langue dans laquelle le test PISA de culture mathématique est administré.

$<$ Autre(s) langue(s) nationale(s) officielle(s) ou dialecte(s)> - S'il n'y a pas d'autre langue officielle dans le pays, cette catégorie sera omise. S'il existe plus d'une langue officielle ou d'un dialecte dans le pays, il faudra utiliser le nom de la deuxième (et troisième) langue(s) officielle(s) ou du deuxième (et troisième) dialecte(s).

Q6 Quel âge aviez-vous quand vous avez commencé à apprendre <la langue de l'évaluation> ?

(EC06) (Ne cochez qu'une seule case.)

\begin{tabular}{|l|c}
\hline Entre 0 et 3 ans. & $\square_{1}$ \\
\hline Entre 4 et 6 ans. & $\square_{2}$ \\
\hline Entre 7 et 9 ans. & $\square_{3}$ \\
\hline Entre 10 et 12 ans. & $\square_{4}$ \\
\hline 13 ans ou plus. & $\square_{5}$ \\
\hline
\end{tabular}

Note : Veuillez vous référer à la Question 5 pour une définition de la <langue de l'évaluation>.

Quelle langue parlez-vous habituellement avec les personnes suivantes?

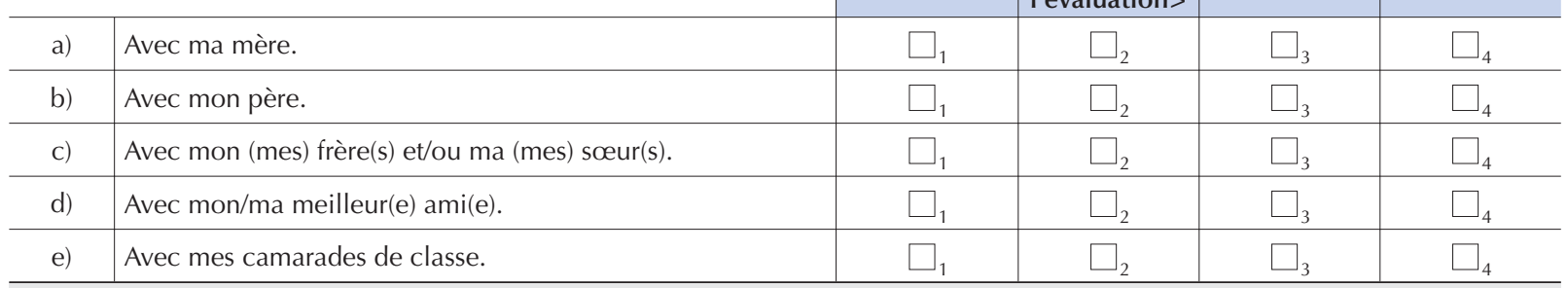

Notes : Veuillez vous référer à la Question 5 pour une définition de la <langue de l'évaluation>.

La <langue d'origine>, parfois appelée « langue maternelle », fait référence à la langue apprise en premier lieu par l'élève au cours de son enfance qui n'est pas la langue de l'évaluation. 
Q8 En quelle langue pratiquez-vous généralement les activités suivantes?

\begin{tabular}{|c|c|c|c|c|c|}
\hline a) & Lire des livres, des magazines ou des journaux. & $\square_{1}$ & $\square_{2}$ & $\square_{3}$ & $\square_{4}$ \\
\hline b) & Regarder la télé ou des films. & $\square_{1}$ & $\square_{2}$ & $\square_{3}$ & $\square_{4}$ \\
\hline c) & Surfer sur Internet. & $\square_{1}$ & $\square_{2}$ & $\square_{3}$ & $\square_{4}$ \\
\hline d) & Rédiger des courriers électroniques ou des lettres. & $\square_{1}$ & $\square_{2}$ & $\square_{3}$ & $\square_{4}$ \\
\hline
\end{tabular}

Notes : Veuillez vous référer à la Question 5 pour une définition de la <langue de l'évaluation>.

Veuillez vous référer à la Question 7 pour une définition de la <langue d'origine>.

Q9 Avez-vous déjà suivi l'un des types de cours suivants, à l'école ou en dehors de l'école ?

(EC09) (Cochez une case par ligne.)

a) J'ai suivi des <cours de soutien $>$ en <langue de l'évaluation>.

Q10 Avez-vous déjà suivi l'un des types de cours suivants, à l'école ou en dehors de l'école ?

(EC11) (Cochez une case par ligne.)

b) J'ai suivi des cours de ma <langue d'origine> (axés principalement sur la lecture,

b) l'expression écrite ou la grammaire).

c) J'ai suivi des cours où les matières scolaires sont enseignées dans ma <langue d'origine > (axés principalement sur le contenu des matières).

Notes : Veuillez vous référer à la Question 5 pour une définition de la <langue de l'évaluation>.

Veuillez vous référer à la Question 7 pour une définition de la <langue d'origine>.

$<$ Cours de soutien $>-$ Tout cours s'ajoutant aux cours ordinaires et destiné à faciliter la remise à niveau d'élèves qui accusent un retard par rapport à leurs camarades de classe.

Cette <année scolaire-ci>, combien d'heures par semaine recevez-vous un soutien systématique pour améliorer vos compétences en <langue de l'évaluation>, à l'école ou en dehors de l'école?

(Ne cochez qu'une seule case.)

Aucune.

Moins de 2 heures par semaine.

2 heures ou plus mais moins de 4 heures par semaine.

4 heures ou plus mais moins de 6 heures par semaine.

6 heures ou plus par semaine.

Notes : Veuillez vous référer à la Question 5 pour une définition de la <langue de l'évaluation>.

$<$ Cette année scolaire-ci > - L'année scolaire en cours ne correspond pas nécessairement à une année civile. 
Cette <année scolaire-ci>, combien d'heures par semaine suivez-vous généralement des cours de votre <langue d'origine> ou un enseignement dans votre <langue d'origine>, à l'école ou en dehors de l'école ?

(Ne cochez qu'une seule case.)

Aucune.

Moins de 2 heures par semaine.

2 heures ou plus, mais moins de 4 heures par semaine.

4 heures ou plus, mais moins de 6 heures par semaine.

6 heures ou plus par semaine.

Notes : Veuillez vous référer à la Question 7 pour une définition de la <langue d'origine>.

Veuillez vous référer à la Question 11 pour une définition de <cette année scolaire-ci>

Q13 Votre mère est-elle née en <pays évalué> ?

(Ne cochez qu'une seule case.)

Non.

Oui.

Si la réponse est Oui, vous avez fini de remplir ce questionnaire. Merci de votre participation.

Vous trouverez ci-dessous des affirmations concernant votre $<$ culture d'accueil $>$ et votre $<$ culture d'origine $>$. La $<$ culture d'accueil> fait référence à la culture du pays dans lequel vous vivez actuellement. La $<$ culture d'origine> fait référence à la culture du pays dans lequel votre mère est née.

Dans quelle mesure êtes-vous d'accord avec les affirmations suivantes?

(Cochez une case par ligne.)

\begin{tabular}{c|l|c|c|c|c}
\multicolumn{2}{l|}{} & $\begin{array}{c}\text { Tout à fait } \\
\text { d'accord }\end{array}$ & D'accord & Pas d'accord & $\begin{array}{c}\text { Pas du tout } \\
\text { d'accord }\end{array}$ \\
\hline a) & J'aime bien avoir des amis de ma <culture d'accueil>. & $\square_{1}$ & $\square_{2}$ & $\square_{3}$ & $\square_{4}$ \\
\hline b) & J'aime bien avoir des amis de ma <culture d'origine>. & $\square_{1}$ & $\square_{2}$ & $\square_{3}$ & $\square_{4}$ \\
\hline c) & J'aime bien participer à des fêtes liées à ma <culture d'accueil>. & $\square_{1}$ & $\square_{2}$ & $\square_{3}$ & $\square_{4}$ \\
\hline d) & J'aime bien participer à des fêtes liées à ma <culture d'origine>. & $\square_{1}$ & $\square_{2}$ & $\square_{3}$ & $\square_{4}$ \\
\hline e) & $\begin{array}{l}\text { Je passe beaucoup de temps avec des amis de ma <culture } \\
\text { d'accueil>. }\end{array}$ & $\square_{1}$ & $\square_{2}$ & $\square_{3}$ & $\square_{4}$ \\
\hline f) & $\begin{array}{l}\text { Je passe beaucoup de temps avec des amis de ma <culture } \\
\text { d'origine>. }\end{array}$ & $\square_{1}$ & $\square_{2}$ & $\square_{3}$ & $\square_{4}$ \\
\hline g) & Je participe à des fêtes liées à ma <culture d'accueil>. & $\square_{1}$ & $\square_{2}$ & $\square_{3}$ & $\square_{4}$ \\
\hline h) & Je participe à des fêtes liées à ma <culture d'origine>. & $\square_{1}$ & $\square_{2}$ & $\square_{3}$ & $\square_{4}$ \\
\hline
\end{tabular}

Les affirmations suivantes concernent les différences entre votre <culture d'accueil> et votre <culture d'origine>

(Cochez une case par ligne.)

\begin{tabular}{|c|c|c|c|c|c|}
\hline & & $\begin{array}{l}\text { Tout à fait } \\
\text { d'accord }\end{array}$ & $\mathrm{D}^{\prime}$ accord & Pas d'accord & $\begin{array}{l}\text { Pas du tout } \\
\text { d'accord }\end{array}$ \\
\hline a) & $\begin{array}{l}\text { Les valeurs sont les mêmes dans ma <culture d'accueil }> \\
\text { et dans ma }<\text { culture d'origine }>\text {. }\end{array}$ & $\square_{1}$ & $\square_{2}$ & $\square_{3}$ & $\square_{4}$ \\
\hline b) & $\begin{array}{l}\text { Les mères de ma }<\text { culture } d^{\prime} \text { accueil }>\text { et les mères de ma } \\
<\text { culture d'origine }>\text { traitent leurs enfants de la même façon. }\end{array}$ & $\square_{1}$ & $\square_{2}$ & $\square_{3}$ & $\square_{4}$ \\
\hline c) & $\begin{array}{l}\text { Les élèves de ma <culture d'accueil> et les élèves de ma } \\
<\text { culture d'origine> se comportent de la même façon avec } \\
\text { leurs professeurs. }\end{array}$ & $\square_{1}$ & $\square_{2}$ & $\square_{3}$ & $\square_{4}$ \\
\hline
\end{tabular}




\section{QUESTIONNAIRE SUR LES TIC}

Le questionnaire sur la maîtrise des technologies de l'information et de la communication (TIC) comporte des questions sur l'accès des élèves aux $\mathrm{TIC}$, les usages qu'ils en font et leurs attitudes à l'égard de l'informatique. Ce questionnaire est administré aux élèves après le questionnaire « Élève ». Il faut cinq minutes environ pour le remplir.

Le questionnaire sur les TIC porte sur divers aspects :

- accès aux TIC ;

- utilisation générale de l'informatique ;

- utilisation de l'informatique en dehors de l'école ;

- utilisation de l'informatique à l'école ; et

- attitudes à l'égard de l'informatique.

\section{Accès au matériel d'information et de communication (TIC)}

Q1 À la maison, avez-vous la possibilité d'utiliser les équipements suivants ?

(IC01) (Cochez une case par ligne.)

\begin{tabular}{|c|c|c|c|c|}
\hline & & $\begin{array}{l}\text { Oui, et je } \\
\text { I'utilise }\end{array}$ & $\begin{array}{c}\text { Oui, mais je } \\
\text { ne I'utilise } \\
\text { pas }\end{array}$ & Non \\
\hline a) & Un ordinateur de bureau. & $\square_{1}$ & $\square_{2}$ & $\square_{3}$ \\
\hline b) & Un ordinateur portable ou un agenda électronique. & $\square_{1}$ & $\square_{2}$ & $\square_{3}$ \\
\hline c) & $\begin{array}{l}\text { Une }<\text { tablette } \mathrm{PC}>\text { (par exemple, un }<\text { iPad }^{\circ}>\text { ou un }<\text { BlackBerry }^{\circ} \\
\text { PlayBook }{ }^{\top M}>\text { ). }\end{array}$ & $\square_{1}$ & $\square_{2}$ & $\square_{3}$ \\
\hline d) & Une connexion à Internet. & $\square_{1}$ & $\square_{2}$ & $\square_{3}$ \\
\hline e) & Une $<$ console de jeux vidéo $>$, par exemple une $<$ PlayStation $^{\circ}$ Sony $^{\oplus}>$. & $\square_{1}$ & $\square_{2}$ & $\square_{3}$ \\
\hline f) & Un < téléphone portable> (sans connexion à Internet). & $\square_{1}$ & $\square_{2}$ & $\square_{3}$ \\
\hline g) & Un <téléphone portable> (avec connexion à Internet). & $\square_{1}$ & $\square_{2}$ & $\square_{3}$ \\
\hline h) & $\begin{array}{l}\text { Un lecteur de musique portable (lecteur Mp3 ou Mp4, un } \mathrm{iPod}^{\circ} \text { ou } \\
\text { équivalent). }\end{array}$ & $\square_{1}$ & $\square_{2}$ & $\square_{3}$ \\
\hline i) & Une imprimante. & $\square_{1}$ & $\square_{2}$ & $\square_{3}$ \\
\hline j) & Une clé USB. & $\square_{1}$ & $\square_{2}$ & $\square_{3}$ \\
\hline k) & Un $<$ lecteur électronique $>$, par exemple, un $<$ Amazon $^{\circ}$ Kindle $^{\mathrm{TM}}>$. & $\square_{1}$ & $\square_{2}$ & $\square_{3}$ \\
\hline
\end{tabular}

Q2 À l'école, avez-vous la possibilité d'utiliser les équipements suivants ?

(IC02) (Cochez une case par ligne.)

\begin{tabular}{|c|c|c|c|c|}
\hline & & $\begin{array}{l}\text { Oui, et je } \\
\text { I'utilise }\end{array}$ & $\begin{array}{c}\text { Oui, mais je } \\
\text { ne l'utilise } \\
\text { pas }\end{array}$ & Non \\
\hline a) & Un ordinateur de bureau. & $\square_{1}$ & $\square_{2}$ & $\square_{3}$ \\
\hline b) & Un ordinateur portable ou un agenda électronique. & $\square_{1}$ & $\square_{2}$ & $\square_{3}$ \\
\hline c) & $\begin{array}{l}\text { Une }<\text { tablette PC }>\text { (par exemple, un }<\text { iPad }^{\Theta}>\text { ou un }<\text { BlackBerry }^{\circ} \\
\text { PlayBook }{ }^{\mathrm{TM}}>\text { ). }\end{array}$ & $\square_{1}$ & $\square_{2}$ & $\square_{3}$ \\
\hline d) & Une connexion à Internet. & $\square_{1}$ & $\square_{2}$ & $\square_{3}$ \\
\hline e) & Une imprimante. & $\square_{1}$ & $\square_{2}$ & $\square_{3}$ \\
\hline f) & Une clé USB. & $\square_{1}$ & $\square_{2}$ & $\square_{3}$ \\
\hline g) & Un $<$ lecteur électronique $>$, par exemple, un $<$ Amazon $^{\odot}$ Kindle $^{T M}>$. & $\square_{1}$ & $\square_{2}$ & $\square_{3}$ \\
\hline
\end{tabular}




\section{Utilisation des ordinateurs en général}

Q3

(IC03)

À quel âge avez-vous utilisé un ordinateur pour la première fois ?

Si vous n'avez jamais utilisé d'ordinateur, vous avez fini de remplir ce questionnaire. Merci de votre participation.

(Ne cochez qu'une seule case.)

\begin{tabular}{|l|c}
\hline À 6 ans ou moins. & $\square_{1}$ \\
\hline Entre 7 et 9 ans. & $\square_{2}$ \\
\hline Entre 10 et12 ans. & $\square_{3}$ \\
\hline À 13 ans ou plus. & $\square_{4}$ \\
\hline Je n'ai jamais utilisé d'ordinateur. & $\square_{5}$ \\
\hline
\end{tabular}

Q4 À quel âge vous êtes-vous servi d'Internet pour la première fois ?

(IC04) (Ne cochez qu'une seule case.)

\begin{tabular}{l|c}
\hline À 6 ans ou moins. & $\square_{1}$ \\
\hline Entre 7 et 9 ans. & $\square_{2}$ \\
\hline Entre 10 et12 ans. & $\square_{3}$ \\
\hline À 13 ans ou plus. & $\square_{4}$ \\
\hline Je ne me suis jamais servi d'Internet. & $\square_{5}$ \\
\hline
\end{tabular}

Un jour de semaine ordinaire, combien de temps utilisez-vous Internet à l'école ?

(Ne cochez qu'une seule case.)

Pas du tout.

De 1 à 30 minutes par jour.

De 31 à 60 minutes par jour.

Entre 1 et 2 heures par jour.

Entre 2 et 4 heures par jour.

Entre 4 et 6 heures par jour.

Plus de 6 heures par jour.

Q6 Un jour de semaine ordinaire, combien de temps utilisez-vous Internet en dehors de l'école ?

(Ne cochez qu'une seule case.)

\begin{tabular}{|l|c}
\hline Pas du tout. & $\square_{1}$ \\
\hline De 1 à 30 minutes par jour. & $\square_{2}$ \\
\hline De 31 à 60 minutes par jour. & $\square_{3}$ \\
\hline Entre 1 et 2 heures par jour. & $\square_{4}$ \\
\hline Entre 2 et 4 heures par jour. & $\square_{5}$ \\
\hline Entre 4 et 6 heures par jour. & $\square_{6}$ \\
\hline Plus de 6 heures par jour. & $\square_{7}$ \\
\hline
\end{tabular}


Un jour de week-end ordinaire, combien de temps utilisez-vous Internet en dehors de l'école ?

(Ne cochez qu'une seule case.)

\begin{tabular}{|l|c}
\hline Pas du tout. & $\square_{1}$ \\
\hline De 1 à 30 minutes par jour. & $\square_{2}$ \\
\hline De 31 à 60 minutes par jour. & $\square_{3}$ \\
\hline Entre 1 et 2 heures par jour. & $\square_{4}$ \\
\hline Entre 2 et 4 heures par jour. & $\square_{5}$ \\
\hline Entre 4 et 6 heures par jour. & $\square_{6}$ \\
\hline Plus de 6 heures par jour. & $\square_{7}$ \\
\hline
\end{tabular}

\section{Utilisation de l'informatique en dehors de l'école}

Q8 En dehors de l'école, à quelle fréquence utilisez-vous un ordinateur pour les activités suivantes ?

(IC08) (Cochez une case par ligne.)

\begin{tabular}{|c|c|c|c|c|c|c|}
\hline & & $\begin{array}{l}\text { Jamais ou } \\
\text { presque } \\
\text { jamais }\end{array}$ & $\begin{array}{l}\text { Une ou deux } \\
\text { fois par mois }\end{array}$ & $\begin{array}{l}\text { Une ou deux } \\
\text { fois par } \\
\text { semaine }\end{array}$ & $\begin{array}{l}\text { Presque tous } \\
\text { les jours }\end{array}$ & Tous les jours \\
\hline a) & Jouer à des jeux à un seul joueur. & $\square_{1}$ & $\square_{2}$ & $\square_{3}$ & $\square_{4}$ & $\square_{5}$ \\
\hline b) & $\begin{array}{l}\text { Jouer à des jeux à plusieurs (sur Internet } \\
\text { ou en réseau). }\end{array}$ & $\square_{1}$ & $\square_{2}$ & $\square_{3}$ & $\square_{4}$ & $\square_{5}$ \\
\hline c) & Utiliser le courrier électronique (e-mail). & $\square_{1}$ & $\square_{2}$ & $\square_{3}$ & $\square_{4}$ & $\square_{5}$ \\
\hline d) & $\begin{array}{l}<\text { Chatter sur Internet }>\text { (par exemple, } \\
\text { sur }<\mathrm{MSN}^{\circ}>\text { ). }\end{array}$ & $\square_{1}$ & $\square_{2}$ & $\square_{3}$ & $\square_{4}$ & $\square_{5}$ \\
\hline e) & $\begin{array}{l}\text { Participer à des réseaux sociaux } \\
\text { (par exemple, <Facebook }>,<\text { MySpace }>\text { ). }\end{array}$ & $\square_{1}$ & $\square_{2}$ & $\square_{3}$ & $\square_{4}$ & $\square_{5}$ \\
\hline f) & $\begin{array}{l}\text { Surfer sur Internet pour m'amuser } \\
\text { (par exemple, pour regarder des vidéos sur } \\
\left.<\text { YouTube }^{T M}\right\rangle \text { ). }\end{array}$ & $\square_{1}$ & $\square_{2}$ & $\square_{3}$ & $\square_{4}$ & $\square_{5}$ \\
\hline g) & Suivre I'actualité sur Internet. & $\square_{1}$ & $\square_{2}$ & $\square_{3}$ & $\square_{4}$ & $\square_{5}$ \\
\hline h) & $\begin{array}{l}\text { Obtenir des informations pratiques sur } \\
\text { Internet (par exemple, le lieu et la date d'un } \\
\text { événement). }\end{array}$ & $\square_{1}$ & $\square_{2}$ & $\square_{3}$ & $\square_{4}$ & $\square_{5}$ \\
\hline i) & $\begin{array}{l}\text { Télécharger de la musique, des films, } \\
\text { des jeux ou des logiciels à partir d'Internet. }\end{array}$ & $\square_{1}$ & $\square_{2}$ & $\square_{3}$ & $\square_{4}$ & $\square_{5}$ \\
\hline j) & $\begin{array}{l}\text { Mettre en ligne mes propres créations pour } \\
\text { les partager (par exemple, de la musique, } \\
\text { des poèmes, des vidéos, des programmes } \\
\text { informatiques). }\end{array}$ & $\square_{1}$ & $\square_{2}$ & $\square_{3}$ & $\square_{4}$ & $\square_{5}$ \\
\hline
\end{tabular}


Q9 En dehors de l'école, à quelle fréquence utilisez-vous un ordinateur pour les activités suivantes ?

(IC09) (Cochez une case par ligne.)

\begin{tabular}{c|l|c|c|c|c|c}
\hline \multicolumn{2}{c|}{} & $\begin{array}{c}\text { Jamais ou } \\
\text { presque } \\
\text { jamais }\end{array}$ & $\begin{array}{c}\text { Une ou deux } \\
\text { fois par mois }\end{array}$ & $\begin{array}{c}\text { Une ou deux } \\
\text { fois par } \\
\text { semaine }\end{array}$ & $\begin{array}{c}\text { Presque tous } \\
\text { les jours }\end{array}$ & Tous les jours \\
\hline a) & $\begin{array}{l}\text { Surfer sur Internet pour le travail scolaire } \\
\text { (pour préparer une rédaction ou un exposé, } \\
\text { par exemple). }\end{array}$ & $\square_{1}$ & $\square_{2}$ & $\square_{3}$ & $\square_{4}$ & $\square_{5}$ \\
\hline b) & $\begin{array}{l}\text { Échanger des e-mails avec d'autres élèves à } \\
\text { propos du travail scolaire. }\end{array}$ & $\square_{1}$ & $\square_{2}$ & $\square_{3}$ & $\square_{4}$ & $\square_{5}$ \\
\hline c) & $\begin{array}{l}\text { Communiquer par e-mail avec les } \\
\text { professeurs et rendre mes devoirs ou autres } \\
\text { travaux. }\end{array}$ & $\square_{1}$ & $\square_{2}$ & $\square_{3}$ & $\square_{4}$ & $\square_{5}$ \\
\hline d) & $\begin{array}{l}\text { Télécharger, consulter des documents sur } \\
\text { le site web de l'école (par exemple, des } \\
\text { horaires ou des documents de cours, etc.) ou } \\
\text { bien y déposer des fichiers. }\end{array}$ & $\square_{1}$ & $\square_{2}$ & $\square_{3}$ & $\square_{4}$ & $\square_{5}$ \\
\hline e) & $\begin{array}{l}\text { Consulter le site web de l'école pour } \\
\text { connaître les dernières informations (par } \\
\text { exemple, les absences de professeurs). }\end{array}$ & $\square_{1}$ & $\square_{2}$ & $\square_{3}$ & $\square_{4}$ & $\square_{5}$ \\
\hline f) & Faire mes devoirs sur ordinateur. & $\square_{1}$ & $\square_{2}$ & $\square_{3}$ & $\square_{4}$ & $\square_{5}$ \\
\hline g) & $\begin{array}{l}\text { Partager des documents scolaires avec } \\
\text { d'autres élèves. }\end{array}$ & $\square_{1}$ & $\square_{2}$ & $\square_{3}$ & $\square_{4}$ & $\square_{5}$ \\
\hline
\end{tabular}

\section{Utilisation de l'informatique à l'école}

Q10 À l'école, à quelle fréquence utilisez-vous un ordinateur pour les activités suivantes ?

(IC10) (Cochez une case par ligne.)

\begin{tabular}{|c|c|c|c|c|c|c|}
\hline & & $\begin{array}{l}\text { Jamais ou } \\
\text { presque } \\
\text { jamais }\end{array}$ & $\begin{array}{l}\text { Une ou deux } \\
\text { fois par mois }\end{array}$ & $\begin{array}{l}\text { Une ou deux } \\
\text { fois par } \\
\text { semaine }\end{array}$ & $\begin{array}{l}\text { Presque tous } \\
\text { les jours }\end{array}$ & Tous les jours \\
\hline a) & $<$ Chatter sur Internet $>$ à I'école. & $\square_{1}$ & $\square_{2}$ & $\square_{3}$ & $\square_{4}$ & $\square_{5}$ \\
\hline b) & Utiliser le courrier électronique (e-mail). & $\square_{1}$ & $\square_{2}$ & $\square_{3}$ & $\square_{4}$ & $\square_{5}$ \\
\hline c) & Surfer sur Internet pour un travail scolaire. & $\square_{1}$ & $\square_{2}$ & $\square_{3}$ & $\square_{4}$ & $\square_{5}$ \\
\hline d) & $\begin{array}{l}\text { Télécharger, consulter des documents sur } \\
\text { le site web de l'école }\left(I^{\prime}<\text { intranet }>\text {, par }\right. \\
\text { exemple) ou y déposer des fichiers. }\end{array}$ & $\square_{1}$ & $\square_{2}$ & $\square_{3}$ & $\square_{4}$ & $\square_{5}$ \\
\hline e) & Déposer mes travaux sur le site web de l'école. & $\square_{1}$ & $\square_{2}$ & $\square_{3}$ & $\square_{4}$ & $\square_{5}$ \\
\hline f) & Utiliser des logiciels de simulation à l'école. & $\square_{1}$ & $\square_{2}$ & $\square_{3}$ & $\square_{4}$ & $\square_{5}$ \\
\hline g) & $\begin{array}{l}\text { Faire des exercices (par exemple, pour } \\
\text { le cours de langue étrangère ou celui de } \\
\text { mathématiques). }\end{array}$ & $\square_{1}$ & $\square_{2}$ & $\square_{3}$ & $\square_{4}$ & $\square_{5}$ \\
\hline h) & Faire mes devoirs sur un ordinateur de l'école. & $\square_{1}$ & $\square_{2}$ & $\square_{3}$ & $\square_{4}$ & $\square_{5}$ \\
\hline i) & $\begin{array}{l}\text { Utiliser les ordinateurs de l'école pour un } \\
\text { travail de groupe ou pour communiquer } \\
\text { avec d'autres élèves. }\end{array}$ & $\square_{1}$ & $\square_{2}$ & $\square_{3}$ & $\square_{4}$ & $\square_{5}$ \\
\hline
\end{tabular}


Q11 Le mois dernier, l'ordinateur a-t-il été utilisé dans l'un des buts suivants pendant vos cours de mathématiques ?

(IC11) (Cochez une case par ligne.)

\begin{tabular}{|c|c|c|c|c|}
\hline & & $\begin{array}{l}\text { Oui, par les } \\
\text { élèves }\end{array}$ & \begin{tabular}{|c|} 
Oui, mais \\
seulement \\
par le \\
professeur \\
pour montrer \\
aux élèves \\
comment \\
faire
\end{tabular} & Non \\
\hline a) & Pour dessiner le graphe d'une fonction (par exemple, $y=4 x+6$ ). & $\square_{1}$ & $\square_{2}$ & $\square_{3}$ \\
\hline b) & Pour faire des calculs avec des nombres (par exemple, calculer $5 * 233 / 8$ ). & $\square_{1}$ & $\square_{2}$ & $\square_{3}$ \\
\hline C) & $\begin{array}{l}\text { Pour construire des figures géométriques (par exemple, un triangle } \\
\text { équilatéral dont la longueur des côtés est donnée). }\end{array}$ & $\square_{1}$ & $\square_{2}$ & $\square$ $\square_{3}$ \\
\hline d) & $\begin{array}{l}\text { Pour saisir des données dans une feuille de calcul (par exemple, dans } \\
<\text { Excel }^{\mathrm{TM}}>\text { ). }\end{array}$ & $\square_{1}$ & $\square_{2}$ & $\square_{3}$ \\
\hline e) & $\begin{array}{l}\text { Pour reformuler des expressions algébriques et résoudre des équations (par } \\
\text { exemple, } a^{2}+2 a b+b^{2} \text { ). }\end{array}$ & $\square_{1}$ & $\square_{2}$ & $\square_{3}$ \\
\hline f) & $\begin{array}{l}\text { Pour dessiner des histogrammes (graphiques montrant comment se distribuent } \\
\text { les données). }\end{array}$ & $\square_{1}$ & $\square_{2}$ & $\square$ \\
\hline g) & $\begin{array}{l}\text { Pour trouver comment le graphe d'une fonction comme } y=a x^{2} \text { change en } \\
\text { fonction de la valeur de } a \text {. }\end{array}$ & $\square_{1}$ & $\square_{2}$ & $\square_{3}$ \\
\hline
\end{tabular}

\section{Attitudes à l'égard de l'informatique}

Pensez à l'expérience que vous avez de l'informatique. Dans quelle mesure êtes-vous d'accord avec les affirmations suivantes?

(Cochez une case par ligne.)

\begin{tabular}{|c|c|c|c|c|c|}
\hline & & $\begin{array}{l}\text { Tout à fait } \\
\text { d'accord }\end{array}$ & D'accord & Pas d'accord & $\begin{array}{l}\text { Pas du tout } \\
\text { d'accord }\end{array}$ \\
\hline a) & L'ordinateur est un outil très utile pour mon travail scolaire. & $\square_{1}$ & $\square_{2}$ & $\square_{3}$ & $\square_{4}$ \\
\hline b) & Faire mes devoirs sur ordinateur est plus amusant. & $\square_{1}$ & $\square_{2}$ & $\square_{3}$ & $\square_{4}$ \\
\hline c) & $\begin{array}{l}\text { Internet est une formidable mine d'informations que je peux } \\
\text { utiliser pour mon travail scolaire. }\end{array}$ & $\square_{1}$ & $\square_{2}$ & $\square_{3}$ & $\square_{4}$ \\
\hline d) & Il est pénible d'utiliser l'ordinateur pour apprendre. & $\square_{1}$ & $\square_{2}$ & $\square_{3}$ & $\square_{4}$ \\
\hline e) & $\begin{array}{l}\text { Puisque n'importe qui peut mettre des informations en } \\
\text { ligne, en général, il vaut mieux ne pas utiliser Internet pour } \\
\text { le travail scolaire. }\end{array}$ & $\square_{1}$ & $\square_{2}$ & $\square$ 唯 & $\square_{4}$ \\
\hline f) & $\begin{array}{l}\text { Les informations trouvées sur Internet sont généralement } \\
\text { trop peu fiables pour être utilisées pour le travail scolaire. }\end{array}$ & $\square_{1}$ & $\square_{2}$ & $\square_{3}$ & $\square_{4}$ \\
\hline
\end{tabular}




\section{QUESTIONNAIRE " PARENTS *}

Un exemplaire de ce questionnaire est distribué par élève. II faut environ 20 minutes pour répondre au questionnaire

«Parents ». Le questionnaire « Parents » porte sur divers aspects :

- la situation des parents ;

- le coût des services d'éducation ;

- I'opinion des parents sur l'établissement de leur enfant ;

- I'implication des parents dans la vie de l'établissement ;

- le choix de l'établissement ;

- le soutien parental pour l'apprentissage à la maison ;

- I'utilité des mathématiques sur le marché du travail et pour l'avenir professionnel de l'enfant ;

- les attentes des parents à l'égard des études et de la profession de l'enfant ;

- les résultats scolaires de l'enfant ;

- les ambitions professionnelles pour l'enfant; et

- I'origine des parents.

\section{La situation des parents}

\section{Q1 Qui remplira ce questionnaire ?}

(PA01) (Cochez autant de cases que nécessaire.)

\begin{tabular}{c|l|c} 
a) & La mère (ou une autre femme tenant lieu de mère). & $\square_{1}$ \\
\hline b) & Le père (ou un autre homme tenant lieu de père). & $\square_{2}$ \\
\hline c) & Une autre personne. & $\square_{3}$ \\
\hline & (Dans ce dernier cas, veuillez préciser la personne dont il s'agit) : & \\
\hline
\end{tabular}

\section{Q2 Quel âge ont les parents de l'enfant ?}

(PA02) (Cochez une case par ligne.)

\begin{tabular}{c|l|c|c|c|c|c}
\multicolumn{2}{l|}{} & $\begin{array}{c}\text { Moins de } \\
36 \text { ans }\end{array}$ & $\begin{array}{c}\text { Entre } \\
36 \text { et } 40 \text { ans }\end{array}$ & $\begin{array}{c}\text { Entre } \\
41 \text { et } 45 \text { ans }\end{array}$ & $\begin{array}{c}\text { Entre } \\
46 \text { et } 50 \text { ans }\end{array}$ & $\begin{array}{c}51 \text { ans } \\
\text { ou plus }\end{array}$ \\
\hline a) & Père. & $\square_{1}$ & $\square_{2}$ & $\square_{3}$ & $\square_{4}$ & $\square_{5}$ \\
\hline b) & Mère. & $\square_{1}$ & $\square_{2}$ & $\square_{3}$ & $\square_{4}$ & $\square_{5}$ \\
\hline
\end{tabular}

\section{Q3 Le père de l'enfant a-t-il obtenu un des diplômes suivants ?}

(PA03) (Cochez une case par ligne.)

\begin{tabular}{|c|c|c|c|}
\hline & & Oui & Non \\
\hline a) & $<$ Niveau CITE 5A ou 6>. & $\square_{1}$ & $\square_{2}$ \\
\hline b) & $<$ Niveau CITE 5B $>$. & $\square_{1}$ & $\square_{2}$ \\
\hline C) & $<$ Niveau CITE 4>. & $\square_{1}$ & $\square_{2}$ \\
\hline d) & $<$ Niveau CITE 3A $>$. & $\square_{1}$ & $\square_{2}$ \\
\hline
\end{tabular}

Notes : Veuillez vous référer à la Question 7 du questionnaire "Élève » pour une définition du $<$ niveau CITE 3 A $>$.

Veuillez vous référer à la Question 14 du questionnaire «Élève " pour une définition du $<$ niveau CITE $4>$ au $<$ niveau CITE $6>$. 
Quel est l'emploi principal du père de l'enfant? (Par exemple, enseignant, aide-cuisinier, directeur des ventes)

( $S^{\prime}$ il ne travaille pas actuellement, veuillez indiquer son dernier emploi principal.)

Écrivez le nom du métier :

Que fait le père de l'enfant dans le cadre de son emploi principal ? (Par exemple, il donne cours à des élèves du secondaire, il aide le cuisinier d'un restaurant à préparer les repas, il dirige une équipe de ventes.)

En une phrase, indiquez en quoi consiste ou consistait son métier :

Q5 La mère de l'enfant a-t-elle obtenu un des diplômes suivants ?

(PA05) (Cochez une case par ligne.)

\begin{tabular}{c|l|c|c}
\hline \multicolumn{2}{l|}{} & Oui & Non \\
\hline a) & <Niveau CITE 5A ou 6>. & $\square_{1}$ & $\square_{2}$ \\
\hline b) & <Niveau CITE 5B $>$. & $\square_{1}$ & $\square_{2}$ \\
\hline c) & $<$ Niveau CITE 4>. & $\square_{1}$ & $\square_{2}$ \\
\hline d) & <Niveau CITE 3A $>$. & $\square_{1}$ & $\square_{2}$ \\
\hline
\end{tabular}

Notes : Veuillez vous référer à la Question 7 du questionnaire "Élève » pour une définition du $<$ niveau CITE 3 A $>$.

Veuillez vous référer à la Question 14 du questionnaire «Élève » pour une définition du $<$ niveau CITE $4>$ au $<$ niveau CITE $6>$.

Quel est l'emploi principal de la mère de l'enfant ? (Par exemple, enseignante, aide-cuisinière,

(Si elle ne travaille pas actuellement, veuillez indiquer son dernier emploi principal.)

Écrivez le nom du métier : à des élèves du secondaire, elle aide le cuisinier d'un restaurant à préparer les repas, elle dirige une équipe de ventes.)

En une phrase, indiquez en quoi consiste ou consistait son métier : 
Q7

(PA07)

\section{Quel est le revenu annuel de votre ménage ?}

Additionnez le revenu global avant impôts de tous les membres de votre ménage.

Souvenez-vous que nous vous demandons de répondre à ces questions seulement si cela ne vous embarrasse pas de le faire et que toutes vos réponses seront gardées strictement confidentielles.

(Ne cochez qu'une seule case.)

\begin{tabular}{l|c} 
Moins de $<$ A euros $>$. & $\square_{1}$ \\
\hline$<$ A euros $>$ ou plus, mais moins de $<$ B euros $>$. & $\square_{2}$ \\
\hline$<$ B euros $>$ ou plus, mais moins de $<$ C euros $>$. & $\square_{3}$ \\
\hline$<$ C euros $>$ ou plus, mais moins de $<$ D euros $>$. & $\square_{4}$ \\
\hline$<$ D euros $>$ ou plus, mais moins de $<$ E euros $>$. & $\square_{5}$ \\
\hline$<$ E euros $>$ ou plus. & $\square_{6}$
\end{tabular}

Notes : <Euros $>$ - cette devise est remplacée par la devise nationale du pays ou de l'économie participant.

$<$ C euros $>$ est la valeur arrondie correspondant au revenu médian des ménages du pays. Dans un pays, le revenu médian est un montant de référence calculé tous ménages confondus : la moitié des ménages affichent un revenu supérieur à ce montant, et l'autre moitié, un revenu qui y est inférieur.

$<$ A euros $>$ est la valeur arrondie correspondant à environ la moitié du revenu médian national ; $<$ B euros $>$ est la valeur correspondant à environ trois quarts du revenu médian national ; $<\mathbf{D}$ euros $>$ est la valeur correspondant à environ cinq quarts du revenu médian national ; $<\mathbf{E}$ euros $>$ est la valeur correspondant à une fois et demie le revenu médian national.

\section{Le coût des services d'éducation}

\begin{tabular}{|c|c|c|}
\hline & \multicolumn{2}{|c|}{$\begin{array}{l}\text { Veuillez répondre à la question suivante en tenant compte uniquement des dépenses relatives à <l'élève qui vous a remis ce } \\
\text { questionnaire }>\text {. }\end{array}$} \\
\hline \multirow{4}{*}{$\begin{array}{c}\text { Q8 } \\
\text { (PA08) }\end{array}$} & \multicolumn{2}{|c|}{$\begin{array}{l}\text { Au cours des douze mois écoulés, à peu près combien d'argent avez-vous dépensé pour la scolarité de } \\
\text { votre enfant? }\end{array}$} \\
\hline & \multicolumn{2}{|c|}{$\begin{array}{l}\text { Pour déterminer ce montant, veuillez tenir compte de tous les frais de scolarité que vous avez réglés à l'établissement de votre } \\
\text { enfant, en y ajoutant les honoraires payés pour des cours particuliers éventuellement donnés à votre enfant par des professeurs } \\
\text { de l'établissement ou par tout autre professeur, ainsi que les montants versés pour tout cours éventuel de bachotage. }\end{array}$} \\
\hline & \multirow{2}{*}{\multicolumn{2}{|c|}{$\begin{array}{l}\text { Ne tenez pas compte des dépenses engagées pour l'achat de matériel (par ex., équipements de sport, uniforme de l'école, } \\
\text { ordinateur ou manuels scolaires) si vous avez effectué ces achats séparément et que leur prix n'est pas inclus dans le montant } \\
\text { global payé à l'établissement. } \\
\text { (Ne cochez qu'une seule case.) }\end{array}$}} \\
\hline & & \\
\hline & Rien. & $\square_{1}$ \\
\hline & $<$ Plus de 0 euro, mais moins de $\mathrm{W}$ euros $>$. & $\square_{2}$ \\
\hline & $<W$ euros ou plus, mais moins de $X$ euros $>$. & $\square_{3}$ \\
\hline & $<\mathrm{X}$ euros ou plus, mais moins de $\mathrm{Y}$ euros $>$. & $\square_{4}$ \\
\hline & $<$ Y euros ou plus, mais moins de $\mathrm{Z}$ euros $>$. & $\square_{5}$ \\
\hline & $<Z$ euros $>$ ou plus. & $\square_{6}$ \\
\hline $\begin{array}{l}\text { Notes } \\
<\mathbf{Z} \text { eu } \\
\text { particu } \\
5 \% \text {. } \\
\text { les pay } \\
\text { tenir c } \\
\text { Le mol } \\
\text { selon }\end{array}$ & $\begin{array}{l}\text { Euros }>\text { : cette devise est remplacée par la devise } \mathrm{n} \text {. } \\
\text { est la valeur arrondie correspondant au montar } \\
\text { s). À titre indicatif, on peut considérer que le pour } \\
\text { euros }>\text { est la valeur arrondie correspondant au mo } \\
\text { ù l'enseignement est entièrement gratuit, il y a lieu } \\
\text { pte des frais occasionnels. } \\
\text { th des valeurs intermédiaires, }<\mathbf{X} \text { euros }>\text { et }<\mathbf{Y} \text { eur } \\
\text { incréments équivalents. }\end{array}$ & $\begin{array}{l}\text { des cours } \\
\text { d'environ } \\
\text { res). Dans } \\
\text { uros) pour } \\
\text { W euros }>\text {, }\end{array}$ \\
\hline
\end{tabular}




\section{L'opinion des parents sur l'établissement de leur enfant}

Nous souhaiterions connaître votre opinion sur l'établissement fréquenté par votre enfant.

Dans quelle mesure êtes-vous d'accord ou non avec les énoncés suivants ?

(Cochez une case par ligne.)

\begin{tabular}{|c|c|c|c|c|c|}
\hline a) & $\begin{array}{l}\text { La plupart des enseignants de l'établissement fréquenté par } \\
\text { mon enfant paraissent compétents et motivés. }\end{array}$ & $\square_{1}$ & $\square_{2}$ & $\square_{3}$ & $\square_{4}$ \\
\hline b) & Le niveau d'exigences est élevé dans cet établissement. & $\square_{1}$ & $\square_{2}$ & $\square_{3}$ & $\square_{4}$ \\
\hline c) & $\begin{array}{l}\text { Je suis satisfait(e) du contenu des cours et des méthodes } \\
\text { d'enseignement utilisées dans cet établissement. }\end{array}$ & $\square_{1}$ & $\square_{2}$ & $\square_{3}$ & $\square_{4}$ \\
\hline d) & Je suis satisfait(e) du climat de discipline de cet établissement. & $\square_{1}$ & $\square_{2}$ & $\square_{3}$ & $\square_{4}$ \\
\hline e) & Les progrès de mon enfant sont suivis de près par l'établissement. & $\square_{1}$ & $\square_{2}$ & $\square_{3}$ & $\square_{4}$ \\
\hline f) & $\begin{array}{l}\text { Je reçois de l'établissement des informations régulières et utiles } \\
\text { sur les progrès scolaires de mon enfant. }\end{array}$ & $\square_{1}$ & $\square_{2}$ & $\square$ प & $\square_{4}$ \\
\hline g) & Cet établissement donne une bonne formation aux élèves. & $\square_{1}$ & $\square_{2}$ & $\square_{3}$ & $\square_{4}$ \\
\hline
\end{tabular}

\section{L'implication des parents dans la vie de l'établissement}

Q10

(PA10)

Au cours de <l'année scolaire> précédente, avez-vous pris part aux activités suivantes en rapport avec l'établissement de votre enfant?

(Cochez une case par ligne.)

\begin{tabular}{|c|c|c|c|}
\hline & & Oui & Non \\
\hline a) & Discuter du comportement de mon enfant avec un professeur, sur mon initiative. & $\square_{1}$ & $\square_{2}$ \\
\hline b) & Discuter du comportement de mon enfant avec un professeur, sur l'initiative de ce dernier. & $\square_{1}$ & $\square_{2}$ \\
\hline c) & $\begin{array}{l}\text { Me porter volontaire pour des tâches manuelles, par exemple, travaux de menuiserie, de jardinage } \\
\text { ou d'entretien du bâtiment ou de la cour. }\end{array}$ & $\square_{1}$ & $\square_{2}$ \\
\hline d) & $\begin{array}{l}\text { Me porter volontaire pour des activités parascolaires, par exemple, club de lecture, pièce de } \\
\text { théâtre, sports, excursion. }\end{array}$ & $\square_{1}$ & $\square_{2}$ \\
\hline e) & Me porter volontaire pour travailler à la bibliothèque ou à la médiathèque de l'établissement. & $\square_{1}$ & $\square_{2}$ \\
\hline f) & $<$ Aider un professeur de l'établissement $>$. & $\square_{1}$ & $\square_{2}$ \\
\hline g) & Donner une conférence. & $\square_{1}$ & $\square_{2}$ \\
\hline h) & $\begin{array}{l}\text { Participer à des activités liées à la <gestion> de l'école, par exemple, faire partie d'un comité } \\
\text { consultatif de parents d'élèves ou du comité de direction de l'établissement. }\end{array}$ & $\square_{1}$ & $\square_{2}$ \\
\hline i) & Discuter des progrès de mon enfant avec un professeur, sur mon initiative. & $\square_{1}$ & $\square_{2}$ \\
\hline j) & Discuter des progrès de mon enfant avec l'un de ses professeurs, sur l'initiative de ce dernier. & $\square_{1}$ & $\square_{2}$ \\
\hline k) & Me porter volontaire pour travailler à la <cantine $>$. & $\square_{1}$ & $\square_{2}$ \\
\hline
\end{tabular}




\section{Le choix de l'établissement}

Q11

(PA11)

Nous souhaiterions connaître les possibilités de choix que vous aviez, en tant que parents, au moment où vous avez choisi l'établissement que fréquente actuellement votre enfant.

Parmi les énoncés suivants, lequel décrit le mieux les choix d'établissement qui sont offerts aux élèves là où vous vivez?

(Ne cochez qu'une seule case.)

Il y a dans cette zone deux autres établissements, ou davantage, qui sont en concurrence avec l'établissement fréquenté actuellement par mon enfant.

Il y a dans cette zone un autre établissement qui est en concurrence avec l'établissement fréquenté actuellement par mon enfant.

Il n'y a dans cette zone aucun autre établissement en concurrence avec l'établissement fréquenté

actuellement par mon enfant.

Q12 Dans quelle mesure les critères suivants sont-ils importants pour choisir un établissement pour votre enfant ?

(PA12) (Cochez une case par ligne.)

\begin{tabular}{|c|c|c|c|c|c|}
\hline & & $\begin{array}{l}\text { Pas } \\
\text { important }\end{array}$ & $\begin{array}{l}\text { Assez } \\
\text { important }\end{array}$ & Important & $\begin{array}{c}\text { Très } \\
\text { important }\end{array}$ \\
\hline a) & L'établissement est proche de notre domicile. & $\square_{1}$ & $\square_{2}$ & $\square_{3}$ & $\square_{4}$ \\
\hline b) & L'établissement a bonne réputation. & $\square_{1}$ & $\square_{2}$ & $\square \square_{3}$ & $\square$ प \\
\hline c) & L'établissement propose des cours ou des matières spécifiques. & $\square_{1}$ & $\square_{2}$ & $\square_{3}$ & $\square_{4}$ \\
\hline d) & $\begin{array}{l}\text { L'établissement adhère à une <« philosophie » religieuse> } \\
\text { particulière. }\end{array}$ & $\square_{1}$ & $\square_{2}$ & $\square_{3}$ & $\square_{4}$ \\
\hline e) & $\begin{array}{l}\text { L'établissement a une approche <pédagogique ou didactique> } \\
\text { particulière, par exemple, <exemple>. }\end{array}$ & $\square_{1}$ & $\square_{2}$ & $\square_{3}$ & $\square_{4}$ \\
\hline f) & $\begin{array}{l}\text { D'autres membres de la famille fréquentent cet établissement, ou } \\
\text { I'ont fréquenté. }\end{array}$ & $\square_{1}$ & $\square_{2}$ & $\square_{3}$ & $\square_{4}$ \\
\hline g) & $\begin{array}{l}\text { Les < frais sont peu élevés> (par exemple, droits de scolarité, coût } \\
\text { des manuels, frais de logement et de nourriture). }\end{array}$ & $\square_{1}$ & $\square_{2}$ & $\square_{3}$ & $\square_{4}$ \\
\hline h) & $\begin{array}{l}\text { L'établissement propose une <aide financière> (par ex., prêt } \\
\text { scolaire, bourse ou subvention). }\end{array}$ & $\square_{1}$ & $\square_{2}$ & $\square_{3}$ & $\square_{4}$ \\
\hline i) & Il règne dans l'établissement une ambiance dynamique et agréable. & $\square_{1}$ & $\square_{2}$ & $\square 3$ & $\square_{4}$ \\
\hline j) & Les résultats scolaires des élèves de l'établissement sont bons. & $\square_{1}$ & $\square_{2}$ & $\square$ 唯 & $\square_{4}$ \\
\hline k) & La sécurité y est bonne. & $\square_{1}$ & $\square_{2}$ & $\square_{3}$ & $\square_{4}$ \\
\hline
\end{tabular}




\section{Le soutien des parents pour l'apprentissage à la maison}

Q13

(PA13)

\begin{tabular}{|c|c|c|c|c|c|c|}
\hline & & $\begin{array}{l}\text { Jamais ou } \\
\text { presque } \\
\text { jamais }\end{array}$ & $\begin{array}{l}\text { Une ou deux } \\
\text { fois par an }\end{array}$ & $\begin{array}{l}\text { Une ou deux } \\
\text { fois par mois }\end{array}$ & $\begin{array}{l}\text { Une ou deux } \\
\text { fois par } \\
\text { semaine }\end{array}$ & $\begin{array}{l}\text { Chaque jour } \\
\text { ou presque } \\
\text { chaque jour }\end{array}$ \\
\hline a) & $\begin{array}{l}\text { Discuter avec mon enfant de la qualité } \\
\text { de son travail scolaire. }\end{array}$ & $\square_{1}$ & $\square 2$ & $\square 3$ & $\square_{4}$ & $\square_{5}$ \\
\hline b) & $\begin{array}{l}\text { Prendre le <repas principal> à table } \\
\text { avec mon enfant. }\end{array}$ & $\square$ & $\square 2$ & $\square 3$ & $\square$ $\square$ & $\square$ \\
\hline c) & $\begin{array}{l}\text { Passer du temps simplement à parler } \\
\text { avec mon enfant. }\end{array}$ & $\square$ & $\square 2$ & $\square 3$ & $\square 4$ & $\square_{5}$ \\
\hline d) & $\begin{array}{l}\text { Aider mon enfant à faire ses devoirs } \\
\text { de mathématiques. }\end{array}$ & $\square_{1}$ & $\square 2$ & $\square 3$ & $\square_{4}$ & $\square$ \\
\hline e) & $\begin{array}{l}\text { Discuter avec mon enfant de ses résultats } \\
\text { en mathématiques. }\end{array}$ & $\square$ & $\square 2$ & $\square 3$ & $\square$ प & $\square_{5}$ \\
\hline f) & $\begin{array}{l}\text { Me procurer du matériel d'apprentissage } \\
\text { en mathématiques (par exemple, des } \\
\text { programmes, des logiciels, des guides } \\
\text { d'études, etc.) pour mon enfant. }\end{array}$ & $\square$ & $\square 2$ & $\square 3$ & $\square$ प & $\square_{5}$ \\
\hline g) & $\begin{array}{l}\text { Discuter avec mon enfant de l'application } \\
\text { des mathématiques dans la vie de tous } \\
\text { les jours. }\end{array}$ & $\square$ & $\square 2$ & $\square$ $\square$ & $\square$ $\square$ & $\square$ $\square$ \\
\hline
\end{tabular}

\section{L'utilité des mathématiques sur le marché du travail et pour l'avenir professionnel de l'enfant}

Nous souhaitons connaître votre opinion sur l'utilité des compétences mathématiques sur le marché actuel du travail. Dans quelle mesure êtes-vous d'accord avec les affirmations suivantes?

(Cochez une case par ligne.)

\begin{tabular}{|c|c|c|c|c|c|}
\hline & & $\begin{array}{l}\text { Tout à fait } \\
\text { d'accord }\end{array}$ & D'accord & Pas d'accord & $\begin{array}{l}\text { Pas du tout } \\
\text { d'accord }\end{array}$ \\
\hline a) & $\begin{array}{l}\text { À l'heure actuelle, il est important d'avoir de solides } \\
\text { connaissances et compétences en mathématiques pour avoir } \\
\text { un bon travail. }\end{array}$ & $\square_{1}$ & $\square_{2}$ & $\square_{3}$ & $\square_{4}$ \\
\hline b) & $\begin{array}{l}\text { En général, les employeurs apprécient que leur personnel ait } \\
\text { de solides connaissances et compétences en mathématiques. }\end{array}$ & $\square_{1}$ & $\square_{2}$ & $\square_{3}$ & $\square_{4}$ \\
\hline c) & $\begin{array}{l}\text { À l'heure actuelle, il faut des connaissances et des compétences } \\
\text { en mathématiques pour la plupart des métiers. }\end{array}$ & $\square_{1}$ & $\square_{2}$ & $\square_{3}$ & $\square_{4}$ \\
\hline d) & $\begin{array}{l}\text { Avoir de bonnes connaissances et compétences en } \\
\text { mathématiques constitue un atout sur le marché du travail. }\end{array}$ & $\square_{1}$ & $\square_{2}$ & $\square_{3}$ & $\square_{4}$ \\
\hline
\end{tabular}




\section{Études et professions à caractère mathématique : les attentes des parents}

Q15

Les questions suivantes portent sur les <professions à caractère mathématique>. Nous entendons par <profession à caractère mathématique> une profession qui exige un niveau universitaire en mathématiques.

Comme exemples de <professions à caractère mathématique>, citons les métiers de professeur de mathématiques, d'économiste, d'analyste financier et d'informaticien.

Parmi les <professions à caractère mathématique>, nous retrouvons également beaucoup de métiers scientifiques, comme ceux d'ingénieur, de météorologue et de médecin. Tous ces métiers peuvent être considérés comme des <professions à caractère mathématique>.

Veuillez répondre aux questions suivantes.

(Cochez une case par ligne.)

\begin{tabular}{c|l|c|c}
\hline \multicolumn{1}{c|}{} & Oui & Non \\
\hline a) & $\begin{array}{l}\text { Y a-t-il dans votre famille quelqu'un (vous compris) exerçant une <profession à caractère } \\
\text { mathématique> ? }\end{array}$ & $\square_{1}$ & $\square_{2}$ \\
\hline b) & Votre enfant montre-t-il un intérêt pour les <professions à caractère mathématique> ? & $\square_{1}$ & $\square_{2}$ \\
\hline c) & Pensez-vous que votre enfant s'orientera vers une <profession à caractère mathématique> ? & $\square_{1}$ \\
\hline d) & $\begin{array}{l}\text { Votre enfant a-t-il évoqué la possibilité d'étudier les mathématiques après l'<école } \\
\text { secondaire>? }\end{array}$ & $\square_{2}$ \\
\hline e) & $\begin{array}{l}\text { Pensez-vous que votre enfant s'orientera vers des études en mathématiques après l'<école } \\
\text { secondaire> ? }\end{array}$ & $\square_{1}$ \\
\hline
\end{tabular}

\section{Les résultats scolaires de l'enfant}

\section{Q16 Votre enfant a-t-il déjà redoublé un <grade> ?}

(PA18) (Cochez une case par ligne.)

\begin{tabular}{|c|c|c|c|c|}
\hline & & Non, jamais & Oui, une fois & $\begin{array}{l}\text { Oui, deux } \\
\text { fois ou plus }\end{array}$ \\
\hline a) & Au $<$ niveau CITE $1>$. & $\square_{1}$ & $\square_{2}$ & $\square_{3}$ \\
\hline b) & Au $<$ niveau CITE $2>$. & $\square_{1}$ & $\square_{2}$ & $\square_{3}$ \\
\hline c) & Au $<$ niveau CITE $3>$. & $\square_{1}$ & $\square_{2}$ & $\square_{3}$ \\
\hline
\end{tabular}

Notes : <Grade > - Fait référence au niveau administratif auquel l'élève est inscrit dans l'établissement. Dans de nombreux pays, le nombre d'années de scolarité correspond à la mesure habituelle du grade.

Veuillez vous référer à la Question 6 du questionnaire " Élève » pour une définition du <niveau CITE 1>.

Veuillez vous référer à la Question 7 du questionnaire "Élève » pour une définition du $<$ niveau CITE $2>$ et du $<$ niveau CITE 3 $>$.

\section{Les ambitions professionnelles pour l'enfant}

\section{Q17 Lequel ou lesquels des diplômes suivants souhaitez-vous que votre enfant obtienne ?}

(PA19) (Cochez autant de cases que nécessaire.)

\begin{tabular}{|c|c|c|}
\hline a) & $<$ Niveau CITE 2>. & $\square_{1}$ \\
\hline b) & $<$ Niveau CITE 3B ou C>. & $\square_{1}$ \\
\hline c) & $<$ Niveau CITE $3 \mathrm{~A}>$. & $\square_{1}$ \\
\hline d) & $<$ Niveau CITE 4>. & $\square_{1}$ \\
\hline e) & $<$ Niveau CITE 5B $>$. & $\square_{1}$ \\
\hline f) & $<$ Niveau CITE 5 A ou $6>$. & $\square_{1}$ \\
\hline
\end{tabular}

Notes : Veuillez vous référer à la Question 7 du questionnaire «Élève » pour une définition du $<$ niveau CITE $2>$ et du $<$ niveau CITE $3>$.

Veuillez vous référer à la Question 14 du questionnaire « Élève » pour une définition du $<$ niveau CITE $4>$ au $<$ niveau CITE $6>$. 
Q18 Quelle profession pensez-vous que votre enfant exercera lorsqu'il aura environ 30 ans ?

Inscrivez le nom du métier :

\section{L'origine des parents}

Q19 Dans quel pays chacun des membres suivants de la famille de l'enfant est-il né ?

(PA21) (Cochez une case par colonne.)

\begin{tabular}{|c|c|c|c|c|c|c|}
\hline & $\begin{array}{l}\text { Mère de } \\
\text { I'enfant }\end{array}$ & $\begin{array}{l}\text { Père de } \\
\text { l'enfant }\end{array}$ & $\begin{array}{l}\text { Grand-mère } \\
\text { maternelle } \\
\text { de l'enfant }\end{array}$ & $\begin{array}{c}\text { Grand-père } \\
\text { maternel de } \\
\text { I'enfant }\end{array}$ & $\begin{array}{c}\text { Grand-mère } \\
\text { paternelle de } \\
\text { I'enfant }\end{array}$ & $\begin{array}{l}\text { Grand-père } \\
\text { paternel de } \\
\text { I'enfant }\end{array}$ \\
\hline <Pays évalué>. & $\square_{1}$ & $\square_{1}$ & $\square_{1}$ & $\square_{1}$ & $\square_{1}$ & $\square_{1}$ \\
\hline$<$ Pays A >. & $\square_{2}$ & $\square_{2}$ & $\square_{2}$ & $\square_{2}$ & $\square_{2}$ & $\square_{2}$ \\
\hline$<$ Pays B>. & $\square_{3}$ & $\square_{3}$ & $\square_{3}$ & $\square_{3}$ & $\square_{3}$ & $\square_{3}$ \\
\hline$<$ Pays C > & $\square_{4}$ & $\square_{4}$ & $\square_{4}$ & $\square_{4}$ & $\square_{4}$ & $\square_{4}$ \\
\hline$<$ Pays D>. & $\square_{5}$ & $\square_{5}$ & $\square_{5}$ & $\square_{5}$ & $\square_{5}$ & $\square_{5}$ \\
\hline$<$ Pays E $>$. & $\square_{6}$ & $\square_{6}$ & $\square_{6}$ & $\square_{6}$ & $\square_{6}$ & $\square_{6}$ \\
\hline$<$ Pays F>. & $\square_{7}$ & $\square_{7}$ & $\square_{7}$ & $\square_{7}$ & $\square_{7}$ & $\square_{7}$ \\
\hline
\end{tabular}

Notes : $<$ Pays évalué $>-$ Fait référence au pays participant à l'évaluation.

$<$ Pays $\mathbf{X}>-$ Les pays ou économies participants peuvent choisir plusieurs pays pour cette question.

Si le père de l'enfant N'EST PAS né en <pays évalué>, quel âge avait-il au moment de son arrivée en <pays évalué>?

(S'il avait moins de 12 mois à ce moment-là, veuillez indiquer "zéro » [0].)

Note : $<$ Pays évalué $>$ - Fait référence au pays participant à l'évaluation.

Si la mère de l'enfant N'EST PAS née en <pays évalué>, quel âge avait-elle au moment de son arrivée en <pays évalué> ?

(Si elle avait moins de 12 mois à ce moment-là, veuillez indiquer "zéro »[0].)

Note : Veuillez vous référer à la Question 19 pour une définition du <pays évalué>.

(Cochez toutes les cases qui conviennent.)

\begin{tabular}{c|l|c|c}
\multicolumn{2}{l|}{} & Mère & Père \\
\hline a) & <Pays évalué $>$. & $\square_{1}$ & $\square_{1}$ \\
\hline b) & <Pays A $>$. & $\square_{2}$ & $\square_{2}$ \\
\hline c) & <Pays B $>$. & $\square_{3}$ & $\square_{3}$ \\
\hline d) & <Pays C $>$. & $\square_{4}$ & $\square_{4}$ \\
\hline
\end{tabular}

Note : Veuillez vous référer à la Question 19 pour une définition du <pays évalué>. 


\section{Q23 Quelle langue le père et la mère de l'enfant parlent-ils la plupart du temps à la maison ?}

(PA25) (Cochez une case par colonne.)

\begin{tabular}{|c|c|c|}
\hline & Mère & Père \\
\hline$<$ Langue de l'évaluation>. & $\square_{1}$ & $\square_{1}$ \\
\hline$<$ Autres langues nationales officielles $>$. & $\square_{2}$ & $\square_{2}$ \\
\hline$<$ Autres langues ou dialectes nationaux $>$. & $\square_{3}$ & $\square_{3}$ \\
\hline$<$ Autre langue $1>$. & $\square_{4}$ & $\square_{4}$ \\
\hline$<$ Autre langue $2>$ & $\square_{5}$ & $\square_{5}$ \\
\hline$<$ Autre langue $3>$. & $\square_{6}$ & $\square_{6}$ \\
\hline Autre langue. & $\square_{7}$ & $\square_{7}$ \\
\hline
\end{tabular}

Note : < Langue de l'évaluation> - Doit être remplacée par le nom de la langue dans laquelle l'évaluation PISA de la compréhension de l'écrit est administrée. Remplacer cette expression par l'intitulé national des cours de "langue d'enseignement ». 


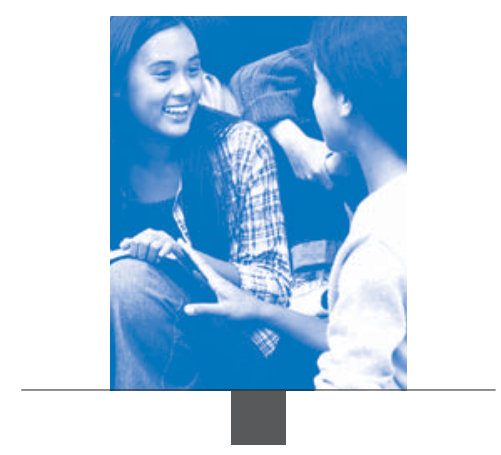

\section{Annexe $B$}

\section{GROUPES D'EXPERTS PISA 2012}

L'Annexe B présente la liste des membres des groupes d'experts qui ont contribué à l'élaboration du cadre d'évaluation du domaine majeur en 2012 (culture mathématique), des nouveaux domaines (résolution de problèmes et culture financière) et des questionnaires. La liste des experts qui ont participé à l'élaboration du cadre d'évaluation de la compréhension de l'écrit du cycle PISA 2009 et du cadre d'évaluation des sciences du cycle PISA 2006 est disponible dans deux publications de I'OCDE : Le Cadre d'évaluation de PISA 2009 - Les compétences clés en compréhension de l'écrit, en mathématiques et en sciences (2009) et Le Cadre d'évaluation de PISA 2006 - Les compétences en sciences, lecture et mathématiques (2006). 
Groupe d'experts en charge

des mathématiques

Kaye Stacey - Présidente

Melbourne Graduate School of Education

(Science and Mathematics Education)

Université de Melbourne

Australie

\section{Caroline Bardini}

Melbourne Graduate School of Education

Université de Melbourne

Australie

\section{Werner Blum}

Département de mathématiques

Université de Kassel

Allemagne

\section{Joan Ferrini-Mundy}

College of Education

Université de l'État du Michigan

États-Unis d'Amérique

\section{Solomon Garfunkel}

COMAP

États-Unis d'Amérique

\section{Toshikazu Ikeda}

Yokohama National University

Japon

\section{Zbigniew Marciniak}

Institut de mathématiques

Université de Varsovie

Pologne

\section{Mogens Niss}

IMFUFA, Département des sciences

Université de Roskilde

Danemark

\section{Martin Ripley}

World Class Arena Limited

Royaume-Uni

\section{William Schmidt}

College of Education

Université de l'État du Michigan

États-Unis d'Amérique

\section{Groupe d'experts en charge} de la résolution de problèmes

\section{Joachim Funke - Président}

Institut de psychologie

Université de Heidelberg

Allemagne

\section{Benö Csapó}

Institute of Education

Université de Szeged

Hongrie

\section{John Dossey}

Département des sciences mathématiques

Université de l'État de l'Illinois

États-Unis d'Amérique

\section{Arthur Graesser}

Psychology, Computer Science, and Institute for Intelligent Systems

Université de Memphis

États-Unis d'Amérique

\section{Detlev Leutner}

Department of Instructional Psychology

Université de Duisburg-Essen

Allemagne

\section{Romain Martin}

Université de Luxembourg FLSHASE

Luxembourg

\section{Richard Mayer}

Département de psychologie

Université de Californie, Santa Barbara

États-Unis d'Amérique

\section{Ming Ming Tan}

Sciences, Curriculum Planning and Development Division Ministère de l'Éducation

Singapour

\section{Groupe d'experts en charge}

\section{de la culture financière}

\section{Annamaria Lusardi - Présidente}

The George Washington University School of Business

États-Unis d'Amérique

Jean-Pierre Boisivon

Université de Paris II Panthéon-Assas

France

\section{Diana Crossan}

Commission for Financial Literacy and Retirement Income Nouvelle-Zélande

\section{Peter Cuzner}

Australian Securities and Investments Commission

Australie

\section{Jeanne Hogarth}

Federal Reserve System

États-Unis d'Amérique

\section{Dušan Hradil}

Ministère des Finances

République tchèque

Stan Jones

Consultant

\section{Canada}

Sue Lewis

Consultante

Royaume-Uni 
Groupe d'experts en charge des questionnaires

Eckhard Klieme - Président

Institut national de recherche pédagogique internationale (DIPF)

Francfort-sur-le Main

Allemagne

\section{Eduardo Backhoff}

University of Baja California at the Institute of Educational

Research and Development

Mexique

\section{Ying-yi Hong}

Nanyang Business School of Nanyang Technological University Singapour

\section{David Kaplan}

Département de psychologie pédagogique

Université du Wisconsin

États-Unis d'Amérique

\section{Henry Levin}

Teachers College, Columbia University

États-Unis d'Amérique

\section{Jaap Scheerens}

Faculté des sciences comportementales

Université de Twente

Pays-Bas

\section{William Schmidt}

College of Education

Université de l'État du Michigan

États-Unis d'Amérique

\section{Fons van de Vijver}

Faculty of Social and Behavioural Studies

Université de Tilburg

Pays-Bas 


\section{ORGANISATION DE COOPÉRATION ET DE DÉVELOPPEMENT ÉCONOMIQUES}

L'OCDE est un forum unique en son genre où les gouvernements oeuvrent ensemble pour relever les défis économiques, sociaux et environnementaux que pose la mondialisation. L'OCDE est aussi à l'avant-garde des efforts entrepris pour comprendre les évolutions du monde actuel et les préoccupations qu'elles font naître. Elle aide les gouvernements à faire face à des situations nouvelles en examinant des thèmes tels que le gouvernement d'entreprise, l'économie de l'information et les défis posés par le vieillissement de la population. L'Organisation offre aux gouvernements un cadre leur permettant de comparer leurs expériences en matière de politiques, de chercher des réponses à des problèmes communs, d'identifier les bonnes pratiques et de travailler à la coordination des politiques nationales et internationales.

Les pays membres de l'OCDE sont : l'Allemagne, l'Australie, l'Autriche, la Belgique, le Canada, le Chili, la Corée, le Danemark, l'Espagne, l'Estonie, les États-Unis, la Finlande, la France, la Grèce, la Hongrie, l'Irlande, l'Islande, Israël, l'Italie, le Japon, le Luxembourg, le Mexique, la Norvège, la Nouvelle-Zélande, les Pays-Bas, la Pologne, le Portugal, la République slovaque, la République tchèque, le Royaume-Uni, la Slovénie, la Suède, la Suisse et la Turquie. L’Union européenne participe aux travaux de l'OCDE.

Les Éditions OCDE assurent une large diffusion aux travaux de l'Organisation. Ces derniers comprennent les résultats de l'activité de collecte de statistiques, les travaux de recherche menés sur des questions économiques, sociales et environnementales, ainsi que les conventions, les principes directeurs et les modèles développés par les pays membres. 


\section{Cadre d'évaluation et d'analyse du cycle PISA 2012 COMPÉTENCES EN MATHÉMATIQUES, EN COMPRÉHENSION DE L'ÉCRIT, EN SCIENCES, EN RÉSOLUTION DE PROBLĖMES ET EN MATIĖRES FINANCIÈRES}

Les élèves sont-ils bien préparés à relever les défis que l'avenir leur réserve ? Sont-ils capables d'analyser, de raisonner et de communiquer leurs idées de manière efficace ? Ont-ils cerné les centres d'intérêt qu'ils cultiveront toute leur vie en tant que membres productifs de la société et de l'économie ? Le Programme international de l'OCDE pour le suivi des acquis des élèves (PISA) cherche à répondre à ces questions au travers d'une évaluation des savoirs et savoir-faire des élèves, la plus exhaustive et la plus rigoureuse qui existe à l'échelle internationale. Le Cadre d'évaluation et d'analyse du cycle PISA 2012 présente le cadre conceptuel qui sous-tend le cinquième cycle de l'enquête PISA. Comme les cycles précédents, le cycle PISA 2012 couvre les mathématiques, son domaine majeur d'évaluation, la compréhension de l'écrit et les sciences, ainsi que deux autres domaines : la résolution de problèmes et la culture financière. Par ailleurs, un questionnaire contextuel et, à titre d'option, un questionnaire sur le parcours scolaire et un questionnaire sur la maîtrise des technologies de l'information et de la communication (TIC) sont administrés aux élèves. Des informations supplémentaires sont recueillies auprès de la direction des établissements d'enseignement via le questionnaire "Établissement » et auprès des parents, via le questionnaire « Parents ", le troisième qui soit proposé à titre d'option. Soixante-six pays et économies,

dont les trente-quatre pays membres de l'OCDE, ont pris part au cycle PISA 2012.

\section{Sommaire}

Chapitre 1. Cadre d'évaluation de la culture mathématique du cycle PISA 2012

Chapitre 2. Cadre d'évaluation de la compréhension de l'écrit du cycle PISA 2012

Chapitre 3. Cadre d'évaluation de la culture scientifique du cycle PISA 2012

Chapitre 4. Cadre d'évaluation de la résolution de problèmes du cycle PISA 2012

Chapitre 5. Cadre d'évaluation de la culture financière du cycle PISA 2012

Chapitre 6. Cadre conceptuel des questionnaires contextuels du cycle PISA 2012

\section{LE PROGRAMME INTERNATIONAL POUR LE SUIVI DES ACQUIS DES ÉLÈVES (PISA) DE L'OCDE}

L'enquête PISA cherche à évaluer la capacité des jeunes à utiliser leurs connaissances et compétences pour relever les défis du monde réel. Cette approche reflète l'évolution des objectifs des programmes de cours : la priorité va désormais à ce que les élèves savent faire avec ce qu'ils ont appris à l'école plutôt qu'à la mesure dans laquelle ils ont assimilé des matières spécifiques. L'enquête PISA est unique en son genre, comme le montrent ses grands principes :

- Son bien-fondé pour l'action publique : les données sur les acquis des élèves sont rapportées à des données sur leurs caractéristiques personnelles et sur des facteurs clés qui façonnent leur apprentissage à l'école et ailleurs pour repérer des différences dans les profils de compétence et identifier les caractéristiques des élèves, des établissements et des systèmes d'éducation qui se distinguent par des niveaux élevés de performance.

- Son approche novatrice basée sur la notion de "littératie ", qui renvoie à la capacité des élèves d'exploiter des savoirs et savoir-faire dans des matières clés, et d'analyser, de raisonner et de communiquer lorsqu'ils énoncent, résolvent et interprètent des problèmes qui s'inscrivent dans divers contextes.

- Sa pertinence par rapport à l'apprentissage tout au long de la vie : l'enquête PISA ne se limite pas à évaluer les compétences des élèves dans des matières scolaires, mais demande également à ceux-ci de décrire leur envie d'apprendre, leur perception d'eux-mêmes et leurs stratégies d'apprentissage.

- Sa périodicité, qui permet aux pays de suivre leurs progrès sur la voie de l'accomplissement d'objectifs clés de l'apprentissage.

- Sa grande couverture géographique et son principe de collaboration : les 34 pays membres de l'OCDE ainsi que 32 pays et économies partenaires ont participé au cycle PISA 2012.

Consulter cette publication en ligne : http://dx.doi.org/10.1787/9789264190559-fr

Cet ouvrage est publié sur OECD iLibrary, la bibliothèque en ligne de l'OCDE, qui regroupe tous les livres, périodiques et bases de données statistiques de l'Organisation. Rendez-vous sur le site www.oecd-ilibrary.org pour plus d'informations.

\section{3}

éditionsOCDE

www.oecd.org/editions
ISBN 978-92-64-19054-2

$982013012 P$ 